Florida International University

FIU Digital Commons

FIU Electronic Theses and Dissertations

University Graduate School

$10-26-2017$

\title{
Resilient and Real-time Control for the Optimum Management of Hybrid Energy Storage Systems with Distributed Dynamic Demands
}

Christopher R. Lashway

Florida International University, clash006@fiu.edu

DOI: $10.25148 /$ etd.FIDC004048

Follow this and additional works at: https:// digitalcommons.fiu.edu/etd

Part of the Electrical and Electronics Commons, Electromagnetics and Photonics Commons, Electronic Devices and Semiconductor Manufacturing Commons, Energy Systems Commons, Engineering Physics Commons, Power and Energy Commons, and the Transport Phenomena Commons

\section{Recommended Citation}

Lashway, Christopher R., "Resilient and Real-time Control for the Optimum Management of Hybrid Energy Storage Systems with Distributed Dynamic Demands" (2017). FIU Electronic Theses and Dissertations. 3515.

https://digitalcommons.fiu.edu/etd/3515

This work is brought to you for free and open access by the University Graduate School at FIU Digital Commons. It has been accepted for inclusion in FIU Electronic Theses and Dissertations by an authorized administrator of FIU Digital Commons. For more information, please contact dcc@fiu.edu. 


\section{FLORIDA INTERNATIONAL UNIVERSITY}

Miami, Florida

\section{RESILIENT AND REAL-TIME CONTROL FOR THE OPTIMUM MANAGEMENT OF HYBRID ENERGY STORAGE SYSTEMS WITH DISTRIBUTED DYNAMIC DEMANDS}

A dissertation submitted in partial fulfillment of the requirements for the degree of DOCTOR OF PHILOSOPHY in ELECTRICAL ENGINEERING by Christopher R. Lashway 
To: Dean John Volakis

College of Engineering and Computing

This dissertation, written by Christopher R. Lashway, and entitled Resilient and Real-time Control for the Optimum Management of Hybrid Energy Storage Systems with Distributed Dynamic Demands, having been approved in respect to style and intellectual contents, is referred to you for judgment.

We have read this dissertation and recommend its approval.

Sakhrat Khizroev

Bilal El-Zahab

Jean Andrian

Mark Roberts

Osama A. Mohammed, Major Professor

Date of Defense: October 26, 2017

The dissertation of Christopher R. Lashway is approved.

Dean John Volakis

College of Engineering and Computing

Andrés G. Gil

Vice President for Research and Economic Development and Dean of the University Graduate School

Florida International University, 2017 
Copyright (C) 2017 by Christopher R. Lashway

All rights reserved. 


\section{DEDICATION}

This dissertation is entirely dedicated to the Lashway family and my girlfriend, Ms. Rachel

Van Buren. Without their unending motivation, support, and love, achieving this lifelong goal would not have been possible. 


\section{ACKNOWLEDGMENTS}

This dissertation and research would have not been made possible without the support and dedication of my major advisor, Prof. Osama Mohammed. I wish to express my gratitude and appreciation for his guidance, encouragement, expert technical adivise and financial support throughout the course of my $\mathrm{PhD}$. Having an opportunity to work at the Energy Systems Research Laboratory and access to wide variety of hardware and software resources was crucial to my success and ultimately led to a successful experience with more output than I could have ever imagined. As my doctoral work progressed and my research gained traction, Prof. Mohammed increasingly provided me opportunities to grow within my professional career inside and outside the university. These not only include taking the lead as the IEEE Power and Energy Society Student Branch Chapter of Miami, but also hosting a variety of seminars, tutorials, and serving as a frequent guest lecturer in his Numerical Analysis course and to students in undergraduate courses.

Secondly, I would like to express my appreciation for my dissertation committee members, Dr. Sakhrat Khizroev, Dr. Mark Roberts, Dr. Bilal El-Zahab and Dr. Jean Andrean. Each have provided their own unique support within either the research or moral support realm. In addition, each has provided insightful comments and suggestions during my initial research proposal. These suggestions were taken very seriously while carrying out the heart of my research over the past three years, research that would not have been possible without the aid of various funding resources. I would like to thank the Office of Naval Research (ONR), which has funded two major projects under my major advisor that I have worked on and supported since 2013. ONR funding has been crucial to meeting my goals as a researcher, push the boundaries of technology, and ultimately providing me 
exposure to new fields. In my final semesters, I also thank support by the Dissertation Year Fellowship (DYF) from the University Graduate School during my last year or reasearch. Although the journey of this $\mathrm{PhD}$ has been met with a wide range of challenges, it was ultimately perserverence that transpired in a way to reach a stunning lifelong accomplishment. This would have not been made possible without a number of others who believed in me since the beginning. First, I want to thank my colleageus at the Energy Systems Research Laboratory for their technical support, which spawned unique and novel research collaborations during my graduate studies. Next, I thank my colleagues from the Naval Surface Warfare Center Dahlgren Division (NSWCDD) for maintaining my position and supporting my personal aspirations to obtain a $\mathrm{PhD}$, particularly Mr. Luis Andrade and Mr. Vince Vendetti, as they have served as both colleageues, mentors, and friends. Finally, I express my sincere graditude to the endless, caring support of the Lashway family and girlfriend, Ms. Rachel Van Buren, who stood by me throughout this journey. 


\section{ABSTRACT OF THE DISSERTATION \\ RESILIENT AND REAL-TIME CONTROL FOR THE OPTIMUM MANAGEMENT \\ OF HYBRID ENERGY STORAGE SYSTEMS WITH DISTRIBUTED DYNAMIC \\ DEMANDS \\ by}

Christopher R. Lashway

Florida International University, 2017

Miami, Florida

Professor Osama A. Mohammed, Major Professor

A continuous increase in demands from smart grid and traction applications have steered public attention toward integrating energy storage (ES) and hybrid energy storage system (HESS) solutions. Modern technologies are no longer limited to a single battery chemistry and size, but can include supercapacitors (SC) and flywheel energy storage systems (FESS) as well. However, insufficient control devices and algorithms to monitor ES and HESS can result in a wide range of operational issues. A modern day ES control platform must have a deep understanding of the source. Furthermore, an optimization of ES types and effective modeling of these devices is crucial. In this dissertation, a specialized modular ES management controller coined as the Energy Storage Management Controller (ESMC) is developed to interface with a variety of ES devices. The EMSC provides the capability to monitor and control a wide range of different ES, while including maintenance and safety features. Uniqueness in the ESMC is a mechanism to completely isolate an ES device, even if it is connected in series, to conduct maintenance or charging while allowing the remaining ES network to continue to operate. A focus is placed upon 
the philosophy and development of the ESMC, where laboratory prototypes lead to an upgraded commercialized design for large-scale systems.

The EMSC is deployed in a wide range of ES and HESS for a number of applications. First, it is tested on a series-connected lead acid battery array, verifying its capabilities and showcasing tools that can be used to improve a battery SoH. SoH is first studied with an introduction to a direct method called electrochemical impedance spectroscopy (EIS), highlighting how its circuit model will provide a basis for the battery models in this work and how a simplified version of EIS could be included in an extension of the ESMC. Next, SoH extraction through the use of pulsed loading is proposed as a simplier and less expensive method to not only obtain battery equivalent circuit models, but also autonomously determine the chemistry. To accomplish this, pseudo 2D (P2D) physics based models (PBM) of lead acid and lithium ion batteries are derived and utilized to improve current battery management software and study SoH impacts. Insight from these models was then applied in the experimental acquisition and development of a comprehensive simulation model.

The concept of HESS is then introduced, where their interfacing power electronics lead to a deep study using PBMs of their switching devices and how they can be used to improve system efficiency. Three unique HESS are tested and evaluated utilizing the ESMC. First, a lead acid battery, lithium ion battery, and SC series-connected HESS is designed and tested for shipboard power system applications. Next, a lithium ion battery and SC parallelconfigured HESS is utilized for an electric vehicle application. Finally, a lead acid battery and FESS parallel-configued HESS is analyzed for how the inclusion of a battery with a FESS can provide a dramatic improvement in the power quality versus a FESS alone. 


\section{TABLE OF CONTENTS}

CHAPTER

PAGE

Chapter 1 Introduction and Research Background ..................................................... 1

1.1 Problem Statement ................................................................................. 1

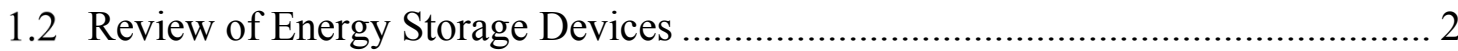

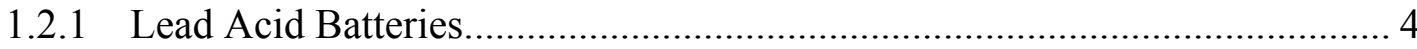

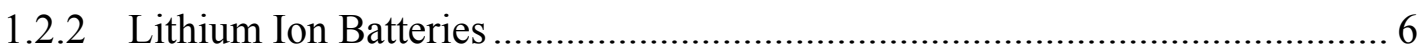

1.2.3 Supercapacitor Energy Storage ............................................................... 7

1.2.4 Flywheel Energy Storage Systems........................................................... 9

1.3 Introduction to Hybrid Energy Storage Systems ……......................................... 10

1.4 Research Objective .................................................................................... 12

1.5 Original Contributions of the Dissertation.......................................................... 13

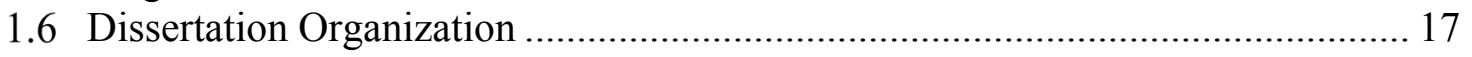

Chapter 2 Developing an Advanced Modular Energy Management System................ 20

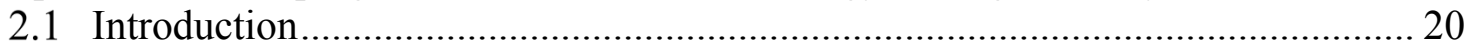

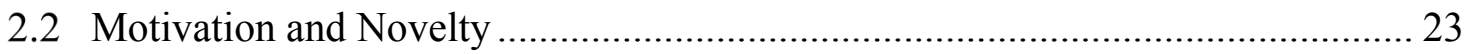

2.3 Importance of State of Charge Equalization ......................................................... 25

2.4 Energy Storage Management Controller Design ................................................. 26

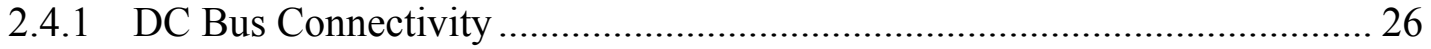

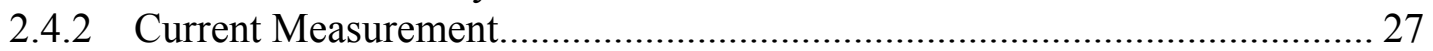

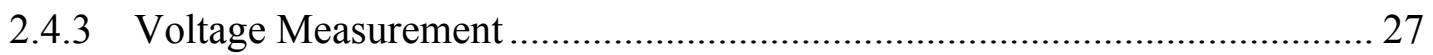

2.4.4 Charging and Diagnostic Bus Connectivity................................................ 28

2.5 Operation Modes ...................................................................................... 29

2.5.1 Normal Operation (Discharging) ………………..................................... 29

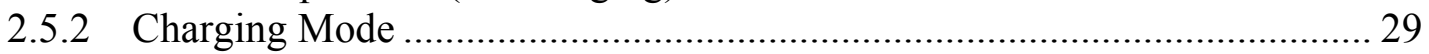

2.5.2.1 Constant Charging ........................................................................... 30

2.5.2.2 Pulsed Charging .................................................................................... 30

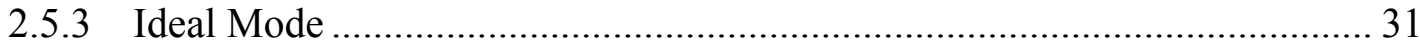

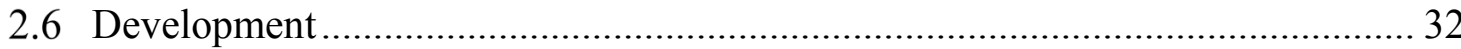

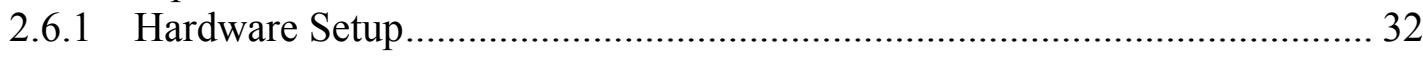

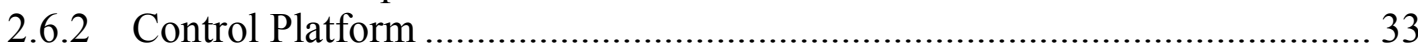

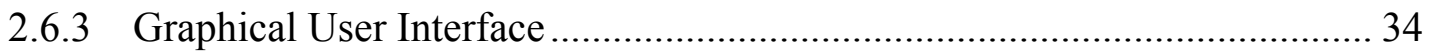

2.6.3.1 Main Load and System Control ......................................................... 35

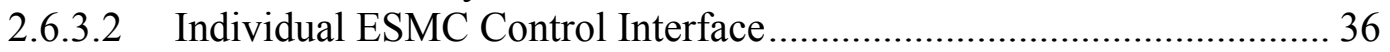

2.7 System Expandability for Commercialization ......................................................... 37

2.7.1 Voltage and Current Sensor Upgrades.......................................................... 37

2.7.2 Switching Component Upgrades .............................................................. 38

2.7.3 Thermocouple Addition ................................................................................ 39

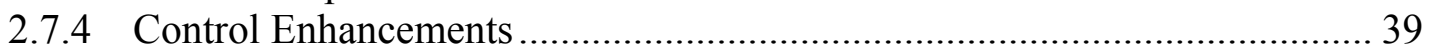

2.7.5 Graphical User Interface (GUI) Development.............................................. 41

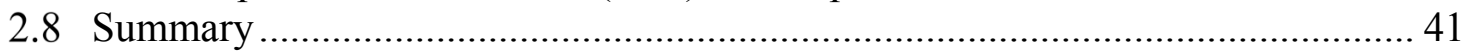


Chapter 3 Energy Storage Management Controller Testing on Lead Acid Batteries ... 42

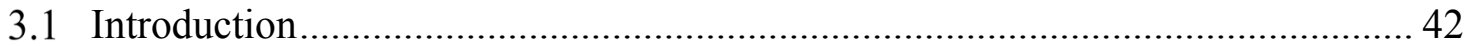

3.2 Basic Equivalent Circuit Model for the Lead Acid Battery................................. 43

3.3 Lead Acid Battery Bank under Test................................................................. 46

3.4 Energy Storage Management Controller Implementation .................................. 48

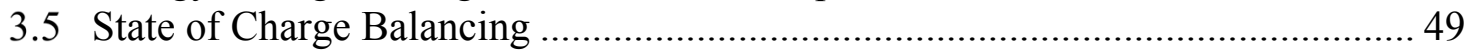

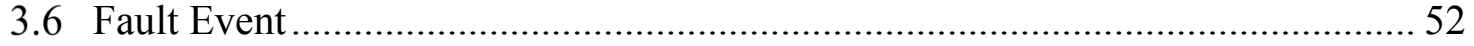

3.7 Discharging under Heavy Pulsed Load.......................................................... 54

3.8 Endurance and Robustness Test...................................................................... 58

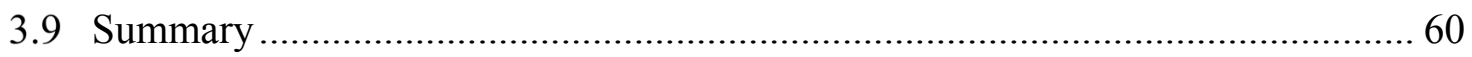

Chapter 4 Battery State of Health using Impedance Spectroscopy ........................... 61

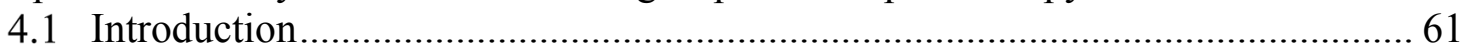

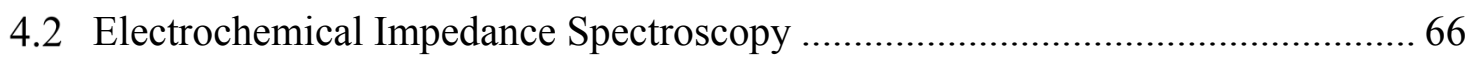

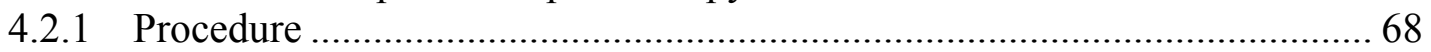

4.2.2 Selected Electrochemical Impedance Spectroscopy Technique ...................69

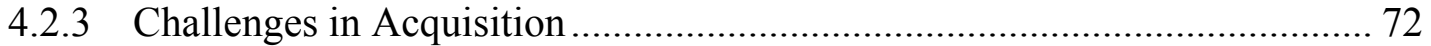

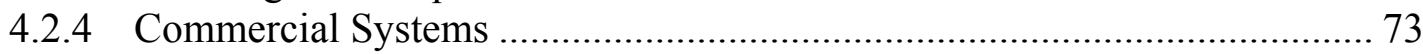

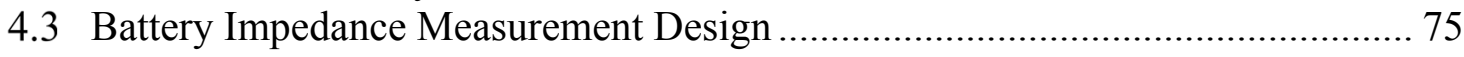

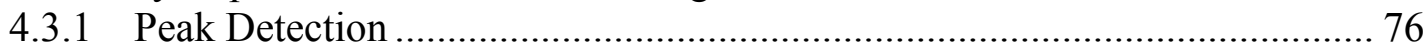

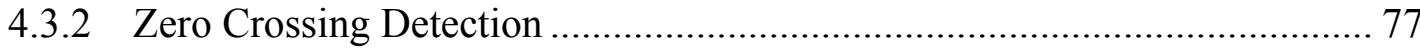

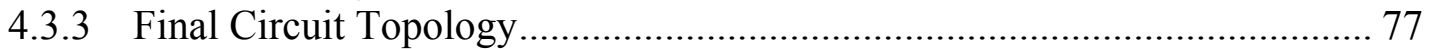

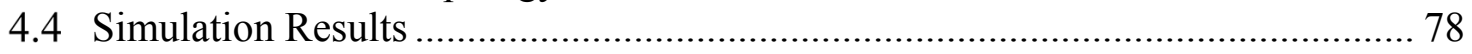

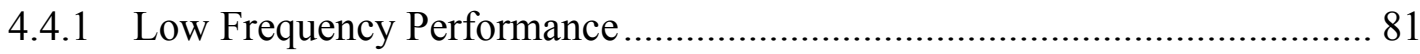

4.4.2 Mid Frequency Performance................................................................... 82

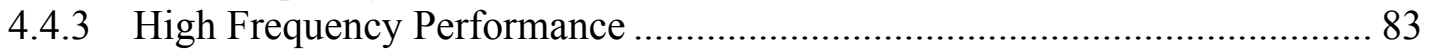

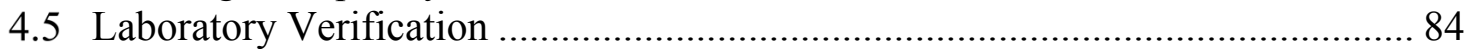

4.6 Proposed Printed Circuit Board Design and Integration.................................... 85

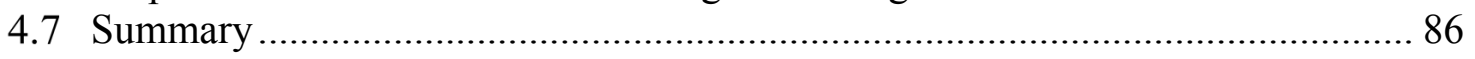

Chapter 5 Physics Based Modeling of Lead Acid and Lithium Ion Batteries.............. 88

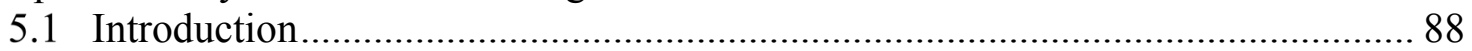

5.2 Physics Based Battery Modeling ................................................................... 91

5.2.1 Lead Acid Cell ............................................................................... 92

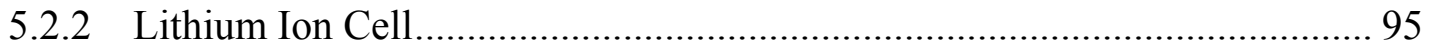

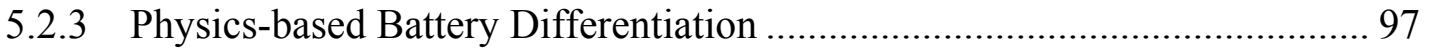

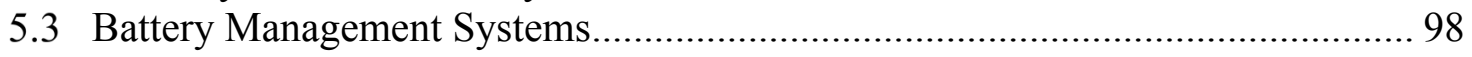

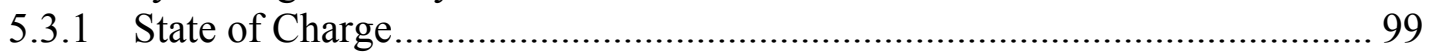

5.3.1.1 Initial Voltage-Based Measurements ............................................... 99

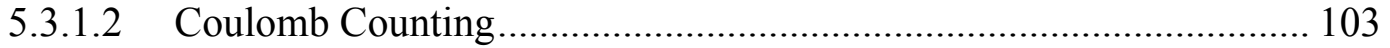

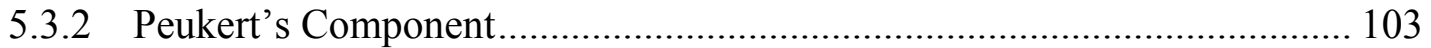

5.3.3 Quantitative Definition of the State of Health ......................................... 104

5.3.4 Generating the $1^{\text {st }}$-Order Randles Equivalent Circuit Model...................... 104

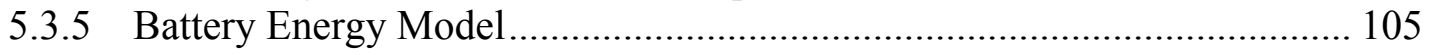

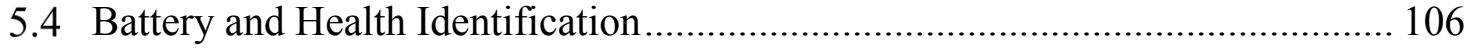




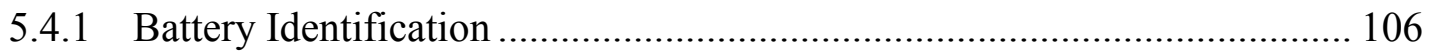

5.4.2 Calculating $1^{\text {st }}$ Order Randles Equivalent Circuit Parameters ..................... 109

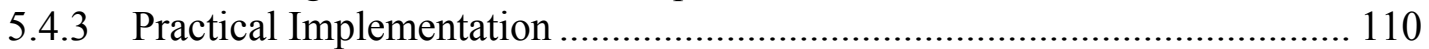

5.4.3.1 Accuracy Variance versus Sampling Frequency ................................ 111

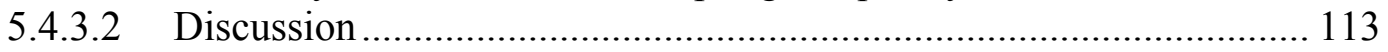

5.5 Battery Management System Implementation............................................. 114

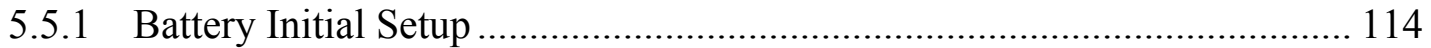

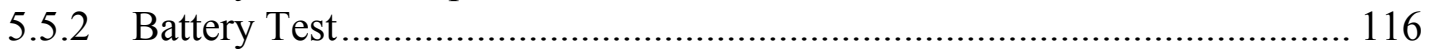

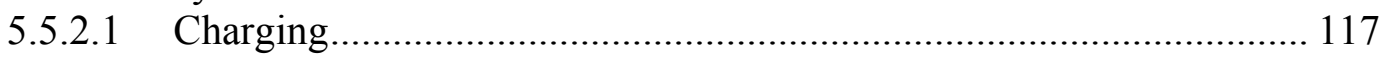

5.5.2.2 Perform Pulse Test............................................................................ 118

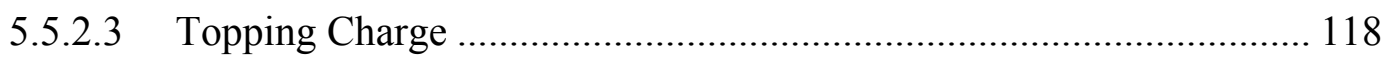

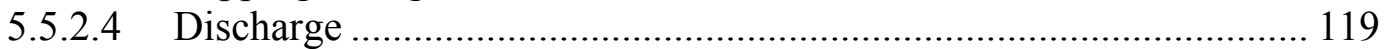

5.5.2.5 Calculate Circuit Parameters and Usable Capacity ............................ 119

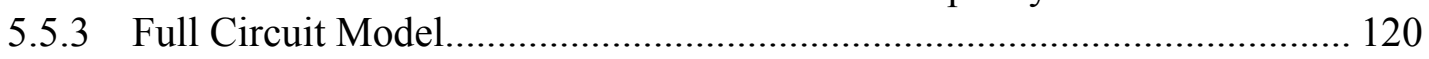

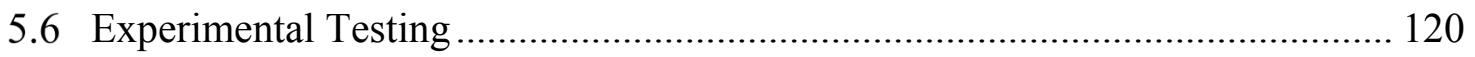

5.6.1 Lithium Ion Battery Testing................................................................ 120

5.6.1.1 3.7 V Lithium Ion Battery in Good Condition.................................... 120

5.6.1.2 3.7 V Lithium Ion Battery in Bad Condition ..................................... 121

5.6.2 Lead Acid Battery Testing ................................................................... 122

5.6.2.1 12 V Lead Acid Battery in Good Condition ....................................... 122

5.6.2.2 12 V Lead Acid Battery in Bad Condition......................................... 123

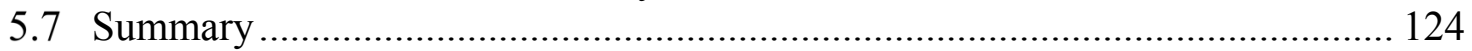

Chapter 6 Extending Lithium Ion Finite Element Models into 3D ............................. 126

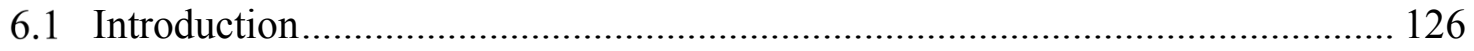

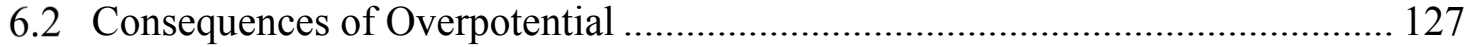

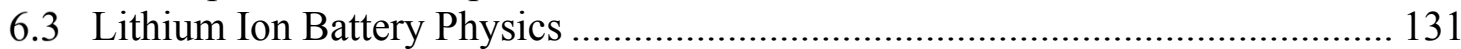

6.3.1 3D Physics Based Model Formulation ........................................................ 133

6.3.2 Finite Element Modeling ...................................................................... 137

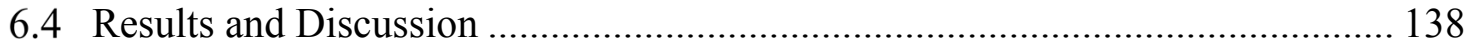

6.4.1 Terminal Voltage and Analysis of Overpotential ...................................... 139

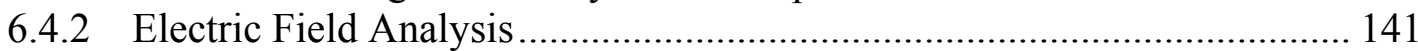

6.4.3 Magnetic Field Analysis and Gradient Currents........................................ 141

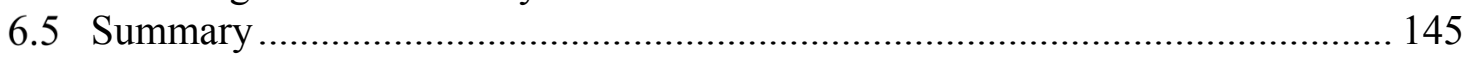

Chapter 7 Developing a Comprehensive Battery Simulation Model ........................... 147

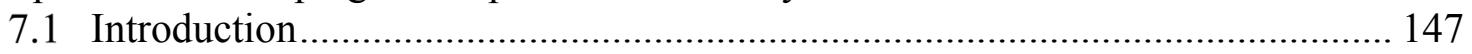

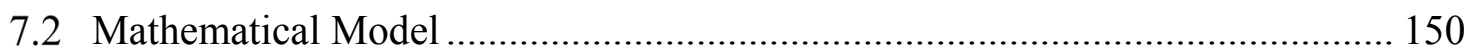

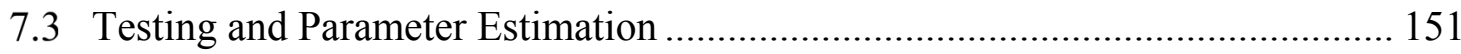

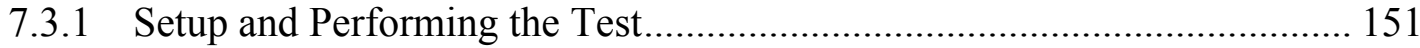

7.3.2 Extracting Circuit Parameters and Open Circuit Voltage............................. 153

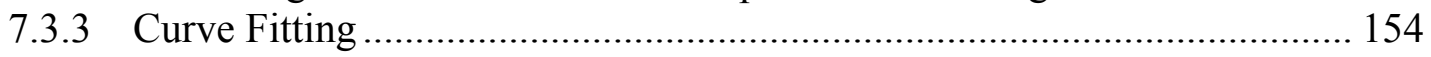

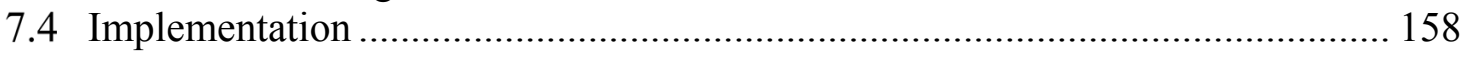

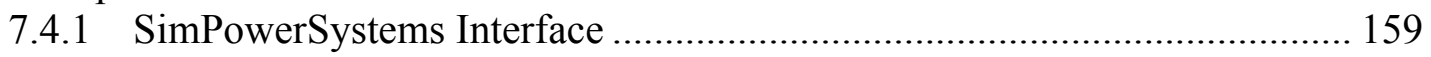

7.4.2 Simscape SimElectronics...................................................................... 160 


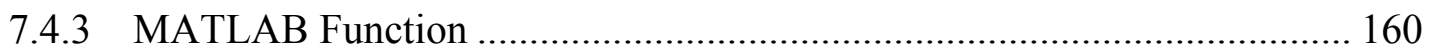

7.4.4 Final MATLAB/Simulink Implementation ............................................... 162

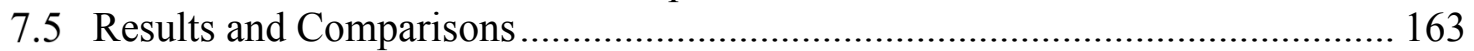

7.6 Modeling an Electric Vehicle Battery and Wireless Power Transfer System .... 166

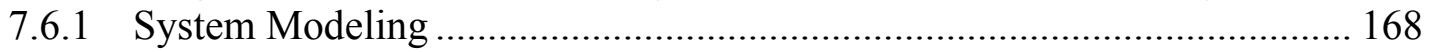

7.6.1.1 Configuration of the Electric Vehicle Battery Bank........................... 168

7.6.1.2 Electric Vehicle Powertrain Model..................................................... 170

7.6.1.3 Wireless Power Transfer System Model............................................ 171

7.6.2 Testing the Quasi-Dynamic Wireless Charging System............................. 172

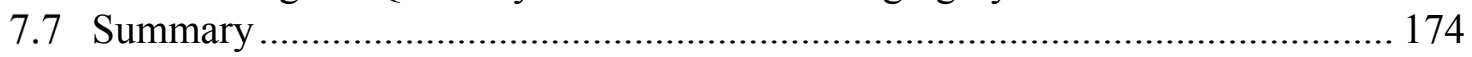

Chapter 8 Interfacing Converters to Support Hybrid Energy Storage Systems .......... 177

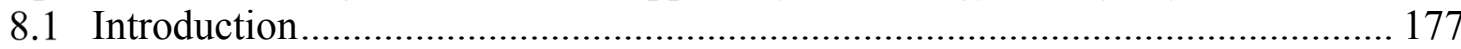

8.2 Basic Power Electronic Devices to Interface Energy Storage ........................... 180

8.2.1 Basic DC-DC Buck Converter............................................................ 180

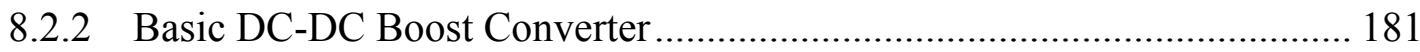

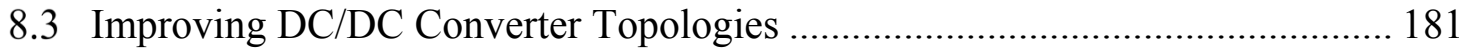

8.4 Simulating the Synchronous Buck Converter using Si versus GaN .................... 183

8.4.1 Initial Simulation in Simulink SimPowerSystems Blockset........................ 185

8.4.2 Improved Dynamics under Simulink SimElectronics Blockset................... 187

8.5 Strengths and Limitations of Switching Models............................................... 193

8.5.1 Evaluating Limitations of the GaNSys GS66508T SimElectronics Model 195

8.5.2 Utilizing Manufacture SPICE Models for Improved I-V Performance...... 196

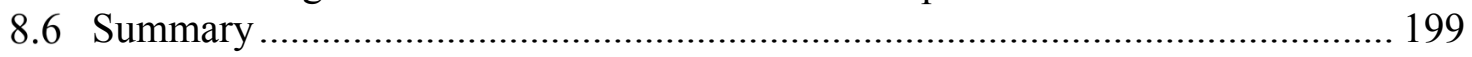

Chapter 9 Physics-based Modeling of the GaN High Electron Mobility Transistor... 201

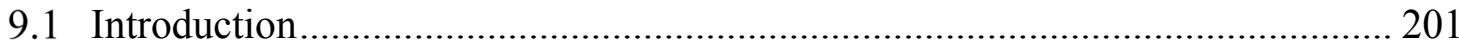

9.2 The eGaN HEMT Physics Based Model ........................................................... 202

9.3 Evaluating Ways to Increase the Breakdown Using the Physics Based Model . 205

9.4 Result Analysis of the GaN HEMT ............................................................ 208

9.5 Further Investigation of the Field Plate Case..................................................... 211

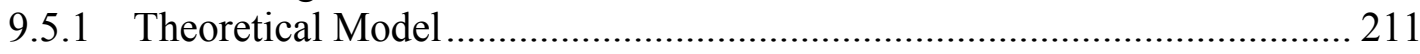

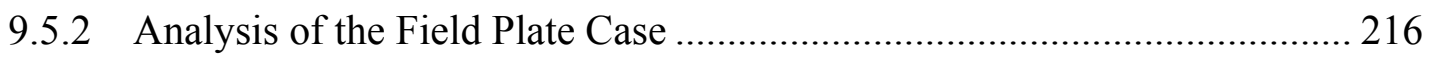

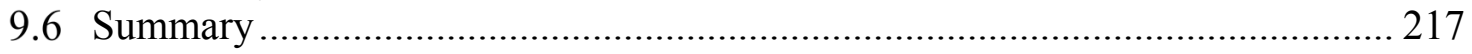

Chapter 10 Hybrid Energy Storage Systems for Shipboard Power Systems and

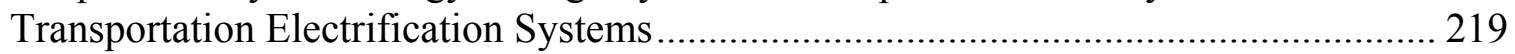

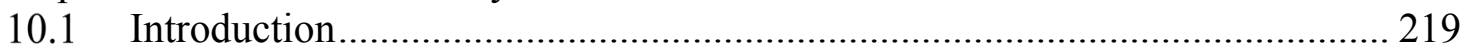

10.2 Modeling of Multiple Energy Storage Types ................................................. 223

10.2.1 Lead Acid Battery Shipboard Power System Model .............................. 223

10.2.2 Lithium Ion Battery Shipboard Power System Model............................. 224

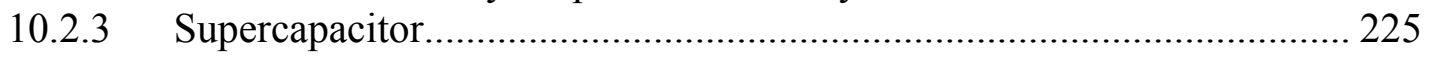

10.3 Equivalent Models for Hybrid Energy Storage Systems …………………..... 227

10.3.1 Case I: 4 Lead Acid Batteries .............................................................. 228

10.3.2 Case II: 2 Lithium Ion and 2 Lead Acid Batteries .................................. 229 
10.3.3 Case III: 2 Lithium Ion and a Lead Acid Battery with Supercapacitor .. 230

10.3.4 Analytical Results Discussion.......................................................... 232

10.4 Coordinated Control of Hybrid Energy Storage Systems .............................. 235

10.4.1 Rolling Charging Operation................................................................ 235

10.4.2 Charging Constraints .......................................................................... 236

10.4.3 Control System and Graphical User Interface Upgrades ...................... 237

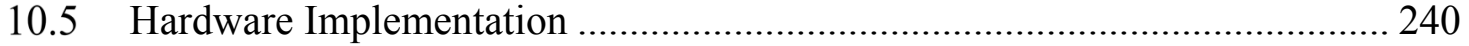

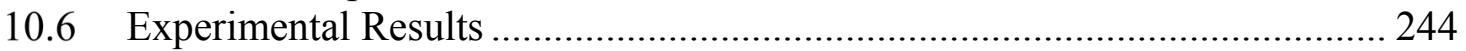

10.6.1 Test I: 2 Lithium Ion and 2 Lead Acid Batteries .................................. 244

10.6.2 Test II: Endurance Test.................................................................... 246

10.6.3 Test III: 2 Lithium Ion Batteries, Lead Acid Battery, and a

Supercapacitor without Rolling Charging ..................................................... 250

10.6.4 Test IV: 2 Lithium Ion Batteries, Lead Acid Battery, and a

Supercapacitor with Supercapacitor in Rolling Charging ................................. 252

10.6.5 Test V: 2 Lithium Ion Batteries, a Lead Acid Battery, and a

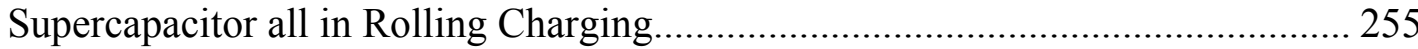

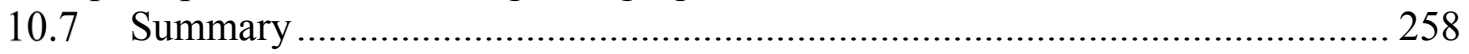

Chapter 11 Lithium Ion Battery - Supercapacitor Hybrid Energy Storage Systems .... 260

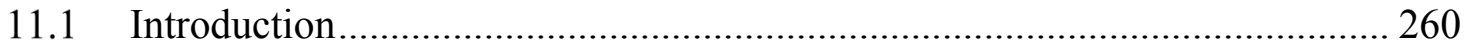

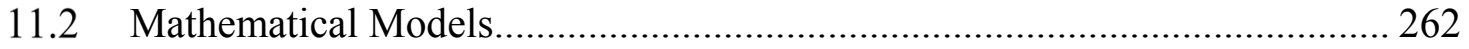

11.2.1 Lithium Ion Battery Cycle Life Analysis............................................ 263

11.2.2 Lithium Ion Battery Electric Vehicle Model ....................................... 268

11.2.3 Supercapacitor Electric Vehicle Model ............................................... 269

11.2.4 Hybrid Lithium Ion - Supercapacitor System ................................. 271

11.3 Hybrid Pulse Power Characterization Test .............................................. 272

11.4 Proposed Hybrid Energy Storage Management Scheme ............................... 273

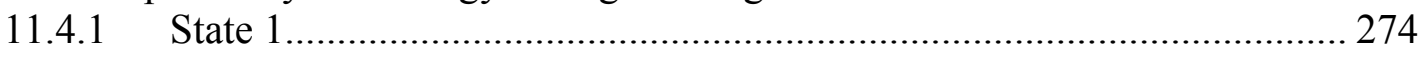

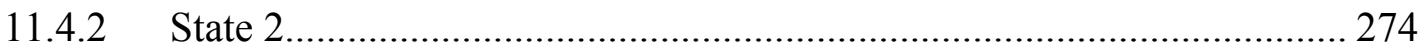

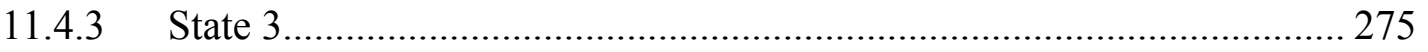

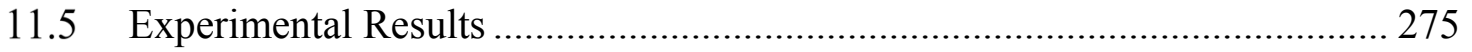

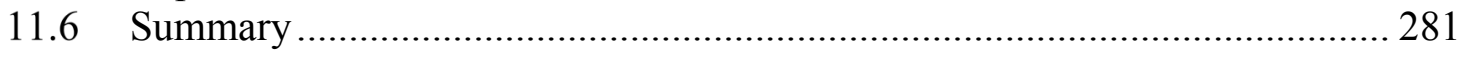

Chapter 12 Flywheel - Lead Acid Battery Hybrid Energy Storage Systems ................ 282

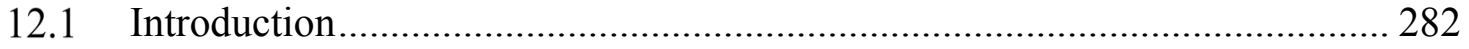

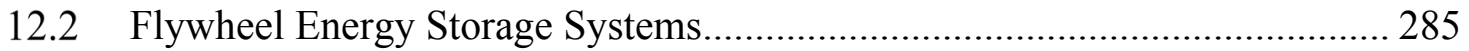

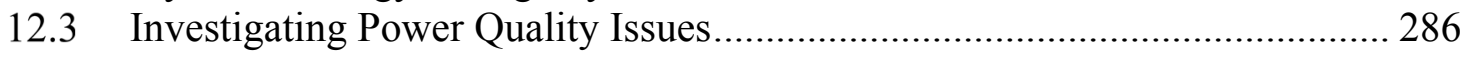

12.3.1 Machine Speed Multiple .................................................................. 286

12.3.2 Mathematical Modeling of the Hybrid Energy Storage System ............. 288

12.3.3 Power Quality Issues with Flywheel DC-DC Converter Interfacing...... 288

12.3.4 Battery Model and Characteristics for Power Quality Improvement .... 289

12.4 Experimental Test Setup ...................................................................... 290

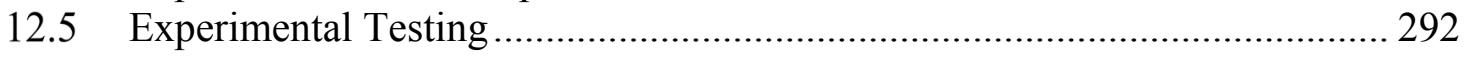

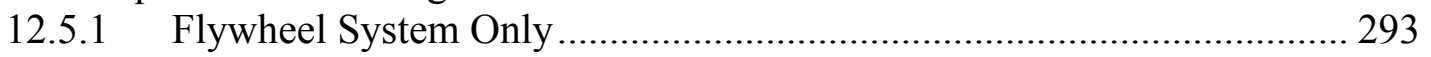

12.5.2 5\% Battery / 95\% Flywheel Current Contribution ............................... 295 
12.5.3 25\% Battery / 75\% Flywheel Current Contribution .............................. 297

12.5.4 50\% Battery / 50\% Flywheel Current Contribution ............................... 299

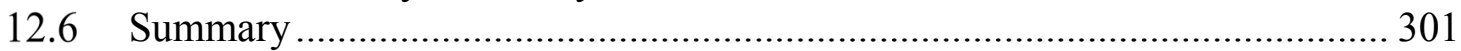

Chapter 13 Conclusions and Future Work ............................................................ 302

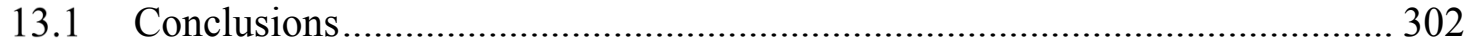

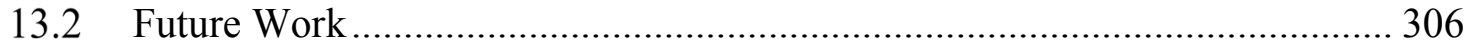

LIST OF REFERENCES ............................................................................. 311

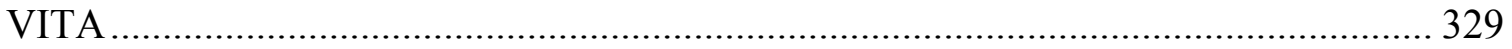




\section{LIST OF TABLES}

TABLE

PAGE

Table 1-1. Comparison of Four Prominent Energy Storage Resources............................ 3

Table 3-1. UPG 121100110 Ah 12V Lead Acid Battery Specifications........................ 47

Table 5-1. Tested Batteries to Acquire Timing Constants......................................... 108

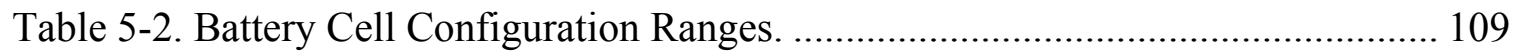

Table 5-3. Generated Time Constant per Sampling Frequency................................. 112

Table 5-4. Calculated Polarization Resistance per Sampling Frequency...................... 113

Table 5-5. Calculated Polarization Capacitance per Sampling Frequency.................... 113

Table 6-1. FEM Simulation Parameters for the Lithium Ion Cobalt Polymer Battery... 135

Table 6-2. 8048168C Lithium Ion Cobalt Polymer Battery Specifications................... 137

Table 6-3. Experimental, Pseudo 2DFEA, and 3DFEA Model Comparison. ................ 139

Table 7-1. PL8048168 51.8V Lithium Ion Cobalt Polymer Battery Coefficients.......... 156

Table 8-1. Synchronous Buck Converter Design Parameters..................................... 183

Table 8-2. Silicon and Gallium Nitride-Based Synchronous Buck Converter

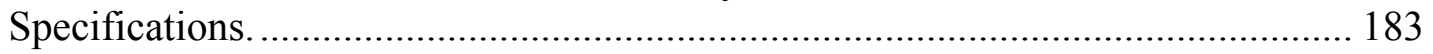

Table 9-1. eGaN HEMT Design Parameters. ....................................................... 206

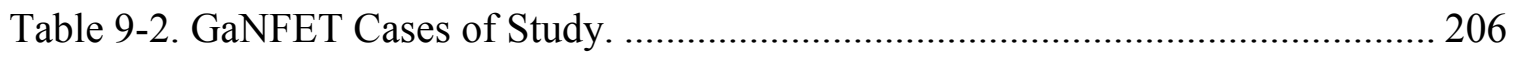

Table 9-3. eGaN HEMT PBM Breakdown Voltage and Maximum Electric Field for

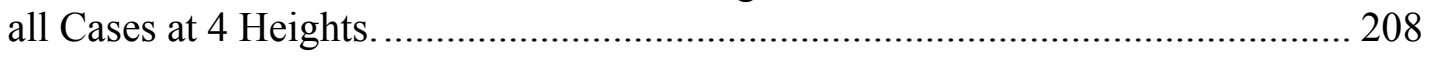

Table 9-4. Polarization Parameters for the Electrostatic Potential on AlGaN Devices.. 213

Table 9-5. GaN HEMT with Field Plate Design Parameters for Theoretical Model. .... 213

Table 10-1. Batteries and Supercapacitor Simulation Parameters.............................. 227

Table 10-2. Shipboard Power System Load Profiles.............................................. 242 
Table 11-1. Cycle Life until 30\% Capacity Loss $(70 \% \mathrm{SoH})$ as a Function of Depth

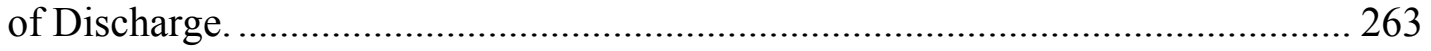

Table 11-2. Switching States for Energy Storage Management Controllers in Each Operating Mode ............................................................................................. 274

Table 12-1. Hampden DYN-300X Motor Specifications............................................... 286

Table 12-2. Power Quality Analysis of the Voltage at the Load................................... 296 


\section{LIST OF FIGURES}

FIGURE

PAGE

Figure 1.1. Normalized Energy Storage Device Performance Snapshot. ........................ 3

Figure 1.2. Lead Acid Batteries: Flooded 12V 6-cell Module, General Operational Schematic 4

Figure 1.3. Lead Acid versus Lithium Ion Battery Discharge Curve Comparison............ 5

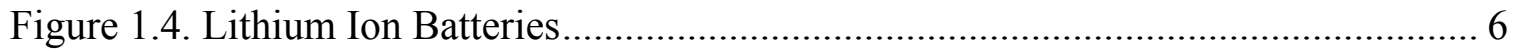

Figure 1.5. Supercapacitor versus Flywheel Energy Storage Discharge Curve

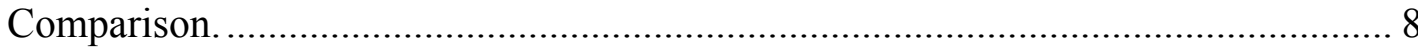

Figure 1.6. Supercapacitor Energy Storage ......................................................... 8

Figure 1.7. Flywheel Energy Storage ….......................................................... 9

Figure 1.8. Theoretical Hybrid Energy Storage Systems Performance Snapshot. ........... 11

Figure 2.1. Proposed Energy Storage Management Controller Unit Schematic. ............. 26

Figure 2.2. LEM LA 25-NP Current Transducer..................................................... 27

Figure 2.3. LEM LV 25-P Voltage Transducer....................................................... 28

Figure 2.4. Energy Storage Management Controller in Normal Operation..................... 29

Figure 2.5. Energy Storage Management Controller in Charging Operation................... 30

Figure 2.6. Energy Storage Management Controller in Ideal Mode.............................. 31

Figure 2.7. Energy Storage Management Controller Unit Prototype. ............................. 32

Figure 2.8. Hardware Setup with 4 ESMC Units.................................................... 33

Figure 2.9. ESMC Hardware/Software Control Platform............................................. 34

Figure 2.10. ESMC Main Load and System Control GUI......................................... 35

Figure 2.11. Individual ESMC Control Interface GUI. ............................................ 36

Figure 2.12. Reduced Cost Voltage and Current Measurement Circuit from ESMC....... 38 
Figure 2.13. Hierarchy of the Proposed Upgraded ESMC System............................... 40

Figure 3.1. Deconstructed Single-cell Lead Acid Battery with Heavy Sulfation............. 43

Figure 3.2. Basic Equivalent Circuit Model for a Lead Acid Cell Based on Electrochemical Impedance Spectroscopy. .................................................... 44

Figure 3.3. Pulsed Load Response on Healthy \& Damaged Battery............................. 45

Figure 3.4. 10 - 12V 110 Ah Lead Acid Battery Bank.............................................. 47

Figure 3.5. ESMC Implemented in a $4-12 \mathrm{~V}$ Lead Acid Battery Bank. ....................... 49

Figure 3.6. SoC Balancing Scenario ................................................................. 51

Figure 3.7. Protection against Fault Events in the Charging Circuit ............................. 53

Figure 3.8. Discharging under a Heavy Pulsed Load ............................................. 55

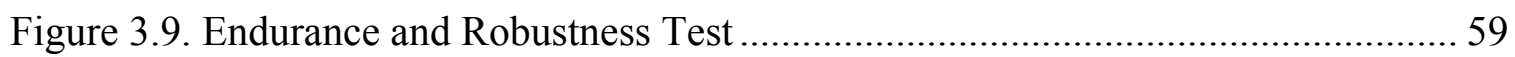

Figure 4.1. Lead Acid Battery Charge/Discharge State of Health Curve Comparison. ... 63

Figure 4.2. Example of Changes in the Randles Equivalent Circuit as a Battery Ages. .. 64

Figure 4.3. Example Randles $1^{\text {st }}$ Order Equivalent Circuit Frequency Response ............ 67

Figure 4.4. Electrochemical Impedance Spectroscopy Circuit Fit Examples................... 68

Figure 4.5. Electrochemical Impedance Spectroscopy Circuit Topology. ...................... 70

Figure 4.6. Electrochemical Impedance Direct Method Determination........................ 71

Figure 4.7. Experimental Test Circuit to Verify Topology Circuit ............................... 71

Figure 4.8. BioLogic EC Lab Electrochemical Impedance Spectroscopy Test............... 73

Figure 4.9. EC Lab Software During 1.5 Ah Lithium Ion Polymer Test. ...................... 74

Figure 4.10. Peak Detection Circuit Topology.................................................... 76

Figure 4.11. Zero Crossing Detection Circuit Topology .......................................... 77

Figure 4.12. Final Test Circuit Topology......................................................... 78 
Figure 4.13. Frequency Response of the Final Circuit Topology. 79

Figure 4.14. Implementation of EIS Test Circuit Topology in National Instruments Multisim 80

Figure 4.15. EIS Circuit Topology Simulation at Low Frequency $(1 \mathrm{~Hz})$..................... 81

Figure 4.16. EIS Circuit Topology Simulation at Mid Frequency $(1 \mathrm{kHz})$.................... 82

Figure 4.17. EIS Circuit Topology Simulation at High Frequency $(100 \mathrm{kHz}) \ldots \ldots \ldots \ldots \ldots . . . . . .83$

Figure 4.18. Experimental testing of EIS Circuit on a Breadboard.............................. 84

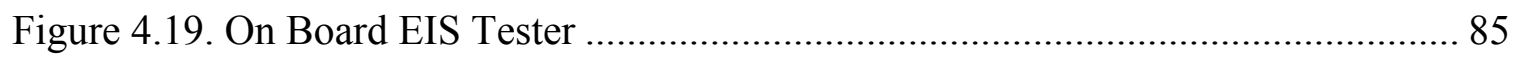

Figure 5.1. Physics-based Model to Experimental Comparison under a C/10 0.005 Hz 50\% Duty Load Pulse for a Lead Acid Cell. ........................................................... 94

Figure 5.2. Physics-based Model to Experimental Comparison under a C/10 $0.005 \mathrm{~Hz}$ $50 \%$ Duty Load Pulse for a Lithium Ion Cell.................................................. 97

Figure 5.3. Lead Acid versus Lithium Ion Cell Voltages under $0.005 \mathrm{~Hz}$ C/10 Load Pulse

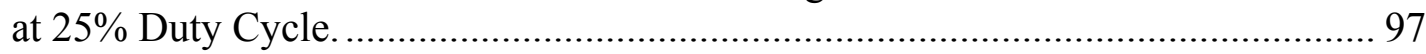

Figure 5.4. Open Circuit Voltage and Temperature versus SoC function per cell for a

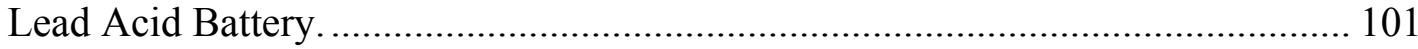

Figure 5.5. Open Circuit Voltage and Temperature versus SoC function per cell for a Lithium Ion Battery.................................................................................... 102

Figure 5.6. Battery Equivalent Model for Multichemistry System. ........................... 105

Figure 5.7. Batteries Tested for Chemistry Identification Mapped to Table 5-1........... 108

Figure 5.8. Battery Test Stand Hardware.............................................................. 111

Figure 5.9. Normal Detection of a 12 V Lead Acid Battery..................................... 115

Figure 5.10. Misidentification of an 8 V (4-cell) Lead Acid Battery for a 12V (6-cell) Lead Acid Battery with Custom Entry and User Warning. .................................. 115

Figure 5.11. Autonomous Battery Management System Cycling Flow Chart. ............. 116

Figure 5.12. Graphical User Interface during a Battery Test................................... 117 
Figure 5.13. Pulse Test Example on a 6 V Lead Acid Battery. 118

Figure 5.14. BMS Software Platform and Equivalent Circuit Generation for a $6 \mathrm{~V}$ Lead Acid Module.

Figure 5.15. 3.7 V 21 Ah Lithium Ion Battery Type Identification.....

Figure 5.16. 3.7 V 21 Ah Lithium Ion Battery Curve Fit and Final Equivalent Circuit for a Battery in Good Condition.

Figure 5.17. 3.7 V 21 Ah Lithium Ion Battery Curve Fit and Final Equivalent Circuit for a Battery in Bad Condition

Figure 5.18. 12 V 21 Ah Lead Acid Battery Curve Fit and Final Equivalent Circuit for a Battery in Good Condition. 123

Figure 5.19. 12 V 21 Ah Lead Acid Battery Type Identification. 123

Figure 5.20. 12 V 21 Ah Lead Acid Battery Curve Fit and Final Equivalent Circuit for a Battery in Good Condition. 124

Figure 6.1. Samsung Galaxy S7 Battery Fast Charging Profile 128

Figure 6.2. Thermal Imaging of Samsung Galaxy S7 Battery During Fast Charging Profile.

Figure 6.3. Pseudo 2D FEM to 3D FEM Representation of the Lithium Ion Battery Cell.

Figure 6.4. Ionic Conductivity of the Lithium Salt Electrolyte versus its Conductivity. 135

Figure 6.5. 8048168C Lithium Ion Cobalt Oxide (LCO) Polymer Battery. 137

Figure 6.6. Electrostatic 3D FEM of the LCO Battery at Full Charge. 138

Figure 6.7. Electric Potential at the Electrodes and Electrolyte of the LCO Battery under Charging. 140

Figure 6.8. Electric Potential at the Electrodes and Electrolyte of the LCO Battery under Discharging. 140

Figure 6.9. Current Density at the Electrodes and Electrolyte of the LCO Battery under Charging. 143 
Figure 6.10. Current Density at the Electrodes and Electrolyte of the LCO Battery under Discharging.

Figure 7.1. PL8048168 51.8 V 21 Ah Lithium Ion Cobalt Polymer Battery Module.... 150

Figure 7.2. $2^{\text {nd }}$-Order Dynamic Battery Equivalent Circuit Model. .............................. 151

Figure 7.3. BatterySpace KP4818C Universal Smart Charger.................................. 151

Figure 7.4. LabVIEW Battery Test Front Panel. .................................................. 152

Figure 7.5. Dynamic Behavior of Battery Voltage under Current Pulse...................... 154

Figure 7.6. Ohmic Resistance Function Curve Fit for Charging and Discharging........ 155

Figure 7.7. Open Circuit Voltage Curve Fit for Charging and Discharging.................. 156

Figure 7.8. Curve-Fitted Polarization Resistance Parameters under Seconds Timespan for Charging and Discharging................................................................ 157

Figure 7.9. Curve-Fitted Polarization Capacitance Parameters under Seconds

Timespan for Charging and Discharging.

Figure 7.10. Curve-Fitted Polarization Resistance Parameters under Minutes

Timespan for Charging and Discharging.......................................................... 158

Figure 7.11. Curve-Fitted Polarization Capacitance Parameters under Minutes Timespan for Charging and Discharging .................................................... 158

Figure 7.12. MATLAB/Simulink Model of the Battery Block Implementation........... 159

Figure 7.13. MATLAB/Simulink Dynamic Battery Model.................................... 163

Figure 7.14. Battery Model Impulse Response Verification under Short Discharge Pulse. 164

Figure 7.15. Battery Model Impulse Response Verification under Long Discharge Pulse. 165

Figure 7.16. Battery Model Impulse Response Verification under Short Charge Pulse. 165

Figure 7.17. Battery Model Impulse Response Verification under Long Charge Pulse. 165

Figure 7.18. Proposed Wireless Power Transfer Traffic Intersection Architecture. ...... 167 
Figure 7.19. Block Diagram of an Electric Vehicle with a Wireless Power Transfer Network 168

Figure 7.20. Dynamic Battery Equivalent Circuit Model Integration with Electric Vehicle System. 169

Figure 7.21. FTP-72 Driving Profile 169

Figure 7.22. Driving Performance at Fixed Power Charging at WPT4. 173

Figure 7.23. Electric Vehicle Driving Performance at Different Standardized Wireless

Power Transfer Charging Levels 174

Figure 8.1. Basic DC-DC Buck Converter Circuit Topology 180

Figure 8.2. Basic DC-DC Boost Converter Circuit Topology. 181

Figure 8.3. Synchronous Buck Converter. 182

Figure 8.4. Synchronous Buck Converter Design in Simulink SimPowerSystems. 184

Figure 8.5. GaN-based and Si-Based Synchronous Buck Converter Voltage Output under SimPowerSystems Simulation. 185

Figure 8.6. GaN-based and Si-Based Synchronous Buck Converter Charging Current Output under SimPowerSystems Simulation. 186

Figure 8.7. GaN-based and Si-Based Synchronous Buck Converter Efficiency under SimPowerSystems Simulation. 186

Figure 8.8. Synchronous Buck Converter Design in Simulink SimElectronics. 187

Figure 8.9. GaN-based and Si-Based Synchronous Buck Converter Voltage Response under SimElectronics Simulation. 188

Figure 8.10. GaN and Si-Based Synchronous Buck Converter Switch Junction Temperature.

Figure 8.11. GaN-based and Si-Based Synchronous Buck Converter Switch Case Temperatures.... 190

Figure 8.12. GaN and Si-Based Synchronous Buck Converter Efficiency under SimElectronics Simulation. 
Figure 8.13. Si and GaN-based Synchronous Converter Efficiencies versus Charging Current.

Figure 8.14. Field Effect Transistor Structure.

Figure 8.15. GaNSys GS66508T eGaN HEMT SimElectronics I-V Characterization. . 195

Figure 8.16. I-V Characteristic Curves for GaNSys GT66508T. 196

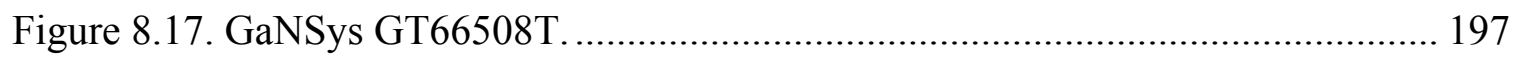

Figure 8.18. GaNSys GT66508T Breakdown Voltage Simulation in LT SPICE. ......... 198

Figure 9.1. General eGaN HEMT Structure............................................................... 202

Figure 9.2. eGaN HEMT Silvaco Solid Model........................................................... 203

Figure 9.3. eGaN HEMT Finite Element Meshing...................................................... 204

Figure 9.4. eGaN HEMT Breakdown Characteristics. ............................................... 205

Figure 9.5. New eGaN HEMT Schematic Layer Structures.......................................... 206

Figure 9.6. eGaN HEMT PBM Breakdown Voltage Analysis for all Cases of Study... 207

Figure 9.7. Locations of the Electric Field Cross-Sections. ........................................... 208

Figure 9.8. eGaN HEMT PBM Electric Field Distributions along the x-axis................ 209

Figure 9.9. Drain, Source and Gate currents for the Field Plate Case.......................... 210

Figure 9.10. Illustration of the eGaN HEMT Charge Distribution Modeling. ............... 212

Figure 9.11. Cross-section of the HEMT with a Field Plate (FP-HEMT) ..................... 214

Figure 9.12. eGaN HEMT PBM Electric Field Distribution for the Field Plate Case at its Individual Breakdown Voltage for all y-distances........................................... 215

Figure 9.13. eGaN HEMT PBM 2D Electric Field Distribution for the Field Plate Case

Figure 10.1. Open Circuit Voltage versus State of Charge for Lead Acid \& Lithium

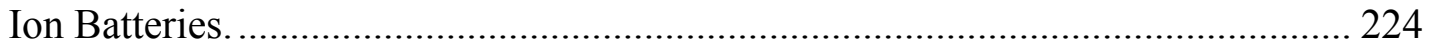

Figure 10.2. Shipboard Power System Equivalent Circuit Models. ................................ 225 
Figure 10.3. Lumped Parameter Model for Case I. 228

Figure 10.4. Verification of Voltage and Current under $0.1 \mathrm{~Hz}$ Test Pulse for Case I... 228

Figure 10.5. Lumped Parameter Model for Case II..................................................... 229

Figure 10.6. Verification of Voltage and Current under 0.1 Hz Test Pulse for Case II. 229

Figure 10.7. Lumped Parameter Model for Case III...................................................... 231

Figure 10.8. Verification of Voltage and Current under 0.1 Hz Test Pulse for Case III.232

Figure 10.9. Comprehensive Simulink Simulation Platform to Evaluate all Cases. ...... 233

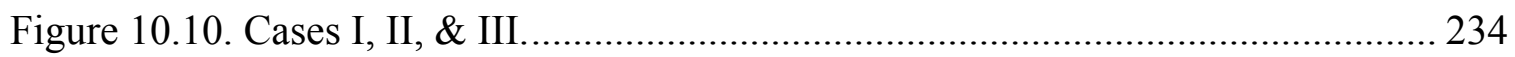

Figure 10.11. Upgraded ESMC Control Platform to Support Hybrid Energy Storage and Shipboard Power System Loads.................................................................... 239

Figure 10.12. Experimental Setup of the ESMC, Converter, Supercapacitors, Lead Acid Battery, and Lithium ion Batteries............................................................... 240

Figure 10.13. Configuration of ESMC Controllers in Series.......................................... 241

Figure 10.14. USS Zumwalt DDG-1000 and Modeled Shipboard Loads...................... 241

Figure 10.15. Test I: Individual Energy Storage Modules........................................... 243

Figure 10.16. Test I: 72-second Close-up of Currents of Individual Energy Storage

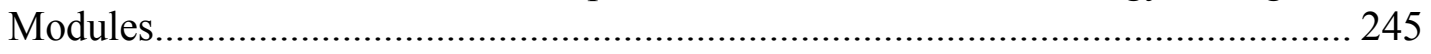

Figure 10.17. Test I: Energy Output from Currents of Individual Energy Storage Modules. 245

Figure 10.18. Test II without Rolling Charging. 247

Figure 10.19. Test II with Rolling Charging: Individual Energy Storage Modules ....... 248

Figure 10.20. Test II with Rolling Charging: Energy Output from Individual Energy Storage Modules. ………………………………………............................. 250

Figure 10.21. Test III: Individual Energy Storage Modules ......................................... 251

Figure 10.22. Test IV: Voltage from Individual Energy Storage Modules.................... 253 
Figure 10.23. Test IV: Voltages from the ESMC Input Array and DC Bus. 253

Figure 10.24. Test IV: 30 min Zoom from Individual Energy Storage Modules ........... 254

Figure 10.25. Test V: Individual Energy Storage Modules ...................................... 256

Figure 10.26. Test V: Voltages from the ESMC Input Array and DC Bus. .................. 257

Figure 10.27. Test V: Power Output to the DC Bus................................................ 257

Figure 11.1. Modified Lithium Ion Battery Psuedo-2D Model Including Solid

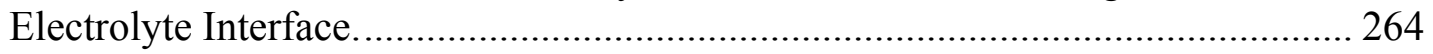

Figure 11.2. Voltage and Current Profiles for the Lithium Ion Battery Degradation

Model in the First and Last Cycle.................................................................... 266

Figure 11.3. Cycle Comparison of Voltage Drop across the SEI Layer after $100 \mathrm{~s}$ into

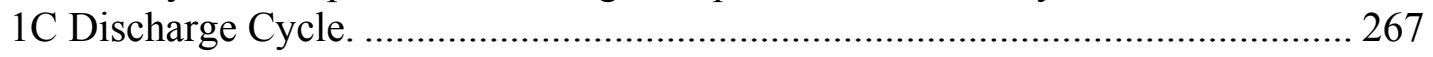

Figure 11.4. Discharge Voltage Profile Comparison and Capacity Retention. .............. 267

Figure 11.5. Open Circuit Voltage versus State of Charge for a 4-cell Lithium Ion Battery and 58 F (16.2 V) Supercapacitor. ...................................................... 268

Figure 11.6. Lithium Ion Battery Equivalent Circuit Model Utilized for the Electric

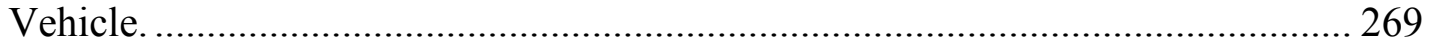

Figure 11.7. Supercapacitor Equivalent Circuit Model Utilized for the Electric

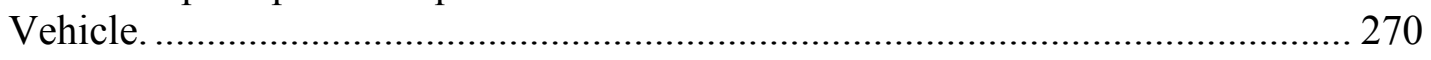

Figure 11.8. Hybrid Pulse Power Characterization Test Profile................................. 272

Figure 11.9. Schematic Diagram for Energy Storage Modular Controllers Connected in Parallel for Electric Vehicle Testing........................................................ 273

Figure 11.10. Individual Energy Storage Voltage Experimental Results..................... 276

Figure 11.11. Individual Energy Storage State of Charges Experimental Results........ 276

Figure 11.12. Individual Energy Storage Currents under Electric Vehicle

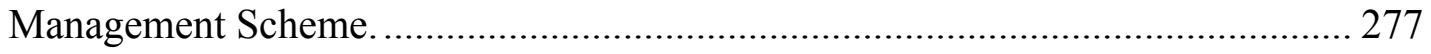

Figure 11.13. Individual Energy Storage Power under Electric Vehicle Management Scheme. 
Figure 11.14. Four Minute Close-up of all States.

Figure 11.15. DC Bus and Energy Storage Array Voltages under Electric Vehicle Management Scheme.

Figure 12.1. Formula One Kinetic Energy Recovery System Flywheel Energy Storage Device 282

Figure 12.2. Significant Factors Inducing Ripple Frequencies in the DC Machine...... 285

Figure 12.3. Flywheel - Lead Acid Battery Hybrid Energy Storage System. ............... 287

Figure 12.4. Lead Acid Battery Energy and Lifetime and Randles Circuit Models Utilized for Flywheel Integrated Hybrid Energy Storage Systems. 289

Figure 12.5. Initial Testing and Power Quality Analysis of the Flywheel. 291

Figure 12.6. Custom LabVIEW Flywheel and Battery Data Acquisition Interface...... 291

Figure 12.7. Voltage and Current of Flywheel Energy Storage Only. 293

Figure 12.8. Discrete Fourier Transform of the Voltage and Current of the Flywheel Energy Storage.

Figure 12.9. Voltage Ripple Distortion versus Percentage of the DC Component. 295

Figure 12.10. Discrete Fourier Transform of Load Voltage with 5\% Battery Current Contribution.

Figure 12.11. Voltage Percentage versus DC Component under 5\% Battery Current Contribution.

Figure 12.12. Departure of Ripple Voltage at each MSM from 2\% Compliance under 5\% Battery Current Contribution.

Figure 12.13. Discrete Fourier Transform of Load Voltage with 25\% Battery Current Contribution.

Figure 12.14. Voltage Percentage versus DC Component under 25\% Battery Current Contribution.

Figure 12.15. Departure from 2\% Compliance under 25\% Battery Contribution. 299

Figure 12.16. Discrete Fourier Transform of Load Voltage with 50\% Battery Current Contribution. 
Figure 12.17. Voltage Percentage versus DC Component under 50\% Battery Current

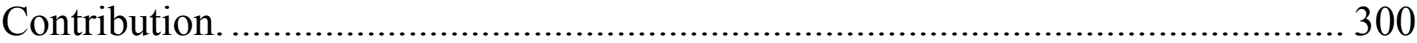

Figure 12.18. Departure from 2\% Compliance under 50\% Battery Current

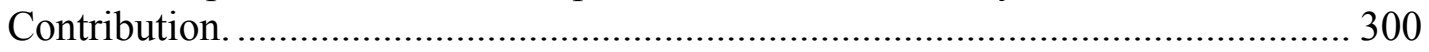




\section{LIST OF ACRONYMS}

\begin{tabular}{|c|c|}
\hline 2DEG & 2-Dimensional Electron Gas \\
\hline $\mathrm{ADC}$ & Analog to Digital Converter \\
\hline $\mathrm{Ah}$ & Amp-Hour \\
\hline $\mathrm{BEV}$ & Battery Electric Vehicle \\
\hline BMS & Battery Management System \\
\hline $\mathrm{BV}$ & Breakdown Voltage \\
\hline $\mathrm{CC}$ & Constant Current \\
\hline $\mathrm{CV}$ & Constant Voltage \\
\hline DAQ & Data Acquisition \\
\hline DFT & Discrete Fourier Transform \\
\hline DoD & Depth of Discharge \\
\hline DOE & Department of Energy \\
\hline EIS & Electrochemical Impedance Spectroscopy \\
\hline ES & Energy Storage \\
\hline ESMS & Energy Storage Management System \\
\hline ESS & Energy Storage System \\
\hline $\mathrm{EV}$ & Electric Vehicle \\
\hline FEM & Finite Element Modeling \\
\hline FESS & Flywheel Energy Storage System \\
\hline $\mathrm{FP}$ & Field Plate \\
\hline GA & Genetic Algorithm \\
\hline $\mathrm{eGaN}$ & enhancement-mode Gallium Nitride \\
\hline HEMT & High Electron Mobility Transistor \\
\hline HESS & Hybrid Energy Storage System \\
\hline $\mathrm{HEV}$ & Hybrid Electric Vehicle \\
\hline HPF & High Pass Filter \\
\hline
\end{tabular}




\begin{tabular}{|c|c|}
\hline HPPC & Hybrid Pulse Power Characterization \\
\hline IC & Integrated Circuit \\
\hline LPF & Low Pass Filter \\
\hline $\mathrm{NC}$ & Normally Closed \\
\hline NO & Normally Open \\
\hline OCV & Open Circuit Voltage \\
\hline LSM & Least Squares Method \\
\hline $\mathrm{MCU}$ & Microcontroller Unit \\
\hline MG & Microgrid \\
\hline MOCVD & Metal Oxide Chemical Vapor Deposition \\
\hline MOSFET & Metal Oxide Semiconductor Field Effect Transistors \\
\hline MSM & Machine Speed Multiple \\
\hline ODE & Ordinary Differential Equation \\
\hline $\mathrm{P} 2 \mathrm{D}$ & Pseudo 2-Dimensional \\
\hline PBM & Physics Based Model \\
\hline PCB & Printed Circuit Board \\
\hline PHEV & Plug-in Hybrid Electric Vehicle \\
\hline PI & Porportional Integral \\
\hline PWM & Pulse Width Modulation \\
\hline QDWPT & Quasi Dyanamic Wireless Power Transfer \\
\hline $\mathrm{RC}$ & Resistor-Capacitor \\
\hline RMSE & Root-Mean-Square Error \\
\hline $\mathrm{SC}$ & Supercapacitor \\
\hline SEI & Solid Electrolyte Interface \\
\hline SMD & Surface Mount Devices \\
\hline SPICE & Simulation Program with Integrated Circuit Emphasis \\
\hline SoC & State of Charge \\
\hline $\mathrm{SoH}$ & State of Health \\
\hline SPS & Shipboard Power System \\
\hline SSR & Solid State Relay \\
\hline
\end{tabular}


TCAD

THD

TTL

WBG

WPTS

Wh
Technology Computer Aided Design

Total Harmonic Distortion

Transistor-Transistor Logic

Wide Band-Gap

Wireless Power Transfer System

Watt-hour 


\section{Chapter 1 Introduction and Research Background}

\subsection{Problem Statement}

The structure and resiliency of the emerging electrical grid will rely heavily on energy storage (ES) to provide uninterrupted service to the customer. The usage of ES continues to grow due to its capability in restoring system voltage and frequency following an outage [1]-[5]. As the modern electrical grid continues to increase in its complexity, so does the inclusion of renewable ES, which are inherently intermittent. A grid which derives a large fraction of its energy from solar photovoltaics, wind turbine generators, and/or fuel cells have the major drawback of not being dispatchable [6]. Without the aid of ES devices, energy must be either drawn from a traditional non-renewable source on-demand, or ES units must be prepared and deployed effectively. This requirement only becomes more critical when applied to localized microgrids such as on an electric vehicle (EV), shipboard power system (SPS), or an aircraft.

Although the causes of both the Northeast blackout of 1965 and 2003 were different, the lack of grid resilience and backup energy could have avoided tens of millions of customers losing power [7]-[8]. A versatile and modular grid with ES could have aided in preventing these cascading failures. The integration of a robust ES system can have similar impacts on grid support during natural disasters. The record-breaking 2005 hurricane season, in particular, tested the limitations of the utility grid across the United States [9]. In South Florida, Hurricane Wilma was responsible for the largest disruption to electrical service ever experienced to date in the region, where up to $98 \%$ lost electrical service across 42 counties. With a localized ES system, major transmission lines, which took several 
months to reconnect, could have provided backup energy to critical loads and emergency services. It is important to mention, however, that a simple ES system without adequate control measures is insufficient. Advanced ES control and management could have played a significant role in relief after these events.

While the role of ES on utility power systems becomes more crucial, an inherently offgrid application such a SPS or EV places them at the center of attention. Naval propulsion systems, such as the DDG 1000 requires an incredible 100,000 hp of total shaft horsepower in propulsion [10]. To reduce its massive fuel burden, ES must be deployed to maintain primary shipboard operations. Without intimate control metrics and maintenance procedures, millions of dollars would be wasted on ineffective sources that not only result in reduced effectiveness and lifespans of ES, but also permit unsafe operation that can result in shock and fire hazards. This is especially true with sensitive electrochemical sources such as lithium ion batteries [11]. In January 2013, the Boeing 787 Dreamliner aircraft suffered a short circuit across one its new lithium-ion 8-series cell battery modules while in operation. A lack of adequate control caused a cascading failure resulting in a fire that grounded all 787 aircraft for over three months. ES management in electric and hybrid vehicles follows a similar criticality. In 2013, Tesla motors recalled 439 of their Roadster models due to fire hazards over a hybrid lithium ion and lead acid battery system [12]. An adequate Energy Management System (EMS) to control each module or individual battery cell could have helped prevent these disasters.

\subsection{Review of Energy Storage Devices}

Prior to a discussion of how ES devices can be effectively utilized and managed, a brief overview of four common ES types utilized in this dissertation is discussed including: lead 
acid batteries, lithium ion batteries, Supercapacitors (SC), and Flywheel ES Systems (FESS). In this section, a particular focus is placed upon six major categories of interest that should be considered in the selection of an ES device that are summarized in Table 1-1. Figure 1.1 provides a comparative performance snapshot with a scale normalized amongst all four ES devices of interest. The six performance categories are as follows: 1) Energy Density or the amount of energy that can be stored per unit volume (or mass), 2) Power Density or how fast the energy can be extracted per unit mass (or volume), 3) Energy Cost, 4) Response Time, 5) Self Discharge Rate, and 6) Lifespan. A significant part of this dissertation investigates lifespan, or the State of Health ( $\mathrm{SoH})$ impacts and how they can be minimized for ES devices.

Table 1-1. Comparison of Four Prominent Energy Storage Resources.

\begin{tabular}{|c|c|c|c|c|c|c|c|}
\hline \multirow{2}{*}{$\begin{array}{c}\text { Energy } \\
\text { Storage } \\
\text { Type }\end{array}$} & \multirow{2}{*}{$\begin{array}{l}\text { Energy } \\
\text { Density } \\
\text { (Wh/L) }\end{array}$} & \multirow{2}{*}{$\begin{array}{l}\text { Power } \\
\text { Density } \\
\text { (W/kg) }\end{array}$} & \multirow{2}{*}{$\begin{array}{c}\text { Energy } \\
\text { Cost } \\
(\$ / \mathbf{k W h})\end{array}$} & \multirow{2}{*}{$\begin{array}{c}\text { Response } \\
\text { Time }\end{array}$} & \multirow{2}{*}{$\begin{array}{c}\text { Self } \\
\text { Discharge } \\
\text { Rate }\end{array}$} & \multicolumn{2}{|c|}{ Lifespan } \\
\hline & & & & & & $\begin{array}{c}\text { Maximum } \\
\text { Cycles }\end{array}$ & $\begin{array}{l}\text { Service } \\
\text { (years) }\end{array}$ \\
\hline $\begin{array}{r}\text { Lead } A \\
\text { Batte }\end{array}$ & 85 & 180 & 50 & Slow & $3-20^{\circ}$ & 1,500 & $3-12$ \\
\hline $\begin{array}{r}\text { Lithiu } \\
\text { Bat }\end{array}$ & 463 & 295 & 250.00 & Medium & 6-8\%/mo & 4,200 & $5-20$ \\
\hline Superc & 10 & 0 & $1,000.00$ & Ver? & -2 & 1,00 & 10 \\
\hline Flywheel & 8 & 5,000 & 500.00 & Fast & $3-40 \% / \mathrm{hr}$ & $10,000,000$ & $20-35$ \\
\hline
\end{tabular}
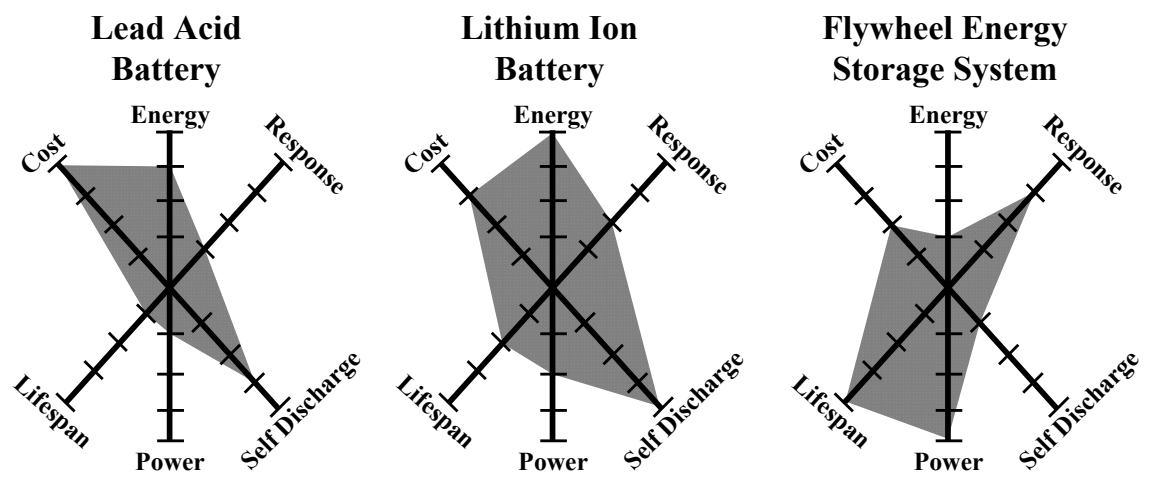

\section{Supercapacitor}

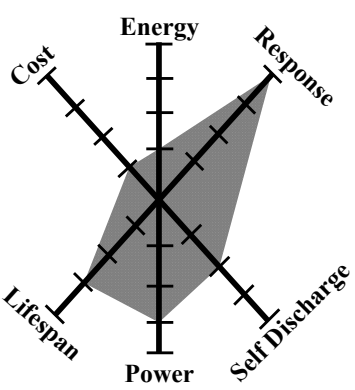

Figure 1.1. Normalized Energy Storage Device Performance Snapshot. 


\subsubsection{Lead Acid Batteries}

The lead acid battery has maintained a strong hold in the market as a result of its simplicity in design and availability of inexpensive materials [13]. Despite the fact that emerging hybrid electric and EV have moved onto other technologies such as nickel metal hydride and lithium ion, the lead acid market remains strong as it is still the most common starter battery and primary backup support for uninterruptible power supplies (UPS) [14]. The chemical formula for a lead acid battery cell operation is defined in Equation (1-1), where porous lead $\mathrm{Pb}$ (Negative) and lead dioxide $\mathrm{PbO}_{2}$ (Positive) electrodes are placed in an electrolyte of sulfuric acid $\mathrm{H}_{2} \mathrm{SO}_{4}$ and water to precipitate the storage and removal of electrons.

$$
\mathrm{Pb}+\mathrm{PbO}_{2}+2 \mathrm{H}_{2} \mathrm{SO}_{4}+2 e^{-} \rightleftharpoons 2 \mathrm{PbSO}_{4}+2 \mathrm{H}_{2} \mathrm{O}+2 e^{-}
$$

A fully charged battery has an electrolyte composed of approximately $60 \%$ sulfuric acid and a discharged battery is primarily composed of water. A removal of electrons from the sulfuric acid in the discharging phase precipitates in the production of solid sulfate $\mathrm{PbSO}_{4}$ at the battery plates. The charging process removes the solid sulfate and places it back into the electrolyte. To the right in Figure 1.2, a pictorial demonstration of this process is depicted.
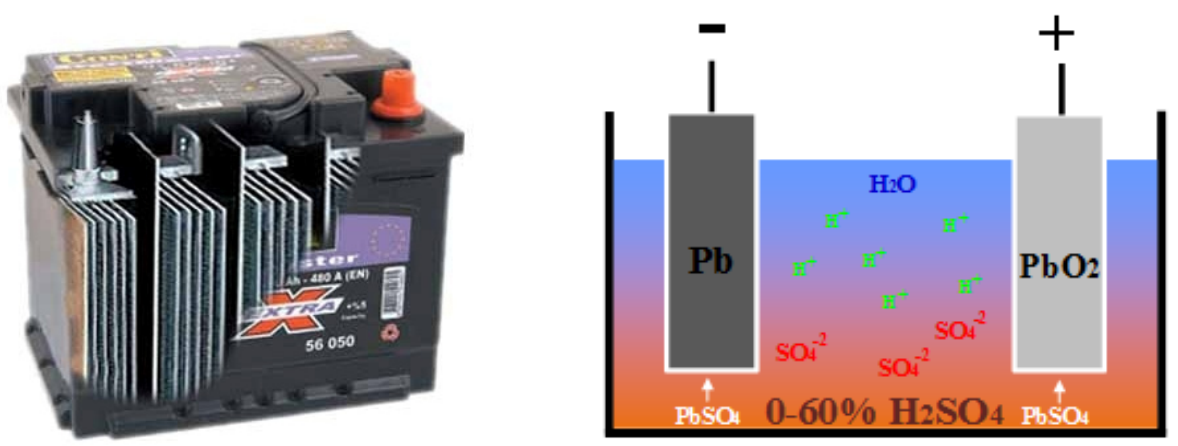

Figure 1.2. Lead Acid Batteries: Flooded 12V 6-cell Module (Left), General Operational Schematic (right). 
The lead acid battery has been demonstrated as a dependable resource in stationary grid applications to smoothen the energy harvested by renewables, but has been more universally accepted in restoring system frequency and voltage following an outage $[5],[15],[16]$. Despite its strengths in reliability and low cost, lead acid batteries have a relatively low energy and power density and suffer from a number of other drawbacks. First, they are not ideal to source pulsed loads or a load that contains a very high power demand over a relatively short period of time, as a result of their large internal double layer capacitance [17]. Second, their operational current is severely limited, as an increase from a conservative 20-hour Coulombic (C/20) discharge current (C-rate) will result in a reduced usable capacity and increase ageing. An example discharge curve comparison is depicted to the left in Figure 1.3, where a C-rate increase to $\mathrm{C} / 2(0.5 \mathrm{C})$ results in a $50 \%$ loss of usable capacity [18]. This phenomenon reduces their practicality in a number of applications, particularly EVs. Second, the Depth of Discharge (DoD), or the inverse of State of Charge ( $\mathrm{SoC}$ ), must be limited as an exponential falloff occurs as the DoD increases. Finally, their shelf and cycle life is highly limited as well.
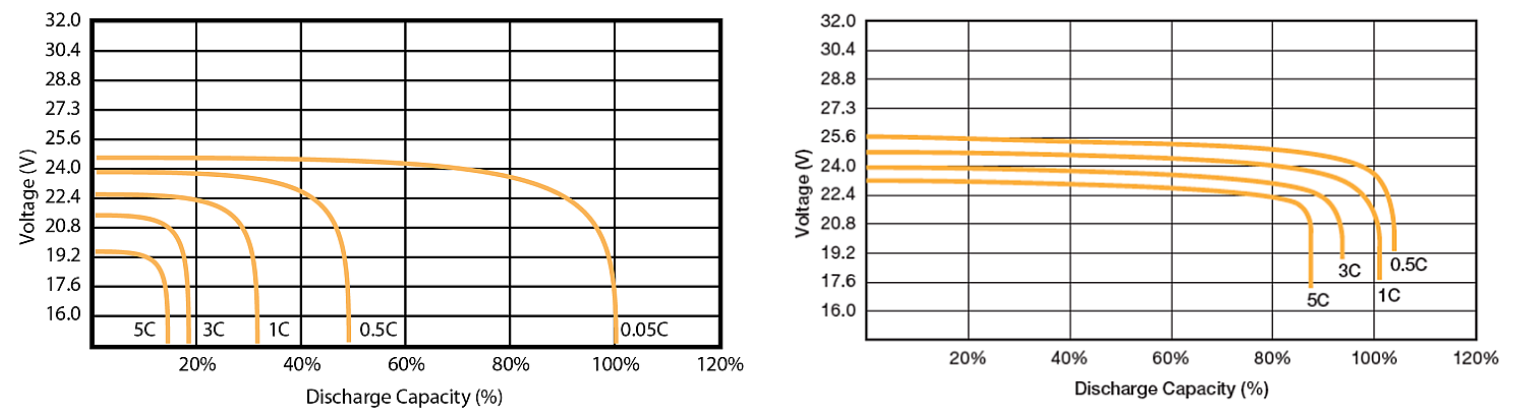

Figure 1.3. Lead Acid (left) versus Lithium Ion (right) Battery Discharge Curve Comparison [18]. 

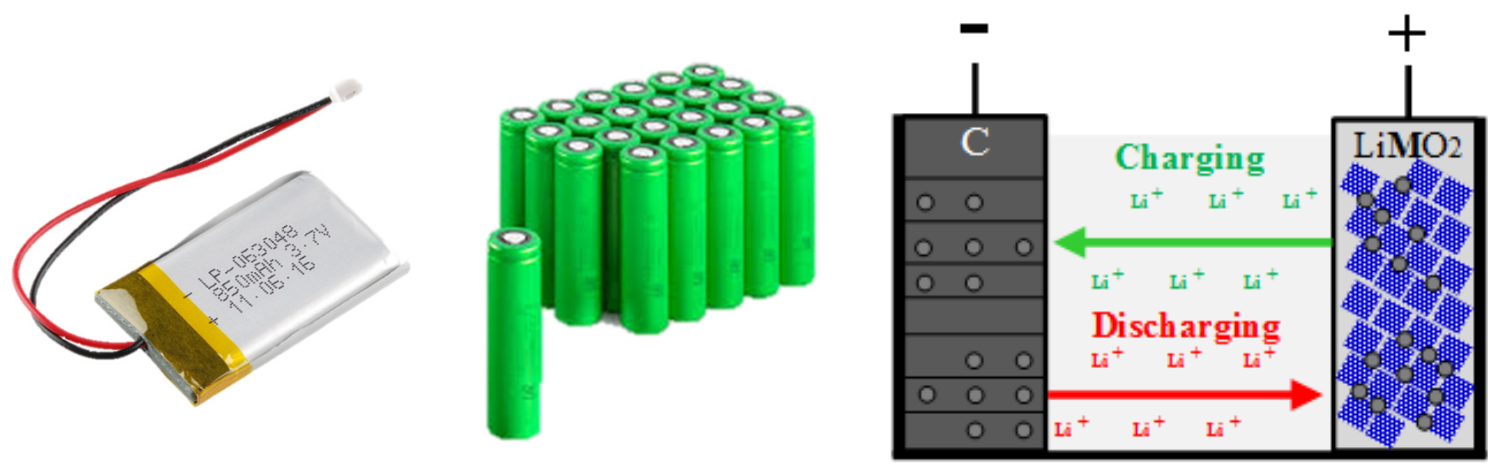

Figure 1.4. Lithium Ion Batteries: Polymer Cell Type (Left), Cylindrical Cell Type (Center), and General Operational Schematic (right).

\subsubsection{Lithium Ion Batteries}

Lithium ion battery usage has surged in recent years not only in portable electronic devices, but also large scale EVs, SPS, and grid storage [19]. They have been featured in a wide variety of packaging types, though the polymer and cylindrical (e.g. 18650) types have been the most popular. Polymer cell types are popular in consumer electronics and compact applications. As a result of their compact design, they can typically store more energy than the cylindrical-type cells. However, polymer-type discharge currents are typically limited to $1 \mathrm{C}$, whereas cylindrical cells can operate at $5 \mathrm{C}$ or greater in some cells.

Advanced lithium ion battery management systems (BMS) have been demonstrated in microgrid applications for both islanded and grid-connected modes to provide voltage and frequency support [4],[20]. Their operation is significantly different from that of lead acid, where the energy is stored inside its electrodes, utilizing the electrolyte as simply a transfer layer [21]. Many types exist and are characterized by differences in the metal (M) oxide used in their positive electrode (LixMO2). The electrolyte is a lithium salt in an organic solvent and negative electrode is a porous carbon graphite (LixC6) [22]. The chemical formula representing the defining the operation of a common lithium ion cell is: 


$$
\mathrm{LiMO}_{2}+\mathrm{C}+\mathrm{xLi}^{+}+\mathrm{xe}^{-} \rightleftharpoons \mathrm{CLi}_{x}+\mathrm{Li}_{1-x} \mathrm{MO}_{2}+\mathrm{xLi}^{+}+\mathrm{xe}^{-}
$$

where the charging process is shown from left to right and the discharging process from right to left. A graphical demonstration of the process is demonstrated to the right in Figure 1.4 .

Since lithium ion battery management is more complex than that of the lead acid, studies have looked at the best method to control these schemes [23],[24]. This trait combined with a smaller cell construction allow them to respond faster to demands. Lithium ion batteries offer a significant improvement in their capability to source high current without having to make the same trade-offs in operational impacts as the lead acid battery. An example discharge curve comparison is depicted to the right in Figure 1.3, where the total cell capacity can be extracted even at a C/2 (0.5C) discharge current [18]. The first reduction of capacity does not occur until 1C, and may only experience a $10 \%$ capacity reduction at 5C (in cylindrical cells) making them very attractive for $\mathrm{EV}$ applications. Furthermore, their lifespans are much longer. Unfortunately, similar to the lead acid battery, their lifespans are still limited by their DoD and temperature [25]. Also, the BMS cost for lithium ion batteries increases as a result of required crucial cell balancing and thermal control for safety. A number of companies have been working to reduce the cost of grid-scale lithium ion battery arrays, however, wide-scale lithium ion battery deployment cost is still at a premium as compared to lead acid batteries.

\subsubsection{Supercapacitor Energy Storage}

The SC provides a significant improvement in the response time versus any electrochemical battery. Although their construction is chemical in nature, no electrochemical reaction takes place. This enables them to respond extremely fast to a 
demand, while their lifetime is left minimally affected, even under a heavy current demand and a deep DoD [26]. Shown to the right in Figure 1.6, the SC is composed of two porous electrodes divided by a separator soaked in a solvent electrolyte [27]. This construction enables a much higher charge density versus the traditional capacitor as a result of an increased active surface area [28]. Their typical charging and discharging process is shown to the left in Figure 1.5, resembling a similar operational profile as compared to the traditional capacitor.
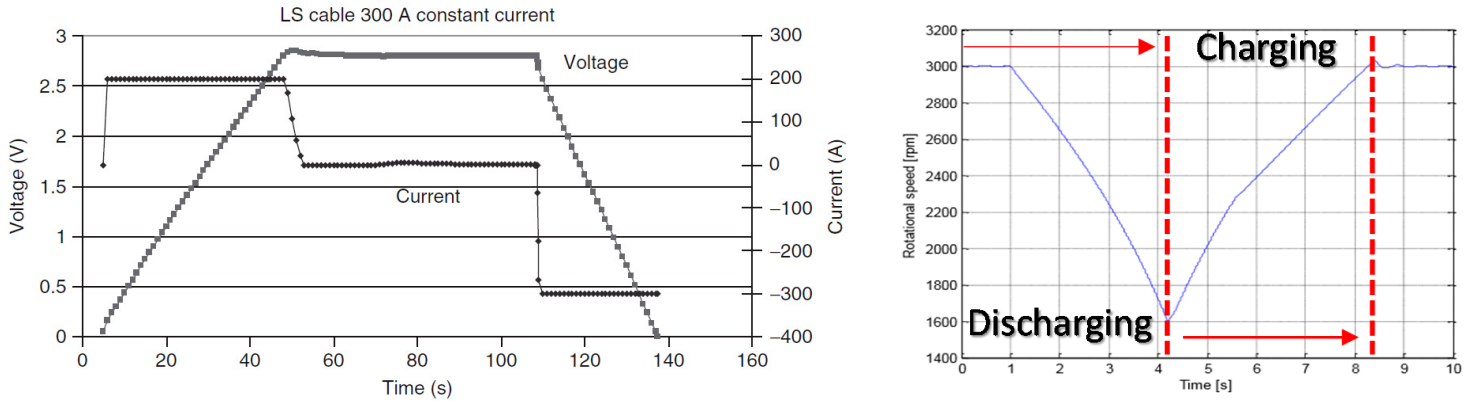

Figure 1.5. Supercapacitor [28] (left) versus Flywheel Energy Storage (right) Discharge Curve Comparison.
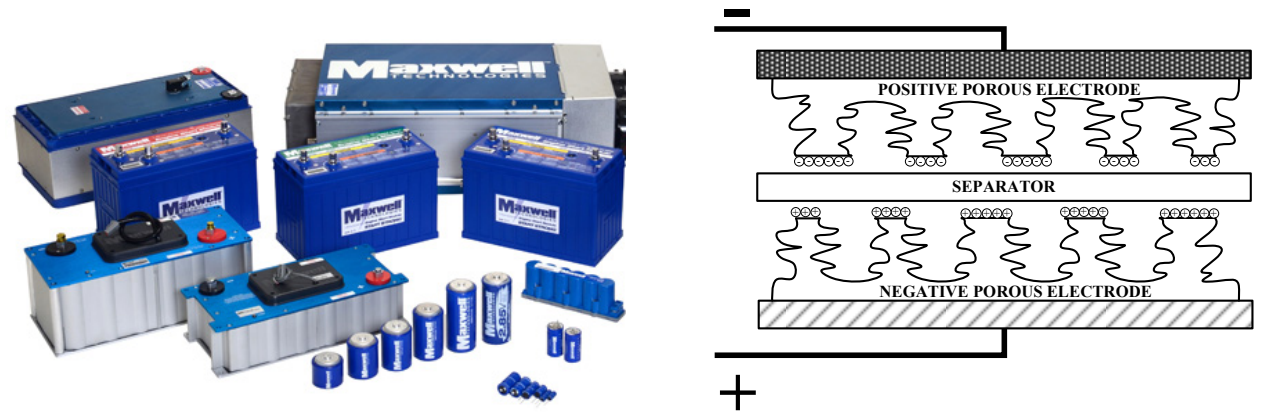

Figure 1.6. Supercapacitor Energy Storage: Maxwell Supercapacitors [29] (left) and Operational Construction (right).

Their usage has been studied in mobile SPS applications where weight is a concern, but also in some grid applications with multiple renewable energy resources. In these applications, the SC provides short term storage to supply the deficiency power [30],[31]. Unfortunately, their low energy density can require an enormous capacitance to be 
effective, which may prove to be impractical as a sole ES device [32]. Its energy density is only around $10 \%$ of that of the lead acid battery and only $2 \%$ of a lithium ion battery. Furthermore, its self-discharge rate is relatively high. Finally, of all the ES devices included in this survey, they currently have the highest energy cost per $\mathrm{kWh}$.
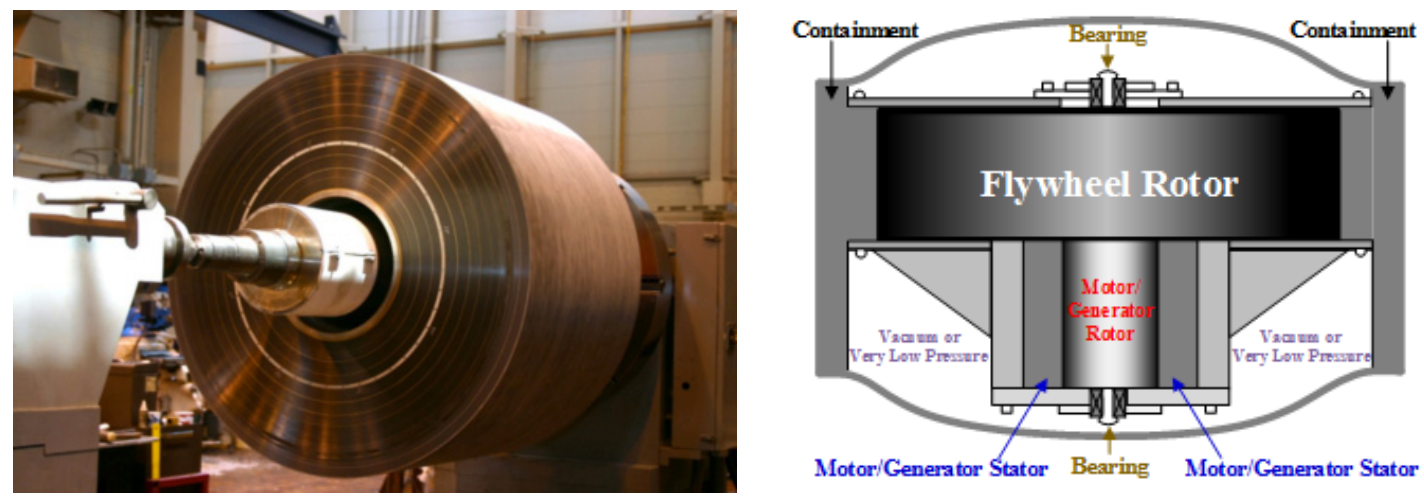

Figure 1.7. Flywheel Energy Storage: Utility Grid Storage Device [33] (left) and Basic Layout (right).

\subsubsection{Flywheel Energy Storage Systems}

Similar to the lead acid battery, FESS have been synonymous with industrial UPS systems for quite some time, but for a very different purpose. FESS provides some of the highest power density in this survey and are primarily purposed to support pulsed loads [34]. Analogous to an electromechanical battery, FESS store kinetic energy in a high inertia rotating mass, where an electric machine operates simultaneously as a motor during charging and a generator during discharging. A view of the basic FESS construction is depicted to the right in Figure 1.7 [35]. Its power output is a function of the square of its rotational speed, which allows FESS to provide extremely high power density. This has made them an excellent solution for maintaining power quality, particularly in voltage sag or swell cases where a great deal of power is required or must be extracted quickly [36][37]. This has been particularly of interest in shipboard propulsion systems, as it has been 
shown to not only improve SPS power quality but also increase its reliability [38]. FESS have also been tested for their usage in volatile applications such as wind and solar energy as an excellent mechanism to quickly store and expend energy [39].

Unfortunately, FESS have a very high self-discharge rate as a result of friction losses. For this reason, lately a focus has been placed on the introduction of low loss machinery and composite materials, which would not only help to alleviate some of these concerns, but also increase the potential energy density, another significant drawback [40]. Their cost is half than that of the SC and their response time is still very fast, limited only by the initial inertia required to start moving the rotating mass. However, a major drawback in FESS is their very low energy density, some $20 \%$ further below the SC. Furthermore, if FESS are not utilized often, their high self-discharge rate could reduce their feasibility.

\subsection{Introduction to Hybrid Energy Storage Systems}

Although some ES devices, such as the lithium ion battery, can provide a rather balanced contribution of energy versus power density, cost and lifetime aspects can jeopardize their sole integration. Similarly, the integration of a FESS alone would provide excellent voltage and frequency support on a grid system, but would fail to fill energy demands over longer periods without a huge system. For this reason, Hybrid ES Systems (HESS) can provide a more balanced solution in terms of not only power and energy density, but also cost, lifespan, and self-discharge rates. HESS have emerged in an effort to utilize the strengths of multiple ES devices in a way that is not only more efficient, but potentially cost and lifetime effective.

Recent HESS work in microgrid applications has focused on the collaboration of batteries with SCs, evaluating both lead acid and lithium ion batteries [41],[42]. A recent 
focus has been placed on how to minimize losses, while ensuring an optimum power split between the two sources [43]. A great deal of work has focused specifically on pulsed load management through the deployment of HESS systems [44],[45]. Unfortunately, reaching the required level of power and filling the energy demand over such a short period of time can be challenging. Examples of these applications are featured throughout this dissertation, from naval weapons platforms to the sporadic demands placed upon an electric motor in EV applications.

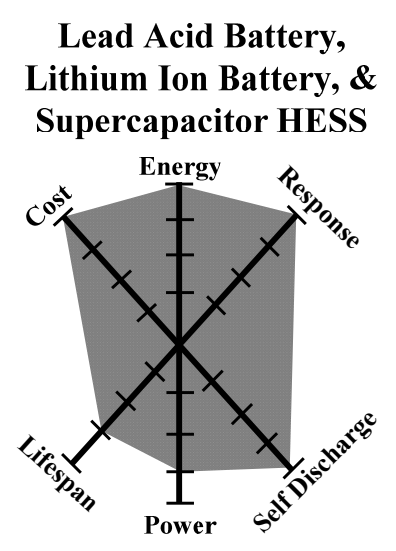

(Featured in Chapter 10)
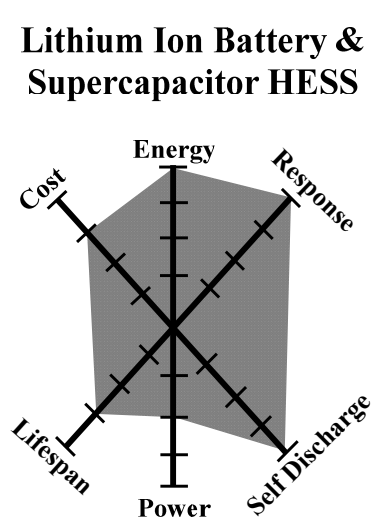

(Featured in Chapter 11)

\section{Flywheel \& Lead Acid \\ Battery HESS}

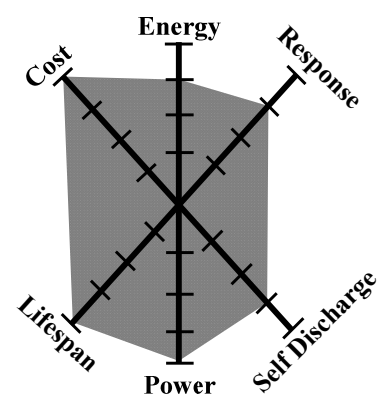

(Featured in Chapter 12)

Figure 1.8. Theoretical Hybrid Energy Storage Systems Performance Snapshot.

For this reason, three different HESS systems are designed, modeled, and tested in later chapters. In Chapter 10, a lead acid battery, lithium ion battery, and SC HESS designed for SPS applications. In Chapter 11, a lithium ion battery and SC HESS is utilized for an EV application, while in Chapter 12, a lead acid battery and FESS HESS is tested and evaluated for power quality improvements. A revised six-part normalized performance snapshot for each of these HESS is depicted in Figure 1.8. Figure 1.8 takes into account the theoretical best case, given each ES device is managed and controlled effectively, another major topic of this dissertation. 


\subsection{Research Objective}

Insufficient control devices and algorithms to monitor ES and HESS can result in a wide range of operational issues. A modern day ES control platform must have a deep understanding of the source. Furthermore, the optimization of ES types and configurations play a pivotal role in efficient energy transfer. Precision SoC and SoH of each ES device is needed to manage each unit effectively. Control and protection measures inside series ES configurations also have the advantage of reducing fire and shock hazards. If properly deployed, these measures can improve and sustain robustness in the modern day electric grid, but also avert potentially catastrophic scenarios in SPS and EV power systems. These systems must be prepared to handle each operating scenario to maintain stability and critical operations. With stiff new demands requiring multiple high-energy pulsed and constant loads, a BMS or EMS must be highly efficient and capable of responding instantaneously to a need. Without proper management, large, expensive ES cannot be utilized effectively. Furthermore, the inability to directly control the inclusion or exclusion of available sources on a bus reduces the reliability of the system.

Without optimal ES devices charged and available, there are a number of consequences. First, the remaining energy in the system will either be incapable to supply an upcoming demand, or, the need is filled by an ES that has already been deeply discharged accelerating its aging and eventually, reducing its effectiveness. Given these needs, the aim of this dissertation is to develop a new modular infrastructure to effectively manage multiple types of ES in a number of different applications and scenarios through the integration of an advanced EMS device. An effective EMS will be engineered to handle a wide range of ES devices including protection under multiple voltage, current, and 
capacity configurations. Meanwhile, advanced maintenance and balancing techniques shall be developed through the seamless integration of software and hardware capabilities, ensuring efficient and safe operation with an intimate understanding of unique ES system dynamics.

The main research problem is composed of three main objectives. The first objective is geared toward the development of an advanced EMS device called the Energy Storage Management Controller (ESMC) to interact with and provide optimal management and maintenance to an ES device with real-time control and monitoring capabilities. This objective will initially focus upon single ES devices, and identify how its design can be commercialized. In the second objective, its initial functionality will be extended to support a multitude of different applications while providing distributed control, protection, and support for load profiles containing multiple loads and dynamic elements. The final objective is focused upon examining the implementation of the ESMC in a variety of HESS, including battery banks of multiple chemistries, SCs, and FESS.

\subsection{Original Contributions of the Dissertation}

A majority of the contributions in this dissertation can be mapped back to the core modular ESMC. The initial ESMC was developed and verified with the capability to extract series-connected ES devices from an array. The design provides a circuit topology along with the proper hardware and software to effectively manage individual ES devices inside the array, enabling total isolation amongst modules when necessary. A bypass circuit provides a path for the series-connected ES system to continue functioning at a reduced voltage level, where the ES device can be charged by a dedicated isolated charging circuit or enable maintenance operations. The unique topology and control of the ESMC enables 
the user to apply custom charging profiles to each individual ES. The original contribution and studies of this dissertation are outlined in the following list:

- Following the initial test and demonstration of the ESMC, its concept was extended to provide a commercial platform that can support larger power systems and zonal networks with a communications platform. The ESMC establishes a critical landmark in this dissertation, as many chapters utilized one or more multiple ESMC devices to conduct laboratory testing and invent or test new management schemes. EMSC initial testing was conducted on lead acid batteries, where its features and concepts were demonstrated as well as a unique pulsed charging method that was used to improve the lead acid battery SoH.

- A focus is then placed upon enhancing software and management algorithms as well as $\mathrm{SoH}$ and acquiring real-time equivalent circuits. First, a circuit topology was proposed as a future hardware and software extension to apply a version of Electrochemical Impedance Spectroscopy (EIS) to batteries to gain a reduced-order circuit model. An alternative solution was then designed as a comprehensive hardware and software platform to autonomously determine between a lithium ion or lead acid battery and its series-cell configurations through the deployment of a single standardized pulsed load. The same load is also utilized as another way to obtain a basic set of equivalent circuit parameters. The method was developed and validated by way of a pseudo 2D (P2D) physics-based model (PBM) of both the lead acid and lithium ion battery. This work also contributed by acquiring insight into the $\mathrm{SoH}$ of the battery connected using multiple methods: a dynamic equivalent circuit model and a usable energy tracker. Along with enhanced $\mathrm{SoH}$ metrics it improved modern $\mathrm{SoC}$ algorithms 
by providing a new voltage and temperature-based initial $\mathrm{SoC}$ mechanism for both chemistries.

- An extension was then made into the lithium ion battery PBM into 3D, where a contribution was made in highlighting a new advantage that 3D PBMs can provide in being able to visualize and study the generation of undesired current gradients across a lithium ion cell surface. High levels of normalized cell currents (high C-rates) are a staple in EV and SPS applications and generate gradient currents, which contribute to uneven thermodynamic and material stress and can have a profound impact on the battery SoH. Continuing on a focus placed upon the demand for accurate battery equivalent circuit models, a comprehensive testing platform was designed based on the contribution of two different battery equivalent circuit acquisition procedures. A dynamic $2^{\text {nd }}$ order model of a lithium ion battery module was obtained, capturing a "fingerprint" of the battery for accurate simulations. The all-inclusive model was implemented within MATLAB/Simulink as a drop-in replacement to the legacy SimPowerSystems battery block. The novelties within this work were particularly focused upon the development of the model within Simulink and the method to which such an advanced model could be integrated with a variety of applications without requiring a great deal of computational overhead.

- The next major contribution integrated, modeled, tested, and evaluated three unique series-configured HESS for SPS consisting of lead acid batteries, lithium ion batteries, and SCs. The ESMC was tested and validated with the connection of lithium ion batteries, SC, and FESS. The uniqueness in this work is in the development of specialized software that was able to utilize many features of the ESMC to execute 
constant and multiple SPS pulsed loads and enable the execution of a new control concept coined as "rolling charging." Novelties in this work include modeling and evaluation of multiple new series-configured HESS architectures composed of lead acid batteries, lithium ion batteries, and SCs, modeling and testing of multiple naval SPS pulsed loads with varying frequencies and magnitudes via per unit system, and the introduction of "rolling charging" to coordinate charging and discharging of individual ES units while in operation to extend runtime while acknowledging SoH trade-offs.

- SoH trade-offs remain a common theme in the final contribution, where a new control algorithm is designed for a lithium ion battery and SC HESS for EVs. A particular focus is placed upon preserving the SoH of the lithium ion battery by reducing cycling and stress upon the battery as it toggles between motoring (discharge) and regenerative braking (charging) power. The management scheme was accomplished through the use of ESMC devices and another version of the control software, which also resulted in an increased energy harvesting efficiency from regenerative braking. The final contribution in this dissertation studied and quantified power quality impacts of a DC network consisting of a FESS and lead acid battery HESS. Voltage and current ripple disturbance frequencies are characterized by a new metric to target and reduce noise frequencies inherent to FESS operation. The ESMC software platform was extended to include a live frequency analysis platform similar to harmonic analysis in the $\mathrm{AC}$ system, where a linear load was used as a reference to balance FESS and lead acid battery current contribution. Multiple lead acid battery contribution levels were tested concluding that even a modest injection of current from the lead acid battery can dramatically improve the power quality of a FESS. 


\subsection{Dissertation Organization}

The first half of this dissertation introduces the ESMC device design, placing a focus on how it was designed with modularity to be interfaced with single ES devices. This laid the groundwork for not only supporting multiple types of ES, but also a mechanism to improve and enhance modeling and software. A transition is then made in the second half, introducing a discussion on HESS which is broken into two focuses: improving aspects of interfacing power electronic converters and the optimal selection and control of ES elements for each application.

In Chapter 2, the concept and development of the ESMC is presented. The first ESMC test application is performed on a lead acid battery in Chapter 3, where the basic operation of the lead acid battery leads to a discussion of its traditional Randles equivalent circuit model and the concept of SoH. SoH is further expanded in Chapter 4, as some of its electrochemical driving mechanisms are discussed as well as the most accurate procedure to date for estimation, EIS is introduced. A direct correlation between Randles equivalent circuit model and EIS is depicted, while a circuit topology is proposed to implement the procedure on a real-time controller.

The following three chapters dive far deeper into battery operation, modeling, and technology. A detailed overview of the usage of P2D battery PBMs is conducted in Chapter 5, highlighting their strengths in deeper analysis for BMS, off-line analysis to study internal characteristics, and providing enhanced models that can serve as a base for new enhanced battery chemistries. In this chapter, both the lead acid and lithium ion battery PBMs are introduced as well as a dynamic Randles equivalent circuit model. Through the application of these two models, these PBMs provide inputs to autotomize the ESMC controller, 
providing an algorithm to determine the chemistry and automatically configure the controller as well as a mechanism to estimate circuit parameters and the battery $\mathrm{SoH}$.

Chapter 6 extends the lithium ion PBM into 3D, recognizing its future implementation in EV and SPS applications justifies the need for providing a deeper analysis of where a SoH impact originates. The 3D PBM also reveals new features internal to the battery operation, which are difficult to measure experimentally while providing a comparison to previous P2D model in Chapter 5. Chapter 7 consolidates all the lessons learned from the modeling tactics and experimental verification from previous chapters to focus specifically on the development of a comprehensive battery equivalent circuit model for a $51.8 \mathrm{~V} 21$ Amp-hour lithium ion battery module. The acquisition of a dynamic $2^{\text {nd }}-$ order equivalent circuit is then applied to a future quasi-dynamic wireless power transfer system for EVs.

Chapter 8 marks the transition into HESS, first focusing upon their interfacing power electronic converters. An overview of basic converters shifts to improving the switching technology and replacing legacy silicon-based switches with wide band-gap (WBG) gallium nitride (GaN)-based semiconductors. A review of the application performance and the use of current GaN switching models reveals a weakness in the usage of basic Simulation Programs with Integrated Circuit Emphasis (SPICE) models, highlighting the need for a GaN PBM. Chapter 9 demonstrates how PBMs are not only useful in modeling of ES devices, but can also be helpful in improving modeling capabilities and understanding of semiconductor switching devices as well. A PBM of the GaN High Electron Mobility Transistor (HEMT) is introduced, where its construction is analyzed using FEM providing a platform where material and geometric design changes are evaluated. 
In the next three chapters, a variety of advanced HESS are introduced for a variety of applications and purposes. Chapter 10 introduces a SPS HESS system consisting of lead acid and lithium ion batteries paired with supercapacitors (SC). An SPS load platform is used, where multiple types of scenarios test and evaluate the HESS performance and extend the system runtime, while remaining mindful of battery SoH impacts. SoH preservation remains a common theme into Chapter 11, where an EV lithium ion battery and SC HESS is studied. A management scheme is introduced with a particular focus on preserving the battery SoH, while maximizing the efficiency of the energy required for propulsion and that which can be captured through regenerative braking. In Chapter 12, a HESS consisting of a lead acid battery and a FESS provides yet another approach in the advantages of HESS, particularly in the practical integration of FESS. The advantages of this system in terms of balancing power versus energy density as well as how the connection of lead acid battery can improve the power quality of the system is quantified. Finally, the conclusions and recommendations for further research are presented in Chapter 13. 


\section{Chapter 2 Developing an Advanced Modular Energy Management System}

\subsection{Introduction}

Modern day energy management systems (EMS) must have a deep understanding of each energy storage (ES) device while being capable to go far beyond basic switching, State of Charge (SoC), as well as voltage and current measurements. Insufficient control devices and algorithms to monitor ES devices can result in a wide range of operational issues. With a complex network of new hybrid ES systems potentially consisting of multiple types of ES devices or battery chemistries, a modular, adaptable EMS is needed that can manage a wide variety of devices. These systems must be prepared to handle each unique operating scenario to maintain stability and critical operations. Without proper management, large, expensive ES cannot be utilized effectively leading to a reduced efficiency and even fire hazards.

A number of studies have been presented in literature on EMS architectures. The research, however, has traditionally been limited to batteries, highlighting the importance in obtaining accurate SoC and lifetime or State of Health $(\mathrm{SoH})$ measurements. In reference [46], a management system solution was presented that demonstrated the importance of including $\mathrm{SoH}$ in measurements for grid applications. Focusing on a range of discharge rates and the Depth of Discharge (DoD) for two parallel-configured lead-acid batteries, a Life Consumption Rate (LCR) factor was defined and modeled for each of the two batteries independently. Battery stack configurations were regulated to produce a total SoC through the means of the current integration method, or in some simplified cases, voltage-based measurements [47]. In Reference [4], an advanced method to depict the SoC of a lithium- 
ion utility array was tested in both grid-connected and islanded modes for microgrid (MG) operations. Three modes of operation were proposed to indirectly measure the $\mathrm{SoC}$ of the battery array. The system was able to demonstrate the effectiveness of its control strategy, but it was still unable to access individual battery modules.

Though individual modules have been difficult to access in series configurations, research has demonstrated SoC balancing for parallel connections. In Reference [48], a supervisory EMS algorithm was designed to regulate charge flow to a bank of three batteries. A constant current was used to charge each battery independently with respect to the load profile and individual $\mathrm{SoC}$ measurements. Balancing of the array was accomplished simply through a means of switching and was unable to adjust the charging current. Different methods of cell equalization for lithium ion batteries including the flying capacitor charge shuttling method, shared single, and multiple transformer methods were discussed in Reference [49]. A charge equalization technique similar to the transformer based one was used in a battery management system (BMS) proposed in Reference [50]. This technique employed a topology that was developed specifically for lithium ion battery cells. In this topology, the charging voltage is pulsed through a control signal and passed through a transformer. The current from the cell stack then induced currents in each of the secondary coil windings, where the secondary (connected to each battery) with the least reactance received the highest induced current. This topology ensured that each cell had a charge current proportional to its SoC.

In Reference [51], a comprehensive review of existing BMS for grid-scale applications was provided. A notional model for implementing a BMS into a battery energy storage system (BESS) was presented. In this model, a number of objectives are identified 
including source peak power demand, cell balancing, and thermal control, but still lacked the capability of extracting individual cells from a stack or even in a parallel configuration. In this case, $\mathrm{SoH}$ and $\mathrm{SoC}$ methods were identified for lithium ion and redox flow batteries only. Two simplified BMS schemes were presented in References [52] and [53]. The latter emphasized the importance of not neglecting differences in each battery's internal resistance during charging.

In Reference [54], an EMS was developed with a focus placed on extending the lifetime and efficiency of an ES system (ESS). Using the Peukert Lifetime Energy Throughput (PLET) model, the energy efficiency of the ESS was improved. An optimization algorithm was presented in Reference [55], where a discrete-time model of an electrochemical storage device was developed to introduce a battery system, but was limited to simply a wind turbine and sodium nickel chloride battery combination. The use of pulsed charging, however, has surfaced as a more direct method improve battery lifetimes and charging efficiency.

Pulsed charging has introduced a revolutionary control over battery charging behaviors by accelerating charging rates while providing battery charge balancing [56]. In Reference [57], a battery equalization method was proposed utilizing a positive and negative pulsed charger to balance cells in an electric vehicle (EV). Pulsed charging was not only used as a method to regulate charging current, but also improve $\mathrm{SoH}$. In the case of a damaged battery, charging current pulses can be used in an attempt to characterize or improve the lead acid battery SoH by neutralizing the internal electrolyte [58]-[60]. Introducing this capability to each battery in a stack configuration would not only provide controlled current charging, but also provide a tool to potentially revive batteries in the event of a failure. 
In this chapter, an advanced ES management controller (ESMC) is developed to monitor individual ES inside a series configuration and identify independent voltages, current contributions, and SoC levels for each ES device. The proposed EMS has the capability to fully decouple an ES device from the system and apply it to a charging and diagnostics bus while still maintaining a connection to the load even within a series configuration. The system bypasses the decoupled ES device to guarantee the continuity of the supply and maintain normal operation of the whole stack. The proposed system offers the capability of charging more than one ES device at the same time with different charging levels through applying pulsed charging currents with different duty cycles and frequencies. The voltage fluctuations accompanied by the coupling and decoupling of ES devices are mitigated by a DC-DC boost converter to maintain bus voltages preventing the propagation of problems from the ESMC to the utility side.

\subsection{Motivation and Novelty}

The ESMC is motivated by the imperative need for obtaining individual and independent control of each ES unit (i.e. ES device or stack of ES devices) in an array or bank to achieve efficient operation. Another objective is to prevent failures on a single ES device from impacting the operation of the entire system. The system presented can be suitable in applications ranging from a small mobile MG such as an EV or shipboard power system up to utility scale. The proposed topology offers a variety of features and capabilities, which can be summarized in the following:

1. The ESMC is capable of controlling each individual unit within a series/parallel array. The unit can range from a single ES device in small arrays or a stack (i.e. sub-array) in large arrays. In other words, each single unit can be treated, controlled, 
and monitored separately from the others presenting a contribution to the enhancement of distributed operation.

2. The ESMC has the capability to charge some units within an ES array while other units can continue to serve the load. By applying a pulsed charging profile at different frequencies and duty cycles, the ESMC can control and regulate the charging energy to each unit. Hence, SoC balancing can be accomplished without a need for proprietary power electronic converters.

3. The ESMC is capable to electrically isolate a unit allowing the operator to perform required maintenance or replace the device without affecting the performance of the remaining array elements.

4. The ESMC incorporates a protection scheme that can determine a faulty bus on either the load or charging side and isolate it.

5. Considering an appropriate selection of relays and other components, the ESMC can be expanded to control ES devices of a wide range of capacities and voltage levels at a relatively low cost.

The aforementioned capabilities can increase the reliability, efficiency, and lifetime of an ES array. These capabilities and features are verified experimentally in the next chapter. As reviewed in the previous section, most EMS focus on either cell equalization, SoC and SoH estimation, or pulsed charging, but do not include all features. Furthermore, many of these schemes involve transformers or large, complicated power electronic devices that introduce more points of failure. 


\subsection{Importance of State of Charge Equalization}

The system under study involves the connection of an ES bank, which is divided into an extendable number of stacks where each stack contains $N_{E S}$ ES devices. A common misconception is that each ES device in the array introduces identical aging and current distributions when their in-service dates are similar. Without accurate measurements of voltage and current from each ES device, there is no guaranteed method to determine the SoC. This is particularly of concern in battery ES devices where $\mathrm{SoH}$ is a major consideration.

Consider a simple $4-12 \mathrm{~V}$ lead acid battery array (48 VDC) with a 110 Amp-hour (Ah) capacity. When charging the array, it is found that only one battery achieves a true full charge level while the remaining batteries are cutoff prematurely to around $90 \%$ SoC. Equation (2-1) presents an averaging function that represents the SoC of the entire array:

$$
S O C_{t o t}=\frac{1}{N_{E S}} \sum_{i=1}^{N_{E S}} S O C_{i}
$$

where $S O C_{i}$ represents the SoC of each ES device. It can be shown that the actual SoC of this configuration would be reduced by $5 \%$ until individual battery charging levels are corrected. A 5\% miscalculation of SoC appears to be minimal, except when compared to the 110 Ah capacity of the array, which would result in 5.5 Ah of energy left unutilized. Furthermore, the continued undercharging of batteries $2-4$ to only $90 \%$ SoC would eventually result in the permanent inability to charge these batteries to their full capacities [13]. This performance shift is driven by differences in the SoH of each battery. Varying material impurities, thermal stresses, and minute offsets in manufacturing processes can 
produce a wide range of results. These metrics are further complicated when nonlinear or dynamic loads are introduced.

\subsection{Energy Storage Management Controller Design}

The schematic for a single ESMC unit is shown in Figure 2.1. The ES device is placed in-between a network of relays in order to achieve isolation for complete coupling and decoupling.

\subsubsection{Bus Connectivity}

In order to achieve full isolation, two normally-closed (NC) relays connect the positive and negative terminals of the ES device to the DC bus. A normally-open (NO) relay connects the positive terminal of the ESMC to its negative terminal to offer the ES device a bypass circuit, decoupling it from the array while still providing an alternative path to maintain continuity of supply. An interlock is included between the three relays to avoid simultaneous connection that would fully isolate the battery in the case of performing maintenance or coupling in the charging circuit.

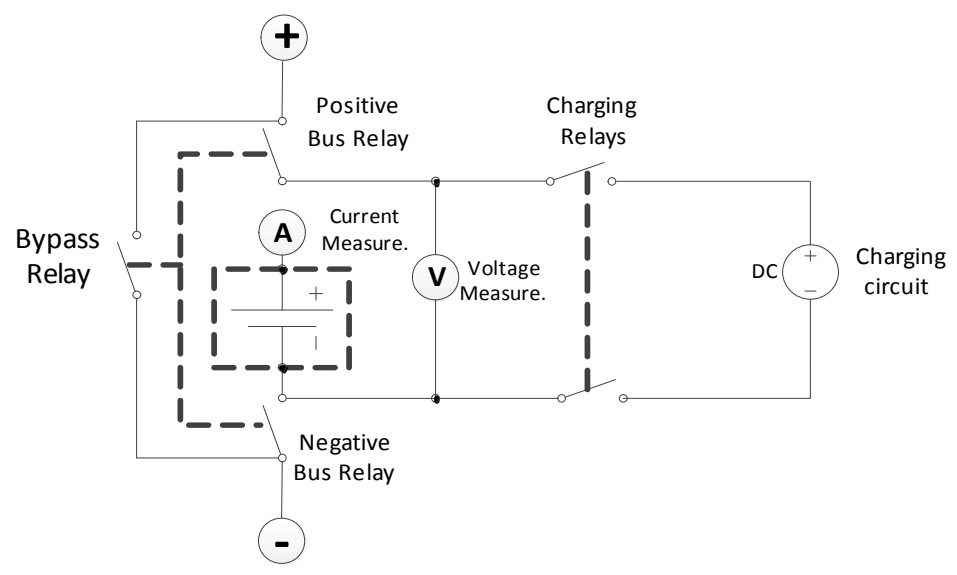

Figure 2.1. Proposed Energy Storage Management Controller Unit Schematic. 


\subsubsection{Current Measurement}

A current measurement is provided directly at the battery terminals. A LA 25-NP current transducer based on the Hall effect is installed in series in the current path from the positive terminal [62]. The LA 25-NP can measure a current of up to $36 \mathrm{~A}$, a level more than adequate for laboratory testing, by properly setting the primary insertion inductance. Tuning of this value is accomplished by connecting pins $1-5$ as well as pins 6-10 together as shown in Figure 2.2. The measurement of a voltage drop across $R_{M}$ provides a near linear representation of the current $\mathrm{I}_{\mathrm{p}}$.
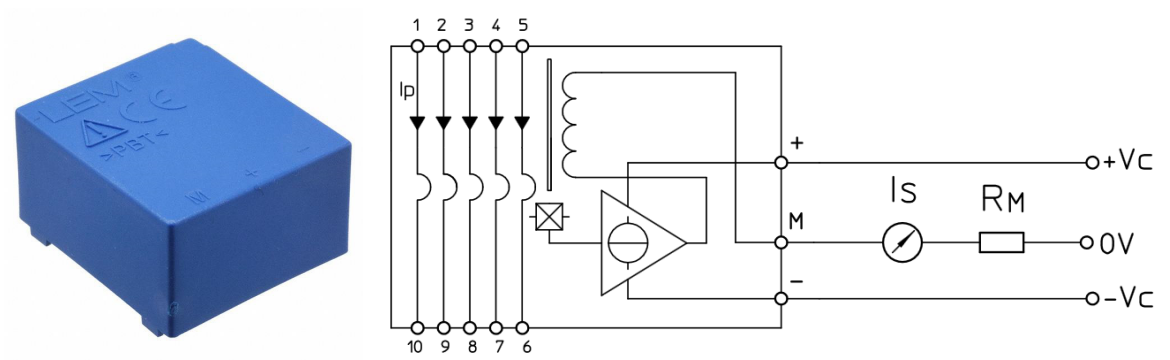

Figure 2.2. LEM LA 25-NP Current Transducer.

\subsubsection{Voltage Measurement}

Due to limitations of the Data Acquisition (DAQ) equipment, a voltage range of \pm 10 VDC must be adhered to. In order to handle a wide range of ES devices and/or module voltages, a LV25-P voltage transducer was used also based on Hall Effect [63]. Unfortunately, the implementation of a simple voltage divider circuit would not be possible because of a lack of the required isolation. A picture of the LV25-P and its general schematic is shown in Figure 2.3. A primary resistor, $R_{1}$, is calculated in order to tune the nominal voltage to be measured. The maximum voltage per module has been tuned to handle up to $\leq 29.6 \mathrm{VDC}$, a voltage equivalent to the maximum charging voltage of a 7-cell 
lithium ion series battery configuration; thus $R_{1}$ is selected to be $3 \mathrm{k} \Omega$. However, the sensor can handle much higher voltages. Although both the LV25-P and LA 25-NP are powerful and nonintrusive sensors, they represent a majority of the cost in designing an ESMC prototype.

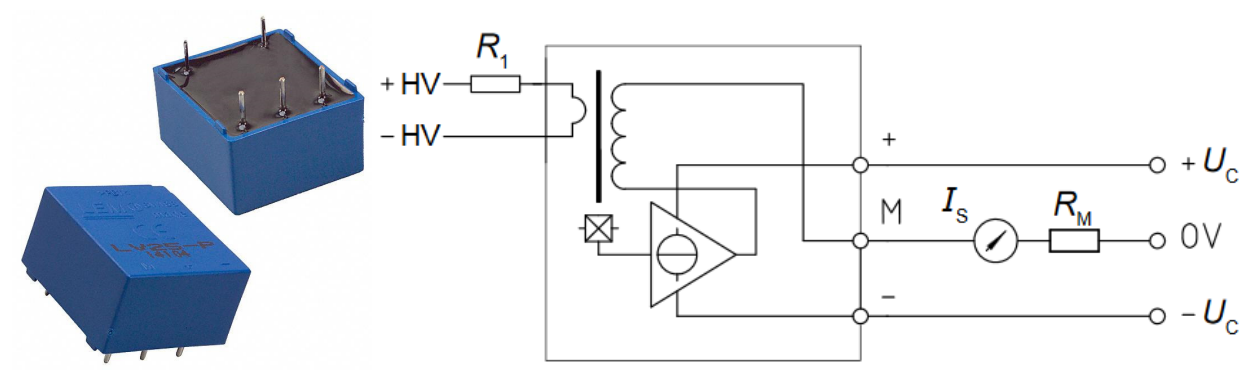

Figure 2.3. LEM LV 25-P Voltage Transducer.

\subsubsection{Charging and Diagnostic Bus Connectivity}

The ESMC is connected to the load via two switches to couple and decouple the output load on the DC bus. One of the major flexibilities added by the ESMC is its versatility in the charging bus to operate over a very wide range of voltages to accommodate various ES devices. Two NO relays offer a connection or isolation from this bus depending on the operating scenario. This bus can provide the charging current for multiple ES in parallel, or the isolation of a single ES device where diagnostics can be performed. This feature is particularly useful for batteries where monitoring the $\mathrm{SoH}$ is crucial for ensuring efficient operation. In this case, diagnostic signals can be sent directly to the battery to evaluate its performance or individual SoH. This useful feature can allow an operator to initiate test procedures and identify a consistently failing battery while the system is running. These relays are interlocked with the other relays to prevent the simultaneous charging or discharging of the ES device or module. 


\subsection{Operation Modes}

The ESMC can operate in three distinct modes: Normal Operation, Charging, and Ideal. The following subsections discuss each mode in detail.

\subsubsection{Normal Operation (Discharging)}

In order to achieve full isolation, two $\mathrm{NC}$ relays connect the positive and negative terminals of the ES to the DC bus. A NO relay bypasses the ES to provide an alternative path to maintain a continuity of supply. An interlock is provided between the three relays to avoid the simultaneous connection. In this mode, the positive and negative bus relays are closed and the bypass relay is open as shown in Figure 2.4.

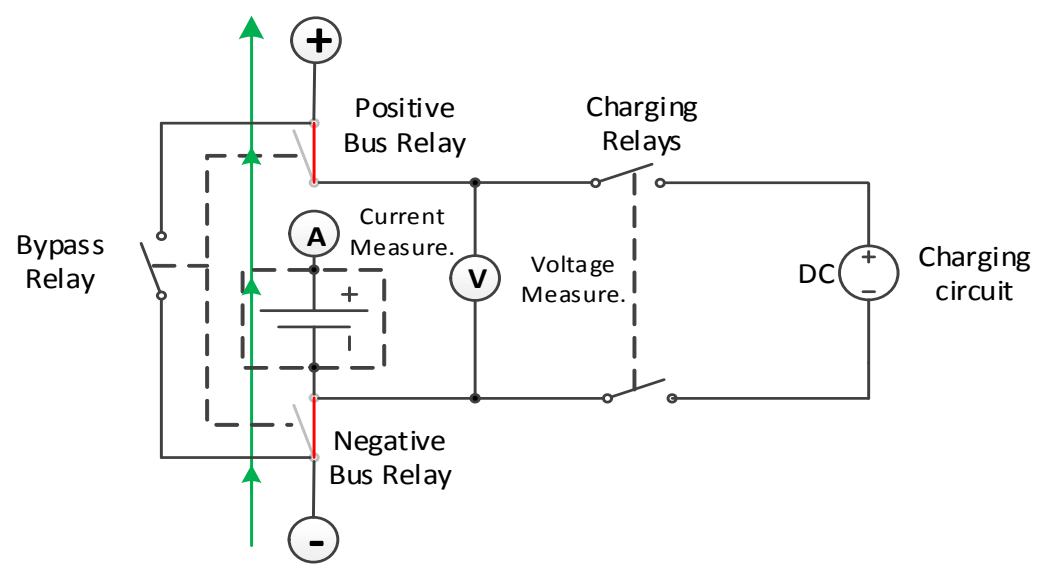

Figure 2.4. Energy Storage Management Controller in Normal Operation.

\subsubsection{Charging Mode}

One of the important features of the ESMC is the capability to charge one ES element while the rest of the array remains in normal operation. In this mode, the positive and negative bus relays are open, while the bypass relay is closed to offer an alternative path for the current to flow. After an adjustable short delay, the ES element is connected to the charging circuit via the charging relays. Figure 2.5 depicts the path of the current in this 
mode. In this case, the ES device is connected to the Charging Circuit in order to be charged. The operator can choose to apply either constant or pulsed charging.

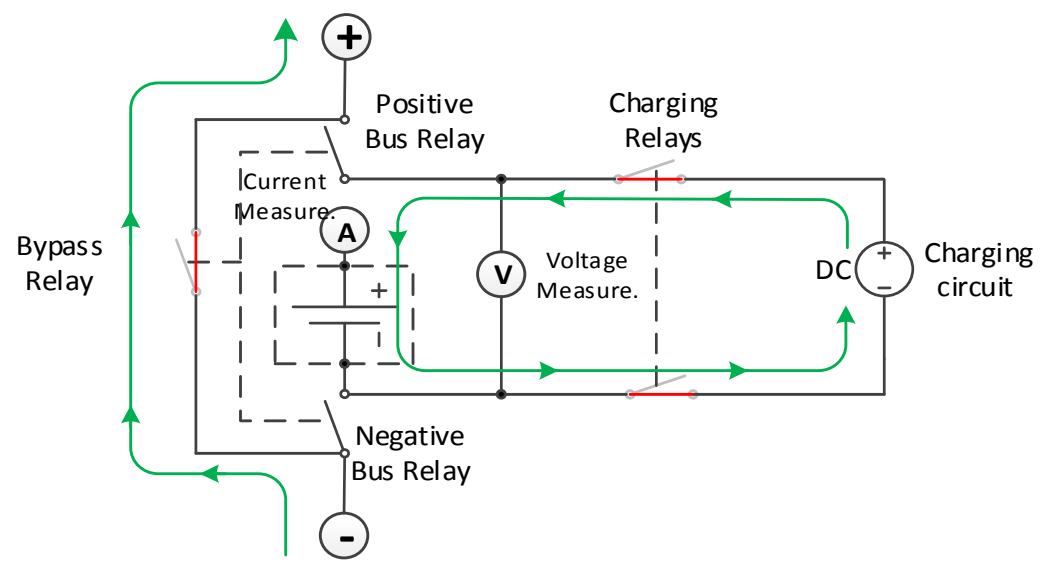

Figure 2.5. Energy Storage Management Controller in Charging Operation.

\subsubsection{Constant Charging}

The Constant Charging mode presents a basic connection to the Charging Circuit where current flow is limited by two major components: the charging source and the number of ES devices operating in Charging mode connected to the same bus. In the event that multiple ES devices are placed in charging mode and are connected to the same supply, the current flow behavior will become highly nonlinear. The magnitude will be a result of many factors, most notably the internal resistance of the ES device. This is a factor of the type of source and, in the case of batteries, its SoH and SoC. For this reason, in these scenarios, pulsed charging can be implemented to provide a better balance of the current flow amongst ES devices.

\subsubsection{Pulsed Charging}

In the Pulsed Charging mode, relays are switched periodically at a designated 
frequency and duty cycle that can be set by the user. Pulsed charging provides a number of new capabilities to the user. First, as was discussed previously, balancing current amongst multiple ES devices on the same fixed charging bus can be accomplished by identifying the optimal pulse frequency and duty cycle for both ES devices connected to the charging bus. Secondly, as discussed earlier, in the case of a damaged battery, pulsed charging can be utilized in an attempt to improve the SoH of the ES device, particularly in lead acid batteries. This is particularly useful as it can break a layer of sulfate from flooded lead acid battery cell plates. The operation of the lead acid battery, the causes of sulfation, and initial experimental testing results are discussed in greater detail in the following chapter.

\subsubsection{Ideal Mode}

This mode may be utilized for maintenance purposes or enable the possibility of online ES replacement by offering complete isolation. This topology is as depicted in Figure 2.6 where the positive and negative bus relays are open to isolate the ES device from the DC bus. The charging relays are open as well to isolate the ES device from the charging circuit, while the bypass relay is closed to provide an alternative path for the current to flow.

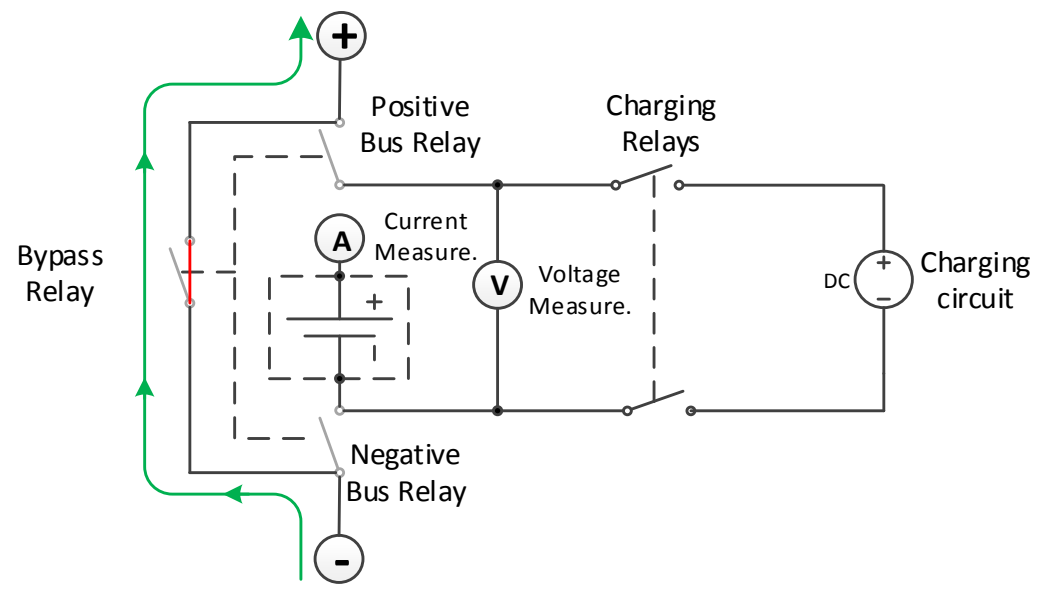

Figure 2.6. Energy Storage Management Controller in Ideal Mode. 


\subsection{Development}

Figure 2.7 depicts the implemented laboratory prototype for an ESMC unit that is fabricated on a $10 \times 16 \mathrm{~cm}$ printed circuit board (PCB), which reflects the compactness and simplicity of the proposed design. The components on the board are numbered in the figure as follows: 1) bypass relay, 2\&3) positive and negative DC bus relays, respectively, 4) freewheeling diode for DAQ device protection [64], 5) positive and negative ES terminals, 6) the LA 25-NP current transducer, 7) the LV25-P voltage transducer, 8) positive and negative DC bus terminals, 9) charging bus relays, and 10) positive and negative charging bus terminals. A unified color coding is followed for all terminals, red for positive and black for negative [65]. In order to test and verify the applicability of the developed design, a testing platform consisting of 4 ESMC controllers were setup in our laboratory.

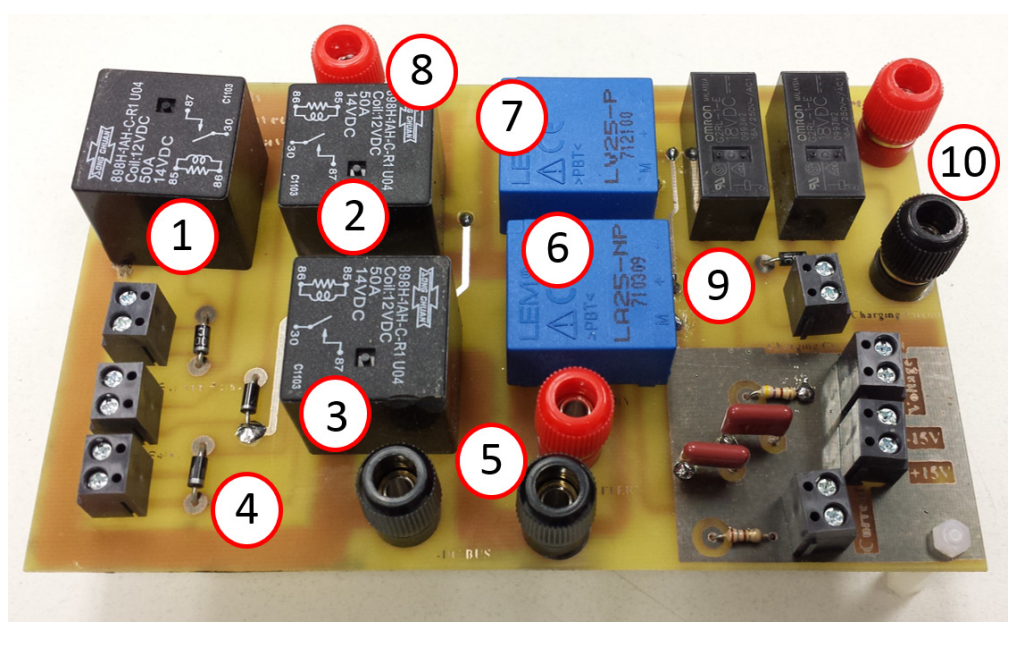

Figure 2.7. Energy Storage Management Controller Unit Prototype.

\subsubsection{Hardware Setup}

Four complete ESMC units have been built in our laboratory and the hardware setup is shown in Figure 2.8. The system features 4 ESMC units connected in series to support a 
48 VDC bus, which is widely used in telecommunication applications [66]. This system will be utilized in the following chapter to conduct a series of tests on a lead acid battery array. In this scenario, a DC-DC converter is used to maintain a constant bus voltage during switching or in the event a reduced array (e.g. one or more batteries decoupled from the stack). The input and output voltages to the DC-DC converter are shown on two Fluke 289 multimeters from left to right, respectively. The current bus voltage is at $49.002 \mathrm{~V}$. Only two batteries are being connected to the DC-DC converters to serve the bus. Three commercially available DC-DC boost converters are connected in parallel to handle an output current up to $30 \mathrm{~A}$. The input battery bus voltage is only $20.107 \mathrm{~V}$.

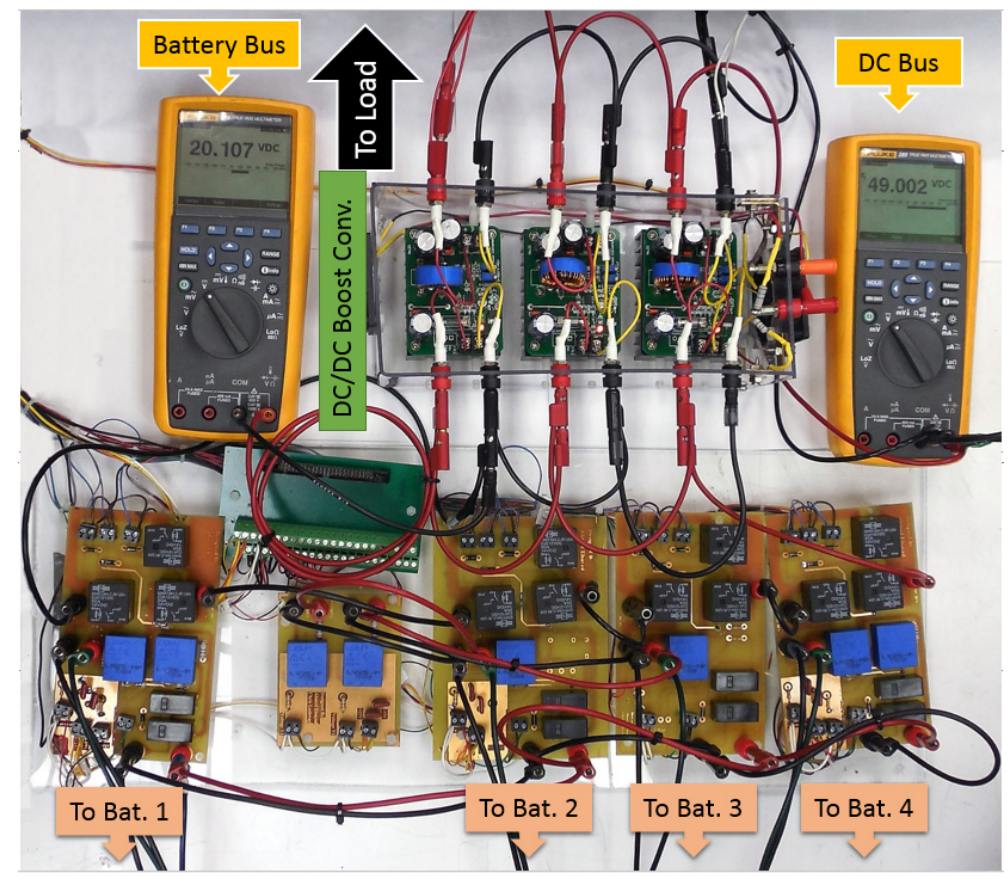

Figure 2.8. Hardware Setup with 4 ESMC Units.

\subsubsection{Control Platform}

The ESMC hardware and software control platform to support 4 ESMC devices is shown in Figure 2.9. Voltage and current measurements are collected from each ESMC 
and hardwired to a National Instruments NI-9206 DAQ module [65]. This module is a 32channel single ended/16-channel differential analog input module. The voltage and current on the low and high voltage sides of the boost converter are measured as well. Control commands are passed to the switching relays from a NI 16 Digital Input/16 Digital Output channel module. The two modules are connected to a PC via a NI 9174 4-slot USB chassis. Real time measurements are then provided to the operator scaled to the appropriate level to provide synchronized control and monitoring of the relays during coupling and decoupling of the DC and charging buses.

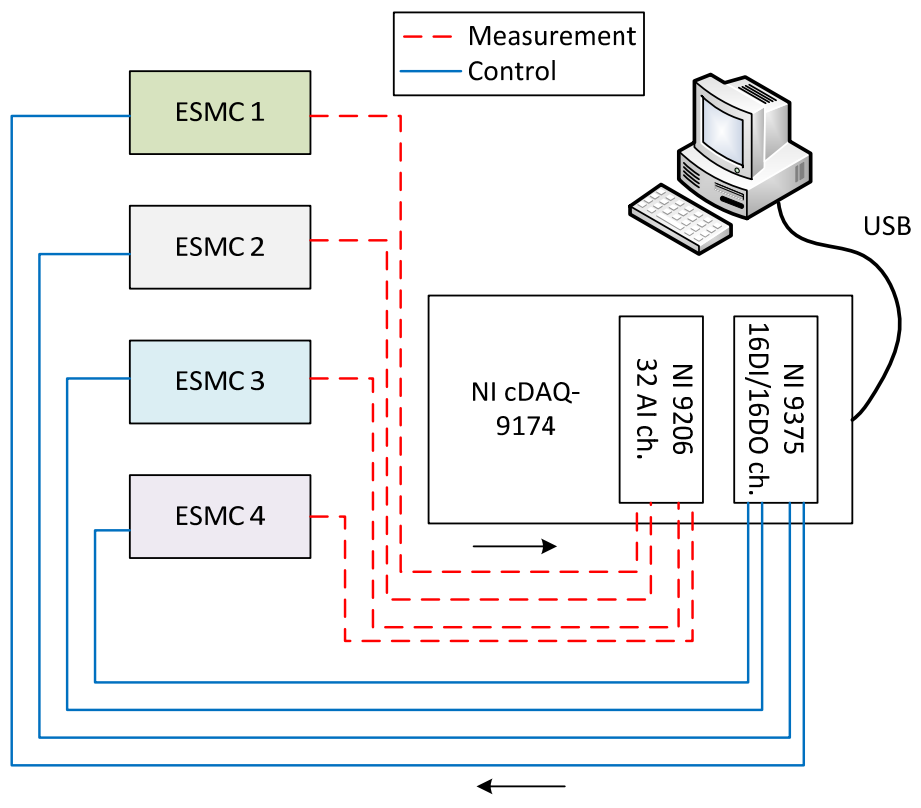

Figure 2.9. ESMC Hardware/Software Control Platform.

\subsubsection{Graphical User Interface}

An initial graphical user interface (GUI) has been designed to be clear and user friendly. The display is split between two distinct control panels: the Main Load and System Control and Individual ESMC Control Interfaces. The following subsections will discuss both in detail. 


\subsubsection{Main Load and System Control}

The main system control panel provides the general status of the load bus as well as the input from the ESMC array and is shown in Figure 2.10 with the following features numbered: DC Bus parameters and a graph depicting the input (1) and output voltages from the DC/DC converter (2) as well as the load current (3). Several controls are provided to operate the load in either constant or pulsed mode (4) where the frequency (5) and duty cycle (6) of the pulsed load can be controlled. The main load and system control panel also allows the user to set absolute maximum values for overcurrent (7) and overvoltage (8) protection with an alarm indicator (9) as well. Since the platform is designed for DAQ, the user can select the path where the data is to be saved (10) and toggle the data logging operation (11).

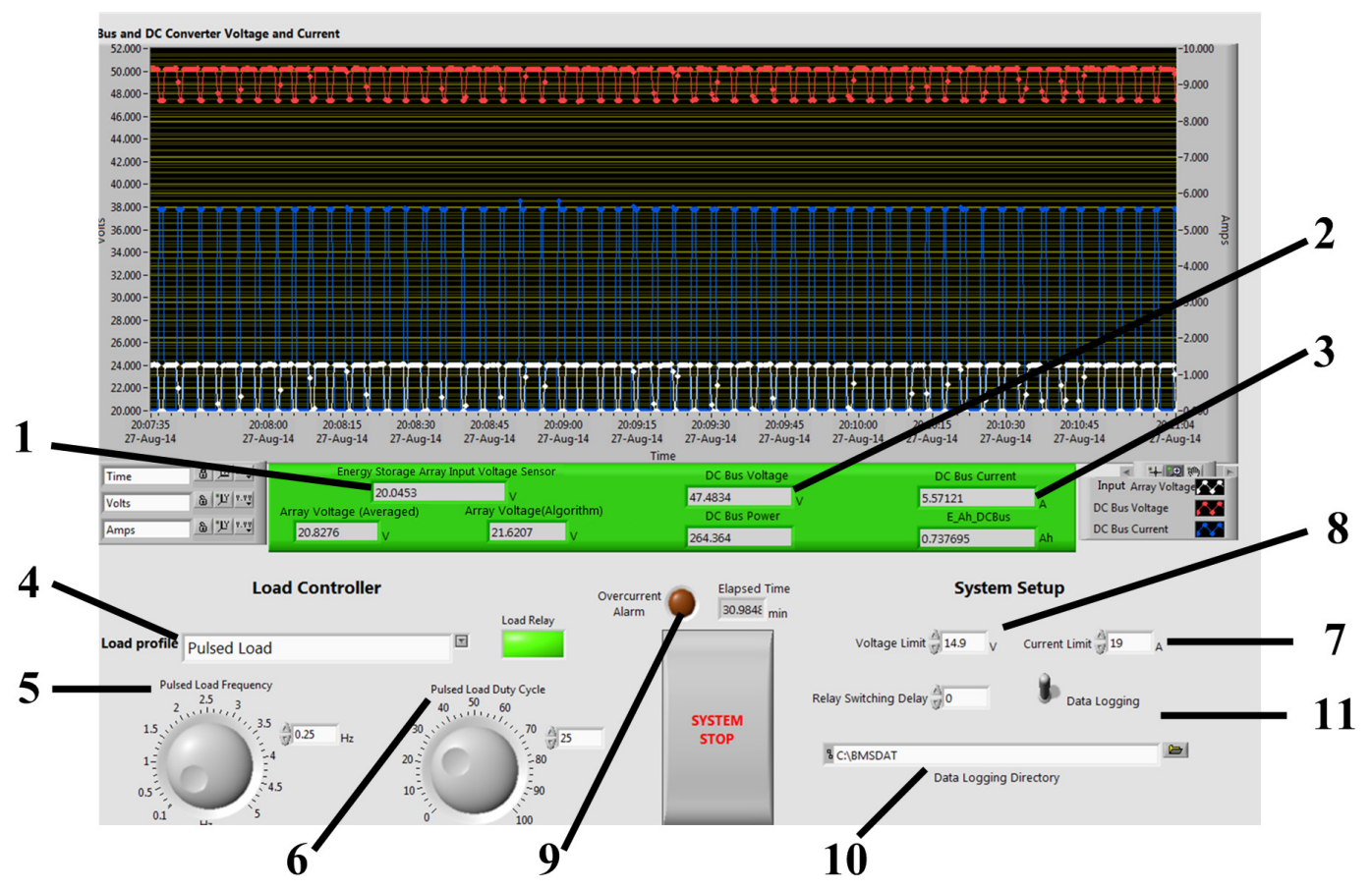

Figure 2.10. ESMC Main Load and System Control GUI. 


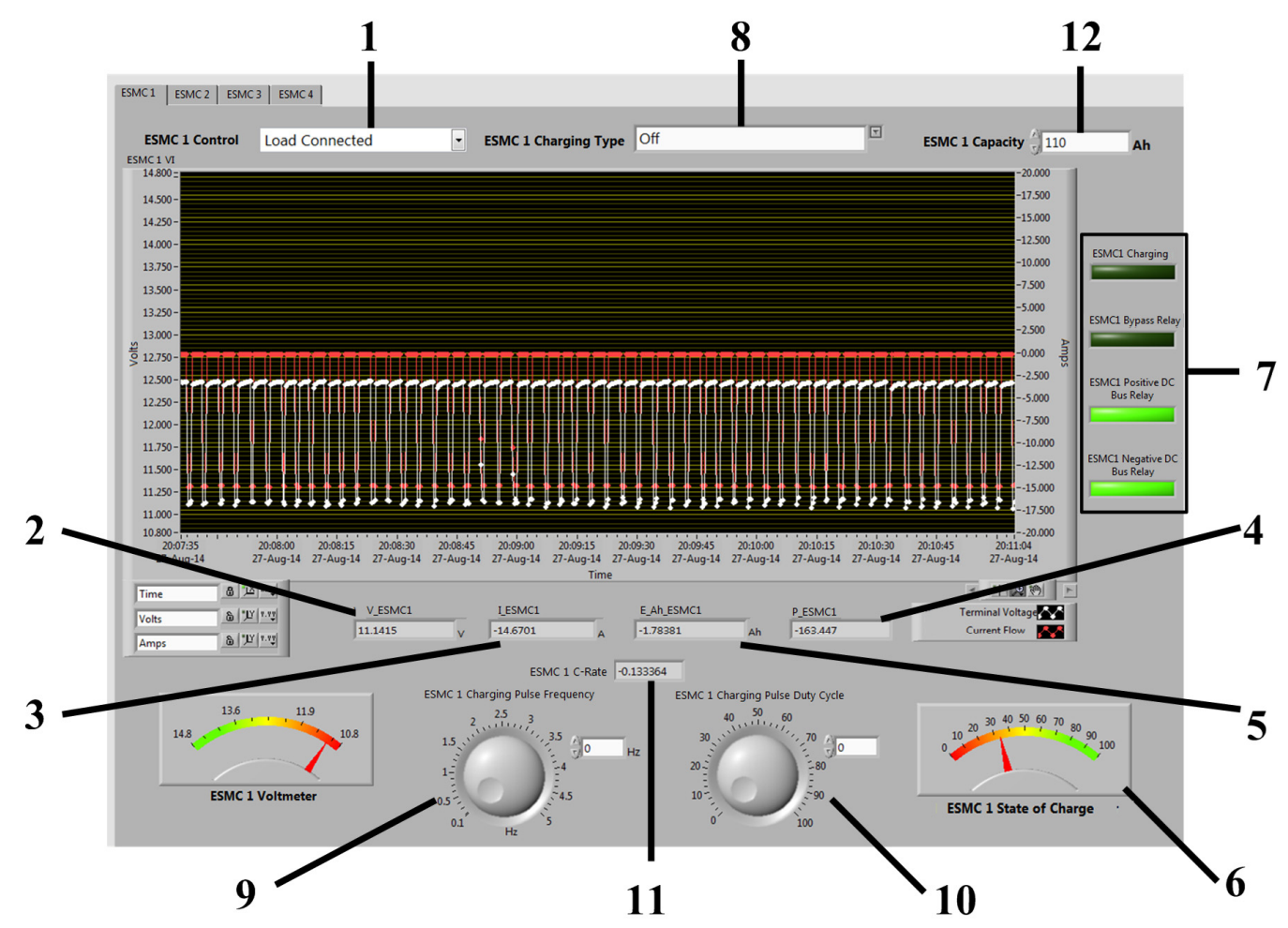

Figure 2.11. Individual ESMC Control Interface GUI.

\subsubsection{Individual ESMC Control Interface}

In the second panel, an individual ESMC device is tabbed out allowing the user to monitor the status and control each ES device individually. The individual ESMC Control Interface is shown in Figure 2.11 with the following features numbered: the operational mode setting (1) and crucial measurements of Voltage (V) (2), current (A) (3), power (W) (4), Ampere-hour (Ah) energy indicators (5) and SoC (6) are provided for each ES device. On the control front, the status of each relay is provided for verification and troubleshooting (7). The user has the ability to designate an independent charging method for each ESMC (8): Off, Constant Charging, and Pulsed Charging, where pulsed charging knobs allow the user to select the frequency (9) and duty cycle (10) of the pulse. The Coulombic or C-rate 
(11) measures the current into or out of the ES device with respect to its capacity setting (12) and is a particularly useful value when monitoring battery ES as it can be used as a metric to analyze performance. As the C-rate increases, the available usable capacity in a battery will decrease. This value is most useful in the discharge phase as it will influence run-times, operation voltage ranges, and $\mathrm{SoH}$ progression. This is discussed further in Chapter 3.

\subsection{System Expandability for Commercialization}

In this section, the concept of the ESMC is expanded to make it more suitable as a commercial product. A US patent was approved on 15 August 2017 (US Patent Number: $14 / 848,711$ ), which analyzed a number of features within the design and recommended alternative configurations or components to reduce the cost and size of the proposed prototype. Five major areas have been reviewed extensively, where hardware and software improvements have been investigated and are vdiscussed in the following subsections.

\subsubsection{Voltage and Current Sensor Upgrades}

In the developed prototype, voltage and current measurements were accomplished using two Hall Effect sensors, but as mentioned previously, a large percentage of the prototype cost is owed to these components. Alternative methods have been investigated to reduce the cost while still maintaining measurement accuracy. In order to lower the cost of the system, an alternative measuring circuit has been investigated where a high level schematic version is shown in Figure 2.12. For the current sensor, a precision shunt resistance is used $R_{s}$ where the voltage drop is fed to an AMC 1200 optically isolated differential amplifier and a small load resistance $\mathrm{R}_{\mathrm{L}_{\mathrm{I}}}$ is placed in series [67]. The current through $\mathrm{R}_{\mathrm{L}_{\mathrm{I}}}$ is then proportional to the current across $\mathrm{R}_{\mathrm{S}}$ where the voltage drop $\mathrm{V}_{\mathrm{I}}+, \mathrm{V}_{\mathrm{I}}-$ 
is measured and fed to a microcontroller unit (MCU). The AMC 1200 is also an applicable alternative in the voltage sensing application. In this case, the voltage across the ES is passed through a voltage divider circuit utilizing high resistances $\mathrm{R}_{1 \mathrm{~V}}, \mathrm{R}_{2 \mathrm{~V}}$. Contrary to the basic circuit topology outlined in Section 2.4.3, in this case, it is referred to an isolated ground $\left(\mathrm{GND}_{1}\right)$. The current through $\mathrm{R}_{\mathrm{LV}}$ is proportional to the voltage across the ES terminals where the differential voltage $V_{V}+, V_{V}-$ is measured and fed to the MCU. These circuits are based on commercial-off-the-shelf isolated amplifiers and can achieve a total cost reduction of around $70 \%$.

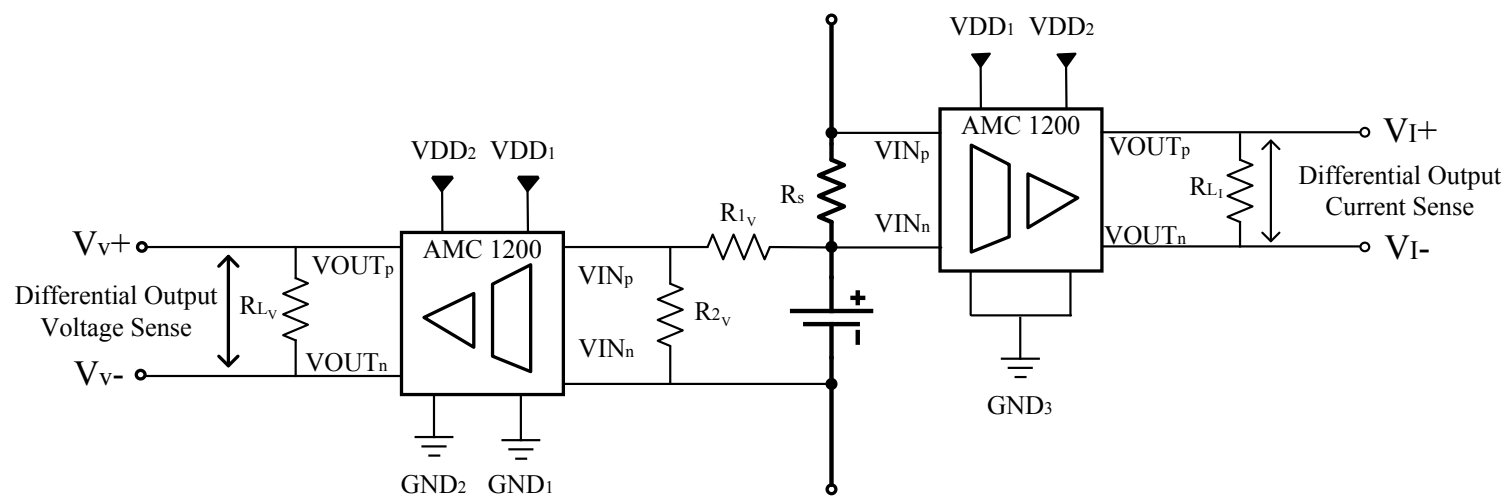

Figure 2.12. Reduced Cost Voltage and Current Measurement Circuit from ESMC.

\subsubsection{Switching Component Upgrades}

In the existing prototype, the switching actions were accomplished by electromechanical relays; however, these pose a number of challenges in a commercial device. In order to further reduce cost and particularly, the weight and overall footprint of an ESMC device, relays can be replaced with high powered metal oxide semiconductor field effect transistors (MOSFETs). The usage of these devices would also reduce parasitic power consumption as well. 


\subsubsection{Thermocouple Addition}

All ES devices are influenced by temperature fluctuations that can impact their performance. Electrochemical ES devices, in particular, are sensitive and in some cases such as in lithium ion batteries, temperature regulation is essential when attempting to charge at voltages near their maximum level. Without precise measurements of voltage, current, and temperature, a generation of solid lithium metal can result in the production of carbon dioxide causing a dangerous thermal runaway condition [68]. Aside from the internal operating temperature, small variations in the ambient temperature can also have a strong influence on SoC deviation, which is investigated in detail later in Chapter 4. For these reasons, a thermocouple is included in the commercialized version of the ESMC where the current voltage and current protection schemes are updated to include thermal limits on the ES device.

\subsubsection{Control Enhancements}

The current control algorithm was designed for and is running on a PC-based system. However, in order to achieve autonomous operation, major modifications need to be completed to the control algorithm. First, the control algorithm can be implemented on a MCU chip which is solely responsible for collecting voltage and current measurements from ES devices to calculate SoC. Based on measured and calculated quantities, the controller can take actions directly and simply have a master supervisory (or zonal) controller to exchange control commands. For example, in the case of a low SoC, the controller can either connect the ES to a charger or completely disconnect it from the array.

Initial testing and verification was completed implementing the ESMC control and monitoring software under a Qt Linux-based framework [69]. The software was 
programmed to run on a portable Beaglebone by beagleboard device running the Linux Ubuntu operating system. As shown in the high level schematic in Figure 2.13, the Beaglebone served as the supervisory controller for Zone $Z$. For future large scale implementations, an architecture like this is necessary to manage large ES arrays installed in various zones. This hierarchy could support a number of future zones that would be present in shipboard power systems and utility grid applications. Measurements from each ESMC are collected using a STMicroelectronics STM32 Discovery board MCU running on a $168 \mathrm{MHz}$ STM32F407 chip and transmitted to the supervisory controller via serial communication (RS-232) under a defined instruction set [70].

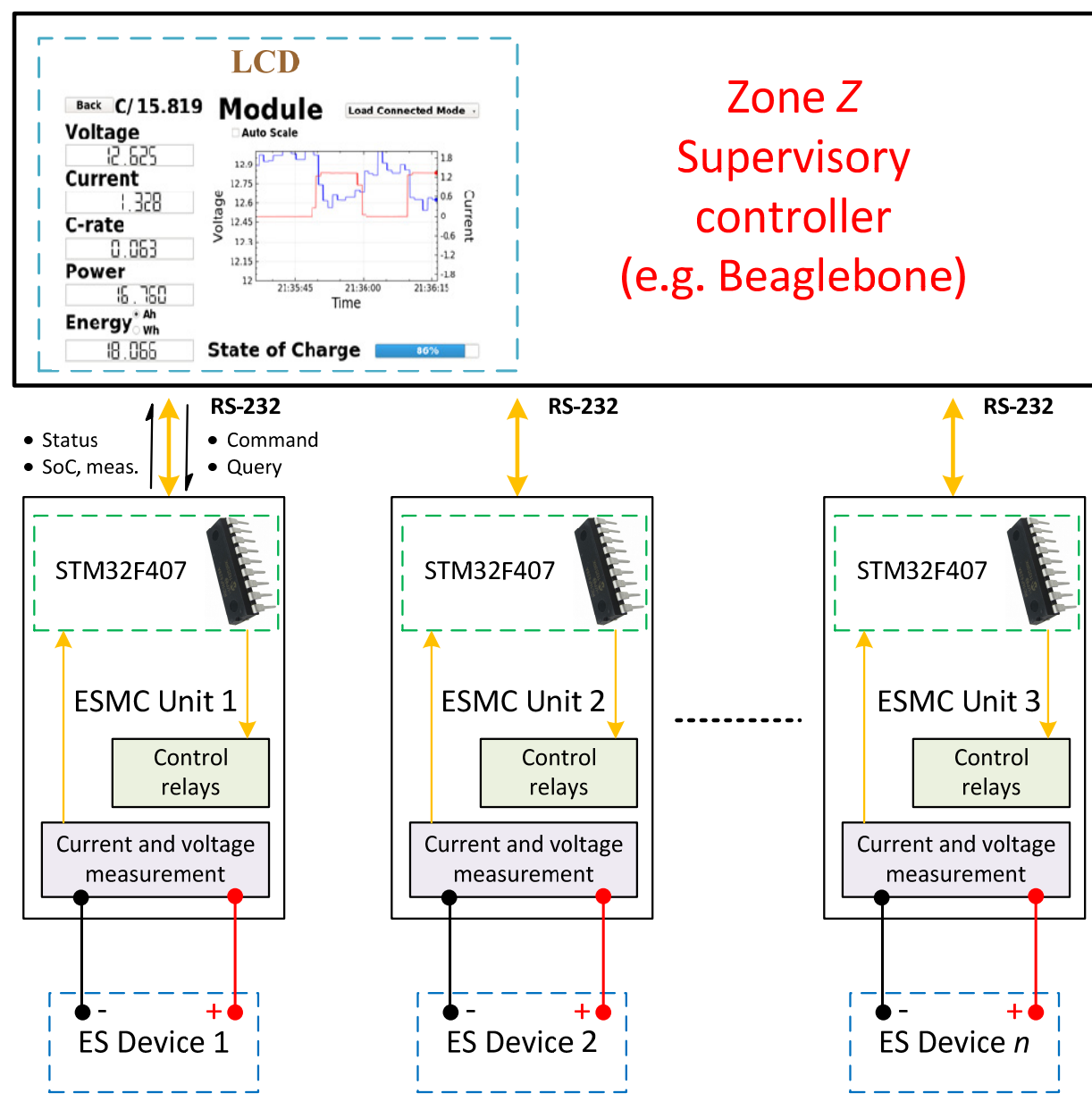

Figure 2.13. Hierarchy of the Proposed Upgraded ESMC System. 


\subsubsection{Graphical User Interface (GUI) Development}

The existing prototype system GUI was developed under a LabVIEW environment, however, a more portable GUI requiring less overhead is needed for commercial operation. To accomplish this, a beta version of a revised GUI software was tested operating under the Qt Linux application framework. The framework was developed for a touchscreen LCD screen to view live measurements, graphs, and status of the system and is shown at the top in Figure 2.13. The upgraded GUI provides a more simplified layout in a clear, easy, and flexible way to provide crucial performance details to the user.

\subsection{Summary}

In this chapter, a specialized ES management controller (ESMC) was developed, discussed, and compared to state of the art BMS and EMS devices found in previous literature. The significance of the ESMC device was addressed in its unique capability to decouple an ES device from the load while still providing an alternative path for current to flow. In addition, a Charging Circuit connection provided charging, balancing, and other maintenance tactics to be orchestrated on a single ES device. The different components, modes, as well as its potential for future expandability and commercialization were discussed in detail. The ESMC provides a significant baseline platform for a number of chapters in this dissertation from individual to hybrid ES testing and evaluation to improving the management system in terms of $\mathrm{SoC}, \mathrm{SoH}$, and introducing autonomous features. In the following chapter, the first test of 4 series-connected ESMC prototypes managing $4-12 \mathrm{~V}$ lead acid batteries is evaluated and a wide range of experimental results are presented. 


\section{Chapter 3 Energy Storage Management Controller Testing on Lead Acid Batteries}

\subsection{Introduction}

The Energy System Management Controller (ESMC) proposed in the previous chapter is tested for is usage in a lead acid battery array in this chapter. The first particular focus has been placed on its integration with lead acid batteries as a result of their high susceptibility to lifetime, or State of Health $(\mathrm{SoH})$ impacts, a major topic of this dissertation. Currently, lead acid batteries are being widely deployed for their usage in grid energy storage (ES) due to their versatility and low cost, but are burdened by a number of factors which result in a declination of their performance. As a lead acid ages, its series resistance will begin to increase as it is susceptible to many factors that impact its lifespan [71]. Most notably, the number of cycles that it has charged and discharged, effects of temperature, and the types or levels of discharge currents it has been exposed to [72]. These factors lead to electrochemical changes inside the battery which result in a decreased usable capacity and inefficient charging.

In the following section, a basic review of lead acid battery operation is discussed as well as its basic equivalent circuit model based on Electrochemical Impedance Spectroscopy (EIS). Through the utilization of the EIS model, differences in the battery performance as it begins to age are reflected by varying component parameters within the circuit. EIS presents the first basic model of battery performance which offers a glimpse into battery lifetime or SoH, a topic which will be covered in deep detail in later chapters. To conduct adequate testing, four prototype ESMC devices were developed attached to 4 - 12V 110 Amp-hour (Ah) deep-cycle lead acid batteries. Some of the specialized features 
proposed by the EMSC are evaluated by conducting four important test cases. First, the SoC balancing example is demonstrated amongst series-connected batteries. Next, the ESMC behavior and handling under a fault is analyzed. Third, a management scenario of lead acid batteries operating under a heavy pulsed load is shown followed by a comprehensive endurance and robustness evaluation.

\subsection{Basic Equivalent Circuit Model for the Lead Acid Battery}

In this subsection, the basic operation of the lead acid battery is discussed as well as its basic electrochemical equivalent model based on EIS. Composed of lead (Negative) and lead dioxide (Positive) electrodes placed into an electrolyte reservoir of sulfuric acid, its operation is governed by changes in the sulfuric acid concentration. A fully charged battery has an electrolyte composed of approximately $60 \%$ sulfuric acid and a discharged battery is primarily composed of water. Discharging precipitates the removal of electrons from the sulfuric acid hereby resulting in the production of solid sulfate at the battery plates. The charging process removes the solid sulfate and places it back into the electrolyte, but the process is not $100 \%$ efficient and some mass remains.

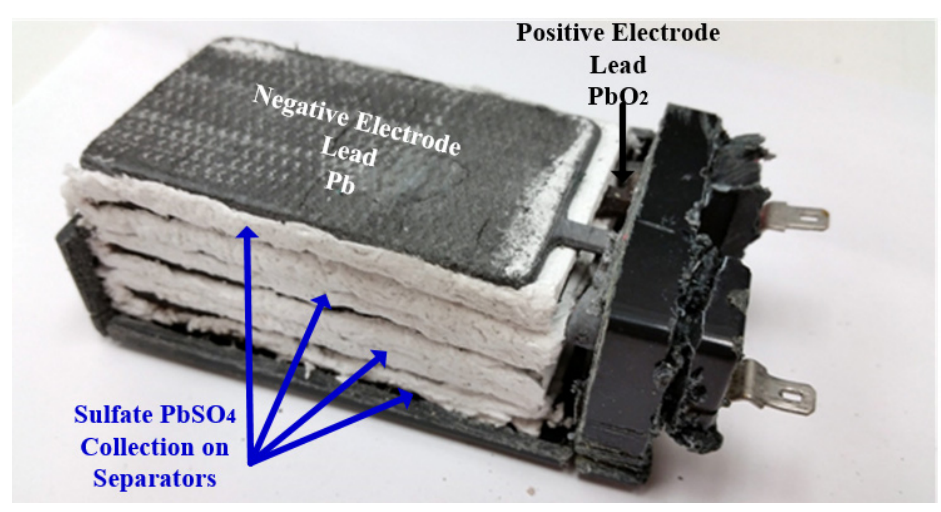

Figure 3.1. Deconstructed Single-cell Lead Acid Battery with Heavy Sulfation. 
Over many cycles, the sulfate mass increases and this phenomenon, known as sulfation, contributes as one of the largest culprits impacting reduction of $\mathrm{SoH}$ in terms of performance as well as a reduced usable capacity. An example of heavy sulfation production is depicted in Figure 3.1. In Figure 3.1, a fully discharged single-cell lead acid battery has been deconstructed revealing the collection of white sulfate $\left(\mathrm{PbSO}_{4}\right)$ on the separators, which provide the isolation between the positive and negative electrodes.

Some works have been published to find an electrochemical method to essentially break down the collection of sulfate at the battery plates [13],[59],[60]. If breaking down solid sulfate is successful, it can be introduced back into the electrolyte hereby increasing the usable capacity. The pulsing of high electrical current is analogous to repetitively pressing against the sulfate layer at a certain pulse frequency. The material stress associated with this can result in cracking of the sulfate layer. Through the application of pulsed charging using the ESMC, this process will be tested and evaluated.

Analyzing battery behavior with varying $\mathrm{SoH}$ with respect to a current pulse requires an extension from the common battery equivalent circuit to account for an impedance variance present at the interface between the electrode and electrolyte. EIS introduces kinetics to solve for three new parameters governing lead acid cell behavior [61].

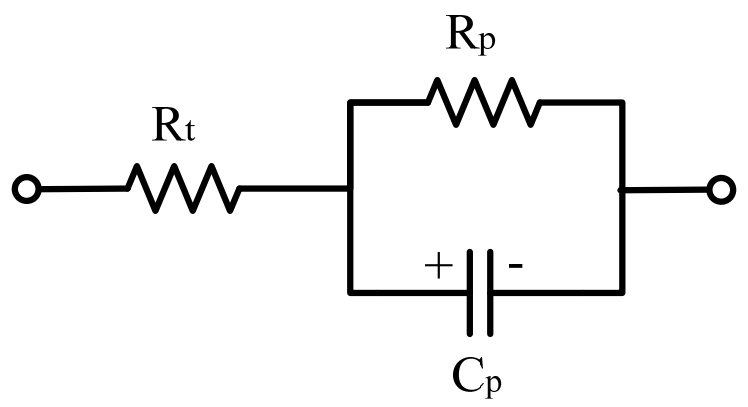

Figure 3.2. Basic Equivalent Circuit Model for a Lead Acid Cell Based on Electrochemical Impedance Spectroscopy. 
Shown in Figure 3.2, a modified resistor-capacitor (RC) circuit is placed in series with the resistance of the electrolyte $\left(\mathrm{R}_{t}\right)$ where $\mathrm{R}_{p}$ represents a resistive element in parallel with a non-faradic capacitance $\left(\mathrm{C}_{p}\right)$ value to explain the reduced absorption and extraction of electrons from the electrodes into the electrolyte. This single order model also provides the first basis in capturing the impulse response of a battery cell or module. The transfer function depicting the overall impedance is:

$$
Z(s)=\frac{\mathrm{R}_{p}}{\mathrm{C}_{p} \mathrm{R}_{p} s+1}+\mathrm{R}_{t}
$$

The voltage $V(t)$ response on the battery cell $V_{c}$ is:

$$
V(t)=V_{c}-\frac{1}{\mathrm{C}_{p}}\left[e^{-\frac{t}{\mathrm{C}_{p} \mathrm{R}_{p}}}+\mathrm{C}_{p} \mathrm{R}_{t} \delta(t)\right] i(t)
$$

where $C_{p}$ is chosen to model the capacitive response on the battery during and after a pulse, $\mathrm{R}_{p}$ controls the voltage drop following a pulse train, and $\mathrm{R}_{t}$ controls the steepness of the voltage drop $\Delta V$ in each pulse. A comparison is shown in Figure 3.3 where the solid and dashed lines depict pulsed load responses for a healthy versus damaged battery.

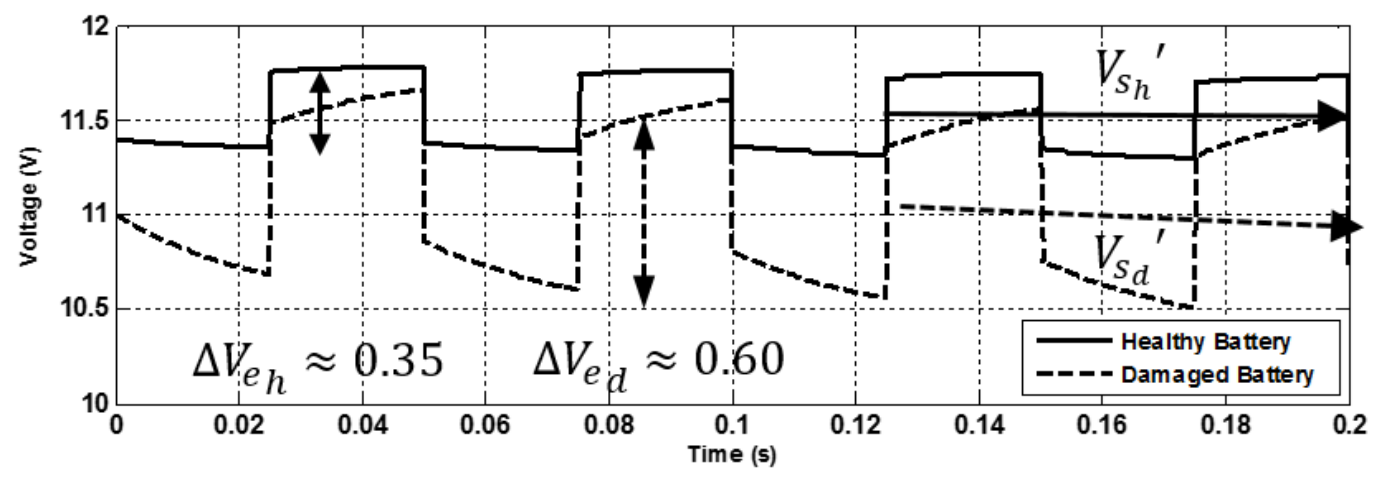

Figure 3.3. Pulsed Load Response on Healthy \& Damaged Battery. 
Minute adjustments are made to $\mathrm{C}_{p}$ to depict a reduced response and recovery time following a pulse on the damaged battery, but $\mathrm{R}_{t}$ and $\mathrm{R}_{p}$ represent the most prominent characteristics. The voltage drop after applying a pulse to a healthy battery $\Delta V_{e_{h}}$ increases significantly as the battery ages where a damaged battery voltage drop is much sharper $\Delta V_{e_{d}}$. In addition, impacts of the pulse train on a healthy battery $V_{s_{h}}{ }^{\prime}$ present a much gentler voltage slope than that of a damaged battery $V_{s_{d}}{ }^{\prime}$. The steeper downward trend is directly correlated to a SoC impact. Without accurate measurements for each battery, one would be unable to detect these characteristics.

\subsection{Lead Acid Battery Bank under Test}

A bank of deep cycle lead acid batteries has been utilized for experimental testing of the ESMC devices. The bank is pictured in Figure 3.4 and consists of 10 UPG 121100110 Ah 12V Lead Acid Batteries all with varying SoH levels [73]. In order to introduce a wide range of testing conditions, different combinations of these 12 batteries were selected for each experiment. Table 3-1 provides a summary of the specifications for each battery. Although at a conservative Coulombic (C-rate) of C/20 (a 20-hour discharge), the full discharge can theoretically source $110 \mathrm{Ah}$ of energy; this value is reduced as the discharge current increases. This is a common phenomenon which is present in all batteries and one which is particularly of a concern in the lead acid battery, which carries with it a significant drop-off as the discharge current increases. Table 3-1 reveals how as the discharge current increases, the available energy decreases, where $40 \%$ of the nameplate capacity is reduced at the maximum discharge rate of $1 \mathrm{C}$. 


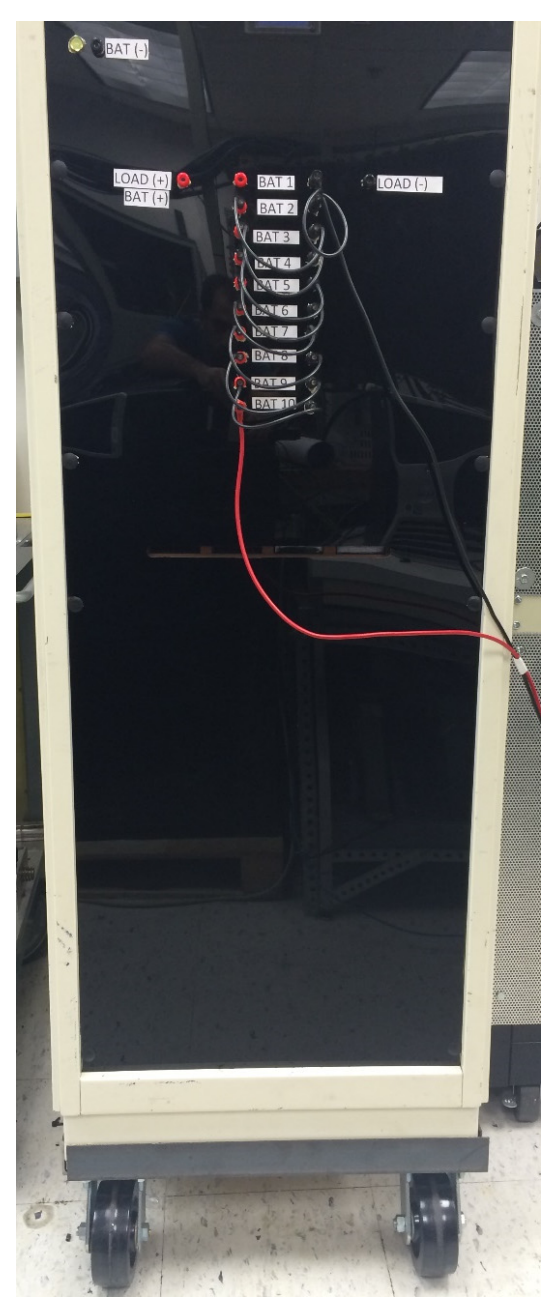

Figure 3.4. 10 - 12V 110 Ah Lead Acid Battery Bank.

Table 3-1. UPG 121100110 Ah 12V Lead Acid Battery Specifications.

\begin{tabular}{|c|c|c|c|}
\hline \multirow{4}{*}{ 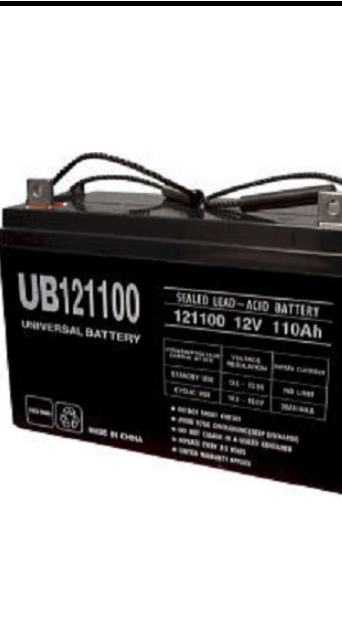 } & \multirow[b]{2}{*}{$\begin{array}{c}\text { Nominal Voltage } \\
\text { Nominal } \\
\text { Capacities } \\
\text { at } 25^{\circ} \mathrm{C} \\
* \text { Rated }\end{array}$} & \multicolumn{2}{|r|}{$12 \mathrm{~V}$} \\
\hline & & $\begin{array}{c}\text { C-Rate } \\
* \mathrm{C} / 20(5.5 \mathrm{~A}) \\
\mathrm{C} / 10(10.2 \mathrm{~A}) \\
\mathrm{C} / 5(18.7 \mathrm{~A}) \\
1 \mathrm{C}(66.0 \mathrm{~A})\end{array}$ & $\begin{array}{l}\text { Energy } \\
* 110.0 \mathrm{Ah} \\
102.3 \mathrm{Ah} \\
93.5 \mathrm{Ah} \\
66.0 \mathrm{Ah} \\
\end{array}$ \\
\hline & Charge & $\begin{array}{l}\text { Voltage } \\
\text { Current }\end{array}$ & $\begin{array}{r}14.4 \leq \mathrm{V} \leq 15.0 \\
\leq \mathrm{C} / 3(33 \mathrm{~A})\end{array}$ \\
\hline & Discharge & $\begin{array}{c}\text { C-Rate } \\
\mathrm{C} / 20 \\
\mathrm{C} / 10 \\
\mathrm{C} / 2 \\
1 \mathrm{C}\end{array}$ & $\begin{array}{r}\text { Cutoff Voltage } \\
10.5 \mathrm{~V} \\
10.2 \mathrm{~V} \\
9.6 \mathrm{~V} \\
7.8 \mathrm{~V}\end{array}$ \\
\hline
\end{tabular}


Another important aspect to consider is an adjustment of the full discharge cutoff, or the minimum voltage level to which the battery can operate safely without being over discharged. Although the cutoff defining the $0 \%$ SoC level at $\mathrm{C} / 20$ is $10.5 \mathrm{~V}$, an increased voltage drop under load will be present at higher loading currents as a result of high polarization and ohmic losses resulting in a lower terminal voltage. This topic is discussed extensively in Chapter 5, where the physics of the lead acid battery demonstrate the reason for this large voltage drop. Under heavy pulsed loading and particularly during the endurance test section, variances in the discharge voltage cutoff will be apparent.

\subsection{Energy Storage Management Controller Implementation}

In the following scenario, 4 ESMC devices are used to manage a $4-12 \mathrm{~V}$ lead acid battery system. The overall topology for connecting ESMC units to four devices is shown in Figure 3.5. Based on the range shown in Table 3-1, the charging bus voltage has been set to $14.7 \mathrm{~V}$. Although the current transducers installed on the ESMC have been tuned to accept the maximum charging current of $33 \mathrm{~A}$, the test current is limited to $16.5 \mathrm{~A}$ [62]. In Figure 3.5, the 14.7 V Charging and Diagnostic Bus is connected in parallel to all ESMC links. The terminals of the ES array are connected to a DC-DC boost converter to stabilize the Bus DC voltage. The converter is unidirectional as the charging of the batteries is accomplished through another bus. In order to validate the plausibility and prove the capabilities of the developed ESMC, ESMC units are tested experimentally under conditions similar to real world conditions. The four ESMC units are connected to lead acid batteries from Figure 3.4 containing varying $\mathrm{SoC}$ and $\mathrm{SoH}$ levels. Different scenarios are created with diverse events and load profiles. 


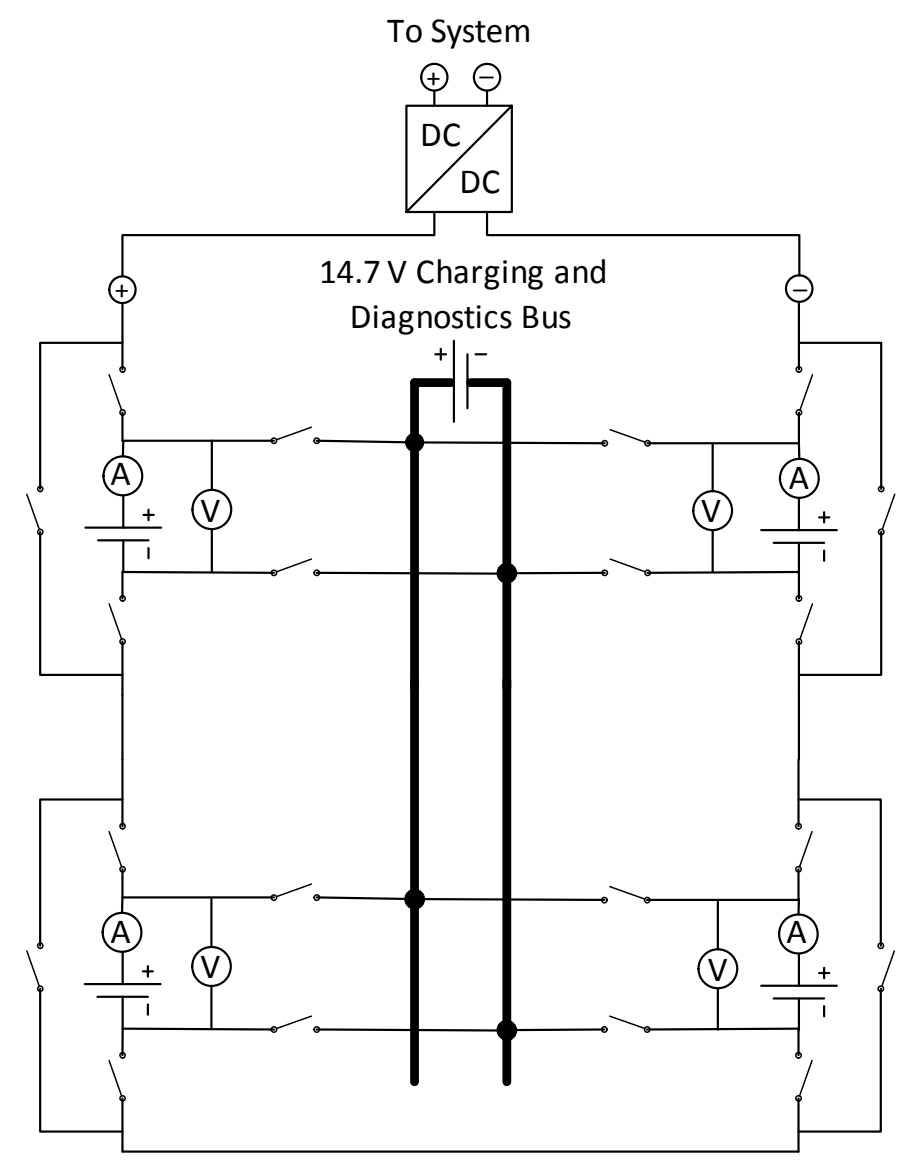

Figure 3.5. ESMC Implemented in a $4-12 \mathrm{~V}$ Lead Acid Battery Bank.

\subsection{State of Charge Balancing}

When more than one battery is placed on charge, current regulation is not possible and the magnitude of current absorbed by each battery will vary based on many characteristics. These range from a simple deviation in $\mathrm{SoC}$ to a mismatch in their internal impedance as was previously discussed in Chapter 2 . A drift in the internal impedance would allow the battery with the lowest impedance to absorb the highest level of current in the stack, thereby limiting the energy that is charged by the other batteries. However, with the individual charging control capability introduced by the ESMC, the operator can control the energy injected into each battery regardless of its individual characteristics. 
Consider a scenario as the one depicted in Figure 3.6. Initially, four batteries are connected in series to serve a load. At minute 5 , the SoC of two batteries (BATT3 \& BATT4) are decreased falling below a certain pre-specified threshold. Keeping a battery running with low SoC would increase its Depth of Discharge (DoD), DoD $=100 \%-$ SoC, and can cause permanent damage. To solve this, these two batteries are changed from Normal Operation to Charging mode. Figure 3.6(a) shows the energy withdrawn from each battery (negative sign) and the charged (positive sign) over a 1-hour operation cycle.

Both batteries are connected to regular constant charging at minute 30 due to the aforementioned reasons where both batteries were not withdrawing the same energy: battery 3 (BATT3) withdrew more energy than 4 (BATT4). Hence, in order to regulate the energy injected into each battery, a pulsed charging profile is applied to both batteries. The pulsed charging currents were under the same frequency $(0.25 \mathrm{~Hz})$, but utilized different duty cycles to control the average injected energy to the battery. The duty cycles were set to $25 \%$ and $75 \%$ for batteries 3 and 4 , respectively.

It can be seen that the slopes of the curves have changed indicating variation in the amount of absorbed charging energy. At minute 45, the injected energy to battery 4 exceeded the energy of battery 3 , so battery 4 is selected to be returned to the stack to share the load with batteries 1 (BATT1) and 2 (BATT2). Consequently, the slope of the energy curve changes to negative which indicates it is losing energy. The energies of batteries 1 and 2 are shown in Figure 3.6(a) with a negative slope over the entire operation cycle. It should be noted that connection, disconnection, and transition of a battery from one state to another are occurring seamlessly while the other two batteries continue to supply the pulsed load. The load pattern is depicted in Figure 3.6(d). 

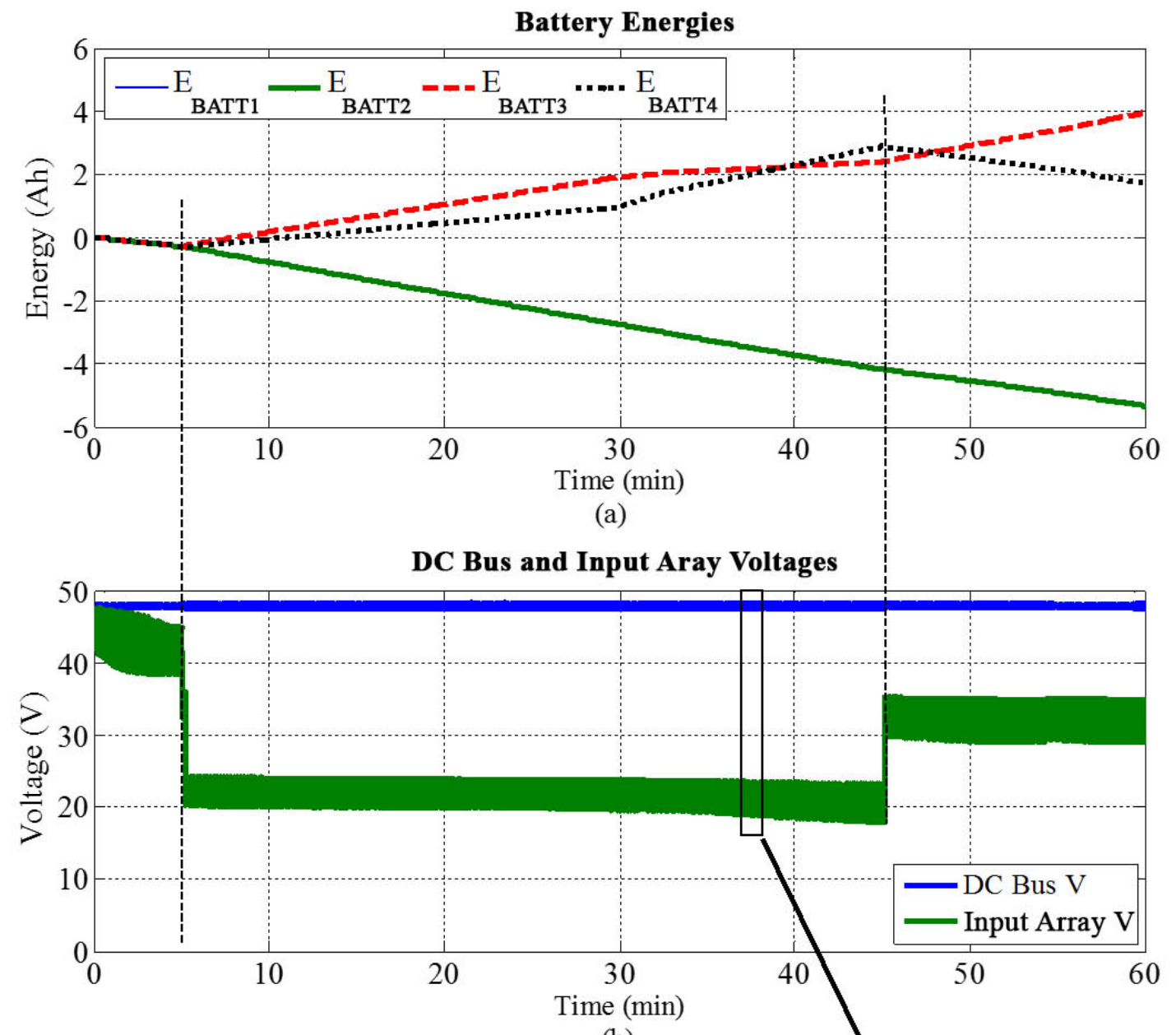

(b)

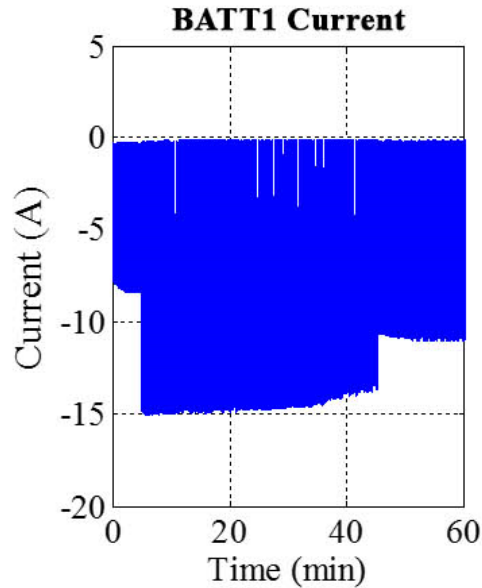

(c)

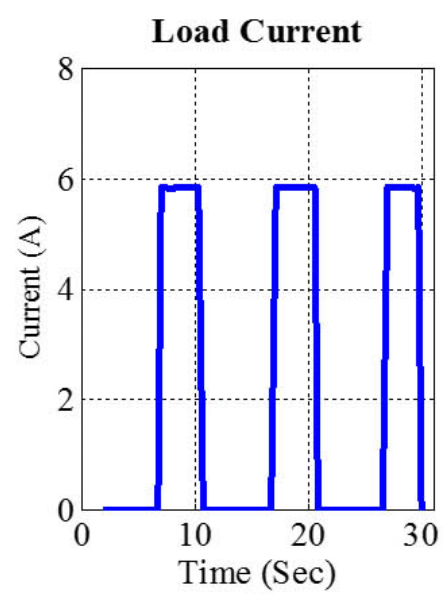

(d)

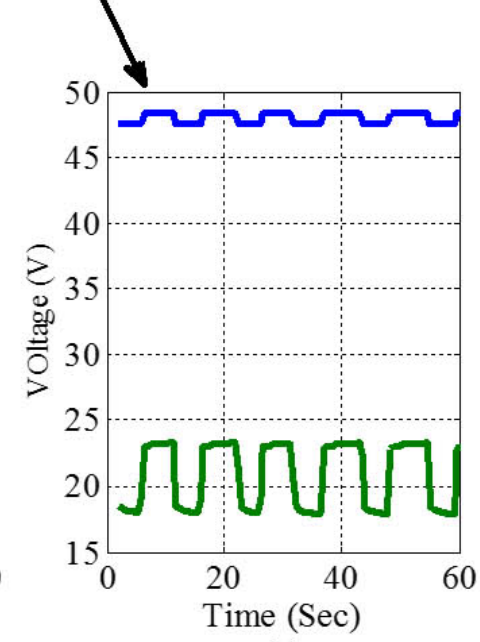

(e)

Figure 3.6. SoC Balancing Scenario (a) Battery Energies, (b) DC Bus and Input Array Voltages (c) Current of First Battery (BATT1), (d) Pulsed Load Current (e) DC Bus and Input Array Voltages (60s close up). 
The DC bus voltage and terminal voltage of the battery bank (input array) are shown in Figure 3.6(b). It can be seen that the voltage of the battery bank dropped from $48 \mathrm{~V}$ to $24 \mathrm{~V}$ when 2 batteries were disconnected. Returning the third battery to the stack brought the battery bank back to around $35 \mathrm{~V}$. High voltage fluctuations are associated with the pulsed load as shown in Figure 3.6(e). The DC-DC boost converter successfully regulated the DC bus voltage isolating any voltage variations from propagating to the load side. The current withdrawn from battery 1 is shown in Figure 3.6(c) which changed from $7 \mathrm{~A}$ in the first 5 min to $15 \mathrm{~A}$ between minutes 5 and 45 , then reducing to $11 \mathrm{~A}$ when the third battery was connected.

Pulsed charging provides a metric to not only regulate current into the battery, but also provide mitigation dynamics in the case of a faulty battery based on the EIS concept mentioned previously in Section 3.2. The pulsed charging capability is also introduced for usage in SoH mitigation. In the case of a defective battery, the pulsed charging concept can be tested at a variety of frequencies (up to $10 \mathrm{~Hz}$ ), currents (up to $10 \mathrm{~A}$ ), and duty cycles in an attempt to crack sulfation. In this case, the Charging and Diagnostic Circuit would be connected only to that particular battery where a maximum safe charging current is used to generate thermodynamic stress. It is worth mentioning that this added functionality not only allows balancing the $\mathrm{SoC}$, but also distributes the stresses on the batteries as those serving the load for longer periods can be put to charge, while the other charged units can be connected to the load, and so on.

\subsection{Fault Event}

A protection scheme is designed as an additional functionality for the ESMC. This scheme was designed in a way to protect the system and reduce interruptions. If a fault 
occurs in the charging circuit, the system disconnects the charging relays to isolate all ESMC devices from the 14.7 V Charging and Diagnostics Bus. The system is able to detect the fault location by checking the current sign, where a positive sign denotes a current injected into the battery. As shown in Figure 3.7, three batteries are connected to the charger, while only one battery (battery 1) is supplying a 0.9 A load. As shown in Figure 3.7(c), an event is created to increase the charging current in battery 4 where the current rises rapidly to 13.5 A reaching a pre-specified threshold.

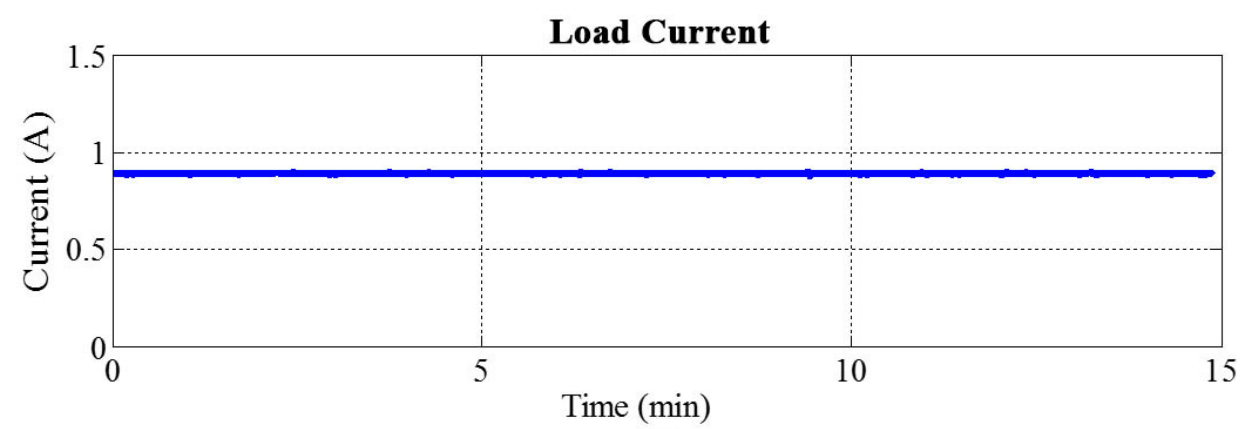

(a)

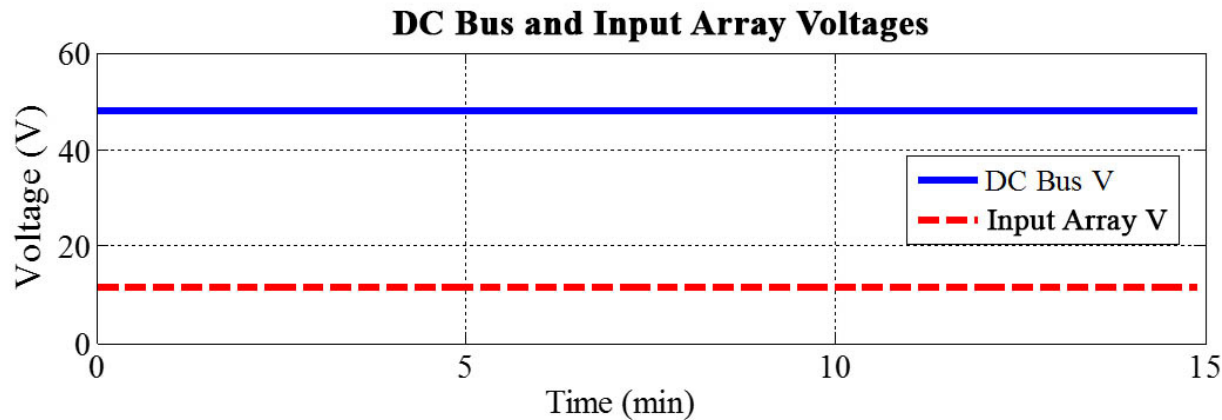

(b)

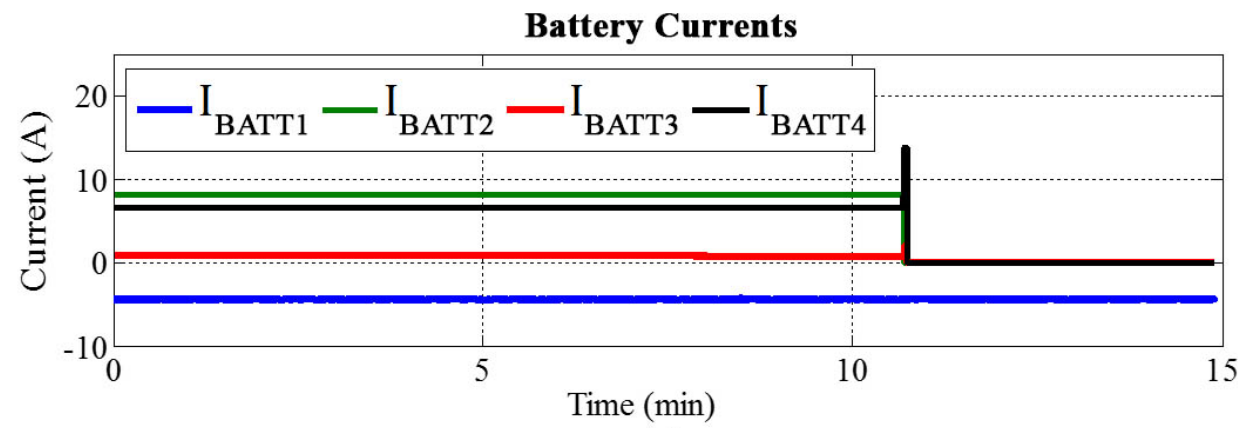

(c)

Figure 3.7. Protection against Fault Events in the Charging Circuit (a) Load Current (b) DC bus and Input Array Voltages (c) Current for the 4 Batteries. 
The system detected this case and sent an "off" command to the charging relays. The current of all the charging batteries fell to zero, while the current of the battery supplying the load remained unaffected. The charging relays for battery 1 are opened as well, but do not affect its operation in Normal Operation mode. Figure 3.7(b) reveals that the DC bus voltage remained constant. The input array voltage is constant at $11.4 \mathrm{~V}$, equal to the voltage of battery 1 .

This scenario demonstrates the effectiveness and reliability of the designed protection scheme. It should be noted that if the fault occurred at the load side, all ESMC units and the load would be disconnected. Also, it emphasizes the previously mentioned points and the importance of individual pulsed charging. When three batteries are connected to charging, each battery withdrew a different current. The charging currents were $8.1 \mathrm{~A}, 0.8$

A and $6.5 \mathrm{~A}$ for batteries 2, 3 and 4, respectively. The lowest charging current on battery 3 indicates a high internal impedance and low $\mathrm{SoH}$. The current withdrawn from battery 1 is almost four times the load current (considering the converter efficiency) as the voltage on the battery side is one quarter of that which is present at the load side.

\subsection{Discharging under Heavy Pulsed Load}

The performance of the ESMC is now investigated under a heavy pulsed load condition. A pulsed load requires very high power for a relatively short period of time [74]. These are often periodic, operating over a small percentage (duty cycle) of a period of 10 $\mathrm{s}$ or less. Although the pulse period is typically short requiring a relatively small amount of energy, reaching the power demand is challenging and places a great deal of stress on the ES sources. 


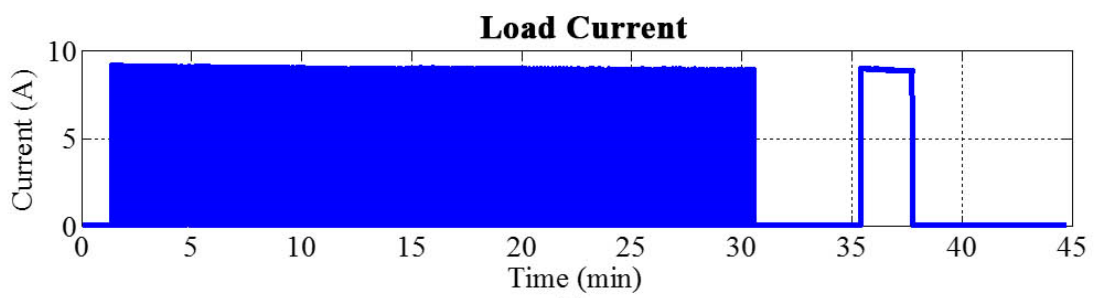

(a)

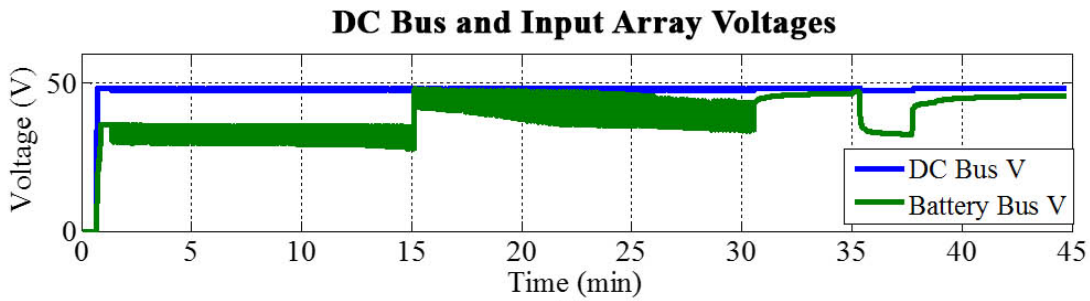

(b)

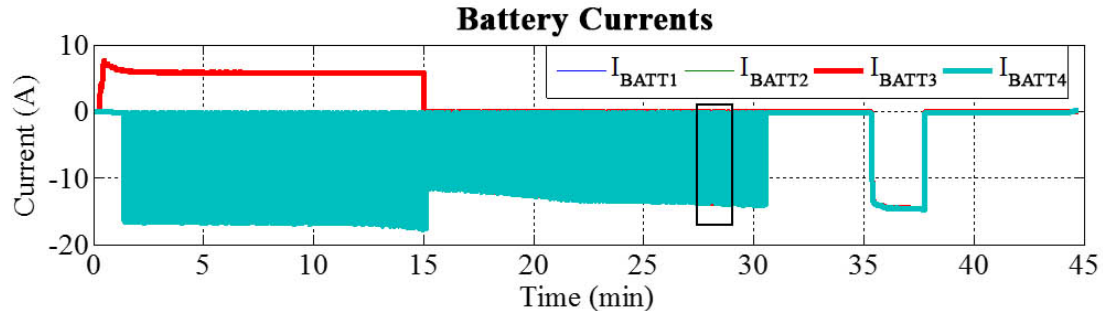

(c)

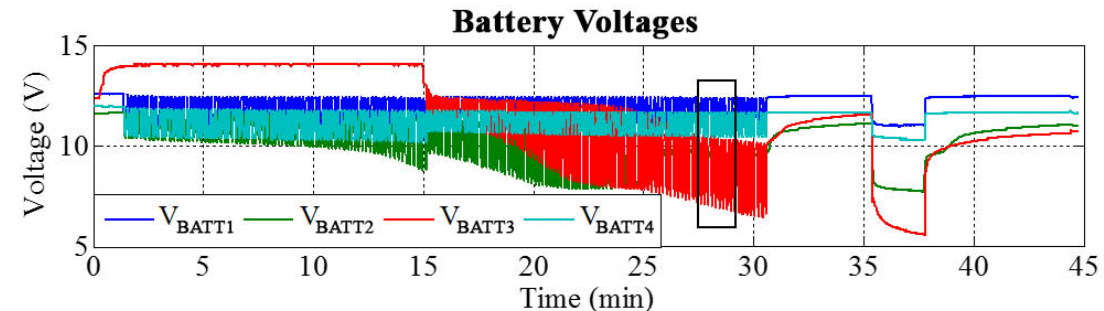

(d)
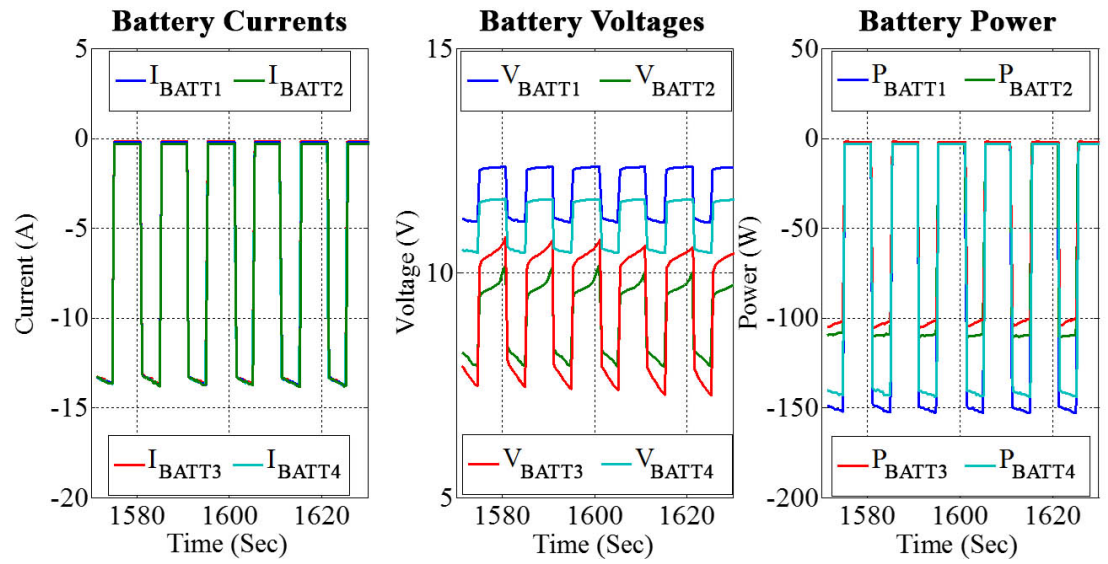

Figure 3.8. Discharging under a Heavy Pulsed Load (a) Load Current (b) DC Bus and Input Array Voltages (c) Battery Currents (d) Battery Voltages (e) Battery Currents (1min zoom from (c)) (f) Battery Voltages (1-min zoom from (d)) (g) Battery Power. 
The pulsed load test is important as its dynamics are present in a wide variety of applications which are addressed in this dissertation, from the utility grid to EVs and shipboard power systems. In this scenario, the pulsed load amplitude is set to $9 \mathrm{~A}$ at a frequency of $0.1 \mathrm{~Hz}$ and $50 \%$ duty cycle for approximately 30 minutes. The complete load profile is shown in Figure 3.8(a). It can be seen that the operation cycle is divided into three sections where each section is approximately 15 minutes long. In the first section, it was found that battery 3 had a low voltage due to excessive aging as opposed to the other batteries which reflects on its SoH and SoC. Hence, battery 3 was placed in charging mode to improve its SoC where a constant charging current of approximately $6 \mathrm{~A}$ is applied for the first 15 minutes. The other three batteries remained connected to serve the pulsed load. The terminal voltage of the stack is $36 \mathrm{~V}$ under no load dropping to $30 \mathrm{~V}$ during the heavy loading period under the pulse. The voltages of the DC bus and the input array are shown in Figure 3.8(b).

Figure 3.8(c) depicts a compilation of the currents measured from each of the four batteries. The first 15-minute segment shows the pulse being supported by only three batteries, or a $36 \mathrm{~V}$ bus. The levels of current contributed from battery 1 , battery 2 , and battery 4 are somewhat balanced at a high pulse current of $16.5 \mathrm{~A}$ at the battery side. This current corresponds to a C-rate of $\mathrm{C} / 6.67$, or a 6.67 hour discharge. Looking at the envelope formed by the amplitude of the pulsating battery currents, the current drawn from the remaining battery stack is stable for the first 15 minutes.

Following the charging of battery 3 , at minute 15 the battery is placed back in to serve the load, reducing each battery current to approximately 13 A. Initially, this reduces the burden and C-rates on the batteries, but as the system continues to operate, the current 
amplitude begins to expand demonstrating that a weak battery is still present in the system. To compensate for a continuously dropping voltage on battery 3 , the remaining batteries are forced to increase their currents to supply the required energy and fill the pulse. This is explained by looking at Figure 3.8(d). It can be seen that the voltage of battery 3 is drastically decaying. A close up of the voltage waveform is shown in Figure 3.8(f), where a steep decay of the voltage during the pulse is present indicating that the battery cannot sustain the heavy loading current for a longer period.

This rate of change can be used to determine parasitic parameters of the battery using the equations provided in Section 3.2 and EIS methods. A closer look into these figures reveal a wide range of information about the characteristics of the batteries and their expected lifetimes. The voltage profile of battery 3 (red) compared to that of battery 1 (blue) can give a realistic indication for relative differences in their characteristics. The voltage of battery 1 is consistent and steady. This can be used to easily identify an unhealthy or low-performing battery inside a large stack. The current and power injected by each battery are depicted in Figure 3.8(e) and Figure 3.8(g), respectively.

During a short rest period, it is shown that the voltages of batteries are recovering to their initial values. The slowest recovery rates were seen on batteries 3 and 4 . A final test is applied where a constant current of $9 \mathrm{~A}$ is applied for 2 minutes. Using the current sourced during the constant current stage in Figure 3.8(a), the load is essentially stable, but should be lower with all four batteries contributing. A load current of 15 A reveals that one battery is faulty. By analyzing the bus voltages in Figure 3.8(d), one can identify a weaker input array voltage as the slope of the voltage drop during the pulse is substantial. The voltages of batteries 3 and 4 drop to $6 \mathrm{~V}$ and $8 \mathrm{~V}$, respectively, far below the minimum 
discharge cutoff for a load of $\mathrm{C} / 5$ or greater as designated earlier in Table 3-1. Batteries 1 and 2 are relatively healthy.

The output DC bus voltage is shown in Figure 3.8(b) in green with respect to the input array voltage in blue. A closer look at Figure 3.8(b) in the pulsed load region is shown, demonstrating the stability of the DC bus and voltage variation present on the array input as the terminal voltage of the battery array transitions from $45 \mathrm{~V}$ to $33 \mathrm{~V}$. The input voltage depicts the voltage stabilization and number of batteries connected to the stack. In addition, though the DC bus voltage is impacted by the magnitude of the pulse, the voltage variation remains relatively small and within standard limits [75]. Thus, a change in the battery array size and input voltage only introduces a small deviation on the output DC bus voltage.

\subsection{Endurance and Robustness Test}

In order to examine the endurance and robustness of the developed prototype, it was put in operation for a continuous 40-hour test. Measurements were collected via LabVIEW and saved in a database. During this test, the system performed the different functions (supplying a load, pulsed charging, supplying a load again, and constant charging) without recording any issues or failures. The voltage and current data is shown in Figure 3.9(a) and Figure 3.9(b), respectively. Figure 3.9(c) shows a close-up of the voltage in the first 45 minutes of the test. The test was performed by selecting a battery with very low SoH. A secondary purpose was to investigate the effectiveness of pulsed charging.

It can be seen from Figure 3.9(c) that initially the open circuit terminal voltage of the battery was $8.5 \mathrm{~V}$. When a load was applied to the battery, the voltage dropped significantly reaching around $6.75 \mathrm{~V}$, far below the absolute minimum discharge voltage cutoff for the UB 121100. Pulsed charging was applied to the battery, injecting maximum current at a 
14.7 $\mathrm{V}$ charging voltage under a $1 \mathrm{~Hz}$ pulse frequency and duty cycle of $75 \%$. After charging, the same load was applied again, where the voltage dropped drastically in the beginning reaching around $7.1 \mathrm{~V}$. This test shows improvement in the battery voltage (around $+0.35 \mathrm{~V}$ ) due to a partial cracking of the accumulated sulfate layer.

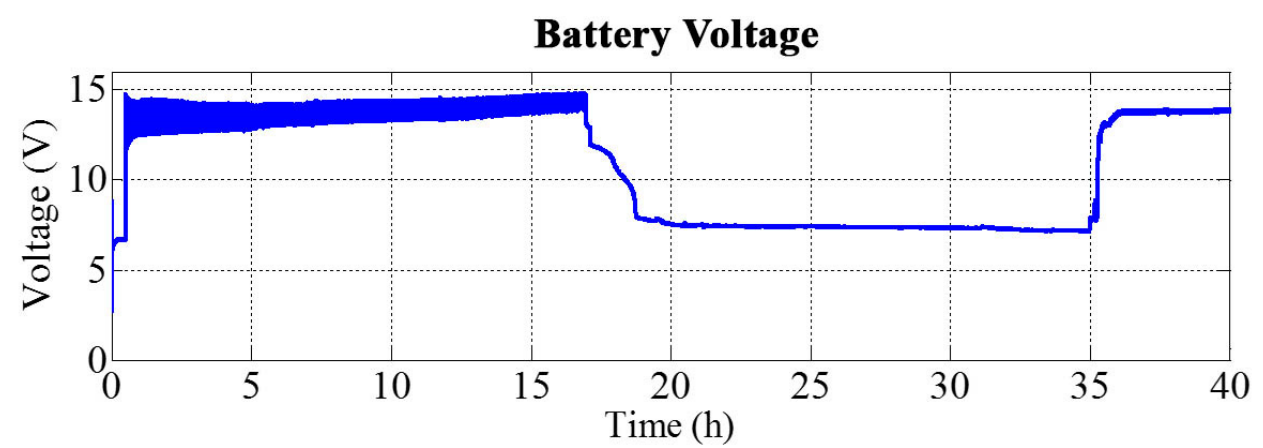

(a)

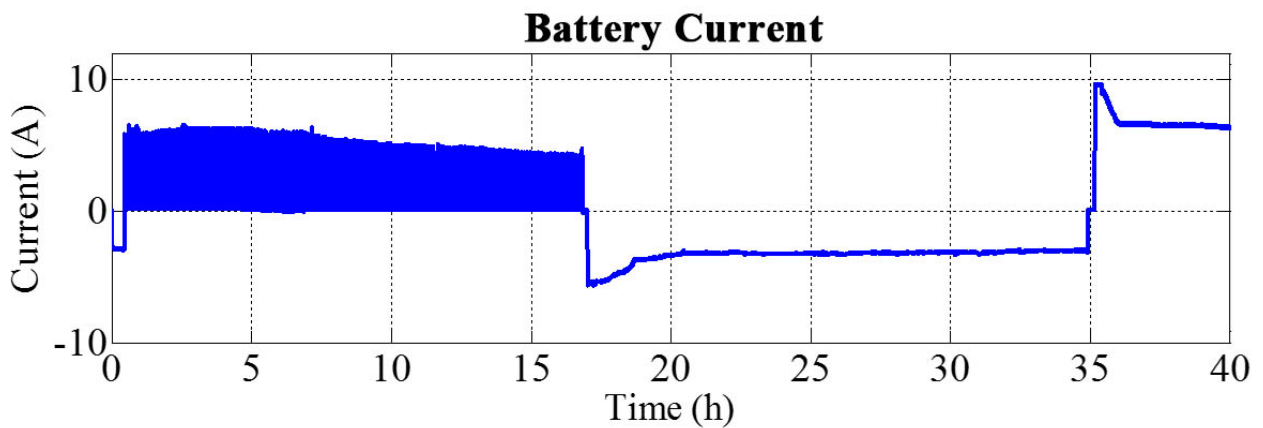

(b)

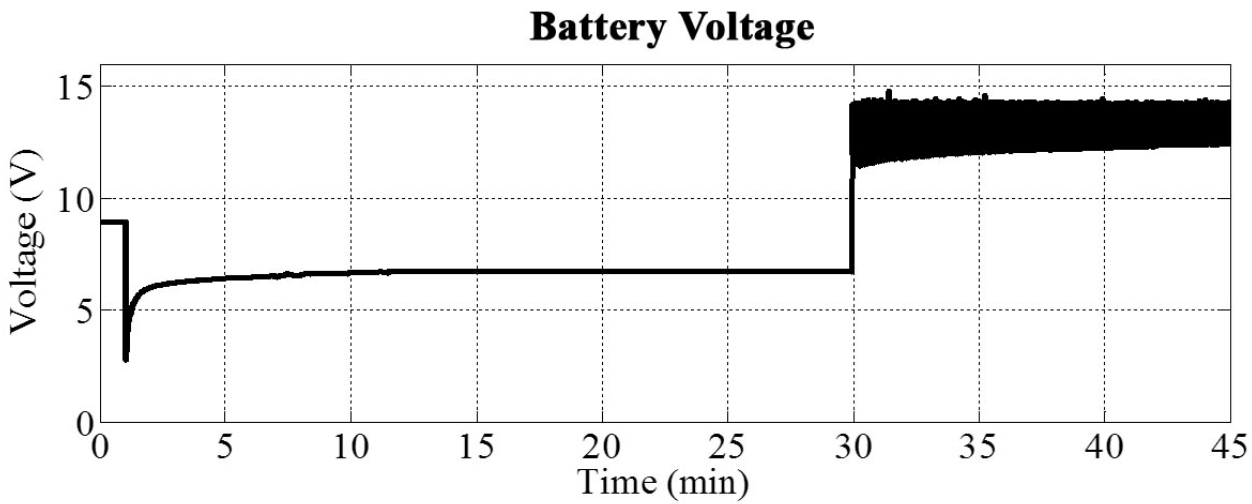

(c)

Figure 3.9. Endurance and Robustness Test (a) Battery Voltage (b) Battery Current (c) Battery Voltage (first $45 \mathrm{~min}$ ). 


\subsection{Summary}

In this chapter, four laboratory ESMC prototypes were placed through four initial tests to verify their performance and functionality under different loading conditions. Each ESMC was capable of monitoring the voltage, current, power, energy and SoC for each battery in the array and independently controlled individual batteries. Through the isolation of individual batteries, one or more lead acid batteries in a series stack configuration were charged, while the system continued to supply the load. A number of studies were conducted revealing the new topology can significantly increase the reliability of the system. The first scenario revealed how the collaboration of multiple ESMC devices can be used to conduct SoC balancing. The second and third evaluated the ESMC behavior and performance under a fault event as well as a heavy pulsed load. The fourth and final test was particularly important and served multiple purposes. First, to evaluate the ESMC performance over an endurance period and second, to test the capability of utilizing certain features to improve a battery $\mathrm{SoH}$.

In Section 3.2, a battery SoH was introduced and a discussion of how battery performance can be evaluated through tracking circuit components in a basic EIS model. This dissertation will introduce multiple methods in which EIS circuit component values can be obtained and accurate values are crucial for conducting meaningful simulations. In the next chapter, the direct approach at acquiring EIS values is taken through the implementation of a traditional EIS procedure. The history and theory of EIS and how component values can be obtained experimentally though the aid of an AC voltage disturbance will be discussed in detail. 


\section{Chapter 4 Battery State of Health using Impedance Spectroscopy}

\subsection{Introduction}

All rechargeable batteries have an associated lifespan or State of Health $(\mathrm{SoH})$ which will result in a reduction of their performance, usable capacity, and runtime over their calendar life and usage [77]. When a battery is first placed into service, charging and discharging voltage curves will behave near their ideal case. However, as a battery ages, this curve becomes unpredictable and highly nonlinear. Accurate and reliable estimations of SoH and performance are crucial in the development of a smart battery management system (BMS). Without insight into a battery SoH, a BMS cannot adjust to new operational parameters which will result in inaccurate State of Charge (SoC) readings thereby inaccurately estimating the remaining usable capacity.

The batteries leading the market today are sealed lead acid and lithium ion batteries. Although the lead acid battery suffers from a number of health-related effects as was addressed in the previous chapter, its popularity still remains high due to a low cost of materials [71]. Their service to the electric vehicle (EV) industry, however, is limited as a shift has been made to various lithium ion compounds due to serious $\mathrm{SoH}$ impacts as a result of a required deep Depth of Discharge (DoD) and the associated high discharge currents required for EV operation. Lithium ion batteries have been introduced as an alternative, where their operating ranges can be constrained to increase cycle life, a tactic which is more complicated with lead acid batteries as they must be charged in full regularly to maintain their full usable capacity. Although lithium ion batteries offer an improvement, they are not immune to many of the same SoH issues [78]. While deep DoD continues to 
hold a strong bearing on the lithium ion battery cycle life, new SoH problems arise from thermal expansion and contraction of materials during cycling, especially at high currents. These operating scenarios increase the impedance of the battery.

Although a quantitative definition of $\mathrm{SoH}$ has not yet been standardized, it can traditionally be referred to as a percentage of remaining usable capacity versus the nameplate. Some of the earliest procedures were introduced in the late 1990s through higher level algorithms [79]. In Reference [79], six years of lead acid battery data was analyzed to characterize the health of the battery into three SoH phases: starting, working, and capacity drop. Reference [80] analyzed the coup de fouet, or the initial discharge voltage drop behavior as a method to estimate the remaining capacity.

A detailed example of capacity loss in a lead acid battery is shown in Figure 4.1 where the charging and discharging voltage and current progressions for both a healthy and damaged UB 6120 lead acid battery are compared over time [81]. The time scales have been matched in order to better highlight differences in the charging and discharging periods. Both batteries were charged under a standard two-stage charging scenario: Constant Current (CC) where approximately $75 \%$ of the capacity is transferred followed by Constant Voltage (CV), which injects less energy but is required by the lead acid battery to maintain its full capacity [82]. Notice that the CC stage in the healthy battery lasted for approximately one third of the charging period followed by $\mathrm{CV}$ for the remaining time. The CV period also dropped off gradually, where the slope of the battery current (shown in orange) eventually reached a minimum value where charging ceased. As shown, the charging period lasted for 15.3 hours transferring 11.2 Amp-hours (Ah) into the battery followed by a Constant Resistance (CR) discharge phase. Over the discharge period, the 
battery operated at its rated current for 17.9 hours discharging $11.0 \mathrm{Ah}$, or approximately $98 \%$ of the charged energy.
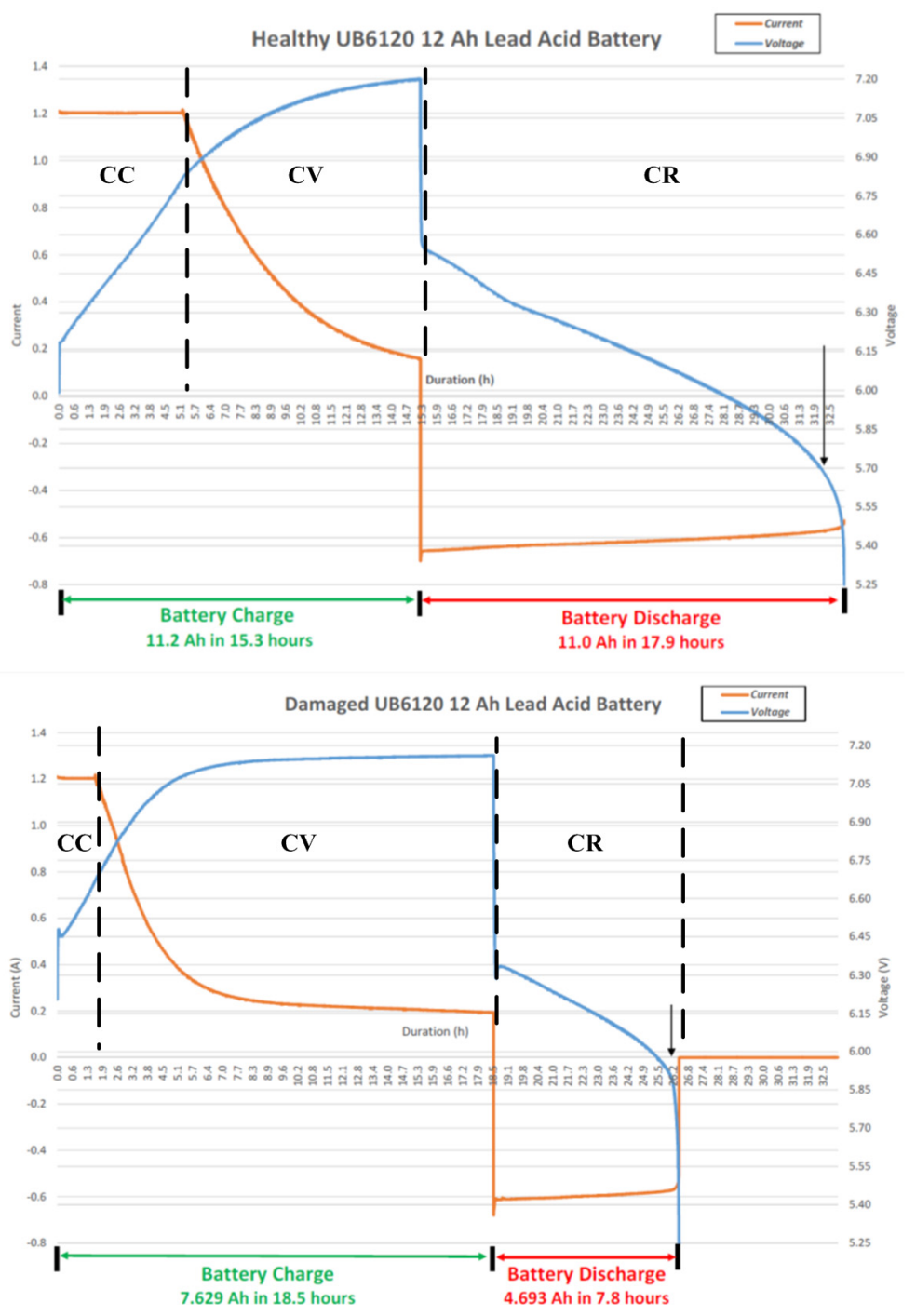

Figure 4.1. Lead Acid Battery Charge/Discharge State of Health Curve Comparison.

The same battery is shown below in Figure 4.1 following many charge/discharge cycles. This battery was intentionally exposed to heavy discharging currents and was over discharged numerous times leading to internal damage. Notice a significant increase in the length of the charging period where the duration in $\mathrm{CC}$ mode was reduced by $70 \%$ and the 
$\mathrm{CV}$ period featured a steep falloff in the charging current. Although the battery charged for 18.5 hours, only 7.629 Ah of capacity was absorbed. The most noticeable result is present in the CR discharge phase, which only lasted for 7.8 hours discharging $57 \%$ less energy than the new battery (4.693 Ah). Furthermore, the discharge period was significantly less than the charging period.

Capacity may be the most useful to the end user, but a degradation in performance is a result of complex electrochemical processes which impact far more than the available capacity. As a basic introduction to this concept, the $1^{\text {st }}$ order Randles equivalent circuit model which was introduced in the previous chapter is revisited. Accompanying the capacity loss phenomenon in the UB 6120, component values in its equivalent circuit model have shifted as well. A Randles $1^{\text {st }}$ order representation is shown in Figure 4.2. As can be seen, many values can change as the battery ages.

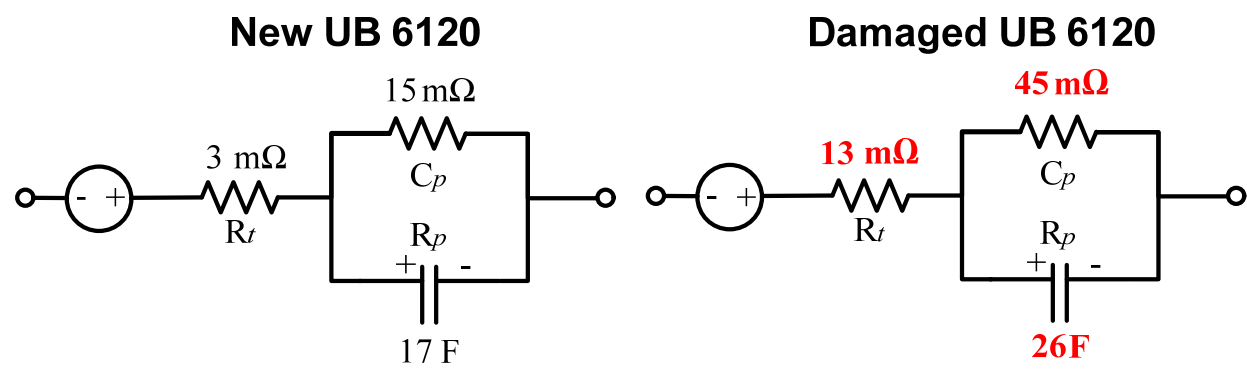

Figure 4.2. Example of Changes in the Randles Equivalent Circuit as a Battery Ages.

In this case, both the ohmic resistance $R_{t}$ and impulse parameters $R_{p}, C_{p}$ are drastically different between the new and damaged UB 6120 battery. First, $R_{t}$ has increased by almost three times its original value which will result in a much heavier voltage drop when the battery is placed under the same load. Next, both impulse parameters have increased 
suggesting the battery recovery following a load or charging current will take much longer to recover. A simple calculation of a resistor-capacitor (RC) time constant $\tau_{p}=R_{p} C_{p}$ reveals the recovery period between the new and damaged batteries increased from 0.255 $\mathrm{s}$ to $1.17 \mathrm{~s}$. The lack of a fast recovery period also suggests the battery condition is poor.

A number of techniques have been proposed to offer greater insight into a battery $\mathrm{SoH}$ [83]-[85]. In Reference [86], two techniques were investigated in an attempt to track and detect capacity decay and compute SoH. The first technique makes use of fuzzy logic to compute a health index; whereas, the second relies upon a neural network topology. Both techniques required early stage characterization of the battery, but were able to track the usable capacity to within $\pm 5 \%$ of the actual value. However, these procedures were only evaluated on batteries of the same type, capacity, and manufacturer. Furthermore, determination of the current usable capacity cannot reveal insight into the battery impulse response or its equivalent circuit parameters. In order to extract signature information related to a battery condition, Electrochemical Impedance Spectroscopy (EIS) must be performed.

EIS presents the most effective method to date in extracting equivalent circuit parameters, where a wide range of $\mathrm{AC}$ voltage frequencies are passed across the electrodes and the battery current response and phase shift is recorded [87]. The use of EIS has been a powerful diagnostic tool in the material science field to analyze developmental materials, sensors, and emerging electro-chemistries. However, once the device or battery has left fabrication, it will rarely be exposed to this characterization again. EIS remains a premium in the operational battery industry where the implementation of a low-cost solution is still a challenge [88],[89]. However, with the availability of low-cost, small printed circuit 
boards (PCB), this technology could be made available to future EVs providing near realtime battery health analysis to the driver and even anticipate failures.

In this chapter, the core concept of battery EIS is investigated as one of two methods that are addressed in this dissertation to obtain the battery equivalent circuit. EIS is considered a noninvasive technique, as the current it applies is extremely small operating on a low power AC circuit, which is separate from a DC bus, load, or charger. The theory of EIS is discussed next as well as its common challenges revealing the necessity to explore an alternative method as will be presented in the next chapter. A simplified EIS topology for a precision on-board measurement circuit application is proposed. The considerations and trade-offs associated in its development will be discussed while a simulation platform will test and verify the proposed circuit topology through simulation and initial laboratory testing.

\subsection{Electrochemical Impedance Spectroscopy}

The process of EIS is highly correlated to electrochemical processes as it can measure the dielectric properties of a medium as a function of frequency. The impedance of the battery electrodes are measured under a wide range of different AC frequencies [90]. Traditionally, anywhere from $1 \mathrm{mHz}$ up to $10 \mathrm{MHz}$ is applied at the battery terminals. At each frequency, the magnitude and phase difference between the system $\mathrm{AC}$ voltage and current response is recorded and constructed into a Bode plot similar to that which is shown in Figure 4.3(a).

A key characteristic of EIS when it is applied to a specific type of electrochemistry is a correlation between the frequency test range and particular components of the electrochemical conversion process. For the lithium ion battery, electrochemical kinetics, 
or the movement of lithium ions between electrodes can be revealed at lower frequencies; whereas, ohmic loss though the electrolyte can be obtained at much higher frequencies. Similar characteristics can be extracted from the lead acid battery where ohmic losses are present at higher frequencies as well but differ from that of lithium ion. Furthermore, each battery will feature a signature range as a result of the chemistry type. With high enough fidelity and a model of sufficient order given the application, it will become unique to only one specific battery.
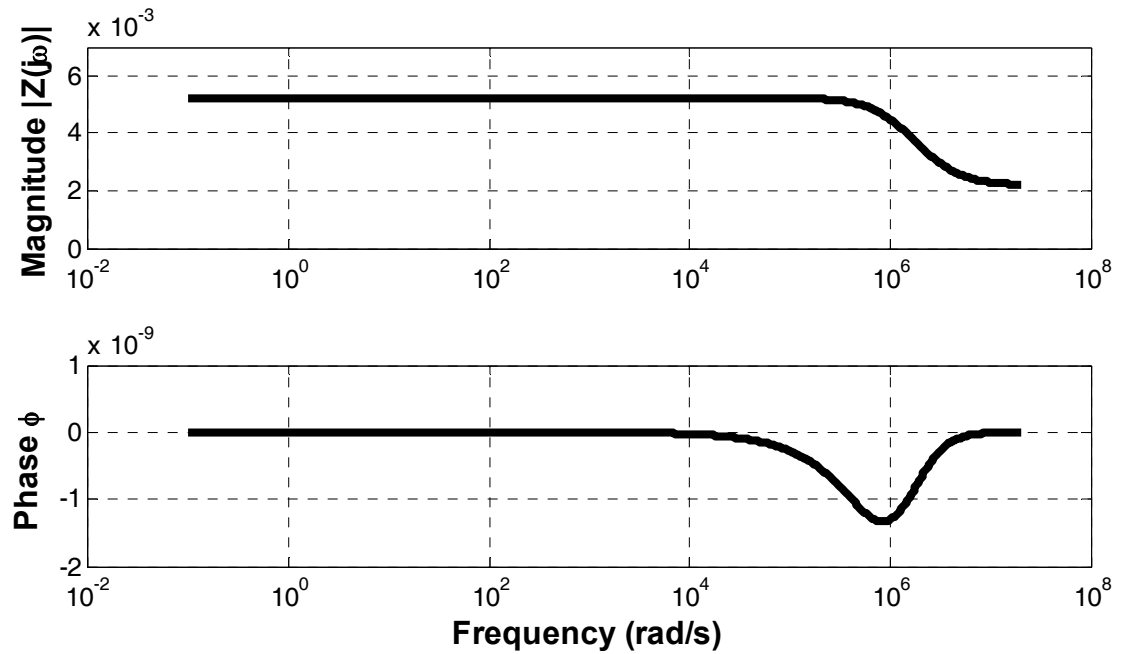

(a)

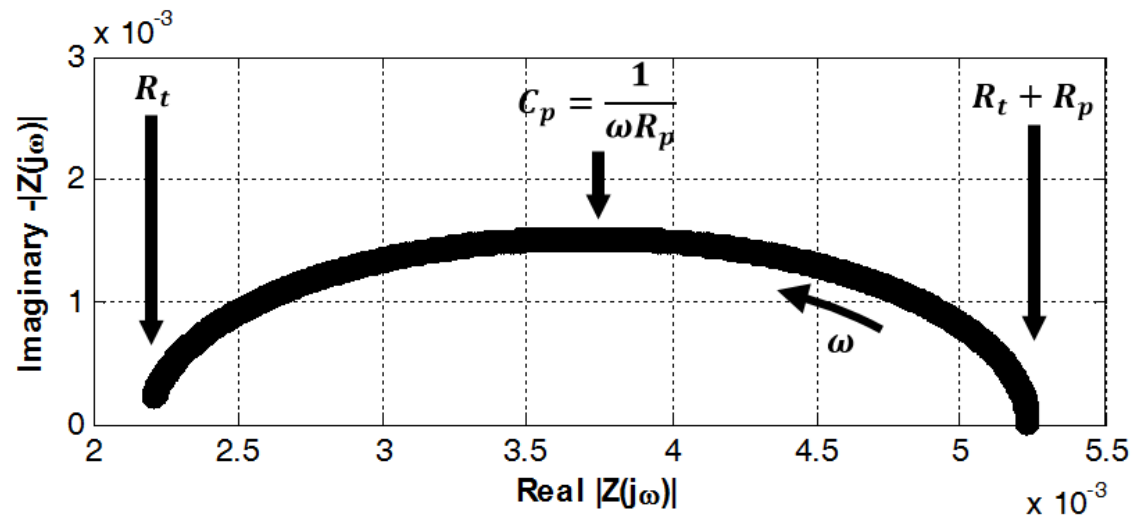

(b)

Figure 4.3. Example Randles $1^{\text {st }}$ Order Equivalent Circuit Frequency Response a) Bode Plot and b) Nyquist Plot. 


\subsubsection{Procedure}

The Bode plot output in Figure 4.3(a) depicts an example lithium ion battery test scenario where an AC signal is passed from $100 \mathrm{mHz}$ to $10 \mathrm{MHz}\left(\sim 10^{-1} \leq \omega \leq 10^{7} \mathrm{rad} / \mathrm{s}\right)$ [91]. To adjust this plot for easier readability and provide a distinction of equivalent circuit parameters, the Bode plot is consolidated to form a Nyquist impedance plot as shown in Figure 4.3(b), where the real $\operatorname{Re}|Z(j \omega)|$ and imaginary $\operatorname{Im}|Z(j \omega)|$ components are defined by their rectangular coordinates. Since the battery impedance is primarily capacitive, the imaginary axis is mirrored to plot $-\operatorname{Im}|\mathrm{Z}(j \omega)|$ where $\emptyset$ is the phase shift from the reference signal. The real and imaginary components follow the traditional relation:

$$
\left\{\begin{array}{l}
\operatorname{Re}|\mathrm{Z}(j \omega)| \equiv|\mathrm{Z}| \cos \emptyset \\
\operatorname{Im}|\mathrm{Z}(j \omega)| \equiv|\mathrm{Z}| \sin \emptyset
\end{array}\right.
$$

Figure 4.3(b) provides enough information to construct a basic Randles equivalent circuit representation for a battery shown in Figure 4.4. Both lead acid and lithium ion batteries can follow this basic form, but each produce very different component values. As covered previously, $\mathrm{R}_{t}$ represents the ohmic resistance of the electrolyte while $\mathrm{R}_{p}$ and $\mathrm{C}_{p}$ represent the polarization resistance and capacitance across the electrodes governing the impulse response.

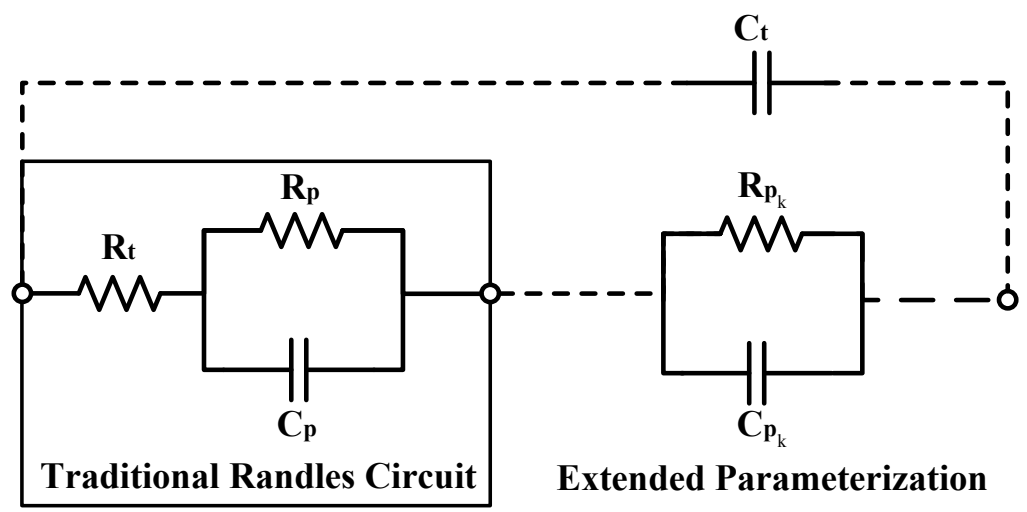

Figure 4.4. Electrochemical Impedance Spectroscopy Circuit Fit Examples. 
The traditional Randles circuit in Figure 4.4 presents the simplest output form from EIS, but it is important to mention that many fitted circuit models exist. Since RC parameters can typically be tied to the interfacial region of two materials, or electrodes in this case, the equivalent circuit parameters can be extended to include a number of RC components designated by $\mathrm{R}_{p_{k}}$, and $\mathrm{C}_{p_{k}}$, respectively. In addition, depending on the chemistry, measurement fidelity, and required depth of the model, the capacitance of the electrolyte $\mathrm{C}_{t}$ may be included as well.

For the raw impedance output analyzed in Figure 4.3, the three basic components of the Traditional Randles Circuit in Figure 4.4 are solved for. Looking back to Figure 4.3(b), at low frequencies $(\omega \rightarrow 0)$, the capacitive component $C_{p}$ is virtually removed solving for the sum of the polarization and ohmic resistances $\mathrm{R}_{t}+\mathrm{R}_{p}$. As the frequency $\omega$ increases, a sharp phase shift occurs corresponding to the time constant $\tau_{p}$ of the polarization capacitance $\mathrm{C}_{p}$ at the center of the arc. Obtaining $\mathrm{C}_{p}$ is achieved following the traditional $\mathrm{RC}$ circuit relation:

$$
\omega=\frac{1}{\tau_{p}}=\frac{1}{R_{p} C_{p}} \therefore \quad C_{p}=\frac{1}{\omega R_{p}}
$$

This transition point would expect to drift slightly from battery to battery and drastically from chemistry to chemistry. As the frequency progresses toward infinity ( $\omega \rightarrow$ $\infty$ ), a short circuit is applied at $C_{p}$ leaving only the ohmic loss through the electrolyte $R_{t}$.

\subsubsection{Selected Electrochemical Impedance Spectroscopy Technique}

A number of EIS measurement techniques have been proposed utilizing both frequency and time domain methods [90]. Wheatstone bridges were popular under frequency domain analysis, but were typically limited to audio frequency ranges preventing high frequency 
impedance measurements. Resistance arms were later substituted for transformer legs where the impedance ratio was proportional to the square of the transformer turns ratio. Other variants were produced later but continued to feature complex, bulky measurement apparatuses. The introduction of potentiostatic control has enabled the most convenient method of obtaining the impedance.

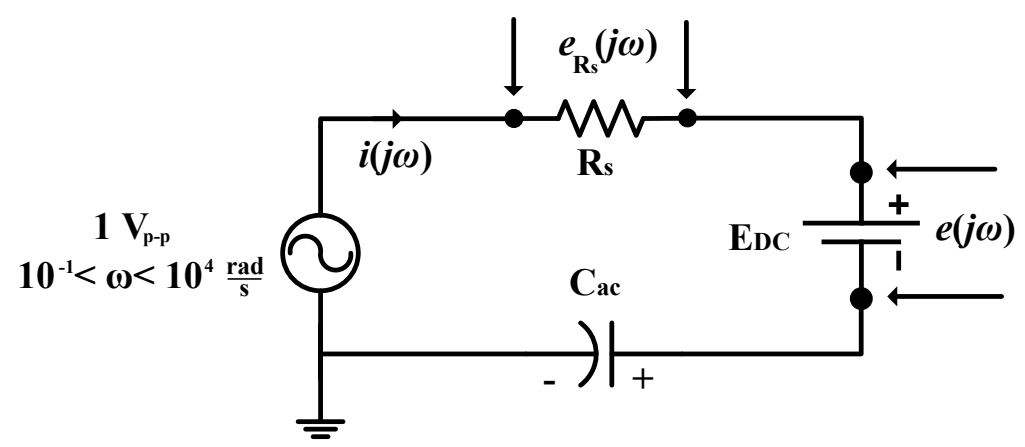

Figure 4.5. Electrochemical Impedance Spectroscopy Circuit Topology.

Shown in Figure 4.5, a $1 \mathrm{~V}_{p-p}$ AC voltage is passed through a current shunt resistance $\mathrm{R}_{s}$ and connected to the anode of the battery. The cathode is then passed through a large filtering capacitor $C_{a c}$ to block a DC voltage loop. Since the output signal is the system voltage $e(j \omega)$ (battery AC voltage) and input is the current response $i(j \omega)$, the transfer function is merely the impedance $\mathrm{Z}(j \omega)$. The equation is simply modified to replace $i(j \omega)$ with the voltage $e_{R_{s}}(j \omega)$ across the shunt resistance $\mathrm{R}_{s}$. This renders the modified formula to be:

$$
Z(j \omega)=\frac{e(j \omega)}{i(j \omega)}=\frac{R_{s} e(j \omega)}{e_{R_{S}}(j \omega)}
$$

To satisfy Equation (4-3), the direct impedance measurement method was implemented. 


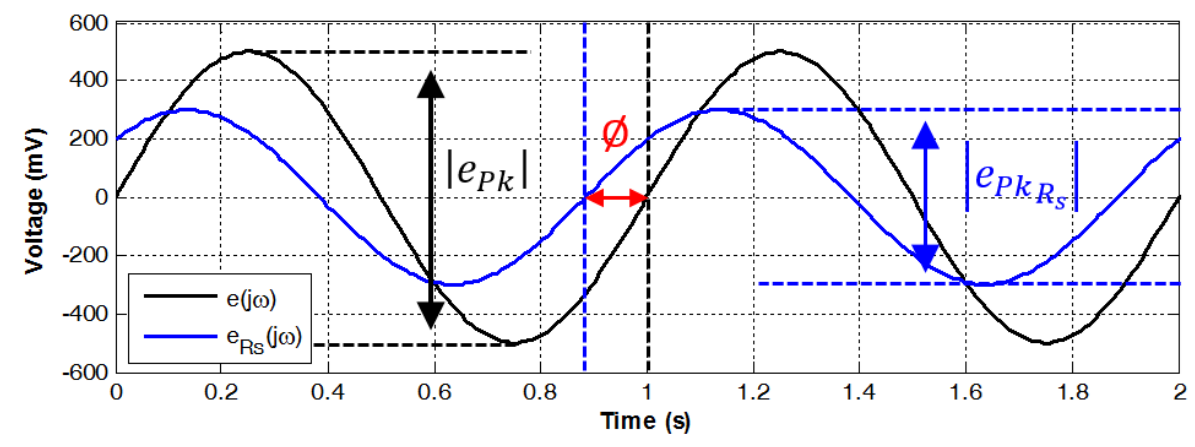

Figure 4.6. Electrochemical Impedance Direct Method Determination.
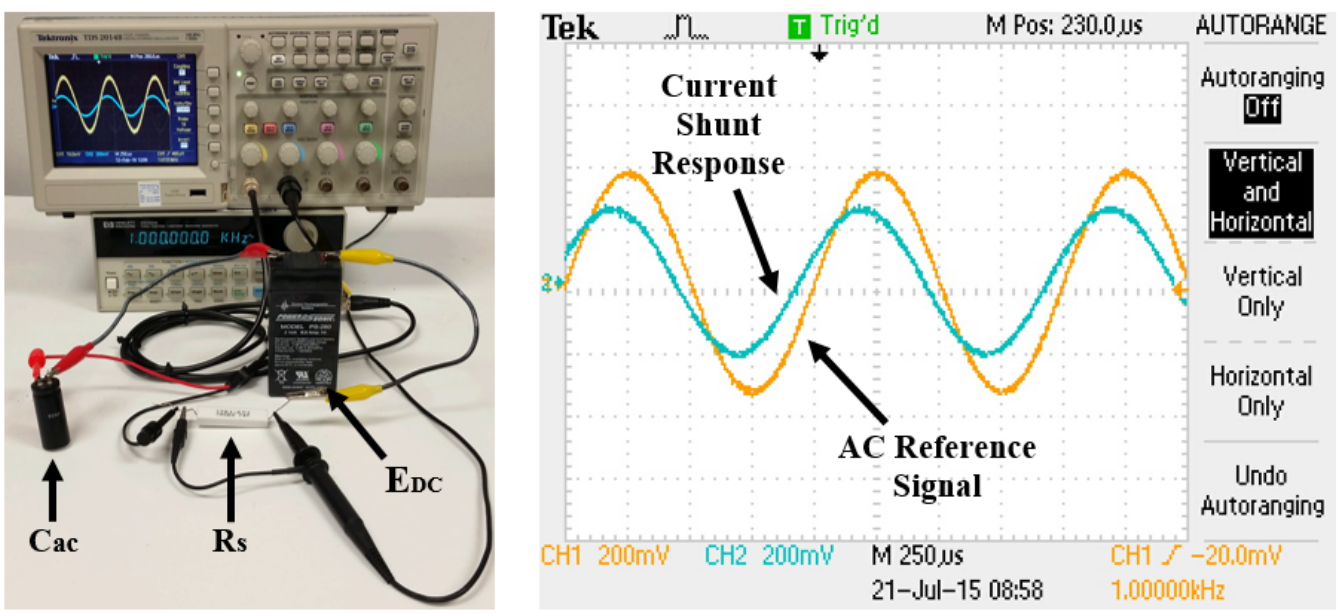

Figure 4.7. Experimental Test Circuit to Verify Topology Circuit Design (left) and 1 $\mathrm{kHz}$ AC Disturbance Frequency and Current Response (right).

Shown in Figure 4.6, the intended design compares peak magnitudes of both the AC voltage across the battery terminals $\left|e_{P k}\right|$ and shunt $\left|e_{P k_{R_{S}}}\right|$ to obtain the impedance magnitude $|Z|$. By tracking the zero crossing of both signals, the phase shift $\emptyset$ is calculated. The final output for the phasor impedance $\overline{Z(j \omega)}$ is then:

$$
\overline{Z(J \omega)}=\frac{R_{s}\left|\mathrm{e}_{\mathrm{Pk}}\right|}{\left|\mathrm{e}_{\mathrm{Pk}_{\mathrm{R}_{\mathrm{S}}}}\right|} \angle \varnothing
$$

To confirm the functionality of the topology in Figure 4.5, the circuit was constructed in a laboratory and tested on a variety of battery cells. Shown in Figure 4.7, a single-cell 
6.0 Ah lead acid battery was tested at a variety of different frequencies where the reference wave and voltage across the current shunt was recorded. Shown to the right in Figure 4.7 reveals the current shunt response (blue) as a result of a $1 \mathrm{kHz}$ AC reference wave (yellow). As depicted in the figure, the magnitude of the current response is slightly reduced to that of the reference with a minor lagging phase shift.

\subsubsection{Challenges in Acquisition}

There are several challenges involved in acquiring accurate and meaningful EIS measurements. One objective of this effort is to present an EIS board which limits the amount of signal conditioning or processing required to obtain the Nyquist plot. In this way, the circuit topology can be engineered to provide a minimized set of analog outputs, where a BMS system like the Energy Storage Management Controller (ESMC) presented in previous chapters can use real-time information to quickly calculate the impedance. To accomplish this, precision measurements of the AC system voltage across the battery and shunt signal are crucial as $\mathrm{R}_{t}$ and $\mathrm{R}_{p}$ can typically drop well below $10 \mathrm{~m} \Omega$, as was shown previously in Figure 4.2. Second, careful consideration must be taken in selecting the frequency steps as extremely low frequencies will introduce long downtimes while taking into account that the lack of an adequate wide test frequency spectra will prevent an accurate identification of $\mathrm{R}_{t}$ and $\mathrm{R}_{p}$.

Although an adequate function generator like the one pictured to the right in Figure 4.7 can produce a reference signal which is easy to preserve and analyze, the current response across the battery is very small thereby producing an even smaller voltage drop. A shunt resistance with an extremely tight tolerance $(\leq 0.1 \%)$ must be used while in a PCB design stage, signal tracks and the ground plane must be optimized to reduce the noise and stray 
inductance exposed at very high frequencies. Finally, the process of noise filtering is complex as a result of testing over such a wide frequency range.

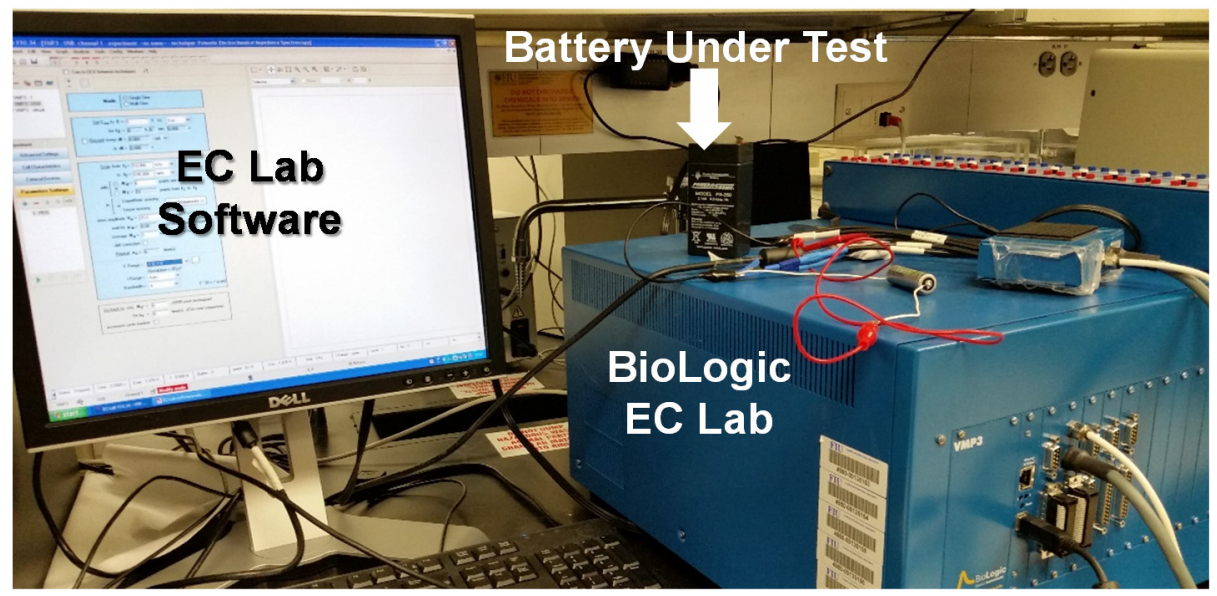

Figure 4.8. BioLogic EC Lab Electrochemical Impedance Spectroscopy Test.

\subsubsection{Commercial Systems}

A number of EIS systems are available in the commercial market, but are accompanied by a high price tag and are typically cumbersome as shown in Figure 4.8 [92]. As previously mentioned, many of these tools are popular in material research fields. EIS provides a high quality method to characterize new biomedical devices to semiconductors. The EIS system type, cost, and size can typically be mapped to the required excitation current magnitude to pass through associated materials of the test subject to conduct an EIS procedure [93]. A device with a small surface area would require less current to flow through its associated materials while conducting a test, making impedance measurements simpler. However, as the surface area becomes larger, the level of current required to perform the test increases as well that further increases the complexity and cost of the EIS system. Since the surface area of the active regions inside a battery are considerably larger than typical samples generated when conducting material research, the high excitation 
current warrants the need for very specialized equipment which can cost tens of thousands of dollars [92].

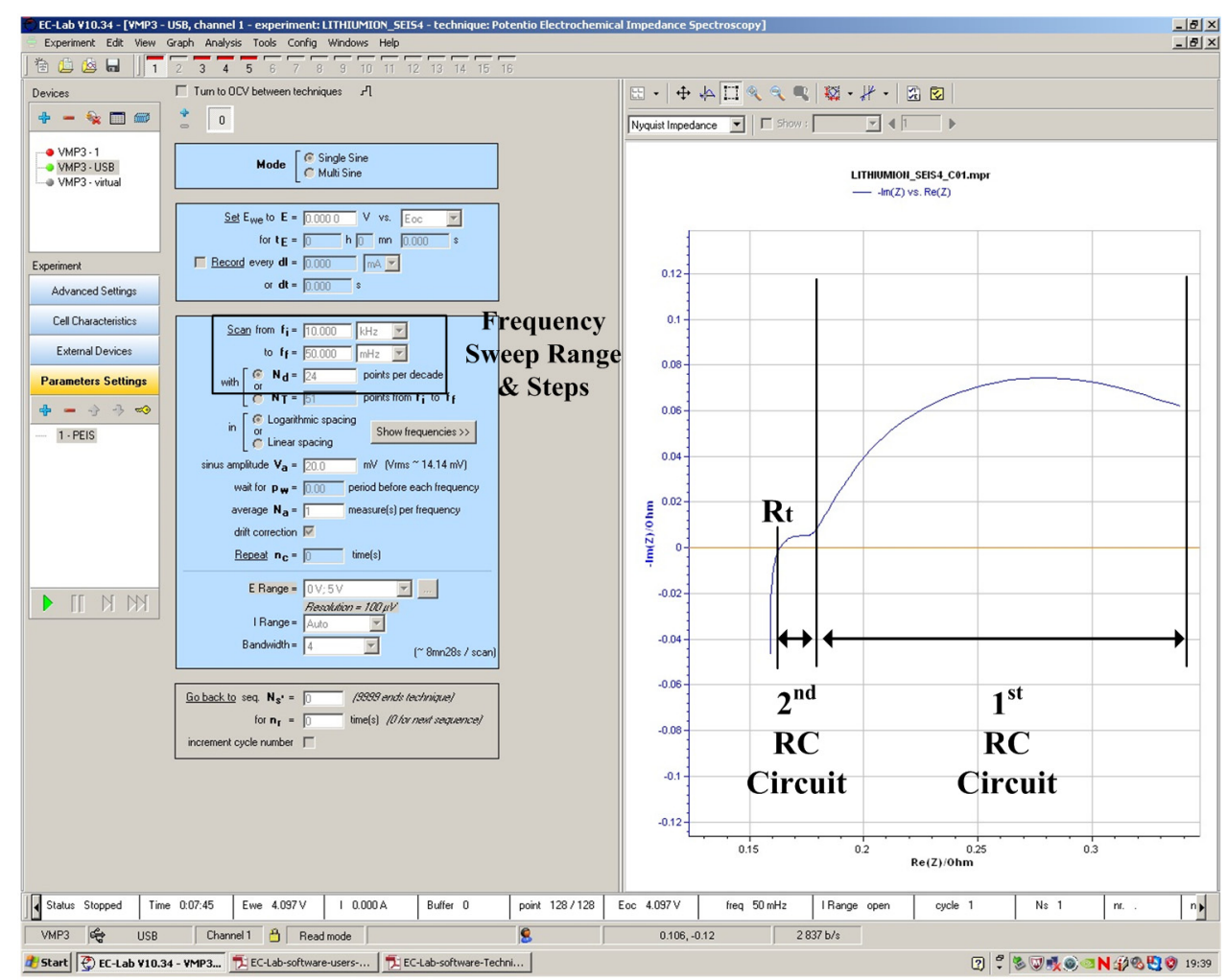

Figure 4.9. EC Lab Software During 1.5 Ah Lithium Ion Polymer Test.

To demonstrate the output from a commercial EIS procedure, the BioLogic EC Lab EIS system was available to obtain the impedance curve of a small, 1.5 Ah lithium ion battery [94]. Since the excitation current of the BioLogic EC is limited to $100 \mathrm{~mA}$ or less, testing the 6 Ah lead acid cell from the previous section exceeded this limitation. Shown in Figure 4.8, the BioLogic EC Lab device is connected to a PC, where its associated proprietary EC Lab Software was used to perform the test. The user can assign their own frequency sweep and the number of steps or use a default range. For this test, $R_{t}$ can be clearly identified via inspection at the intersection of the imaginary axis at $165 \mathrm{~m} \Omega$. Since 
the BioLogic EC Lab is not limited to EIS testing of batteries, the user is then able to select from a wide variety of circuit topologies that could potentially fit the Nyquist curve. In this case, the progression is fairly consistent with a Randles $2^{\text {nd }}$ order equivalent circuit model featuring $2 \mathrm{RC}$ time constants.

\subsection{Battery Impedance Measurement Design}

In this section, a circuit topology to develop an effective technique for administering EIS on a compact, low-cost controller board is discussed. Previously in Figure 4.4, the traditional Randles equivalent circuit and some potential expansions were introduced. In this design, a high-speed, low-cost controller would not be expected to yield the highest fidelity, thus an extended parameterization is not expected. However, in a complex battery array for an EV or shipboard power system, the Randles equivalent circuit model would be sufficient to offer insight into SoH without adding extensive overhead. It is anticipated that an external PCB following this circuit topology could offer a convenient expansion module to the ESMC and a number of BMS systems on the market.

The battery testing circuit was designed as shown previously in Figure 4.5 where initially, a basic function generator is used to provide a reference signal. The shunt resistance $\mathrm{R}_{s}$ is chosen to be $10 \Omega$ as to ensure a large magnitude of $\left|e_{R_{S}}\right|$. The design is separated into two major components: peak and zero crossing detection that will be connected to both the system AC voltage across the battery $e(j \omega)$ and the current signal across the shunt $e_{R_{s}}(j \omega)$. The following sections will describe how each component was selected to serve these two functions as well as the special modifications required to the current response signal. 


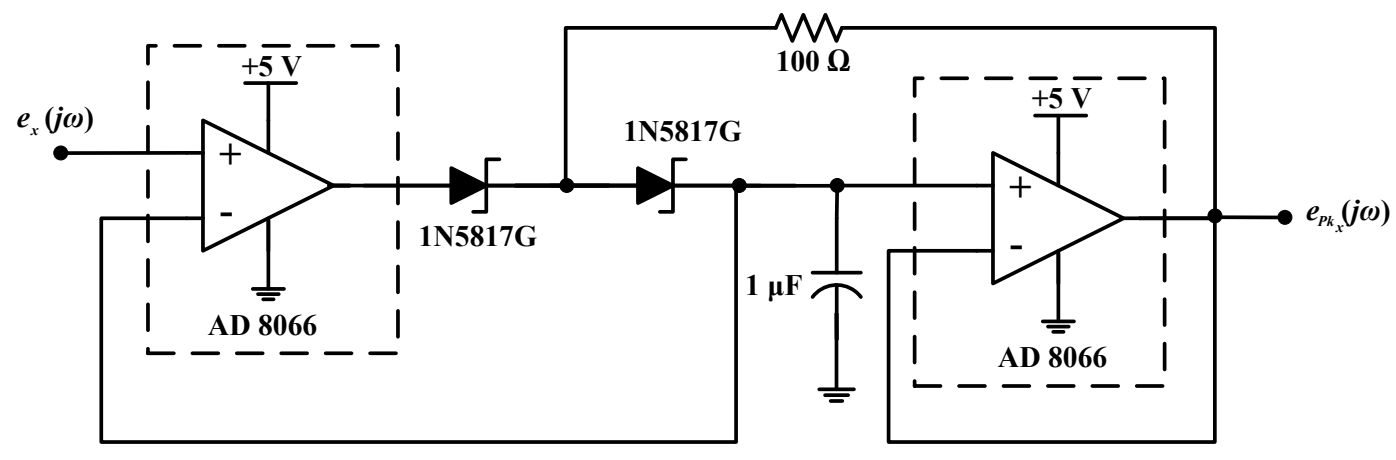

Figure 4.10. Peak Detection Circuit Topology.

\subsubsection{Peak Detection}

Each peak detection circuit consists of a dual-channel Analog Devices AD 8066 FastFET operational amplifier, where the system AC or shunt voltage $e_{x}(j \omega)$ is fed first to the primary amplifier and the output from the secondary amplifier provides the peak signal $e_{P k_{x}}(j \omega)$ [95]. The $\mathrm{AD} 8066$ is known for its high performance and speed but is particularly useful in sensitive applications due to its very high input and low output impedance. Shown in Figure 4.10, two Schottky $1 \mathrm{~N} 5817 \mathrm{G}$ diodes are implemented to minimize the forward voltage drop and enable fast switching action. The $1 \mu \mathrm{F}$ capacitor quickly charges and slowly discharges to the consecutive peak amplitude of the input signal, but remains fully isolated from the input function as to not introduce stray capacitance on the reference signal. These combined features significantly reduce the ripple voltage output over a wide range of sinusoidal input frequencies. The AD 8066 can operate over a wide supply voltage range of $5 \mathrm{~V}$ to $24 \mathrm{~V}$ without the need for a differential supply. To make this integrated circuit package flexible for integration with a microcontroller unit (MCU), the single-ended $+5 \mathrm{~V}$ supply was chosen. 


\subsubsection{Zero Crossing Detection}

The second major component is a precision zero crossing circuit to calculate the phase shift $\varnothing$ between the AC system voltage $e(j \omega)$ and the battery current response $e_{R_{S}}(j \omega)$ across the shunt resistor $\mathrm{R}_{s}$. Shown in Figure 4.11, the system AC or shunt voltage $e_{x}(j \omega)$ is fed to a Linear Technology LT 1116 high speed comparator. The LT 1116 was selected for its stability over a wide range of operating conditions and particularly for low frequency triggering, which presents the greatest challenge [96]. The intended output $e_{0_{x}}(j \omega)$ provides a transistor-transistor logic (TTL) level square wave suitable for passing to either an analog or digital microcontroller. Similar to the AD 8066, the TL 1116 is also capable of operation from both a single or differential $\pm 5 \mathrm{~V}$ supply thus the single-ended $+5 \mathrm{~V}$ output was once again chosen for easy integration with a MCU or BMS.

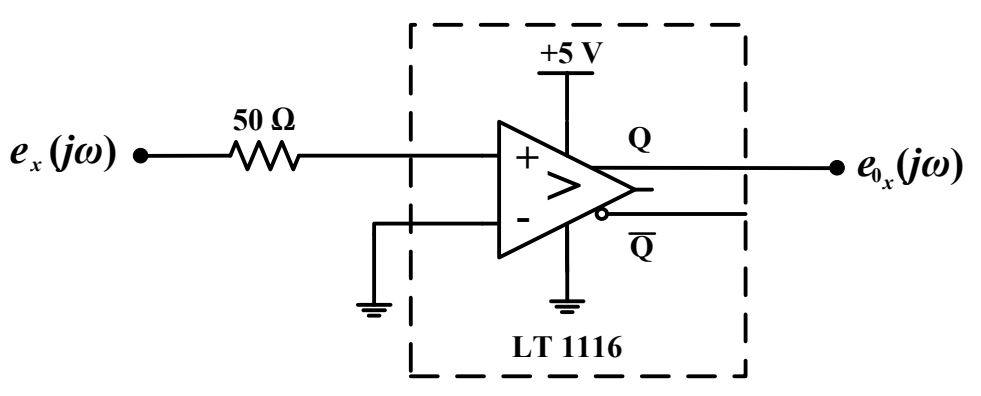

Figure 4.11. Zero Crossing Detection Circuit Topology.

\subsubsection{Final Circuit Topology}

The final circuit topology is shown in Figure 4.12 where the testing circuit from Figure 4.5 is connected to two peak and two zero crossing circuits. For the system AC voltage $e(j \omega)$, the signal is connected directly to the peak $e_{P k}(j \omega)$ and zero crossing $e_{0}(j \omega)$ circuits without the need for further amplification. However, due to the sensitivity of the shunt voltage signal $e_{R_{S}}(j \omega)$, a fully-differential isolation amplifier was needed. The Texas 
Instruments AMC 1200, which was previously discussed for its use in extending the ESMC design in Chapter 2, was used as it provides precision isolation and is optimized for the direct connection to a shunt resistor [67]. The AMC 1200 contains an optocoupler, where the inputs and outputs $x$ are in the form $V_{x_{p}}$ and $V_{x_{n}}$. Since the optocoupler provides total insolation, the output common reference $V_{o_{n}}$ to the shunt voltage measurement is unique to obtain the peak $e_{P k_{R_{S}}}(j \omega)$ and zero crossing $e_{0_{R_{S}}}(j \omega)$ signals. The risk in including an amplifier in this system was an introduction of a nonlinear gain which would render an inaccurate peak detection measurement. The AMC 1200 features a linear gain of $8 \pm 0.01$ enabling a simple correction factor to be applied to the shunt peak detection output following the integration with a MCU.

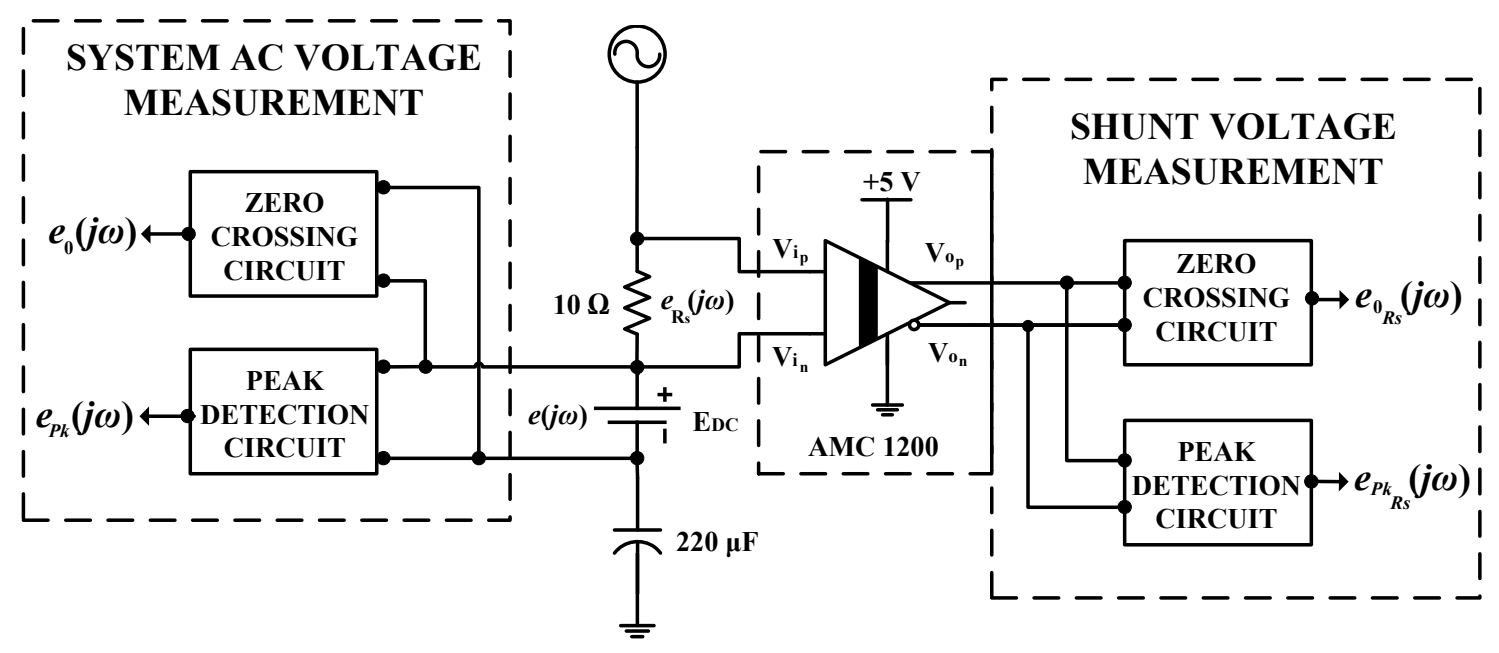

Figure 4.12. Final Test Circuit Topology.

\subsection{Simulation Results}

To demonstrate the operation of the proposed circuit topology, National Instruments (NI) Multisim was used to conduct a comprehensive simulation. All three major integrated circuit (IC) architectures were available to import into the final model. To perform an 
analysis of the accuracy of both the zero crossing and peak detection circuits, the frequency response of both are shown in the Bode plot in Figure 4.13 over the intended operating frequency range of $1 \mathrm{~Hz}$ to $100 \mathrm{kHz}$.
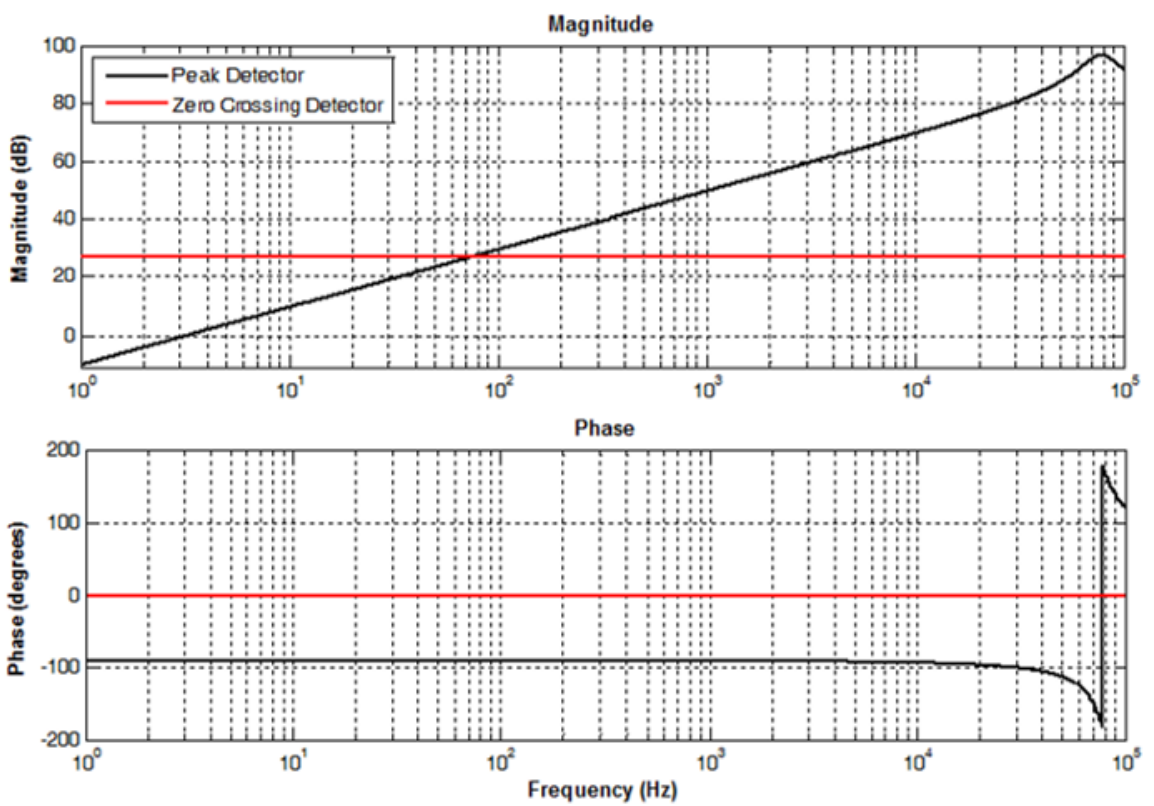

Figure 4.13. Frequency Response of the Final Circuit Topology.

For the zero crossing detector, the magnitude and phase were held relatively constant across the entire frequency span. Although the magnitude was lower than that of the peak detector, there is no presence of a phase shift. The peak detection circuit, however, has a flat phase shift of $-90^{\circ}$ until approximately $10 \mathrm{kHz}$ where it progresses to $-180^{\circ}$. Although this behavior is undesired, the $1 \mu \mathrm{F}$ capacitor is crucial for smoothening the peak signal at lower frequencies. Furthermore, since the ideal peak detection signal is DC in nature, this phase shift has little impact on the performance.

Using the non-ideal battery block in Multisim, a simple impedance and capacity was introduced into the proposed circuit design. Unfortunately, the production of an accurate 
Bode and Nyquist plot for a battery would require actual experimental data or a higherorder physics-based model (PBM) of the battery, thus only the time-domain performance of the system could be evaluated. The following sections evaluate the performance of the circuit at the low $(1 \mathrm{~Hz})$, medium $(1 \mathrm{kHz})$, and maximum $(100 \mathrm{kHz})$ reference frequencies. The Multisim schematic is shown in Figure 4.14 where the main battery is pictured at the center (V1) in series with its associated AC filtering capacitor (C2) at its negative terminal and a $10 \Omega$ shunt (R2) at the positive terminal. The circuit above the battery is purposed to sense the $\mathrm{AC}$ voltage across the battery terminals while the bottom senses the $\mathrm{AC}$ current across R2.

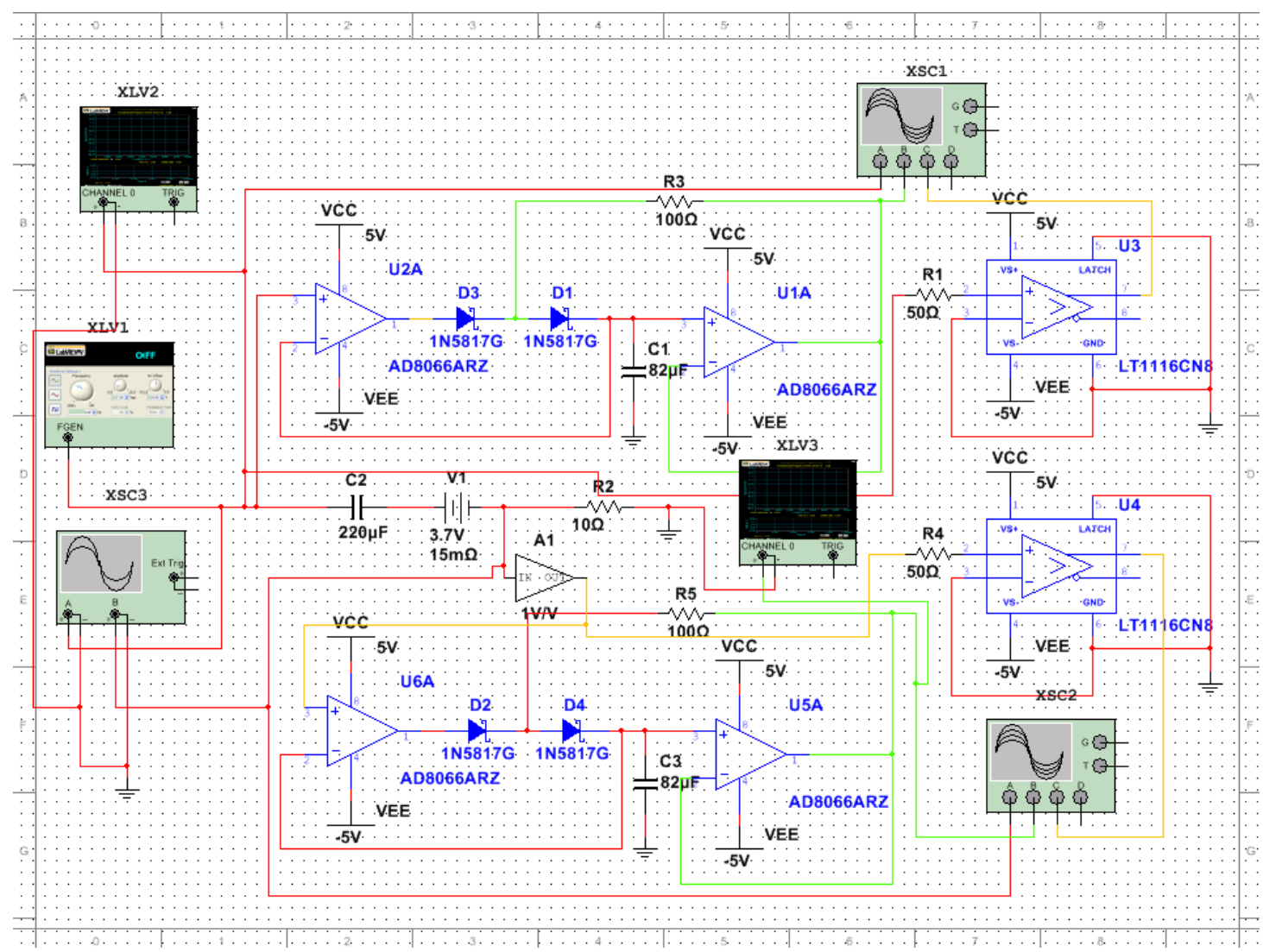

Figure 4.14. Implementation of EIS Test Circuit Topology in National Instruments Multisim. 

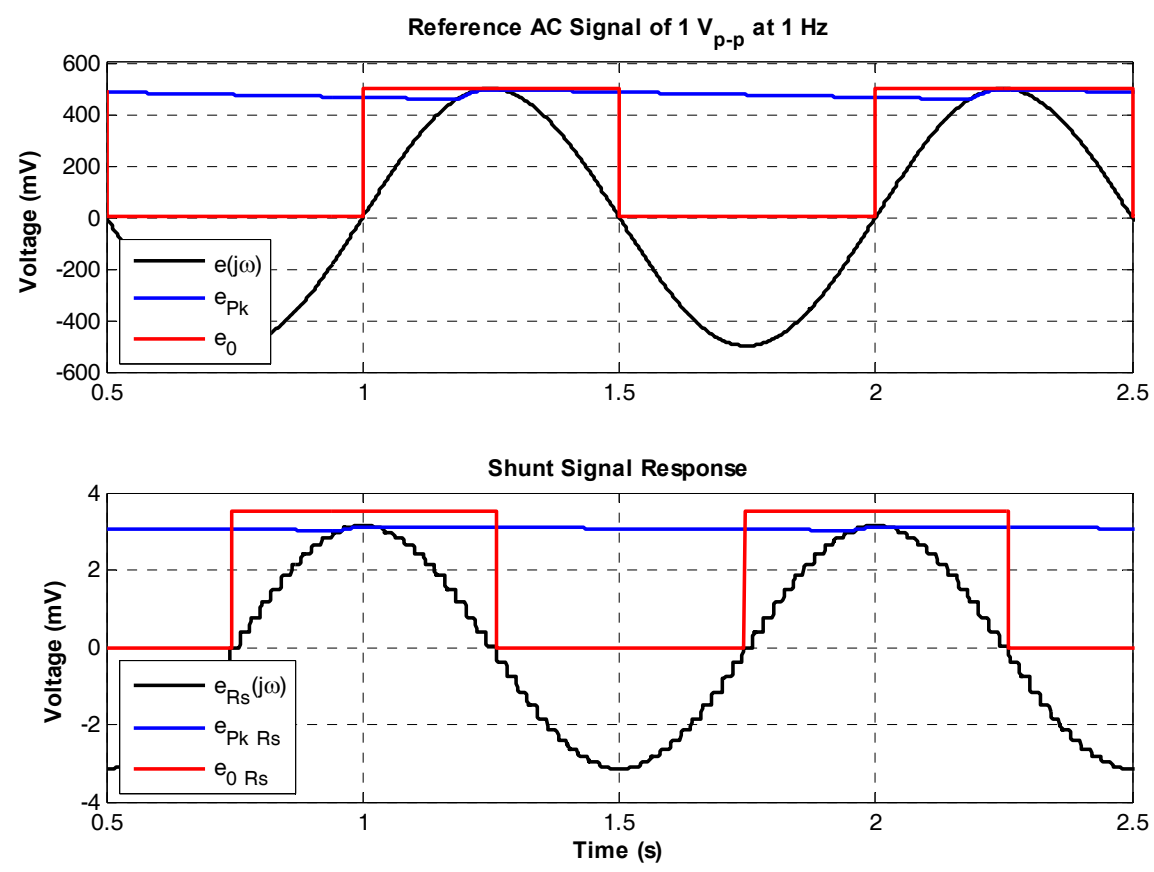

Figure 4.15. EIS Circuit Topology Simulation at Low Frequency (1 Hz).

\subsubsection{Low Frequency Performance}

The first test evaluated the performance of the proposed EIS circuit at low frequencies. Low frequency information is crucial in order to obtain the sum of all resistances in the equivalent circuit, but presents a challenge in terms of the minimal current response magnitude which will appear across the shunt. Figure 4.15 depicts the voltage $e_{x}(j \omega)$ in black and the peak detector $e_{P k_{x}}(j \omega)$ signals in blue where the peak is reduced from 500 $\mathrm{mVp}$ to only $3 \mathrm{mVp}$ after being passed through the shunt. The low frequency performance features the largest error of up to $7 \%$ for both the shunt and system AC voltage signals due to a requirement in limiting the capacitor size (Figure 4.10). For practical implementations, this would be increased for both signals to smoothen the peak or a switch case could be provided to transition between low and high reference frequencies, a tactic that is implemented in the proposed practical implementation in Section 4.6. The zero crossing 
digital signals $e_{0_{x}}(j \omega)$ (red) have been normalized to fit the plot scale and closely follow all zero crossings for both the shunt and AC system voltage signals.
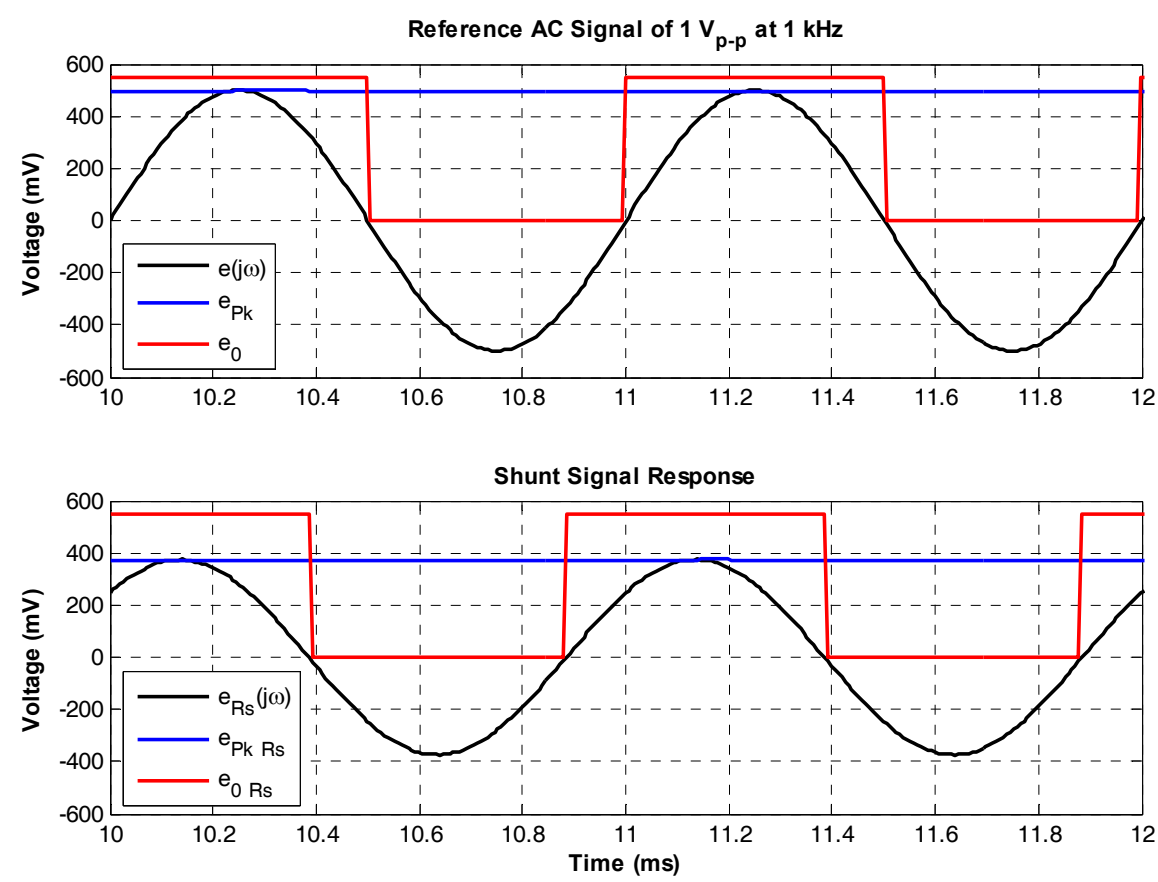

Figure 4.16. EIS Circuit Topology Simulation at Mid Frequency $(1 \mathrm{kHz})$.

\subsubsection{Mid Frequency Performance}

The second test evaluated the performance of the proposed EIS circuit at a mid-level frequency of $1 \mathrm{kHz}$. Mid-frequency information aids in inducing a maximum phase shift to obtain capacitor $\mathrm{C}_{p}$. Figure 4.16 once again depicts the voltage signals in black $e_{x}(j \omega)$ and peak detectors $e_{P k_{x}}(j \omega)$ in blue. At mid-level frequencies, the shunt response increases to around $380 \mathrm{mVp}$. Although a ripple is still present on both peak detectors, it is minimal at $1 \mathrm{kHz}$ at less than $1 \%$. The zero crossing digital signals $e_{0_{x}}(j \omega)$ are once again depicted in red. 


\subsubsection{High Frequency Performance}

The final test evaluated the performance of the proposed EIS circuit at the maximum design frequency of $100 \mathrm{kHz}$. High frequency information aids in revealing the ohmic resistance $R_{t}$. Figure 4.17 once again depicts voltage signals $e_{x}(j \omega)$ in black and peak detectors $e_{P k_{x}}(j \omega)$ in blue. At $100 \mathrm{kHz}$ (in this simulation), the magnitude of both the AC system voltage and shunt are nearly equal revealing that the maximum test frequency for the battery has been reached. A close inspection shows that the peak detection of the AC system voltage and shunt is nearly linear. The zero detection circuits $e_{0_{x}}(j \omega)$ are still operating correctly, but at $100 \mathrm{kHz}$ a slight delay is present as a result of the upper level limitations of the LT 1116 comparator [96].
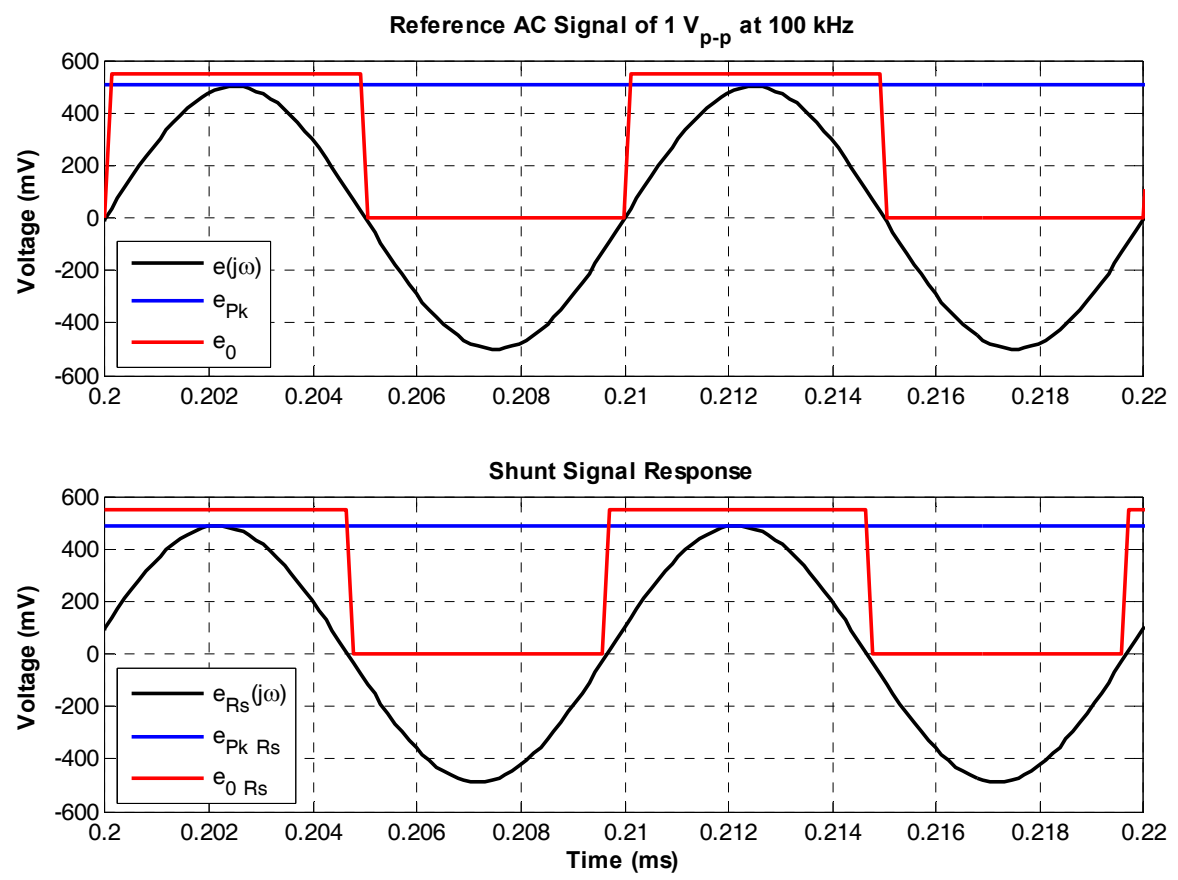

Figure 4.17. EIS Circuit Topology Simulation at High Frequency (100 kHz). 


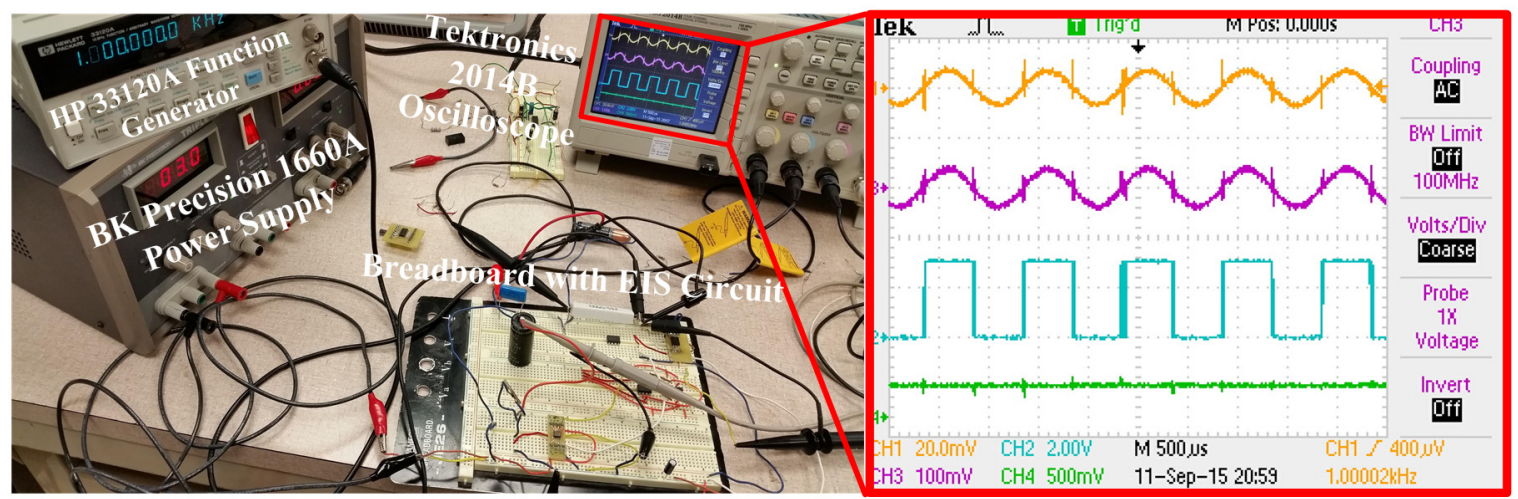

Figure 4.18. Experimental testing of EIS Circuit on a Breadboard.

\subsection{Laboratory Verification}

Following the production of successful experimental results, part of the EIS circuit was tested on a breadboard to provide an initial verification using one set of peak and zero crossing detection components. The setup is pictured in Figure 4.18. To the right, the oscilloscope screen has been expanded for easier recognition. In this scenario, the input from the function generator is shown in orange while the amplified output signal is shown in purple. The scales have been adjusted in order to save space on the scope screen so the purple waveform has been amplified by 5 times $(100 \mathrm{mV} / \mathrm{step}$ versus $20 \mathrm{mV} / \mathrm{step})$. The square wave output from the zero crossing circuit is shown in blue which closely follows the zero crossing points of both the amplified and original $\mathrm{AC}$ wave, hereby following the frequency. Finally, the output from the peak detection circuit is shown in green. Aside from some shot noise at the zero crossing times, the signal reveals a stable constant voltage level that can be isolated with minor hardware or digital filtering. 

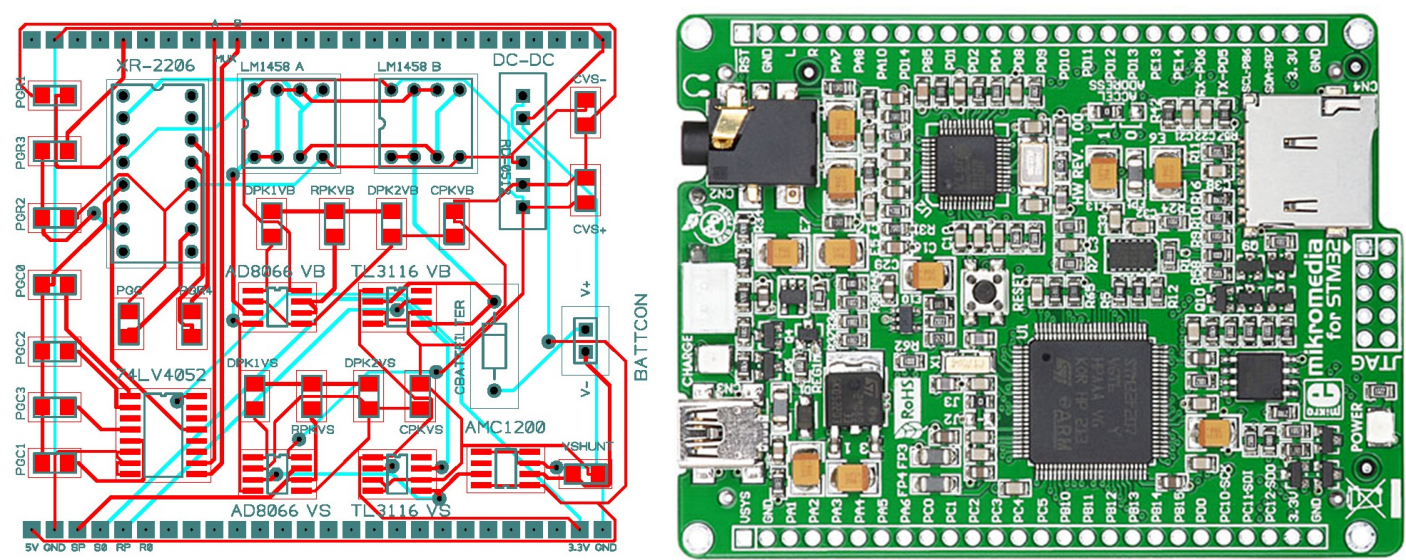

Figure 4.19. On Board EIS Tester: Proposed Printed Circuit Board Design (left) for Integration with the ARM Cortex M4 (right).

\subsection{Proposed Printed Circuit Board Design and Integration}

A practical implementation of the EIS design has been drafted on a PCB that is intended to easily mate with a MikroElektronika STM32 ARM Cortex M4 MCU [97]. The Cortex M4 MCU was selected for integration as it carries with it the same $168 \mathrm{MHz}$ STM32F407 processor as was featured on the STM32 Discovery Board previously investigated for the ESMC. However, the Cortex M4 also includes a 320 x 240 pixel touchscreen interface with a reduced footprint of only $8 \mathrm{~cm} \times 6 \mathrm{~cm}$.

Figure 4.19 shows the proposed PCB layout to the left, which is designed to mate with the Cortex M4 layout pictured to the right. To provide primary power, a differential DCDC converter was favored to convert the supply from the MCU for use of the EIS circuit, while an EXAR XR 2206 monolithic function generator IC is used to generate the AC reference wave [98]. The output frequency of the AC signal generated by the XR 2206 can be pooled into a number of frequency bands dictated by a capacitance value tied to one of its control pins. Since the XR 2206 must output an extremely wide range of frequencies, a 
bank of five different capacitors were needed to cover the range. A Nexperia 74LV4052 4channel multiplexer is purposed to dynamically switch between each capacitor [99]. SOP16 Surface mount device (SMD) packages with a $1.27 \mathrm{~mm}$ pin spacing were selected for the 74LV4052, LT 1116, AD 8066, and AMC 1200. 1206-type (3.2 x $1.6 \mathrm{~mm})$ surface mount resistors, capacitors, and diodes were selected for remaining components.

\subsection{Summary}

In this chapter, one of two methods outlined in this dissertation was explored in detail to obtain a battery equivalent circuit while also shedding more light on the topic of analyzing and assessing a battery SoH. A comprehensive review of the theory, procedure, and challenges involved in the deployment of an EIS device was discussed as well as how it can be used to track the $\mathrm{SoH}$ of a battery. A procedure to obtain a wide range of extended Randles equivalent circuit models was discussed, while the operation and output from a commercial system was demonstrated.

A circuit topology was designed taking into consideration the various challenges involved in the operation and acquisition of EIS signals. In the design, the main areas of interest pointed toward precision peak and zero crossing detection in order to measure how a signal magnitude and phase changes as it passes through the battery at each frequency. Simulation results were presented as well as extended testing of the physical circuit for laboratory verification. Finally, a PCB design was proposed intended for integration with a common Cortex M4 MCU or as a future expansion module for the ESMC.

Although the physics and operation of a battery play a large part in why EIS works, it was only briefly discussed as a way to map out the response of a battery to the extraction of equivalent circuit components. In the next chapter, the physics of the lead acid and 
lithium ion batteries will be discussed in great detail. An alternative method to obtain the battery equivalent circuit and $\mathrm{SoH}$ will be presented through the application of a standardized loading pulse, which will be verified through the development of PBMs of both the lead acid and lithium ion batteries. This same loading pulse also carries with it a second feature: autonomous detection of the battery chemistry. A new, comprehensive battery management scheme is then proposed to decipher the lead acid or lithium ion battery chemistry and determine the cell configuration while applying advanced $\mathrm{SoC}, \mathrm{SoH}$, and battery equivalent circuit determination procedures. 


\section{Chapter 5 Physics Based Modeling of Lead Acid and Lithium Ion Batteries}

\subsection{Introduction}

There has been a great deal of discussion in previous chapters regarding the operation, popularity, and drawbacks surrounding the lead acid battery. Despite its downsides, it still remains the most prevalent vehicle starter battery and a dependable resource to provide auxiliary power support [100]. Lead acid batteries are a cost-effective method to regulate and store the energy generated by renewable resources, particularly in grid-scale applications [14]. Although lithium ion batteries carry with them a much higher price tag, they are far superior in their higher energy and power densities, increased tolerances to heavy discharge currents, and reduced charging periods. Solar applications, in particular, have traditionally deployed advanced lead acid batteries on site, though lithium ion batteries are slowly being added to improve power capacity and reduce concerns over their lifespans [101]. Lithium ion has even begun to appear in the consumer market for residential renewable energy systems, such as the Tesla Powerwall [102]. However, many of these applications still utilize legacy deep cycle lead acid batteries or a hybrid of both to provide affordable backup power [103].

For electric vehicles (EV), the introduction of lithium ion batteries for propulsion has virtually led the growth of the market [25]. Although lithium ion is typically designated as the primary source of power, often other energy storage (ES) devices are included as well, including lead acid batteries, to support auxiliary power and lighting [12]. As future Battery Management System (BMS) devices and algorithms are developed, it is important to continue support for both battery types to enable both an interchangeable ES system and 
hybrid battery systems. The US Navy has begun analyzing hybrid battery storage as well in their commitment toward an all-electric ship with the DDG 1000, while the aeronautical industry is progressing through a transformation of its own from lead acid to lithium ion battery systems [111],[112]. While support for the efficient operation of both battery types is needed, it is also important to consider the need for accurate State of Health $(\mathrm{SoH})$ estimations as well. Both batteries will be susceptible to their own unique SoH impacts. SoH tracking and obtaining a dynamic Randles equivalent circuit model is needed to optimize both the lead acid and lithium ion battery lifespans.

In the previous chapter, the concept of Electrochemical Impedance Spectroscopy (EIS) was explored as an accurate method to determine the battery $\mathrm{SoH}$, however, the process was complex and costly to implement. For this reason, common BMS tools on the market are not usually equipped with an advanced method to obtain SoH. If tracked, it is typically limited to historical cycling information [104]. Furthermore, the BMS is typically specific to a given battery type, cell configuration, and/or the capacity, making a majority of them proprietary. Autonomous detection of the battery type and characteristics would provide a wide range of new capabilities, and depending on the method, could also provide a great deal of insight into the battery SoH. A detection algorithm was proposed for a smart charger in Reference [105] but required a full discharge of the battery. Reference [106] presented a battery chemistry identification scheme through defining a battery voltage gradient, but required heavy discharge rates to work and was only tested and verified on smaller cell capacities.

Recently, new proposed BMS have begun to address the importance of including $\mathrm{SoH}$ inside their platforms [107]. In Reference [108], an adaptive parameter estimation method 
was proposed to monitor $\mathrm{SoH}$, but was computationally intensive. Reference [46] demonstrated the importance of including SoH to continuously adjust the operating range based on the cycle performance. The use of pulsed load testing has surfaced as another option, but has remained limited to lithium ion batteries [110]. An optimal pulse testing solution should be compatible with both battery types. An adaptive controller compatible with both battery chemistries would improve interoperability, while easing the transition to a hybrid battery ES system.

In this chapter, an adaptive battery monitoring, health, and performance analysis technique is proposed and implemented for use in a hybrid battery ES system. An alternative equivalent circuit estimation technique and method to estimate a battery $\mathrm{SoH}$ is proposed as an alternative to EIS. Through the application of a low-frequency pulsed load, Randles $1^{\text {st }}$-order equivalent circuit values are acquired over each full battery cycle, while the same pulse is also used for initial battery chemistry detection. Physics based models (PBM) of both the lead acid and lithium ion battery cells are derived in detail, and are provided as a validation to the pulse test method. These features are then included in a comprehensive BMS platform, a hardware and software platform that could be added to the Energy Storage Management Controller (ESMC).

Another important facet of this dissertation is investigating ways to improve State of Charge (SoC) estimation in battery ES devices, thus this platform has also looked closely at the need for improving SoC accuracies for both battery types. Once the system is in operation, an adaptive algorithm accounts for shifts in the SoH from cycle-to-cycle using two assessment methods: 1) the estimation of equivalent circuit parameters and 2) the update of the usable capacity that is represented by a capacitive energy model. A final 
control platform is implemented, demonstrating the chemistry detection, automatic cell configuration, a refined initial $\mathrm{SoC}$ estimation, and the production of an online Randles equivalent circuit.

\subsection{Physics Based Battery Modeling}

This section will discuss the theory behind the use of pulse testing through the development and utilization of PBMs for both the common lithium ion and lead acid batteries. Through finite element modeling (FEM), insight into the behavior of each cell and how a standardized pulse can be applied regardless of its capacity will result in the same behavior. The basis for each battery interface was developed in COMSOL Multiphysics through a coupling between electrochemistry and electromagnetics [113],[114]. A pseudo two-dimensional (P2D) spatial representation of the negative electrode, electrolyte, and positive electrode provide a FEM environment to describe the behavior of each cell under a normalized discharge pulse. Various standardized discharge pulses were tested, but a Coulombic Rate (C-rate) of C/10 (10-hour discharge) over a $50 \mathrm{~s}$ period $(0.005 \mathrm{~Hz})$ was ultimately selected, as it applied a strong enough current density to push each cell out of equilibrium without causing unnecessary harm to the battery. A $25 \%$ duty cycle was chosen to elongate the recovery voltage period, which will be used to generate time constants for both the lead acid $\left(\tau_{P b}\right)$ and lithium ion $\left(\tau_{L i}\right)$ battery. This will be discussed in detail later.

Although the operation of the lead acid and lithium ion battery cells is drastically different, both are governed by Ohm's law, which describes the transport of charge in each electrode $i_{s}$ and electrolyte $i_{l}[115]$ :

$$
\nabla \cdot \boldsymbol{i}_{s}=Q_{s}
$$




$$
\nabla \cdot \boldsymbol{i}_{l}=Q_{l}
$$

where $Q_{s}$ and $Q_{l}$ represent a sum of all current contributions in the electrodes and electrolyte, respectively. The total active induced currents in each cell are:

$$
i_{v, t o t}=\sum_{m} a_{v} i_{l o c}+i_{v, d l}
$$

where $a_{v}$ is the active surface area under all $m$ reactions and $i_{v, d l}$ represents an induced current as a result of the double layer capacitance in the electrodes. Electrode kinetics are depicted by the localized current produced at the electrodes $i_{l o c}$ and are described by the Butler-Volmer expression:

$$
i_{l o c}=i_{0}\left[e^{\frac{\alpha_{a} \eta F}{\mathrm{RT}}}+e^{\frac{\alpha_{c} \eta F}{\mathrm{RT}}}\right]
$$

where $i_{0}$ is the exchange current density, $\alpha_{a}$ and $\alpha_{c}$ are charge transfer coefficients, $R$ is the universal gas constant $(8.314 \mathrm{~J} / \mathrm{mol} \cdot \mathrm{K}), F$ is Faradays constant $(96,485$ Coulombs $/ \mathrm{mol})$, $T$ is the absolute temperature, and $\eta$ is the activation overpotential describing how the cell voltage behavior will deviate from its electrochemical equilibrium potential $E_{e q}$. Equation (5-4) reveals how the current generated at the electrodes will result in a voltage drop $\Delta V$ in each of the battery types as a result of the overpotential $\eta$, which is calculated by the following:

$$
\eta=\Delta V=\varphi_{s}-\varphi_{l}-E_{e q}
$$

where $\varphi_{s}$ and $\varphi_{l}$ are the electric potentials of the electrode and electrolyte, respectively.

\subsubsection{Lead Acid Cell}

The lead acid battery operation was briefly discussed in Chapter 3 in the development of a preliminary equivalent cell model. In this section, its electrochemical formula and 
function is reviewed and mapped to a crucial set of differential equations governing its operation. As previously mentioned, the lead acid battery operation is primarily governed by changes in the sulfuric acid electrolyte concentration $c_{l}$, which decreases during discharging and increases during charging. The chemical formula defining the charge and discharge processes is as in Equation (5-6), where lead and lead dioxide electrodes are placed in an electrolyte reservoir to precipitate the storage and removal of electrons:

$$
\mathrm{Pb}+\mathrm{PbO}_{2}+2 \mathrm{H}_{2} \mathrm{SO}_{4}+2 e^{-} \rightleftharpoons 2 \mathrm{PbSO}_{4}+2 \mathrm{H}_{2} \mathrm{O}+2 e^{-}
$$

A fully charged battery has an electrolyte concentration $c_{l}$ of approximately $60 \%$ sulfuric acid by volume $(20 \mathrm{~mol} / \mathrm{L})$ and a discharged battery is primarily water $(\sim 0 \mathrm{~mol} / \mathrm{L})$. Three governing equations limit the speed of the electrochemical conversion process. First, $R_{l_{P b}}$ represents an electrochemical reaction source term describing how charge is transferred to the electrodes. A superficial velocity vector $\mathbf{u}$ limits the electrode reaction speed [116]:

$$
R_{l_{P b}}=\frac{\partial c_{l}}{\partial t}+\nabla \cdot\left(-D_{l} \nabla c_{l}\right)+\mathbf{u} \cdot \nabla c_{l}
$$

where $D_{l}$ represents the diffusion coefficient at each concentration $c_{l}$. The speed of the transport process is further constrained by the molar flux $\mathbf{N}_{l_{P b}}$ generated inside the electrolyte:

$$
\mathbf{N}_{l_{P b}}=-D_{l} \nabla c_{l}+\mathbf{u} c_{l}
$$

a quantity that is also impacted by $D_{l}$ and $c_{l}$. A third component impacting the voltage response is a considerable double layer capacitance $i_{v, d l}$ :

$$
i_{v, d l}=\left[\frac{\partial \varphi_{s}}{\partial t}-\frac{\partial \varphi_{l}}{\partial t}\right] a_{v} C_{d l}
$$

which is a function of the changing electrode and electrolyte potentials, the double layer capacitance $C_{d l}(\mathrm{~F} / \mathrm{m})$, and the active surface area $a_{v}$, which is directly proportional to the 
cell capacity. The active surface area for the lead acid battery cell $a_{v_{P b}}$ is:

$$
a_{v_{P b}}=a_{v, \max }\left[\frac{\varepsilon-\varepsilon_{0}}{\varepsilon_{\max }-\varepsilon_{0}}\right]
$$

where the difference in the porosity $\varepsilon$ of the lead and lead dioxide electrodes from full charge $\varepsilon_{\max }$ to full discharge $\varepsilon_{0}$ limit the maximum surface area $a_{v, \text { max }}$, hereby reducing the total active induced current in Equation (5-10). In the lead acid cell, a higher overpotential $\eta_{P b}$ is required in order to generate the same current, thus Equation (5-4) is modified to include a constraint based on $c_{l}$. The localized current produced at the lead acid battery cell electrodes is:

$$
i_{l o c_{P b}}=i_{0}\left[e^{\frac{\alpha_{a} \eta F}{\mathrm{RT}}}+e^{\frac{\alpha_{c} \eta F}{\mathrm{RT}}}\right]\left[\frac{c_{l}}{c_{l, r e f}}\right]
$$

where the lead acid exchange current density $i_{0}$ is a constant.

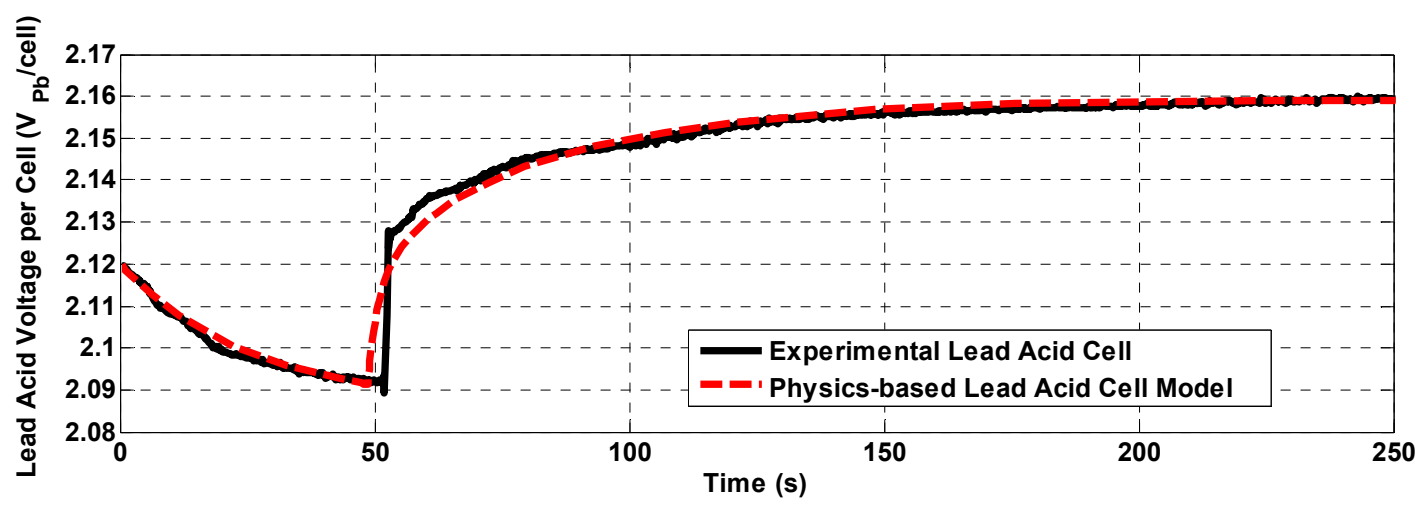

Figure 5.1. Physics-based Model to Experimental Comparison under a C/10 0.005 Hz $50 \%$ Duty Load Pulse for a Lead Acid Cell.

The PBM versus experimental lead acid cell voltage is shown in Figure 5.1, where a normalized $\mathrm{C} / 10$ discharge pulse is applied at $0.005 \mathrm{~Hz}$ under a $25 \%$ duty cycle to a fully charged cell. The single-cell lead acid battery used in this verification has a 6 Amp-hour (Ah) capacity. A close inspection reveals a long voltage recovery time $\tau_{P b}$ as well as a 98 
$\mathrm{mV}$ voltage drop from equilibrium (i.e. open circuit). Since this behavior will differ when the battery is not at full charge, to obtain equivalent circuit parameters, the pulse must be applied at $100 \%$ SoC. However, a lack of full charge will not heavily impact $\tau_{P b}$, thus battery identification can be accomplished by widening the tolerance. The model follows a close approximation to that of the experimental test, except at the pulse transition period. This is likely a limitation in the P2D model and/or differences in manufacturing of the electrolyte reservoir in the test battery. A correlation between $a_{v_{P b}}$, rated capacity $E_{\text {rated }}$, and $i_{\text {loc }_{P b}}$ reveals the voltage under a $\mathrm{C} / 10$ discharge pulse results in a similar behavior regardless of the cell capacity.

\subsubsection{Lithium Ion Cell}

In this section, the chemistry and operation of the lithium ion battery cell is introduced. The lithium ion cell operates very differently, as it primarily stores charge in its electrodes, utilizing a lithium salt electrolyte purely as a transport layer [21]. The chemical formula defining the operation of the common lithium ion cobalt oxide (LCO) cell is:

$$
\mathrm{LiCoO}_{2}+\mathrm{C}+\mathrm{xLi}^{+}+\mathrm{xe}^{-} \rightleftharpoons \mathrm{CLi}_{x}+\mathrm{Li}_{1-x} \mathrm{CoO}_{2}+\mathrm{xLi}^{+}+\mathrm{xe}^{-}
$$

where the charging process is shown from left to right and the discharging process from right to left. Its transport properties are revealed by the lithium ion molar flux equation in Reference [21]:

$$
\mathbf{N}_{l_{L i}}=-D_{l} \nabla c_{l}+\frac{\mathbf{i}_{l} t_{+}}{F}
$$

where the reaction speed is limited by the transport number $t_{+}$as current $\mathbf{i}_{l}$ is carried across the electrolyte. The lithium ion cell reaction source term $R_{l_{L i}}$ is still a function of a varying electrolyte concentration, but is now impacted by changes in $\mathbf{N}_{l_{L i}}$ as well. 


$$
R_{l_{L i}}=\frac{\partial c_{l}}{\partial t}+\nabla \cdot \mathbf{N}_{l_{L i}}
$$

One can observe that $\mathbf{N}_{l_{L i}}$ and $R_{l_{L i}}$ contain no superficial speed terms $\mathbf{u}$ as were present in the lead acid cell. In addition, the double layer capacitance from Equation (5-9) is very low as compared to the lead acid $C_{d l_{P b}} \gg C_{d l_{L i}}$, thus it has been neglected.

Other major differences are present in the operation of a lithium ion battery that result in a shallower voltage drop under load. The active surface area in Equation (5-3) has no immediate constraints $a_{v_{L i}} \cong a_{v_{\max }}$, reducing $\eta_{L i}$. Furthermore, the Butler-Volmer expression is left unmodified, removing its dependence on the electrolyte concentration. The exchange current density, $i_{0_{L i}}$ varies based on kinetics:

$$
i_{0_{L i}}=F\left(k_{c}\right)^{\alpha_{a}}\left(k_{a}\right)^{\alpha_{c}}\left(c_{s, \max }-c_{s}\right)^{\alpha_{a}}\left(c_{s}\right)^{\alpha_{c}}\left(c_{l}\right)^{\alpha_{a}}
$$

where $k_{a}$ and $k_{c}$ represent charge rate constants and $c_{s}$ and $c_{s, \max }$ represent the current and maximum concentration of the electrodes, respectively. Equation (5-15) demonstrates how the primary current generated at the load is a result of the concentration of lithium salt in the electrodes. The conductivity, however, can be impacted later in life as a solidelectrolyte interphase (SEI) layer forms with respect to cycle life and operation. However, this has minor impacts on the timing constant primarily used in lithium ion battery detection.

The PBM versus experimental lithium ion cell voltage is shown in Figure 5.2, where the same normalized $\mathrm{C} / 10$ discharge pulse is applied to a fully charged cell. The single lithium ion cell under test is nearly equivalent in capacity to the lead acid test cell at 6.4 Ah. A much shorter voltage recovery time $\tau_{L i}$ and voltage drop is present from equilibrium $(68 \mathrm{mV})$ than that of the lead acid cell. Figure 5.2 reveals a closer approximation to that of 
the experimental test as a result of minimal dependence on the nonlinear double layer capacitance. Since the same general set of equations are solved for, the lithium ion cell has the same dependence over $a_{v_{L i}}, E_{\text {rated }}$, and $i_{l o c_{L i}}$, revealing the voltage behavior under a standardized discharge pulse behaves in a similar manner regardless of the cell capacity.

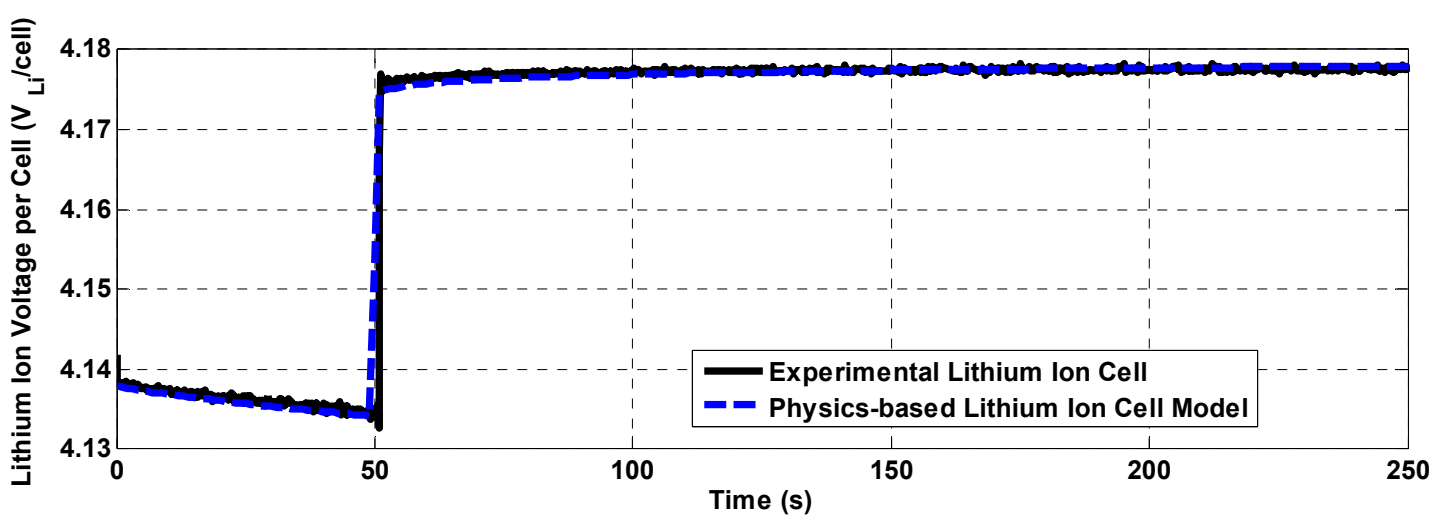

Figure 5.2. Physics-based Model to Experimental Comparison under a C/10 $0.005 \mathrm{~Hz}$ $50 \%$ Duty Load Pulse for a Lithium Ion Cell.

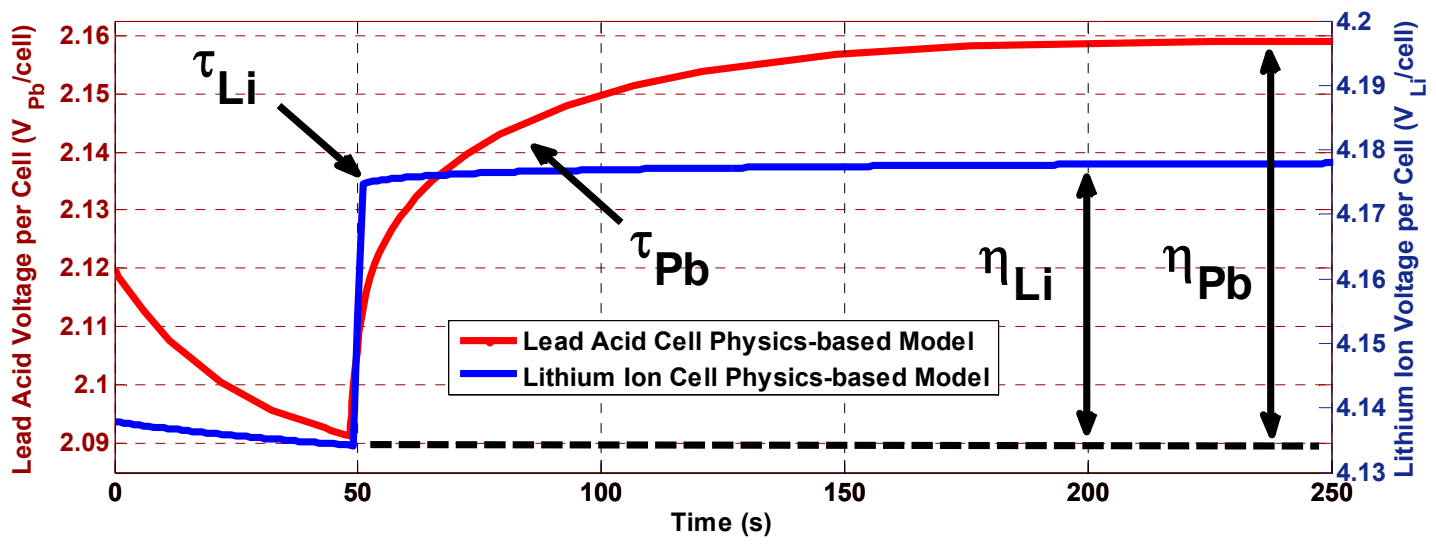

Figure 5.3. Lead Acid versus Lithium Ion Cell Voltages under $0.005 \mathrm{~Hz}$ C/10 Load Pulse at $25 \%$ Duty Cycle.

\subsubsection{Physics-based Battery Differentiation}

A comparison between both the lead acid and lithium ion PBMs under the $\mathrm{C} / 10$ discharge pulse is shown in Figure 5.3. One can observe a striking difference in the voltage recovery periods of the lead acid $\tau_{P b}$ versus lithium ion $\tau_{L i}$ battery cells, as well as a $30 \%$ 
reduction in the overpotential $\eta$ when comparing the lithium ion cell to the lead acid cell. Although there are a number of advantages in obtaining the full PBM, particularly in the design and off-line estimation domain, it is challenging and computationally-intensive to utilize it in an online controller.

One of the major novelties of the developments in this chapter is recognizing that a full physics-based controller is unnecessary to identify the battery chemistry and obtain a Randles $1^{\text {st }}$-order equivalent circuit, but can be used as an intermediary to link between the physics and electrical domains. Differences in the electrochemical source terms $R_{l}$ and double layer capacitances $i_{v, d l}$ are connected to the recovery voltage, generating a time constant $\tau$ as a means for chemistry detection. In the same way, a scaling of the ButlerVolmer expression $i_{l o c}$, in conjunction with a different active surface area $a_{v}$ while in operation, provides the two driving factors to link a standardized C-rate pulse to a predictable response.

Ideally, if each battery type were tested in new condition or at a matching SoC, a single standard response would be expected from each chemistry. Using this as a reference, differences in the response from an ideal (expected) state would reveal signature values for an electrochemical equivalent circuit while providing a secondary measure to assess and track the SoH. Through establishing a firm relationship to predict the behavior using this PBM representation, the development of a real-time BMS can be accomplished based on a relatively simple foundation. This process is discussed in detail later in Section 5.4.

\subsection{Battery Management Systems}

All battery ES require a specialized BMS to provide a robust monitoring and protection platform. This information, however, only provides accuracy when a method has been 
implemented to provide insight into its lifetime, performance, and voltage stability to properly control and maintain its SoH. This is particularly the case in an EV, shipboard, or aeronautical power system application, where high C-rates and a deep Depth of Discharge (DoD) would be frequently observed [117]. The following subsections discuss how this unique BMS and cycling platform will increase accuracy in terms of SoC measurement, differentiating battery types, and assessing $\mathrm{SoH}$.

\subsubsection{State of Charge}

The open circuit voltage (OCV) is an excellent source to obtain the initial SoC, assuming it is taken at a state of equilibrium. Following this, an enhanced coulomb counting mechanism can be deployed. The combination of both OCV and coulomb counting has been standardized in industry, but both can suffer from a number of inaccuracies that have led to a variety of improvements in initial and online SoC estimation. One way is through utilization of an Extended Kalman Filter (EKF), but its success strongly depends on the accuracy of the battery model and a predetermined system noise matrix [118]. For the applications addressed in this dissertation, the noise content is expected to be substantial. In Reference [119], an adaptive EKF was introduced to improve this issue, but could not be applied to an aged cell. Since the proposed system has been designed to have a wide SoH and noise tolerance, the EKF-based controllers have been avoided. However, two core factors have been involved in the preliminary design of these controllers: a temperature-dependent initial OCV-based SoC estimation and a coulomb counting scheme adjusted based on the recent estimated usable capacity.

\subsubsection{Initial Voltage-Based Measurements}

OCV estimations are highly dependent on the chemistry and ambient temperature, and 
recent works have explored new methods to improve them [120],[121]. In Reference [120], extensive testing was conducted to assess performance over a wide temperature range, presenting the concept of a multistate OCV-based SoC estimation dependent on whether the battery was previously in the charging or discharging phase. Reference [121] presented a revised method acknowledging the resting time can have an impact on the OCV-based SoC for lithium ion phosphide ( $\mathrm{LiFePO} 4)$ batteries. Although both systems revealed an improvement, they required previous knowledge of the battery state or how it was used. Furthermore, both had heavy computational requirements. In the development of this system, a focus has been placed on optimizing the tradeoff between accuracy and simplicity to make a package suitable for a microcontroller unit (MCU)-based system similar to the commercial ESMC. These systems are assumed to have no previous knowledge of the battery connected. Since the temperature dependence is significant and does not require previous data, the OCV-based initial SoC equations for both lead acid and lithium ion cells account for temperature.

\subsection{Lead Acid Batteries}

The lead acid battery cell OCV $V_{o c_{P b}}$ measurements are based on the Nernst equation, a fundamental relationship between the electromotive force of the cell, its electrochemical reactions, and thermodynamics [47]. As opposed to a curve fitting procedure resulting in coefficients with no physical meaning, the Nernst equation provides a bridge to the electrochemical realm. The OCV of a single lead acid cell $V_{o c_{P b}}$ under no load is:

$$
V_{o c_{P b}}=\xi^{0}-2.303 \frac{R T}{n F} \log c_{l}
$$

where $\xi^{0}$ is the electrode potential of a lead acid cell $(1.931 \mathrm{~V})$ and $c_{l}$ represents the molar 
concentration of the electrolyte. Since the $\mathrm{SoC}$ of a lead acid cell is directly proportional to $c_{l}$, Equation (5-16) is rearranged to solve for SoC taking into consideration both a fluctuating $V_{o c_{P b}}$ and temperature $T$ :

$$
S o C_{P b}\left(V_{o c_{P b}}, T\right)=a c_{l}\left(V_{o c_{P b}}, T\right)=a e^{\left[\frac{n F\left(V_{o c_{P b}}-\xi^{0}\right)}{2.303 R T}\right]}
$$

where $a$ is a linear scaling factor between $c_{l}$ and SoC equal to 5 , since the concentration range is $0<c_{l} \leq 20 \mathrm{~mol} / \mathrm{L}$, as was established in Section 5.2.1. A surface plot of Equation (5-17) is shown in Figure 5.4, where the temperature is varied from $-20^{\circ} \mathrm{C}$ to $+45^{\circ} \mathrm{C}$, a reasonable range of operation. Under this range, $V_{O c_{P b}}$ remains mostly linear over the temperature range, but reduces the OCV reference defining $100 \%$ SoC. Since the full discharge OCV is fixed, lower temperatures will dilate the operating voltage range, as can be shown in Figure 5.4.

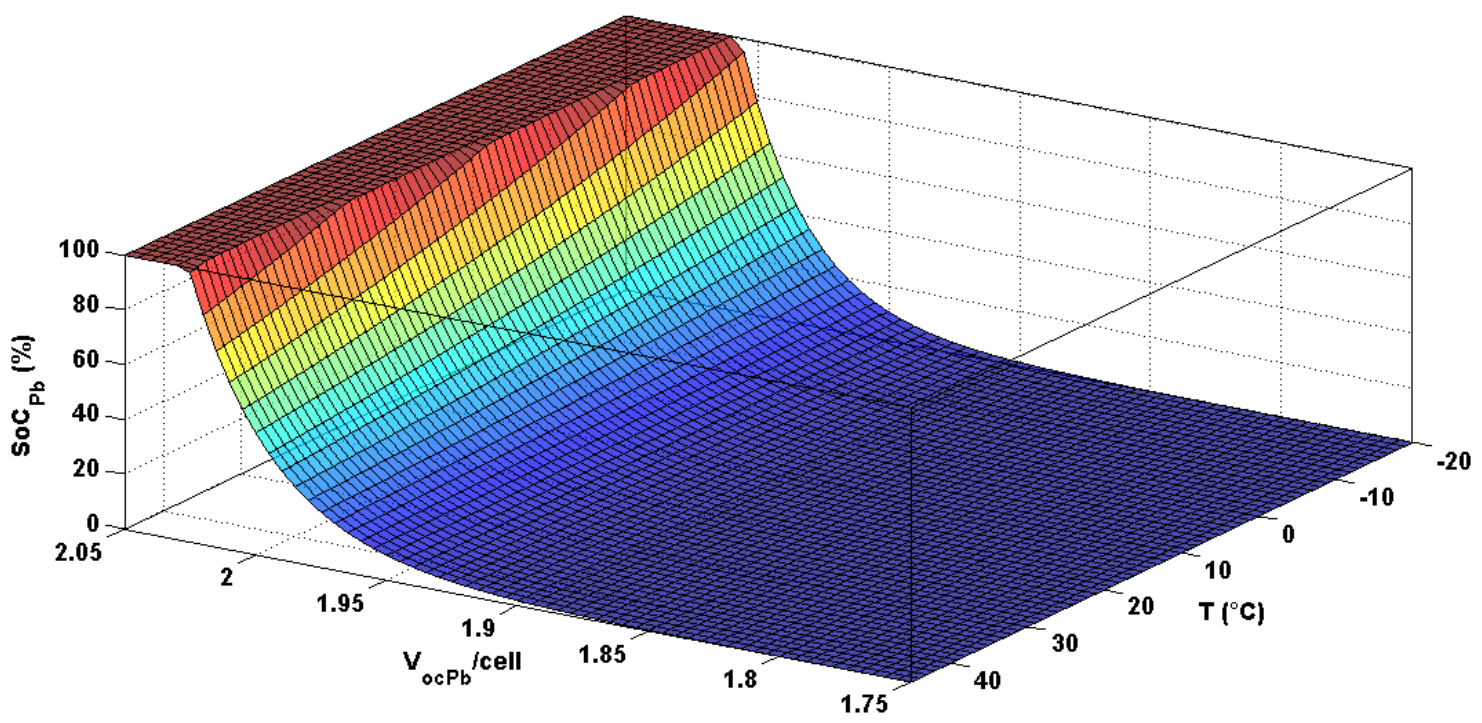

Figure 5.4. Open Circuit Voltage and Temperature versus SoC function per cell for a Lead Acid Battery. 


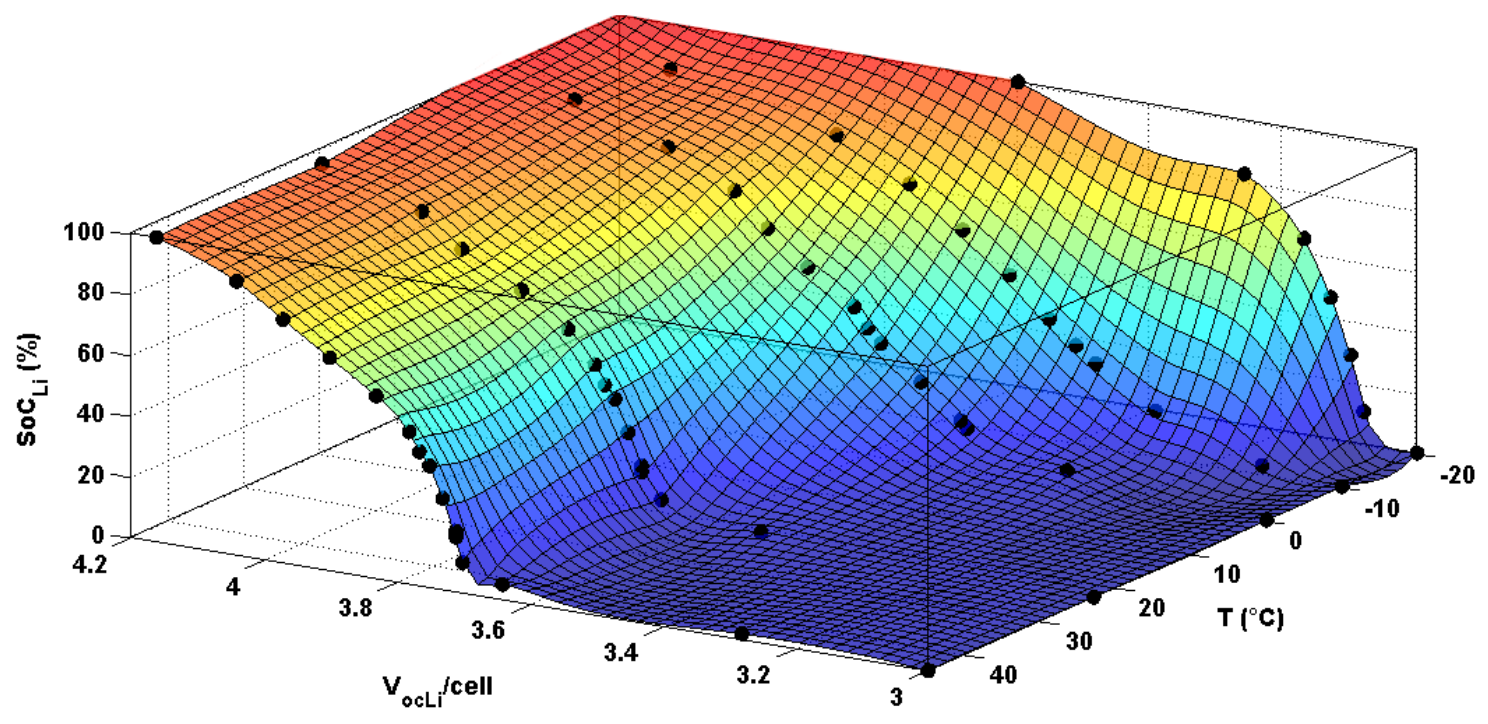

Figure 5.5. Open Circuit Voltage and Temperature versus SoC function per cell for a Lithium Ion Battery.

\subsection{Lithium Ion Batteries}

The lithium ion battery OCV $V_{o c_{L i}}$ measurements are different from that of the lead acid battery cell as a result of its very different operation. The relationship between the SoC $S o C_{L i}$, OCV $V_{o c_{L i}}$, and temperature $T$ is highly nonlinear. Since its dependence cannot be conveniently mapped to the Nernst equation, curve-fitting was selected to generate a lookup table to determine $S o C_{L i}\left(V_{o c_{L i}}, T\right)$. For the lithium ion cell, $V_{o c_{L i}}$ versus SoC curves were replicated at five different temperatures: $-20^{\circ} \mathrm{C},-10^{\circ} \mathrm{C}, 0^{\circ} \mathrm{C},+23^{\circ} \mathrm{C}$, and $+45^{\circ} \mathrm{C}[122]$. A three-dimensional (3D) curve fitting procedure was then applied using a thin-plate spline function to interpolate values along the surface, shown in Figure 5.5. Original data points extracted from the each of the five curves are superimposed in black. Through the use of a lookup table, the computational requirement is dramatically reduced from the methods that were proposed in Reference [121]. 


\subsubsection{Coulomb Counting}

Following an estimation of the SoC at the OCV, charging and discharging energy in the system is monitored through coulomb counting. Using a timing reference, the current is integrated over a fixed sampling period to determine the capacity that was removed or replaced, but this method alone is insufficient. Enhanced coulomb counting methods have been addressed previously in References [119]-[121], highlighting the need to account for

the shift in the usable capacity $E_{u s a b l e}$ (in Ah) over time. Thus, $E_{u s a b l e}$ has been used as the reference in the online algorithm [123]:

$$
\operatorname{SoC}(t)=\operatorname{SoC}_{x}\left(t_{0}\right)-\int_{0}^{t} \frac{\eta I_{\text {batt }}}{3600 E_{\text {usable }}}(\tau) d \tau
$$

where $\operatorname{SoC}_{x}\left(t_{0}\right)$ represents the initial SoC OCV-based measurement for each battery type $x(\mathrm{~Pb}$ or $\mathrm{Li})$ integrated over a 1 -second period $d \tau$, where $I_{\text {batt }}$ is the battery current and $\eta$ is the cycle efficiency.

\subsubsection{Peukert's Component}

As the discharge current increases from the rated value of a battery, the available energy will decrease as a result of an increased overpotential [91]. Similarly, as the discharge current decreases, the overpotential is reduced, providing an increase in the available energy and runtime. To account for this phenomenon, Peukert's component has been implemented to correct the BMS for an altered expectation in the usable energy. Peukert's law provides a capacity adjustment $E_{\text {usable }}$ based on the following relation:

$$
E_{\text {usable }_{P}}=E_{\text {usable }}\left(\frac{E_{\text {usable }}}{I_{\text {batt }} H}\right)^{k-1}
$$

where $H$ is the rated discharge time in hours and $k$ is the Peukert constant. For this system, 
$k$ is assumed to be a constant value of 1.0909 , but depending on the application, this value would be adjusted by the battery manufacturer.

\subsubsection{Quantitative Definition of the State of Health}

While SoC is the most common active assessment for a battery, insight into performance and condition is crucial to maintain efficient operation and has already been a major topic of interest in previous chapters. Degradation and aging of a battery is a complex process that involves many parameters, but the most of interest to the user is the usable capacity. Although as previously mentioned, $\mathrm{SoH}$ has not yet been formally defined, in this system, a quantitative definition of $\mathrm{SoH}$ has been established. $\mathrm{SoH}$ is defined by the usable capacity $E_{\text {usable }}$ of the battery under the most recent full discharge cycle versus its rated capacity $E_{\text {rated }}$ :

$$
\text { SoH }=\left(\frac{E_{\text {usable }}}{E_{\text {rated }}}\right) \times 100 \%
$$

$E_{\text {rated }}$ can be set to the full nameplate (i.e. peak) or nominal capacity. The nominal rating presents a reduced operating capacity in order to preserve the life of the battery for applications with high DoD. Depending on the application, a few minor adjustments would need to be made to the BMS to restrict the battery operating range. First, a simple offset can be applied to the initial OCV-based curves from the lead acid and lithium ion batteries in Section 5.3.1.1, and second, a shallower discharge voltage cutoff and higher charging current cutoff would need to be observed.

\subsubsection{Generating the $1^{\text {st }}$-Order Randles Equivalent Circuit Model}

An alternative method to EIS is proposed in this subsection for estimating the component parameters through the use of a low frequency $\mathrm{C} / 10$ discharge pulse test. Recall 
the basic $1^{\text {st }}$-order Randles equivalent circuit that has been slightly modified in Figure 5.6. The resistance $R_{t}$ still represents the average ohmic resistance of the electrolyte, while $\mathrm{R}_{p}$ and $\mathrm{C}_{p}$ still represent the polarization resistance and capacitance, respectively. A method to estimate $\mathrm{R}_{p}$ and $\mathrm{C}_{p}$ will be discussed in detail in the following subsection. However, without highly accurate sensing and a precision load resistance, the ohmic resistance $R_{t}$ is challenging to obtain. Alternatively, a sum of the $\mathrm{C} / 10$ load $R_{L}$ and ohmic resistance $R_{t}+R_{L}$ can be monitored cycle-to-cycle by noting a reference value from the initial cycle. Using the Randles equivalent circuit parameters, the operator can monitor the specific aging processes of each battery module.

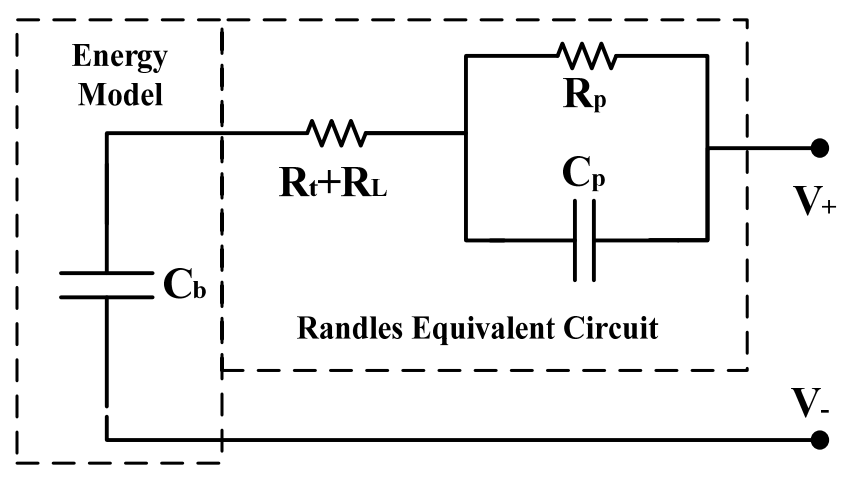

Figure 5.6. Battery Equivalent Model for Multichemistry System.

\subsubsection{Battery Energy Model}

The complete battery model is divided into two parts: the equivalent circuit parameter estimation and a lifetime energy model, which has been previously neglected. The common energy model depicted in Reference [109] models the battery as a large capacitor $C_{b}$ in parallel with a self-discharge resistor. However, for this system, the battery is assumed to be in operation anytime it is connected and due to high self-discharge resistances, has been 
omitted. The capacitance is referred to the OCV of each battery at full charge $V_{o c_{F C}}$, thus the equivalent charge storage capacitance $C_{b}$ is calculated by the following formula:

$$
C_{b}=3600 V_{o c_{F C}} E_{\text {usable }}
$$

\subsection{Battery and Health Identification}

Through applying a low frequency $\mathrm{C} / 10$ load, both an initial identification of the lead acid or lithium ion battery is accomplished as well as estimating its Randles equivalent circuit parameters to gain insight into aging. This section describes how these procedures can be implemented on a real-time BMS. The real-time BMS has been demonstrated using a battery testbed that is pictured later in Figure 5.8.

\subsubsection{Battery Identification}

The timing constant $\tau$ is generated through exponential curve fitting of the battery under test. $\tau$ is extracted and used as the primary metric to determine the battery type. Online curve fitting using the Least Squares Method (LSM) has been utilized in a number of BMS applications for a wide range of purposes, primarily for accurate capacity estimation [125],[126]. Reference [126] demonstrated how LSM could be implemented with the coulomb counting method, in a goal to reduce accumulated error in measurements. Reference [127] used LSM to extract equivalent circuit parameters online without the assistance of a loading or charging disturbance (generation of overpotential), but resulted in relatively high error and required over $15 \mathrm{~min}(1000 \mathrm{~s})$ to converge, whereas the proposed system takes $7 \mathrm{~min}$ under two passes of $3.5 \mathrm{~min}$ each. In this work, the traditional LSM method is sufficient since the $\mathrm{C} / 10$ discharge pulse instigates a considerable voltage drop and one which can produce a measurement immediately following the voltage recovery period. 
The basic formula for the traditional LSM is:

$$
\frac{1}{N} \sum_{i=0}^{N-1} w_{i}\left(f_{i}-V_{i}\right)^{2}
$$

where $N$ is the length of voltage $V_{i}$ samples, $w_{i}$ is the $i$ th weighting element, $f_{i}$ is the $i$ th element of the best exponential fit, and $V_{i}$ is the $i$ th element of the voltage input vector. The resulting exponential fit of the recovery period following the $0.005 \mathrm{~Hz}$ pulse under a $25 \%$ duty cycle can be reduced to a basic form:

$$
V[n]=a e^{-b t[n]}+c
$$

Following the calculation of the $a, b$, and $c$ values in Equation (5-23), the voltage response follows:

$$
[n]=V_{L}\left(1-e^{-\frac{t[n]}{\tau}}\right)
$$

Relating the curve fitted form of Equations (5-23) and (5-24), the generated time constant $\tau$ is extracted from $b$ and forced positive $|\tau|=\left|-b^{-1}\right|$.

Table 5-1 depicts a wide range of different lead acid and lithium ion batteries of different capacities, voltages, and known health conditions, where each battery is shown with its corresponding number in Figure 5.7. Each battery was tested three times to ensure consistent results. The lithium ion batteries were revealed to traditionally yield time constants below $1 \mathrm{~s}$, whereas lead acid batteries were found to yield time constants above $20 \mathrm{~s}$ thus $\tau=20 \mathrm{~s}$ was chosen as the threshold value. Under a closer inspection, $\tau$ can also approximate the condition of the battery. Generally, $\tau$ will begin to increase as the condition of the battery decreases. 
Table 5-1. Tested Batteries to Acquire Timing Constants.

\begin{tabular}{l|c|r|r|r|r|r|r}
\hline \hline Battery Type & Condition & Cells & $\begin{array}{c}\text { Nominal } \\
\text { Voltage }\end{array}$ & $\begin{array}{c}\text { Test } \\
\text { Current }\end{array}$ & Capacity & $\begin{array}{c}\text { Timing } \\
\text { Constant }\end{array}$ & $\begin{array}{c}\text { Battery } \\
\text { Number }\end{array}$ \\
\hline Li-ion - NMC & Fair & 1 & $3.7 \mathrm{~V}$ & $0.150 \mathrm{~A}$ & $1.5 \mathrm{Ah}$ & $3.869 \mathrm{~s}$ & 1 \\
\hline Li-ion - NMC & Good & 1 & $3.7 \mathrm{~V}$ & $0.200 \mathrm{~A}$ & $2.0 \mathrm{Ah}$ & $1.650 \mathrm{~s}$ & 2 \\
\hline Li-ion - LCO & Good & 1 & $3.7 \mathrm{~V}$ & $0.640 \mathrm{~A}$ & $6.4 \mathrm{Ah}$ & $0.245 \mathrm{~s}$ & 3 \\
\hline Li-ion - LCO & Bad & 1 & $3.7 \mathrm{~V}$ & $2.100 \mathrm{~A}$ & $21.0 \mathrm{Ah}$ & $14.292 \mathrm{~s}$ & 4 \\
\hline Li-ion - LCO & Good & 1 & $3.7 \mathrm{~V}$ & $2.100 \mathrm{~A}$ & $21.0 \mathrm{Ah}$ & $0.208 \mathrm{~s}$ & 5 \\
\hline Li-ion - LCO & Good & 2 & $7.4 \mathrm{~V}$ & $2.100 \mathrm{~A}$ & $21.0 \mathrm{Ah}$ & $0.208 \mathrm{~s}$ & $* 5$ \\
\hline Li-ion - LCO & Good & 3 & $11.1 \mathrm{~V}$ & $2.100 \mathrm{~A}$ & $21.0 \mathrm{Ah}$ & $0.477 \mathrm{~s}$ & $* 5$ \\
\hline Lead Acid & Good & 1 & $2.0 \mathrm{~V}$ & $0.600 \mathrm{~A}$ & $6.0 \mathrm{Ah}$ & $21.033 \mathrm{~s}$ & 6 \\
\hline Lead Acid & Good & 3 & $6.0 \mathrm{~V}$ & $0.450 \mathrm{~A}$ & $4.5 \mathrm{Ah}$ & $29.242 \mathrm{~s}$ & 7 \\
\hline Lead Acid & Fair & 3 & $6.0 \mathrm{~V}$ & $1.200 \mathrm{~A}$ & $12.0 \mathrm{Ah}$ & $33.013 \mathrm{~s}$ & 8 \\
\hline Lead Acid & Bad & 3 & $6.0 \mathrm{~V}$ & $1.200 \mathrm{~A}$ & $12.0 \mathrm{Ah}$ & $24.003 \mathrm{~s}$ & 9 \\
\hline Lead Acid & Good & 6 & $12.0 \mathrm{~V}$ & $2.100 \mathrm{~A}$ & $21.0 \mathrm{Ah}$ & $56.546 \mathrm{~s}$ & 10 \\
\hline Lead Acid & Fair & 6 & $12.0 \mathrm{~V}$ & $2.100 \mathrm{~A}$ & $21.0 \mathrm{Ah}$ & $34.150 \mathrm{~s}$ & 11 \\
\hline \multicolumn{7}{c}{ *Same cell type with similar age connected in series } &
\end{tabular}

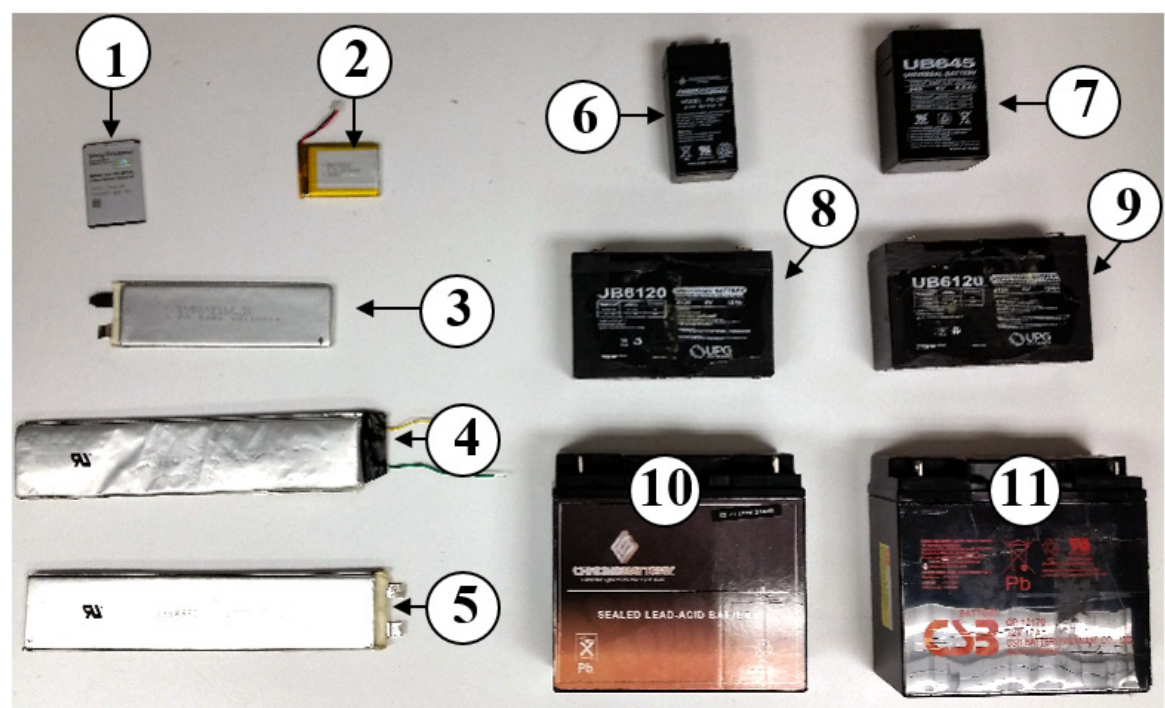

Figure 5.7. Batteries Tested for Chemistry Identification Mapped to Table 5-1.

Following the pulses and the OCV reaching an equilibrium state, the series-cell configuration of the battery can be determined. All batteries have nominal voltages dependent upon the chemistry. As a result, individual battery cell voltages and ranges can 
be classified within a normal range of operation when the source is in good condition. Table 5-2 depicts the different nominal voltages and operation ranges associated with each type of battery where $V_{d c}, V_{n o m}$, and $V_{c}$ represent the discharge cutoff, nominal, and charging voltages of the battery, respectively. The actual detection ranges were extended to account for batteries that have a reduced $\mathrm{SoH}$ or suffered from overcharge or over-discharge, where $V_{\min }$ and $V_{\max }$ are the minimum and maximum detection voltages under each configuration. Following a successful detection, $V_{d c}, V_{n o m}$, and $V_{c}$ are established in optimal operating zones to preserve the battery $\mathrm{SoH}$. The total battery identification process takes three minutes to complete, where the best results are found at a high SoC. However, it is important to mention that this test is designed to cover the entire operating range.

Table 5-2. Battery Cell Configuration Ranges.

\begin{tabular}{c|c|r|r|r|r|r}
\hline \hline Battery & \multirow{2}{*}{ Cells } & \multicolumn{3}{|c|}{ Operation Ranges } & \multicolumn{2}{c}{ Detection Range } \\
\cline { 3 - 8 } & & \multicolumn{1}{|c|}{$V_{d c}$} & $V_{\text {nom }}$ & \multicolumn{1}{c}{$V_{c}$} & $V_{\min }$ & $V_{\max }$ \\
\hline Lithium Ion & 1 & 3.300 & 3.700 & 4.200 & 2.900 & 4.399 \\
\hline Lithium Ion & 2 & 6.600 & 7.400 & 8.400 & 4.400 & 8.699 \\
\hline Lithium Ion & 3 & 9.900 & 11.100 & 12.600 & 8.700 & 13.199 \\
\hline Lithium Ion & 4 & 13.200 & 14.800 & 16.800 & 13.200 & 16.499 \\
\hline Lead Acid & 1 & 1.750 & 2.000 & 2.10 & 1.200 & 2.299 \\
\hline Lead Acid & 2 & 3.500 & 4.000 & 4.20 & 2.300 & 4.599 \\
\hline Lead Acid & 3 & 5.250 & 6.000 & 8.40 & 4.600 & 6.899 \\
\hline Lead Acid & 4 & 7.000 & 8.000 & 10.50 & 6.900 & 8.599 \\
\hline Lead Acid & 5 & 8.750 & 10.000 & 12.60 & 8.600 & 10.499 \\
\hline Lead Acid & 6 & 10.250 & 12.000 & 14.70 & 10.500 & 13.699 \\
\hline
\end{tabular}

\subsubsection{Calculating $1^{\text {st }}$ Order Randles Equivalent Circuit Parameters}

The voltage drop and recovery response after the $\mathrm{C} / 10$ pulse can provide parameter estimation as well. Pulse frequencies from $0.001 \mathrm{~Hz}$ to $500 \mathrm{~Hz}$ were tested, but revealed that as the period of the pulse fell below the timing constant of most batteries, the disturbance was virtually absorbed at the battery terminals. This refocused attention to 
pulses of $\leq 0.1 \mathrm{~Hz}$. To maximize the recovery voltage period while limiting the total time of the test, the same $0.005 \mathrm{~Hz}$ pulse at a $25 \%$ duty cycle was chosen and initiated twice for verification limiting the total test time to just under $7 \mathrm{~min}$. The parameters must be applied with equivalent $\mathrm{SoC}$ levels, thus this pulse test is applied when the battery is at full charge.

Multiple low frequency pulsed loads are then applied while the system notes the initial voltage, initial voltage drop, and recovery period. To solve for individual resistor-capacitor (RC) components, Equation (5-24) is used where $V[n]$ is replaced by the initial voltage drop $\Delta V$ between the open circuit $V_{o c}$ and loading $V_{L}$ voltage under the $\mathrm{C} / 10$ loading current $I_{L}$. Following an exponential fit using Equations (5-22) to (5-24), the result is equated to $\Delta V$ and $C_{p}$ is quickly solved for from $\tau$. The Randles equivalent circuit estimation is then accomplished by:

$$
\begin{gathered}
R_{p}=\frac{\Delta V}{I_{L}} \\
C_{p}=\frac{\tau}{R_{p}}=\frac{b^{-1}}{R_{p}}
\end{gathered}
$$

\subsubsection{Practical Implementation}

All features have been verified using a battery testing bed developed to test the new control, management, and analysis techniques, as well as evaluate cycling performance. This battery testing platform, shown in Figure 5.8, features solid state relays (SSR) to initiate discharging and charging pulses all connected to a main DC bus. The discharging SSR is connected to a mechanically controlled resistive load, whereas the charging SSR is connected to a BK Precision 1761 DC Power Supply [128]. The entire platform is monitored and controlled by a National Instruments (NI) LabVIEW DAQ platform featuring 12-bit $\pm 10 \mathrm{~V}$ Analog to Digital Converters (ADCs) at a sampling frequency of 
$20 \mathrm{kHz}$ to measure voltage and current. To handle $\mathrm{C} / 10$ currents over a wide range of capacity ratings, a LEM LA-25 current transducer has been utilized [62]. Although this test and evaluation platform is not portable, attention was placed on making the final system suitable for implementation on an embedded controller such as the ESMC.

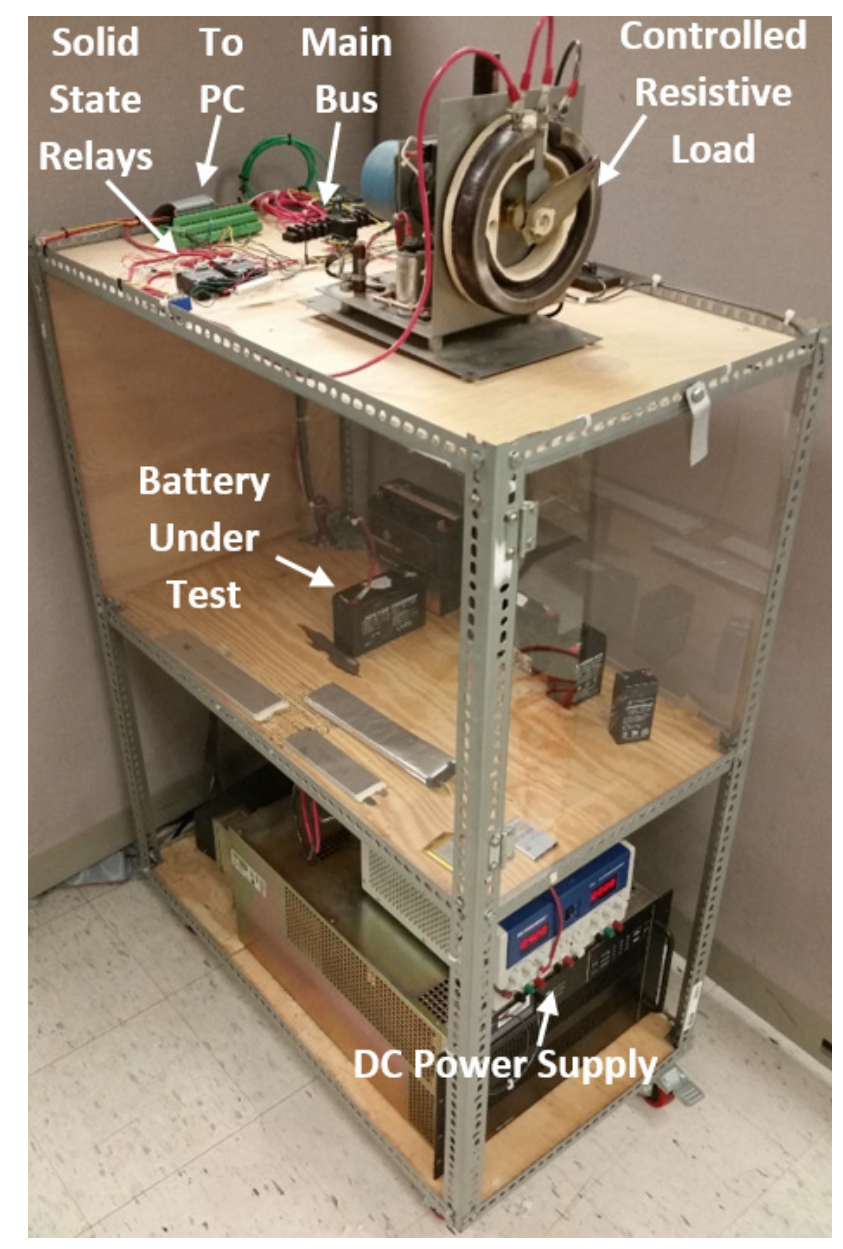

Figure 5.8. Battery Test Stand Hardware.

\subsubsection{Accuracy Variance versus Sampling Frequency}

A series of tests were performed at much lower sampling rates to evaluate the performance of the system at computational speeds that are suitable for an embedded controller. In an effort to demonstrate a comparison, a subset of batteries were introduced 
to lower sampling frequencies of $2 \mathrm{kHz}, 200 \mathrm{~Hz}$, and $20 \mathrm{~Hz}$. Although a $20 \mathrm{~Hz}$ sampling frequency would be far too slow in most applications, the electrochemical response of the battery (taking into account the Nyquist criterion) is slower than $10 \mathrm{~Hz}(\tau>0.1 \mathrm{~s})$, thus, it is still sufficient to determine the battery chemistry.

Table 5-3. Generated Time Constant per Sampling Frequency.

\begin{tabular}{|c|r|r|r|r|r|}
\hline Battery Type & $\begin{array}{c}\text { Size } \\
\text { (Ah) }\end{array}$ & $20 \mathrm{kHz}$ & $2 \mathrm{kHz}$ & $200 \mathrm{~Hz}$ & $20 \mathrm{~Hz}$ \\
\hline Li-ion - LCO & 21.0 & $0.658 \mathrm{~s}$ & $0.642 \mathrm{~s}$ & $0.632 \mathrm{~s}$ & $0.568 \mathrm{~s}$ \\
\hline Lead Acid & 21.0 & $24.999 \mathrm{~s}$ & $31.434 \mathrm{~s}$ & $27.197 \mathrm{~s}$ & $33.882 \mathrm{~s}$ \\
\hline Li-ion - LCO & 6.4 & $0.510 \mathrm{~s}$ & $0.509 \mathrm{~s}$ & $0.499 \mathrm{~s}$ & $0.511 \mathrm{~s}$ \\
\hline Lead Acid & 6.0 & $20.115 \mathrm{~s}$ & $21.493 \mathrm{~s}$ & $20.661 \mathrm{~s}$ & $17.462 \mathrm{~s}$ \\
\hline
\end{tabular}

\section{Legend: Correctly Identified, Incorrectly Identified}

Table 5-3 depicts a table of the generated time constants for lead acid and lithium ion batteries with small (6-6.4 Ah) and medium (21 Ah) capacities. Since the generated time constant cutoff between lead acid and lithium ion batteries is set relatively high at $20 \mathrm{~s}$, smaller lead acid batteries can run a risk of being misidentified. This is purely due to adding a wide range of tolerance for the lithium ion batteries that are in much worse condition (i.e. low $\mathrm{SoH})$. Generally, when the $\mathrm{SoH}$ is low, the response of the battery following a disturbance is slower, thereby generating a longer time constant. Table 5-3 also shows that both lithium ion batteries under test generated time constants far below $1 \mathrm{~s}$, where an increase in the time constant was minimal even with a capacity over three times larger. By further optimizing the tolerance of the time constant cutoff or establishing a range of acceptable battery conditions that the intended application can be exposed to, this could be easily modified. 
When calculating equivalent circuit values, the sampling frequency tolerance becomes more critical. In the case of calculating the polarization resistance $R_{p}$, the values are fairly consistent, even at much lower sampling frequencies since the timing element is not as prevalent. Table 5-4 depicts the calculated values for $R_{p}$ from the software platform for each of the four frequencies. Even at $20 \mathrm{~Hz}$, the highest error is only around $5 \%$. However, when looking at the polarization capacitance $C_{p}$, the deviation is much wider. Shown in Table 5-5, both batteries introduce a higher error than what was observed by $R_{p}$, but the proposed system produces values that are still within an acceptable range of accuracy $(<8 \%)$ at a sampling frequency of $200 \mathrm{~Hz}$ or higher.

Table 5-4. Calculated Polarization Resistance per Sampling Frequency.

\begin{tabular}{|c|c|c|c|c|c|c|c|c|}
\hline \multirow{3}{*}{$\begin{array}{c}\text { Battery } \\
\text { Type }\end{array}$} & \multirow{3}{*}{$\begin{array}{l}\text { Size } \\
\text { (Ah) }\end{array}$} & $20 \mathrm{kHz}$ & \multicolumn{2}{|c|}{$2 \mathrm{kHz}$} & \multicolumn{2}{|c|}{$200 \mathrm{~Hz}$} & \multicolumn{2}{|c|}{$20 \mathrm{~Hz}$} \\
\hline & & Measured & Measured & Error & Measured & Error & Measured & Error \\
\hline & & $\mathrm{m} \Omega$ & $\mathrm{m} \Omega$ & $\underline{\%}$ & $\mathrm{~m} \Omega$ & $\underline{\%}$ & $\mathrm{~m} \Omega$ & \% \\
\hline Li-ion - LCO & 21.0 & 54 & 54 & $0.0 \%$ & 55 & 1.8 & 56 & 3.7 \\
\hline Lead Acid & 21.0 & 200 & 200 & $0.0 \%$ & 203 & 1.5 & 205 & 2.5 \\
\hline Li-ion - LCO & 6.4 & 57 & 56 & $1.7 \%$ & 60 & 5.2 & 60 & 5.2 \\
\hline Lead Acid & 6.0 & 110 & 110 & $0.0 \%$ & 111 & 0.9 & 116 & 5.4 \\
\hline
\end{tabular}

Table 5-5. Calculated Polarization Capacitance per Sampling Frequency.

\begin{tabular}{|c|c|c|c|c|c|c|c|c|}
\hline \multirow{2}{*}{$\begin{array}{l}\text { Battery } \\
\text { Type }\end{array}$} & \multirow{2}{*}{$\begin{array}{l}\text { Size } \\
\text { (Ah) }\end{array}$} & \multirow{2}{*}{\begin{tabular}{|c|}
$20 \mathrm{kHz}$ \\
$\frac{\text { Measured }}{(\mathrm{F})}$ \\
\end{tabular}} & \multicolumn{2}{|c|}{$2 \mathrm{kHz}$} & \multicolumn{2}{|c|}{$200 \mathrm{~Hz}$} & \multicolumn{2}{|l|}{$20 \mathrm{~Hz}$} \\
\hline & & & $\frac{\text { Measured }}{(\mathrm{F})}$ & \begin{tabular}{|l|} 
Error \\
$\%$
\end{tabular} & $\frac{\text { Measured }}{(\mathrm{F})}$ & $\frac{\text { Error }}{\%}$ & $\frac{\text { Measured }}{(\mathrm{F})}$ & $\frac{\text { Error }}{\%}$ \\
\hline Li-ion - LCO & 21.0 & 14.5 & 14.5 & 0.0 & 13.9 & 4.1 & 17.8 & 22.7 \\
\hline Lead Acid & 21.0 & 134.5 & 136.2 & 1.2 & 130.9 & 2.6 & 111.1 & 17.3 \\
\hline Li-ion - LCO & 6.4 & 12.7 & 12.3 & 3.1 & 11.7 & 7.8 & 14.6 & 14.9 \\
\hline Lead Acid & 6.0 & 309.1 & 307.2 & 0.6 & 319.2 & 3.2 & 292.2 & 5.4 \\
\hline
\end{tabular}

\subsubsection{Discussion}

In the previous subsection, it was shown that high accuracy can still be maintained even when the DAQ sampling rate is 100 times lower than the current test configuration. This 
point is important to note in the practical implementation of this system when addressing the required onboard memory to store the data cache. When moving from $20 \mathrm{kHz}$ to 200 $\mathrm{Hz}$, a data cache of $50-60 \mathrm{MB}$ per curve fit is reduced significantly to only $1 \mathrm{MB}$ per curve fit. The hardware in Figure 5.8 would also need to be revised to make a practical, compact, and cost-effective system. To this point, the controllable electronic load could be replaced with a small network of power resistors. Since the loading period under the C/10 pulse is only $50 \mathrm{~s}$, the voltage reduction over this period is relatively small and with a minor tradeoff in accuracy, it may be neglected. Finally, the LEM LA-25 could be replaced with a precision shunt resistance similar to the implementation that was demonstrated in the ESMC commercialization to significantly reduce the cost and size of the system.

\subsection{Battery Management System Implementation}

In this section, the battery testbed has been extended to test and evaluate the concepts addressed in this chapter. The following subsections cover the program operation.

\subsubsection{Battery Initial Setup}

In this subsection, a full procedure for setup and management of a battery in this BMS is outlined for implementation on an industrial BMS or the ESMC.

1. Connect Battery and Specify Size: The user inputs the battery capacity in Ah.

2. Perform Battery Type ID: The battery chemistry identification process is conducted lasting $200 \mathrm{~s}$.

3. Match to Series Cell Configuration: The voltage is fit into the ranges as was shown previously in Table 5-2 based on the battery type.

4. Verify Configuration with User: A dialog is presented to the user for verification. If both match, as demonstrated in the example shown in Figure 5.9, the system will 
proceed to the next step. If not, as shown in Figure 5.10, the user can manually select the correct battery type and the correct number of cells. If the platform miscalculates both, the system proceeds. If the user selects the same battery type as was scanned but under a different cell configuration, the system identifies the OCV was out of the expected range indicating a defective or damaged battery module.

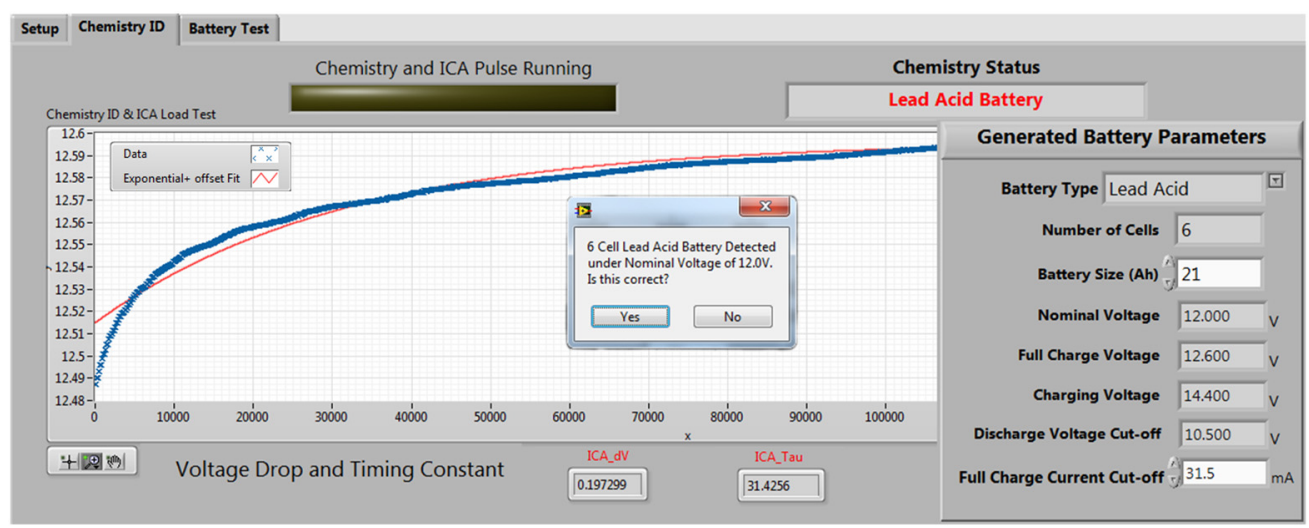

Figure 5.9. Normal Detection of a $12 \mathrm{~V}$ Lead Acid Battery.

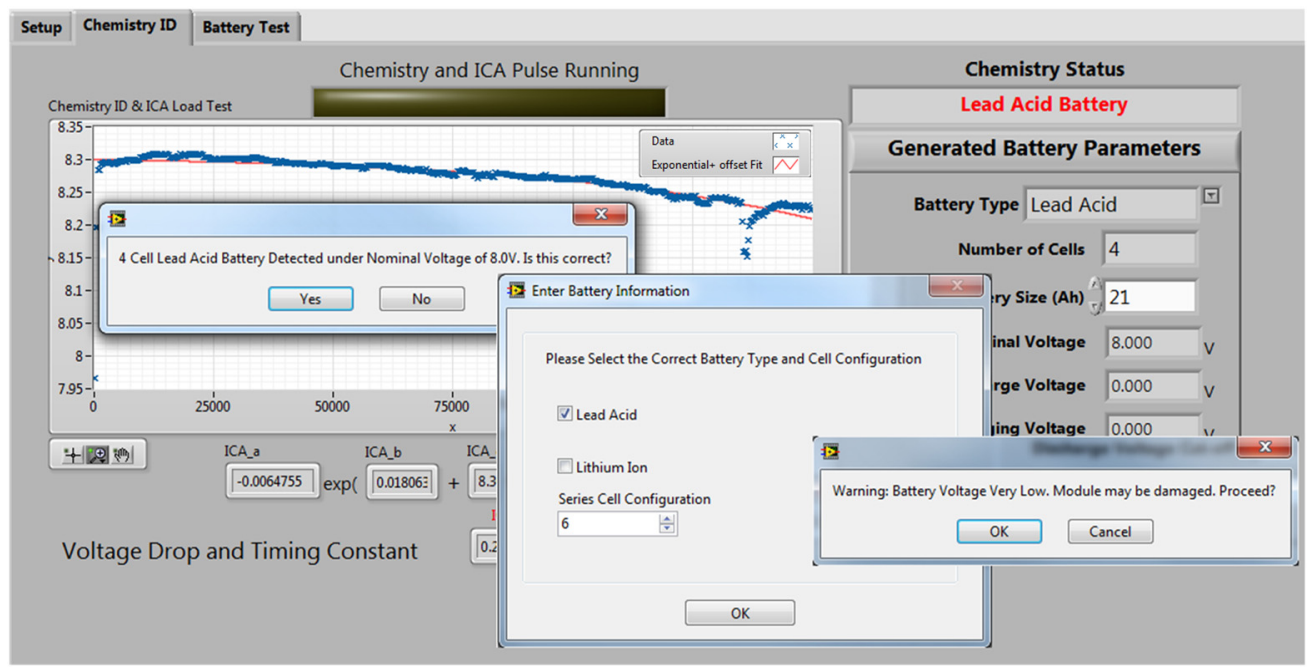

Figure 5.10. Misidentification of an $8 \mathrm{~V}$ (4-cell) Lead Acid Battery for a 12V (6-cell) Lead Acid Battery with Custom Entry and User Warning.

5. Estimate SoC: The SoC is calculated by the OCV method using Equation (5-17) for a lead acid battery or the look-up table $S_{o} C_{L i}\left(V_{o c_{L i}}, T\right)$ for lithium ion. 
6. Begin Test: All charging and discharging parameters have been established and the system can proceed.

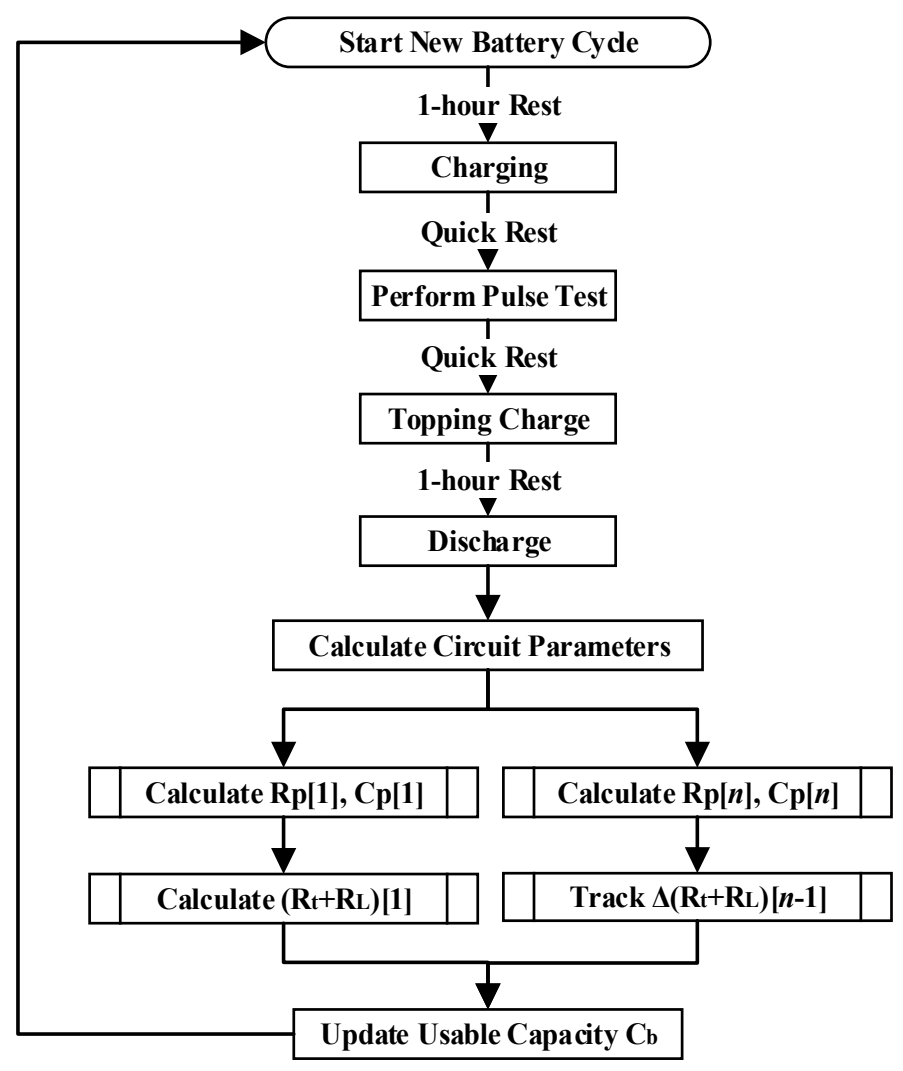

Figure 5.11. Autonomous Battery Management System Cycling Flow Chart.

\subsubsection{Battery Test}

The goal of the battery test procedure is to demonstrate a basic range of functionality in which a single battery or module would be exposed to during a full operation cycle. A flow chart is shown in Figure 5.11. Although a constant load and charging profile has been utilized on this platform, this is primarily to demonstrate how the proposed features could be implemented on a comprehensive online BMS such as the ESMC. However, it is important to mention that this system could also be suitable as a modular maintenance tool, where conservative constant charging and discharging currents would be necessary to 
evaluate the battery performance while minimizing the thermodynamic stress. A screenshot of the Graphical User Interface (GUI) visible to the user while the system is in operation is shown in Figure 5.12. During operation, a wealth of information is provided to the user to obtain the status of the test including: voltage, current, C-rate, power, SoC, energy exchange in Ah, energy exchange in Watt-hours (Wh), SoH, and cycle efficiency.

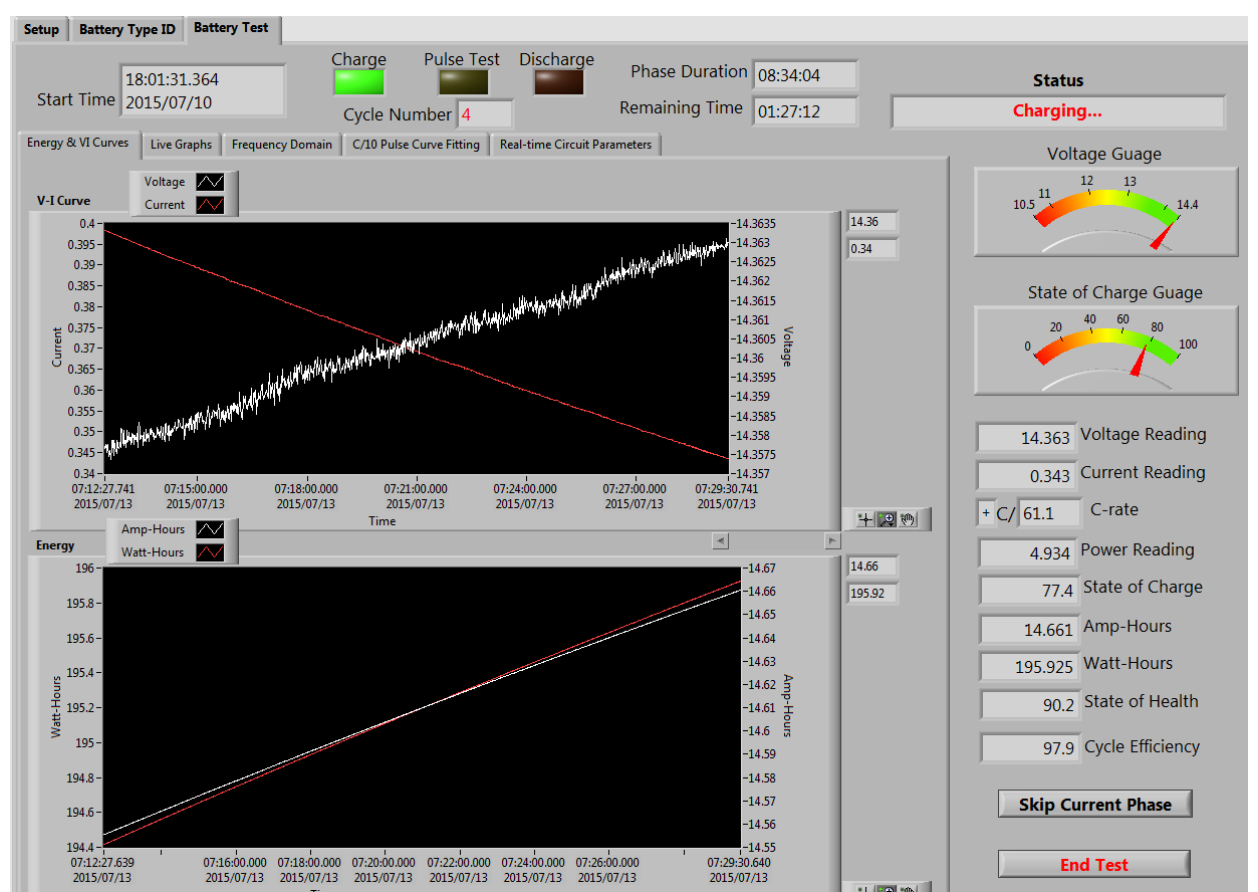

Figure 5.12. Graphical User Interface during a Battery Test.

\subsubsection{Charging}

Using the initial SoC value $\operatorname{SoC}_{x}\left(t_{0}\right)$, the remaining amount of energy stored is estimated in Ah. By Equation (5-18), the system will sum charging energy until one of two stop conditions occur: 1) the battery has reached its full charge current or 2) the energy charged has exceeded the total energy of the battery by $25 \%$. The $+25 \%$ allowance accounts for a reduced roundtrip efficiency and potential thermodynamic losses. Once charging has completed, a 5 min rest period is observed before the pulse test. 


\subsubsection{Perform Pulse Test}

Two $0.005 \mathrm{~Hz}$ pulses at the $\mathrm{C} / 10$ discharge current are applied under a $25 \%$ duty cycle. Following each pulse, an exponential fit is applied as per Equations (5-22) and (5-23) and $R_{p}, C_{p}$ parameters are then estimated based on Equations (5-24) to (5-26). Following the second pulse, these values are averaged to ensure accuracy and passed to the circuit parameter display front-end, where an example performed on a $6 \mathrm{~V}$ 2-cell lead acid battery is shown in Figure 5.13.

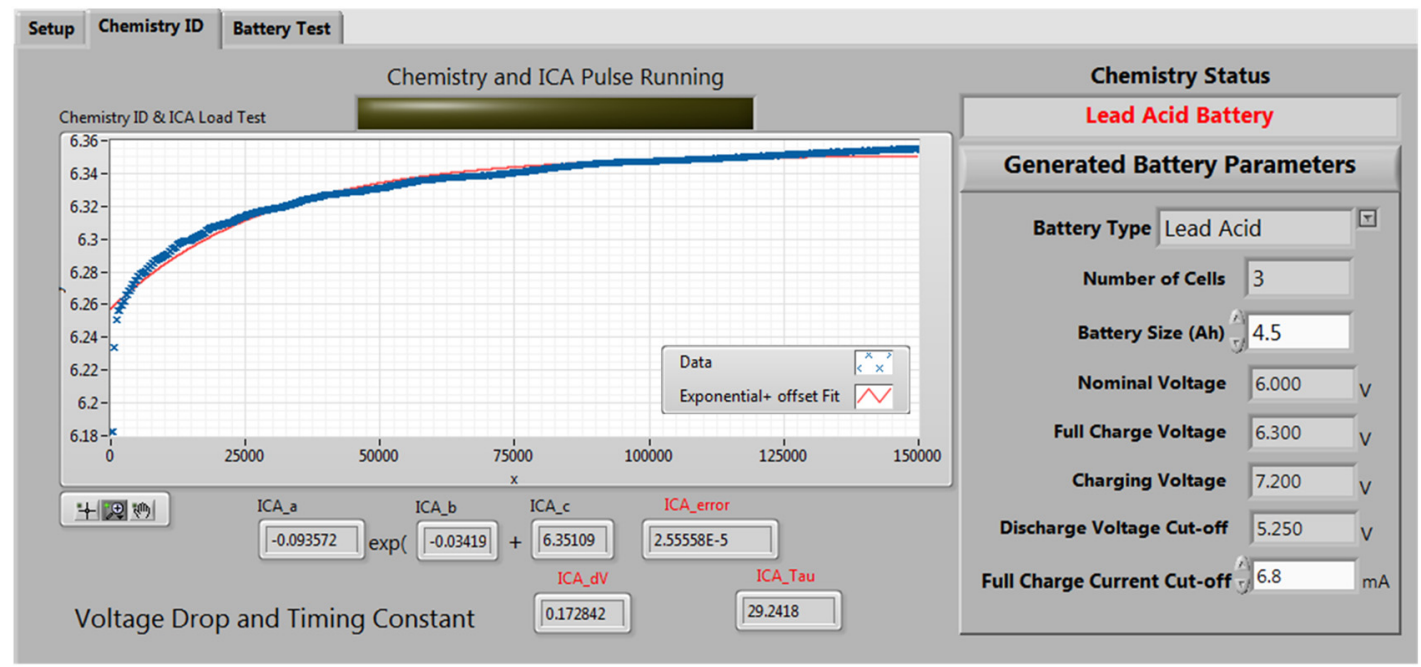

Figure 5.13. Pulse Test Example on a 6 V Lead Acid Battery.

\subsubsection{Topping Charge}

To maintain the highest accuracy in assessing the battery SoH, the energy expended during the pulse tests are replaced with a quick topping charge phase. The stop condition in this phase is further protected by the charging current cutoff. Once this has completed, an extended 1-hour rest period is observed to prepare the battery for a full discharge. Although it is unlikely an online BMS would be able to observe a full 1-hour rest, this would be ideal in a maintenance scenario. 


\subsubsection{Discharge}

The discharge phase applies the Peukert component to correct for the expected usable capacity under discharge. For convenience, once again the $\mathrm{C} / 10$ discharge current is used. The new usable Peukert capacity is used as the SoC reference as well when applying coulomb counting. In the event that a full discharge event occurs, the final calculation of the $\mathrm{SoH}$ in Equation (5-20) is modified to compare the total energy output to the revised Peukert-adjusted capacity.

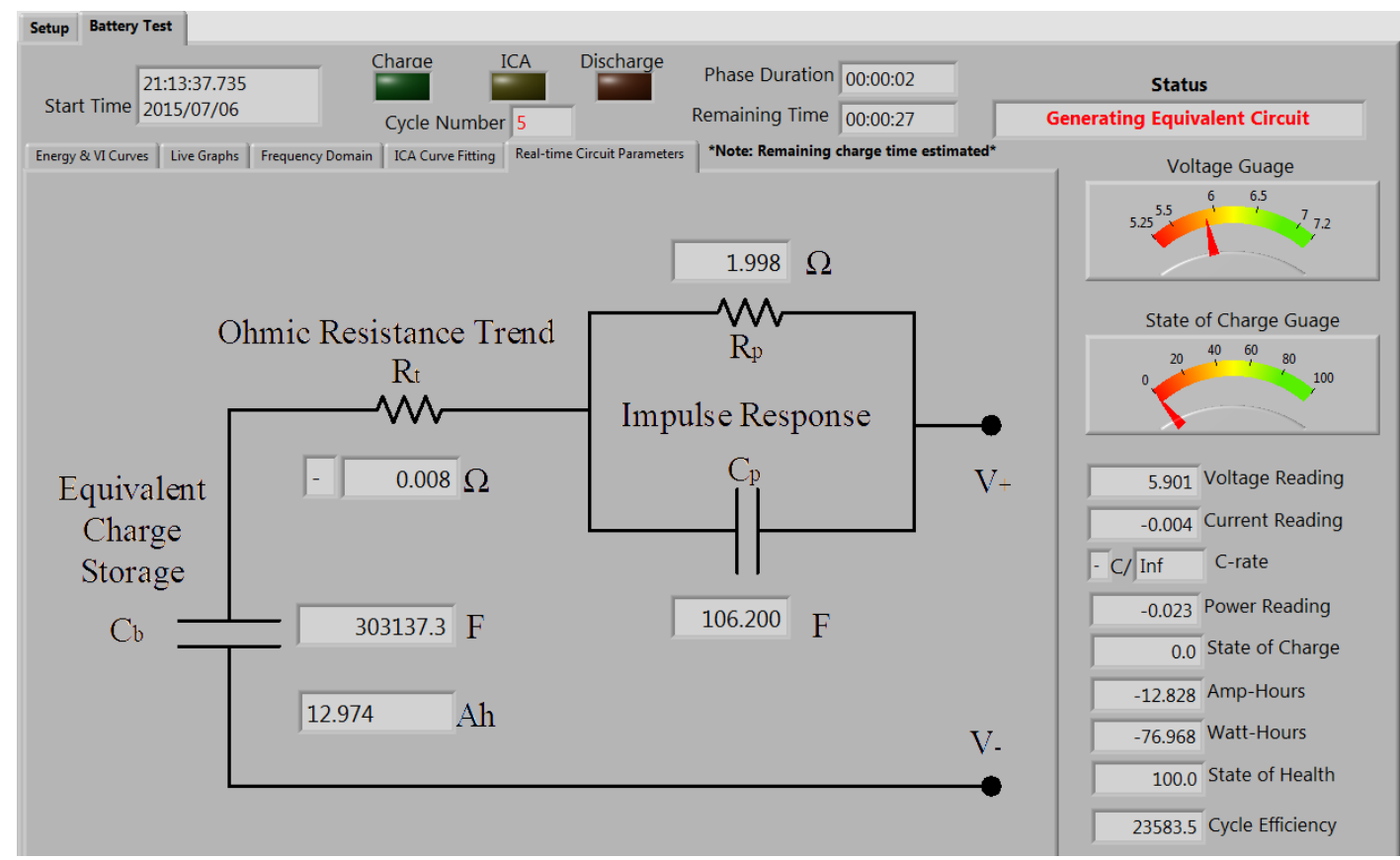

Figure 5.14. BMS Software Platform and Equivalent Circuit Generation for a 6 V Lead Acid Module.

\subsubsection{Calculate Circuit Parameters and Usable Capacity}

Following a full discharge, the usable energy is obtained and the equivalent charge storage capacitor $C_{b}$ is calculated based on Equation (5-21). An example is shown in Figure 5.14 where the equivalent circuit model for a healthy $6 \mathrm{~V}$ lead acid battery is shown with $C_{b}$ filled in revealing the 12.974 Ah energy output during the discharge stage is similar to 
the charge storage of a 303,137.3 F capacitor. The parameterization estimations for $R_{t}, C_{p}$, and $R_{p}$ in the Randles $1^{\text {st }}$-order equivalent circuit have been calculated as well to be 1.998 $\Omega, 106.2 \mathrm{~F}$, and $0.008 \Omega$, respectively.

\subsubsection{Full Circuit Model}

The equivalent circuit model is shown at the end of every cycle and can be accessed anytime thereafter. Cycle 1 would specify 'NaN' for the $R_{t}$ value, but would produce the new charge storage capacitance and impulse response parameters. Thus, for a maintenance scenario, a minimum of two cycles are required to estimate all equivalent circuit parameters.

\subsection{Experimental Testing}

To demonstrate the detailed capabilities of the BMS and testing platform, a combination of both healthy and damaged lead acid and lithium ion batteries were placed on the testbed. All four batteries tested have matching rated capacities of $21 \mathrm{Ah}$.

\subsubsection{Lithium Ion Battery Testing}

Two 3.7-V single-cell 21 Ah lithium ion LCO batteries were placed on the system: one in good health and one defective. The following subsections discuss these test results.

\subsubsection{3.7 V Lithium Ion Battery in Good Condition}

The initial test depicts a battery identification for a single-cell lithium ion battery in good condition. Pictured as Battery Number 5 previously in Figure 5.7, the timing constant $\tau$ is labeled in Figure 5.15 and was found to be $0.437 \mathrm{~s}$ revealing the battery was indeed, lithium ion. From Table 5-2, the OCV fell within the range of a single-cell. Figure 5.16 reveals that after two cycles the system detected $R_{t}$ had a shift of $69 \mathrm{~m} \Omega$. The battery discharged 17.199 Ah of energy in cycle 2 similar to the charge storage in a 209,888.9 F 
capacitor. The impulse response resistance $R_{p}$ and capacitance $C_{p}$ was $51 \mathrm{~m} \Omega$ and 14.687 $F$, respectively. $R_{p}$ is well within the range of expectation for a lithium ion battery, whereas the capacitance is slightly higher than some cells, but still much lower than that of lead acid revealing a young lithium ion cell [91].

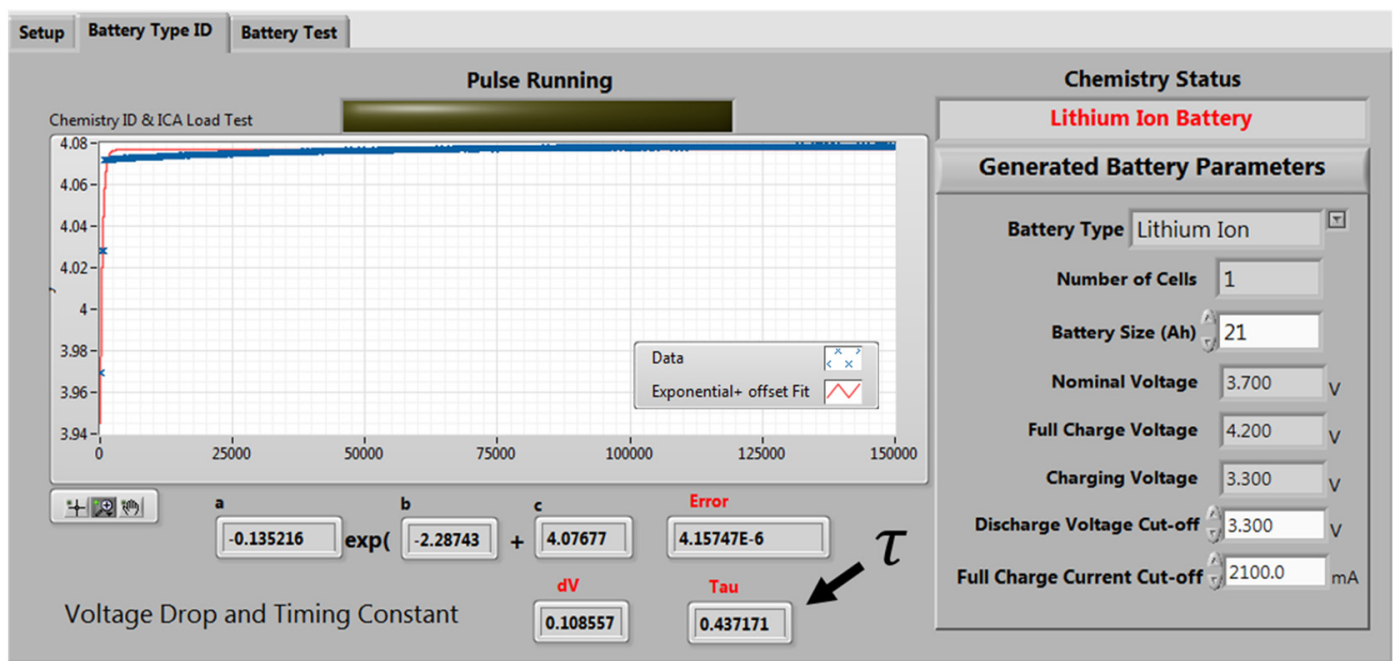

Figure 5.15. 3.7 V 21 Ah Lithium Ion Battery Type Identification.

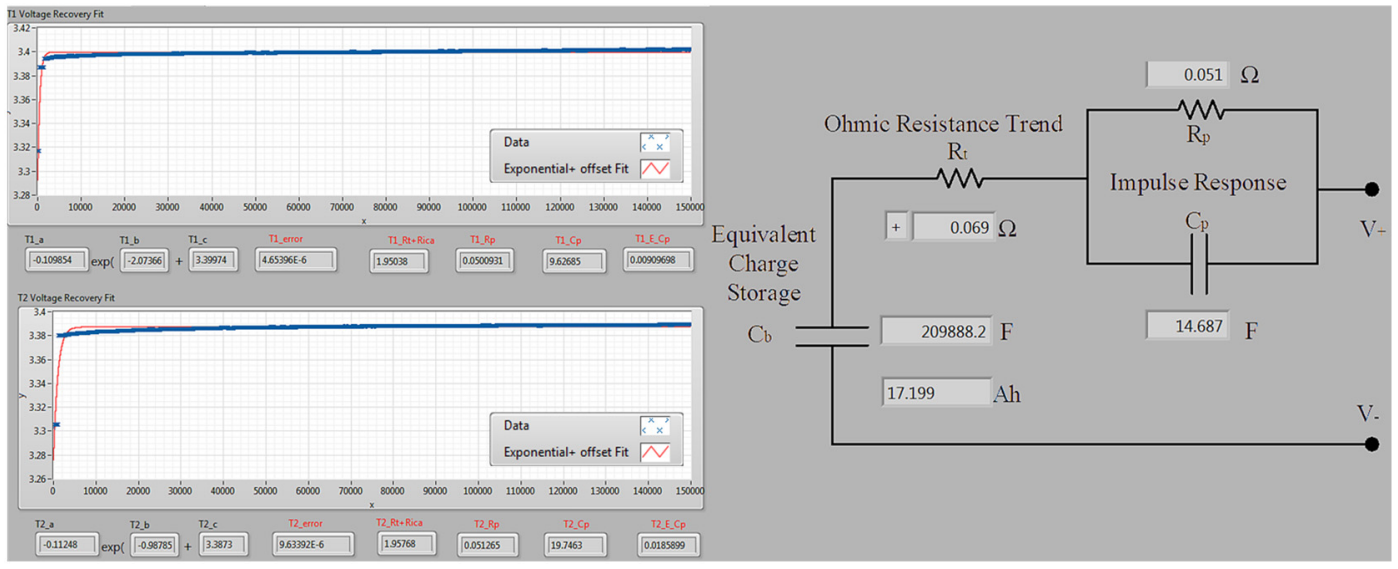

Figure 5.16. 3.7 V 21 Ah Lithium Ion Battery Curve Fit and Final Equivalent Circuit for a Battery in Good Condition.

\subsubsection{3.7 V Lithium Ion Battery in Bad Condition}

Figure 5.17 shows the same single-cell 21 Ah lithium ion battery heavily damaged.

Pictured as Battery Number 4 previously in Figure 5.7, it delivered under 1 Ah in its 
discharge cycle, which is similar in charge storage to a 13,226.8 F capacitor. The battery was only capable of operating for one cycle, thus $R_{t}$ could not be determined. The impulse response resistance $R_{p}$ and capacitance $C_{p}$ were very high, revealing a damaged cell at 837 $\mathrm{m} \Omega$ and $65.155 \mathrm{~F}$, respectively. Although the calculated circuit values quantitatively confirm the battery is in poor health, a qualitative assessment of the two pulse testing waveforms expose a very different response than that of the good battery.

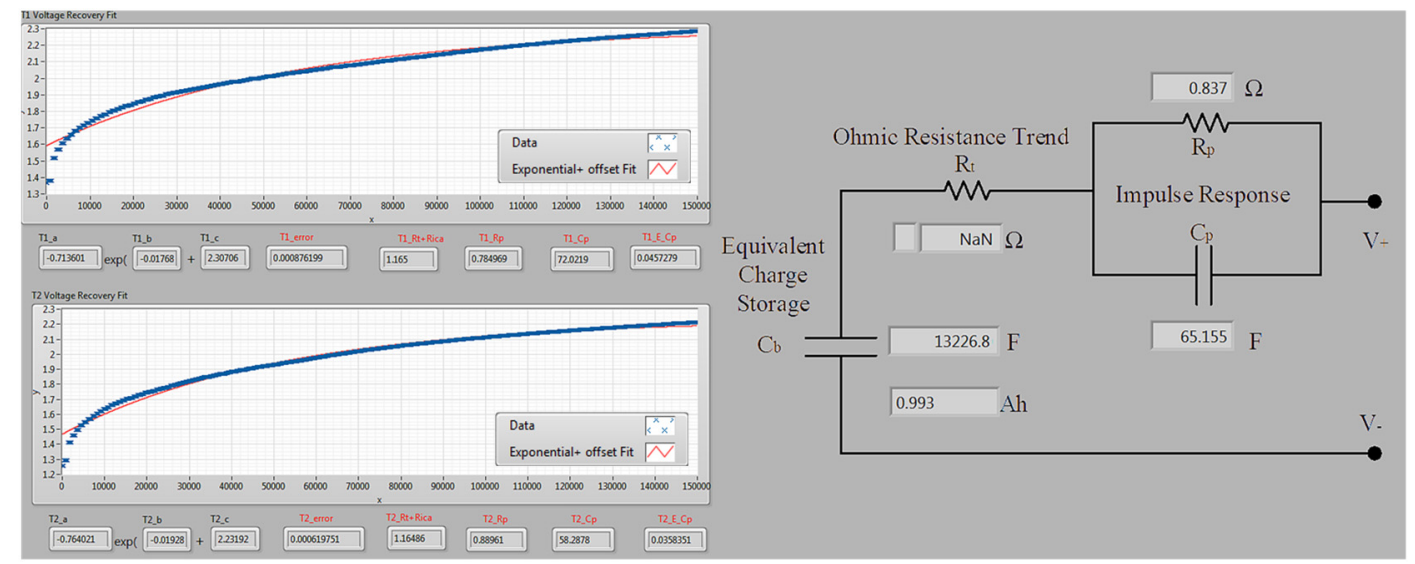

Figure 5.17. 3.7 V 21 Ah Lithium Ion Battery Curve Fit and Final Equivalent Circuit for a Battery in Bad Condition.

\subsubsection{Lead Acid Battery Testing}

Two 12-V 6-cell 21 Ah lead acid batteries were placed on the testbed, one in good health and the other defective. The following subsections discuss these test results in detail.

\subsubsection{12 V Lead Acid Battery in Good Condition}

A healthy $12 \mathrm{~V}$ lead acid battery (depicted as Battery Number 10 previously in Figure 5.7) was placed under test. Figure 5.18 shows the output from the BMS after four cycles, where the total discharge energy was $18.935 \mathrm{Ah}$, similar to the charge storage in a 910,570.5 F capacitor. The shift in the ohmic resistance $R_{t}$ since the first cycle increased 
by $39 \mathrm{~m} \Omega$. The impulse response or polarization resistance $R_{p}$ and capacitance $C_{p}$ was 205 $\mathrm{m} \Omega$ and $286.369 \mathrm{~F}$, respectively, revealing a lead acid battery in good health.

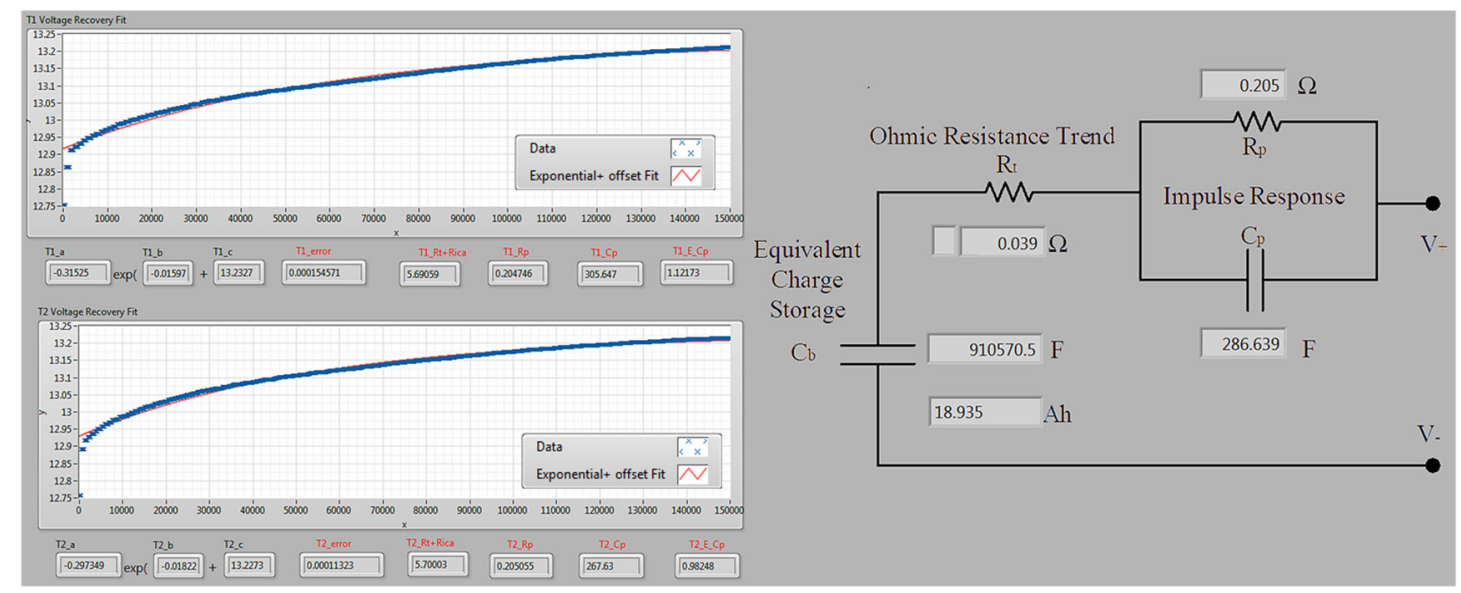

Figure 5.18. 12 V 21 Ah Lead Acid Battery Curve Fit and Final Equivalent Circuit for a Battery in Good Condition.

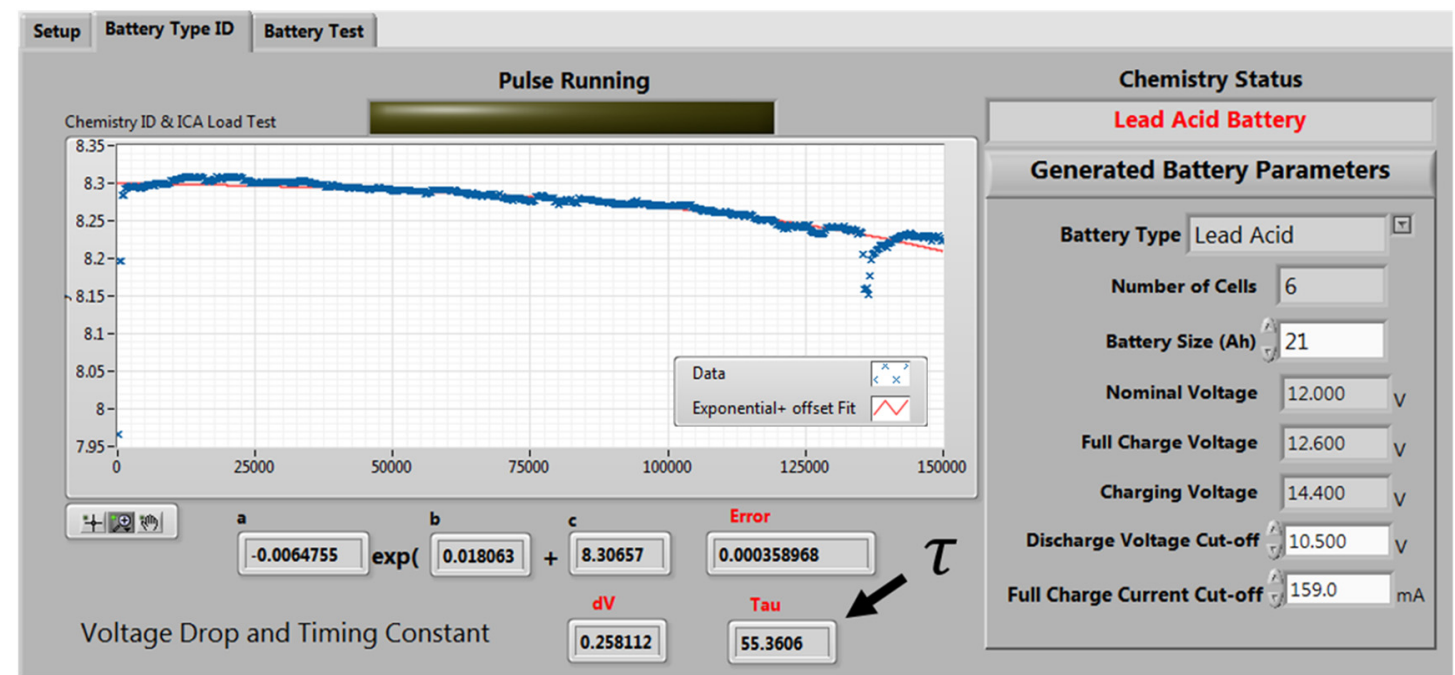

Figure 5.19. 12 V 21 Ah Lead Acid Battery Type Identification.

\subsubsection{12 V Lead Acid Battery in Bad Condition}

In the final scenario, a similar 6-cell lead acid battery (depicted as Battery Number 11 previously in Figure 5.7) was placed under test. Battery Number 11 generated a time constant $\tau>20 \mathrm{~s}$, thus correctly identifying a lead acid battery. However, as shown in Figure 5.19, the voltage did not fall within the expected range for a 6-cell configuration, 
thus the system requested user input providing a warning that the battery may be defective. Since the battery was damaged, once again only one cycle was completed thus $R_{t}$ could not be determined. Figure 5.20 shows the battery only discharged a mere $0.196 \mathrm{Ah}$, which is similar to the columbic storage of a $6,398.5 \mathrm{~F}$ capacitor. The impulse response or polarization resistance $R_{p}$ and capacitance $C_{p}$ was $621 \mathrm{~m} \Omega$ and $35.628 \mathrm{~F}$, respectively. Similarly to the lithium ion battery comparison, a qualitative assessment of the two pulse testing waveforms reveal a very different response than that of a good lead acid battery.

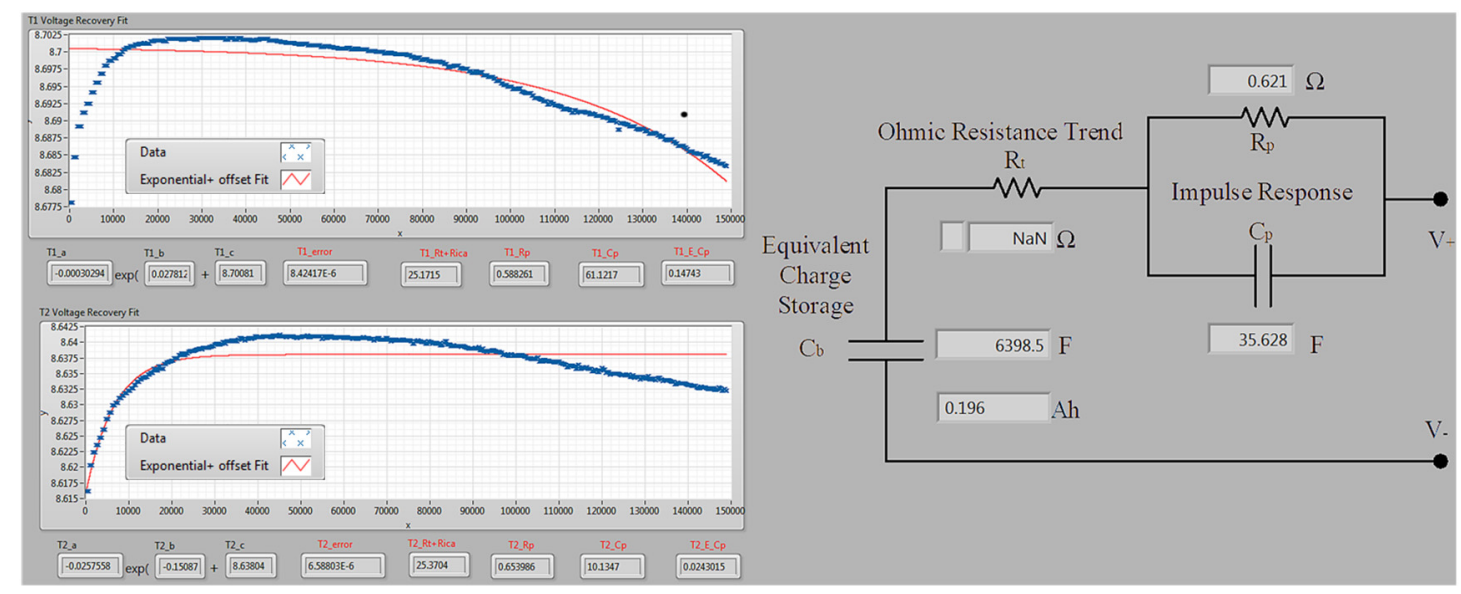

Figure 5.20. 12 V 21 Ah Lead Acid Battery Curve Fit and Final Equivalent Circuit for a Battery in Good Condition.

\subsection{Summary}

This chapter introduced a number of new techniques suitable for implementation on a flexible hybrid BMS. Two new battery detection concepts were developed and verified via PBM to highlight how a standardized $\mathrm{C} / 10$ discharge pulse can be utilized for multiple purposes. The proposed system demonstrated a comprehensive BMS platform with autonomous differentiation of lead acid and lithium ion battery chemistries, determination of the series-cell configuration, and an estimation of a Randles $1^{\text {st }}$-order equivalent circuit 
including an energy tracking model. Furthermore, other aspects of improving a BMS were addressed. Prior to operation, the initial SoC estimation procedure was enhanced through a voltage and temperature-based algorithm for both battery chemistries. These features were tested and evaluated on a final control platform on a wide range of lithium ion and lead acid battery cell configurations, capacities, and health conditions.

In the midst of development, the system was designed keeping computation and memory overhead in mind, where the performance and accuracy was tested under a wide range of conditions accounting for limitations in MCU capabilities. In this way, the platform and procedures can be utilized not only an alternative to EIS on a BMS or the ESMC, but also improve SoC estimations by monitoring historical trends of SoH (usable capacity in this context) and performance. The information determined by this model would provide a useful model for off-line analysis or advanced maintenance.

Although in this chapter the PBM was developed and utilized as a tool to demonstrate the effectiveness of using a pulsed load to obtain the battery chemistry and equivalent circuit model, PBMs were not found to be practical for their implementation inside a realtime controller. However, the offline usage of PBMs can provide powerful simulation tools to very accurately capture the performance of battery ES devices. With a model of sufficient detail, PBMs can offer further insight into battery performance and health mechanisms that are not easy, or even impossible to measure. In the next chapter, the lithium ion battery PBM is expanded considerably into 3D, where a detailed formulation of the 3D PBM is derived. Trade-offs and comparisons in accuracies of the P2D PBM and the 3D PBM are discussed, while the enhanced 3D PBM carries with it a novel capability in visualizing a major contributor to lithium ion battery ageing. 


\section{Chapter 6 Extending Lithium Ion Finite Element Models into 3D}

\subsection{Introduction}

The lithium ion battery was introduced in the previous chapter as an alternative battery energy storage (ES) device. Their rapid deployment in utility, electric vehicle (EV), and shipboard power system applications was discussed, but it is their usage in common everyday consumer electronics devices that has made them commonplace in our daily lives. The mobile phone and laptop industry has utilized lithium ion batteries since the early 2000s. These devices have tested the durability, versatility, and lifespan, or State of Health (SoH), while taking advantage of new capabilities they have to offer. The ability to function regularly at much higher charging and discharging currents as compared to legacy chemistries such as lead acid or nickel cadmium has contributed to their popularity.

In the previous chapter, physics based models (PBM) of both the lithium ion and lead acid batteries were introduced. These models were simplified in this analysis, utilizing a pseudo two-dimensional (P2D) PBM for two reasons: 1) to reduce computational complexity and 2) to provide universal PBMs that were not dependent upon the capacity of the cell. A comparison in P2Ds of the lithium ion and lead acid batteries revealed that the lithium ion battery voltage drop or rise behavior, previously referred to as overpotential, was very different than that from legacy battery chemistries [129]. Although it was not demonstrated in the P2D analysis, in the same light, high charging currents will result in a very different overpotential as well. Lead acid batteries are charged between $2.30 \mathrm{~V}$ to 2.45 V per series connected cell, where excessive voltage can cause a decomposition of the electrolyte and initiate premature aging [130]. Although exposure to higher voltages can 
place a toll on their long-term $\mathrm{SoH}$, lead acid batteries do not pose as much of a safety risk and are far more resilient to these conditions.

The lithium ion battery, on the other hand, is a much more sensitive device. Standard lithium ion cobalt oxide (LCO) batteries are charged at $4.20 \mathrm{~V}$ and can only tolerate up to a $50 \mathrm{mV}$ increase in the terminal voltage before catastrophic affects such as thermal runaway can take place [68],[131]. Excessive charging currents and a lack of adequate control can push the terminal voltage to dangerous levels if not properly handled. PBMs can be used to study these traits while providing a deep investigation into real-time battery performance and its long-term SoH [132]. A PBM provides a mechanism to not only accurately forecast the terminal voltage, but also track how electrochemical reactions impact the cell. This information is crucial in understanding how the design and package impacts the operation, while it also provides a new mechanism to study how these behaviors can impact the SoH.

\subsection{Consequences of Overpotential}

Battery SoH has been discussed a number of times in this dissertation, starting from a generalized definition to mapping its specific impacts to the lead acid battery. In this chapter, a focus will be placed specifically on the lithium ion battery. Although $\mathrm{SoH}$ management in this battery is primarily mapped to regulating its Depth of Discharge (DoD) and enforcing a tight operating temperature range, the degradation of active materials is a complex process that must consider many variables not easily measured [133]. Previously, overpotential was defined simply in the context of the change of voltage $\Delta \mathrm{V}$ present between the open circuit voltage (OCV) and the terminal voltage under loading or charging. In this chapter, the concept of overpotential will be further investigated. A 
voltage drop under load not only results in an altered voltage profile or performance in the electrical domain, but has instant consequences in the thermal domain. The most common, joule heating overpotential (i.e. $\mathrm{I}^{2} \mathrm{R}$ losses), can be observed simply as a result of the power dissipation through an internal resistance. At increased operational currents, the joule heating component is significant, and can be apparent within minutes or less of operation.

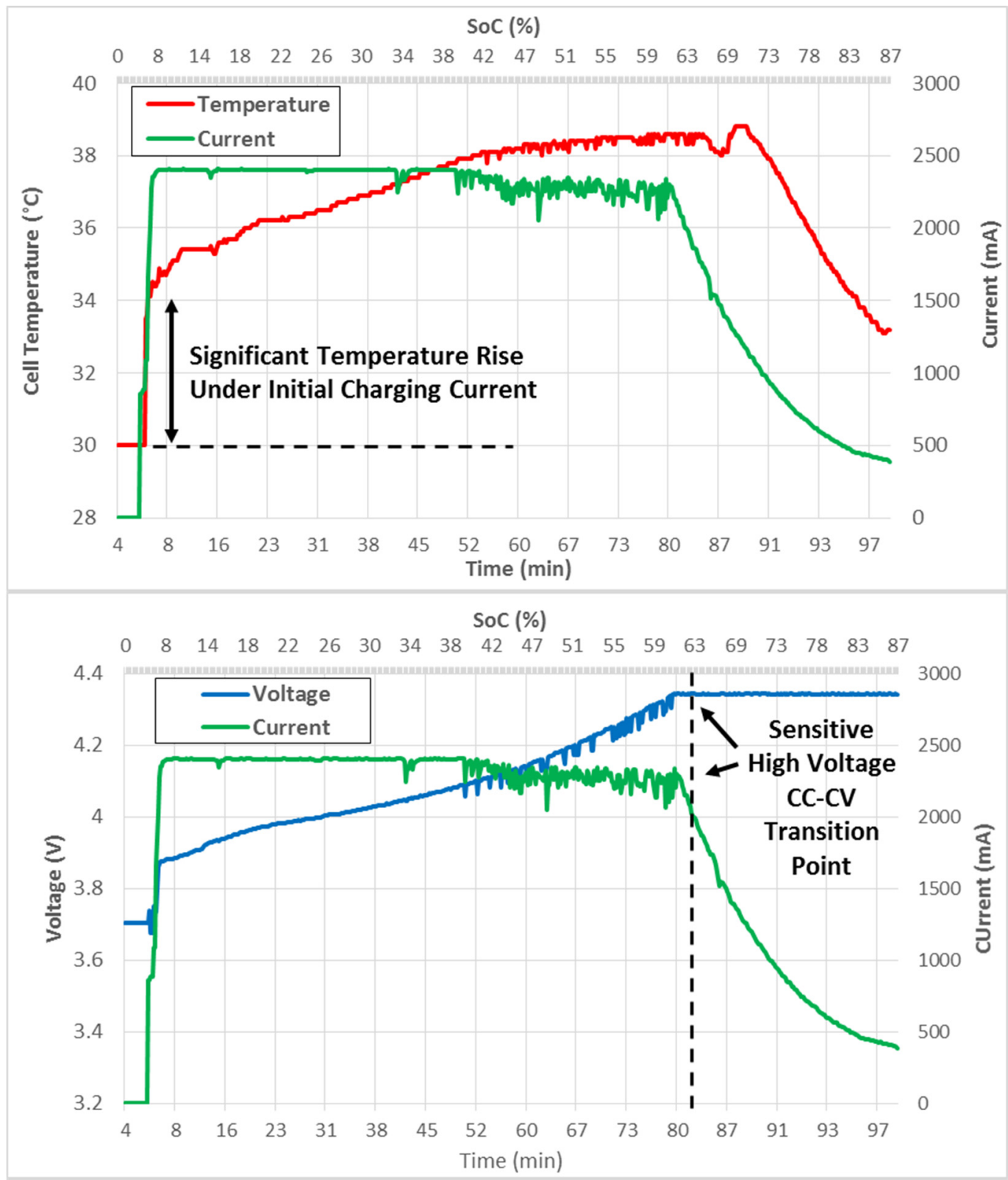

Figure 6.1. Samsung Galaxy S7 Battery Fast Charging Profile: Current versus Temperature (top) and Current versus Voltage (bottom). 
This phenomenon is frequently observed in modern cellular phones, particularly Samsung devices, which utilize an adaptive fast charger to minimize charging times [134]. A significant increase in the charging current results in heating of the battery cell, which takes a toll on its active materials. The charging profile from a Samsung Galaxy S7 utilizing a fast charger to charge a lithium nickel manganese cobalt oxide (NMC) cell was recorded and is shown in Figure 6.1. The charging current versus the temperature rise is shown at the top. The initial fully discharged cell temperature under light loading was $30^{\circ} \mathrm{C}$. After applying a constant current (CC) of $2400 \mathrm{~mA}$, a $5^{\circ} \mathrm{C}$ temperature rise is observed after only 3 min. The charging progression from the S7 fully discharged at rest was repeated and recorded using a thermal imager in Figure 6.2. Figure 6.2 indicates the battery form factor, the location of the positive and negative terminals, and the local temperature. The temperature at rest was $27.8^{\circ} \mathrm{C}$, progressing to $30^{\circ} \mathrm{C}\left(+2.2^{\circ} \mathrm{C}\right)$ after 1 min and $33.2 \mathrm{C}$ $\left(+5.4^{\circ} \mathrm{C}\right)$ after $3 \mathrm{~min}$ of charging. One can also observe how the shape of the battery begins to form along the boundaries of the thermal outline, but are inhomogenous.
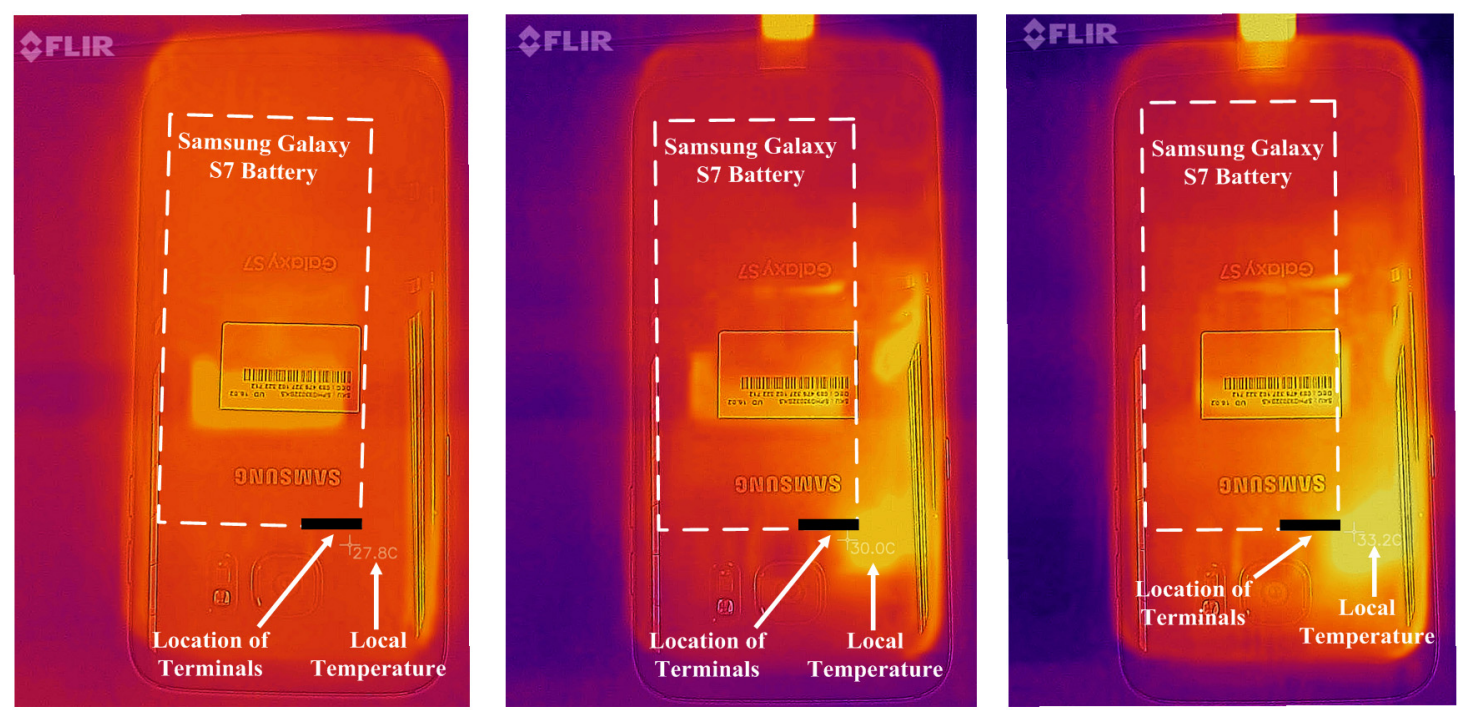

Figure 6.2. Thermal Imaging of Samsung Galaxy S7 Battery During Fast Charging Profile: Initial State (left), After 1 min (center), After 3 min (right). 
A generalized charging profile was discussed previously in Chapter 4, which consists of a CC phase followed by a Constant Voltage (CV) phase. Significant energy is transferred during $\mathrm{CC}$, and since this represents the maximum current, it is also the fastest charging period. However, since the charging current is so high in the case depicted in Figure 6.1, charging in the $\mathrm{CV}$ phase is required or the battery would only reach a maximum of $61 \%$ State of Charge (SoC). This is a result of the much higher overpotential, where the cell reaches its maximum terminal voltage sooner. In order to maximize the period in which the cell remains in $\mathrm{CC}$, the maximum terminal voltage level is also pushed close to its maximum as well (4.35 V in the case of this NMC battery cell). As mentioned previously, exceeding the maximum battery terminal voltage for any period of time can be catastrophic and result in a thermal runaway condition. This sensitive transition zone is shown below in Figure 6.1. Through the aid of an advanced PBM, a more accurate forecast of the overpotential and joule heating during loading and charging could help reduce the risk of a cell in being exposed to these conditions.

Joule heating accounts for only one type of overpotential, but it is important to mention that other types exist and can be mapped directly to the electrochemical conversion process and the active materials. At conservative charge or discharge currents, the balance of current across the battery cell can be assumed to be similar, which would in turn result in a relatively linear thermal heating profile. However, once the cell reaches high current levels, the distribution of current across the cell becomes highly nonlinear as a result of concentration overpotential.

The concept of concentration overpotential is a complex electrochemical phenomenon, which is caused by a number of factors which surface once the lithium ion battery begins 
to operate at high current levels. Previously, the lithium ion battery operation was described in the development of a P2D PBM, where the energy stored in a lithium ion cell is mapped to the concentration of lithium ions at the electrodes. At high current, a variable conductivity of the electrolyte and electrode at different $\mathrm{SoC}$ levels results in gradient currents. Gradient currents at the electrodes cause nonlinear joule heating, leading to uneven material stress that in turn degrades the cell SoH [135]. Through the development of a three-dimensional (3D) Finite Element Model (FEM) of the lithium ion battery, this phenomenon can be visualized and studied. In this chapter, a common LCO battery PBM is constructed using 3D FEM, where an electric and magnetic field analysis is conducted under charging and discharging at Coulombic rates (C-rates) of C/10 (10-hour), C/2 (2hour), and 1C (1-hour) current levels. An experimental analysis is made to verify the model while highlighting new operational features that can have a profound impact on the battery SoH.

\subsection{Lithium Ion Battery Physics}

Lithium ion batteries exchange lithium ions using their electrolyte as a transfer layer between the positive and negative electrodes [132]. Many types exist and are characterized by differences in the metal (M) oxide used in their positive electrode ( $\mathrm{Lix} M \mathrm{MO} 2)$, where the negative electrode is essentially a carbon graphite (LixC6) [22]. As the battery charges, lithium ions move from the positive to the negative electrode, where the process is simply reversed during discharging. A LCO $(\mathrm{LixCoO} 2)$ battery has been modeled and studied in this chapter, as it is not only a popular battery in mobile electronics, but has also been experimented with in transportation electrification because of its extremely high energy density. However, its safety and limited lifespan present a challenge in its utilization. 


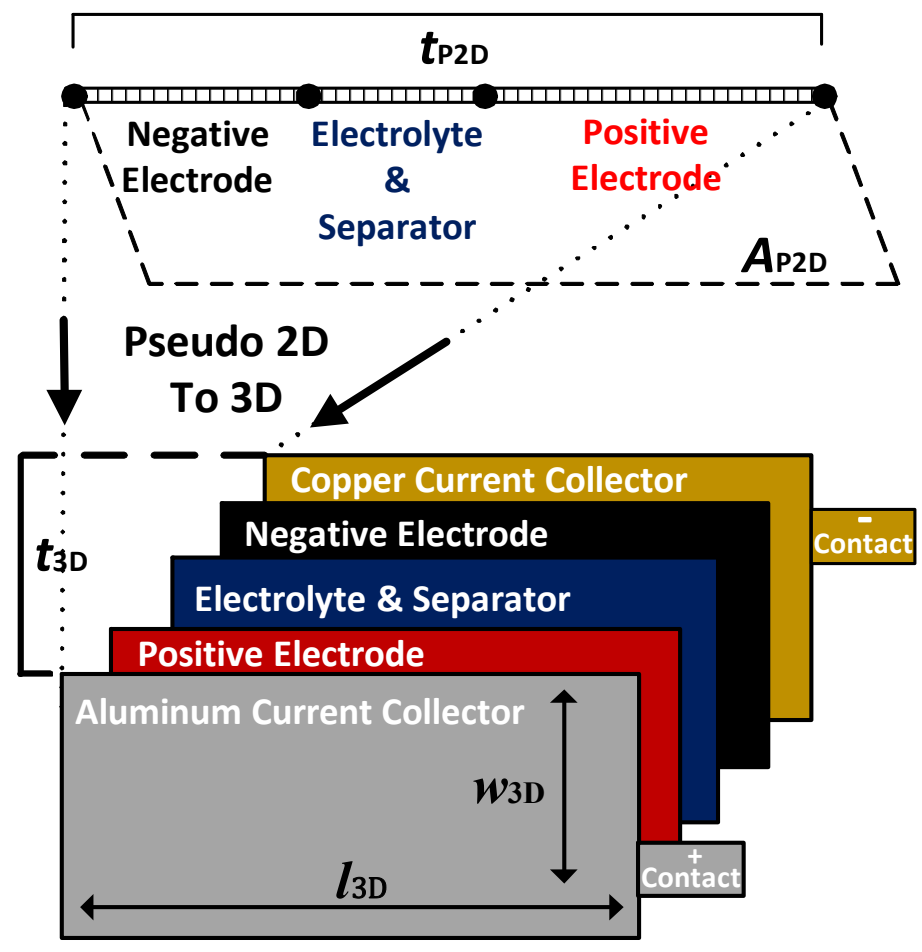

Figure 6.3. Pseudo 2D FEM to 3D FEM Representation of the Lithium Ion Battery Cell.

Traditional FEM of battery physics begins using a P2D representation, as was done in Chapter 5, modeling critical components of the electrochemical process. The FEM is solved along a line, representing a cross section of the battery materials from the edge of the negative electrode to the edge of the positive electrode using a minimum number of meshing points, as shown in Figure 6.3. These models only introduce the battery cell thickness $t_{P 2 D}$ and active surface area $A_{P 2 D}$ to model operation, neglecting dimensions of the package, plates, and current collectors. This formulation is computationally efficient and provides a superior improvement over legacy Randles equivalent circuit models during operation.

Although the P2D is a powerful improvement over the Randles equivalent circuit, it still represents a simplification of the cell operation. The P2D sacrifices a number of other 
details that can provide insight into the internal electromagnetic losses and stresses across the structure of the battery cell [136]. An extension into 3D FEM provides a way to study new operational features of the battery while providing a mechanism to assess voltage losses across the electrolyte, magnetic field propagation, and the generated gradient currents.

\subsubsection{D Physics Based Model Formulation}

The basis of the nonlinear lithium ion 3D FEM formulation is broken into two major parts, solving for both electrochemical and electromagnetic components [114]. In this subsection, the 3D PBM mathematical formulation of the LCO battery is derived. A summary of all associated simulation parameters is provided later in Table 6-1. The insertion and extraction of lithium ions occur at the surface of each electrode and can be represented by spherical particles of radius $r_{p}$ [132]. The molar flux $R_{L i+}$ of lithium at the particle surface can be represented by the following equation:

$$
R_{L i+}=-\sum \frac{v_{L i+} i_{l o c}}{n F} \frac{A_{v} r_{p}}{3 \varepsilon_{S}}
$$

where $v_{L i+}$ is a stoichiometric coefficient, $i_{l o c}$ is the localized current generated as a result of electrode kinetics, $n$ is the number of electrons involved in the reaction, $F$ is Faraday's constant, $A_{v}$ is the active surface area, and $\varepsilon_{s}$ is the electrode volume fraction.

Lithium diffuses $D_{s}$ to and from each surface, altering the total concentration of lithium $c_{s}$ in the solid phase:

$$
\frac{\partial c_{s}}{\partial t}=\nabla \cdot\left(-D_{S} \nabla c_{s}\right)
$$

subject to the following boundary conditions: 


$$
\left\{\begin{array}{c}
\frac{\partial c_{s}}{\partial r}=\left.0\right|_{r=0}=0 \\
-D_{s} \frac{\partial c_{s}}{\partial r}=-\left.R_{L i+}\right|_{r=r_{p}}=0
\end{array}\right.
$$

The ratio of the present concentration of lithium ions in the electrodes $c_{s}$ versus their maximum storage capacity $c_{s, \max }$ defines the SoC of each electrode $s$.

$$
\operatorname{SoC}_{s}=\frac{c_{s}}{c_{s, \max }}
$$

As lithium ions move across the electrolyte, the mass of all reactants is conserved:

$$
\varepsilon_{l} \frac{\partial c_{l}}{\partial t}+\nabla \cdot\left(-D_{l} \nabla c_{l}\right)=R_{l}-\left(\frac{i_{t o t}+Q_{l}}{F}\right) t_{+}
$$

where $\varepsilon_{l}$ is the electrolyte volume fraction, $c_{l}$ is the electrolyte concentration, $D_{l}$ is the electrolyte salt diffusivity, $t_{+}$is the transport number for lithium ions, and $R_{l}$ is the reaction source term. A connection is made to the electromagnetics domain in Equation (6-6). The the total generated cell current $i_{\text {tot }}$ and an arbitrary current $Q_{l}$ is constrained by the following relation:

$$
\nabla \cdot\left(-\sigma_{l} \nabla \phi_{l}+\frac{2 \sigma_{l} R T}{F}\left(1+\frac{\partial \ln f}{\partial \ln c_{l}}\right)\left(1-t_{+}\right) \nabla \ln c_{l}\right)=i_{\text {tot }}+Q_{l}
$$

where $R$ is the universal gas constant, $T$ is the absolute temperature, $f$ is an activity coefficient, and $\sigma_{l}$ and $\phi_{l}$ represent the conductivity and electric potential of the electrolyte, which vary based on the concentration $c_{l}$. This relationship is demonstrated in Figure 6.4.

The lithium ion reaction at the electrode induces an exchange current density $i_{0}$, demonstrating how a primary current generated at the load is a result of the concentration of lithium at the electrodes:

$$
i_{0}=F\left(k_{c}\right)^{\alpha_{a}}\left(k_{a}\right)^{\alpha_{c}}\left(c_{s, \max }-c_{s}\right)^{\alpha_{a}}\left(c_{s}\right)^{\alpha_{c}}\left(c_{l}\right)^{\alpha_{a}}
$$


where $k_{a}, \alpha_{a}$ and $k_{c}, \alpha_{c}$ represent the charge rate $k$ and transfer $\alpha$ coefficients at the anode $a$ and cathode $c$, respectively.

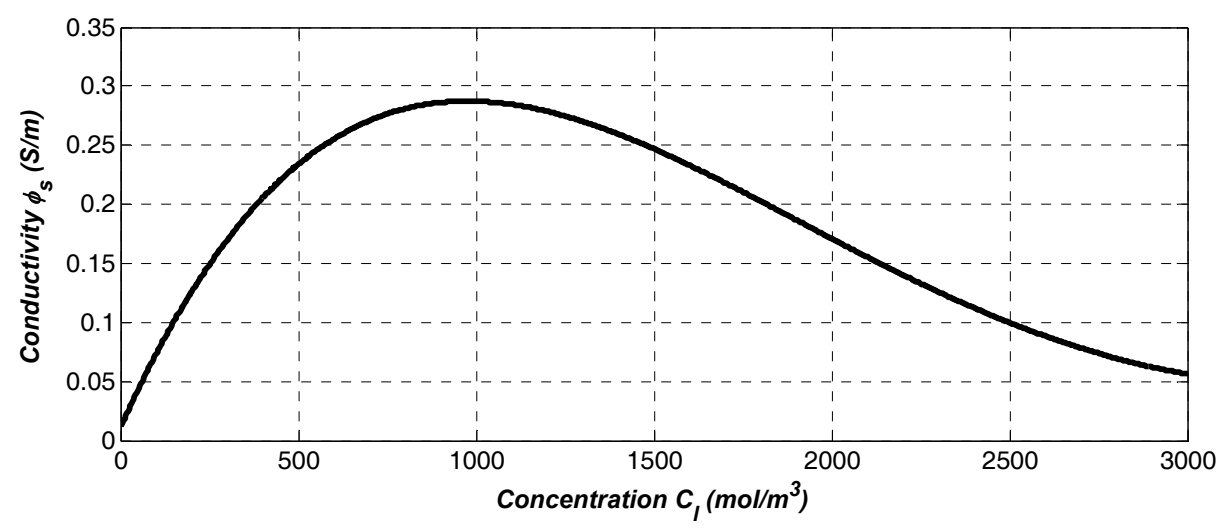

Figure 6.4. Ionic Conductivity of the Lithium Salt Electrolyte versus its Conductivity.

Table 6-1. FEM Simulation Parameters for the Lithium Ion Cobalt Polymer Battery.

\begin{tabular}{|c|c|c|c|}
\hline Description & Variable & \multicolumn{2}{|c|}{ Value } \\
\hline Cell Length & $l_{3 D}$ & & $0.150 \mathrm{~m}$ \\
\hline Cell Width & $w_{3 D}$ & & $0.050 \mathrm{~m}$ \\
\hline Cell Thickness & $t_{3 D}$ & & $0.010 \mathrm{~m}$ \\
\hline Test Temperature & $T$ & & $293.15 \mathrm{~K}$ \\
\hline Faradays Constant & $F$ & 96,48 & 35 Coulombs $/ \mathrm{mol}$ \\
\hline Universal Gas Constant & $R$ & & $8.314 \mathrm{~J} / \mathrm{mol} \cdot \mathrm{K}$ \\
\hline Stoichiometric Coefficient & $v_{L i+}$ & & -1 \\
\hline Number of Electrons Involved in Reaction & $n$ & & 1 \\
\hline Transport Number for Lithium Ions & $t_{+}$ & & 0.363 \\
\hline Anodic Charge Rate Coefficient & $k_{a}$ & & $2 \times 10^{-11} \mathrm{~m} / \mathrm{s}$ \\
\hline Cathodic Charge Rate Coefficient & $k_{c}$ & & $2 \times 10^{-11} \mathrm{~m} / \mathrm{s}$ \\
\hline Anodic Charge Transfer Coefficient & $\alpha_{a}$ & & 0.5 \\
\hline Cathodic Charge Transfer Coefficient & $\alpha_{c}$ & & 0.5 \\
\hline Activity Coefficient & $f$ & & 0 \\
\hline Electrolyte Salt Diffusivity & $D_{l}$ & & $7.5 \times 10^{-11} \mathrm{~m}^{2} / \mathrm{s}$ \\
\hline \multicolumn{2}{|l|}{ Electrode Material-Dependent Values } & $\begin{array}{l}\text { Positive } \\
\text { Electrode }\end{array}$ & $\begin{array}{l}\text { Negative } \\
\text { Electrode }\end{array}$ \\
\hline Spherical Particle Size & $r_{p}$ & $8.0 \mu \mathrm{m}$ & $12.5 \mu \mathrm{m}$ \\
\hline Electrode Volume Fraction & $\varepsilon_{S}$ & 0.297 & 0.471 \\
\hline Electrolyte Volume Fraction & $\varepsilon_{l}$ & 0.444 & 0.357 \\
\hline Maximum Concentration & $c_{s, \max }$ & $3900 \mathrm{~mol} / \mathrm{m}^{3}$ & $14870 \mathrm{~mol} / \mathrm{m}^{3}$ \\
\hline Electrode Conductivity & $\sigma_{s}$ & $3.8 \mathrm{~S} / \mathrm{m}$ & $100 \mathrm{~S} / \mathrm{m}$ \\
\hline Electrode Diffusion Coefficient & $D_{S}$ & $1.0 \times 10^{-13} \mathrm{~m}^{2} / \mathrm{s}$ & $3.9 \times 10^{-14} \mathrm{~m}^{2} / \mathrm{s}$ \\
\hline
\end{tabular}


The Butler-Volmer expression describes how $i_{0}$ contributes to a localized current induced at the electrode surface, which is dispersed in 3D:

$$
i_{l o c}(x, y, z, t)=i_{0}\left[e^{\frac{\alpha_{a} \eta(x, y, z, t) F}{\mathrm{RT}}}+e^{\frac{\alpha_{c} \eta(x, y, z, t) F}{\mathrm{RT}}}\right]
$$

where $\alpha_{a}$ and $\alpha_{c}$ are the anodic and cathodic charge transfer coefficients, respectively, and $\eta$ is the activation overpotential, revealing how the terminal voltage will drop or increase from its standard electric open circuit potential $E_{e q}$ under a load or source.

$$
\eta(x, y, z, t)=\phi_{s}(x, y, z, t)-\phi_{l}(x, y, z, t)-E_{e q}
$$

Table 6-1 provides a summary of all simulation parameters, which were utilized in the FEM development of the 3D PBM. The electric field cannot propagate beyond the total width $w_{3 D}$, length $l_{3 D}$, or thickness $t_{3 D}$ of the cell depicted in Figure 6.3. This results in the electric field boundary condition:

$$
\left\{\begin{array}{l}
\left.\frac{\partial \phi_{s}(x, y, z, t)}{\partial x}\right|_{x=0}=\left.\frac{\partial \phi_{s}(x, y, z, t)}{\partial x}\right|_{x=w_{3 D}} \\
\left.\frac{\partial \phi_{s}(x, y, z, t)}{\partial y}\right|_{y=0}=\left.\frac{\partial \phi_{s}(x, y, z, t)}{\partial y}\right|_{y=l_{3 D}}=0 \\
\left.\frac{\partial \phi_{s}(x, y, z, t)}{\partial z}\right|_{z=0}=\left.\frac{\partial \phi_{s}(x, y, z, t)}{\partial z}\right|_{z=t_{3 D}}
\end{array}\right.
$$

The resulting open circuit voltage of the LCO battery cell is thus:

$$
V_{c e l l}(t)=\phi_{s, p}\left(l_{3 D}, y, z\right)-\phi_{s, n}(0, y, z)-R_{f} I_{b a t t}(t)
$$

where $\phi_{s, p}$ and $\phi_{s, n}$ represent the electrode potentials at the positive $p$ and negative $n$ electrodes, respectively, and $R_{f}$ represents the ohmic loss between the tabs and current collectors. 
Table 6-2. 8048168C Lithium Ion Cobalt Polymer Battery Specifications.

\begin{tabular}{|c|c|c|}
\hline Nominal Voltage & & $3.7 \mathrm{~V}$ \\
\hline Rated Capacity & $\begin{array}{l}0-100 \% \text { SoC Operating } R \\
10-90 \% \text { SoC Operating } R c\end{array}$ & $\begin{array}{l}6400 \mathrm{mAh} \\
5400 \mathrm{mAh}\end{array}$ \\
\hline Cell Dimensions & \multicolumn{2}{|c|}{$168.5 \mathrm{~mm} \times 48.5 \mathrm{~mm} \times 8.0 \mathrm{~mm}$} \\
\hline Mass & & $140 \mathrm{~g}$ \\
\hline Charging & $\begin{array}{r}\text { Charging Voltage } \\
\text { Maximum Current } \\
\text { Standard Current } \\
\text { Temperature Range }\end{array}$ & $\begin{array}{r}4.20 \pm 0.05 \mathrm{~V} \\
1 \mathrm{C}(6.40 \mathrm{~A}) \\
\mathrm{C} / 2(3.20 \mathrm{~A}) \\
0-45^{\circ} \mathrm{C}\end{array}$ \\
\hline Discharging & $\begin{array}{r}\text { Maximum Current } \\
\text { Standard Current } \\
\text { Minimum Cutoff Voltage } \\
\text { Recommended Cutoff } \\
\text { Temperature Range }\end{array}$ & $\begin{array}{r}5 \mathrm{C}(32.00 \mathrm{~A}) \\
\mathrm{C} / 5(1.28 \mathrm{~A}) \\
2.75 \mathrm{~V} \\
3.30 \mathrm{~V} \\
0-60^{\circ} \mathrm{C}\end{array}$ \\
\hline
\end{tabular}

\subsubsection{Finite Element Modeling}

The specifications for the $8048168 \mathrm{C}$ lithium ion battery modeled in this study are summarized in Table 6-2. The $8048168 \mathrm{C}$ has a capacity of $6.4 \mathrm{Ah}$, though the operating SoC range has been constrained to utilize only 5.4 Ah in order to preserve its usable capacity and $\mathrm{SoH}$ [138]. The cell dimensions are $168.5 \mathrm{~mm}$ x $48.5 \mathrm{~mm}$ x $8.0 \mathrm{~mm}$ containing copper-based positive and aluminum-based negative current collectors measuring $10 \mathrm{~mm}$ x $10 \mathrm{~mm}$ x $0.1 \mathrm{~mm}$. However, in order to provide a computationally efficient model, these dimensions were slightly altered to $150 \mathrm{~mm}$ x $50 \mathrm{~mm}$ x $10 \mathrm{~mm}$ to improve proportionality, allowing for reduced 3D meshing elements.

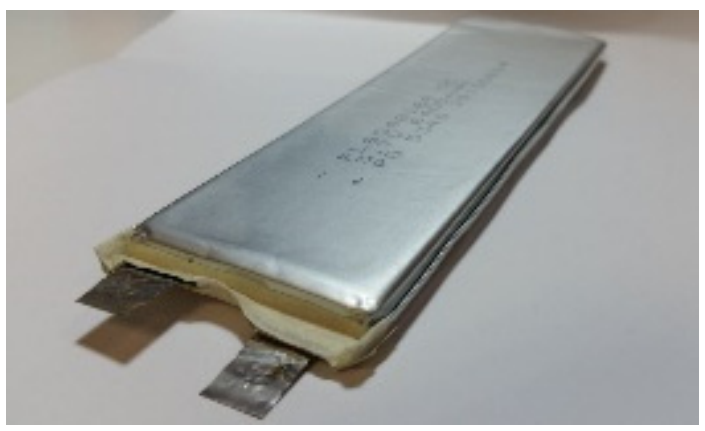

Figure 6.5. 8048168C Lithium Ion Cobalt Oxide (LCO) Polymer Battery. 


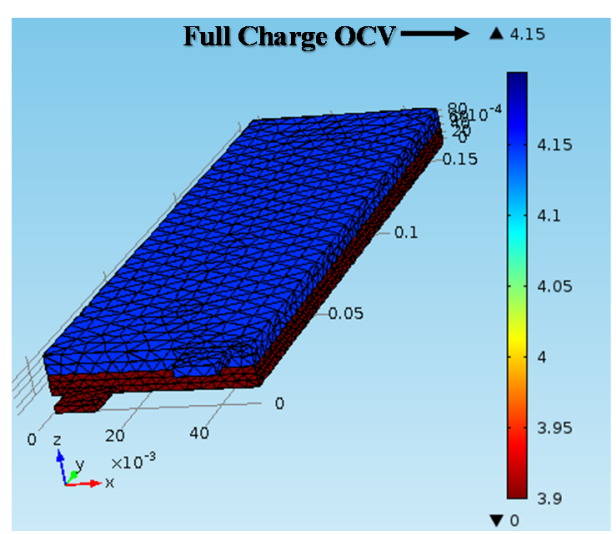

Figure 6.6. Electrostatic 3D FEM of the LCO Battery at Full Charge.

Pictured in Figure 6.5, the LCO cell has no onboard balancing circuitry, thus the length and width of each plate is consistent with its outer dimensions. The thickness of each electrode, electrolyte, and current collectors were estimated by normalizing the dimensions from the P2D model and were depicted in Figure 6.3. These dimensions were then used to partition the cell thickness. Since the protective film is $<0.1 \mathrm{~mm}$, its offset could be neglected. The resulting mesh and the OCV solution at full charge of $4.15 \mathrm{~V}$ is shown in Figure 6.6. Meshing elements were able to remain coarse at the current collectors, reducing the degrees of freedom, but are finer at boundaries where each electrode interfaces with the electrolyte.

\subsection{Results and Discussion}

To quantify the PBM, light, medium, and high loading and charging currents were applied to the terminals of the lithium ion battery, and the results are shown in Table 6-3. Currents were applied at the $\mathrm{C} / 10, \mathrm{C} / 2$, and $1 \mathrm{C}$ rates to both the $\mathrm{P} 2 \mathrm{D}$ and $3 \mathrm{D}$ PBMs. All discharging currents were applied at full charge (100\% SoC) while all charging currents were applied at full discharge $(0 \%$ SoC $)$. 
Table 6-3. Experimental, Pseudo 2DFEA, and 3DFEA Model Comparison.

\begin{tabular}{|c|c|c|c|c|c|c|c|c|c|c|c|}
\hline \multirow[t]{2}{*}{ State } & \multirow{2}{*}{$\begin{array}{l}\text { SoC } \\
(\%)\end{array}$} & \multirow{2}{*}{$\begin{array}{c}\mathrm{C} \\
\text { Rate }\end{array}$} & \multirow{2}{*}{$\begin{array}{c}I_{\text {batt }} \\
\text { (A) }\end{array}$} & \multicolumn{3}{|c|}{ Terminal Voltage } & \multicolumn{3}{|c|}{ Overpotential $\boldsymbol{\eta}(\Delta \mathrm{V})$} & \multicolumn{2}{|c|}{ Error } \\
\hline & & & & $\frac{\text { Exp. }}{(\mathrm{V})}$ & $\frac{\mathrm{P} 2 \mathrm{D}}{(\mathrm{V})}$ & $\frac{3 \mathrm{D}}{(\mathrm{V})}$ & $\frac{\text { Exp. }}{(\mathrm{mV})}$ & $\underline{\mathrm{P} 2 \mathrm{D}}$ & $\frac{3 \mathrm{D}}{(\mathrm{mV})}$ & $\frac{\mathrm{P} 2 \mathrm{D}}{(\mathrm{mV})}$ & $\frac{3 \mathrm{D}}{(\mathrm{mV})}$ \\
\hline $\mathrm{OCV}$ & 100 & -- & - & 4.149 & 4.142 & 4.149 & "-- & "-- & "-- & $\begin{array}{l}- \\
\end{array}$ & "-- \\
\hline & 100 & $-1 \mathrm{C}$ & & 3.9 & & 3.962 & -189.0 & & & +6.5 & +2 \\
\hline Discl & 100 & $-\mathrm{C} / 2$ & -3.20 & 4.085 & 4.045 & 4.064 & -64.0 & -9 & -8 & -33.3 & -20.6 \\
\hline Discharge & 100 & $-\mathrm{C} / 10$ & -0.64 & 4.11 & 4.122 & 4.134 & -13.2 & -20 & -1 & -6.8 & -0.9 \\
\hline $\mathrm{OC}$ & 0 & -- & -- & 3.300 & 3.3 & 3.315 & -- & -- & -- & - & -- \\
\hline Cha & 0 & $+1 \mathrm{C}$ & +6.40 & 3.477 & 3.512 & 3.488 & +177.0 & +194.0 & +172.5 & +17.0 & -4.5 \\
\hline & 0 & $+\mathrm{C} / 2$ & +3.20 & 3.370 & 3.427 & 3.402 & +70.0 & +109.3 & & +39.3 & +16.2 \\
\hline Charge & 0 & $+\mathrm{C} / 10$ & +0.64 & 3.313 & 3.342 & 3.333 & +13.0 & +23.8 & +17.3 & +10.8 & +4.3 \\
\hline
\end{tabular}

\subsubsection{Terminal Voltage and Analysis of Overpotential}

The terminal voltage potential between the electrode plates and overpotential $\eta$ between measurements and each model, as well as the error between the measured $\eta$, is shown in Table 6-3. For discharging, light and heavy loading produces a strong correlation with less than a $3 \mathrm{mV}$ variance, while medium loading introduces the highest error of 20.6 $\mathrm{mV}$. In the charging case, a $5 \mathrm{mV}$ variance is observed from the measured values for light and heavy currents, while the $\mathrm{C} / 2$ charging current drift is around $16 \mathrm{mV}$.

The $\mathrm{P} 2 \mathrm{D}$ follows a similar progression, with its best accuracy at $1 \mathrm{C}$ and $\mathrm{C} / 10$, except with much greater error versus the 3D PBM. There are a number of potential causes for the error observed at $\mathrm{C} / 2$. First, the error could be a result of adjusting the electrode and electrolyte thicknesses to better align with the FEM meshing scale. Adjusting these thicknesses, particularly the thickness of the electrolyte, would vary the ohmic loss. Secondly, both the conductivity of the electrolyte $\sigma_{l}$ and electrodes $\sigma_{s}$ are functions of the electrolyte salt concentration $c_{l}$ and concentration of lithium ions $c_{S}$, respectively. Since the initial states vary greatly when starting from full charge or full discharge, these are driving factors. 


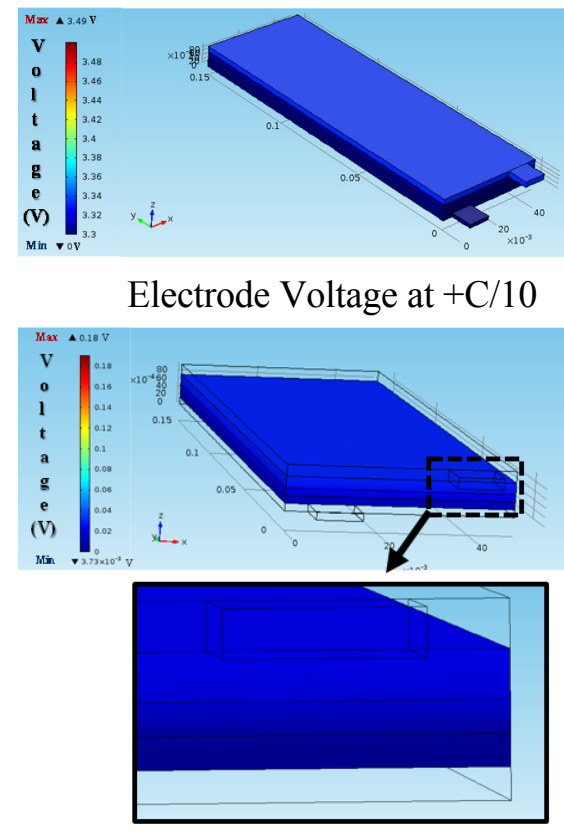

Electrolyte Voltage at $+\mathrm{C} / 10$

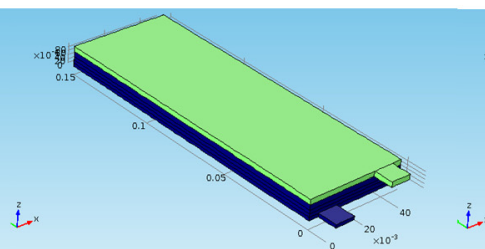

Electrode Voltage at $+\mathrm{C} / 2$

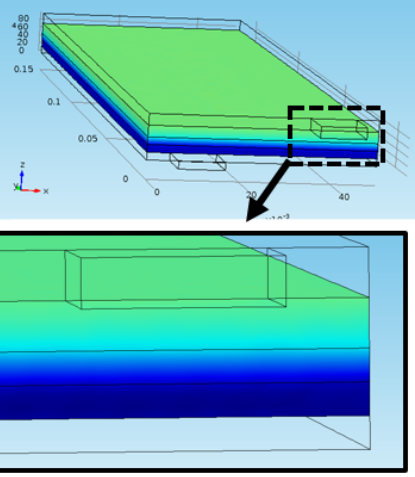

Electrolyte Voltage at $+\mathrm{C} / 2$

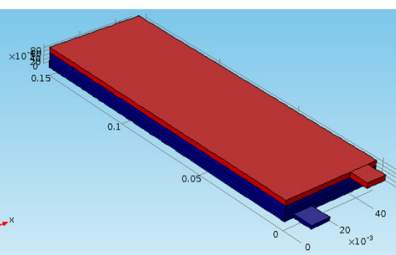

Electrode Voltage at $+1 \mathrm{C}$

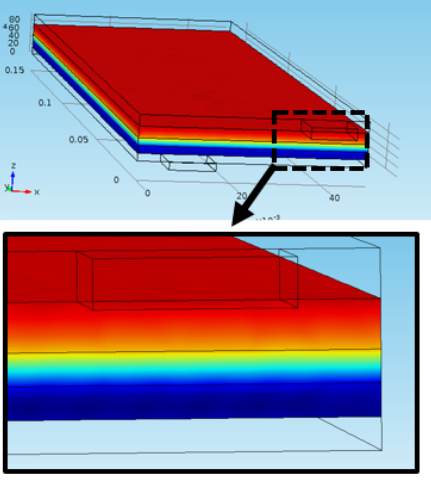

Electrolyte Voltage at $+1 \mathrm{C}$

Figure 6.7. Electric Potential at the Electrodes and Electrolyte of the LCO Battery under Charging.

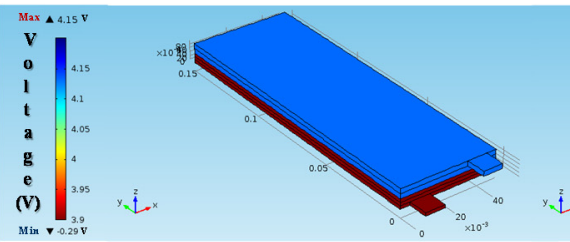

Electrode Voltage at $-\mathrm{C} / 10$

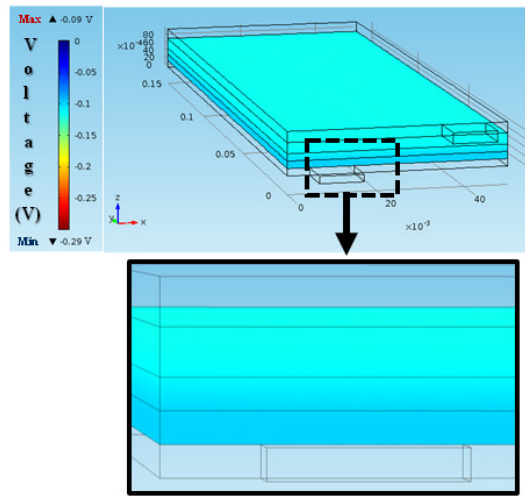

Electrolyte Voltage at $-\mathrm{C} / 10$

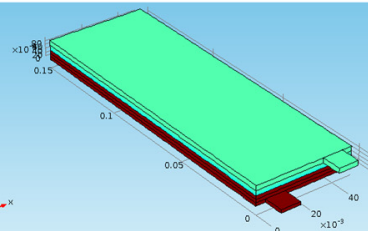

Electrode Voltage at $-\mathrm{C} / 2$
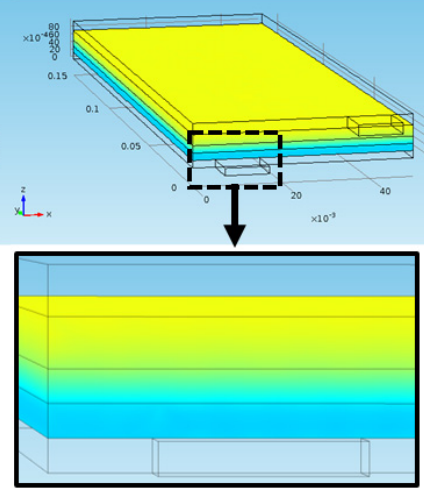

Electrolyte Voltage at $-\mathrm{C} / 2$

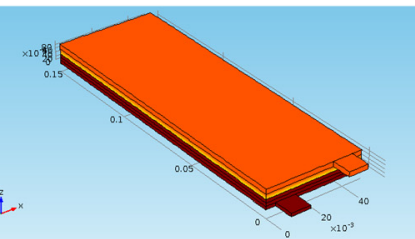

Electrode Voltage at $-1 \mathrm{C}$

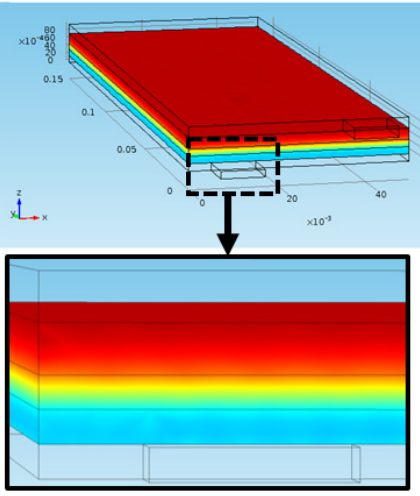

Electrolyte Voltage at $-1 \mathrm{C}$

Figure 6.8. Electric Potential at the Electrodes and Electrolyte of the LCO Battery under Discharging. 


\subsubsection{Electric Field Analysis}

An electric field analysis for the charging and discharging cases is shown in Figure 6.7 and Figure 6.8, respectively. The voltage potential distribution at the electrode plates is shown at the top, revealing the departure from the OCV at each current level. For the charging case, as the current increases, the voltage at the electrodes increases consistent with Table $6-3$ in a fairly linear fashion, from $3.30 \mathrm{~V}(\mathrm{OCV}$ at $0 \% \mathrm{SoC})$ to $3.49 \mathrm{~V}$. In the discharging case, the terminal voltage across the electrodes decreases, dropping from 4.15 $\mathrm{V}(\mathrm{OCV}$ at $100 \% \mathrm{SoC})$ to $3.95 \mathrm{~V}$. The current collectors reveal a potential distribution that is nearly constant across their surfaces. The electrode voltage distribution does not offer a great deal of insight in 3DFEM, however, this is not the case inside the electrolyte. The electric field distribution across the electrolyte is shown at the bottom of Figure 6.7 for charging and at the bottom of Figure 6.8 for discharging.

For charging, $\mathrm{C} / 10$ results in a light voltage drop, where the terminal voltage does not greatly vary. However, at $\mathrm{C} / 2$ and particularly at $1 \mathrm{C}$, a significant variation is present. Close-ups demonstrate a progression in the electrolyte potential, becoming highly nonlinear as it approaches high levels of current. This is visualized to the bottom right of Figure 6.7 and Figure 6.8, where the wide range of colors depicts an electric potential that is very different at each individual cross sectional cut through the electrolyte.

\subsubsection{Magnetic Field Analysis and Gradient Currents}

An extensive magnetic field analysis for the charging and discharging cases is shown in Figure 6.9 and Figure 6.10, respectively, highlighting a strength of the 3D FEM. Since the only measurable current is that which is delivered to the terminals, 3D FEM provides insight into the direction and magnitude of all generated electrochemical currents and the 
locations of the heavy losses. The top plots reveal the normalized (C-rate) current density normal across the cell and terminals under each of the six current levels. Current density vectors are superimposed to illustrate the current propagation across the cell as it is delivered to each electrode. Below, the analysis is extended, viewing the current density normal as a contour plot from 0 to $1,000 \mathrm{~A} / \mathrm{m}^{2}$ in $10 \mathrm{~A} / \mathrm{m}^{2}$ steps.

The charging cases are depicted in Figure 6.9, where all current flows into the positive electrode dispersing across the cell structure. At $\mathrm{C} / 10$, the current density inside the electrodes is nearly equal to that which is delivered at the current collectors. This phenomenon is expected, as at currents below the 5-hour rate $(\mathrm{C} / 5)$, the energy input and output capacities are close to the rated capacity, as verified by the $8048168 \mathrm{C}$ datasheet [138]. Below, the current density norm offers a different perspective. Although the cell current density is calm, some concentrations still form near the junctions where the contacts meet the current collectors. At $\mathrm{C} / 2$, losses begin to increase and the effective $\mathrm{C}$-rate at each electrode shifts, which indicates parasitic losses occurring inside the cell. This phenomenon results in additional energy, which will be needed to inject charge into the battery to effectively charge the cell at the $\mathrm{C} / 2$ current. Another contribution to these losses is caused by gradient currents, which begin to surface near the electrode terminals.

A close-up identifies a development of gradient currents at both electrodes. As charging approaches $1 \mathrm{C}$, differences in the current density at each electrode intensify. Looking at the top plot, although the cell is charged at $1 \mathrm{C}$, the charging current required to overcome parasitic losses is $7 \%$ higher, or an effective $\mathrm{C}$-rate of $1.07 \mathrm{C}$. When analyzing the gradient currents, they develop both at the electrodes and inside the electrolyte, though the magnitude inside the electrolyte is far less. 

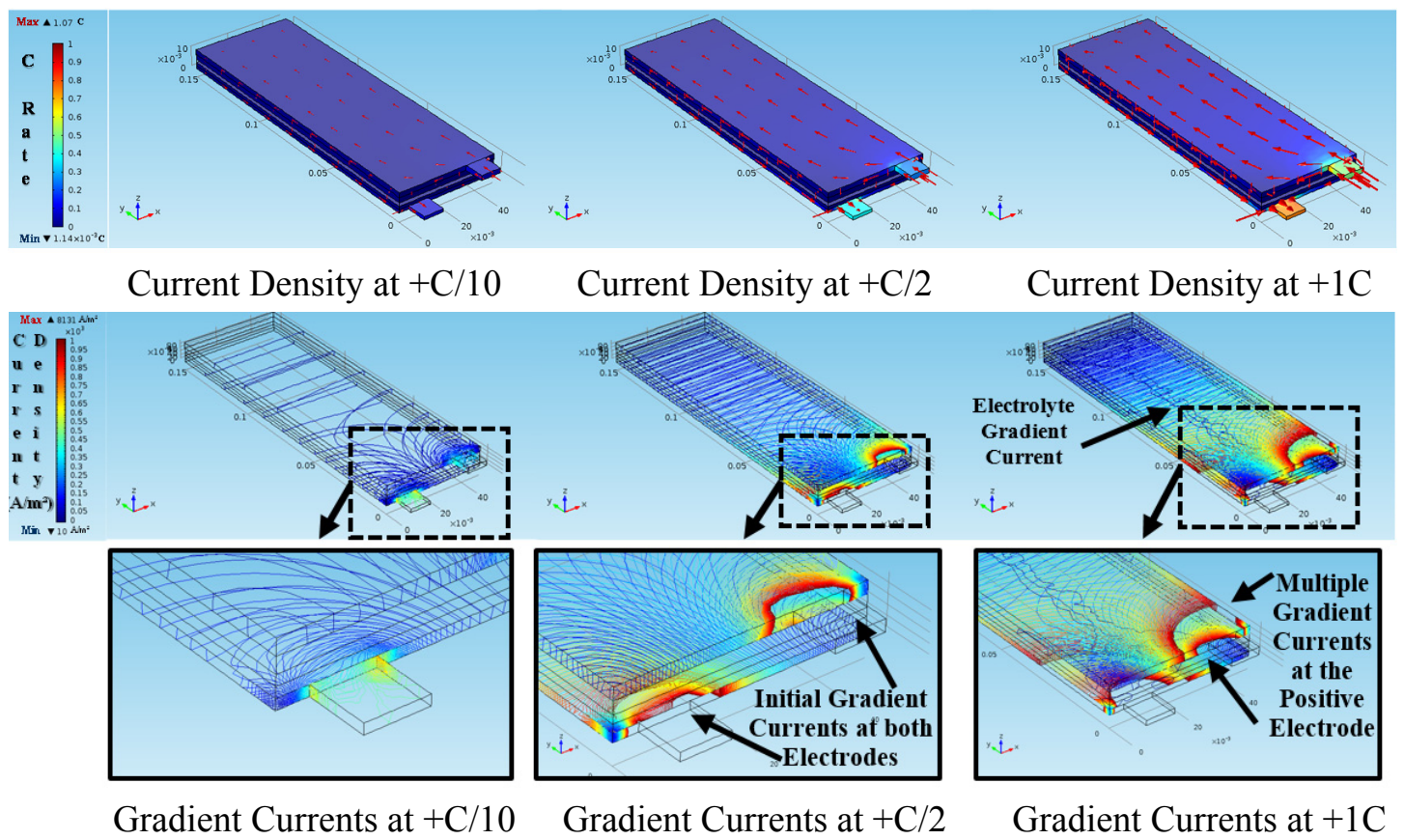

Figure 6.9. Current Density at the Electrodes and Electrolyte of the LCO Battery under Charging.

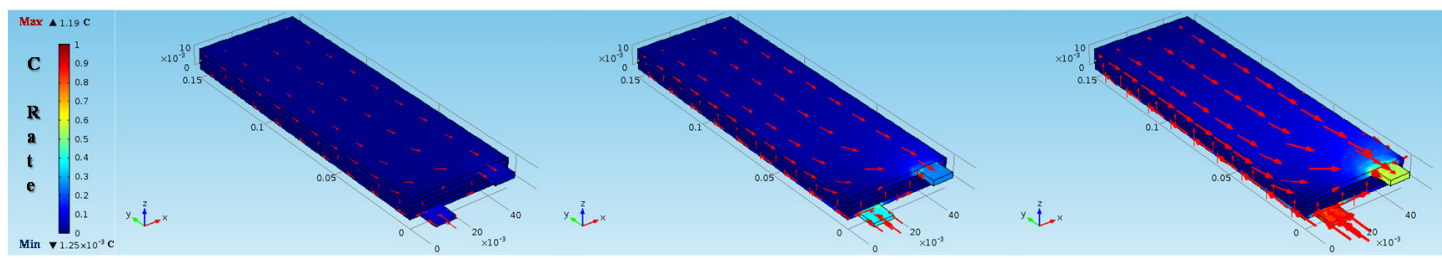

\section{Current Density at $-\mathrm{C} / 10 \quad$ Current Density at $-\mathrm{C} / 2 \quad$ Current Density at $-1 \mathrm{C}$}

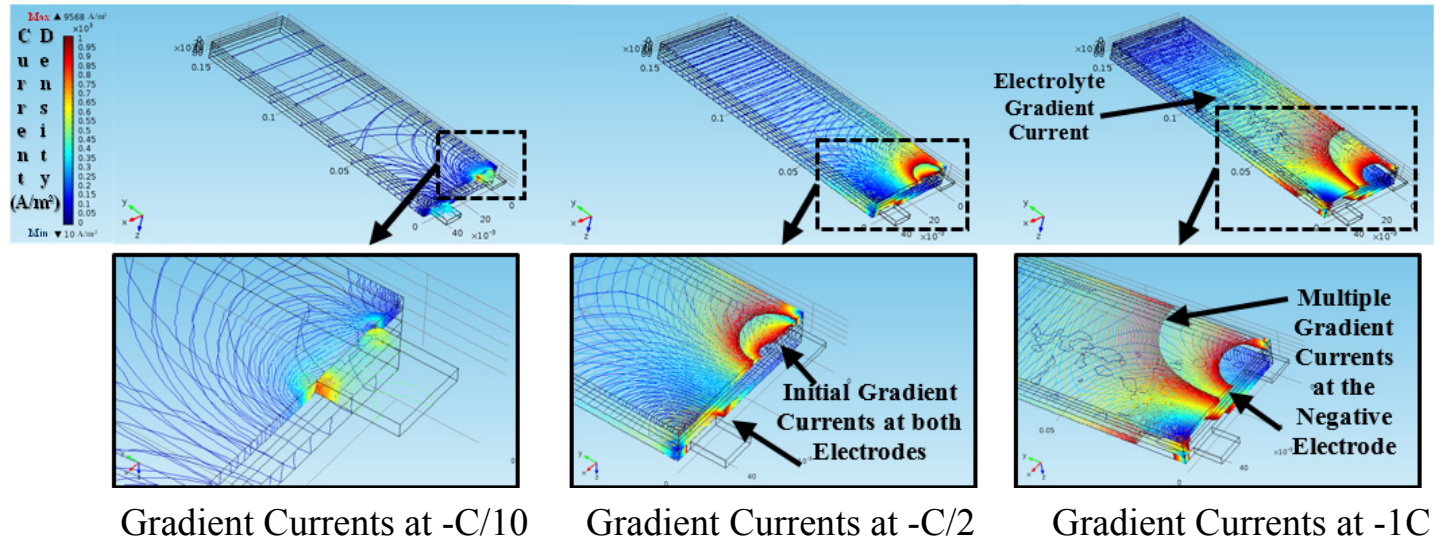

Figure 6.10. Current Density at the Electrodes and Electrolyte of the LCO Battery under Discharging. 
A close-up reveals multiple concentrated currents forming on the electrodes as rings spreading from the positive electrode to nearly half the length of the cell structure. These features can easily go unnoticed without a reduced scale. The reduced scale exposes a maximum current density in during charging of this cell to be at $8,131 \mathrm{~A} / \mathrm{m}^{2}$.

For all discharging cases, current generated inside the cell is dispersed across the cell structure and delivered to the positive electrode. At $\mathrm{C} / 10$, the distribution is similar to that of the charging cases, since $\mathrm{C} / 10$ is less than the rated discharge current of the battery cell. Looking at Figure 6.10, the normalized current density inside the electrodes is nearly equal to that which is delivered at the current collector contacts. Once again, the current density norm offers a different perspective, except concentrations at the junctions are slightly higher in magnitude, with a peak near $1,000 \mathrm{~A} / \mathrm{m}^{2}$ versus the $700 \mathrm{~A} / \mathrm{m}^{2}$ that was observed in the charging case.

At $\mathrm{C} / 2$, the losses increase once again, except the additional energy previously required from the charger is now required by the battery. The top plot reveals an imbalance between the current passing through each electrode. Below, the gradient currents are far worse than what was observed during charging, where multiple concentrated current density rings are already beginning to surface. As the load approaches $1 \mathrm{C}$, the total electrochemical current density required to deliver energy to a load is actually $19 \%$ higher than what is delivered, or an effective C-rate of 1.19C. This case results in the greatest difference between the terminals and is further visualized by the gradient current generation, which once again features both electrode and electrolyte gradients. The current density is high enough that multiple concentrated rings develop, stretching beyond half of the cell length. 


\subsection{Summary}

In this chapter, the concept of the PBM of the lithium ion battery is deeply investigated and expanded from the P2D model. The P2D is used as a foundation to the 3D PBM, where a comprehensive mathematical formulation was made. The 3D PBM was used as a mechanism to study operational characteristics of the common LCO battery cell that are difficult to obtain experimentally, as well as offer an extension of the $\mathrm{SoH}$ analysis.

Terminal voltages during both charging and discharging at $\mathrm{C} / 10, \mathrm{C} / 2$, and $1 \mathrm{C}$ currents were close to measured values. The results highlight new advantages and insight into $\mathrm{SoH}$ impacts on lithium ion batteries that 3D PBMs can offer to study the generation of undesired gradient currents across the battery cell when operating at high charging and discharging currents. A more accurate depiction of overpotential when moving from P2D to $3 \mathrm{D}$ is evident in the results in the form of gradient currents, contributing to thermodynamic and material stress, which contributes to shortening the battery life. This analysis can serve to increase awareness for manufacturers of some of the inherent operational challenges associated with lithium ion batteries in modern applications. Accounting for the lessons learned could help to propose revised geometries to help better distribute gradient currents and generate a more linear current distribution, which would result in a more linear distribution of current across the battery cell.

Some of the strengths of a P2D or 3D PBM have been demonstrated in the last two chapters of this dissertation as a superior way to model accurate depiction of the battery. However, these models are extremely challenging to utilize when conducting deep, comprehensive simulations of an entire electrical power system. Intricate electrical system simulations requiring a great deal of processing power and/or memory can prove to be 
impractical when placing a PBM in the loop.

For these scenarios, a popular option is to generate an enhanced, dynamic Randles equivalent circuit model. An enhanced $2^{\text {nd }}$-order model, which can account for continuously changing component values, and OCV can provide a powerful tool in simulation while capturing the signature dynamics of the battery. Furthermore, it is important to evaluate the required complexity as it relates to the specific application. A close analysis of the application may yield that a dynamic model based on the $\mathrm{SoC}$ is not needed, or a $1^{\text {st }}$ or $2^{\text {nd }}$-order equivalent circuit model is sufficient to obtain an accurate depiction of the terminal voltage and performance once the battery is placed into service. In the next chapter, a dynamic multi time-scale battery model for a $51.8 \mathrm{~V}$ 14-cell LCO battery module is generated to assist in conducting accurate simulations of EVs. A comprehensive data acquisition system is built upon similar concepts as outlined in Chapter 5, where pulsed loading and charging currents are used to extract equivalent circuit component values across the entire battery SoC range. 


\section{Chapter 7 Developing a Comprehensive Battery Simulation Model}

\subsection{Introduction}

The popularity of electrochemical energy storage (ES) continues to grow to support a trend toward transportation electrification [139]. In recent years, electric vehicles (EVs) have become a major topic of discussion, as they are expected to witness a double-digit growth by 2022 [140]. This has placed ES, and particularly battery ES, center stage. As the number of EVs continue to emerge into the market, the concept of hybrid ES consisting of multiple ES devices (e.g. supercapacitors) has been deployed and implemented. The current-voltage (I-V) behavior of supercapacitor (SC) ES can be fairly predictable, as responses in the EV propulsion and traction system fall within the seconds and sub-seconds range [141]. Moreover, the operating State of Charge (SoC) range of the SC remains fairly constant over the course of its lifespan, as a result of an entirely different ageing mechanism. This enables their simulations to be relatively straightforward in most applications. Their performance within hybrid ES systems will be extensively evaluated later in this dissertation for not only EV, but also shipboard power system applications.

In this chapter, a focus has been placed specifically upon the advanced modeling of a battery ES device. In contrast to the SC, battery ES behavior is highly nonlinear and dependent upon many factors [142]. Although battery ES for EV propulsion originally started with lead acid battery testing in the 1990s, this has since moved onto the primary usage of nickel metal hydride (NiMH) and various lithium ion battery chemistries. Six different lithium ion battery chemistries now exist in the market, each with their own signature dynamics [22]. EV manufacturers have explored a wide range of chemistries in 
their aspiration to maximize operating ranges, motoring power, and the battery lifespan. From propulsion and regenerative breaking to a power electronics perspective, advanced models that capture the necessary dynamics are needed. Without a method to extract and implement an advanced battery model capturing these features, an accurate and dependable simulation is not possible.

In Reference [143], a comprehensive test procedure was proposed for building a $2^{\text {nd }}-$ Order dynamic lithium ion battery equivalent circuit model through the introduction of fixed, standardized charging and discharging pulses at high current. During each pulse, Coulombic rates (C-rates) of up to $1 \mathrm{C}$ were imposed at the battery terminals with a goal to amplify the voltage drop or rise response and extenuate its exponential recovery dynamics. Equivalent circuit parameter extraction procedures were discussed, as well as a method to obtain the average open circuit voltage (OCV) trend over the entire SoC span. Unfortunately, the $2^{\text {nd }}$-Order model was unable to map impulse parameters to meaningful time spans, making it difficult when trying to address its adequacy for EV applications.

In Reference [144], a procedure to obtain a $3^{\text {rd }}$-Order dynamic battery model was presented, similarly imposing standardized charge and discharge currents on the battery at multiple SoC levels. In this case, time constants were mapped to the second, minute, and hour ranges. To acquire a time constant within the seconds range, a short, $1.5 \mathrm{~s}$ pulse was applied, followed by a $120 \mathrm{~s}$ rest period. To obtain a time constant within the minutes range, a 6 min pulse was applied, followed by a 20 min rest period, while pulses within the hours range applied pulses for $5 \mathrm{~h}$, followed by a $15 \mathrm{~h}$ rest period. Through analyzing the voltage behavior during and following each pulse, ohmic resistance and impulse parameters were extracted at each time step. 
In this chapter, a hybrid of these previous tests and procedures have been implemented to build and program a battery test stand. The final comprehensive model was fully implemented as a drop-in SimPowerSystems block in MATLAB/Simulink. Conceptually, the process in acquiring the equivalent circuit model is similar to the method addressed previously in Chapter 5, where a $1^{\text {st }}$-Order Randles equivalent circuit model was developed for an autonomous battery management system. However, now a variety of pulse currents are utilized to obtain a $2^{\text {nd }}$-Order model, which is charge or discharge and SoC-dependent. Moreover, by applying a current magnitude of five to ten times greater, the accuracy in extracting its I-V behavior is dramatically increased.

A hybridization and simplification is applied from the procedures in References [143] and [144]. Although these previous systems produced accurate results, specialized equipment with elaborate setups were necessary. In this work, the battery testing system is designed and implemented with relatively low-cost equipment, and programmed and controlled by a National Instruments (NI) LabVIEW data acquisition (DAQ) interface. The battery model addresses the requirements and depth required to conduct an accurate EV simulation. Since EV dynamics primarily impact time constants in the second and minuterange, a $2^{\text {nd }}-$ Order model is acquired. Comprehensive testing is conducted to extract the $\mathrm{OCV}$ and equivalent circuit parameters trends based on the SoC for both charging and discharging operation. The trend for both the OCV and each parameter was individually curve-fit to generate the six functions essential for an accurate simulation. The final model also includes an energy rate adjustment that compensates for the reduced capacity observed at high discharge currents. The model is generated for a PL8048168 21 Amp-hour (Ah) Lithium Ion Cobalt (LCO) Polymer battery module, as shown in Figure 7.1, containing 14 
LCO cells in series to reach a nominal voltage level of $51.8 \mathrm{~V}$ [145]. It is worthy to mention this module contains 14 of the same LCO cells that have already been studied and analyzed in the previous chapters.

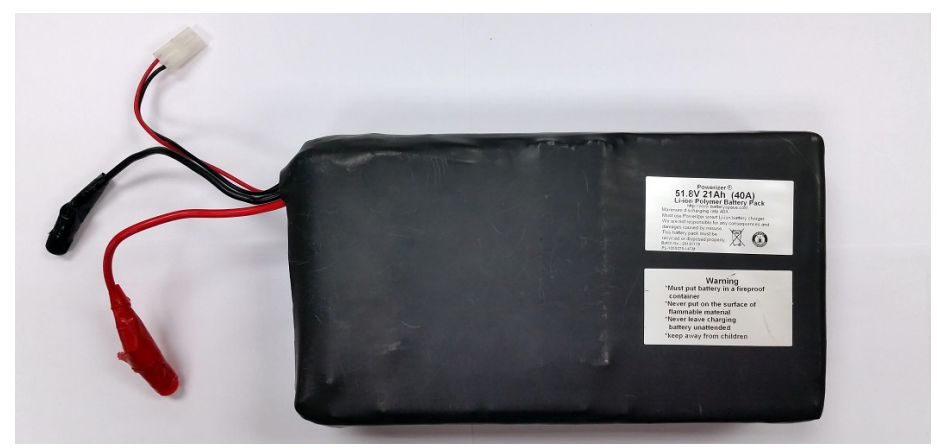

Figure 7.1. PL8048168 51.8 V 21 Ah Lithium Ion Cobalt Polymer Battery Module.

\subsection{Mathematical Model}

The Randles equivalent circuit provides the basis for virtually all battery representation based on Electrochemical Impedance Spectroscopy (EIS), beginning with a $1^{\text {st }}$-Order circuit and typically progressing to $3^{\text {rd }}$-Order [142]. In the development of this battery model, a $2^{\text {nd }}-$ Order equivalent circuit has been selected for two reasons. First, the MATLAB/Simulink environment can require a great deal of overhead and to enable the support for an EV bank, where many modules are utilized in large parallel-series configurations. For these cases, a $2^{\text {nd }}-$ Order model would improve the computational efficiency. Second, when executing active EV simulations, minute and second-range responses are particularly of interest, while hour-range responses only target extremely long stops (e.g. parking overnight). The $2^{\text {nd }}-$ Order equivalent circuit model is shown in Figure 7.2, where dynamic values are passed to variable resistances, capacitances, and a voltage source. 
The terminal voltage response at the battery terminals is:

$$
V_{b}(t)=V_{o c} \pm I_{b}(t)\left[R_{0}+R_{s e c}\left(1-e^{\frac{-t}{\tau_{s e c}}}\right) R_{\min }\left(1-e^{\frac{-t}{\tau_{\min }}}\right)\right]
$$

where the time constants in the seconds and minutes range are $\tau_{s e c}=R_{s e c} C_{s e c}$ and $\tau_{\min }=R_{\min } C_{\min }$, respectively.

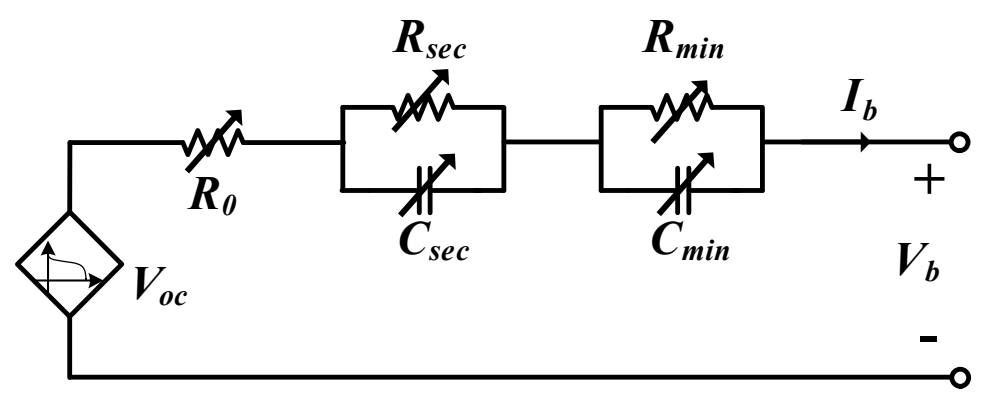

Figure 7.2. $2^{\text {nd }}-$ Order Dynamic Battery Equivalent Circuit Model.

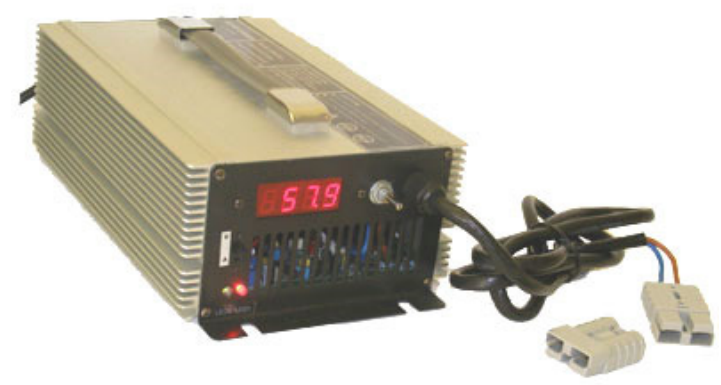

Figure 7.3. BatterySpace KP4818C Universal Smart Charger.

\subsection{Testing and Parameter Estimation}

In this section, the testing system and DAQ is discussed, as well as the process in which equivalent circuit parameters are acquired, processed, and curve-fit to their final functions.

\subsubsection{Setup and Performing the Test}

In order to obtain the equivalent circuit parameters, a battery testing platform was designed that implements a high-powered $3.3 \Omega$ resistive load bank for discharging, and a BatterySpace KP4818C Universal Smart Charger for charging currents (Figure 7.3) [146]. 
During the test, the discharge current magnitude varied between $15.5 \mathrm{~A}(0.74 \mathrm{C})$ at $100 \%$ SoC to $13.1 \mathrm{~A}(0.62 \mathrm{C})$ at $0 \%$ SoC. During charging, a constant current of $18 \mathrm{~A}(0.85 \mathrm{C})$ was held until entering constant voltage mode, where the charger reduced to $1.1 \mathrm{~A}(0.05 \mathrm{C})$ as it approached 100\% SoC. Hall Effect current and voltage transducers were implemented and calibrated to handle the maximum battery operating range between $48.0 \mathrm{~V}$ and $58.5 \mathrm{~V}$ [62],[63].

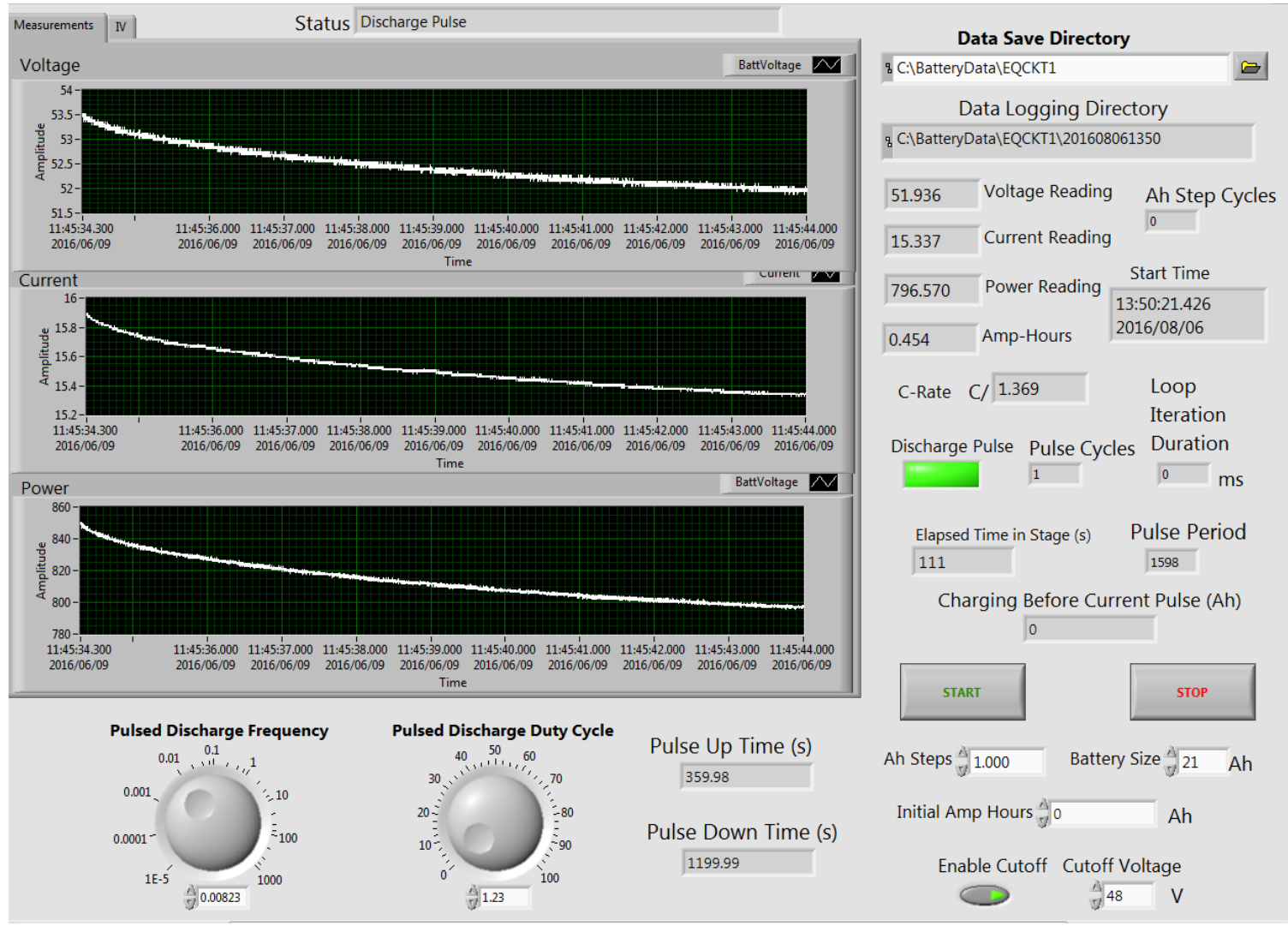

Figure 7.4. LabVIEW Battery Test Front Panel.

A NI PCI-6071E DAQ card was used in conjunction with LabVIEW to implement an automated pulsed and constant charging and discharging system. Custom LabVIEW software was developed to automatically administer the test pulses while logging data. To limit the duration of the testing period, constant charge and discharge phases are observed 
before pulses are administered, where two sequential pulses were run to reduce the possibility of an anomaly. These phases are measured based on the user setting of a number of Ah that will be charged or discharged prior to another set of pulses.

A sample of the LabVIEW front panel during discharging is shown in Figure 7.4. Following user input of the "Data Save Directory," the pulsed discharge frequency and duty cycle is set. The "Ah Step" setting allows the user to designate the amount of energy to be charged or discharged when observing a constant charge or discharge phase. A value of 0 Ah would simply continuously administer pulses. Although this would drastically improve the accuracy of the final curve-fit to SoC procedure, it significantly increases the testing period. An additional indicator entitled "Discharging Before Current Pulses" counts down the remaining energy to be charged or discharged before the next set. To achieve a reasonable balance of test duration to accuracy for this 21 Ah battery, 1 Ah of charging or discharging is observed, generating minimum SoC steps of $4.7 \%$. Although data was logged at all times, only $1 \mathrm{~Hz}$ sampling was observed over the constant loading and charging periods, increasing to $1 \mathrm{kHz}$ during the pulse testing periods.

\subsubsection{Extracting Circuit Parameters and Open Circuit Voltage}

Test data was then analyzed for each pulse to extract each equivalent circuit parameter. The process adhered to is discussed in Reference [143], where the dynamic behavior during and after the pulse is used to extract each parameter. Consider the voltage waveform shown in Figure 7.5, where the initial voltage $V_{i}$ is placed under a pulsed loading or charging current $\pm \mathrm{I}_{\mathrm{p}}$ for $\Delta t_{\text {on }}$ followed by a rest period of $\Delta t_{o f f}$. The duration of both periods are tuned based on whether the user is obtaining the long (min) or short (s) time constant. 
Three simple relations are made to interpret the battery response during and following the pulse in terms of ohmic and resistor-capacitor (RC) impulse parameters. The experimental ohmic resistance $R_{0_{\text {exp }}}$ can be acquired by measuring the terminal voltage drop between the end of the pulse $V_{p}$ and after $V_{a p}$ with respect to current $I_{P}$.

$$
R_{0_{e x p}}=\frac{\left|V_{a p}-V_{p}\right|}{\left|I_{p}\right|}
$$

$\mathrm{RC}$ parameters are obtained by a similar procedure, measuring the voltage drop between the final voltage $V_{f}$ and $V_{a p}$ following $I_{p}$.

$$
R_{t_{\text {exp }}}=\frac{\left|V_{f}-V_{a p}\right|}{\left|I_{p}\right|} ; \quad C_{t_{\text {exp }}}=\frac{\tau_{\text {exp }}}{R_{t_{\text {exp }}}}
$$

In addition to identifying precise values of $V_{a p}, V_{p}$ and $V_{f}, \tau_{\text {exp }}$ is extrapolated from by exponential curve-fitting. These values are then captured for all long and short charging and discharging pulses, along with the initial battery $\mathrm{SoC}$ reference at $V_{i}$.

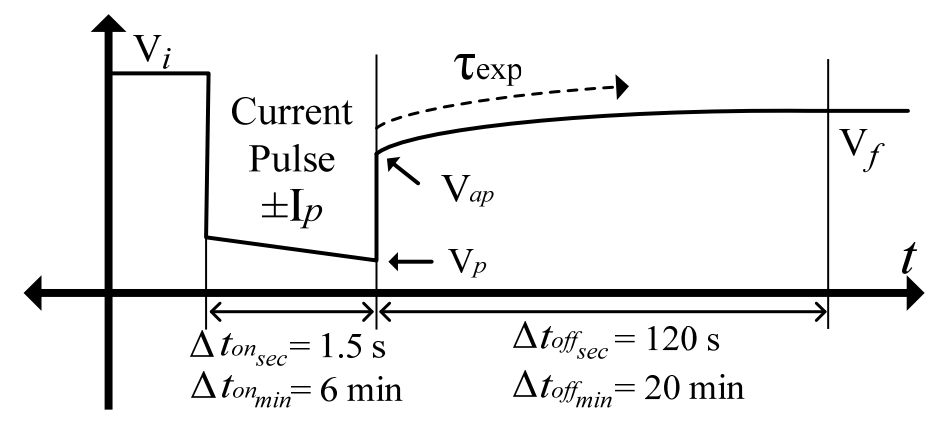

Figure 7.5. Dynamic Behavior of Battery Voltage under Current Pulse.

\subsubsection{Curve Fitting}

Following the calculation and extraction of values for $R_{0_{\text {exp }}}, R_{t_{\text {exp }}}$ and $C_{t_{\text {exp }}}$ at every measured SoC level, the data was curve-fit. As expected, a comprehensive analysis 
revealed that multiple functions are suitable to fit each parameter. Thus, a focus was placed upon a balance between minimizing error and the function simplicity, while giving priority to functions that are suitable to model both the charging and discharging trends.

Shown in Figures 7.6 to 7.11 , the charging experimental points are depicted in blue, while discharging is depicted in red. Since the variance of $R_{0}$ is minimal between charging and discharging, the datasets were combined, and only a single trend exists between SoC and $R_{0}$. The closest fit to the progression observed in Figure 7.6 was consistent with a $4^{\text {th }}$ Order polynomial:

$$
R_{0}(S O C)=a_{1} S O C^{4}+a_{2} S O C^{3}+a_{3} S O C^{2}+a_{4} S O C+a_{5}
$$

where $a_{1}-a_{5}$ represent each of the extracted coefficients, which are shown in Table 7-1.

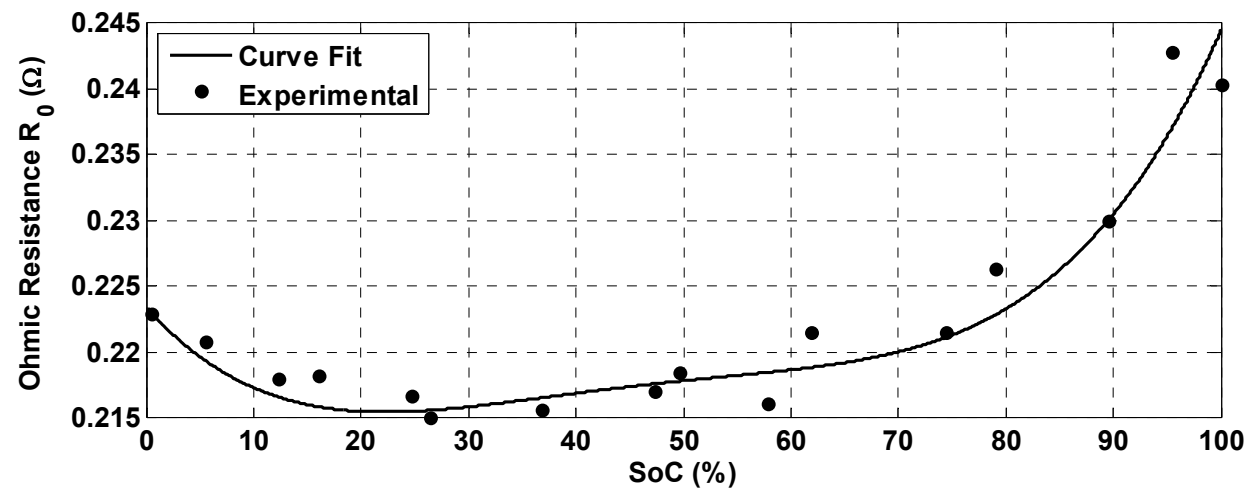

Figure 7.6. Ohmic Resistance Function Curve Fit for Charging and Discharging.

For all remaining functions, different coefficients are required when operating in charging or discharging mode. For the $\mathrm{OCV}$, a complex SoC-voltage relation justifies the need for a $5^{\text {th }}$-Order polynomial, as shown in Figure 7.7:

$$
V_{o c}(S O C)=a_{6 x} S O C^{5}+a_{7 x} S O C^{4}+a_{8 x} S O C^{3}+a_{9 x} S O C^{2}+a_{10 x} S O C+a_{11 x}
$$

where $a_{6 x}-a_{11 x}$ represent the coefficients and the mode is denoted by the $x$ subscript shown in Table 7-1, utilizing $d$ for discharging and $c$ for charging, respectively. 


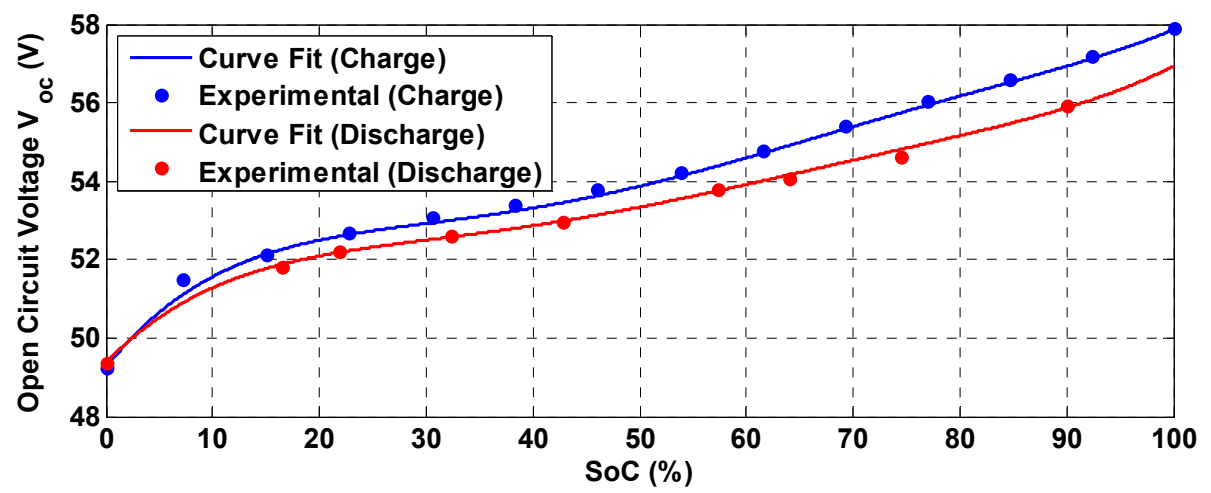

Figure 7.7. Open Circuit Voltage Curve Fit for Charging and Discharging.

Table 7-1. PL8048168 51.8V Lithium Ion Cobalt Polymer Battery Coefficients

\begin{tabular}{c|c|c|c|c|c}
\hline \hline $\begin{array}{c}\text { Coefficient } \\
a_{n x}\end{array}$ & $\begin{array}{c}\text { Charge } \\
a_{n c}\end{array}$ & $\begin{array}{c}\text { Discharge } \\
a_{n d}\end{array}$ & $\begin{array}{c}\text { Coefficient } \\
a_{n x}\end{array}$ & $\begin{array}{c}\text { Charge } \\
a_{n c}\end{array}$ & $\begin{array}{c}\text { Discharge } \\
a_{n d}\end{array}$ \\
\hline$a_{1}$ & \multicolumn{2}{|c|}{0.29500} & $a_{15 x}$ & 0.01895 & 0.02218 \\
\hline$a_{2}$ & \multicolumn{2}{|c|}{-0.53990} & $a_{16 x}$ & -69.94 & 63.22 \\
\hline$a_{3}$ & \multicolumn{2}{|c|}{0.35810} & $a_{17 x}$ & 121 & -113.6 \\
\hline$a_{4}$ & -0.09226 & $a_{18 x}$ & -81.3 & 66 \\
\hline$a_{5}$ & 0.22350 & $a_{19 x}$ & 40.12 & 15.64 \\
\hline$a_{6 x}$ & 78.99 & 70.98 & $a_{20 x}$ & 0.3397 & -0.1734 \\
\hline$a_{7 x}$ & -237.5 & -202.2 & $a_{21 x}$ & -0.4096 & 0.3648 \\
\hline$a_{8 x}$ & 266.3 & 218.2 & $a_{22 x}$ & 0.1516 & -0.1666 \\
\hline$a_{9 x}$ & -133.2 & -107.3 & $a_{23 x}$ & 0.05377 & 0.09134 \\
\hline$a_{10 x}$ & 34.03 & 27.89 & $a_{24 x}$ & $2.184 \mathrm{e}-6$ & 720.8 \\
\hline$a_{11 x}$ & 49.24 & 49.36 & $a_{25 x}$ & 19.25 & 0.6925 \\
\hline$a_{12 x}$ & 0.01715 & -0.04911 & $a_{26 x}$ & 928.7 & 419.6 \\
\hline$a_{13 x}$ & 0.01964 & 0.09523 & $a_{27 x}$ & 0.5496 & -2.012 \\
\hline$a_{14 x}$ & -0.0251 & -0.05824 & - & - & - \\
\hline
\end{tabular}

For impulse parameters in the seconds range, both resistance and capacitance trends are modeled using $3^{\text {rd }}$-Order polynomials shown in Figure 7.8 and Figure 7.9:

$$
\begin{aligned}
& R_{\text {sec }}(S O C)=a_{12 x} S O C^{3}+a_{13 x} S O C^{2}+a_{14 x} S O C+a_{15 x} \\
& C_{\mathrm{sec}}(S O C)=a_{16 x} S O C^{3}+a_{17 x} S O C^{2}+a_{18 x} S O C+a_{19 x}
\end{aligned}
$$

where $a_{12 x}-a_{15 x}$ represent the coefficients required to obtain $R_{s e c}$ and $a_{16 x}-a_{19 x}$ to obtain $C_{\text {sec }}$. 


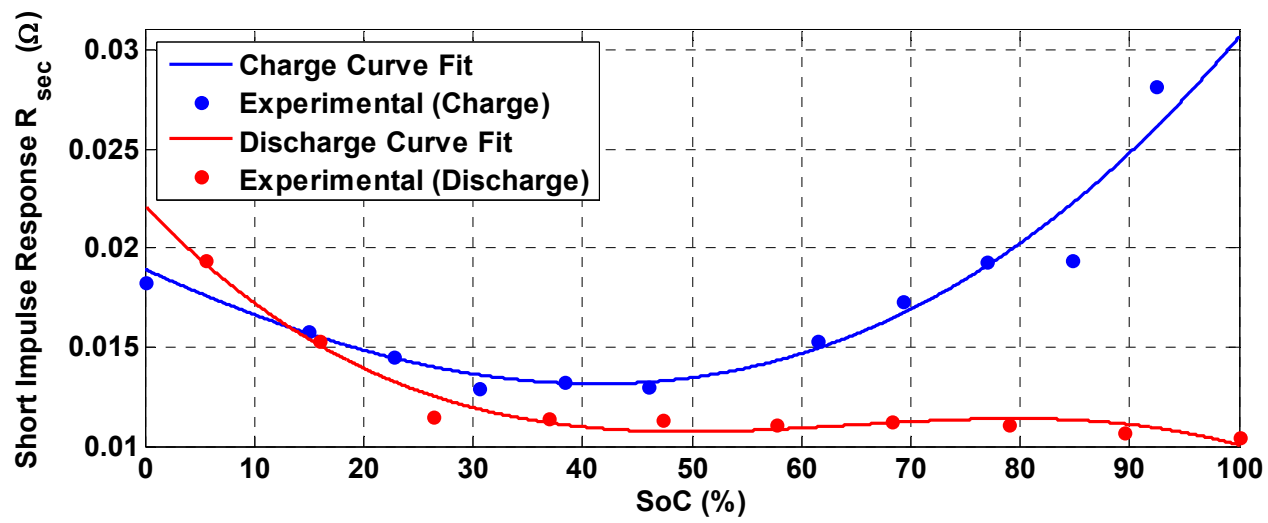

Figure 7.8. Curve-Fitted Polarization Resistance Parameters under Seconds Timespan for Charging and Discharging.

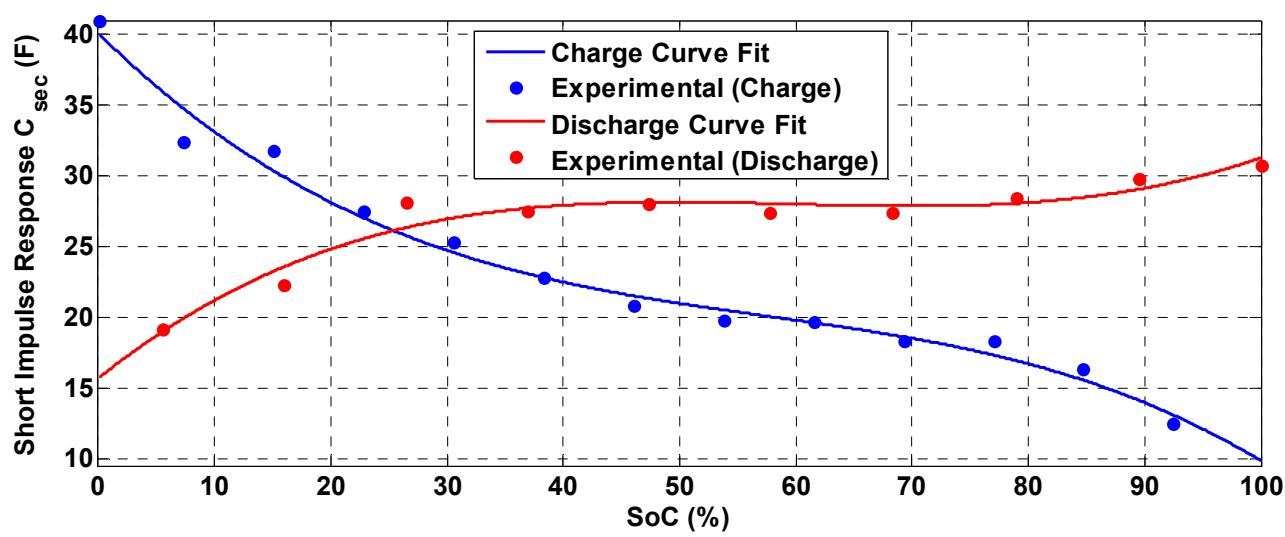

Figure 7.9. Curve-Fitted Polarization Capacitance Parameters under Seconds Timespan for Charging and Discharging.

For impulse parameters in the minutes-range, the resistance $R_{\min }$ shown in Figure 7.10 can be accurately depicted through a $3^{\text {rd }}$-Order polynomial:

$$
R_{\min }(S O C)=a_{20 x} S O C^{3}+a_{21 x} S O C^{2}+a_{22 x} S O C+a_{23 x}
$$

where $a_{20 x}-a_{23 x}$ are the coefficients. However, some complexity in the capacitance trend required a more complex fit, which utilized a 2-term exponential:

$$
C_{\min }(S O C)=a_{24 x} e^{a_{25 x}[S O C]}+a_{26 x} e^{a_{27 x}[S O C]}
$$

where $a_{24 x}-a_{27 x}$ are the final coefficients. The capacitance trend for the minutes impulse response is shown in Figure 7.11. 


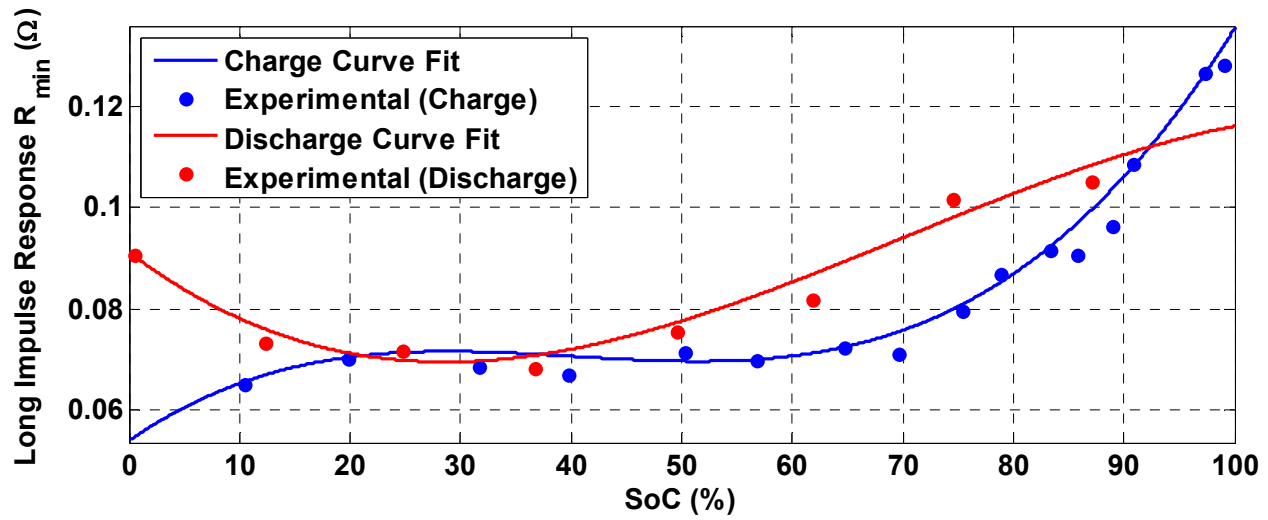

Figure 7.10. Curve-Fitted Polarization Resistance Parameters under Minutes Timespan for Charging and Discharging.

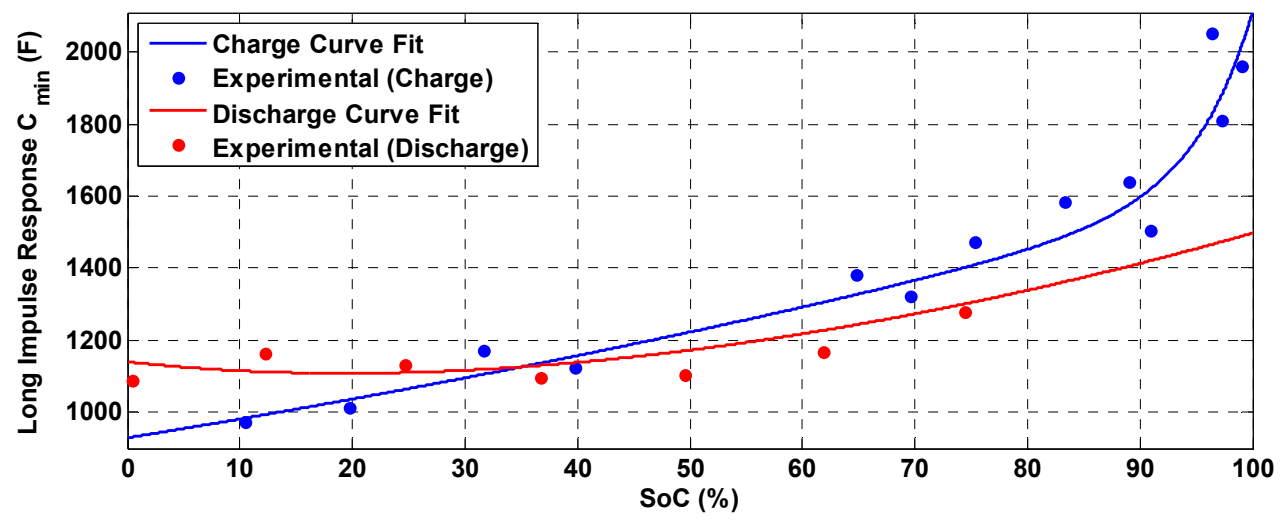

Figure 7.11. Curve-Fitted Polarization Capacitance Parameters under Minutes Timespan for Charging and Discharging.

\subsection{Implementation}

An implementation of the MATLAB/Simulink-based battery model is shown in Figure 7.12. The final model is broken into three major parts: 1) the MATLAB function code block, 2) the Simscape SimElectronics components, and 3) the SimPowerSystems interface. 


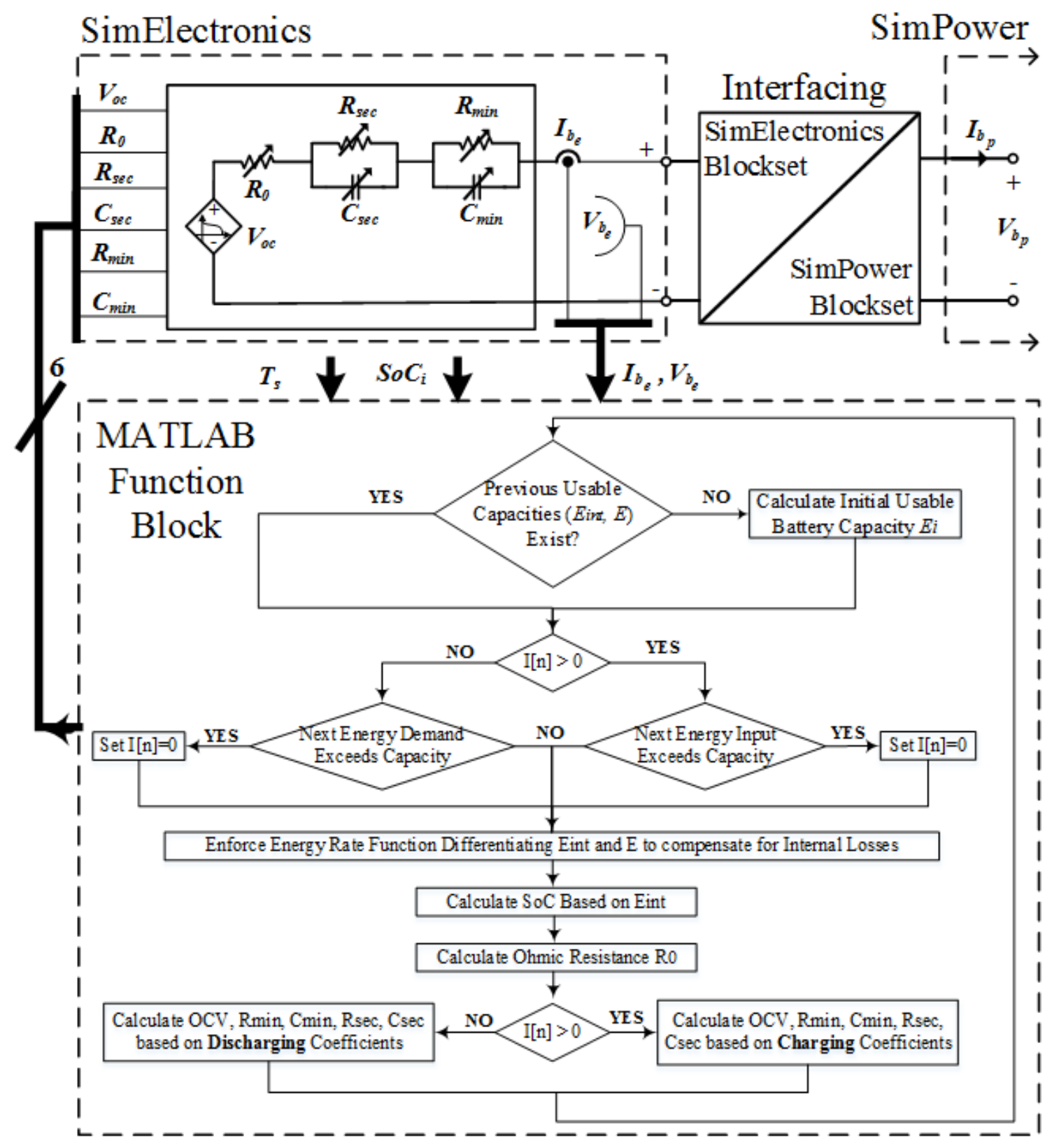

Figure 7.12. MATLAB/Simulink Model of the Battery Block Implementation.

\subsubsection{SimPowerSystems Interface}

SimPower was the most preferred environment for the final battery model, as it can easily interface with a wide range of power and energy components, motor drives, and power electronic devices to conduct an EV simulation. Furthermore, the final block was 
intended to be a drop-in replacement to the battery model already available within the SimPower block set. Unfortunately, SimPower does not feature variable resistors and capacitors, thus the primary battery equivalent circuit had to be designed within Simscape SimElectronics and conditioned to work seamlessly on the SimPower side using a Simscape to SimPowerSystems Interface block, as shown to the right in Figure 7.12. The final model is packaged as a SimPower block.

\subsubsection{Simscape SimElectronics}

In order to implement the dynamic circuit components in the battery equivalent model, a Simscape SimElectronics block set was utilized as variable resistor and capacitor components were available. From the MATLAB Function block, the OCV $V_{o c}$ and values for all remaining components $\left(R_{s e c}, C_{s e c}, R_{\min }, C_{\min }\right)$ are passed to each of the elements, as shown in Figure 7.12. SimElectronics components (e) require their own current $I_{b_{e}}$ and voltage sensors $V_{b_{e}}$, which are interpreted and fed back to the MATLAB function block to keep track of the SoC and battery terminal voltage. Similarly, the voltage $V_{b_{p}}$ and current $I_{b_{p}}$ measurements native to the SimPower block set $(p)$ are shown off to the right in Figure 7.12, which are fed from the final SimPower block.

\subsubsection{MATLAB Function}

A MATLAB function block was needed to apply initial conditions, implement all equations, and keep track of the current energy and SoC of the battery. Prior to the calculation of SoC and component values within the equivalent circuit, the MATLAB function block requires the user to provide the desired discrete solver step time $T_{s}$ in seconds, the battery capacity $E_{c a p}$ in Ah, and the initial SoC $S O C_{i}$ in decimal form. Since 
$\mathrm{T}_{\mathrm{S}}$ must be synchronized with both SimScape and SimPower components, this value is fed to both independent solvers.

A flow chart representing the MATLAB function is shown in Figure 7.12. Using $S O C_{i}$, the initial usable capacity $E_{i}$ is calculated based on the specified initial SoC.

$$
E_{i}=E_{c a p} S O C_{i}
$$

Based on the current requested or injected at the battery terminals at each sample $\mathrm{n}$, some constraints are applied. If the battery is approaching full capacity and the upcoming sample will exceed $E_{c a p}, \mathrm{I}[\mathrm{n}]$ is replaced by 0 to prevent the battery from an overcharge. Similarly, in the case that the battery is approaching full discharge and the upcoming sample could risk an over discharge, $\mathrm{I}[\mathrm{n}]$ is once again replaced by 0 . Next, a primary battery energy (capacity) tracking function is defined, representing the energy delivered to the load or sourced from a charger. The discrete time energy function $E[\mathrm{n}]$ applies current summation with respect to the designated sample step $\mathrm{T}_{\mathrm{s}}$ :

$$
E[\mathrm{n}]=E[\mathrm{n}-1]+\frac{\mathrm{I}[\mathrm{n}] \mathrm{T}_{\mathrm{s}}}{3600}
$$

where $E[\mathrm{n}]$ is tracked in $\mathrm{Ah}$.

Although $E[\mathrm{n}]$ represents the energy sourced from or provided to the user, it is not sufficient to explain the total energy expended internally to the battery. For all batteries, the available usable capacity will vary based on the C-rate applied. At low discharge currents, a full output equal to $E_{c a p}$ is expected, assuming the battery is in relatively good health. However, as the current approaches high levels of $1 \mathrm{C}$ and beyond, a notable difference is observed between the nameplate capacity and the amount of energy available to the load as a result of internal heat losses. To model this phenomenon, full discharges 
were performed at a wide range of C-rates, from $0.05 \mathrm{C}$ to $1 \mathrm{C}$, where the resulting energy output was fit to the following linear function that introduces two new variables. First, an energy rate adjustment $E_{\text {rate }}$ is defined, which represents an offset in the total energy to be expected from the battery given the current C-rate $C_{\text {rate }}[\mathrm{n}]$ applied.

$$
E_{\text {rate }}[\mathrm{n}]=1.019-0.3109 C_{\text {rate }}[\mathrm{n}]
$$

Next, the energy rate is applied to an alternative internal energy function $E_{\text {int }}$ that takes these losses into account. Here, $E_{\text {int }}$ represents a fictitious capacity from the point of view of the battery, which may or may not represent all energy that the user has received. To implement $E_{\text {rate }}$, an adjustment is made to the applied current I[n] in Equation (7-11), which is scaled by $E_{\text {rate }}$ and stored in Ah.

$$
E_{\text {int }}[\mathrm{n}]=E_{\text {int }}[\mathrm{n}-1]+\frac{\mathrm{I}[\mathrm{n}] \mathrm{T}_{\mathrm{s}}}{3600 E_{\text {rate }}[\mathrm{n}]}
$$

The importance of $E_{\text {int }}$ resides in its ability to represent the true SoC level, regardless of the operating conditions. The $\mathrm{SoC}$ is then calculated by the following equation:

$$
\operatorname{SoC}[\mathrm{n}]=\frac{E_{\text {int }}[\mathrm{n}]}{E_{c a p}}
$$

Finally, all remaining parameters are calculated based on the current $\mathrm{SoC} \operatorname{SoC}[\mathrm{n}]$. The OCV $V_{o c}$ and remaining components $\left(R_{s e c}, C_{s e c}, R_{\min }, C_{\min }\right)$ are also dependent on whether they are in charging or discharging operation.

\subsubsection{Final MATLAB/Simulink Implementation}

A view of the MATLAB/Simulink schematic is pictured in Figure 7.13. The SimPower block provided to the user is shown to the left, where the only required input is the initial SoC. Positive (BATT+) and negative (BATT-) output terminals are accompanied by a 
Measurements \& Coefficients output bus that contains all internal operating variables, components values, and measurements. The inside of this block is shown to the right in the Simulink diagram. At the top, the timing reference, the previous current reading, and initial SoC are passed to the MATLAB function block. The output bus from the function block provides all Measurements \& Coefficients values, which are passed to the 2nd-Order Dynamic Randles equivalent circuit shown below. Below, to the left, the SimScape solver configuration is connected to a voltage source and all passive components. To the right, a conversion from the Simscape to SimPowerSystems Interface connects the block to the load or charger.

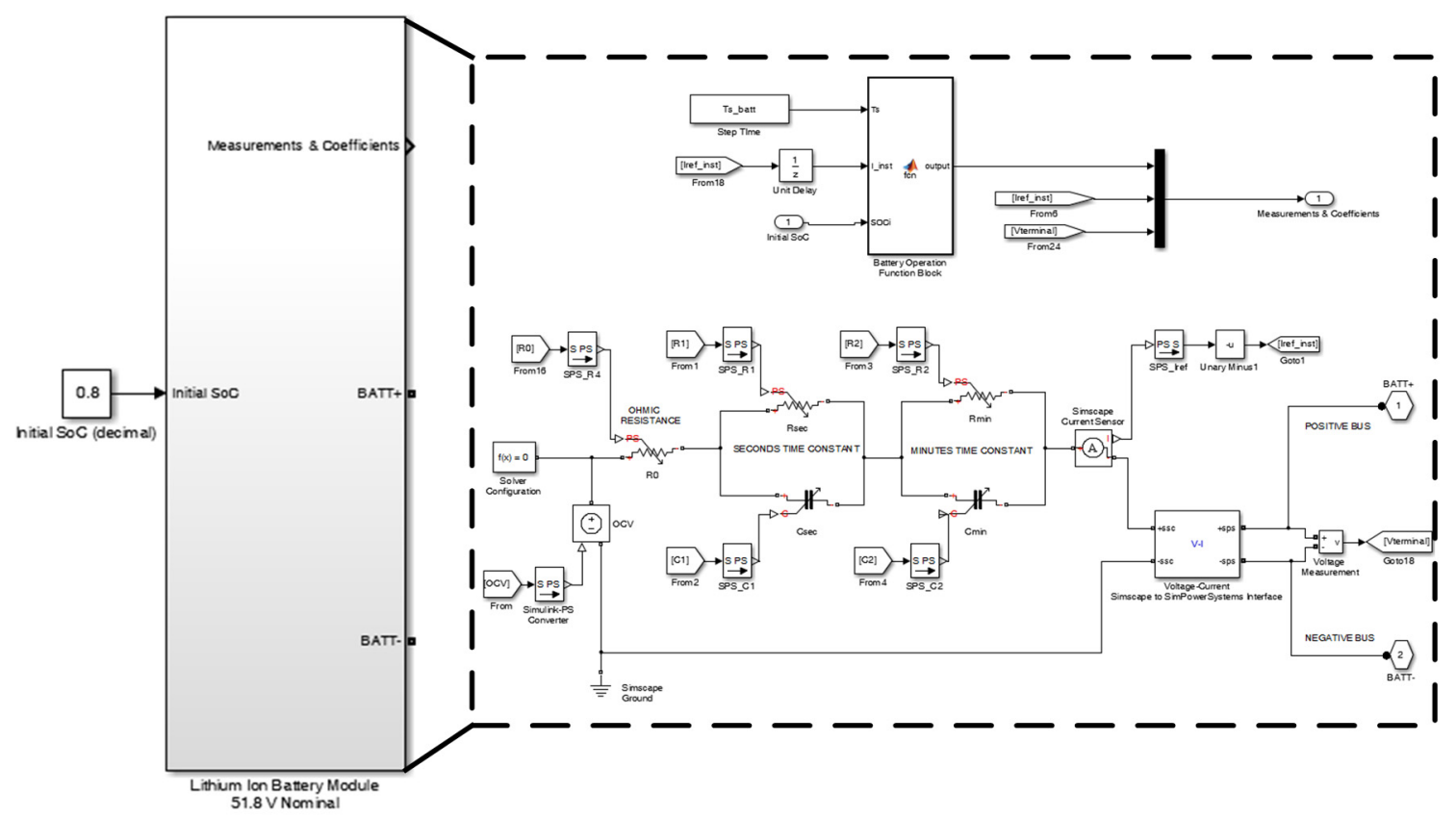

Figure 7.13. MATLAB/Simulink Dynamic Battery Model.

\subsection{Results and Comparisons}

To verify accuracy of the final battery equivalent model, the same $1.5 \mathrm{~s}$ and $6 \mathrm{~min}$ charge and discharge pulses conducted in the equivalent circuit acquisition were applied to 
the battery model at four different $\mathrm{SoC}$ levels across its operating range. Since the number of pulses taken experimentally were finite, the SoC test references were not the same for all four tests, but were targeted to fall within four important regions: near the practical SoC discharge cutoff for EVs (15-25\%), mid-range SoC (45-55\%), high SoC (60-70\%), and near the practical full charge SoC for EVs (75-90\%). The results from each comparison are shown in Figures 7.14 to 7.17.

Figure 7.14 and Figure 7.15 depict the voltage response during and following discharge current pulses of $0.74 \mathrm{C}$, where the measured values at multiple SoC levels are shown in multiple colors compared to the simulation in dashed lines. For the short discharge pulse pictured in Figure 7.14, the measured and simulated voltage responses are very close, confirming the battery model is able to accurately capture high-frequency (sub-seconds range) recovery components of the battery. The long discharge pulse comparison is pictured in Figure 7.15, revealing a close agreement with both ohmic and impulse response components. The most variation was observed at low (22\%) SoC and high SoC (84\%) levels.

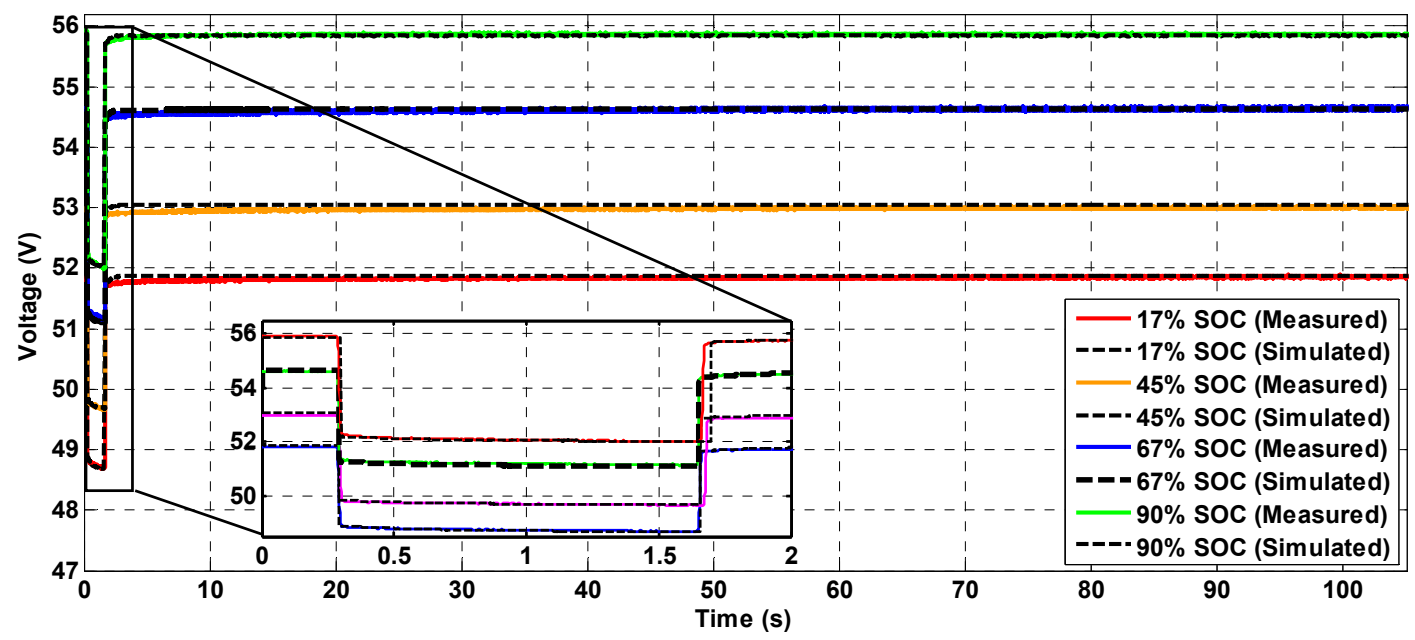

Figure 7.14. Battery Model Impulse Response Verification under Short Discharge Pulse. 


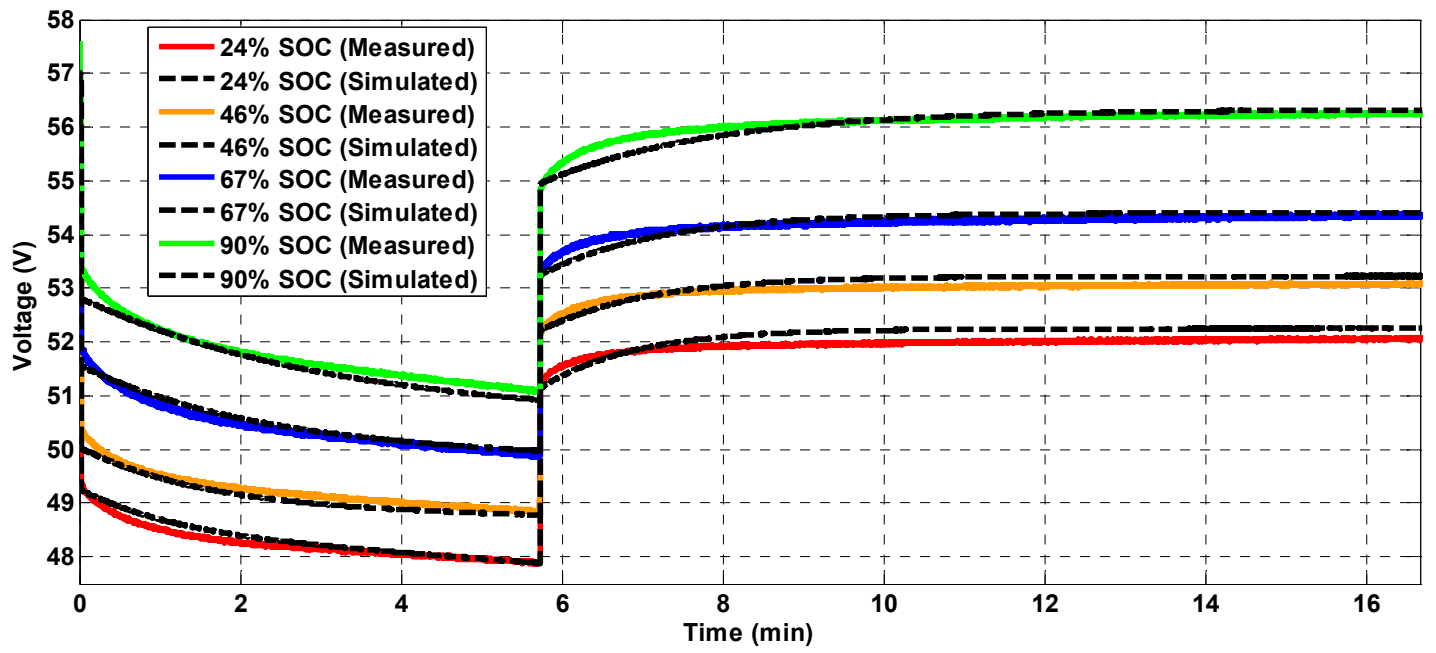

Figure 7.15. Battery Model Impulse Response Verification under Long Discharge Pulse.

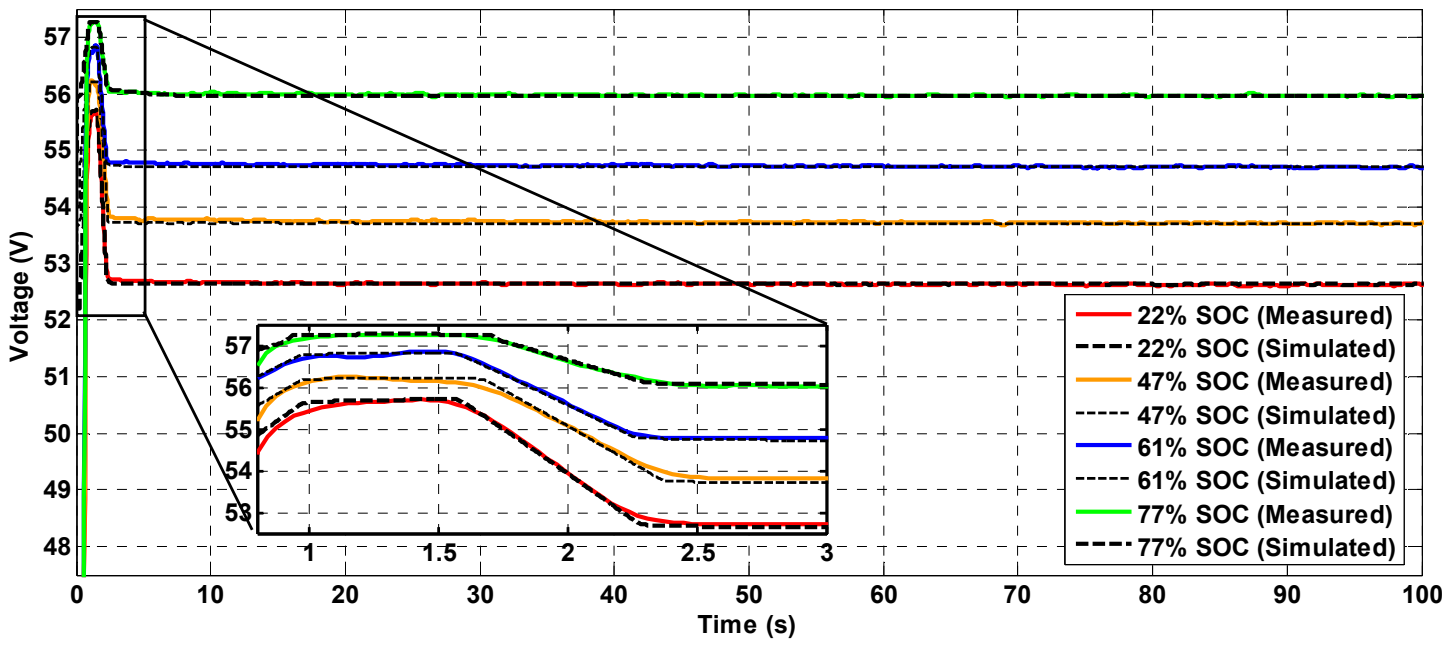

Figure 7.16. Battery Model Impulse Response Verification under Short Charge Pulse.

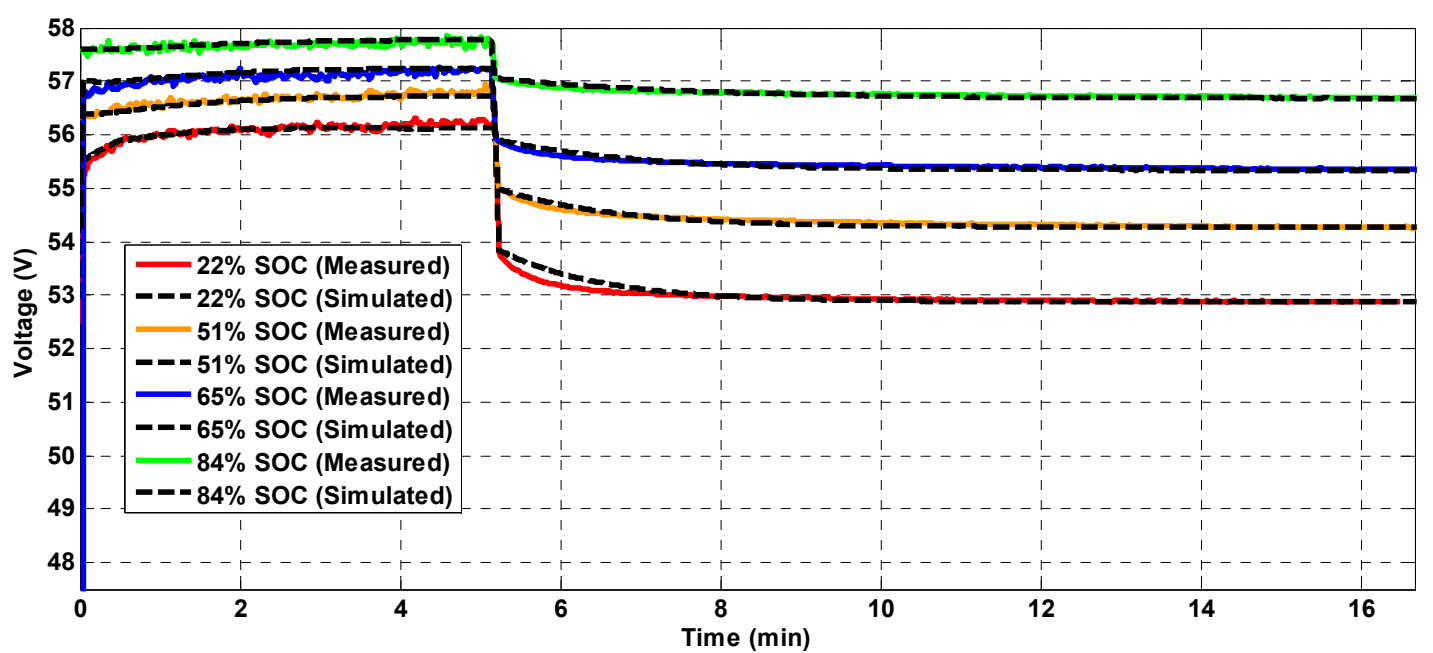

Figure 7.17. Battery Model Impulse Response Verification under Long Charge Pulse. 
The charging current pulses are shown in Figure 7.16 and Figure 7.17, where a maximum charging current of $0.85 \mathrm{C}$ is imposed. As a result of a constant voltage charging transition at high SoC levels, the test current is slightly reduced near the practical full charge values. The short pulse is shown in Figure 7.16, once again indicating a close correlation of charging performance in the sub-second response range. The long charge pulse response is shown in Figure 7.17. The model reveals a closer correlation to experimental values than the discharging pulses. At medium (51\%) to high (84\%) SoC levels, both the ohmic and impulse components show a close correlation between measured and simulated responses. The largest drift is seen in the impulse components at the low $(22 \%)$ SoC level. However, it is important to note that in a practical application, this is near the minimum operating SoC level.

\subsection{Modeling an Electric Vehicle Battery and Wireless Power Transfer System}

In this section, this battery model will be applied to an advanced EV propulsion and wireless charging system to conduct a feasibility study on a wireless charging technique [148]. Wireless charging can be classified into two distinct modes: stationary and dynamic, where the Society of Automotive Engineers (SAE) has designated four standardized charging levels in the new SAE J2954 standard [149]. In both modes, a primary coil is implanted below the vehicle. In stationary mode, energy is transferred to a parked vehicle using a single coil buried under the road surface, while in dynamic mode, energy is transferred to a moving vehicle using a series of buried coils. Dynamic charging would be particularly useful for highway driving, where an EV can operate and charge while making minimal stops. However, controlled charging while operating at high speed remains a challenge. Furthermore, a wide-scale implementation of travel lanes with millions of 
charging coils in the road would be costly. A proposal of this nature could prove to be impractical.

An intermediate solution between stationary and dynamic charging has been explored in this section, which falls into the classification of a quasi-dynamic wireless power transfer (WPT) system. Quasi-dynamic WPT (QDWPT) could provide a balance between the infrastructure cost, while still enabling a majority of the same advantages dynamic charging has to offer. Using QDWPT, an EV could charge during transient stops on the city roads, such as bus stops for electric buses, and traffic signals for EVs. The comprehensive $2^{\text {nd }}-$ Order dynamic battery model developed in this chapter has been utilized to provide an initial feasibility analysis for the future deployment of this concept.

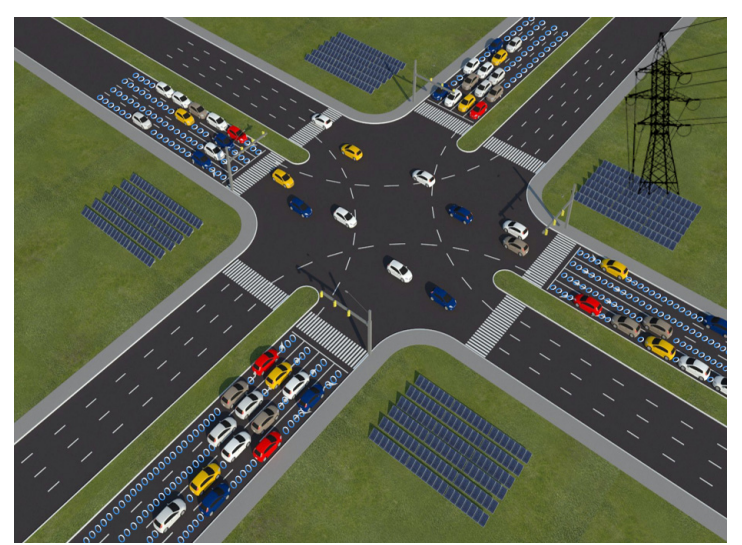

Figure 7.18. Proposed Wireless Power Transfer Traffic Intersection Architecture.

A conceptual drawing is shown in Figure 7.18, where a primary string of wireless pads are placed beneath the pavement in each travel lane at each direction of the intersection, and are depicted in blue. To determine the optimal number of WPT coils to support each lane, a traffic flow analysis can be conducted to define the minimum coverage distance. In this system, it is assumed that over the course of a full traffic light cycle across all directions, wireless coils are available for all stopped traffic (when under a red signal). 


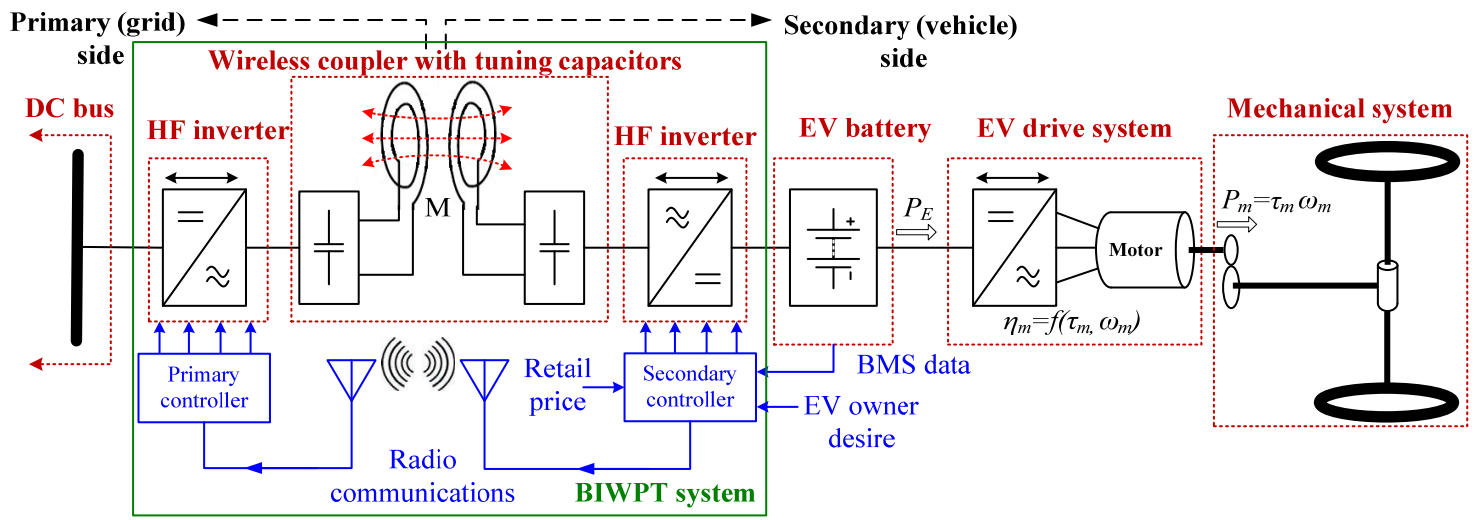

Figure 7.19. Block Diagram of an Electric Vehicle with a Wireless Power Transfer Network.

\subsubsection{System Modeling}

A block diagram of the wirelessly connected EV network is shown in Figure 7.19. The system consists of three main parts: the WPT system (WPTS), the EV battery, and the EV drive system. The WPTS has two isolated sides: the grid and vehicle. The power flow between these sides is managed by a secondary controller. The modeling of each part of the network is described in the following subsections.

\subsubsection{Configuration of the Electric Vehicle Battery Bank}

Present-day EVs are subjected to heavy power and energy demands that are not limited to high transient discharge currents, but also sporadic regenerative braking charge currents. Furthermore, with the inclusion of QDWPT, a battery model accounting for multiple timeconstants is needed to accurately depict the dynamic response of the battery system. For this, the $2^{\text {nd }}$-Order Randles dynamic equivalent circuit model developed in this chapter was utilized. To reach the specifications of a standard EV battery pack voltage and capacity, seven modules were placed in series and three in parallel to reach a nominal voltage of 362.6 V and 63 Ah, respectively. Figure 7.20 depicts a modified version of the equivalent 
circuit model, where a general block diagram indicates how the EV battery model is connected to the traction system driven by a reference speed $v(t)$ from a driving profile. The electric motor loading and regenerative braking power $\left(P_{E}\right)$ is calculated and divided by $V_{b}$ to generate the reference current $I_{b, r e f}$ to the battery bank.

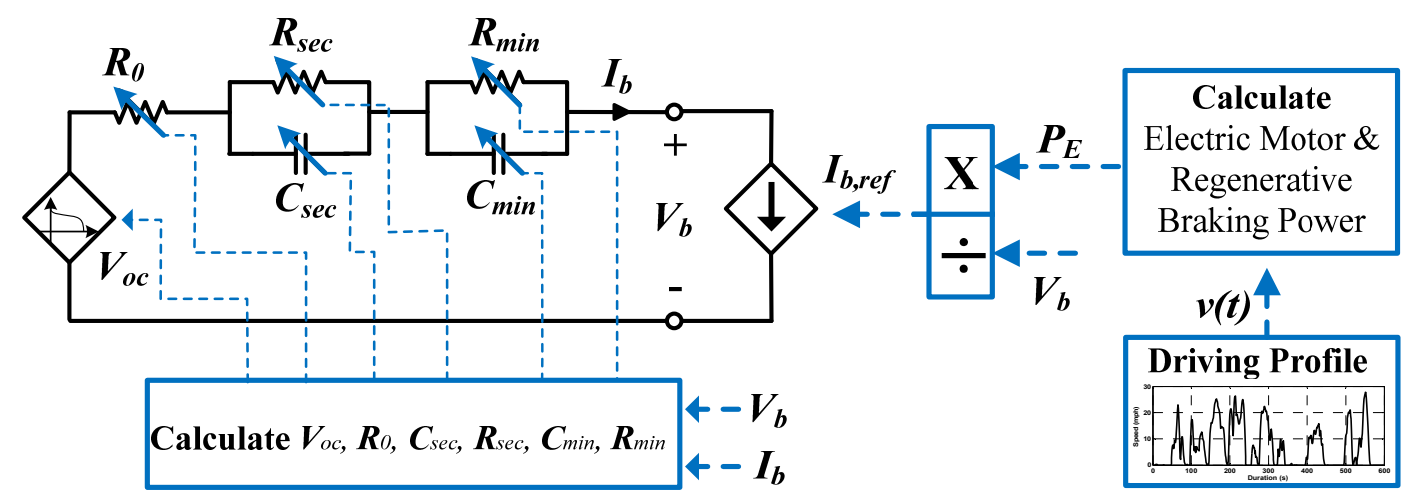

Figure 7.20. Dynamic Battery Equivalent Circuit Model Integration with Electric Vehicle System.
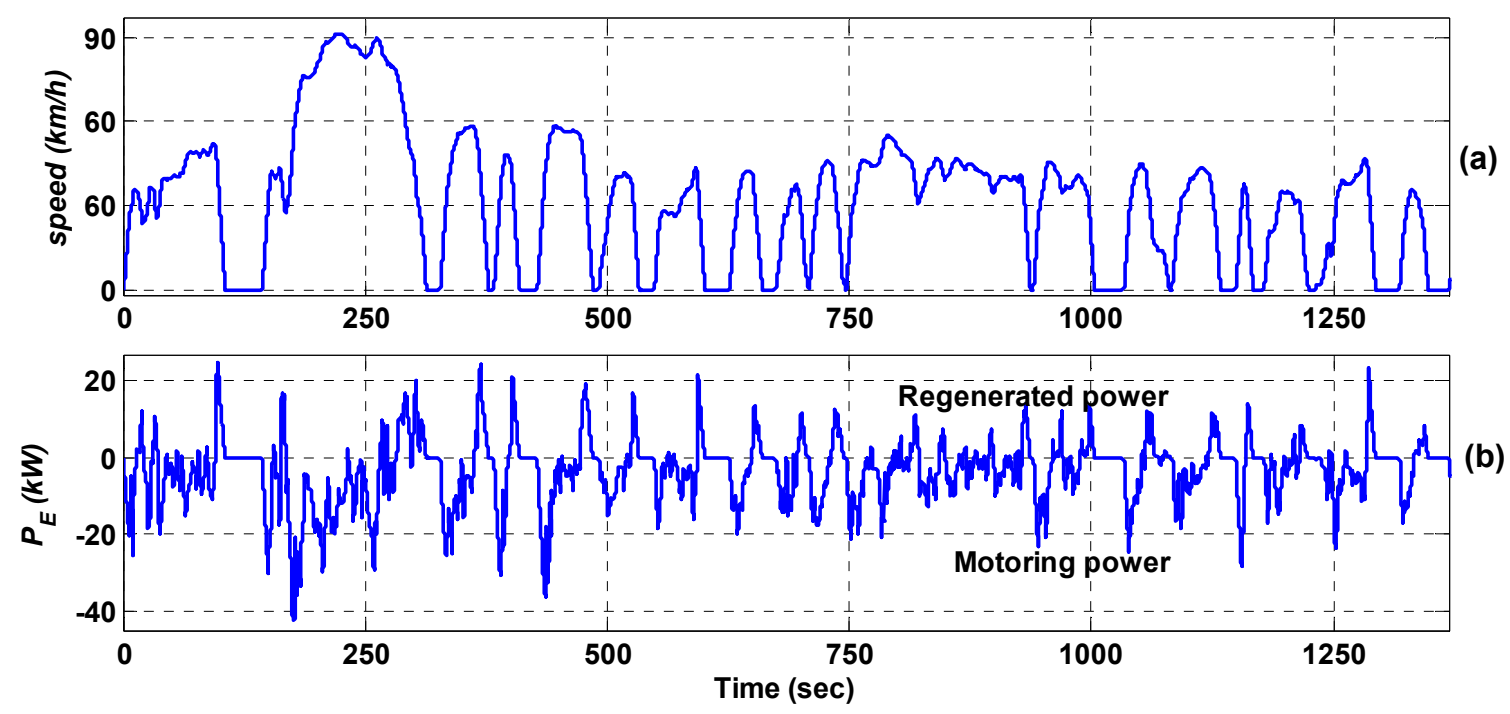

Figure 7.21. FTP-72 Driving Profile: (a) Speed (b) Electric Power.

The EV chosen in this study aligns with provisions published by the US National Highway Traffic Safety Administration (NHTSA), classified as a heavy passenger car similar to small-sized sedan with a curb weight of $1680 \mathrm{~kg}$ [150]. The Federal Test 
Procedure-72 (FTP-72) dynamometer driving profile was chosen, which represents typical city driving test conditions [151]. A $12 \mathrm{~km}$ commute is run over an approximately 22minute period at an average speed of $31.5 \mathrm{~km} / \mathrm{h}$ and is shown in Figure $7.21(\mathrm{a})$. FTP-72 consists of two phases: 1) a 505-second "cold start cycle," taking the vehicle up to 91.2 $\mathrm{km} / \mathrm{h}$, and 2) an 867-second "transient" phase representing stop-and-go city driving. Phase 1 presents the greatest demand on the battery pack in terms of power and energy output, as the high-speed driving portion reduces the availability of QDWPT. Phase 2 subjects the EV to frequent stop-and-go conditions at traffic signals, where WPT will be initiated. The FTP-72 speed profile is passed to an EV powertrain model.

\subsubsection{Electric Vehicle Powertrain Model}

The EV under test is modeled to include both drive power applied to the motor and regenerative braking recovery power. The resistance force $F_{v}$ of the $\mathrm{EV}$ at speed $v(t)$ is calculated by the summation of aerodynamic drag, rolling resistance, and grading resistance at an angle $\vartheta[152]$ :

$$
F_{v}(v(t))=\frac{1}{2} \rho_{a} c_{D} A_{f} v(t)^{2}+F_{R}(v(t))+g m_{t} \sin \vartheta
$$

where $\rho_{a}, c_{D}, A_{f}$, and $m_{t}$ represent the air density $\left(1.205 \mathrm{~kg} / \mathrm{m}^{3}\right)$, drag coefficient $(0.32)$, frontal EV area $\left(2.31 \mathrm{~m}^{2}\right)$, and vehicle mass, respectively. The rolling resistance function $F_{R}(v(t))$ can be found in detail in Reference [152]. Wheel resistance and dynamic torque for acceleration are passed through the gearbox $G_{r}(6.45)$ to calculate motor torque $\tau_{m}$ and speed $\omega_{m}$ :

$$
\tau_{m}(t)=\frac{r_{w h} F_{v}(t)}{G_{r}}+\frac{\theta_{v}}{G_{r} r_{w h}} \frac{d v(t)}{d t}
$$




$$
\omega_{m}(t)=v(t) / r_{w h} G_{r}
$$

where $r_{w h}$ and $\theta_{v}$ represent the radius of the wheels $(0.29 \mathrm{~m})$ and total vehicle inertia (145 $\left.\mathrm{kg} \cdot \mathrm{m}^{2}\right)$, respectively. The resulting battery power flow $\left(P_{E}\right)$ is then:

$$
P_{E}(t)= \begin{cases}\tau_{m} \omega_{m} / \eta_{m}(t), & \tau_{m}(t) \geq 0 \\ \tau_{m} \omega_{m} \eta_{m}(t), & \text { otherwise }\end{cases}
$$

where $0<\eta_{m}(t)<1$ is the motor-inverter efficiency, which is a function of the motor speed and torque interpolated from Reference [152]. The resulting motoring and regenerated power profile from the FTP-72 drive cycle is shown in Figure 7.21(b).

\subsubsection{Wireless Power Transfer System Model}

Typically, a WPTS consists of two sides: a primary (grid) and secondary (vehicle) side. The former is coupled with a DC bus and placed beneath the EV in the road, while the latter is attached to the EV battery and placed inside the vehicle. Each side consists of a high frequency (HF) inverter, controller, compensation circuit, and wireless pad [153]. The two sides are loosely coupled by magnetic induction via a large air gap $(100-250 \mathrm{~mm})$ according to the SAE J2954 standard [149]. During charging operation, the DC bus power is converted to HF AC (20-90 kHz) by the primary inverter to supply the primary pad. The primary power then moves by magnetic induction to the secondary pad through the air gap. The secondary power is rectified by another inverter to supply the EV battery.

Capacitor banks are essential to compensate the large reactive power required to magnetize the wide air gap. The power flow control in the system is achieved by controlling the switching of the two HF inverters based on the phase shift technique. Using this technique, the controllers adjust the phase shift between switching of each inverter leg. The power flow direction is controlled by the phase shift between the two inverter voltages 
[154]. The WPTS is also modeled in MATLAB/Simulink and linked to the battery and driving model to represent the entire performance of the wirelessly-connected EV.

\subsubsection{Testing the Quasi-Dynamic Wireless Charging System}

A large-scale model was used to evaluate the effectiveness of implementing this system at traffic signals. In this scenario, it is assumed that the EV charging will start automatically once the EV stops at the traffic signal. The charging power is kept fixed by the power flow controller of the WPTS. Four different standard charging levels are analyzed: WPT1=3.7 $\mathrm{kVA}$, WPT2 $=7.7 \mathrm{kVA}$, WPT3 $=11.1 \mathrm{kVA}$, and WPT4 $=22 \mathrm{kVA}$ based on the SAE J2954 standard. The EV is assumed to start driving from an initial $\mathrm{SoC}$ of $80 \%$, a reasonable maximum practical SoC for an EV. The driving performance during the WPT4 charging level is depicted in Figure 7.22. As can be observed from the driving profile reference in Figure 7.22(a), charging is initiated when the vehicle speed is $0 \mathrm{~km} / \mathrm{h}$. The terminal voltage of the battery is shown Figure 7.22(b), revealing both the high and low-frequency response components. The EV charging current and power is approximately $52 \mathrm{~A}(0.83 \mathrm{C})$ and 22 $\mathrm{kW}$, respectively. The final plot reveals the SoC progression throughout the driving cycle. Even with the limited availability of applying a charging current at the vehicle stops, the high charging current magnitude is able to recover a majority of the energy that was discharged over the drive cycle.

The same study was conducted for different standard charging levels, and the driving performance is compared with the case while there is no QDWPT charging, as shown in Figure 7.23. In this scenario, the initial SoC is assumed a little below the practical EV full charge at $70 \% \mathrm{SoC}$. This figure shows the EV battery SoC throughout the driving period, without and with implementing a WPT charger at the traffic signal. 

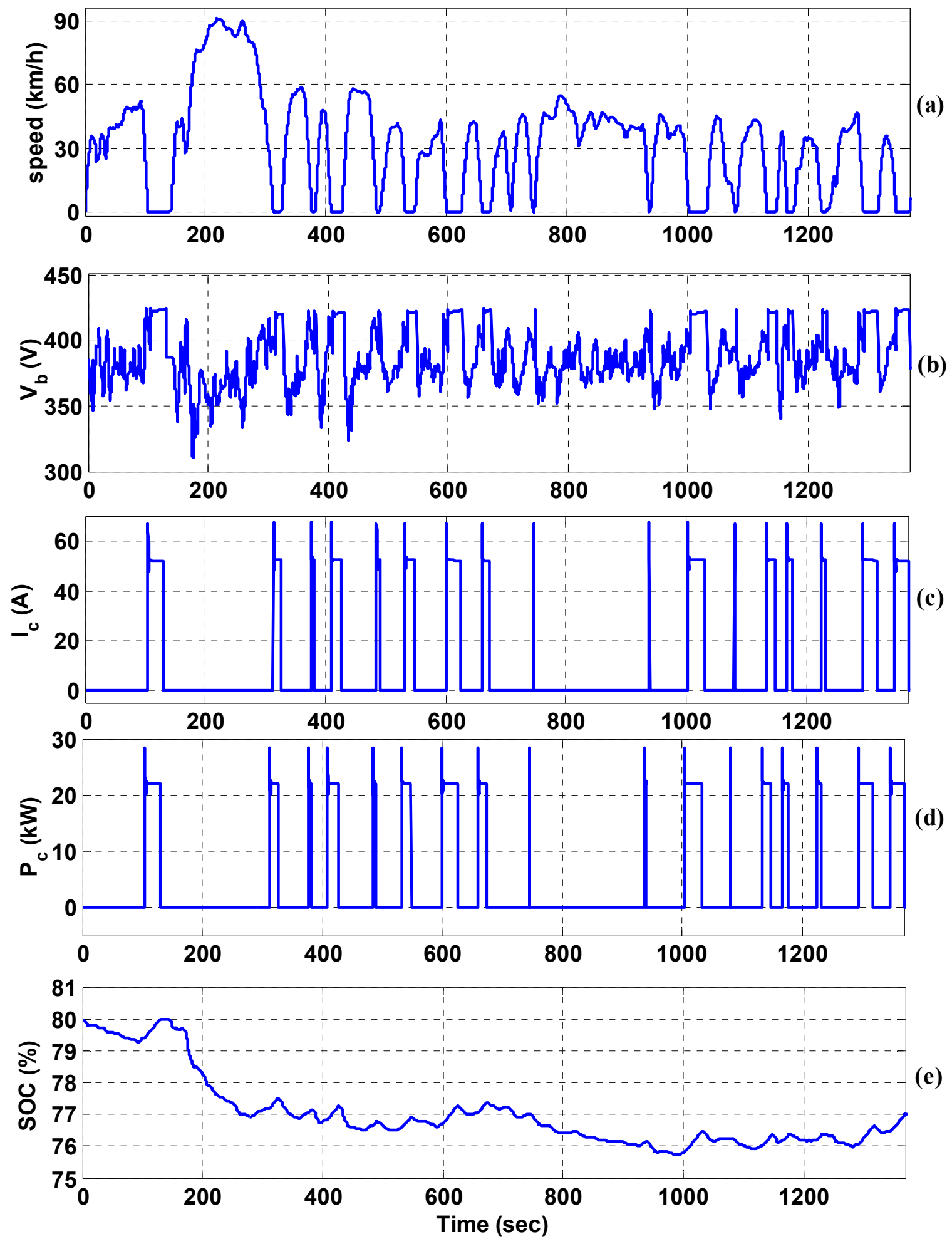

Figure 7.22. Driving Performance at Fixed Power Charging at WPT4: (a) Speed, (b) EV Power, (c) Charger Power, (d) EV SoC, (e) EV Voltage, (f) EV Current. 


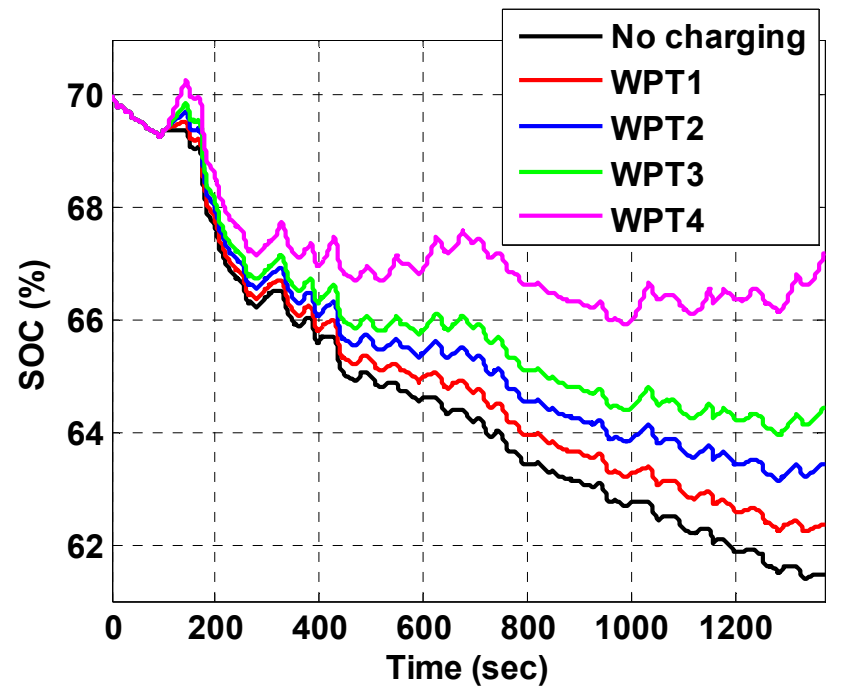

Figure 7.23. Electric Vehicle Driving Performance at Different Standardized Wireless Power Transfer Charging Levels.

It can be observed that through the utilization of QDWPT charging, the driving range is extended as a function of the charging power level for the same stop time. All charging levels exhibit a shallower reduction in the SoC, where WPT4 results in only a net 3\% SoC reduction by the end of the drive cycle. WPT4 and higher levels appear promising for these applications, since the EV may recover its initial SoC by the end of the driving profile. Through the implementation of the advanced battery model developed in this chapter, the battery voltage response over the quickly changing driving and regenerative breaking pulses, as well as the longer response following QDWPT charging periods, were accurate to the performance of the PL8048168 battery module when extended to an array that would support an EV. Furthermore, through the inclusion of the energy rate function, a more accurate depiction of the $\mathrm{SoC}$ was realized over the driving cycle.

\subsection{Summary}

In this chapter, a multiple-time-constant battery model was implemented particularly for use in accurate EV simulations. The unique I-V characteristics of a $51.8 \mathrm{~V} 21 \mathrm{Ah}$ LCO 
battery module was captured using a hybrid modeling procedure, where a $2^{\text {nd }}-$ Order Randles dynamic equivalent circuit contains dynamic RC components mapped to both the minute and second time-scales. A battery test stand composed of relatively inexpensive components and instrumentation administered controlled discharging and charging current pulses over the entire battery SoC operating range. The data was then curve-fitted to extract the dynamic equivalent circuit parameters. The final comprehensive model was implemented in the MATLAB/Simulink environment to be used seamlessly with the SimPowerSystems block set. Results revealed the model to produce a close match to experimental values. Following its development and verification, the model was utilized in the simulation of a future EV wireless charging system. In this concept, the EV drivetrain, propulsion, and wireless charging system was modeled and simulated to evaluate the practically of installing wireless charging at traffic intersections. Some results were presented, revealing the strengths of the battery model in accurately depicting the battery terminal voltage behavior and its SoC progression.

Although a focus was placed primarily upon the advanced modeling of a lithium ion battery system, the concept of hybrid ES systems (HESS) has been introduced, as many EVs and other all-electric drivetrains contain more than one ES type. The introduction of a HESS composed of multiple battery chemistries, SCs, or flywheel ES systems can drastically improve the system performance with the proper design and control. However, it also introduces a dilemma within the power electronics domain, as multiple ES devices require advanced interfacing electronics. Each ES type has its own operating voltage and current range. Without the assistance of a power electronics converter, the combination of a HESS is challenging and if possible, would suffer from low efficiency. In the next 
chapter, the basic interfacing power electronics devices will be discussed, as well as their operation within a HESS system. A particular focus will then be placed on present-day switching devices, their efficiencies, and frequency limitations, as well as how wide-band gap semiconductor switches could offer a notable improvement for future HESS. 


\section{Chapter 8 Interfacing Converters to Support Hybrid Energy Storage Systems}

\subsection{Introduction}

Thus far, energy storage (ES) has been managed, analyzed, and modeled in a variety of different ways. Since the introduction of the Energy Storage Management Controller (ESMC) in Chapter 2, a focus has been particularly placed upon lead acid and lithium ion battery ES systems. Beginning with the implementation of a simple Randles equivalent circuit model to evaluate battery performance under a variety of conditions, an extension to physics based models (PBMs) helped lay the framework to connect a deep relationship between their varying circuit models and lifetime, or State of Health (SoH). Performance

and $\mathrm{SoH}$ degradation concerns are synonymous with all battery ES, and despite a designer's best efforts, all that can be controlled is the rate at which this degradation occurs. Furthermore, the energy and power density of each battery chemistry is fixed. Although some types, such as lithium ion batteries, may offer a reasonable balance between the two, ES devices with far better energy and power densities are available.

A solution to these concerns is the design of a hybrid ES system (HESS), which can combine the strengths of multiple ES devices. This would increase both the energy and power density of the system, while potentially relieving some of the stress placed on the batteries, aiding in reducing their rate of degradation. Electric vehicles (EV), shipboard power systems (SPS), smart home energy systems, and the Smart Grid are some examples that traditionally employ more than one ES type [156]. For example, the integration of a lithium ion battery with a supercapacitor (SC) could purpose the battery as the primary energy source, while the SC could be utilized to handle high-power demands, as their 
power density is one hundred times greater than the battery [28]. This type of integration could decouple or maintain a conservative constant current on the battery, while the SC provides a short duration current to fill a heavy discharge pulse. With the proper control and interfacing power electronics, this basic HESS would avoid exposing the battery to unnecessary high currents, hereby prolonging its lifespan. For these reasons, the second half of this dissertation is focused upon the development of various HESS for different applications.

Although the integration of a HESS system carries with it a number of advantages, it also introduces a new set of challenges. In order to connect ES devices together, a power electronic interfacing converter is required. From common portable computers to cellular phones, to light emitting diode (LED) lighting, efficient conversion is needed to provide clean, lower operational voltage levels to these devices. With the recent surge in renewable energy, EVs, and hybrid AC/DC SPS, a great deal of research is being done in an effort to scale these devices to handle much higher voltage and current levels, while still pushing the boundaries of efficiency. For the US Navy, for instance, current shipboard platforms such as the DDG 1000 have revised legacy power system models, increasing the load potential to serve electromagnetic rail guns and high-powered radar equipment [10], [158]. In the meantime, power electronics have continued to improve as well to handle loads, which would be highly disruptive to the onboard main turbine generators.

To support these demands, an efficient HESS must be designed. Although the HESS design for a structure of this scale has many facets, in this dissertation, a general focus is placed upon two. First, the selection and configuration of the optimal ES types and devices to support these applications, which will be discussed in the next chapter, and second, 
improving their interfacing power electronics. To support a wide range of voltage and current levels for multiple ES devices, while supporting the interfacing of renewables, the demand from the power electronics front is very significant. The efficiency of conversion is only one main aspect. As new mobile applications such as EVs and SPS emerge, the energy and power density of the converters also comes into play. In order to support the buck or boost operation in applications with an extremely large voltage change, while operating at a high level of current, design of the converter becomes complicated and in many cases, requires multiple conversion stages. An increase in the switching speed and minimization of their voltage ripple is crucial, all the while ensuring total harmonic distortion (THD) levels remain at or below their required levels. These categories all fall into the power quality assessment of the HESS, an aspect which will be discussed in detail later in Chapter 10.

In this chapter, an overview of the common DC-DC interfacing electronics will give way to focus upon the synchronous buck converter, a more advanced topology for interfacing HESS. The synchronous buck converter will then be simulated with a lithium ion battery and flywheel ES system HESS. Different simulation platforms will highlight how deeper models of the switching devices can result in not only a more accurate depiction of the circuit performance, but also pinpoint a major aspect where the efficiency of the circuit can be improved. An investigation into the operation of the silicon (Si) metal oxide semiconductor field effect transistors (MOSFET) and its replacement with gallium nitride (GaN) wide band-gap (WBG) semiconductors is two-fold, showcasing the improvement of the circuit performance and efficiency, while identifying the need for deeper models of the GaN switches. 


\subsection{Basic Power Electronic Devices to Interface Energy Storage}

Since each ES device carries with it its own voltage level and operating conditions, a DC-DC converter is traditionally necessary to match voltage levels and manage the power flow between ES devices [161]. Two fundamental topologies are depicted in Figure 8.1, both relying upon the periodic opening and closing of a switch to operate. Since the duration the switch is on dictates the required size of the output capacitor and inductor, DC-DC converters are switched at high speeds of $\mathrm{kHz}$ or greater [162]. The following subsections will briefly describe the operation of each topology.

\subsubsection{Basic DC-DC Buck Converter}

The basic buck converter is utilized to step down from a higher voltage to a lower voltage and its topology is shown in Figure 8.1. A diode $D_{\text {buck }}$ provides a path for the inductor current when the switch $Q_{b u c k}$ is open, simply reverse-biasing when $Q_{b u c k}$ is closed. As $Q_{b u c k}$ opens and closes, the resulting pulsed square wave is smoothened by a capacitor $C_{b u c k}$, maintaining an average value. Some variation, or ripple, is present as a result of the time $C_{\text {buck }}$ takes to charge and discharge, as the pulse varies between on and off. Through a simple modification of the square wave duty cycle (also known as pulse width modulation), the voltage reduction from the source to load side can be easily controlled.

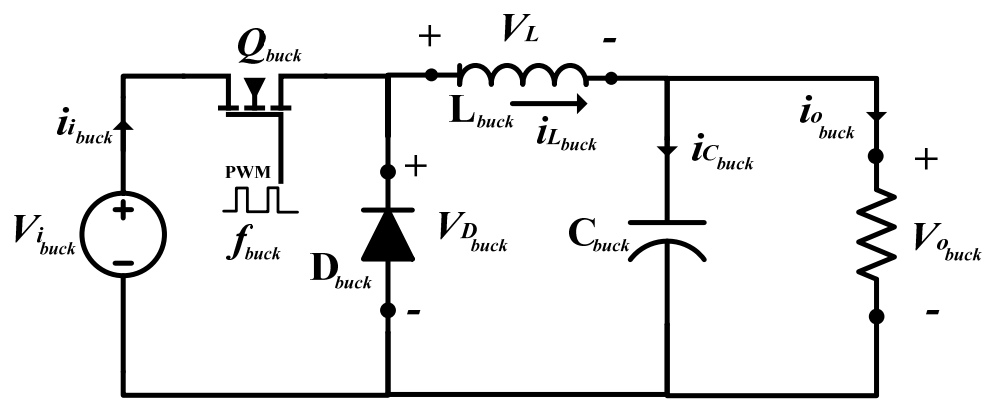

Figure 8.1. Basic DC-DC Buck Converter Circuit Topology. 


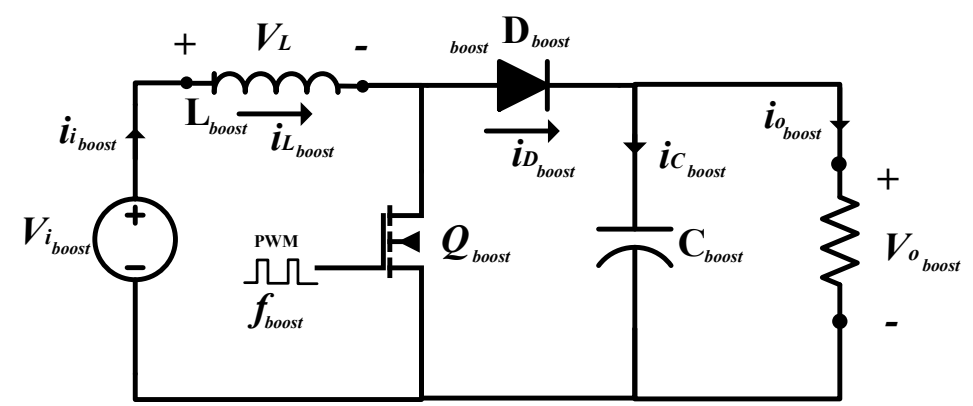

Figure 8.2. Basic DC-DC Boost Converter Circuit Topology.

\subsubsection{Basic DC-DC Boost Converter}

The basic circuit schematic of a boost converter is shown in Figure 8.2. In this scenario, the primary voltage is stepped up to a higher voltage. When the switch $Q_{\text {boost }}$, is closed, current flows through the inductor $L_{\text {boost }}$ to the load, while $L_{\text {boost }}$ stores energy in a magnetic field. When $Q_{\text {boost }}$ opens, the current reduces as a result of a higher impedance, discharging the magnetic energy to maintain the load. Since this reverses the polarity of $L_{\text {boost }}$, its voltage is added in series with the source $v_{i_{\text {boost }}}$, where a capacitor $C_{\text {boost }}$ is charged through diode $D_{\text {boost }}$ to hold the resulting higher voltage. As with the buck converter, some ripple is present as a result of the time $C_{\text {boost }}$ charges and discharges its electric field. Also, similar to the buck converter, control of the pulse width modulation (PWM) duty cycle controls the resulting output voltage.

\subsection{Improving DC/DC Converter Topologies}

Power electronics development within this field is very broad, and has spawned a significant deal of research. An analysis of all features and aspects that can be improved in the design, control, and switch driving circuits of these devices is out of the scope of this chapter. However, in this subsection, a focus is placed on a common enhancement of the buck converter, which is popular in high-efficiency ES interfacing. Modification from the 
legacy buck converter is rather simple, where a second MOSFET is used in place of the diode since it has a much lower voltage drop when conducting. This results in a higher circuit efficiency, especially important in applications with a low-voltage output also featuring high-current, which is why it is commonly used in interfacing lithium ion battery and SC-based HESS [162].

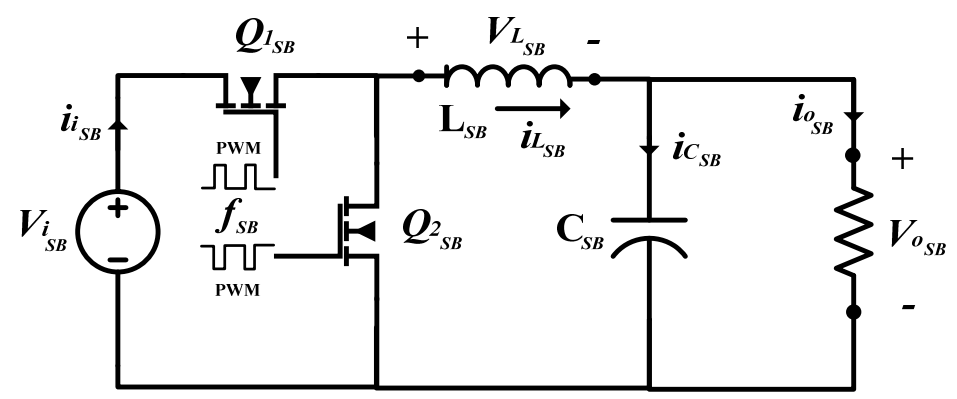

Figure 8.3. Synchronous Buck Converter.

Consider the synchronous buck converter design shown in Figure 8.3. In its traditional operation, two Si MOSFETs are utilized in its operation, where the switching states of $Q_{1_{S B}}$ and $Q_{2_{S B}}$ are simply inverted. Since the on-resistance $R_{o n}$ of a MOSFET is sufficiently small, the $0.3 \mathrm{~V}$ to $0.4 \mathrm{~V}$ diode drop is considerably reduced when the converter is not in a conducting state ( $Q_{1_{S B}}$ off and $Q_{2_{S B}}$ on). However, a sufficiently small $R_{o n}$ yields a number of other advantages as well. When the switch is in its conducting state, a lower $R_{o n}$ contributes to lower losses and better thermal characteristics that in turn enable the converter to operate at higher current levels. Although Si-based MOSFETs are common in the market, there is a desire to obtain a lower $R_{o n}$ and lower input capacitance $C_{i_{s s}}$ with improved thermal characteristics. These needs have inspired researchers to investigate into alternative switching materials. WBG devices such as GaN have emerged as a potential replacement, offering a considerable reduction in $R_{o n}, C_{i_{s s}}$, and better thermal stability. 
Table 8-1. Synchronous Buck Converter Design Parameters.

\begin{tabular}{|c|l|r|}
\hline \hline Input Voltage & $V_{i_{S B}}$ & $125 \mathrm{~V}$ \\
\hline Output Charging Voltage & $V_{O_{S B}}$ & $12.6 \mathrm{~V}$ \\
\hline Output Charging Current & $i_{O_{S B}}$ & $* 63 \mathrm{~A}$ \\
\hline Smoothening Capacitor & $\mathrm{C}_{S B}$ & $10 \mathrm{mF}$ \\
\hline Inductor & $\mathrm{L}_{S B}$ & $5 \mu \mathrm{H}$ \\
\hline Switching Frequency & $f_{S B}$ & $50 \mathrm{kHz}$ \\
\hline
\end{tabular}

*3C Charging Rate for 21 Ah 3-cell Lithium Ion Polymer Battery Module

Table 8-2. Silicon and Gallium Nitride-Based Synchronous Buck Converter Specifications.

\begin{tabular}{|c|c|c|c|}
\hline Description & Variable & $\begin{array}{l}\text { Si MOSFET } \\
\text { Infineon } \\
\text { IPW60R125P6 }\end{array}$ & $\begin{array}{c}\text { eGaN HEMT } \\
\text { GaNSys } \\
\text { GS66508T }\end{array}$ \\
\hline Surface Area & $A$ & $326.56 \mathrm{~mm}^{2}$ & $31.05 \mathrm{~mm}^{2}$ \\
\hline \multicolumn{4}{|c|}{ Electrical } \\
\hline On-Resistance & $R_{\text {on }}$ & $125 \mathrm{~m} \Omega$ & $50 \mathrm{~m} \Omega$ \\
\hline Input Capacitance & $C_{i_{S S}}$ & $2660 \mathrm{pF}$ & $260 \mathrm{pF}$ \\
\hline Reverse Transfer Capacitance & $C_{r_{s S}}$ & $80 \mathrm{pF}$ & $2 \mathrm{pF}$ \\
\hline Maximum Switching Frequency & $f_{\max }$ & $1 \mathrm{MHz}$ & $100 \mathrm{MHz}$ \\
\hline \begin{tabular}{|l|} 
Maximum Voltage \\
\end{tabular} & $V_{d s_{\max }}$ & \multicolumn{2}{|c|}{$650 \mathrm{~V}$} \\
\hline \multicolumn{4}{|c|}{ Thermal } \\
\hline $\begin{array}{l}\text { Junction-to-Case } \\
\text { Thermal Resistance }\end{array}$ & $R_{J C}$ & $0.57 \mathrm{~K} / \mathrm{W}$ & $0.50 \mathrm{~K} / \mathrm{W}$ \\
\hline $\begin{array}{l}\text { Case-to-Ambient } \\
\text { Thermal Resistance }\end{array}$ & $R_{C A}$ & $62 \mathrm{~K} / \mathrm{W}$ & $5 \mathrm{~K} / \mathrm{W}$ \\
\hline Maximum Junction Temperature & $T_{J_{\max }}$ & \multicolumn{2}{|c|}{$+150^{\circ} \mathrm{C}(423.15 \mathrm{~K})$} \\
\hline Heat Transfer Coefficient & $h$ & \multicolumn{2}{|c|}{$20 \mathrm{~W} / \mathrm{m}^{2} \cdot \mathrm{K}$} \\
\hline Initial Temperature & $T$ & \multicolumn{2}{|c|}{$+25^{\circ} \mathrm{C}(298.15 \mathrm{~K})$} \\
\hline
\end{tabular}

\subsection{Simulating the Synchronous Buck Converter using Si versus GaN}

In this section, a synchronous buck converter is designed to interface a flywheel ES system to charge a lithium ion battery module. The circuit architecture for both the Si and GaN-based converter is identical aside from the replacement of the switch. Table 8-1 
provides a summary of the desired input $V_{i_{S B}}$ and output $V_{O_{S B}}$ voltages, charging current $i_{O_{S B}}$, selected capacitor $C_{S B}$ and inductor $L_{S B}$ values, and the PWM switching frequency $f_{S B}$. In this case, the flywheel is expected to input $125 \mathrm{~V}$, while the output of 12.6 $\mathrm{V}$ matches the maximum allowable voltage to charge a 3-cell 21 Amp-hour (Ah) lithium ion phosphate (LiPO4) battery bank connected in series [164]. The charging current is set to $63 \mathrm{~A}$, as this matches an extreme charging Columbic $(\mathrm{C})$ rate of $3 \mathrm{C}\left(1 / 3^{\text {rd }}\right.$-hour rate) on the battery. It is worthy to note that charging at $3 \mathrm{C}$ to $5 \mathrm{C}$ is not uncommon when utilizing LiPO4-type cells for EV or SPS applications.

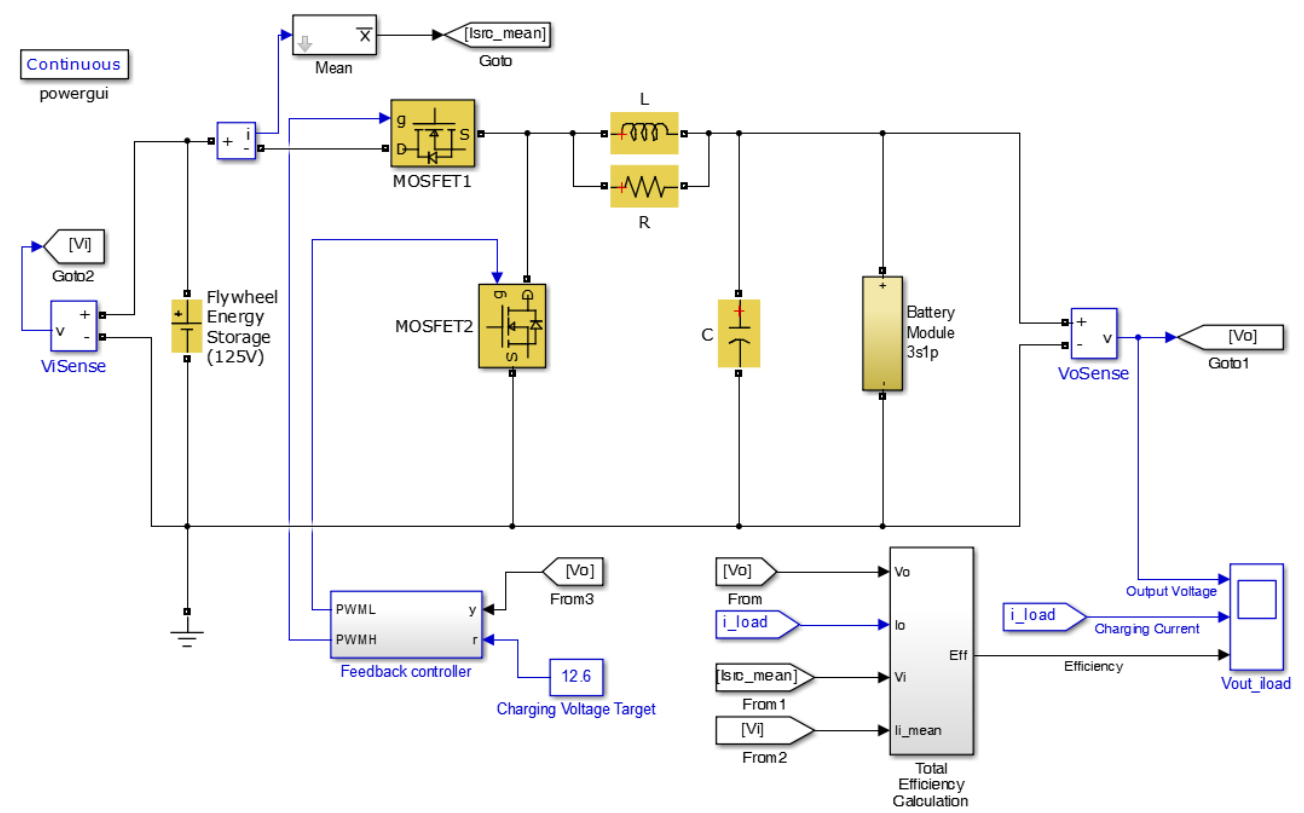

Figure 8.4. Synchronous Buck Converter Design in Simulink SimPowerSystems.

Commercial Si and GaN-based switches have been selected for comparison. Since the availability of commercial GaN is limited, the GaNSys GS66508T enhanced GaN High Electron Mobility Transistor (eGaN HEMT) was first selected, while the comparable Sibased Infineon IPW60R125P6 MOSFET was selected with similar specifications depicted in Table 8-2 [165],[166]. Note the GS66508T features an $R_{\text {on }}$ one-third of its Si-based 
counterpart. Input $C_{i_{S S}}$ and reverse transfer $C_{r_{S S}}$ capacitances are ten to forty times less than that of the IPW60R125P6, while the package size is also one-tenth of the size, featuring a lower thermal resistance. In order to conduct an initial simulation of the synchronous buck converter operation, Simulink SimPowerSystems is initially used, where the circuit configuration is shown in Figure 8.4.

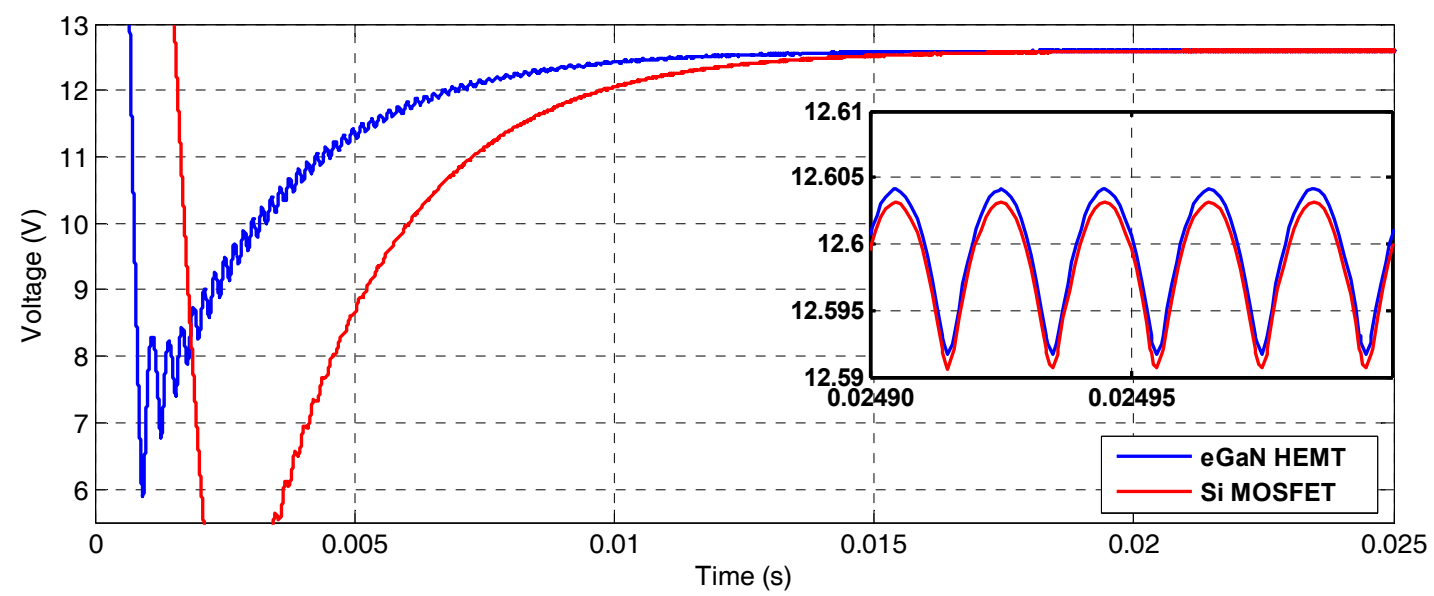

Figure 8.5. GaN-based and Si-Based Synchronous Buck Converter Voltage Output under SimPowerSystems Simulation.

\subsubsection{Initial Simulation in Simulink SimPowerSystems Blockset}

To accurately measure the conversion efficiency, "Mean" blocks are included to obtain the average current sourced and expended over each PWM pulse period. A source conversion was also required to convert the inductor $L$ to a voltage source, achieved by including a large parallel resistance $\mathrm{R}=10 \mathrm{k} \Omega$. The output voltage over the first $25 \mathrm{~ms}$ of operation is shown in Figure 8.5. Notice both converters take approximately $15 \mathrm{~ms}$ to settle to their steady-state targets of $12.6 \mathrm{~V}$. As anticipated, some ripple is present in both converters of approximately $15 \mathrm{mVpp}$. Since the SimPowerSystems design is only capable of taking $R_{o n}$ into account in the switch model, its performance is near ideal and is weak in simulating the accurate frequency and magnitude of the voltage ripple. 


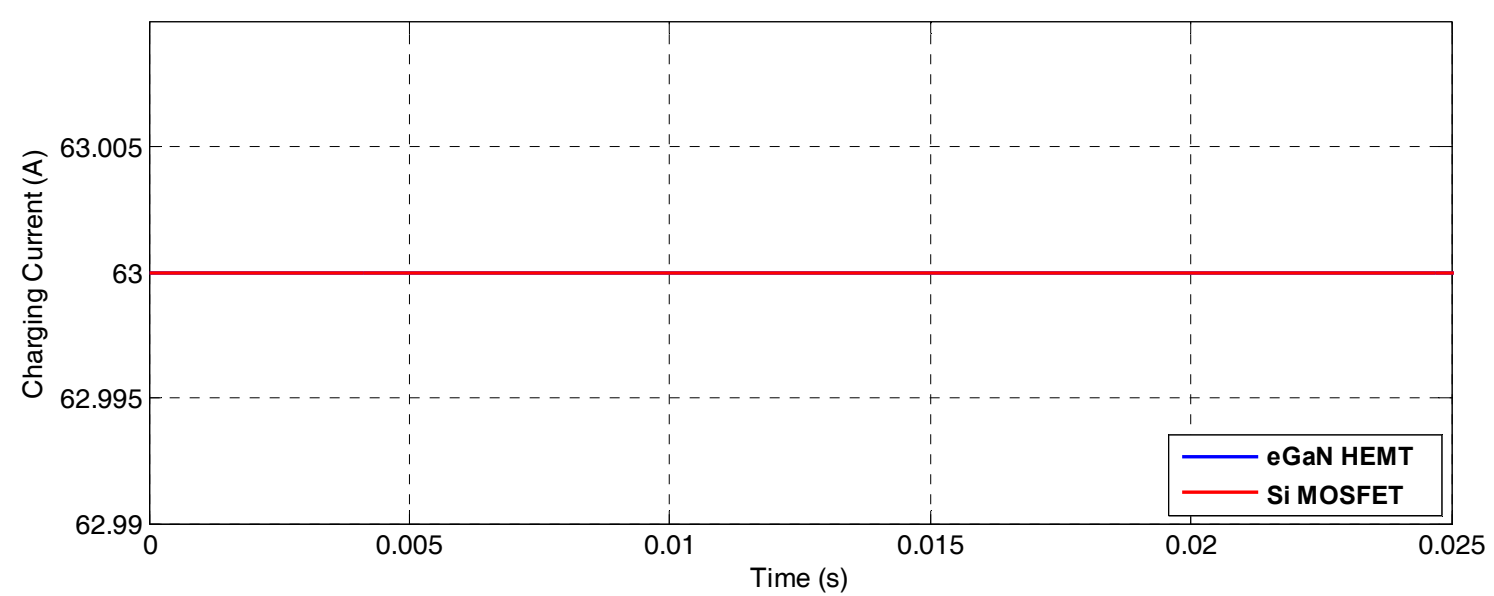

Figure 8.6. GaN-based and Si-Based Synchronous Buck Converter Charging Current Output under SimPowerSystems Simulation.

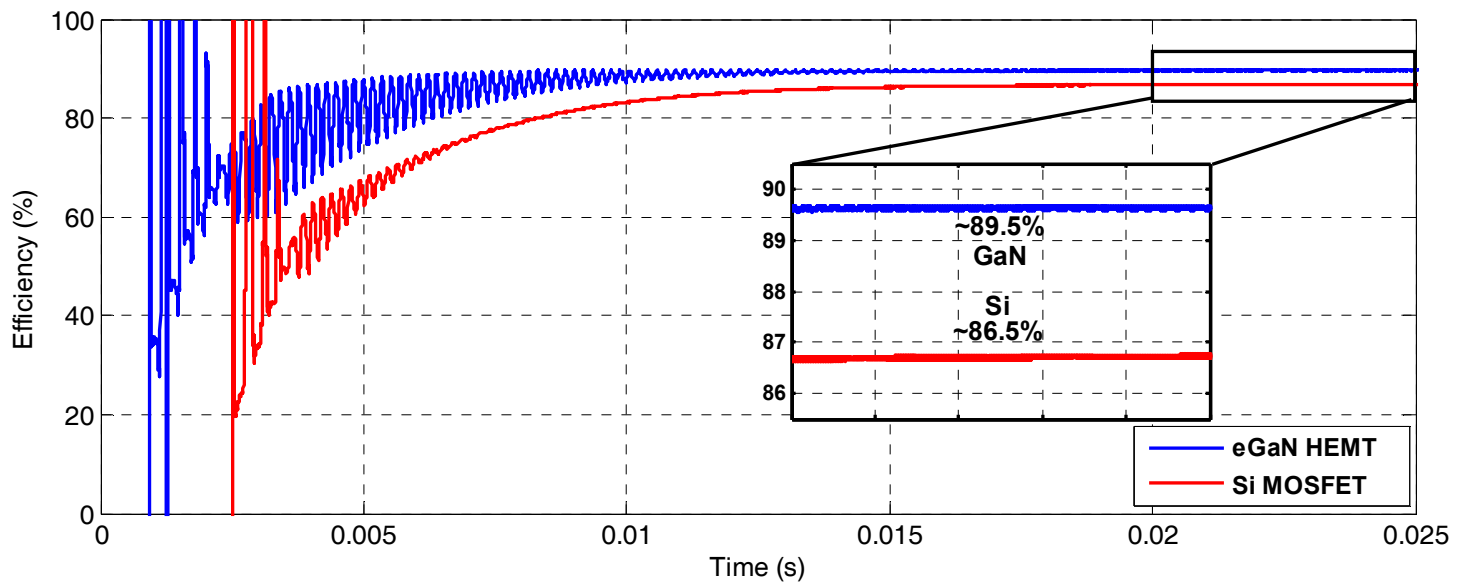

Figure 8.7. GaN-based and Si-Based Synchronous Buck Converter Efficiency under SimPowerSystems Simulation.

Since the output voltage ripple and charging current for the GaN versus Si converters match once they have reached a steady-state point, their output powers are nearly identical. However, as a result of a lower $R_{\text {on }}$, a basic representation of the losses through each converter can be assessed. Figure 8.7 represents the total input-output efficiency of each converter in real-time. Using the SimPowerSystems MOSFET model, the GaN-based converter appears to only offer a $3 \%$ improvement in the total efficiency over the $\mathrm{Si}$ converter. 


\subsubsection{Improved Dynamics under Simulink SimElectronics Blockset}

Although Figure 8.7 demonstrates an improvement in the efficiency when eGaN HEMT switches are used, the SimPowerSystems model neglects a great deal of detail related to differences in their internal capacitance and thermal losses. These factors have a dramatic impact on the voltage response, as well as the real efficiency of the system. Furthermore, minimal differences are observed when the converter charging current is varied, which is not realistic. A higher-level model depicting each switch is required that can account for more physical, electrical, and thermal characteristics of each switch. To achieve this, the same SimPowerSystems circuit designed in Figure 8.4 was rebuilt using the Simulink SimElectronics blockset. SimElectronics not only provides support for more physical, electrical, and thermal MOSFET characteristics, but also has the ability to couple losses into the thermal domain to simulate the switch operating temperature. Figure 8.8 depicts the alternative Simulink synchronous buck converter developed with the SimElectronics blockset.

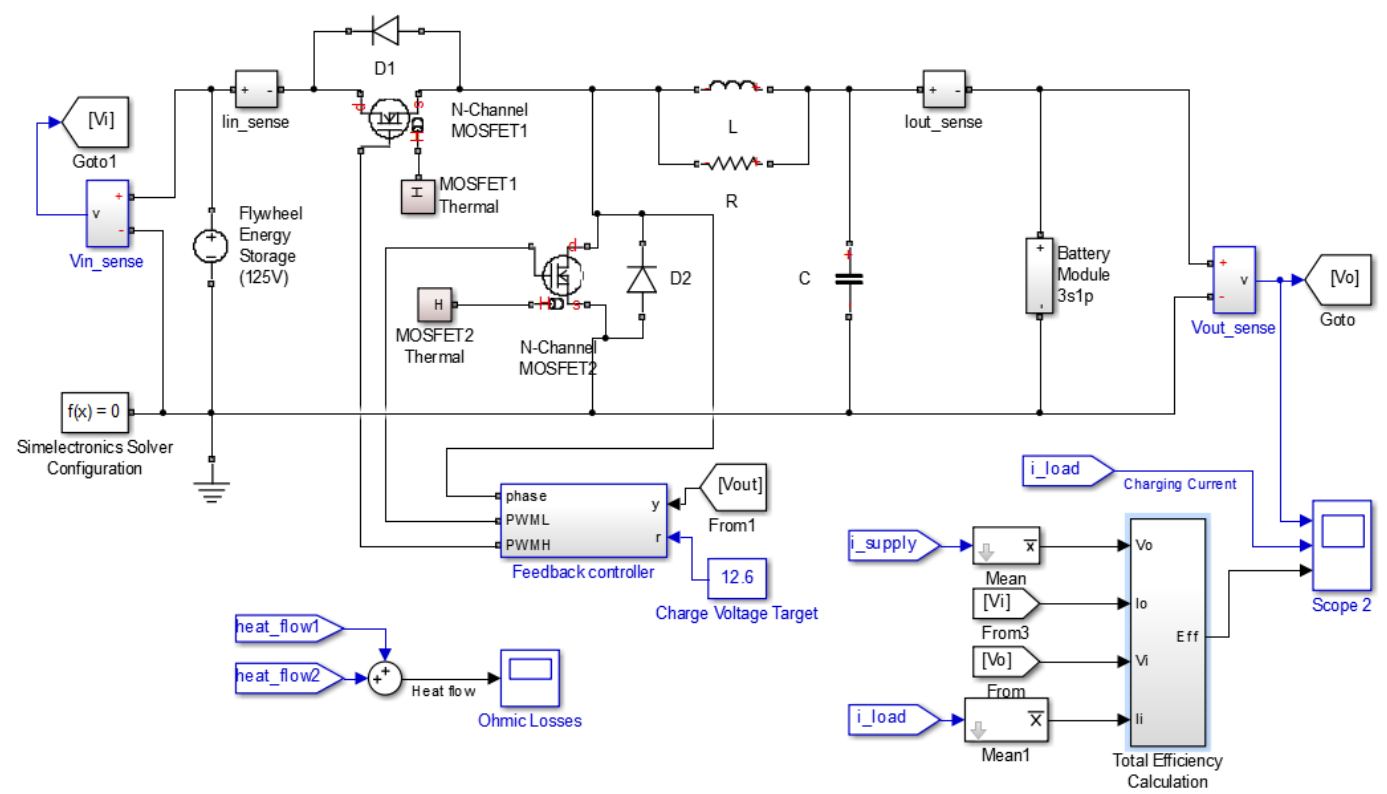

Figure 8.8. Synchronous Buck Converter Design in Simulink SimElectronics. 
Most of the model depicted in Figure 8.8 is similar, with an exception to the depth of the models for MOSFET1 and MOSFET2. Furthermore, characteristics of the body drift diodes inherent to each MOSFET have been separated so they can be modeled in detail from the GS66508T and IPW60R125P6 datasheets. Below MOSFET1 and to the left of MOSFET2, a block represents a coupling of the internal ohmic losses from each MOSFET into the thermal domain. In this case, the thermal model is taken to be relatively simple, where the thermal and dimensional characteristics from Table 8-2 are used to capture the expected temperature rise at the MOSFET junction and case over time. Beginning with the same analysis conducted for the SimPowerSystems simulation, the output voltage of each converter is shown in Figure 8.9. One can notice a striking difference in the waveforms shown over the same initial $25 \mathrm{~ms}$ operating period. At the initial start of each converter, the GaN-based converter responds remarkably faster, reaching near the target output voltage in $0.5 \mathrm{~ms}$. Some oscillation is present, though it is expected as a result of the Proportional Integral (PI) controller driving the PWM signal. The converter completely settles within $10 \mathrm{~ms}$.

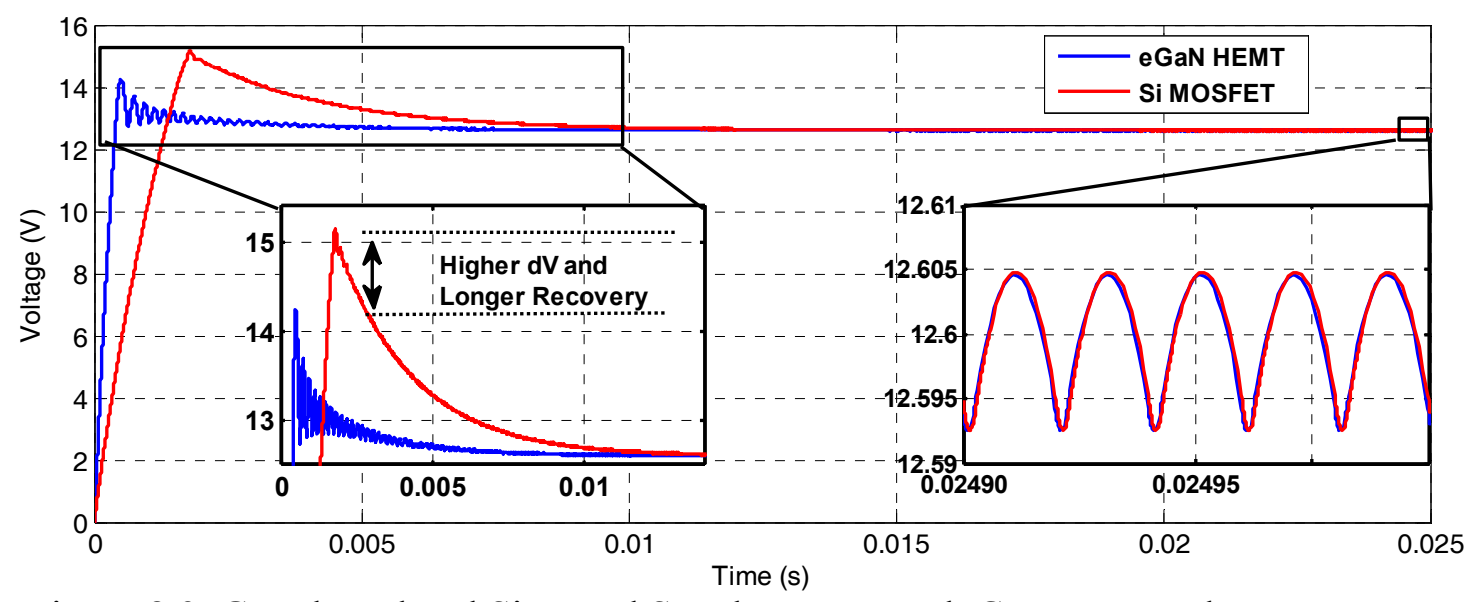

Figure 8.9. GaN-based and Si-Based Synchronous Buck Converter Voltage Response under SimElectronics Simulation. 


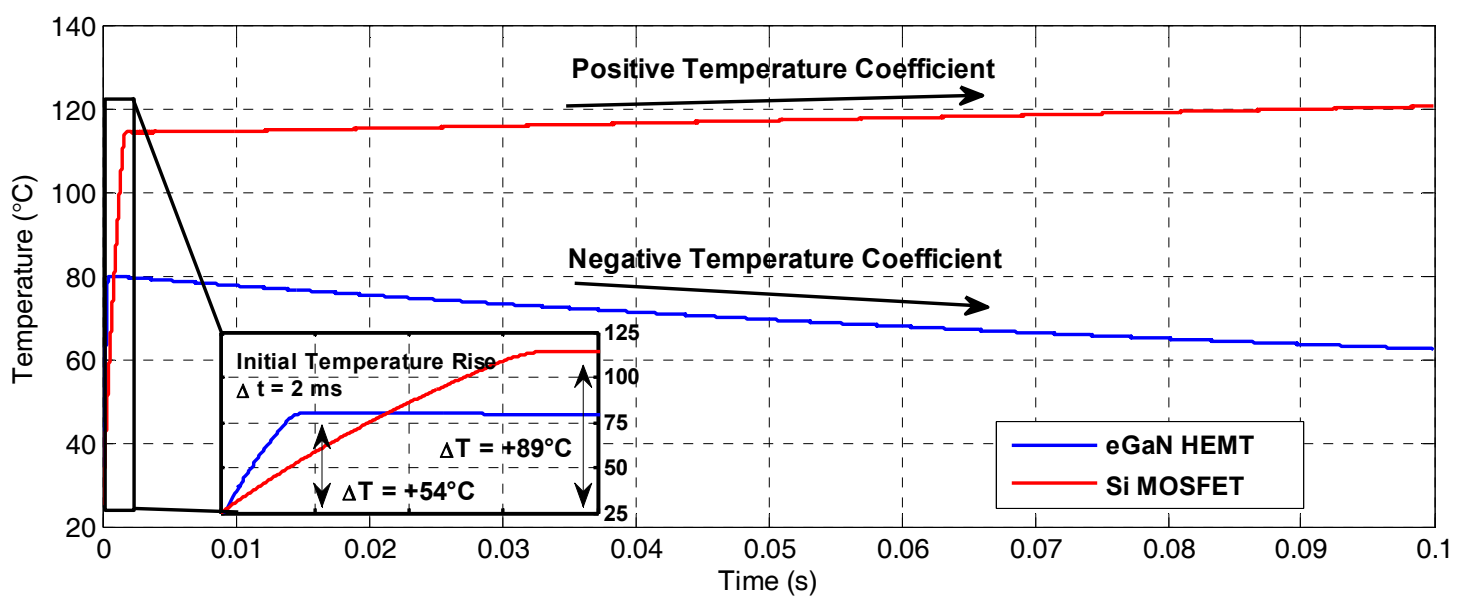

Figure 8.10. GaN and Si-Based Synchronous Buck Converter Switch Junction Temperature.

For the Si-based converter, the settling period is approximately $50 \%$ longer, reaching its steady-state after approximately $15 \mathrm{~ms}$. An initial rise of the voltage to within the range of its target output takes almost four times longer $(1.8 \mathrm{~ms})$. Although the settling period of the Si-based MOSFET is far longer than that of the $\mathrm{GaN}$, it is the higher voltage present at the output terminals that could impact the battery. A maximum sustained voltage of 12.6 $\mathrm{V}$ is exceeded by $2.55 \mathrm{~V}$ in the Si-based design prior to the settling of the converter. In the GaN-based design, only a $1.65 \mathrm{~V}$ voltage rise is observed and placed on the battery bank for a shorter period of time. Although these characteristics occur under very short durations, exposure of lithium ion batteries to high voltage could result in quicker aging in HESS applications or premature failure.

Since switching losses can be coupled into the thermal domain, these internal losses can now be quantified to track the internal MOSFET junction and case temperatures during operation. Figure 8.10 depicts the temperature trend of the GaN-based and Si-based switches for the first $100 \mathrm{~ms}$ of operation. A 63 A current results in an instant rise of the 
junction temperatures, where the Si MOSFET junction increases by $+89^{\circ} \mathrm{C}$ and the eGaN HEMT increases by $+54^{\circ} \mathrm{C}$ during the first $2 \mathrm{~ms}$ of operation. Notice the eGaN HEMT rises only $+35^{\circ} \mathrm{C}$ following its initial turn-on. However, it is the GaN performance once it reaches steady-state that is more notable. Once it reaches a steady-state, the temperature of the eGaN HEMT reduces to a constant level, while the Si MOSFET continues to increase. Figure 8.11 reveals the $\mathrm{Si}$ and $\mathrm{GaN}$ switch case temperature trends over the same period. The Si MOSFET temperature rise is $28 \%$ steeper than that of the eGaN HEMT. Continued operation in these conditions would quickly reach the maximum operating junction temperature of $+150^{\circ} \mathrm{C}$. This point suggests the Si-based synchronous converter would require a significant heat sink, assuming it is capable to handle this operating scenario.

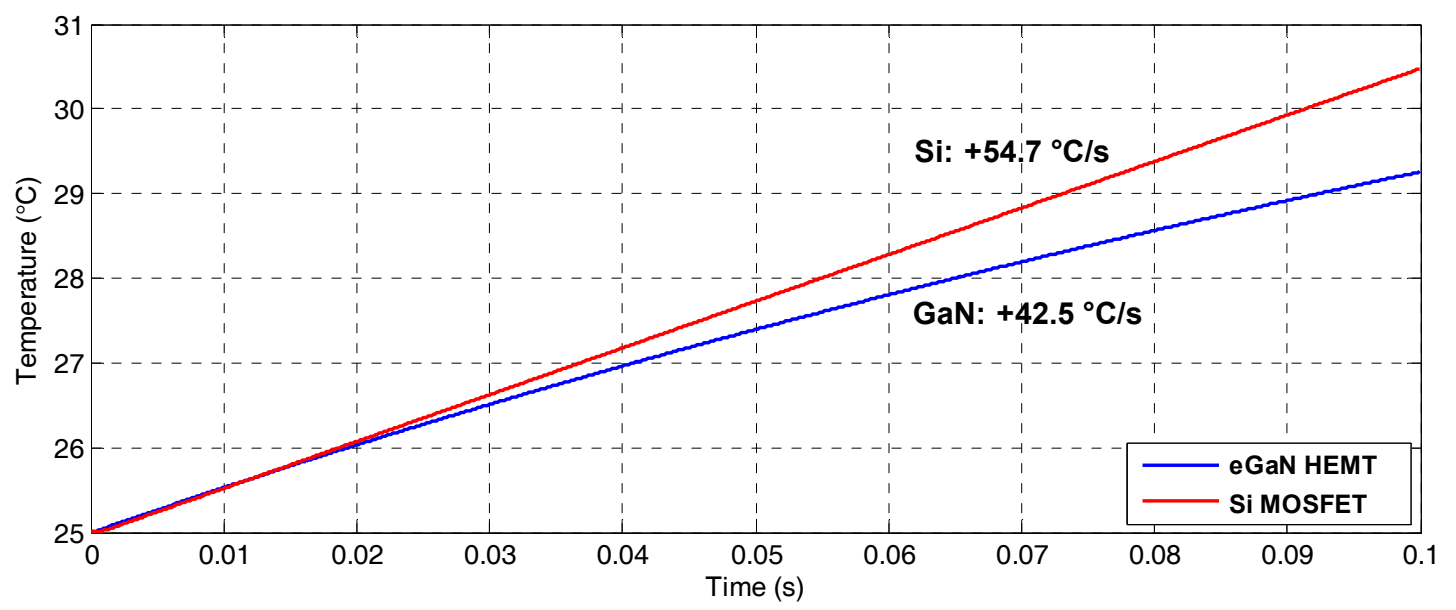

Figure 8.11. GaN-based and Si-Based Synchronous Buck Converter Switch Case Temperatures.

Insight into the thermal domain reveals there is a notable difference in the efficiency of each converter that was not captured in the previous SimPowerSystems simulation. The real-time input-output conversion efficiencies of the $\mathrm{Si}$ versus GaN-based converters are shown in Figure 8.12. By inspection, the inclusion of new switch dynamics yields a 
significant difference in the conversion efficiency. Following each converter reaching steady-state, the GaN-based converter yields nearly a $13 \%$ increase in the conversion efficiency, with a 63 A output charging current.

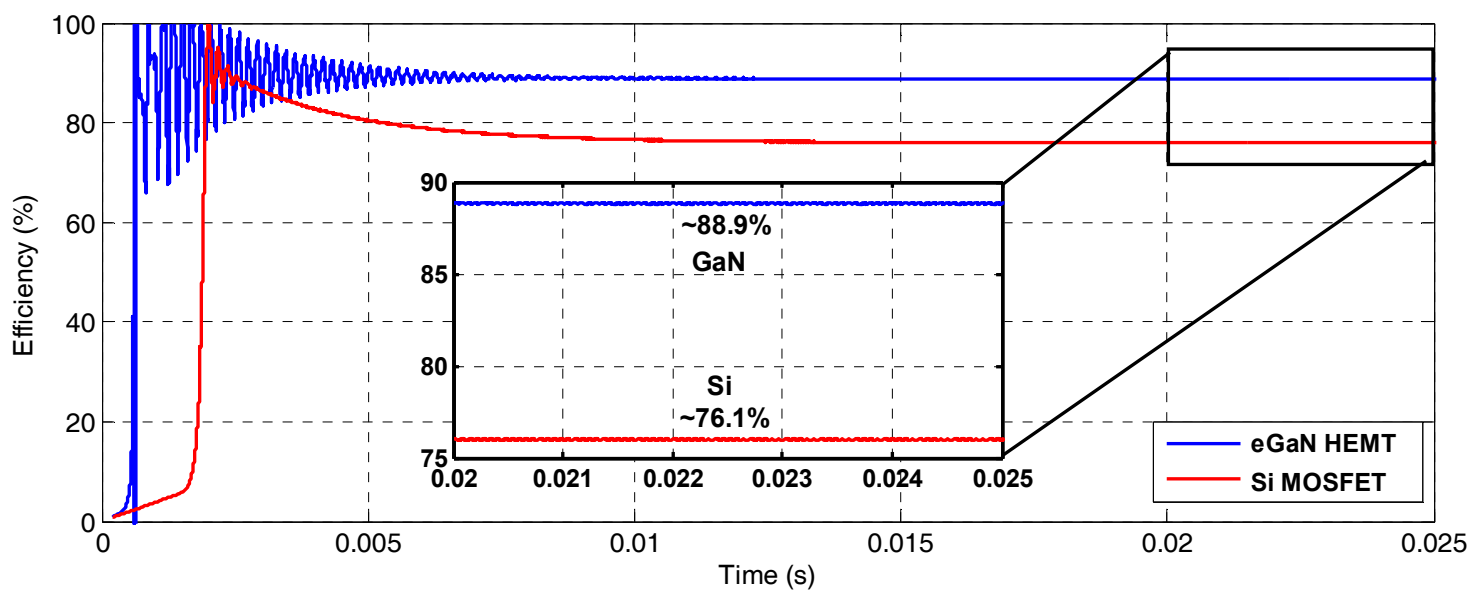

Figure 8.12. GaN and Si-Based Synchronous Buck Converter Efficiency under SimElectronics Simulation.

Since the SimElectronics model is capable of accounting for a number of new characteristics versus the SimPowerSystems model, the efficiency of each converter can now be estimated as a function of the charging current for each converter. This progression is shown in Figure 8.13. The charging current is shown at the bottom, while the normalized charging C-rate of the lithium ion battery module is shown to the top. As depicted, charging currents of a low magnitude result in a significant percentage of heat losses. For the Sibased converter, a $\mathrm{C} / 20$ charging current is barely supported. The GaN-based converter offers an improvement, though the efficiency is still far lower than an acceptable value.

As the current increases to $\mathrm{C} / 2$ and greater, the efficiency of the GaN-based converter reaches nearly $90 \%$ and maintains it until the peak current magnitude of $63 \mathrm{~A}(3 \mathrm{C})$. The Si-based converter follows a similar trend, except with an efficiency between $12 \%$ and $34 \%$ lower. Its performance, particularly within these types of ES interfacing applications, 
demonstrates the potential replacement of Si MOSFETs in traditional power electronics devices. Although in this example the conversion efficiency was focused upon as the primary improvement, eGaN HEMT-based buck converters can also be produced at lower cost with a much greater power density and transient response than their Si counterparts [167]. eGaN HEMTs can also be operated comfortably at much higher switching frequencies. The typical $50 \mathrm{kHz}$ switching frequency selected for a Si-based converter could be realistically increased by five to ten times. However, to achieve this, a complete redesign of the circuit is necessary. This not only includes adjusting the inductor and capacitor values, but also requires a deep understanding of the control and differences in how eGaN HEMT switches will behave under these operating conditions.

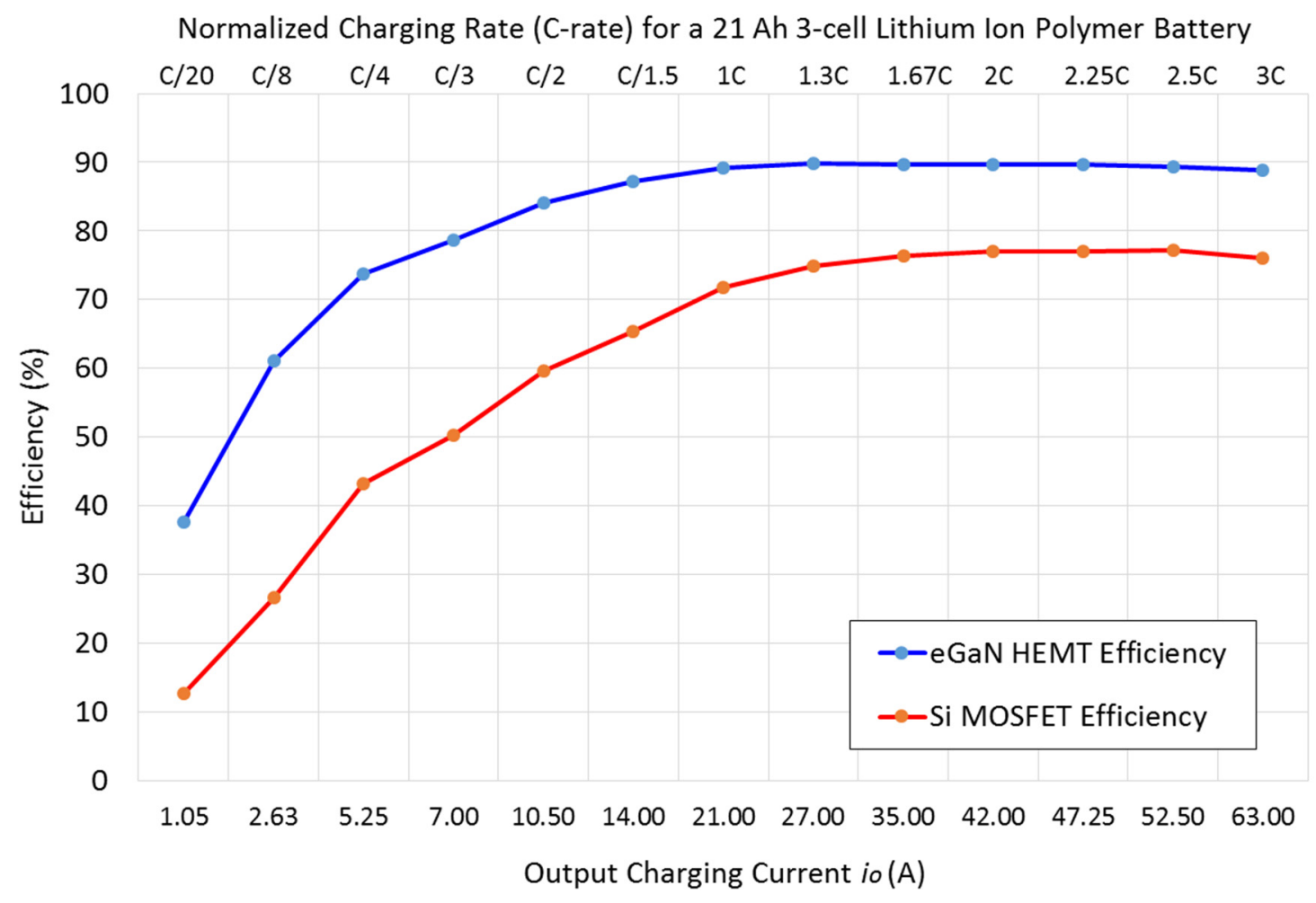

Figure 8.13. Si and GaN-based Synchronous Converter Efficiencies versus Charging Current. 


\subsection{Strengths and Limitations of Switching Models}

In the previous section, differences in the Si MOSFET and eGaN HEMT performance were focused upon at the circuit and application level. This was intended to present an important power electronics application within HESS that viewed the implementation of an eGaN HEMT as a drop-in replacement to Si. Similar to the selection process of an adequate battery equivalent circuit model as covered in previous chapters, the depth of the switch model must be evaluated for its form and function as well. In the case of enhancing power electronic converters, this places a focus on enhancing the switch model. A comparison between the Simulink SimElectronics and SimPowerSystems-based converters revealed how an inadequate model can have major implications, even at the application level. To acquire all of the advantages in enhancing power electronics devices that $\mathrm{GaN}$ has to offer, a focus must now be placed at the device level.

To improve modeling the performance of the GaNSys GS66508T, it is first important to recognize that its philosophy of operation is far different from that of the Si MOSFET [168]. The active regions of each switch are grown on a substrate material and consist of three common terminals, namely: the source $(\mathrm{S})$, where charge carriers enter the channel, a voltage control gate $(\mathrm{G})$, and an output drain (D), where charge exits. Each terminal is then attached to a conductor. Figure 8.14 shows a side-by-side comparison of the switch construction. In the Si MOSFET shown to the left, the gate contact is separated from the channel by a silicon dioxide ( $\mathrm{SiO} 2)$ passivation layer. An inversion charge is established by the charge carriers of the conducting channel. This phenomenon reveals the significance of $n$ or $p$-type doping, or the introduction of impurities to alter the intrinsic states of Si to operate the Si MOSFET. The inversion charge is caused by electrons in the case of a $p$ - 
type substrate, and holes in the case of an $n$-type substrate, induced at the $\mathrm{Si}-\mathrm{SiO} 2$ interface by the voltage applied to the gate. For the $n$-channel Si MOSFET utilized in the previous section, electrons enter and exit the channel at $n+$ type source and drain contacts.
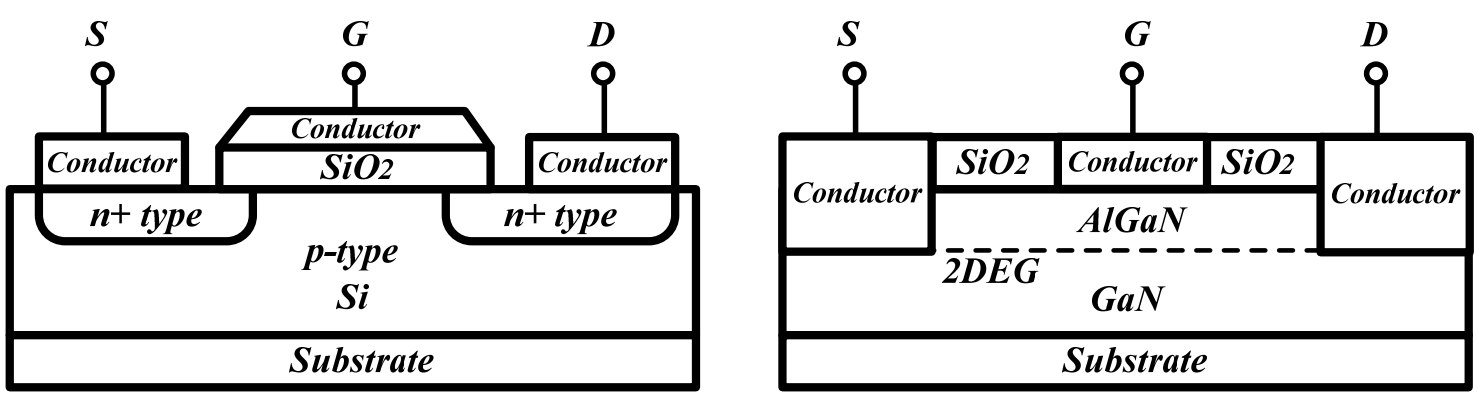

Figure 8.14. Field Effect Transistor Structure: $n$-channel Silicon MOSFET (Left) and Enhancement mode Gallium Nitride High Electron Mobility Transistor (Right).

The operation of the eGaN HEMT is considerably different. Shown to the right in Figure 8.14, no doping is required to induce operation [167]. The operation of the eGaN HEMT is reliant upon an inherent property of $\mathrm{GaN}$ material as being piezoelectric. By stacking aluminum gallium nitride $(\mathrm{AlGaN})$ on top of $\mathrm{GaN}$, a lattice mismatch in their atomic structures induces a strain, which results in the formation of a layer of free electrons between the two materials. This collection of charge along the GaN-AlGaN interface is referred to as the 2-dimensional electron gas (2DEG), creating a channel between the source and drain of the eGaN HEMT. Theoretical limits of $R_{o n}$ in the eGaN HEMT are significantly better, as the mobility of electrons across the 2DEG is huge [169]. Through applying a voltage at the gate contact, electrons across the 2DEG can either be depleted or enhanced by the electric field, providing control of the channel. For the eGaN HEMT, applying a positive voltage to the gate will allow current to flow across the channel. 


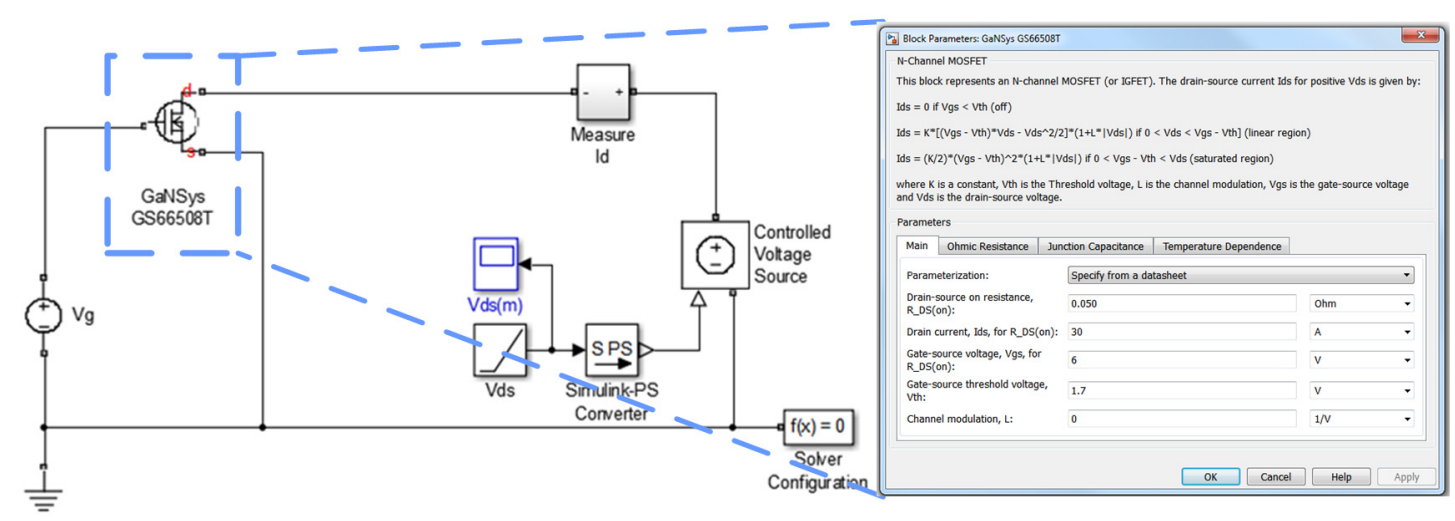

Figure 8.15. GaNSys GS66508T eGaN HEMT SimElectronics I-V Characterization.

\subsubsection{Evaluating Limitations of the GaNSys GS66508T SimElectronics Model}

Now consider the same eGaN HEMT, as was studied in the previous subsection. As a result of the enhanced modeling featured in SimElectronics, basic current-voltage (I-V) characteristic curves for the GS66508T can be generated. In this case, a variety of gate voltages $V_{g}$ are introduced, while a sweep is applied to the drain-to-source voltage $V_{d s}$ to capture the behavior of the drain current $I_{d}$ (output current). Through accurate modeling of the GS66508T I-V curves, a deeper relationship can be drawn to model the electrical behavior between its source, drain, and gate contacts. Modeling at this level can then be used to map out the dependence of the current and voltage between terminals, which can then be represented by an equivalent circuit model inside the simulation platform. Figure 8.15 demonstrates a simple test circuit, in which the characteristic curves are approximated by inputting parameters from the datasheet.

Using a controlled voltage source for $V_{g}, V_{g}$ is set to five typical gate voltages, as specified by the datasheet. For each $V_{g}, V_{d s}$ is swept past its saturation region to demonstrate where a stable operating current has been reached. Figure 8.16 demonstrates 
a comparison between the I-V characteristic curves published on the GS66508T datasheet and the curves generated by the SimElectronics simulation. Although the curves appear to be similar, there are a number of notable differences. First, the current increases in the saturation region for virtually all gate voltages above $+2 \mathrm{~V}$. This makes it difficult to predict its behavior at low $V_{d s}$. Next, although $V_{g}=+6 \mathrm{~V}$ and $V_{g}=+5 \mathrm{~V}$ result in an $I_{d}$ similar to the datasheet, gate voltages below $+5 \mathrm{~V}$ yield very different drain current profiles. Once the gate voltage approaches its minimum turn-on voltage $V_{g}=+2 \mathrm{~V}$, the resulting $I_{d}$ is only around an average of $3 \mathrm{~A}$, far below the near $10 \mathrm{~A}$, which is featured on the datasheet.
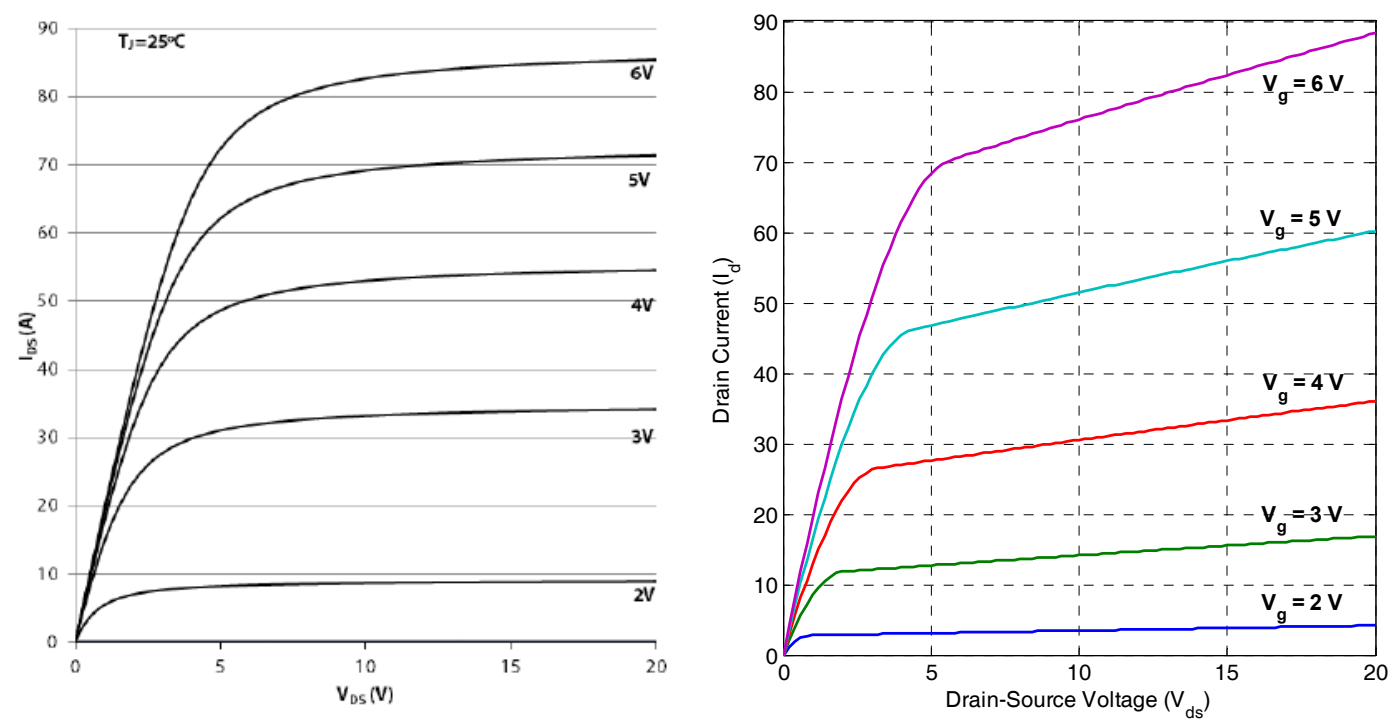

Figure 8.16. I-V Characteristic Curves for GaNSys GT66508T: Datasheet (Left) and SimElectronics Simulation Output (Right).

\subsubsection{Utilizing Manufacture SPICE Models for Improved I-V Performance}

There are a number of causes for the drift in the SimElectronics model, but most can be mapped back to the lack of depth. Since the physics of the eGaN HEMT and its operation differ greatly from $\mathrm{Si}$, an accurate mapping of their I-V behavior requires specialized models from the manufacturer. Furthermore, modeling these characteristics can be difficult 
to simulate within the SimElectronics environment. Fortunately, GaNSys provides a customized Simulation Program with Integrated Circuit Emphasis (SPICE) model for the Linear Technology SPICE (LT SPICE) software environment that is capable of closely modeling I-V behavior. The enhanced SPICE model for the GS66508T is shown to the left in Figure 8.17, while the same test circuit pictured in Figure 8.15 was replicated within LT SPICE to obtain I-V characteristic curves (Figure 8.17). A comparison between those published on the datasheet reveals a close resemblance to its expected I-V behavior.
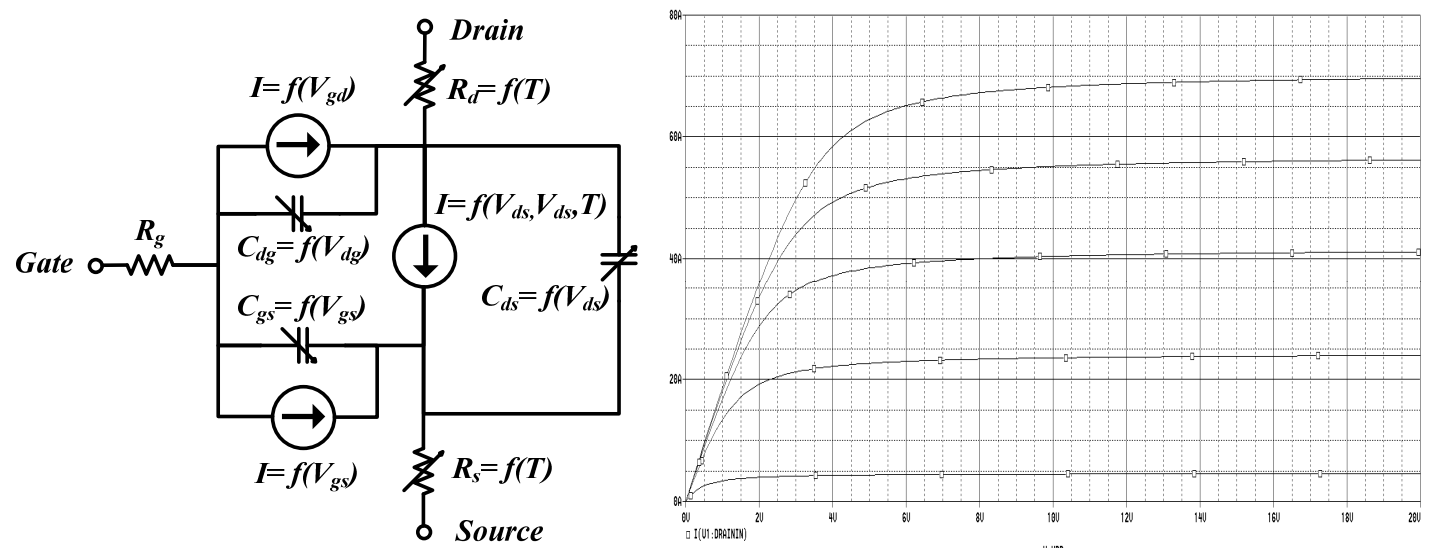

Figure 8.17. GaNSys GT66508T: LT SPICE Model (Left) and I-V Characteristic Curves Output (Right).

The LT SPICE-based GT66508T model presents a dramatic improvement in the dynamics not captured by SimElectronics. This could offer yet another increase in the accuracy of operation at the device level, while aiding to better depict I-V responses across the terminals of the eGaN HEMT while in operation. These dynamics would be particularly useful in redesigning the synchronous buck converter to operate at higher frequencies, as a substantial increase in the PWM frequency would generate very different I-V dynamics across the switch and output. These features can range from a different gate voltage to an expected impact of the switch capacitance, especially at higher frequencies. 


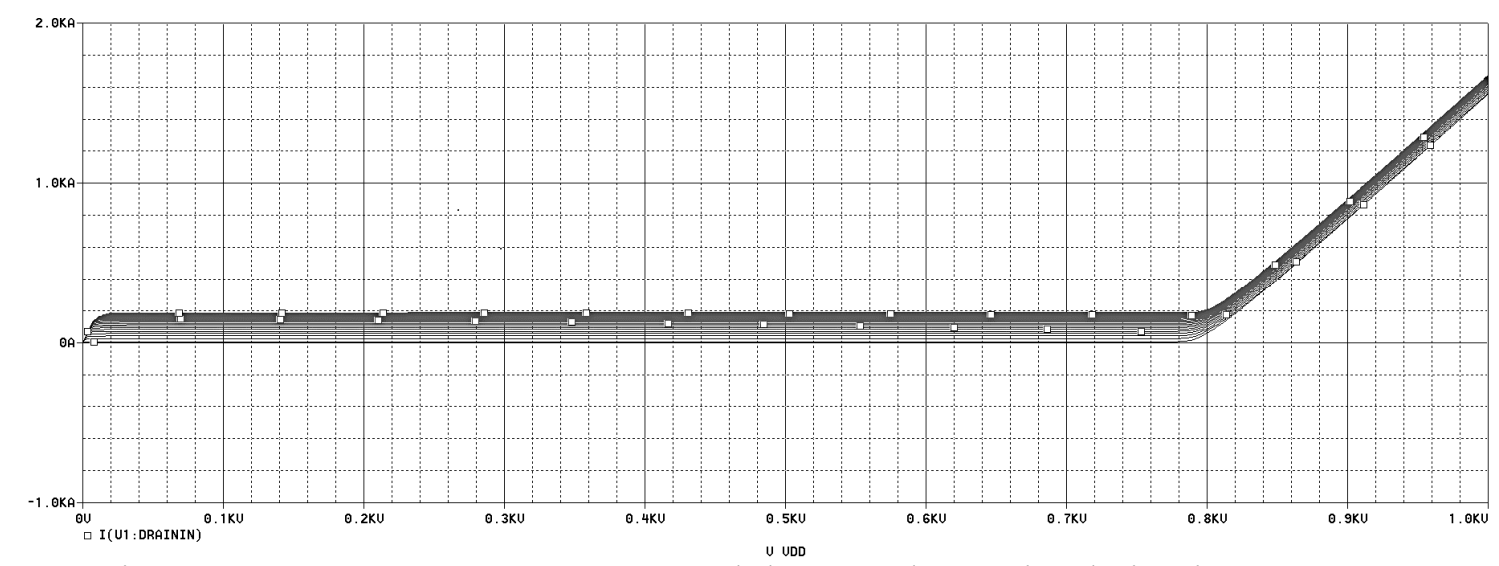

Figure 8.18. GaNSys GT66508T Breakdown Voltage Simulation in LT SPICE.

Although these features are obtained from a closer analysis at the device level and can improve simulations at the application level, they are still limited in pushing the technological boundaries of $\mathrm{GaN}$. In the I-V characterization provided by the datasheet, $V_{d s}$ is only swept up to $20 \mathrm{~V}$. However, even in the synchronous buck circuit, a voltage of $125 \mathrm{~V}$ is expected across the switch between its on and off states. Moreover, the maximum operating voltage $V_{d s_{\max }}$ is over five times higher. $V_{d s_{\max }}$ represents a critical state of operation, where $V_{d s}$ is increased so high, that the eGaN HEMT progresses out of its saturation region and breaks down. At the Breakdown Voltage (BV), where $V_{d s}=V_{d s_{\max }}$, the voltage increases so high that it approaches a major physical limitation of the semiconductor. At this point, the internal reversed-biased body drift diode breaks down and high current flows between the source and drain, causing a short circuit. Since the BV phenomenon is not typically simulated at the application level, LT SPICE also has difficulty in accurately predicting this point.

A simulation beyond the BV of the GS66508T has been conducted in Figure 8.18, revealing a linear increase of current past the $800 \mathrm{~V}$ level. At an application level operating under normal conditions, this is sufficient. However, as the usage of $\mathrm{GaN}$ becomes more 
popular in power electronic devices, an accurate prediction of precisely where the $\mathrm{BV}$ occurs is crucial. In order to obtain this information, an extension to a PBM is needed. Though not always necessary, finding a precise BV location has advantages in both the application and device sides. On the application front, an accurate depiction of where the BV occurs can help to safely increase the operating voltage of the power electronic converter, while providing a comprehensive simulation platform. This platform can also be used to simulate, investigate, forecast, and mitigate failures. On the device front, a PBM provides a direct correlation between the semiconductor physics, materials, and dimensioning of the device, and its operating limitations. In the following chapter, a PBM of the eGaN HEMT will be developed and comprehensively investigated.

\subsection{Summary}

In this chapter, the concept of HESS was expanded upon, as it can take advantage of the strengths from multiple types of ES to maximize both power and energy density of the system. In this dissertation, efficient HESS design has been broken into two categories: the design of the interfacing power electronic converter and the optimal selection and control of ES elements. The first facet was covered in detail, where an overview of the basic buck and boost DC-DC converters paved the way to a specific focus upon the use of a synchronous buck converter, a popular power electronic interfacing device for HESS systems. Following a demonstration of the converter functionality and performance in a scenario where a flywheel ES system is used to charge a lithium ion battery module, an analysis was carried out to calculate the efficiency of the converter. This placed a significant focus upon the losses experienced through the switches. 
Targeting replacement of the Si switches as a solution for improvement, the legacy Si MOSFETs were replaced with WBG eGaN HEMT switches. This revealed a significant improvement in the input-output conversion efficiency across the entire operating range of the converter when operating with identical components and the same switching frequency. However, in order to push the technological boundaries of eGaN HEMT and better understand their true voltage and current limitations, a PBM must be developed to more closely represent its operation. With a validated base eGaN HEMT PBM, a world of opportunities will open at the device level, which can be used to explore ways to increase its BV. In the following chapter, a PBM of the eGaN HEMT will be formulated and an extensive study will be carried out to evaluate breakdown mechanisms in the common eGaN HEMT device. 


\section{Chapter 9 Physics-based Modeling of the GaN High Electron Mobility Transistor}

\subsection{Introduction}

In the previous chapter, the enhancement Gallium Nitride (eGaN) High Electron Mobility Transistor (HEMT) was analyzed with a side-by-side comparison to its traditional Silicon (Si)-based counterpart. Initially, this approach focused on advantages from an applications perspective. However, limitations of various models were exposed in the implementation of eGaN in even the most basic DC-DC power electronic converters. Three different Simulation Program with Integrated Circuit Emphasis (SPICE) models were tested, where none had the ability to forecast the Breakdown Voltage (BV) of the device. In order to further push the boundaries of eGaN and enable their usage in future power electronics applications, a bridge is required between the semiconductor physics realm and the power and electrical engineering sector. This is accomplished through the development of a physics-based model (PBM) of the eGaN HEMT. Accurately modeling its functionality has advantages that far surpass simply those that exist in the application front, but can also provide feedback to material engineers and scientists to optimize the design of the device.

Since the physics of the eGaN HEMT is so different from its Si counterpart, the use of a common metal oxide semiconductor field effect transistor (MOSFET) model in its place is insufficient. Although proprietary-developed SPICE models, such as the LTSpice model used in the previous chapter, reveal an outstanding improvement in capturing I-V characteristics, they have a limited operating range. In order to further technology and push 
the boundaries of the eGaN HEMT performance, while retaining an understanding of why and how they will eventually fail, a PBM is necessary. Similar to the strengths observed in battery PBMs, capturing subtleties of the materials, polarization, and unique physics of the 2-dimensional electron gas (2DEG) is necessary to accurately simulate all operating scenarios, including the BV. With a detailed model, not only can this PBM be utilized to more accurately study the behavior of the switch, but it can provide details difficult or even impossible to obtain experimentally. In this chapter, an extensive study will be conducted to evaluate breakdown mechanisms in GaN HEMT. A comprehensive PBM of the common HEMT will provide the base comparison to conduct a number of different material and geometric investigations in a progression toward the optimal configuration. An electric field distribution across the source, gate, and drain will be analyzed for each case as well as their I-V curves up to the BV to establish the best case for improvement.

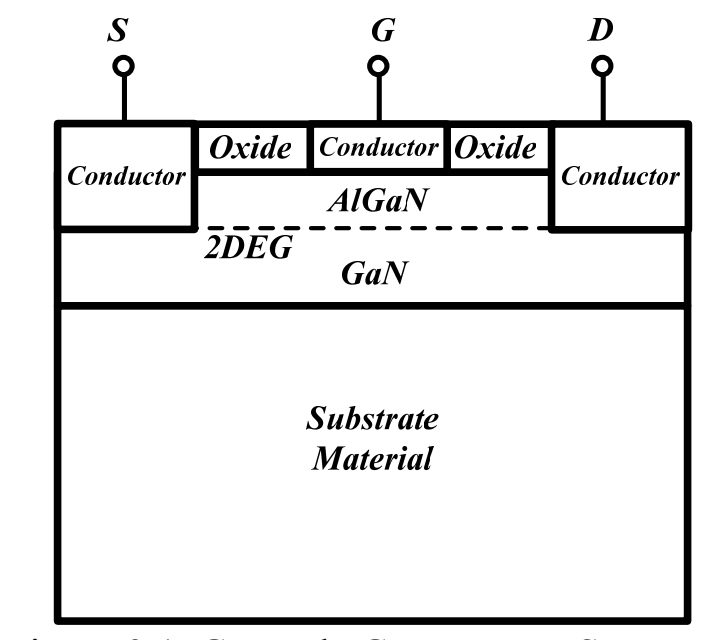

Figure 9.1. General eGaN HEMT Structure.

\subsection{The eGaN HEMT Physics Based Model}

The eGaN HEMT PBM was developed in the Silvaco Atlas Technology Computer Aided Design (TCAD) environment, an advanced platform for semiconductor device 
simulation in a 2D space based on the schematic form shown in Figure 9.1. Recall from the previous chapter that the operation of the eGaN HEMT relies upon piezoelectric properties of the $\mathrm{GaN}$ material. A stacking of aluminum gallium nitride ( $\mathrm{AlGaN}$ ) on top of GaN results in the creation of the $2 \mathrm{DEG}$, operating as the channel. The solid material model of the eGaN in the Silvaco environment is shown in Figure 9.2. First, a substrate material is defined to which the semiconductor is grown on. In this case, Si is shown, as it is a popular material in terms of performance and cost, and is the simplest to grow on.

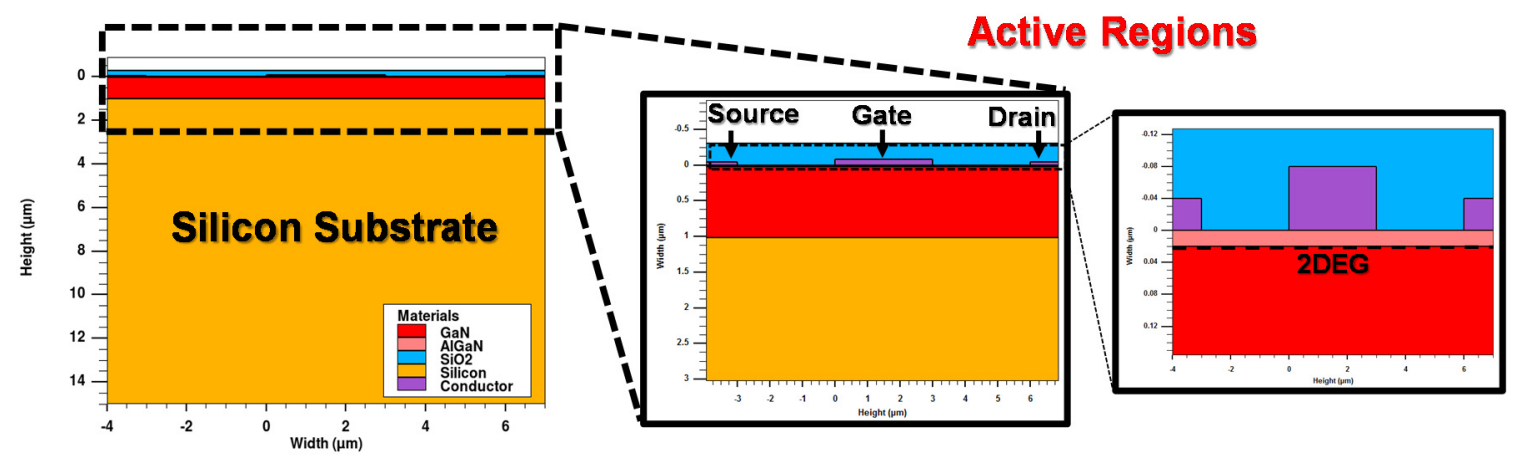

Figure 9.2. eGaN HEMT Silvaco Solid Model.

Shown to the left, the thickness of the substrate is significantly larger than the thickness of the active region, where a majority of the switch physics occurs. In the area shown to the middle, locations of the source, gate, and drain conductors, as well as the active GaN and AlGaN material, are clearly shown. Since the AlGaN layer is typically another magnitude smaller than that of the GaN or electrode contacts, the location of the AlGaN layer separating the $\mathrm{GaN}$ and electrodes forming the heterojunction are shown to the right. It is in this region where the $2 \mathrm{DEG}$ channel will be generated.

To setup an accurate, yet computationally efficient solution interface, two significant things are kept in mind when developing the PBM based on Finite Element Modeling 
(FEM). First, the GaN-AlGaN junction requires fine meshing along the y-axis to accurately model activity in the 2DEG channel. Along the $x$-axis, finer meshing is also made across each conductor, and particularly the conductor edges. The remaining regions can tolerate larger FEM elements, thus using the 2DEG and conductor edges as reference points for fine meshing; the mesh can be scaled up gradually in other regions.

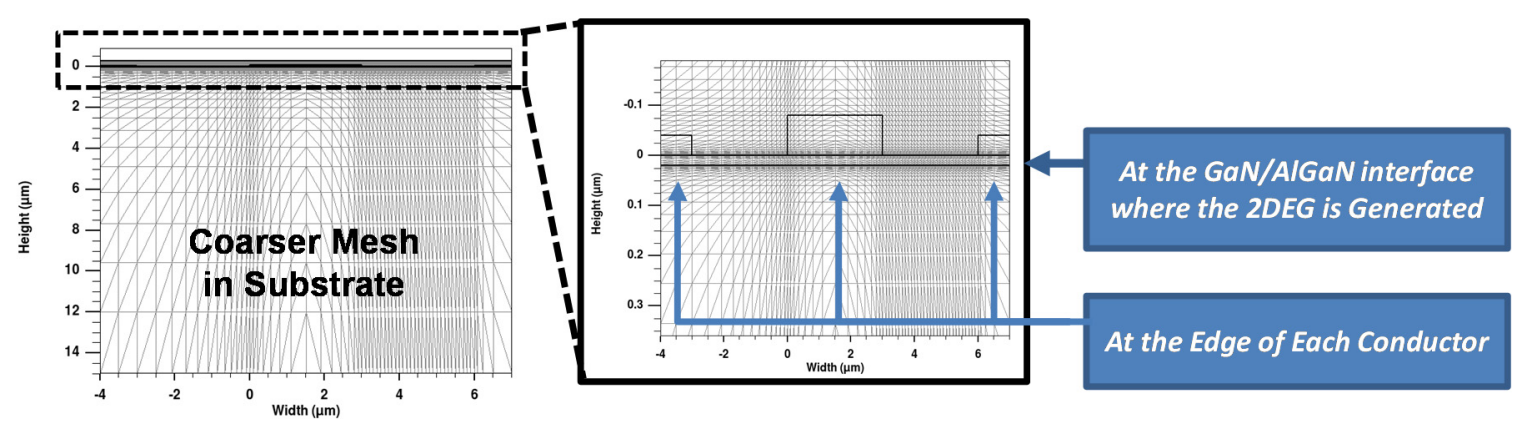

Figure 9.3. eGaN HEMT Finite Element Meshing.

The PBM takes into consideration the general material GaN HEMT structure and dimensions, as shown in Figure 9.3. Using Reference [170] as a basis, the PBM design and performance can be compared to a GaN HEMT grown and verified in a laboratory through the process of metal oxide chemical vapor deposition (MOCVD) where all required fabrication details are provided. Thus, a PBM can be engineered to verify the performance of an actual physical device. Moreover, once this verification has confirmed the correct deployment of the physics, this PBM can then be treated as a base model to experiment how altering parts of the structure, materials, and dimensions can be done to increase the BV [171].

Figure 9.4 depicts a comparison between the experimental breakdown current-voltage (I-V) characteristic curve obtained experimentally from Reference [170], and that which is obtained from simulation of the PBM. Taken in the eGaN HEMT off-state, the average $I_{D S}$ 
current is well under $1 \mathrm{nA}$ until $V_{D S}$ approaches $600 \mathrm{~V}$, where $I_{D S}$ gradually begins to increase, then quickly accelerates as the $V_{D S}$ approaches $800 \mathrm{~V}$. As can be shown, $V_{D S}$ sweeps from the experimental analysis and PBM are similar in shape, as well as identifying an accurate location of the BV at approximately $800 \mathrm{~V}$.
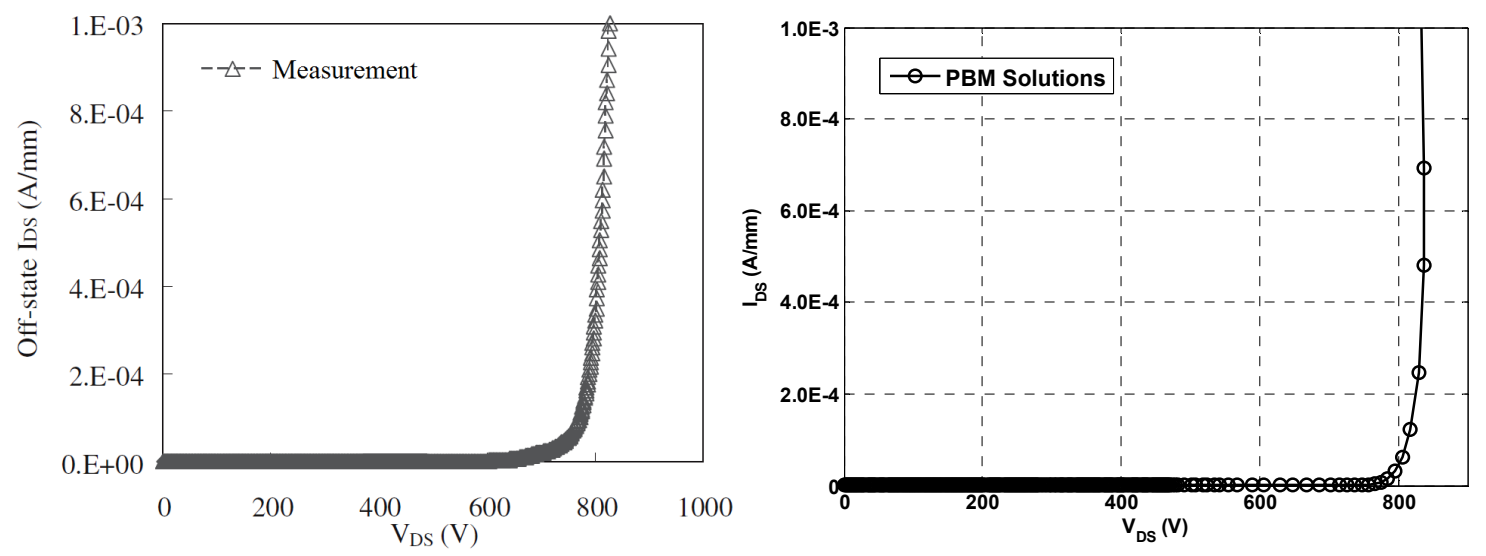

Figure 9.4. eGaN HEMT Breakdown Characteristics: Experimental Measurements (Left) and Physics Based Model Output (Right).

\subsection{Evaluating Ways to Increase the Breakdown Using the Physics Based Model}

One major limitation of the eGaN HEMT is a difficulty in easily scaling them to support higher currents and voltages, as required by electric vehicles (EV) and shipboard power systems (SPS). Since eGaN HEMTs are susceptible to a phenomenon known as surface breakdown, increasing the $\mathrm{BV}$ over a similar footprint is challenging. There are several $\mathrm{BV}$ mechanisms, such as: source-drain breakdown (punch-through), gate-drain breakdown (leakage through the Schottky diode), vertical breakdown (poor compensation of the buffer layer), and impact ionization (an electron-hole pair generation close to the gate) [172]. In impact ionization, the $\mathrm{BV}$ depends on the critical electric field $E_{\text {crit }}$ of the material, or the field strength needed to initiate impact ionization causing an avalanche breakdown [167]. 
Table 9-1. eGaN HEMT Design Parameters.

\begin{tabular}{|c|c|}
\hline Name & Value \\
\hline Total Width & $11.00 \mu \mathrm{m}$ \\
\hline Substrate Thickness & $13.98 \mu \mathrm{m}$ \\
\hline GaNFET Thickness & $16.00 \mu \mathrm{m}$ \\
\hline Source/Drain Width & $1.00 \mu \mathrm{m}$ \\
\hline Gate Width & $3.00 \mu \mathrm{m}$ \\
\hline Active GaN Layer Thickness & $1.00 \mu \mathrm{m}$ \\
\hline AlGaN Layer Thickness & $0.02 \mu \mathrm{m}$ \\
\hline AlGaN Composition & $x=20 \%$ \\
\hline
\end{tabular}

Table 9-2. GaNFET Cases of Study.

\begin{tabular}{|c|c|c|c|c|c|c|}
\hline \multirow{2}{*}{$\begin{array}{c}\text { Case } \\
\text { of } \\
\text { Study }\end{array}$} & \multirow{2}{*}{$\begin{array}{c}\text { Structure } \\
\text { in } \\
\text { Figure } 9.5\end{array}$} & \multirow{2}{*}{$\begin{array}{c}\text { Passivation } \\
\text { Layer }\end{array}$} & $\begin{array}{c}\text { Substrate } \\
\text { Layer }\end{array}$ & \multicolumn{3}{|c|}{$\begin{array}{c}\text { Doping Profiles } \\
\left(\mathbf{c m}^{-3}\right)\end{array}$} \\
\cline { 5 - 7 } & & $\begin{array}{c}\mathrm{GaN} \\
\text { Acceptor }\end{array}$ & $\begin{array}{c}\text { GaN } \\
\text { Donor }\end{array}$ & $\begin{array}{c}\text { AlGaN } \\
\text { Donor }\end{array}$ \\
\hline Original & (a) & $\mathrm{SiO}_{2}$ & $\mathrm{Si}$ & $2 \times 10^{17}$ & $2 \times 10^{16}$ & $1 \times 10^{17}$ \\
\hline Passivation & (a) & $\mathrm{SiN}$ & $\mathrm{Si}$ & $2 \times 10^{17}$ & $2 \times 10^{16}$ & $1 \times 10^{17}$ \\
\hline GaN Substrate & (a) & $\mathrm{SiN}$ & $\mathrm{GaN}$ & $2 \times 10^{17}$ & $2 \times 10^{16}$ & $1 \times 10^{17}$ \\
\hline Light Doping & (a) & $\mathrm{SiN}$ & $\mathrm{GaN}$ & $2 \times 10^{14}$ & $2 \times 10^{13}$ & $1 \times 10^{14}$ \\
\hline Ge Substrate & (a) & $\mathrm{SiN}$ & $\mathrm{Ge}$ & $2 \times 10^{17}$ & $2 \times 10^{16}$ & $1 \times 10^{17}$ \\
\hline Field Plate & (b) & $\mathrm{SiN}$ & $\mathrm{GaN}$ & $2 \times 10^{17}$ & $2 \times 10^{16}$ & $1 \times 10^{17}$ \\
\hline
\end{tabular}

(a) 2DEG

\begin{tabular}{|c|c|}
\hline Source Gate & Passivation \\
\hline & AlGaN \\
\hline & GaN \\
\hline & Substrat \\
\hline
\end{tabular}

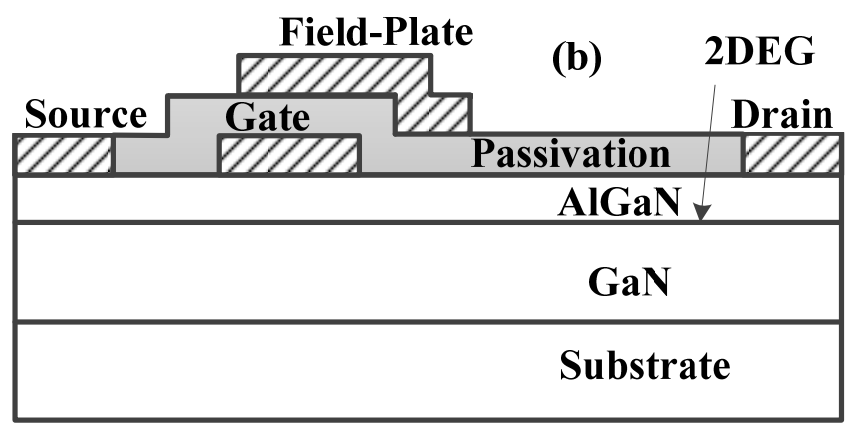

Figure 9.5. New eGaN HEMT Schematic Layer Structures: a) Base and b) Field Plate. 
Thus, there are two ways to avoid the BV: ensuring that the electric field $E_{f}<E_{\text {crit }}$ at the operating point of the eGaN HEMT, or enlarging the drift distance. However, the drift distance is constrained, thus the only solution available is to redistribute $E_{f}$. Following a literature review, three solutions have been suggested to improve the BV: varying the passivation material, changing the substrate material, and the application of a field plate (FP) structure.

In the following sections, different materials and geometric changes have been applied to the base eGaN HEMT PBM to evaluate how much the BV has increased or decreased. A deep analyses of the passivation material, substrate, and doping level are evaluated, as well as the impacts of including a FP contact on top of the HEMT structure. For each case, the BV progression and $E_{f}$ distribution is discussed. Table 9-1 lists the geometry applied to all structures. A comparison of six different structures is presented and summarized in Table 9-2. The schematic layers of these cases are as shown in Figure 9.5. Table 9-2 indicates the structure for each case of study. For the final case shown in Figure 9.5(b) [173], a FP is added to reshape the electric field $E_{f}$ distribution in the channel, and reduce its peak at the point of interest [174].

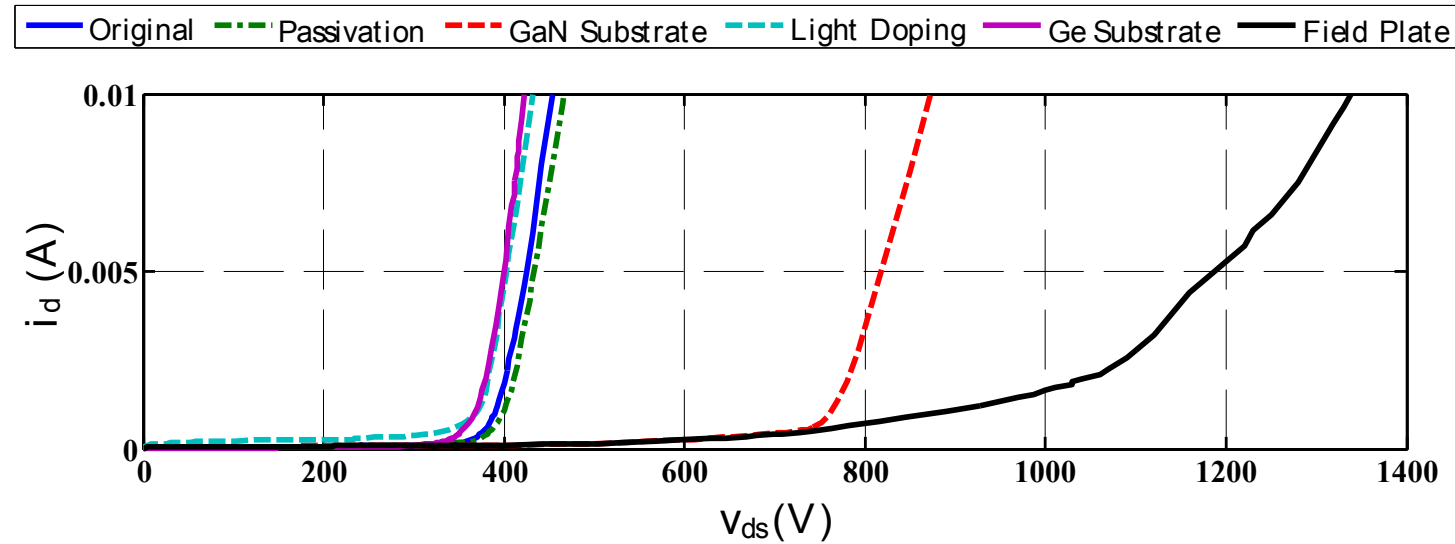

Figure 9.6. eGaN HEMT PBM Breakdown Voltage Analysis for all Cases of Study. 
Table 9-3. eGaN HEMT PBM Breakdown Voltage and Maximum Electric Field for all Cases at 4 Heights.

\begin{tabular}{|c|r|r|r|r|r|r|}
\hline & Original & \multicolumn{1}{|c|}{$\begin{array}{c}\text { Passivation } \\
\text { Change }\end{array}$} & $\begin{array}{c}\text { Light } \\
\text { Doping }\end{array}$ & $\begin{array}{c}\text { Ge } \\
\text { Substrate }\end{array}$ & $\begin{array}{c}\text { GaN } \\
\text { Substrate }\end{array}$ & $\begin{array}{c}\text { Field } \\
\text { Plate }\end{array}$ \\
\hline$E_{{\text {max } y_{1}}(\mathrm{MV} / \mathrm{cm})}$ & 1.893 & 2.098 & 2.229 & 2.494 & 1.366 & 2.150 \\
\hline$E_{{\text {fmax } y_{2}}(\mathrm{MV} / \mathrm{cm})}$ & 2.659 & 2.351 & 2.555 & 2.848 & 1.960 & 5.694 \\
\hline$E_{f \max y_{3}}(\mathrm{MV} / \mathrm{cm})$ & 2.982 & 2.667 & 2.909 & 3.216 & 2.036 & 2.468 \\
\hline$E_{f \max y_{4}}(\mathrm{MV} / \mathrm{cm})$ & 7.439 & 6.233 & 6.737 & 7.218 & 4.467 & 4.622 \\
\hline $\mathrm{BV}(\mathrm{V})$ & 366.5 & 384.3 & 368.7 & 353.7 & 769.2 & 1092.1 \\
\hline$V_{d s}(\mathrm{~V})$ & 354.6 & 351.9 & 354.4 & 353.7 & 354.3 & 350.5 \\
\hline
\end{tabular}

\subsection{Result Analysis of the GaN HEMT}

Results of each BV progression are conducted under the eGaN HEMT off-state at a gate voltage $v_{g}=0 \mathrm{~V}$ and are shown in Figure 9.6. The BV is defined as the maximum slope of the I-V characteristic curve. The $E_{f}$ distribution has been plotted at four different $y$ cross-sections across the structure. The locations are as indicated in Figure 9.7. In Figure 9.7, $y_{1}$ corresponds to a cross section through the passivation layer, $y_{2}$ corresponds to the surface of the device, $y_{3}$ corresponds to the heterojunction where source, gate, and drain contacts as well as the passivation layer meets the $\mathrm{AlGaN}$ layer, and $y_{4}$ corresponds to the 2DEG channel, respectively. For a fair comparison, Figure 9.8 is performed at the lowest BV for all structures, which occurs in the Ge substrate case (353.7 V).

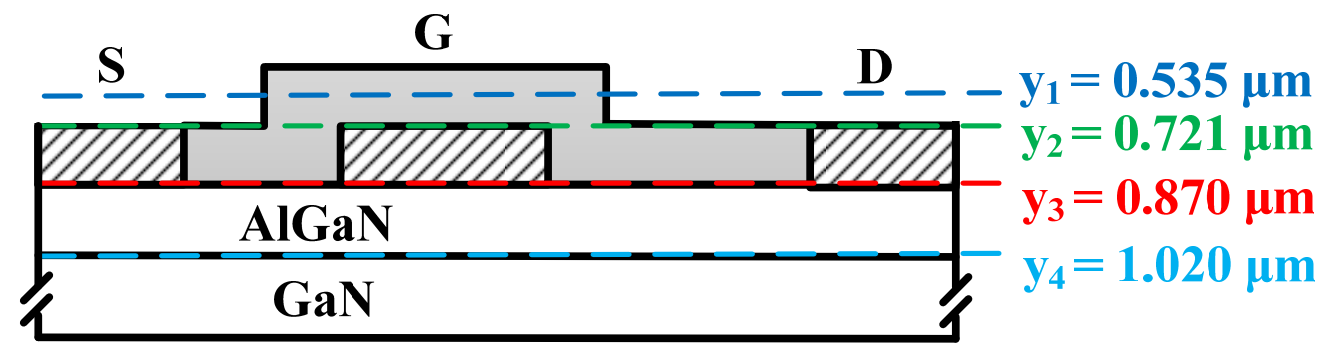

Figure 9.7. Locations of the Electric Field Cross-Sections. 

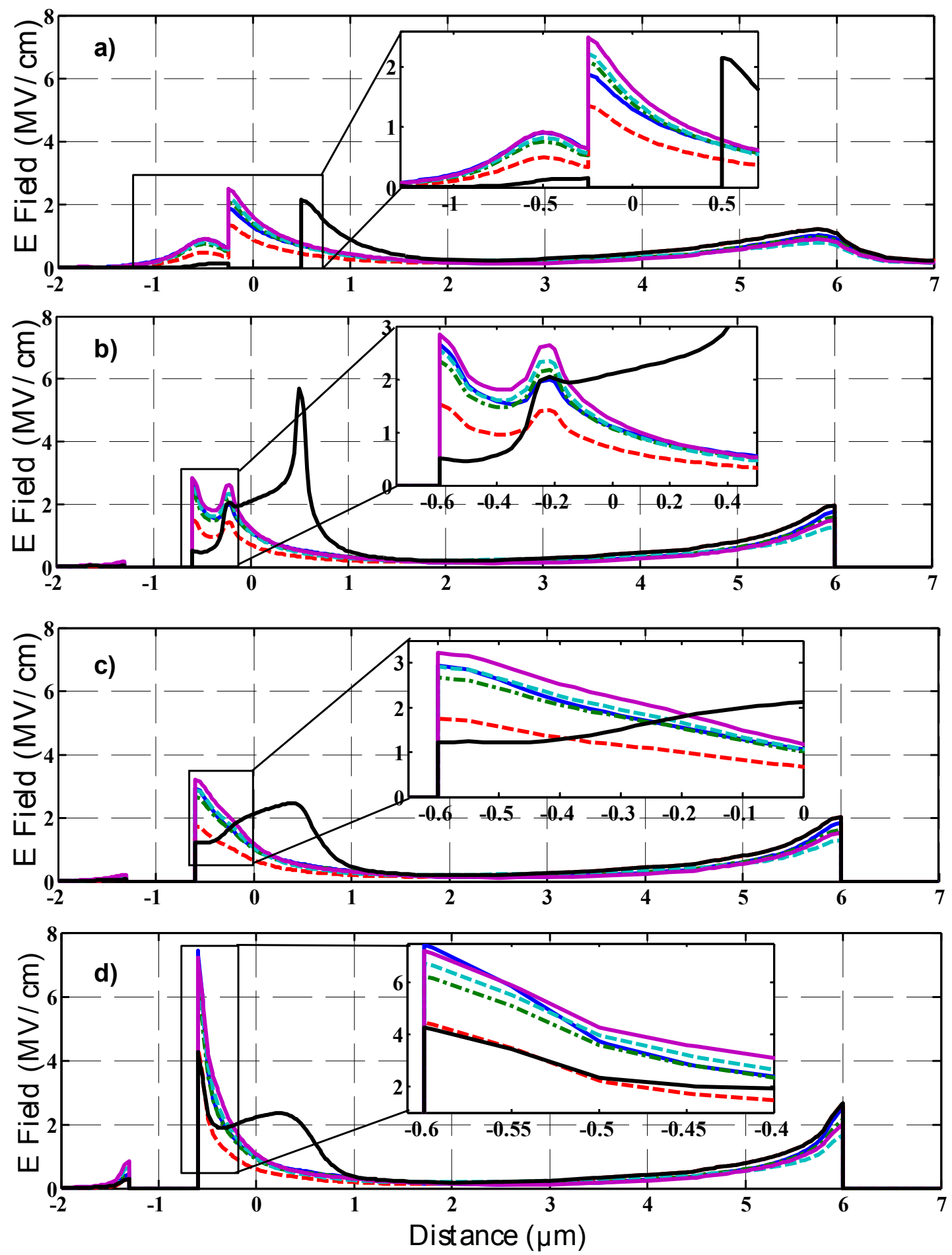

Figure 9.8. eGaN HEMT PBM Electric Field Distributions along the $\mathrm{x}$-axis at a) $y_{1}=$ $0.535 \mu \mathrm{m}$, b) $y_{2}=0.721 \mu \mathrm{m}$, c) $y_{3}=0.870 \mu \mathrm{m}$, and d) $y_{4}=1.020 \mu \mathrm{m}$. 
Changing the passivation from silicon dioxide $(\mathrm{SiO} 2)$ to silicon nitride $(\mathrm{SiN})$ had a small positive effect on the BV and $E_{f \max }$, viewed in Figure 9.8 and summarized in Table 9-3. As a result of its increased performance and reduction of $E_{f \max }, \mathrm{SiN}$ is kept as the insulator for all remaining cases. In the next stage, the Si substrate is removed by etching resulting in a huge increase in the BV, which was to be expected [175].

The fourth case is the study of a doping variation, as outlined previously in Table 9-2 (Light doping). For this case, the typical doping profiles were reduced by $10^{3}$ for all acceptor and donor concentrations. In this case, the variation does not yield a favorable result, presenting one of the smallest $\mathrm{BV}$ and largest $E_{\text {fmax }}$. These characteristics can be observed in Figure 9.8, where the maximum $E_{f}$ at each cross-section is shown in Table 9-3 increasing at all heights as compared to the passivation change case.

Next, another change of the substrate from Si to germanium (Ge) in the base eGaN HEMT was also not positive, resulting in the lowest BV and the reference value for the $E_{f}$ distribution sets. Finally, the addition of a FP to the current best case (Si substrate removal) yielded the best result. The FP result was the most interesting, as it distributed the $E_{f}$ in a way that did not allow for an early breakdown of the device.

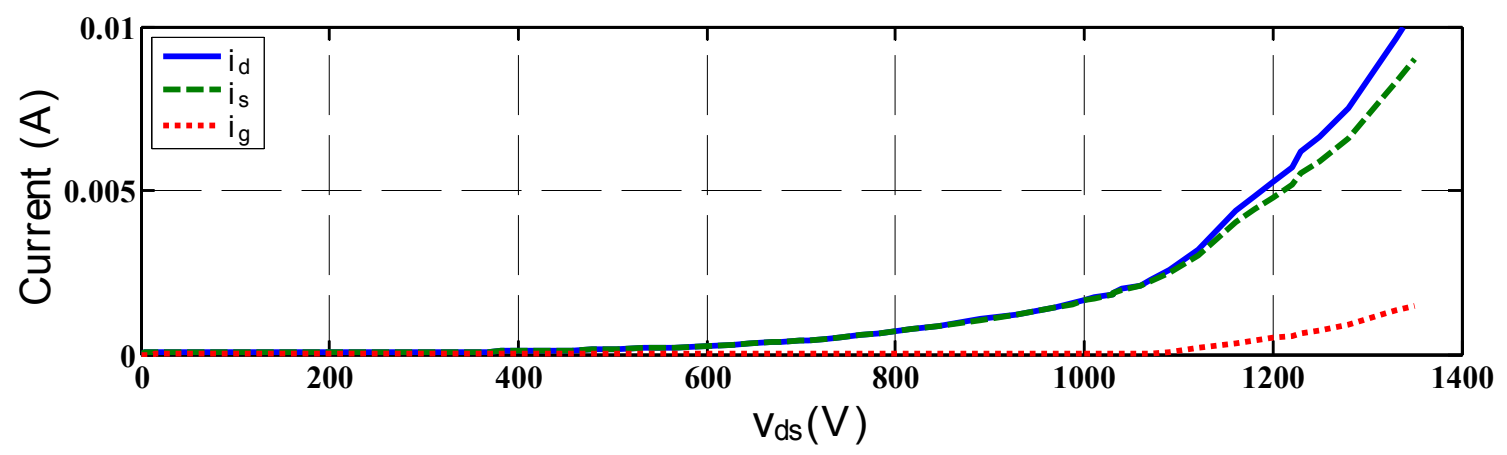

Figure 9.9. Drain, Source and Gate currents for the Field Plate Case. 
Figure 9.9 demonstrates that the origin of the BV for the FP case is drain-source subthreshold leakage [172]. The $E_{f}$ distribution along the $x$-axis is presented at all heights as depicted in Figure 9.8. Distance $y_{2}$ is the height where the $E_{f \max }$ appears for the FP case, while $y_{4}$ is where the $E_{\text {fmax }}$ appears for the remaining cases. For each case, the drain voltage is as indicated in Table 9-3, which is approximately equal to the Ge BV. Thus, from Figure 9.8(b) and Table 9-3, it can be generally noted that as $E_{\text {fmax }}$ decreases, the $\mathrm{BV}$ increases in most cases. This phenomenon was expected. However, $E_{f \max }$ is the highest for the FP device, which appears to be contradictory. This can be explained by noticing that the $E_{f \max }$ is located at $x=0.5 \mu \mathrm{m}$, which is at the end of the FP contact and separated from the 2DEG by the passivation layer ( $\mathrm{SiN}$ insulator) with a very high dielectric strength of $10 \mathrm{MV} / \mathrm{cm}$.

\subsection{Further Investigation of the Field Plate Case}

Following a comprehensive investigation of a number of geometrical, material, and physical changes to the original GaN HEMT, device performance following the application of a FP was found to be the best case. Given these findings, a deeper investigation is conducted into the physics behind the FP performance, where a theoretical model is presented. Following a presentation of a theoretical model, the PBM results from the FP case will be investigated in detail.

\subsubsection{Theoretical Model}

A simple model of the complex charge distribution within the AlGaN donor layer determining $n_{s}$ is proposed in Reference [176]. The components of the charge distribution are as shown in Figure 9.10(a), including the polarization dipole charges $n_{p+}$ and $n_{p-}$ at 
opposite faces of the donor layer, the insulator-donor layer interface charge $n_{t i}$, and the ionized unintentional doping change, $n_{d}$, all per $\mathrm{cm}^{2}$. Hence, the donor layer charge distribution is equivalent to a single positive sheet change located along the heterojunction (see Figure 9.10(b)). The sheet concentration $n_{f}$ is the algebraic sum of the donor layer charges and is equal to $n_{s}$. Note that $n_{f}$ is sensitive to $n_{t i}$. A more accurate but complex model of the charge distribution is presented in Reference [177]. In the reference, the electrostatic potential of the total AlGaN layer is proposed as:

$$
\phi_{\text {AlGaN }}(y)=\left(\frac{P_{\text {spAlGaN }}-P_{\text {spGaN }}}{\varepsilon}+2 \xi \gamma_{x x}-\frac{q n_{s}}{\varepsilon}\right)(y)
$$

where $\varepsilon=\varepsilon_{\text {AlGaN }}+\frac{e_{33}{ }^{2}}{C_{33}}, \xi=\frac{e_{31}-e_{33} C_{13}}{\varepsilon C_{33}}, \gamma_{x x}$ is a strain constant defined as $\gamma_{x x}=$ $\frac{a_{G a N}-a_{\text {AlGaN }}}{a_{\text {AlGaN }}}$, and remaining parameters are summarized in Table 9-4 and Table 9-5.

Figure 9.11 illustrates a cross-section of the HEMT structure with a FP, passivated with $\mathrm{SiN}$ in order to reduce the traps. The FP is deposited like a second gate electrode above the passivation layer. This study follows Reference [174], however, some inconsistences were found preventing a completion of the analytical investigation. Here, a formulation of the problem is established for a future resolution.

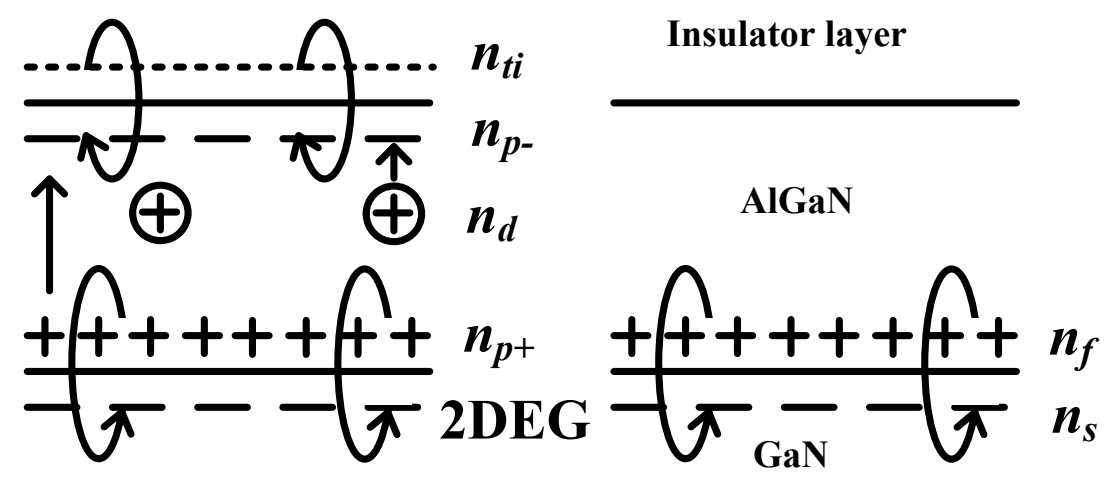

Figure 9.10. Illustration of the eGaN HEMT Charge Distribution Modeling: Detailed picture of the charges in the AlGaN layer (Left) and a Simplified model (Right). 
Table 9-4. Polarization Parameters for the Electrostatic Potential on AlGaN Devices.

\begin{tabular}{|c|c|}
\hline Variable & Description \\
\hline$P_{\text {spalGaN }}(m)=-0.052 m-0.029 \mathrm{C} / \mathrm{m}^{2}$ & AlGaN Spontaneous Polarization \\
\hline$P_{\text {spGaN }}=-0.029 \mathrm{C} / \mathrm{m}^{2}$ & GaN Spontaneous Polarization \\
\hline$a_{\text {GaN }}=3.189 \AA$ & Lattice Constant \\
\hline$a_{\text {AlGaN }}(m)=-0.77 m+3.189 \AA$ & Lattice Constant \\
\hline$e_{31}(m)=-0.11 m-0.49 \mathrm{C} / \mathrm{m}^{2}$ & Piezoelectric Constant \\
\hline$e_{33}(m)=0.73 m+0.73 \mathrm{C} / \mathrm{m}^{2}$ & Piezoelectric Constant \\
\hline$C_{31}(m)=5 m-103 \mathrm{GPa}$ & Elastic constant \\
\hline$C_{33}(m)=-32 m+405 \mathrm{GPa}$ & Elastic constant \\
\hline
\end{tabular}

Table 9-5. GaN HEMT with Field Plate Design Parameters for Theoretical Model.

\begin{tabular}{|c|c|r|}
\hline Name & Variable & \multicolumn{1}{|c|}{ Value } \\
\hline Width of the Source & $L$ & $1.00 \mu \mathrm{m}$ \\
\hline Source-to-Gate Lateral Displacement & $L_{s g}$ & $1.70 \mu \mathrm{m}$ \\
\hline Width of the Field Plate & $L_{f p}$ & $1.40 \mu \mathrm{m}$ \\
\hline AlGaN Thickness & $d_{t}$ & $0.02 \mu \mathrm{m}$ \\
\hline Width of the Gate & $L_{g}$ & $0.70 \mu \mathrm{m}$ \\
\hline Location where Passivation Meets Drain Contact & $L_{2}$ & $6.00 \mu \mathrm{m}$ \\
\hline Gate-to-Drain Lateral Displacement & $L_{g d}$ & $6.90 \mu \mathrm{m}$ \\
\hline Effective Length of the Passivation layer & $L_{e f f}$ & $5.50 \mu \mathrm{m}$ \\
\hline Width of the Drain & $L_{d}$ & $1.00 \mu \mathrm{m}$ \\
\hline Thickness of the Active GaN Layer & $d_{G a N}$ & $16.00 \mu \mathrm{m}$ \\
\hline Relative Permittivity of the AlGaN Layer & $\varepsilon_{A l G a N}$ & 8.82 \\
\hline Field Plate Edge Location Reference to Passivation & $L_{1}$ & $0.50 \mu \mathrm{m}$ \\
\hline Passivation Layer Thickness & $t_{i}$ & $0.30 \mu \mathrm{m}$ \\
\hline Relative Permittivity of the Passivation Layer & $\varepsilon_{t i}$ & 7.50 \\
\hline
\end{tabular}

The origin of the coordinates are placed at the surface of the AlGaN (see Figure 9.11) with a FP length of $L_{1}=L_{F P}$, an insulator length of $L_{2}$, the effective length of the insulator layer $L_{\text {eff }}=L_{2}-L_{1}$, the applied drain voltage $V_{d}$, the total thickness of the AlGaN material layer $d_{T}$, and the thickness of the insulator $t_{i}$. Assuming a linear distribution of the potential along the insulator, we can write: 


$$
\varphi_{f}(x)=\left\{\begin{array}{cc}
0 & x \leq L_{1} \\
\frac{V_{d}\left(x-L_{1}\right)}{L_{e f f}} & L_{1} \leq x \leq L_{2} \\
V_{d} & x \geq L_{2}
\end{array}\right.
$$

where $\varphi_{f}(x)$ represents the potential distribution along the insulator [178]. The potential distribution in the semiconductor $\operatorname{AlGaN} \phi(x, y)$ must satisfy the Poisson equation as in Equation (9-3):

$$
\frac{\partial^{2} \phi(x, y)}{\partial x^{2}}+\frac{\partial^{2} \phi(x, y)}{\partial y^{2}}=-\frac{q n_{s}}{\varepsilon_{A l G a N}}
$$

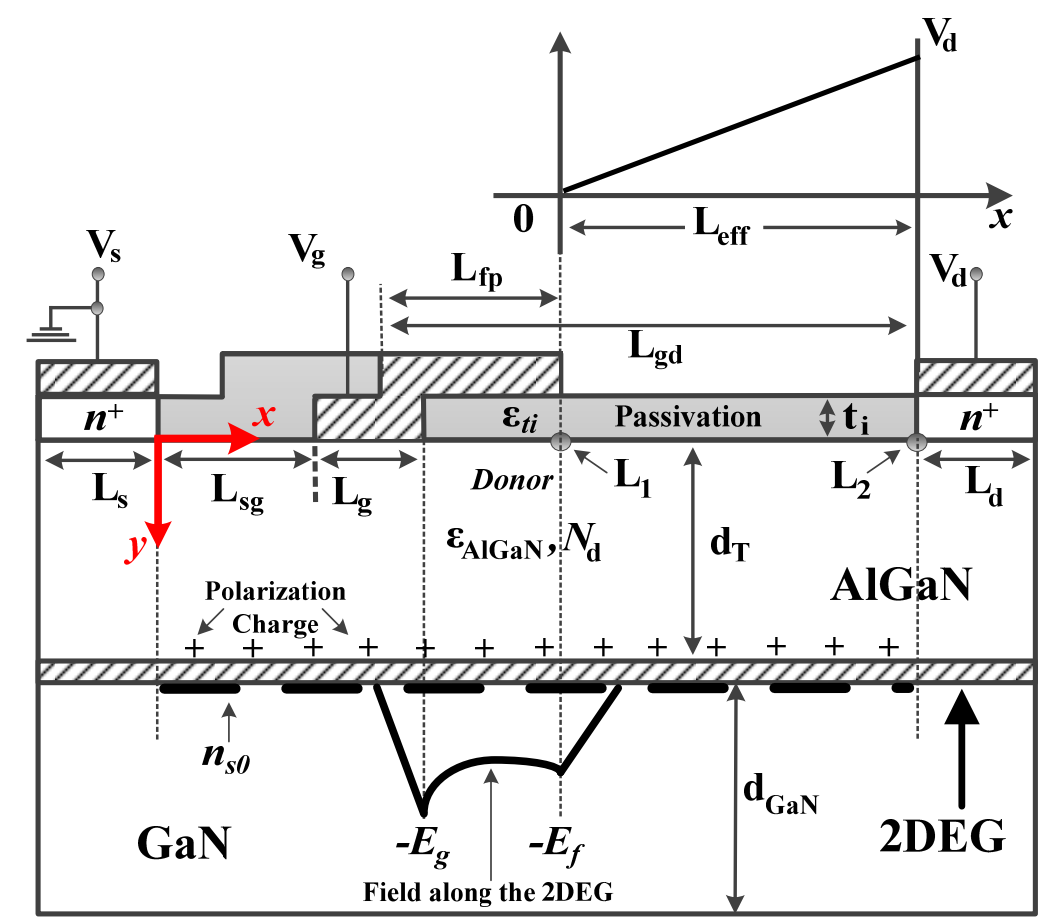

Figure 9.11. Cross-section of the HEMT with a Field Plate (FP-HEMT).

Assuming that the drain region of the structure is completely depleted at the breakdown, the potential function $\phi(x, y)$ can be approximated from References [174], [178], and [179] by an expression for the 2D distribution as a parabolic approximation.

$$
\phi(x, y)=\phi_{0}(x)+\phi_{1}(x) y+\phi_{2}(x) y^{2}+\phi_{A l G a N}(y)
$$


where the coefficients $\phi_{0}(x), \phi_{1}(x)$ and $\phi_{2}(x)$ are only functions of $x$. These coefficients are determined by the boundary conditions, which are applied as follows:

$$
\begin{gathered}
E_{y}(x, 0)=-\left.\frac{\partial \phi(x, y)}{\partial y}\right|_{y=0}=k \frac{\varphi_{f}(x)-\phi_{f}(x)}{t_{i}} \\
\phi\left(x, d_{T}\right)=\phi_{c}(x)
\end{gathered}
$$

where $k=\varepsilon_{i} / \varepsilon_{A l G a N}, \phi_{c}(x)$ is the potential distribution along the 2DEG channel, and $\phi_{f}(x)$ is the potential distribution along the AlGaN surface.

At $y=0, \phi(x, 0)=\phi_{0}(x)=\phi_{f}(x)$ from Figure 9.11. From Equation (9-5), this results in:

$$
\phi_{1}(x)=-k \frac{\varphi_{f}(x)-\phi_{f}(x)}{t_{i}}+\frac{\partial \phi_{A l G a N}}{\partial y}
$$

From Equation (9-6), $\phi_{2}(x)$ is obtained as:

$$
\phi_{2}(x)=\frac{1}{{d_{T}}^{2}}\left(\phi_{c}(x)-\phi_{f}(x)-\phi_{1}(x) d_{T}-\phi_{A l G a N}\left(d_{T}\right)\right)
$$

Introducing $\phi_{0}(x), \phi_{1}(x)$ and $\phi_{2}(x)$ back into Equation (9-4) and solving the Poisson equation results in two ordinary differential equations (ODE) to find the $2 \mathrm{D}$ potential distribution.

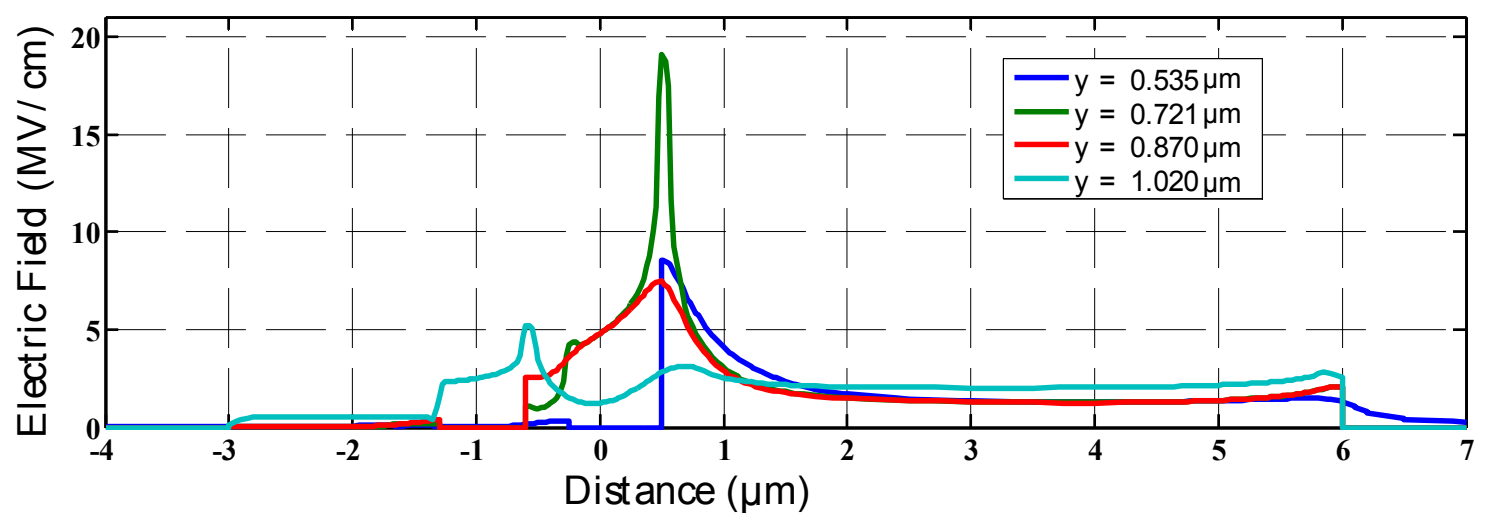

Figure 9.12. eGaN HEMT PBM Electric Field Distribution for the Field Plate Case at its Individual Breakdown Voltage for all y-distances. 

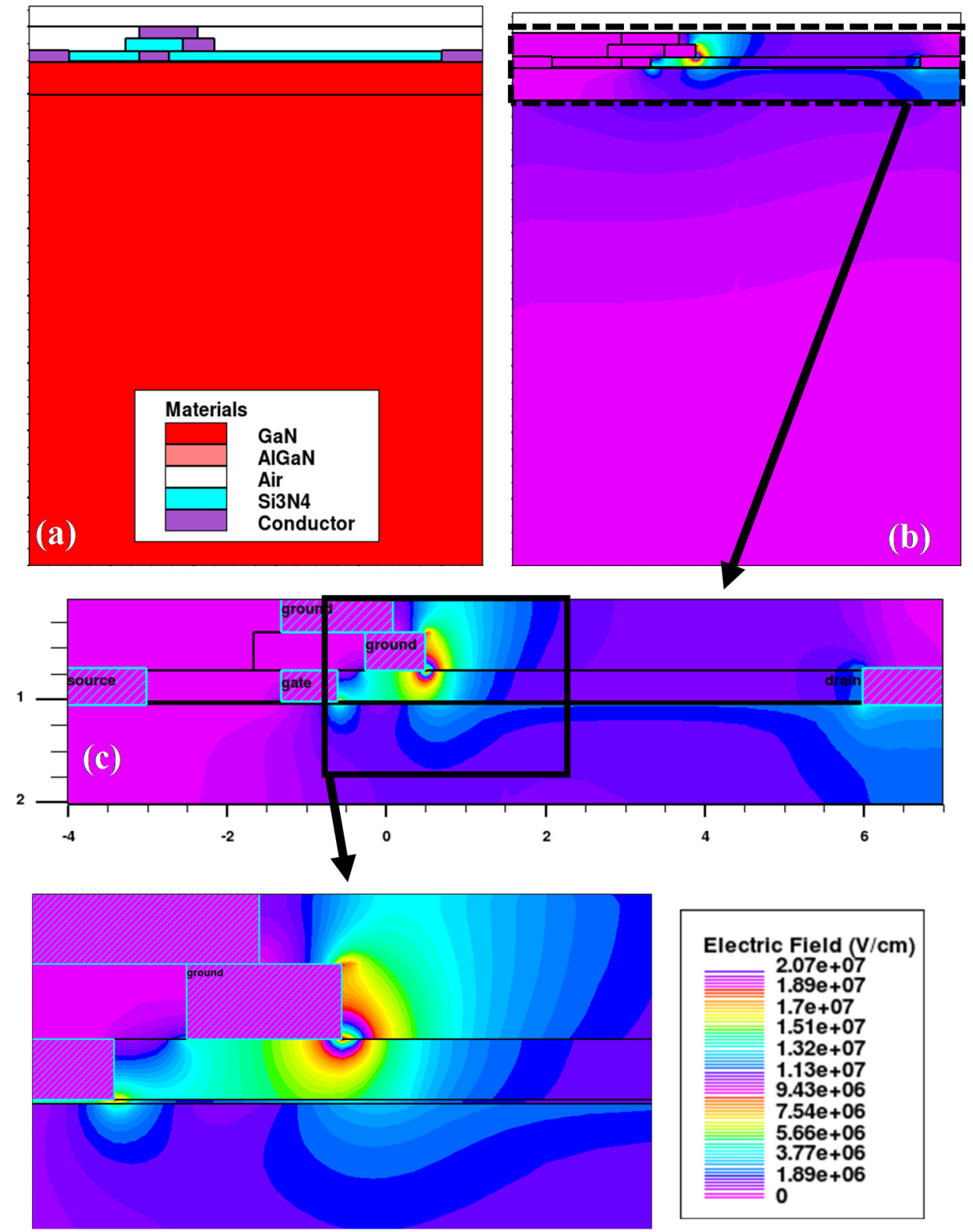

Figure 9.13. eGaN HEMT PBM 2D Electric Field Distribution for the Field Plate Case.

\subsubsection{Analysis of the Field Plate Case}

For the final FP PBM case, the $E_{f}$ distribution along the $x$-direction at different heights has been calculated at its $\mathrm{BV}\left(V_{d s}=B V=1092.1 \mathrm{~V}\right)$ as shown in Figure 9.12. Figure 9.12 shows the key advantage of the FP layout, as it redistributes $E_{f}$ and relocates $E_{f \max }$ along 
the $x$ and $y$ plane. This keeps it below the critical value, hence increasing the BV. The same behavior is more clearly observed in a 2D map in Figure 9.13. It is shown that the highest field strength is present at the corner of the FP-SiN interface on the drain side of the gate, where a field of $20.7 \mathrm{MV} / \mathrm{cm}$ is experienced.

\subsection{Summary}

In this chapter, a PBM was developed to more closely represent the operation of a GaN HEMT device. Using a base PBM, an exploratory section evaluated how modifying the geometry and materials of the eGaN HEMT structure can result in an increase in its maximum operating voltage. Five different modifications were tested on the GaN HEMT base model including passivation, an altered doping profile, an etching and modification of the substrate, and the addition of a FP. For each case, BV curves and an $E_{f}$ analysis was conducted at four important cross-sections across the structure, revealing how each modification impacted the device operation. The best case was established to be a combination of SiN passivation, the application of a GaN substrate, and modification of the geometry to include a FP. This case was further investigated by the introduction of a theoretical model. Through the development of a GaN HEMT with these specifications, future converters for EVs and SPS can contain fewer stages while minimizing through the switches.

In the last two chapters, efficient HESS design has focused upon the design of interfacing power electronic converters, beginning from a power electronics perspective and ending deep into the physics of implementing GaN HEMT switches in place of legacy Si technology. From this point forward, HESS design will progress into development and design with regards to ES device selection, control, and specific applications they support. 
The design and control of a three-device HESS containing both lead acid and lithium ion batteries and a SC will be evaluated in the next chapter for their implementation in modern SPS. A lithium ion battery and SC HESS is designed in Chapter 11 for EV applications, implementing a new control scheme with a goal to reduce cycling on the lithium ion battery. Finally, in Chapter 12 a lead acid battery and flywheel HESS system is evaluated to reveal additional advantages a HESS can have in improving the power quality of a DC system. 


\section{Chapter 10 Hybrid Energy Storage Systems for Shipboard Power Systems and Transportation Electrification Systems}

\subsection{Introduction}

The challenge in installing a wide range of energy storage (ES) types on the utility grid is relatively simpler, as a result of reduced concerns over weight and space. Mobile applications, however, do not have this luxury. The localized microgrid present on a ship, aircraft, or electric vehicle (EV) is susceptible to major operational and logistic challenges. Heavy and frequent pulsed loads, which may present a minimal disturbance to a utility grid-connected system, can prove to be catastrophic when generation resources are limited. A Naval ship is composed of a complex, isolated power system, typically consisting of two main turbine generators (MTG) and two auxiliary turbine generators (ATG) [158]. For example, USS Zumwalt DDG-1000 all-electric ship contains 74.8 MW of onboard total shaft power. Critical loads reserve approximately $15 \%$ of the available energy, but the next generation of equipment introduces loads several magnitudes higher [180]. Energy and power requirements can vary from $100 \mathrm{~kW}$ to $10 \mathrm{GW}$ over durations of microseconds to seconds [181]-[182]. Without proper selection and control, ES units may experience high depth of discharge (DoD), which would reduce their capability of responding quickly to fluctuating demands and significantly reduce their lifespans, or state of health (SoH) [183].

In utility grid applications, hybrid ES system (HESS) deployment and control has recently gained increased attention [42]. These cases have been two-fold: providing a method to reduce the intermittency associated with renewable energy sources, while offering ancillary backup services. The grid-connected system in Reference [184] 
demonstrated a combination of the zinc bromide flow battery (FB) and supercapacitors (SC) to reduce the voltage and frequency instabilities as a result of the variability inherent to wind generation. Several vignettes were tested varying the optimal size of the parallel SC bank, where the SC handled short variations and the FB handled longer variations. Just as ES has been utilized to handle some of the intermittencies associated with generation, their inclusion has been equally as useful to support pulsed loads. Introduced previously in Chapter 3, pulsed loads are commonplace in military applications, but are present in a wide range of other applications and fields as well [185].

The aforementioned grid-connected systems can reduce impacts following a major disturbance or a variance in generation. However, under islanded or stand-alone cases, system stability would rely solely on the support of ES when MTGs and ATGs reach their generation capacities. In Reference [4], a battery management system scheme was demonstrated to control the power flow in a lithium ion-based battery array. The system was tested under both grid-connected and islanded modes of operation. In islanded mode, a battery with an inverter acts as a synchronous generator, providing voltage and frequency support. A number of other control strategies have been demonstrated in literature, but have traditionally only focused on one type of ES [20],[23],[24],[186].

A shipboard power system (SPS) presents unique challenges in terms of ES deployment, since the SPS is inherently an islanded system. Pulsed load management and mitigation is an emerging topic in the future all-electric SPS. In Reference [187], a $0.25 \mathrm{~Hz}$ $36 \mathrm{MW}$ pulsed load was tested on a notional SPS model, where case studies were conducted over the use of a dynamic reactive compensator to maintain bus voltages. However, power demands of multiple pulsed loads present a major challenge in terms of design and 
implementation and are synonymous in SPS. The electromagnetic (EM) railgun and EM catapult were investigated in Reference [188], where short-term pulsed loads were tested both significantly exceeding the available energy from the MTGs when tested independently. ES was proposed as a solution to support both, but was not demonstrated. An extensive review into the impact of multiple pulsed loads on the electric SPS was performed in Reference [189]. EM railgun and free electron laser firing profiles were tested as connected pulsed loads without electrochemical ES, but employed the railgun launcher rotor as flywheel ES. The system proved the current SPS infrastructure could support at least one critical pulsed load, but not both.

Investigations have been performed into deploying electrochemical ES devices as well [30]. In Reference [32], an SC was tested independently with an EM railgun to fill an 800 $\mathrm{kA}$ firing pulse. The topology was capable of supplying the pulse, but required an enormous 500 F SC. Combinations of ES have offered more realistic solutions [41],[44],[45],[190]. In Reference [45], lead acid and sodium sulfide battery banks were simulated in parallel on an SPS to fill a single pulsed load. Each ES bank was installed on a different zonal bus, where it was noted that the ES units were able to respond faster than the MTG to deliver energy. However, HESS support has not yet been evaluated on the same bus or in a series configuration. A control topology for a SPS was proposed in Reference [191], where a parallel-configured battery and SC HESS was simulated with respect to both a constant and pulsed load. Four operation modes were tested to meet critical and pulsed load demands, but only the voltage recovery period following the pulse was discussed and no investigation was provided into the SC or battery performance. Furthermore, the battery type was not identified. 
Typical battery and SC HESS have utilized parallel topologies; however, control of these systems is challenging as a result of the wide voltage operating range of the SC. Without a specialized interfacing converter similar to the synchronous buck introduced in the previous chapter, the SC terminal voltage follows that of the battery. This would leave a significant amount of unutilized energy due to a narrowed operating range [161]. Moreover, a mismatch in the equivalent series resistance (ESR) of each ES device could result in unequal, uncontrolled charging or induce internal circulating currents, a phenomena which parallel-configured lead acid and lithium ion batteries could also be prone to. In Reference [192], a supervisory energy management controller was developed to effectively split an EV load demand between a lithium ion battery and SC in a parallelconfigured HESS. A multiobjective optimization procedure accounting for both the battery and SC equivalent models and converter topology was solved for using dynamic programming. Using these results, a neural network was trained and deployed on the controller with objectives to preserve the battery SoH and enhance total HESS efficiency.

Although literature has demonstrated the impacts of pulsed loads on SPS, it has been limited to testing of each pulsed load independently. In practicality, a robust system requires the capability in handling a variety of SPS equipment over the same period. In this chapter, several series-configured HESS combinations are proposed and tested through the utilization of lead acid and lithium ion batteries as well as a SC bank. The performance of each combination is analyzed. Following the selection of each series-connected HESS, a specialized dispatch control scheme is demonstrated using four Energy Storage Management Controllers (ESMC) to replenish some or all of the energy required to serve one of two pulsed loads. In this effort, an attention is also placed upon the battery SoH 
tradeoffs that are involved in each control scheme. Coined as "rolling charging," a coordination control scheme between the pulsed loads and charging is applied by the ESMC to the heavier pulsed load in an effort to recover part of the discharged energy and reduce the impact of the disturbance on the SPS electrical grid. The dynamics of each ES device are optimized with respect to their operational constraints, while exercising best practices to preserve their SoH.

\subsection{Modeling of Multiple Energy Storage Types}

The following sections describe the selected models for the lead acid and lithium ion batteries as well as the SC in detail. The operational characteristics of each ES play a pivotal role in improving the base case, which is designated as a traditional seriesconnected lead acid battery system. In order to demonstrate the limitations of each ES device, the performance and operational constraints of each are discussed.

\subsubsection{Lead Acid Battery Shipboard Power System Model}

In previous chapters, the depth of the battery model has increased drastically from a basic Randles equivalent circuit model to a comprehensive physics based model. However, it is important to mention these are simulation and application-specific thus for the SPS, the lead acid battery model is broken into two parts. The first part models the ES portion through the application of a very large capacitor in parallel with a self-discharge resistor. Figure 10.1 provides a graphic representation of the relationship between the open circuit voltage (OCV) $\mathrm{V}_{\mathrm{OC}_{\mathrm{Pb}}}(t, \mathrm{I}, \mathrm{SoC}, \mathrm{T})$ and the State of Charge (SoC) for a lead acid battery. The second portion models its unique response. Figure 10.2 depicts the equivalent circuits for the three ES types under study, where the aforementioned energy model is represented by a nonlinear voltage source in Figure 10.2(a). To represent its unique dynamic response, 
lumped parameters based on Electrochemical Impedance Spectroscopy (EIS) were used. For the SPS, a $1^{\text {st }}$-Order Randles equivalent circuit was adequate, as the time constants of both pulsed loads fall within a similar low-frequency timescale. The ohmic resistance of the sulfuric acid electrolyte is represented by $\mathrm{R}_{t_{P b}}$, while the polarization resistance and capacitance are denoted by $\mathrm{R}_{p_{P b}}$ and $\mathrm{C}_{p_{P b}}$, respectively. The transfer function depicting the lead acid battery equivalent impedance is:

$$
\mathrm{Z}_{e q_{P b}}(s)=\mathrm{R}_{t_{P b}}+\frac{\mathrm{R}_{p_{P b}}}{\mathrm{C}_{p_{P b}} \mathrm{R}_{p_{P b}} s+1}
$$

The voltage response $\mathrm{V}_{P b}(t)$ at the battery terminals is:

$$
\mathrm{V}_{P b}(t)=\mathrm{V}_{\mathrm{oc} P \mathrm{~Pb}}(t)-\frac{1}{\mathrm{C}_{p_{P b}}}\left[e^{-\frac{t}{\mathrm{C}_{p_{P b}} \mathrm{R}_{p_{P b}}}}+\mathrm{C}_{p_{P b}} \mathrm{R}_{t_{P b}} \delta(t)\right] \mathrm{I}(t)
$$

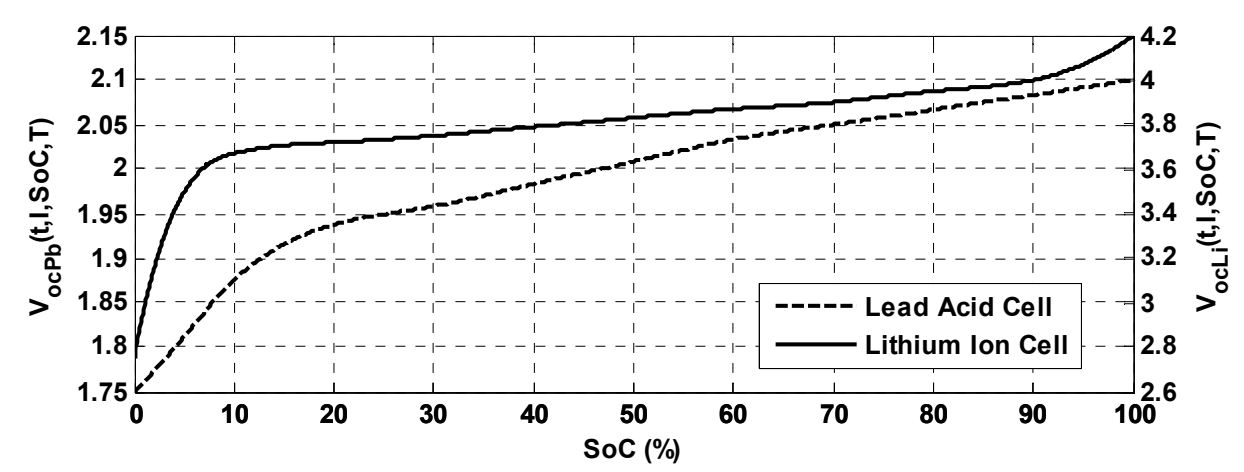

Figure 10.1. Open Circuit Voltage versus State of Charge for Lead Acid \& Lithium Ion Batteries.

\subsubsection{Lithium Ion Battery Shipboard Power System Model}

A two-part model was also used to differentiate the ES and equivalent circuit portions of the lithium ion battery. The ES portion is modeled similarly to that of the lead acid, except the behavior of the voltage versus its SoC is very different. Figure 10.1 depicts the nonlinear lithium ion $\mathrm{OCV} \mathrm{V}_{\mathrm{oc}_{\mathrm{Li}}}(t, \mathrm{I}, \mathrm{SoC}, \mathrm{T})$ versus the SoC as compared to lead acid. The lithium ion equivalent circuit is shown in Figure 10.2(b), following the same $1^{\text {st }}$-Order 
Randles circuit form. The transfer function depicting the equivalent impedance is:

$$
\mathrm{Z}_{e q_{L i}}(s)=\mathrm{R}_{t_{L i}}+\frac{\mathrm{R}_{p_{L i}}}{\mathrm{C}_{p_{L i}} \mathrm{R}_{p_{L i}} s+1}
$$

where $\mathrm{R}_{t_{L i}}$ represents the ohmic resistance of the lithium salt electrolyte and very different polarization resistance $\mathrm{R}_{p_{L i}}$ and capacitance $\mathrm{C}_{p_{L i}}$ values are used. The voltage response $\mathrm{V}_{L i}(t)$ at the battery terminals is:

$$
\mathrm{V}_{\mathrm{Li}}(t)=\mathrm{V}_{\mathrm{oc}_{\mathrm{Li}}}(t)-\frac{1}{\mathrm{C}_{p_{L i}}}\left[e^{-\frac{t}{\mathrm{C}_{p_{L i}} \mathrm{R}_{p_{L i}}}}+\mathrm{C}_{p_{L i}} \mathrm{R}_{t_{L i}} \delta(t)\right] \mathrm{I}(t)
$$

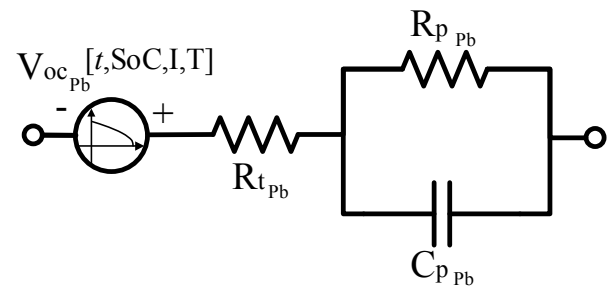

(a)

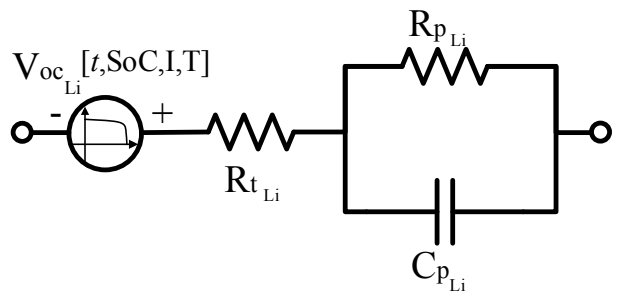

(b)

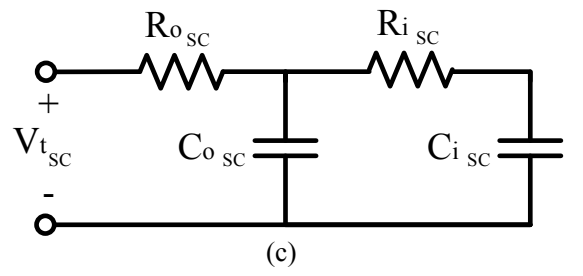

Figure 10.2. Shipboard Power System Equivalent Circuit Models for the (a) Lead Acid $(\mathrm{Pb})$ Battery, (b) Lithium Ion (Li) Battery, and (c) Supercapacitor (SC).

\subsubsection{Supercapacitor}

A SC provides much greater charge storage versus a traditional capacitor as a result of its highly amplified surface area. Composed of two porous electrodes divided by a separator soaked in a solvent electrolyte, no electrochemical reaction takes place. This makes its charge acceptance and delivery much faster than a battery with a cycle life of over 500,000 cycles, even under heavy operation [28]. The drawback, however, is a wide 
operating voltage range, from its maximum rated voltage to $0 \mathrm{~V}$. Furthermore, its energy density is significantly less than that of a battery. Despite the SC operating as an electrochemical ES device, its equivalent circuit model does not follow the same form as that of the batteries.

An extension beyond the common capacitor and ESR is based on a simplified physicsbased representation, where the SC is partitioned into resistor-capacitor (RC) segments associated with outside and inside the core material (Figure 10.2(c)). In Reference [141], four different SC equivalent models were identified from $2^{\text {nd }}$ to $5^{\text {th }}$-Order, dictated by the operating frequency. Since the pulsed loads in this study are of low-frequency, a $2^{\text {nd }}$-Order model is sufficient to model the steady-state and transient voltage fluctuation, where $\mathrm{R}_{o_{S C}}$ and $\mathrm{C}_{O_{S C}}$ represent the resistance and capacitance outside the electrode pore and $\mathrm{R}_{i_{S C}}$ and $\mathrm{C}_{i_{S C}}$ represent the resistance and capacitance inside. The transfer function depicting the SC equivalent impedance is:

$$
\mathrm{Z}_{e q_{S C}}(s)=\mathrm{R}_{o_{S C}}+\frac{\mathrm{R}_{i_{S C}} \mathrm{C}_{i_{S C}} s+1}{\mathrm{C}_{o_{S C}} \mathrm{C}_{i_{S C}} \mathrm{R}_{i_{S C}} s^{2}+\left(\mathrm{C}_{o_{S C}}+\mathrm{C}_{i_{S C}}\right) s}
$$

The voltage response $\mathrm{V}_{S C}(t)$ across the $\mathrm{SC}$ terminals is:

$$
\mathrm{V}_{\mathrm{SC}}(t)=\mathrm{V}_{\mathrm{t}_{S C}}(t)-\left[\frac{\mathrm{C}_{i_{S C}} e^{-\frac{\left(\mathrm{C}_{i_{S C}}+\mathrm{C}_{o_{S C}}\right) t}{\mathrm{C}_{o_{S C}} \mathrm{C}_{i_{S C} \mathrm{R}{ }_{S C}}}}}{\mathrm{C}_{o_{S C}}\left(\mathrm{C}_{o_{S C}}+\mathrm{C}_{i_{S C}}\right)}+\frac{1}{\mathrm{C}_{o_{S C}}+\mathrm{C}_{i_{S C}}}+\mathrm{R}_{o_{S C}} \delta(t)\right] \mathrm{I}(t)
$$

where $\mathrm{V}_{\mathrm{tSC}_{\mathrm{SC}}}(t)$ is the initial terminal voltage of the SC. 
Table 10-1. Batteries and Supercapacitor Simulation Parameters.

\begin{tabular}{l|l|r}
\hline \hline \multicolumn{1}{c|}{ Parameter } & Name & \multicolumn{1}{c}{ Value } \\
\hline Lead Acid Battery Initial Open Circuit Voltage & $\mathrm{V}_{\mathrm{Oc}_{\mathrm{Pb}}}$ & $13.17 \mathrm{~V}$ \\
\hline Lead Acid Battery Ohmic Resistance & $\mathrm{R}_{t_{P b}}$ & $90 \mathrm{~m} \Omega$ \\
\hline Lead Acid Battery Polarization Resistance & $\mathrm{R}_{p_{P b}}$ & $40 \mathrm{~m} \Omega$ \\
\hline Lead Acid Battery Polarization Capacitance & $\mathrm{C}_{p_{P b}}$ & $160 \mathrm{~F}$ \\
\hline Lithium Ion Battery Initial Open Circuit Voltage & $\mathrm{V}_{\mathrm{oc}_{\mathrm{Li}}}$ & $12.80 \mathrm{~V}$ \\
\hline Lithium Ion Battery Ohmic Resistance & $\mathrm{R}_{t_{L i}}$ & $12 \mathrm{~m} \Omega$ \\
\hline Lithium Ion Battery Polarization Resistance & $\mathrm{R}_{p_{L i}}$ & $86 \mathrm{~m} \Omega$ \\
\hline Lithium Ion Battery Polarization Capacitance & $\mathrm{C}_{p_{L i}}$ & $0.015 \mathrm{~F}$ \\
\hline Supercapacitor Initial Terminal Voltage & $\mathrm{V}_{\mathrm{t}_{S C}}$ & $16.13 \mathrm{~V}$ \\
\hline Supercapacitor Resistance Outside Pore & $\mathrm{R}_{o_{S C}}$ & $35 \mathrm{~m} \Omega$ \\
\hline Supercapacitor Capacitance Outside Pore & $\mathrm{C}_{O_{S C}}$ & $42 \mathrm{~F}$ \\
\hline Supercapacitor Resistance Inside Pore & $\mathrm{R}_{i_{S C}}$ & $70 \mathrm{~m} \Omega$ \\
\hline Supercapacitor Capacitance Inside Pore & $\mathrm{C}_{i_{S C}}$ & $18 \mathrm{~F}$ \\
\hline
\end{tabular}

\subsection{Equivalent Models for Hybrid Energy Storage Systems}

In this section, generalized equivalent models for HESS are obtained. Three distinct cases with different combinations are investigated. In order to validate the obtained HESS models, each equivalent circuit is constructed within the MATLAB/Simulink environment. The response of the derived model is then compared to that which is obtained from an experimental setup. In the experimental setup, an $85 \mathrm{~W}$ constant power and $280 \mathrm{~W}$ pulsed power load operating at $0.1 \mathrm{~Hz}$ is used.

Each lead acid battery is a flooded and sealed module containing six cells in series and have a nominal voltage and capacity of $12 \mathrm{~V}$ and $21 \mathrm{Amp}$-hours (Ah), respectively [193]. The lithium ion battery module is composed of three individual cells connected in series, where the nominal voltage of each is $3.7 \mathrm{~V}$, delivering a similar module voltage to the lead acid at $11.1 \mathrm{~V}$ under a matching capacity of $21 \mathrm{Ah}$ [164]. The SC used is manufactured by Maxwell and composed of two $58 \mathrm{~F}$ modules in parallel. Each SC is rated at a maximum 
voltage of $16.2 \mathrm{~V}$ [195]. Table 10-1 shows the equivalent circuit parameters used for each ES type in simulation. Parameters for both lead acid and lithium ion batteries were obtained experimentally and compared to typical values in References [91] and [109], while SC parameters were determined using the procedure outlined in Reference [141]. It is assumed that the SoH of batteries of the same type are close, thus their parameters are similar.

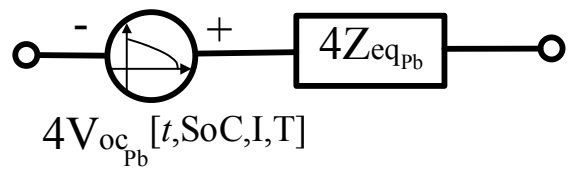

Figure 10.3. Lumped Parameter Model for Case I.
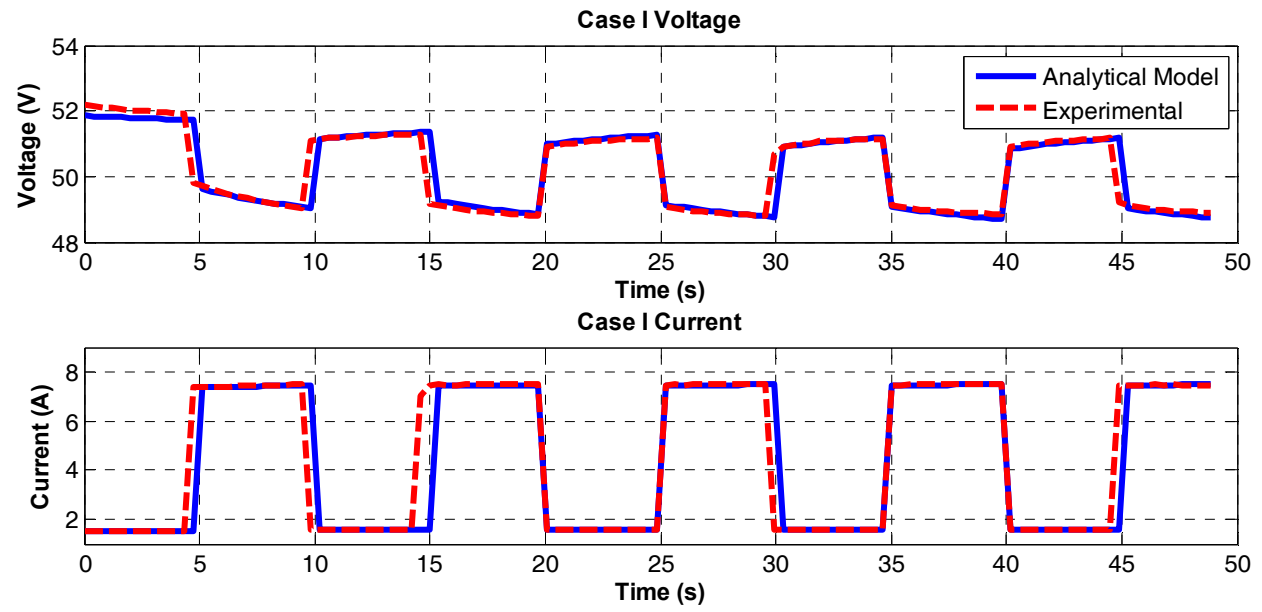

Figure 10.4. Verification of Voltage and Current under $0.1 \mathrm{~Hz}$ Test Pulse for Case I.

\subsubsection{Case I: 4 Lead Acid Batteries}

The first case presents the base case, where a traditional 100\% lead acid battery stack connected in series is tested using four modules of matching voltage and capacity. It is anticipated that without the ESMC, all modules would serve the pulsed load until a prespecified full discharge voltage. Then, to replenish the lost energy, all modules are required to be decoupled and charged offline. The lumped parameter model of this system is shown in Figure 10.3. Since a potentially $4^{\text {th }}$-Order model is condensed to $1^{\text {st }}$-Order, similar to 
Equation (10-1), the equivalent impedance $Z_{e q_{c a s e 1}}$ is:

$$
\mathrm{Z}_{e q_{c a s e 1}}(s)=\frac{4\left[\mathrm{R}_{t_{P b}}+\mathrm{R}_{p_{P b}}+\mathrm{C}_{p_{P b}} \mathrm{R}_{p_{P b}} \mathrm{R}_{t_{P b}} s\right]}{\mathrm{C}_{p_{P b}} \mathrm{R}_{p_{P b}} s+1}
$$

The voltage response on the Case I system $\mathrm{V}_{\text {eq }}(t)$ is then:

$$
\mathrm{V}_{\text {eq } q_{\text {case } 1}}(t)=4 \mathrm{~V}_{\mathrm{oc}_{\mathrm{Pb}}}-\frac{4}{\mathrm{C}_{p_{P b}}}\left[e^{-\frac{t}{\mathrm{C}_{p_{P b}} \mathrm{R}_{p_{P b}}}}+\mathrm{C}_{p_{P b}} \mathrm{R}_{t_{P b}} \delta(t)\right] \mathrm{I}(t)
$$

The voltage and current responses are shown in Figure 10.4. It can be seen that the experimental results validate the accuracy of the developed model. Both voltage responses coincide closely with one other.

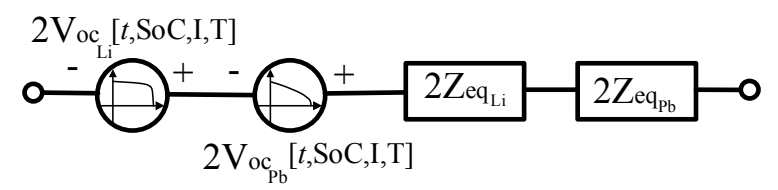

Figure 10.5. Lumped Parameter Model for Case II.

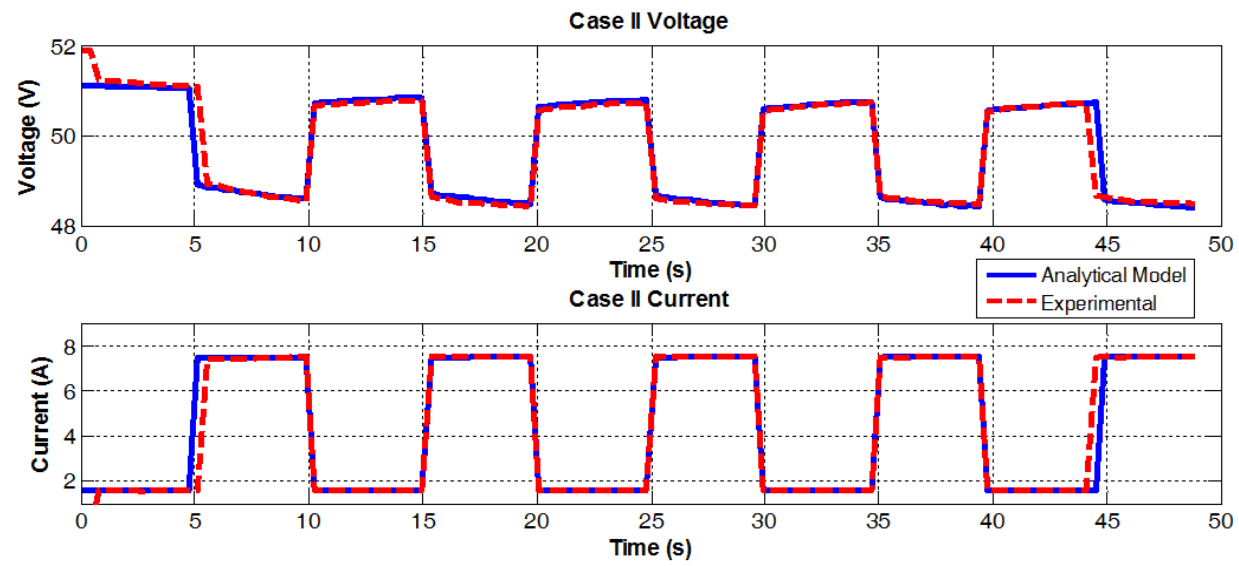

Figure 10.6. Verification of Voltage and Current under $0.1 \mathrm{~Hz}$ Test Pulse for Case II.

\subsubsection{Case II: 2 Lithium Ion and 2 Lead Acid Batteries}

In Case II, $50 \%$ of the lead acid battery modules are replaced with lithium ion modules of matching capacity. Case II introduces a HESS array where half of the modules will now have less susceptibility to SoH impacts as a result of heavy cycling and discharge currents, 
hereby improving performance. This split system introduces a medium, cost-effective solution to improve the array performance without the need for a total replacement of the lead acid batteries. Using this HESS, the system robustness to heavy pulsed loads is improved, while reducing the overall current contribution from each battery. Through utilization of the ESMC, the charging pattern can now be redistributed. The equivalent circuit for Case II is shown in Figure 10.5. The introduction of $\mathrm{C}_{p_{L i}}$ increases $Z_{e q_{c a s e 2}}$ to $2^{\text {nd }}-$ Order:

$$
\begin{aligned}
\mathrm{Z}_{e q_{c a s e 2}}(s)= & 2 \mathrm{R}_{t_{L i}}+2 \mathrm{R}_{t_{P b}} \\
& +\frac{2\left[\left(\mathrm{R}_{p_{P b}} \mathrm{R}_{p_{L i}} \mathrm{C}_{p_{L i}}+\mathrm{R}_{p_{L i}} \mathrm{R}_{p_{P b}} \mathrm{C}_{p_{P b}}\right) s+\mathrm{R}_{p_{L i}}+\mathrm{R}_{p_{P b}}\right]}{\mathrm{C}_{p_{L i}} \mathrm{R}_{p_{L i}} \mathrm{R}_{p_{P b}} \mathrm{C}_{p_{P b}} s^{2}+\left(\mathrm{R}_{p_{L i}} \mathrm{C}_{p_{L i}}+\mathrm{R}_{p_{P b}} \mathrm{C}_{p_{P b}}\right) s+1}
\end{aligned}
$$

The voltage response on the Case II system $V_{\text {case } 2}(t)$ is:

$$
\begin{aligned}
\mathrm{V}_{\text {case } 2}(t)=2 \mathrm{~V}_{\mathrm{oc} L \mathrm{Li}}+2 \mathrm{~V}_{\mathrm{oc}} \mathrm{Pb} \\
-2\left[\frac{e^{-\frac{t}{\mathrm{C}_{L i} \mathrm{R}_{L i}}}}{\mathrm{C}_{p_{L i}}}+\frac{e^{-\frac{t}{\mathrm{C}_{p_{P b}} \mathrm{R}_{P b}}}}{\mathrm{C}_{p_{P b}}}+\left(\mathrm{R}_{t_{L i}}+\mathrm{R}_{t_{P b}}\right) \delta(t)\right] \mathrm{I}(t)
\end{aligned}
$$

Similarly, both the voltage and current responses are shown in Figure 10.6, which validate the accuracy of the developed model.

\subsubsection{Case III: 2 Lithium Ion and a Lead Acid Battery with Supercapacitor}

The final circuit model in Case III replaces one of the remaining two lead acid battery modules with a SC as shown in Figure 10.7. In this HESS, $25 \%$ is now served by SC ES that can withstand cycling in real-time without inflicting major SoH impacts. However, there is a tradeoff in reduced capacity and having to handle a wider operating voltage range. Using four ESMC devices, dynamic charging can be executed efficiently. This will be 
discussed in detail in the following section. The equivalent impedance model $\mathrm{Z}_{e q_{c a s e 3}}$ from the HESS system in Case III is given by:

$$
\begin{aligned}
\mathrm{Z}_{e q_{c a s e}}=2 \mathrm{R}_{t_{L i}}+\mathrm{R}_{o_{S C}}+\mathrm{R}_{t_{P b}} & \\
+\frac{1}{\left(\mathrm{R}_{i_{S C}} \mathrm{C}_{o_{S C}} \mathrm{C}_{i_{S C}}\right) s^{2}+\left(\mathrm{C}_{o_{S C}}+\mathrm{C}_{i_{S C}}\right) s} & +\frac{2 \mathrm{R}_{p_{L i}}}{\mathrm{C}_{p_{L i}} \mathrm{R}_{p_{L i}} s+1}+\frac{\mathrm{R}_{p_{P b}}}{\mathrm{C}_{p_{P b}} \mathrm{R}_{p_{P b}} s+1} \\
& +\frac{\mathrm{C}_{i_{S C}} \mathrm{R}_{i_{S C}}}{\left(\mathrm{R}_{i_{S C}} \mathrm{C}_{o_{S C}} \mathrm{C}_{i_{S C}}\right) s+\mathrm{C}_{o_{S C}}+\mathrm{C}_{i_{S C}}}
\end{aligned}
$$

The voltage response on the Case III system $\mathrm{V}_{e q_{\text {case3 }}}(t)$ is:

$$
\begin{aligned}
& \mathrm{V}_{\mathrm{eq}_{\mathrm{case} 3}}(t)=2 \mathrm{~V}_{\mathrm{oc}_{\mathrm{Pb}}}+\mathrm{V}_{\mathrm{oc}_{\mathrm{Pb}}}+\mathrm{V}_{\mathrm{t}_{\mathrm{SC}}}
\end{aligned}
$$

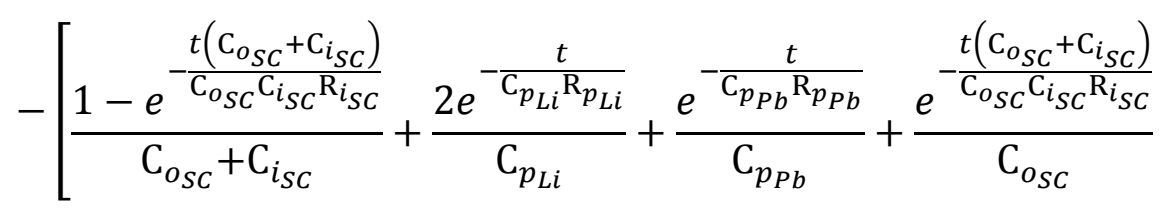

$$
\begin{aligned}
& \left.+\left(2 \mathrm{R}_{t_{L i}}+\mathrm{R}_{o_{S C}}+\mathrm{R}_{t_{P b}}\right) \delta(t)\right]
\end{aligned}
$$

The voltage and current responses are shown in Figure 10.8. It is shown that discharging the SC reduced the overall voltage of the stack, as expected. Nonetheless, the model response tracks the voltage reduction introduced by replacing a lead acid battery with an SC.

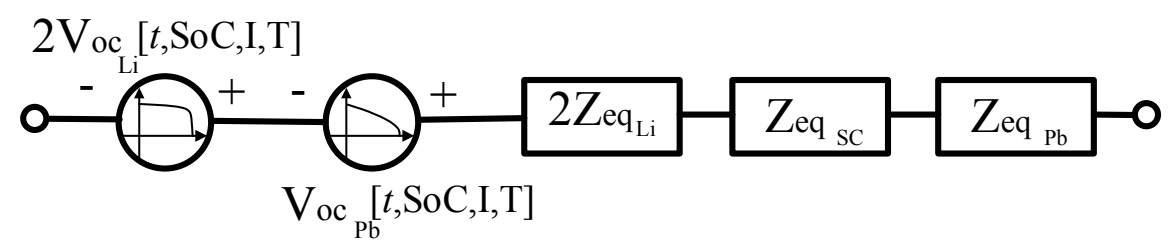

Figure 10.7. Lumped Parameter Model for Case III. 

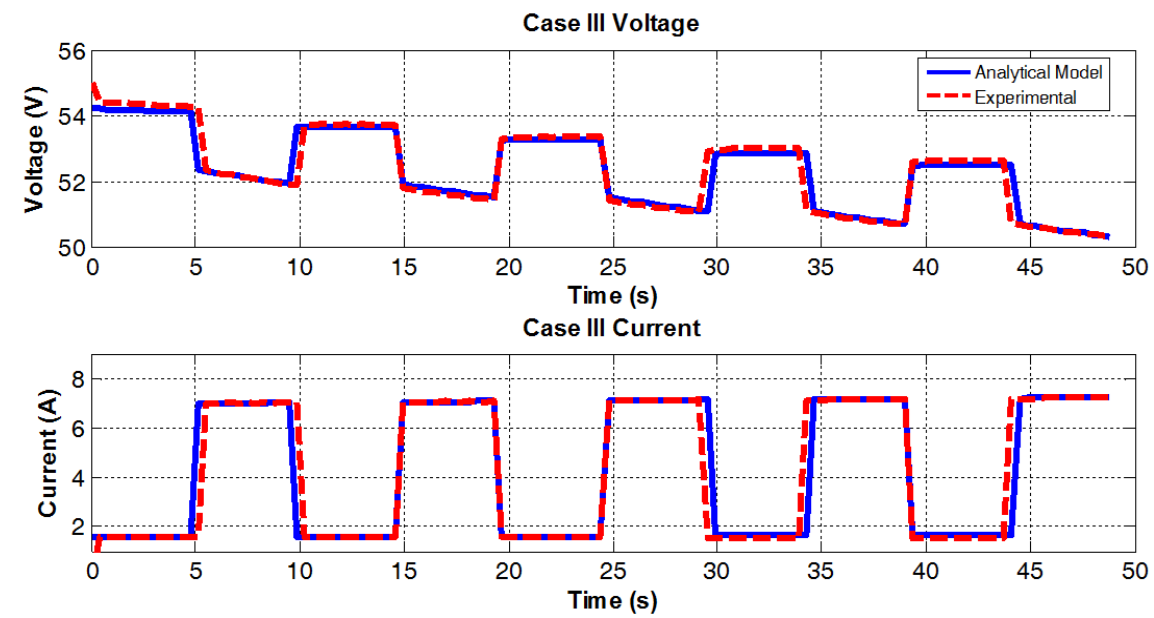

Figure 10.8. Verification of Voltage and Current under $0.1 \mathrm{~Hz}$ Test Pulse for Case III.

\subsubsection{Analytical Results Discussion}

In order to demonstrate the differences in responses for each HESS configuration, a comprehensive Simulink-based simulation platform was designed to test all three cases simultaneously. Shown in Figure 10.9, subsystem blocks were created for each ES device, where the contents of each are shown below the main schematic. Figure 10.10 depicts a comparison of the output power, voltage, and current for all three cases. One can see each HESS discharge voltage trend and pulsed load response differs greatly from case-to-case. Case I follows a steeper discharge voltage trend (higher slope during the pulse) than Case II, where the current steadily increases following each pulse to maintain a constant power delivery to the load. Following each pulse, the recovery voltage is highly nonlinear due to a high timing constant generated by the large lead acid battery capacitance $\mathrm{C}_{p_{P b}}$. Case II has a more linear voltage behavior outside of the pulse, with a voltage drop less sharp than that of Case I due to the reduced ohmic resistance $\mathrm{R}_{t_{L i}}$ of the lithium ion modules. The voltage in Case II ends lower as a result of a lower terminal voltage $\mathrm{V}_{\mathrm{oc}_{\mathrm{Li}}}$ of the lithium ion modules, but the current injected under each pulse is held nearly constant. 


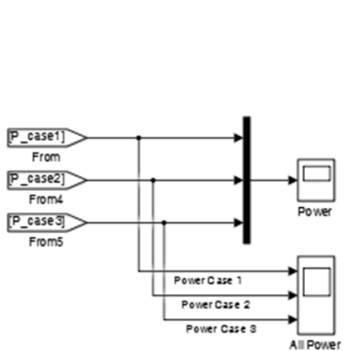

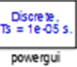

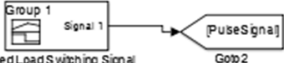
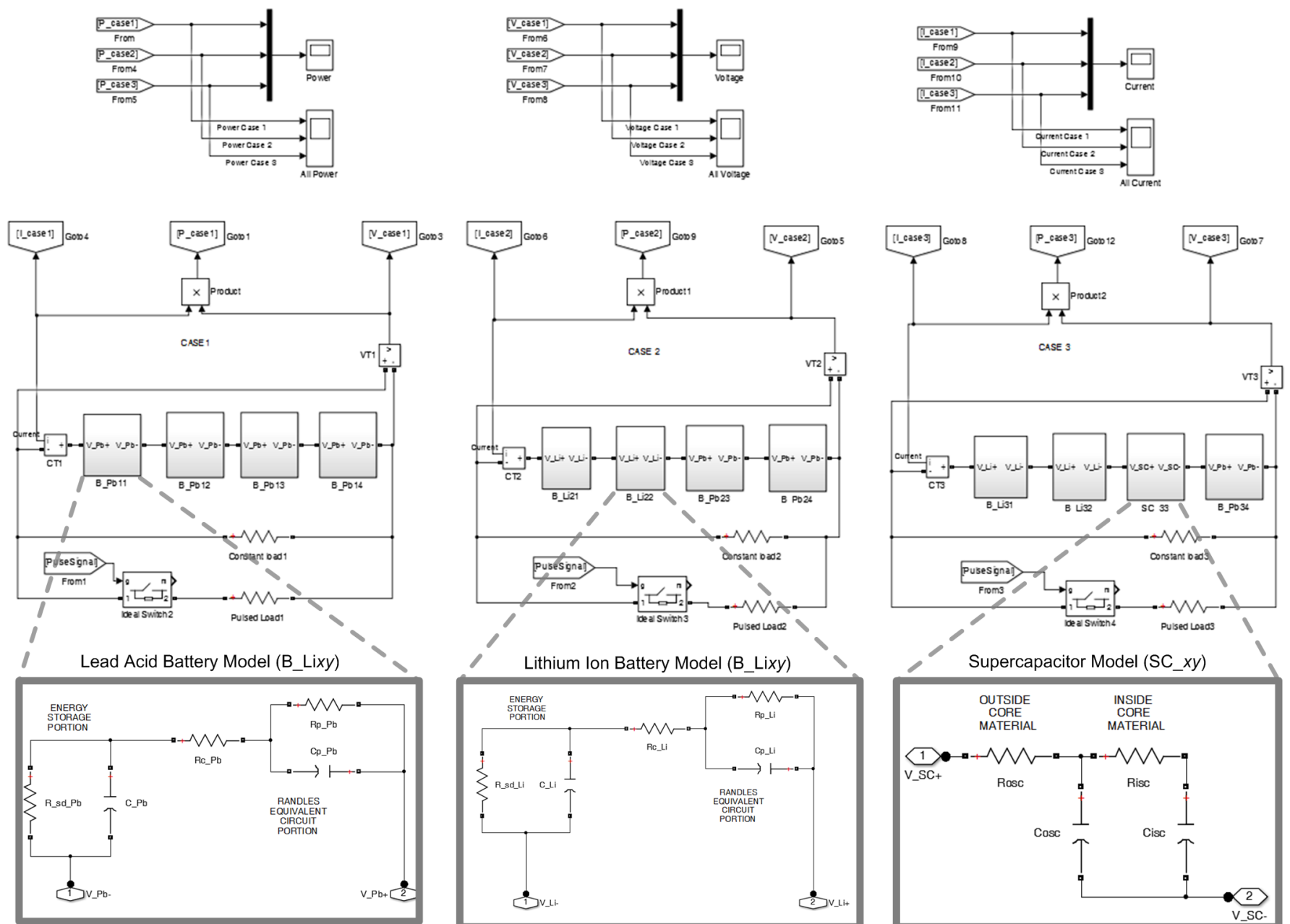

*Where: $x$ represents Case number and $y$ represents Series cell number

Figure 10.9. Comprehensive Simulink Simulation Platform to Evaluate all Cases. 


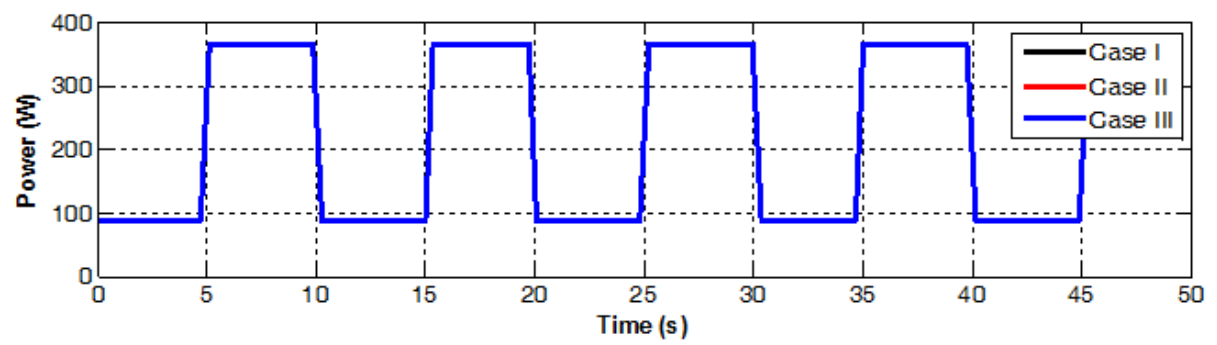

(a)

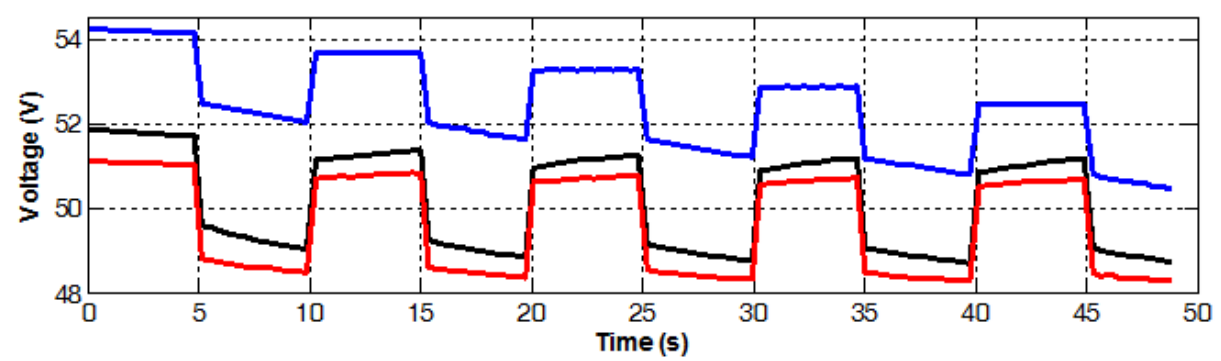

(b)

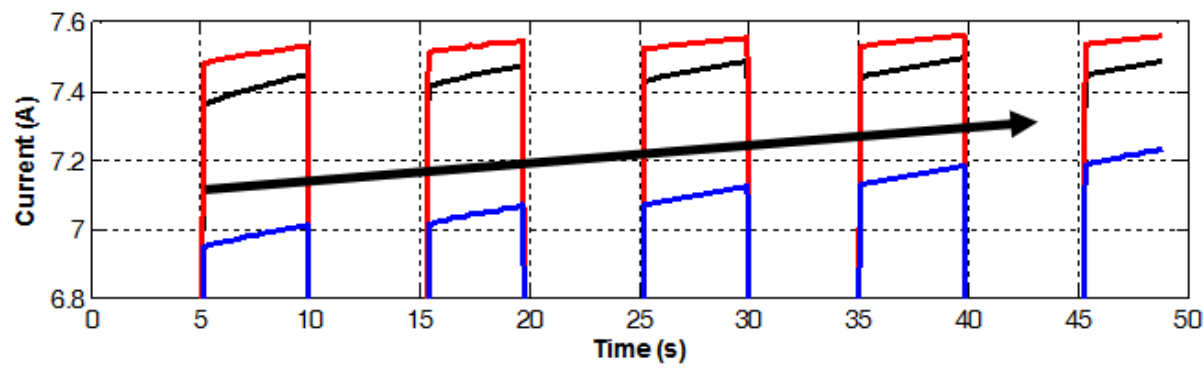

(c)

Figure 10.10. Cases I, II, \& III: a) Power, (b) Voltage, and (c) Current Response.

Case III features the highest initial voltage due to a high full charge terminal voltage of the $\mathrm{SC} \mathrm{V}_{\mathrm{t}_{\mathrm{SC}}}$, hereby reducing the initial current required. Following each pulsed load (during the off-pulse), the stack voltage is nearly flat as shown by the blue line in Figure 10.10(b), but due to the reduced storage capacity of the SC, the long term voltage trend declines at the steepest rate. This causes an increase in the current required from all ES sources to supply the remaining demand (as shown in Figure 10.10(c)). These widely varied characteristics add to the importance and highlight the need for developing a specialized control system for HESS. Without advanced control, over-charging or over- 
discharging of a single ES device within the array could occur, leading to a potential system failure or even permanent damage to the device.

\subsection{Coordinated Control of Hybrid Energy Storage Systems}

To handle the diverse charging characteristics for each different ES type within the HESS stack, an array of ESMC devices are utilized to provide a safe interconnection to each. In this section, the rolling charging concept is discussed, followed by a review of the charging constraints for each ES device. To implement these features as well as manage the discharge limitations of each ES, a number of upgrades were applied to the original National Instruments (NI) LabVIEW interface that was developed previously in Chapter 2. These features are illustrated and described in detail.

\subsubsection{Rolling Charging Operation}

A new concept of "rolling charging" is developed with a goal to extend the operation time of certain HESS to aid in supplying heavy pulsed loads. This concept utilizes the unique capabilities of the ESMC in extracting a weak or discharged ES module from the HESS array and placing it on charge, while maintaining system operation. As an example, in actual SPS, the EM railgun pulsed power requirement would present a challenge in terms of available energy on the HESS and may quickly drop the SoC of an ES module to severely low levels. If the firing frequency and number of shots of the railgun were known, one could anticipate when the energy requirement was needed. Using this information, an ES module could then be extracted to charge when the pulse is off and reconnected again only when the pulse is active. Through advanced control and timing, this could be synchronized with the pulsed load. To balance the impact on all ES modules while also providing each applicable module the opportunity to charge, each is dispatched for a period 
of time and then "rolled" to the next module with the lowest SoC. This tactic can allow the system to sustain itself for extended operation periods, while at the same time, permit the maintenance of some ES units when the stack is still under operation. This could be very beneficial for critical SPS loads.

\subsubsection{Charging Constraints}

Charging currents and voltage levels vary based on each type of ES device. Since the ESMC topology employs two isolated busses, namely a DC Bus and Charging Bus, another major advantage is that it is capable of handling different charging constraints for different types of ES. Consequently, the voltage and current limits of the Charging Bus can be adjusted dynamically based on the type of ES connected. In more complicated or larger systems, multiple busses could be provided with the aid of an ESMC connected to each device. Moreover, the ESMC can set the current limitations to pre-specified values to maintain a safe charging current. This would not only reduce fire hazards, but would also limit the rate of $\mathrm{SoH}$ degradation on the batteries.

For the lead acid battery employed in this experiment, particular care has been taken to regulate its charging current to prolong its $\mathrm{SoH}$. The manufacturer established an absolute maximum charging current at the 4-hour Coulombic (C)-rate (C/4), but to limit thermodynamic and material stress, this has been reduced to a conservative $\mathrm{C} / 6$ current with a maximum charging bus voltage of $14.8 \mathrm{~V}$. The lithium ion batteries, however, have much less susceptibility to higher charging currents as long as charging voltage levels are carefully controlled. For the lithium ion cells deployed in this experiment, a voltage tolerance of $4.20 \pm 0.03 \mathrm{~V} /$ cell or $12.60 \pm 0.09 \mathrm{~V}$ is regulated for the 3-cell series module, where the maximum charging current is increased to $\mathrm{C} / 2$ [164]. The SC module 
is an exception, as the charging current is not referenced to its storage capacity. Theoretically, charging to its full voltage level $\mathrm{V}_{\max _{\mathrm{SC}}}$ is only limited by its ESR $R_{E S R}$, but the International Electrotechnical Commission (IEC) has established a safety limit $\mathrm{I}_{\text {max }_{I E C}}$ for practical applications based on $2.6 \%$ of this current [28].

$$
\mathrm{I}_{\max _{I E C}}=0.026\left(\frac{\mathrm{V}_{\max _{S C}}}{R_{E S R}}\right)
$$

For the SC modules employed in this study, $I_{\max } \cong 19 \mathrm{~A}$.

\subsubsection{Control System and Graphical User Interface Upgrades}

Previously in Chapter 2, a control interface was developed to manage an array of four ESMC devices. A number of upgrades were applied to support not only the new required control demands, but to also provide a platform that has an understanding of the operating limitations for each ES device. Shown in Figure 10.11, this Graphical User Interface (GUI) is once again broken into two portions: the Main Load and System Control and Individual ESMC Control Interfaces. The Main Load and System Control is shown to the left, where a Load Control Center has been added. Tabs are provided to toggle between engaging the constant load and the two pulsed loads.

For each pulsed load, in addition to setting a basic pulse frequency and duty cycle, a section entitled "Pulse Synchronization Dispatch Service" has been included to provide a means to synchronize the pulsed load with individual or multiple ESMC devices. In this case, the user can select to engage or disengage one or more ESMC devices with the pulsed load, where checkboxes grey-out in the event that the ESMC device has already been dispatched with another pulsed load. In the example shown in Figure 10.11, Pulsed Load 2 has been selected to engage ESMC 1 when the pulse is on ("On with Pulse"). Since 
ESMC 1 is already assigned to Pulsed Load 2, ESMC 1 is greyed-out for dispatch by Pulsed Load 1.

Although the dispatch service provides a powerful new feature and enables the capability of rolling charging, multiple new timed loops are required on the LabVIEW control platform to support it. This results in the propagation of some delay between engaging the load and switching modes on each ESMC device; thus, some background timers are provided to verify the duration of the pulse matches the user's setting. Moreover, in this experimental setup, all loads are connected via solid state relays (SSR), while all switching actions on each ESMC is accomplished by much slower electromechanical relays. Since the SSRs will engage far quicker than the electromechanical relays, even if the switching signal were received at the same time, a delay is present. This further increases the total delay between the load and ESMC devices. To manage this issue, each pulsed load has a phase shift setting where a delay can be tuned to match this delay period. The "Signal Charts" tab provides live switching waveforms for each pulsed load as compared to the ESMC. By toggling the phase shift between the output signal to the pulsed load and ESMC dispatch, the user can converge on an optimal phase delay to match the load and ESMC dispatch.

Updates to the Individual ESMC Control Interfaces are shown to the right in Figure 10.11. New configuration tabs separate an automated ES Device Setup and a Charging Setup. For each ESMC, the ES type can be selected as well as its associated number of series cells, capacity, and charging preferences. Using this information, the Full Charge Voltage, Charging Voltage, Discharge Voltage Cut-off, and Full Charge Current Cut-off operating ranges are calculated using those previously defined in Chapter 5 (Table 5-2). 
Main Load and System Control

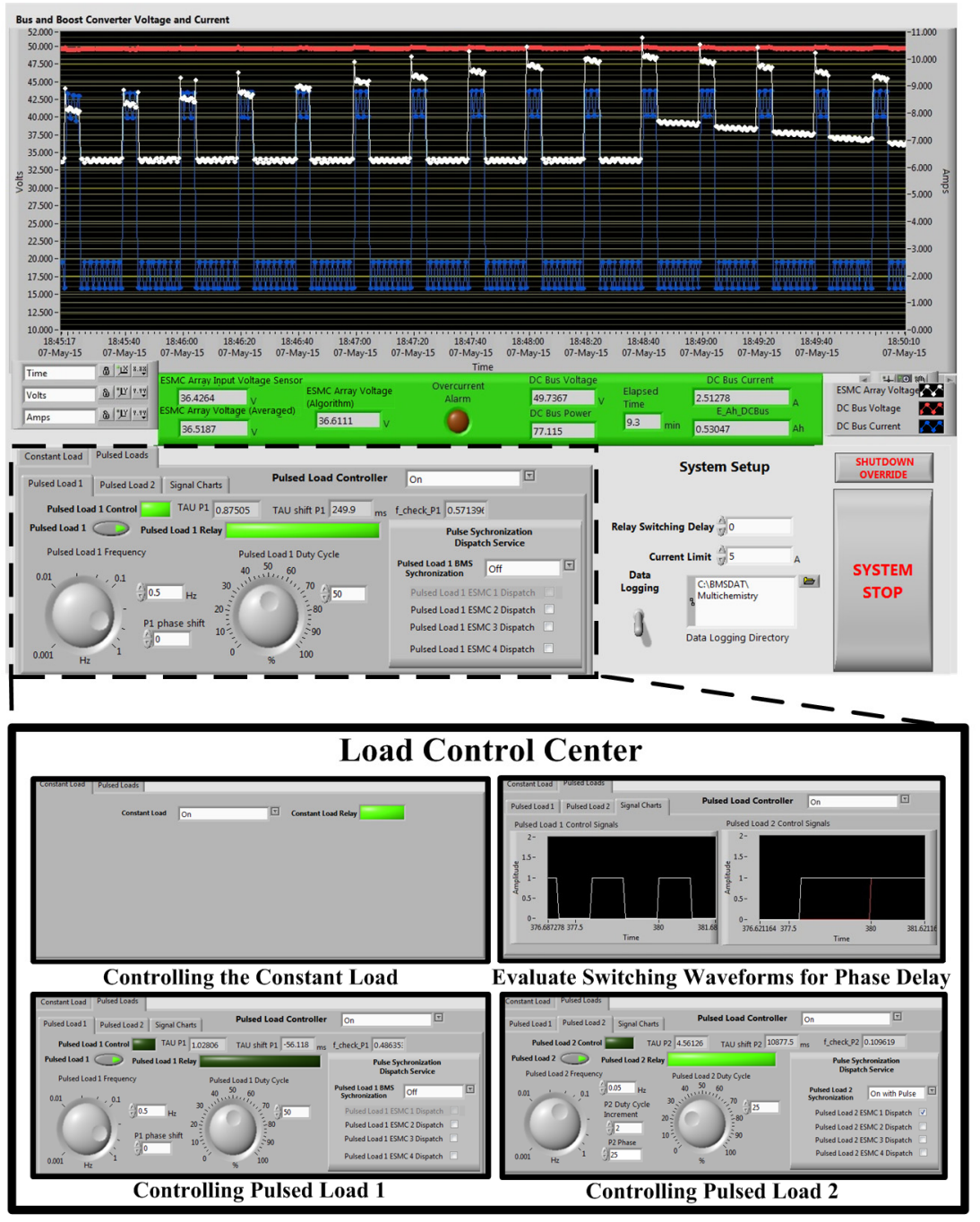

Individual ESMC Control Interfaces

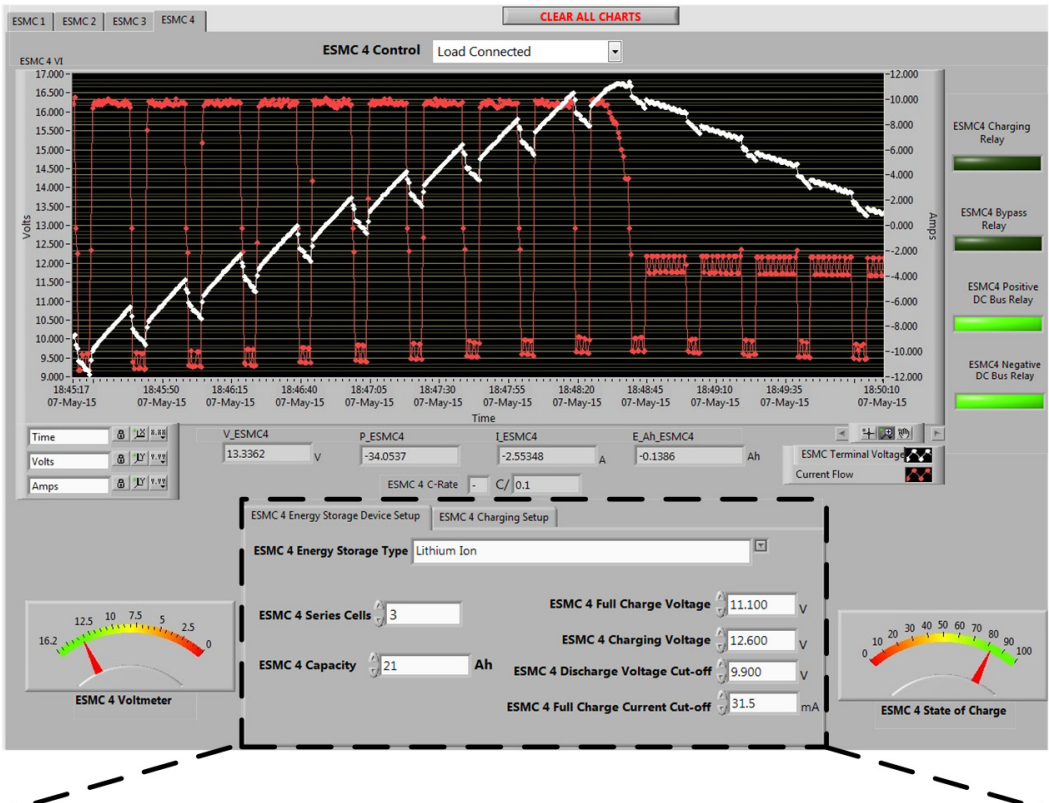

Energy Storage Device Setup and Charging Configuration
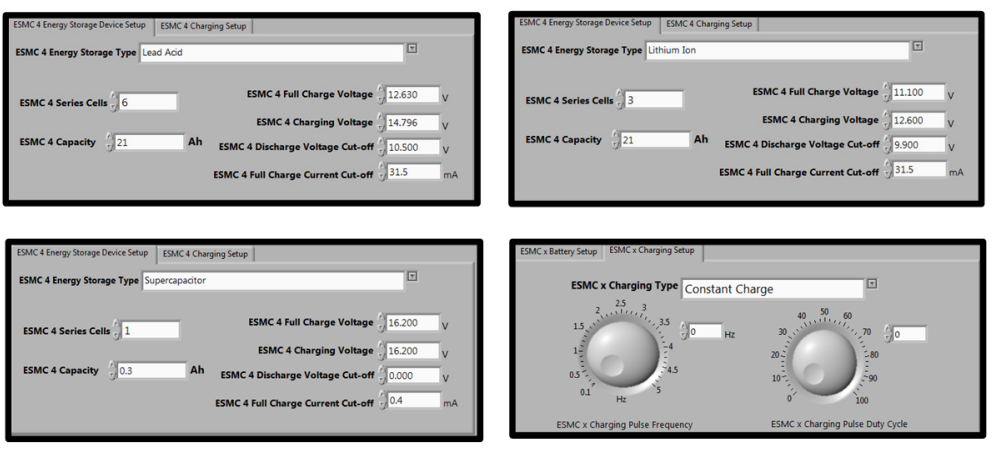

Figure 10.11. Upgraded ESMC Control Platform to Support Hybrid Energy Storage and Shipboard Power System Loads. 
In addition, the SC operating ranges have been added, as well. Operating ranges are then displayed to the user, where if necessary, can be modified. Upgraded SoC measurements have also been included following developments from Chapter 5. For the lead acid and lithium ion batteries, an initial voltage-based estimation gives way to current integration when in operation. For the SC, initial and operating SoC measurements are directly proportional to its terminal voltage. Finally, the Charging Setup tab houses controls developed in the initial version to toggle between constant and pulsed charging modes, where if pulsed charging is activated, its frequency and duty cycle can be set.

\subsection{Hardware Implementation}

To investigate the feasibility of the proposed control technique, a hardware setup has been established as depicted in Figure 10.12 and Figure 10.13. The lead acid and lithium ion batteries as well as the SC are the same types as summarized previously in Table 10-1. The voltage of the DC Bus is $48 \mathrm{~V}$, while the voltage of the charging bus is controlled based on the ES charging requirements. A programmable Magna-Power XR375-15.9 DC power supply was used for the Charging Bus [157].

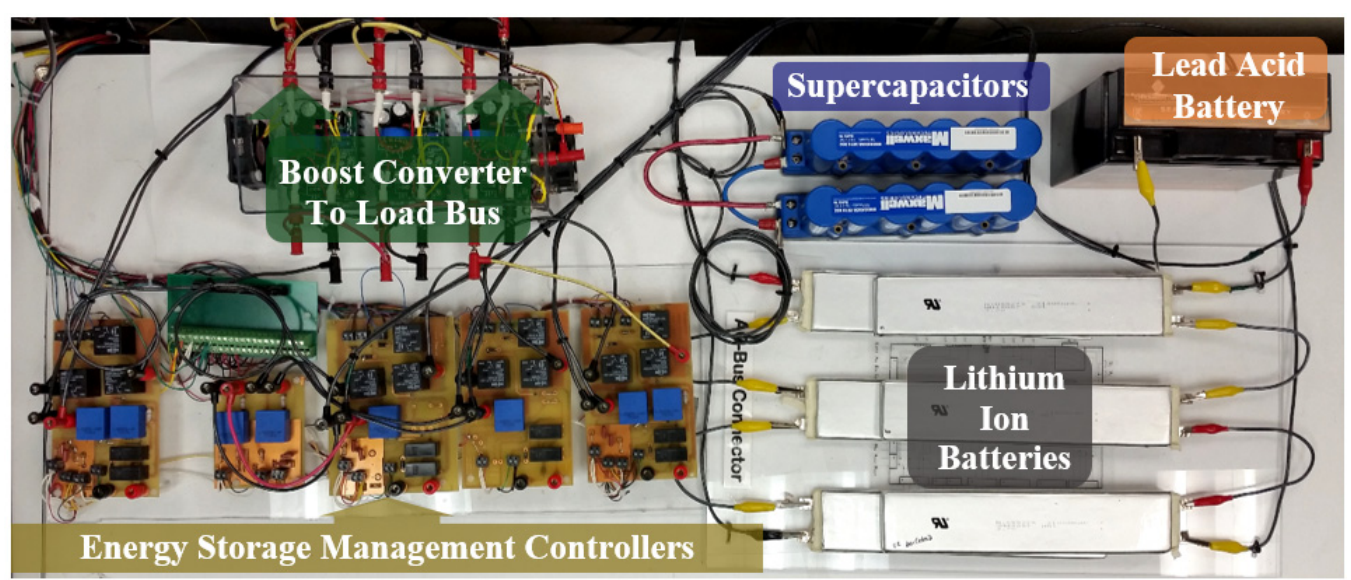

Figure 10.12. Experimental Setup of the ESMC, Converter, Supercapacitors, Lead Acid Battery, and Lithium ion Batteries. 


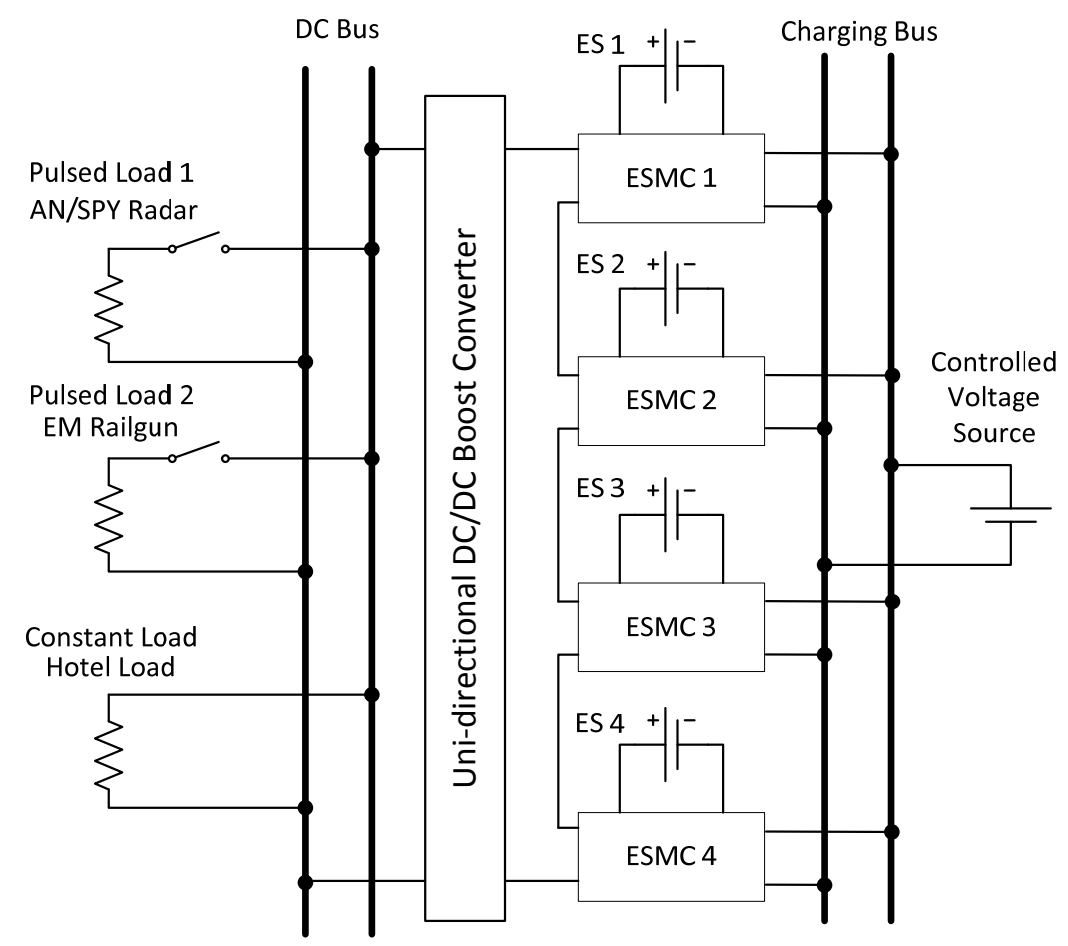

Figure 10.13. Configuration of ESMC Controllers in Series.

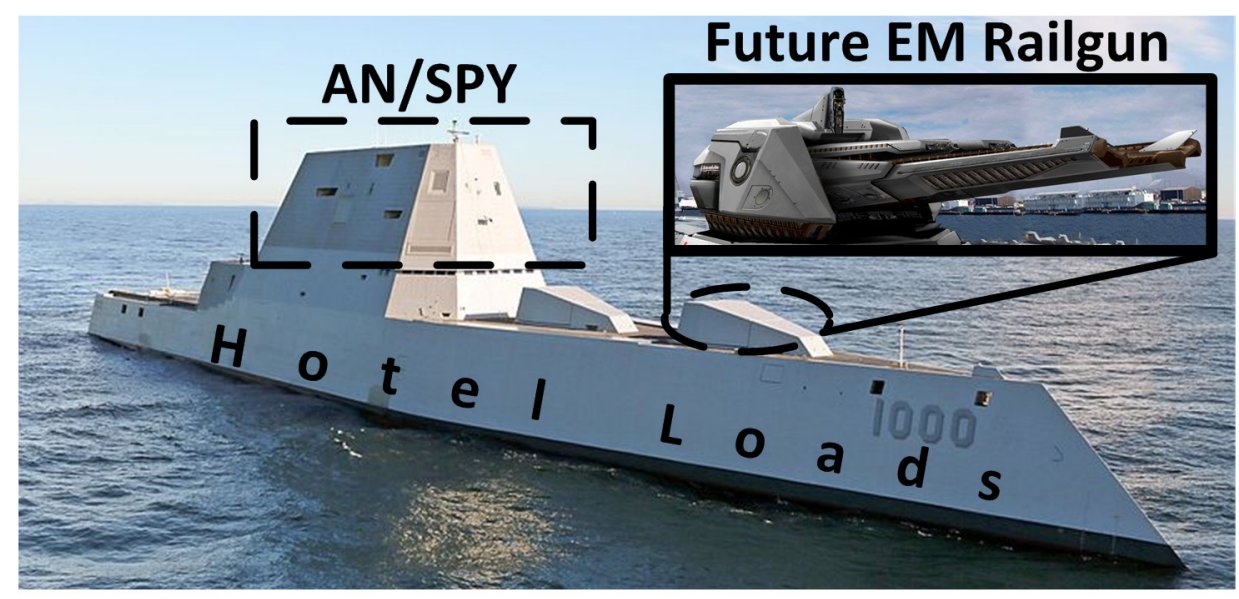

Figure 10.14. USS Zumwalt DDG-1000 and Modeled Shipboard Loads.

Specifications of the loads were selected based on the information gathered from the DDG-1000 [158],[158]. A constant, also referred to as the hotel load, has been selected with an average power demand of 11.22 MW. The parameters of all loads were then translated to the per-unit scale, where the hotel load is designated as the base with the pulsed loads referenced to it as shown in Table 10-2. 
Table 10-2. Shipboard Power System Load Profiles.

\begin{tabular}{c|c|c|c|c|c|c|c}
\hline \hline Load Type & Equipment & $\begin{array}{c}\text { Frequency } \\
(\mathrm{Hz})\end{array}$ & $\begin{array}{c}\text { Duty } \\
\text { Cycle } \\
(\%)\end{array}$ & $\begin{array}{c}\text { Actual } \\
\text { Power } \\
(\mathrm{MW})\end{array}$ & $\begin{array}{c}\text { Per } \\
\text { Unit } \\
(\mathrm{pu})\end{array}$ & $\begin{array}{c}\text { Scaled } \\
\text { Power } \\
(\mathrm{W})\end{array}$ & $\begin{array}{c}\text { Test } \\
\text { Resistance } \\
(\Omega)\end{array}$ \\
\hline Constant Load & Hotel Load & - & - & 11.22 & 1.000 & 105.0 & 32.0 \\
\hline Pulsed Load 1 & AN/SPY Radar & 0.50 & 50 & 6.00 & 0.587 & 60.7 & 55.4 \\
\hline Pulsed Load 2 & EM Railgun & 0.05 & 25 & 38.00 & 3.661 & 384.4 & 8.7 \\
\hline
\end{tabular}

Pulsed Load 1 is modeled after the AN/SPY-1 radar system, originally developed by Lockheed Martin in 1973 [159]. The AN/SPY has gone through a series of variants and versions to reach the current SPY-3 platform installed aboard the DDG-1000. The SPY-3 provides superior medium to high altitude performance over other radar bands and is the primary radar used for missile engagements. Since the SPY-3 specifications represent sensitive information, the AN/SPY-1 was modeled representing the lighter of two considerable pulsed loads installed on the SPS. Operating with a power demand of 0.587 $\mathrm{pu}$, Pulsed Load 1 has a scanning frequency of $0.5 \mathrm{~Hz}$ under a $50 \%$ duty cycle.

Pulsed Load 2 represents a next generation naval gun that is still in testing, but under serious consideration for future deployment aboard the DDG-1000 [160]. The EM railgun represents the most significant challenge in terms of power and energy demand for future naval electrical design. The railgun utilizes an enormous EM force is used to launch a high velocity projectile accelerated along a pair of conductive rails up to $2.1 \mathrm{~km} / \mathrm{s}$. Although no set timeline for installation aboard the DDG-1000 has been set, demonstrations continue to push the boundaries of performance. At the same time, a significant on-going challenge has been faced in designing the electrical architecture needed to support them. As summarized in Table 10-2, the power demand for an EM railgun shot is nearly 3.5x that of the hotel load. Pulsed Load 2 is modeled with a $5 \mathrm{~s}$ active duration and a duty cycle of $25 \%$. 


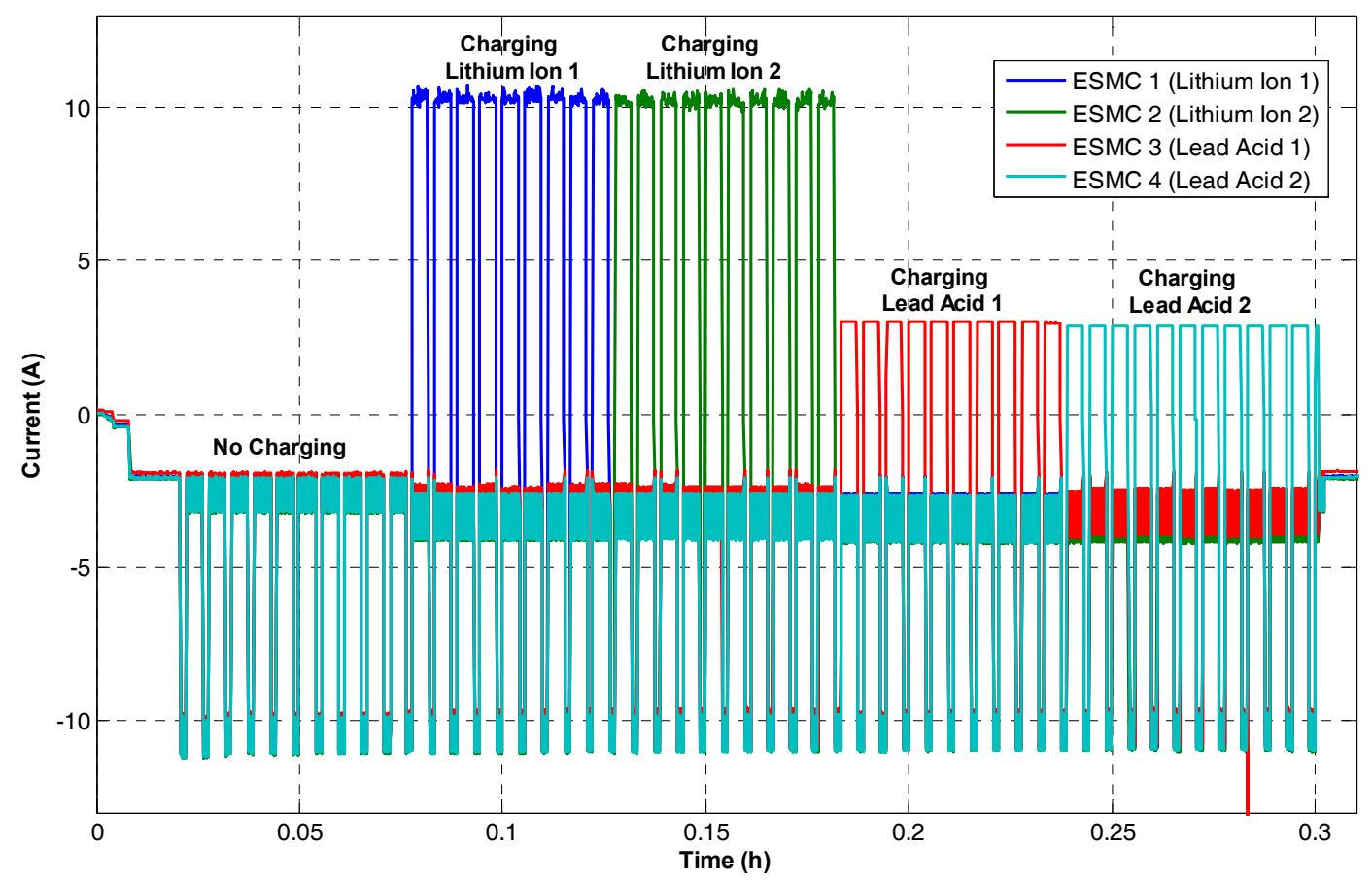

(a)

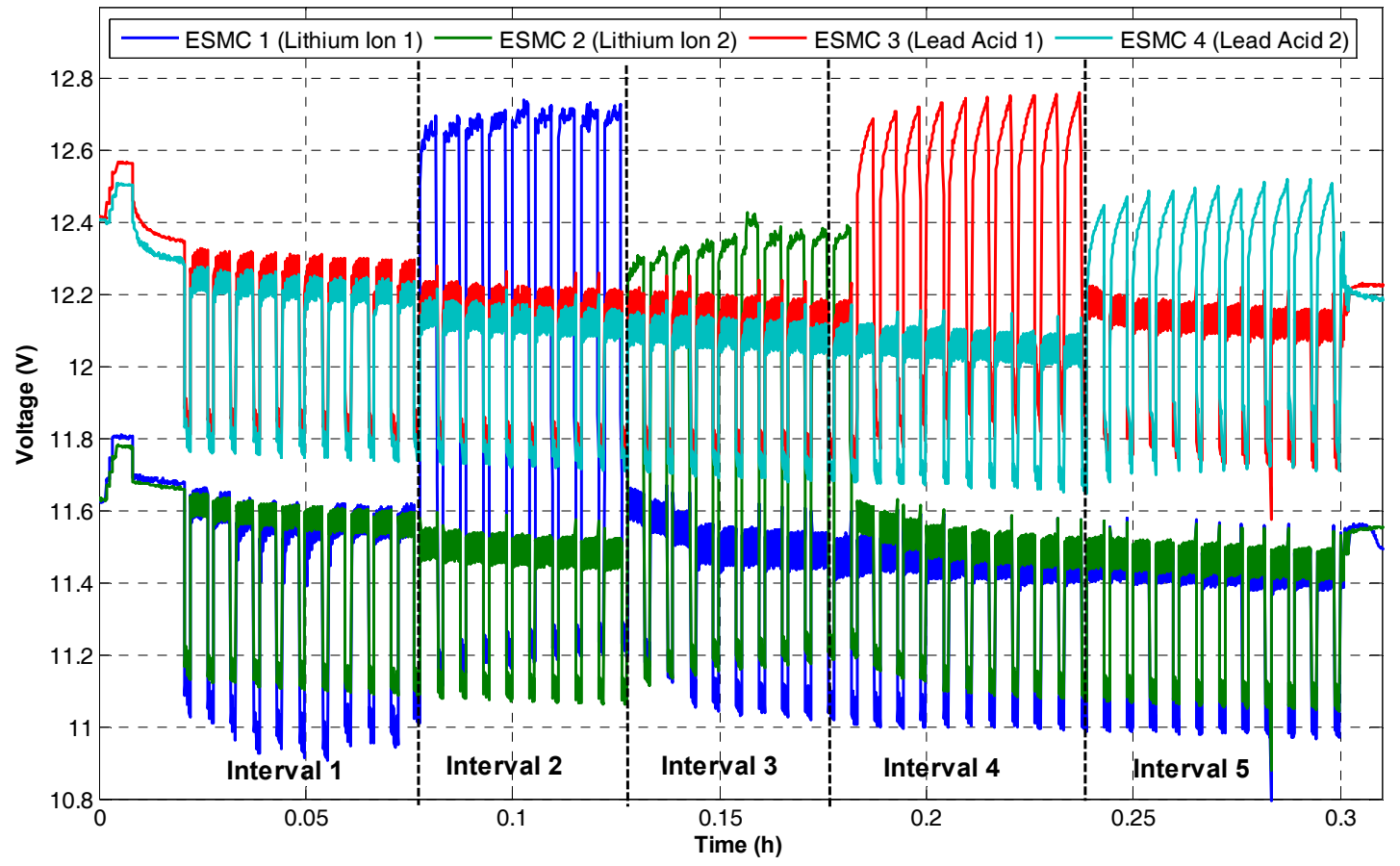

(b)

Figure 10.15. Test I: Individual Energy Storage Modules (a) Currents and (b) Voltages. 


\subsection{Experimental Results}

Five test scenarios are carried out to test the support of the SPS with a variety of different series-configured HESS. In Tests I and II, a balanced 50\% lithium ion and 50\% lead acid battery HESS is evaluated under all SPS loads. An initial dynamic charging test is demonstrated under a short period, then an endurance test operates the hotel load and a single heavy pulsed load until the HESS reaches its full discharge cutoff. In Tests III and IV, a lithium ion and lead acid battery HESS containing the SC is evaluated with all loads connected and without applying the SC to rolling charging. Finally, Test V evaluates the same load profile applying rolling charging to all available ES in the system.

\subsubsection{Test I: 2 Lithium Ion and 2 Lead Acid Batteries}

In this test, the HESS was composed of two lithium ion batteries connected to ESMC 1 \& ESMC 2, and two lead acid batteries connected to ESMC 3 and ESMC 4. The test results are shown in Figure 10.15 to Figure 10.17, where the test duration was around 20 $\min (0.33 \mathrm{~h})$, divided into five intervals. The current and voltage profiles during the test are depicted in Figure 10.15, where it can be seen that in interval 1, all four batteries served the load. Although all batteries contributed equally to the current, their operating voltages differed, even amongst battery modules of the same chemistry. This highlights potential differences in their SoH or a lack of precise cell balancing.

In the second interval, lithium ion battery 1 (ESMC 1) was extracted during the light loading periods (when the large pulsed load was off) and connected to the Charging Bus. This can be further illustrated by looking at a zoom in Figure 10.16, where a close-up of a $72 \mathrm{~s}(0.02 \mathrm{~h})$ period is shown. The effect of the fast pulsed load (of lower amplitude) is clear, and both pulsed loads overlap over some periods. 


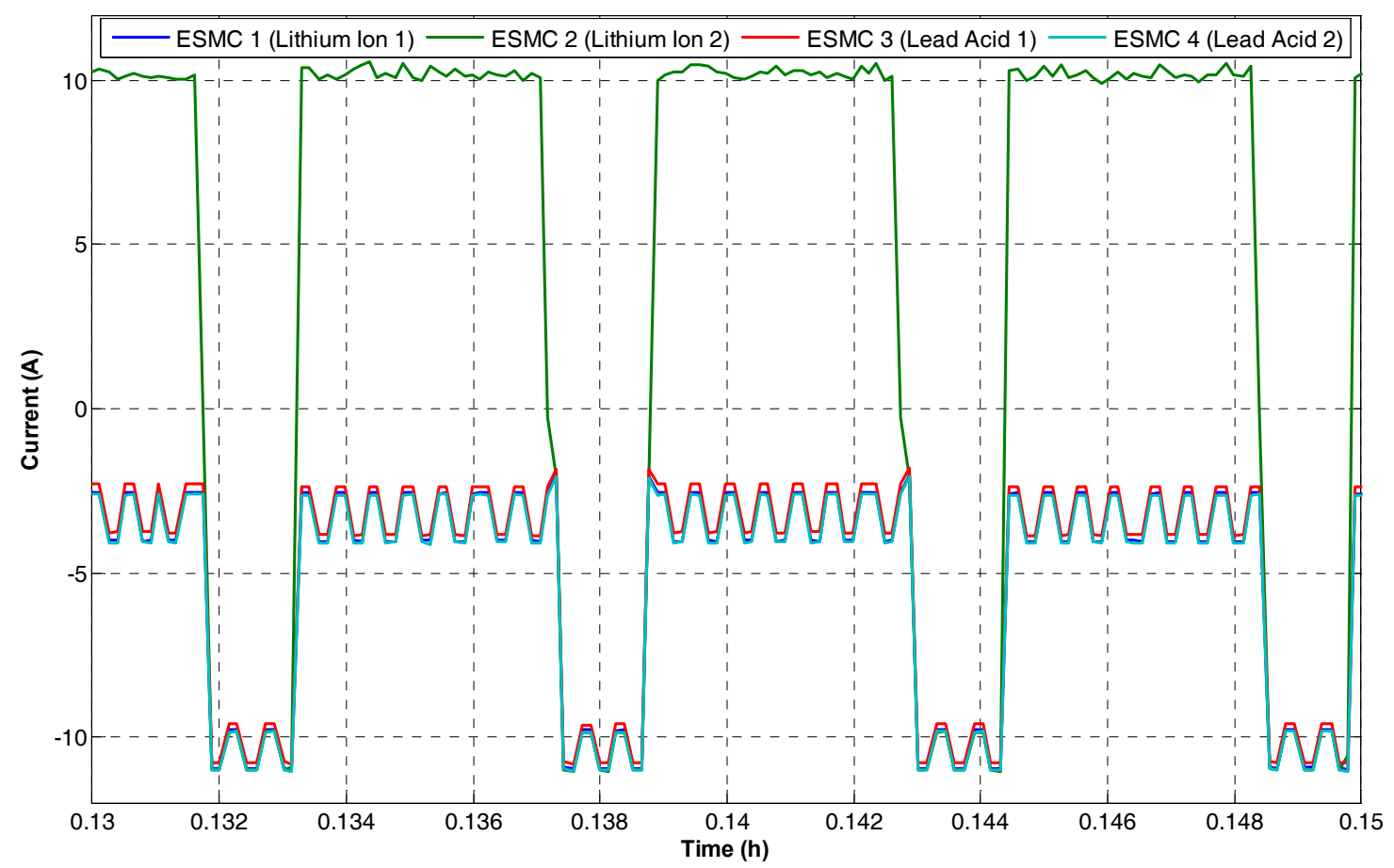

Figure 10.16. Test I: 72-second Close-up of Currents of Individual Energy Storage Modules.

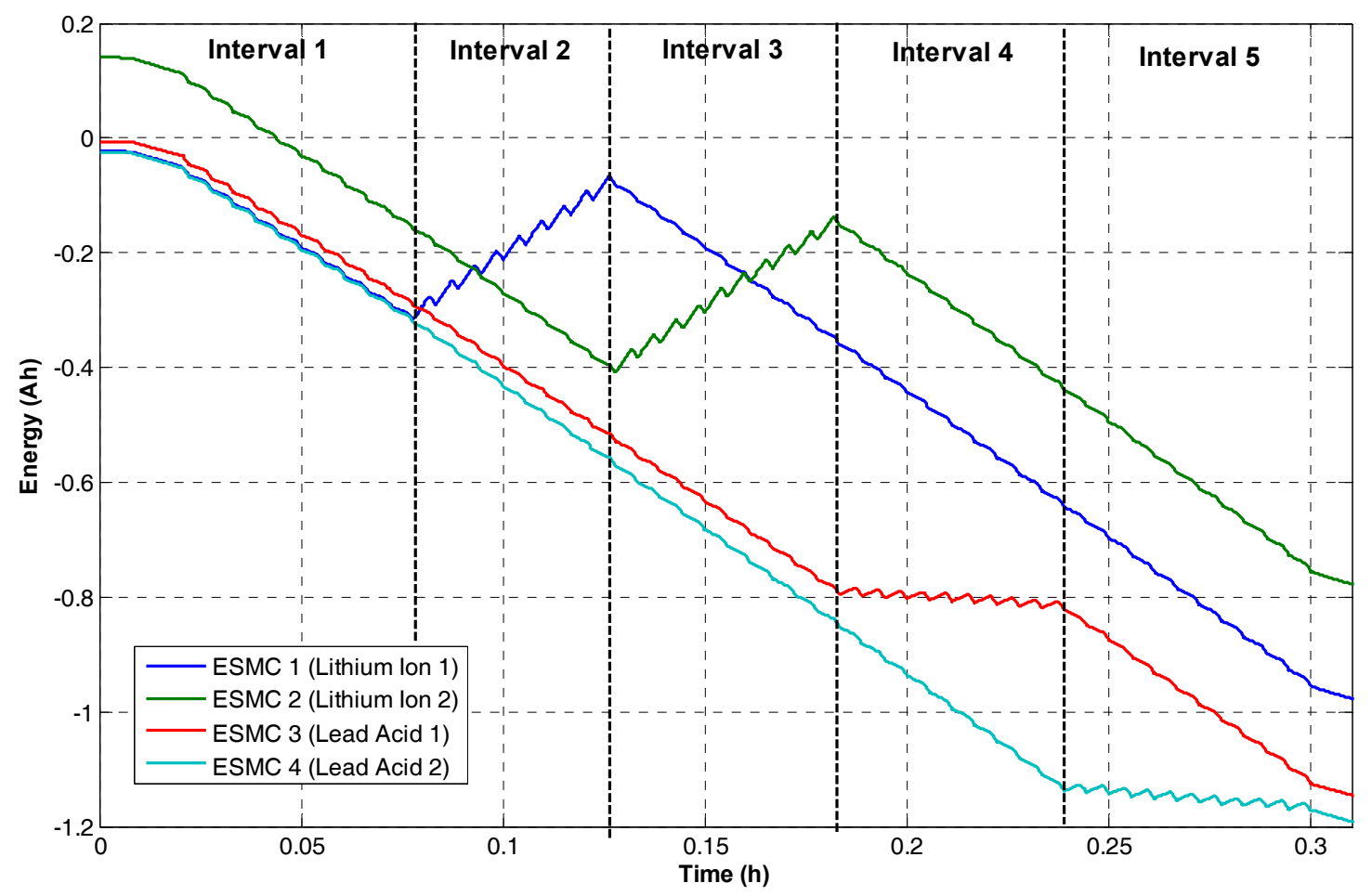

Figure 10.17. Test I: Energy Output from Currents of Individual Energy Storage Modules. 
It should be noted that the negative current indicates discharging the battery, while positive current indicates charging. In the third interval, the second lithium ion battery (ESMC 2) is extracted to be charged, resulting in an identical charging current, but with a lower charging voltage than Lithium Ion 1 . This is due to the increased energy loss that occurred in interval 4 as depicted in Figure 10.17. Lithium Ion 1 had to increase its current contribution during interval 2 to compensate for the loss of Lithium Ion 2.

In the fourth and fifth intervals, the lithium ion batteries return to serve the load, while the lead acid batteries take turns in charging. The signature dynamics of the lead acid are visible in Figure 10.15(b), depicting a slow saturation of their terminal voltages due to the high equivalent capacitance of the lead acid battery. Similarly to what was observed with the lithium ion batteries, the charging voltage of Lead Acid 1 is higher than Lead Acid 2 when the same charging current is applied. The lower charging voltage is once again caused by Lead Acid 2 having to expend more energy by the time it reaches interval 5 as illustrated in Figure 10.17.

\subsubsection{Test II: Endurance Test}

To investigate the effectiveness of the proposed rolling charging technique in extending the "in operation" time of the HESS, an endurance test was performed utilizing the full potential of the ES modules with a $52 \mathrm{~W}$ hotel load and a single heavy $320 \mathrm{~W}$ pulsed load. The HESS configuration is the same as in Test I. In Figure 10.18, the voltage and current off the HESS is shown without the ESMC. Without the control provided by an ESMC connected to each ES, discharge voltage progressions of the lead acid and lithium ion followed close to what was expected. Figure 10.18(a) reveals the voltage progressions for batteries of each chemistry are similar, and Figure 10.18(b) confirms the current 
contribution under a single pulse was equivalent for each battery type. It can be seen from Figure 10.18 that the HESS with ESMC support was discharged, reaching its full voltage discharge cutoff after $164 \mathrm{~min}(2.73 \mathrm{~h})$.

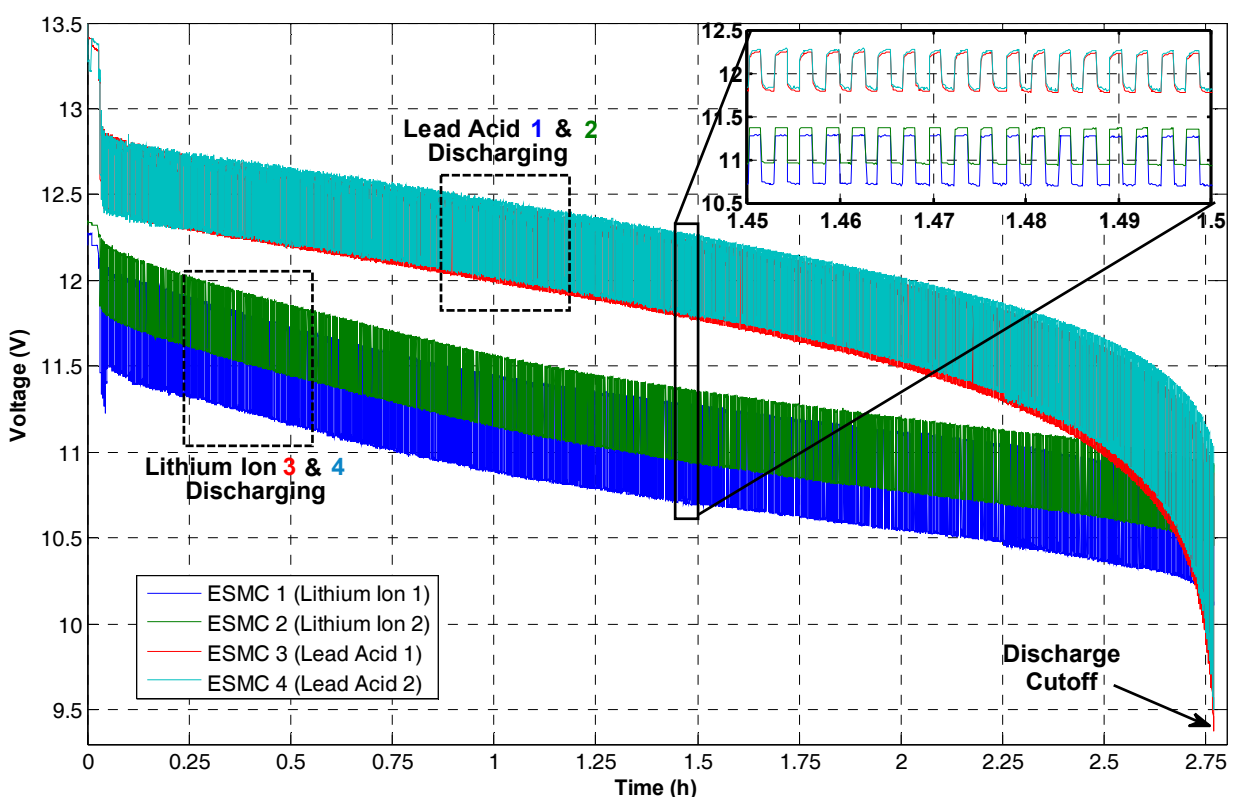

(a)

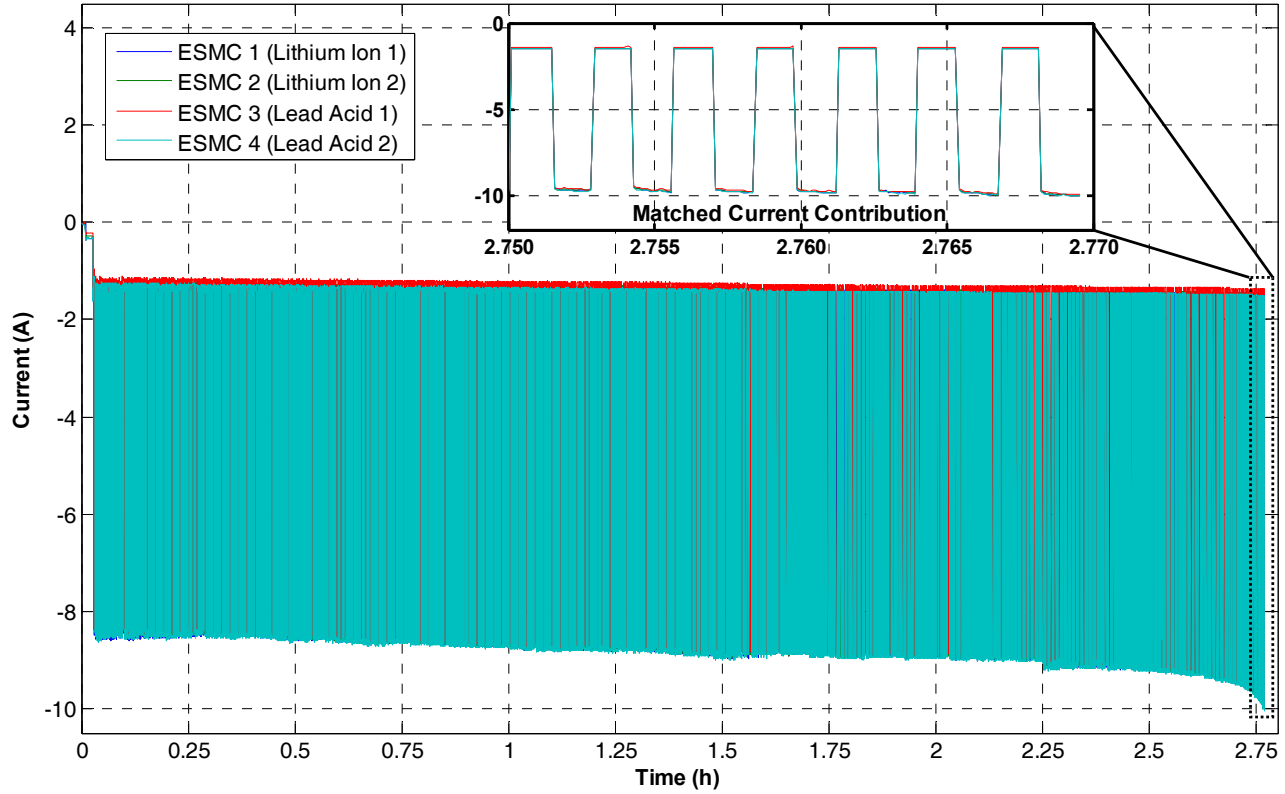

(b)

Figure 10.18. Test II without Rolling Charging: Individual Energy Storage Modules (a) Voltages and (b) Currents. 


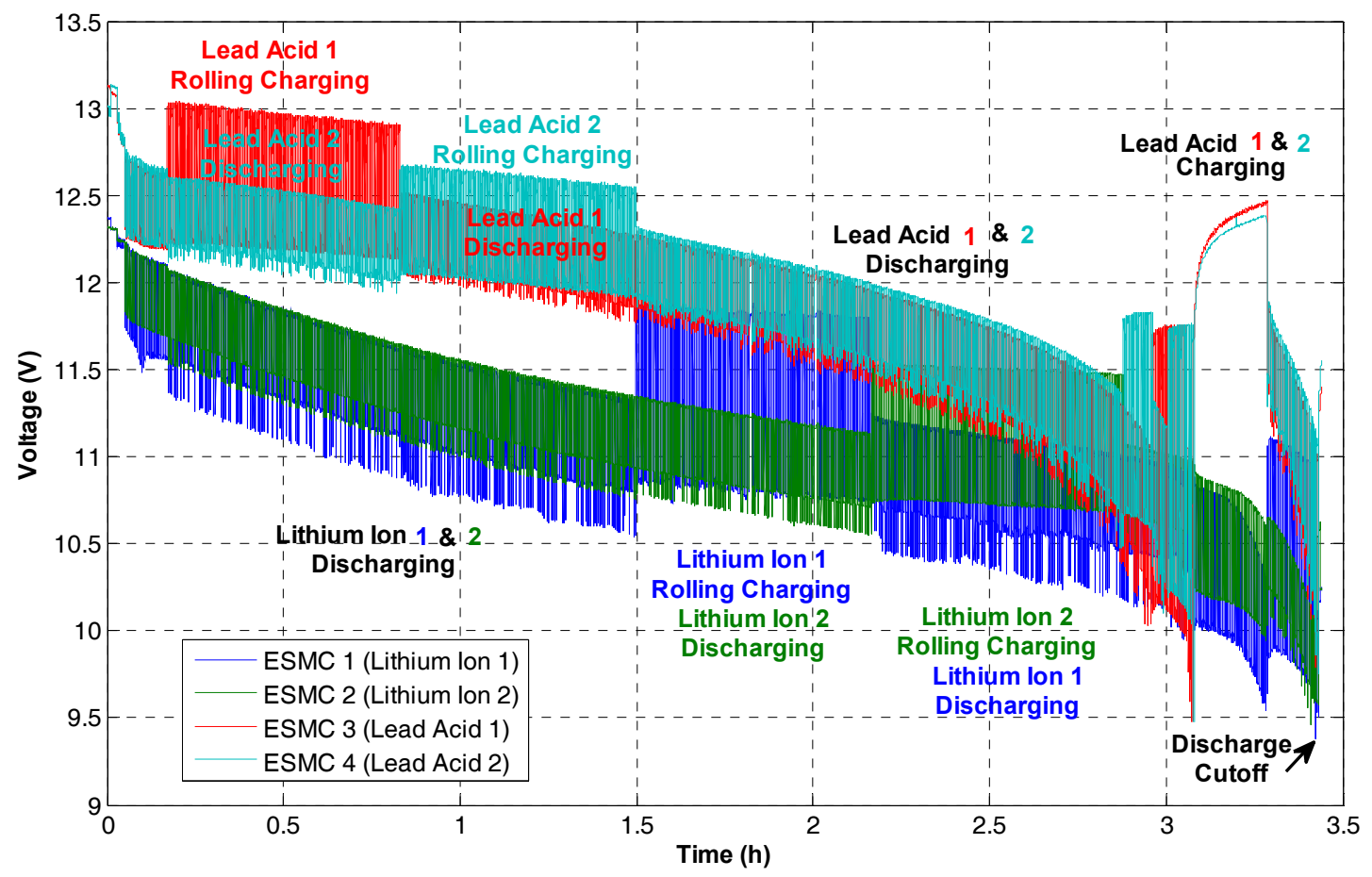

(a)

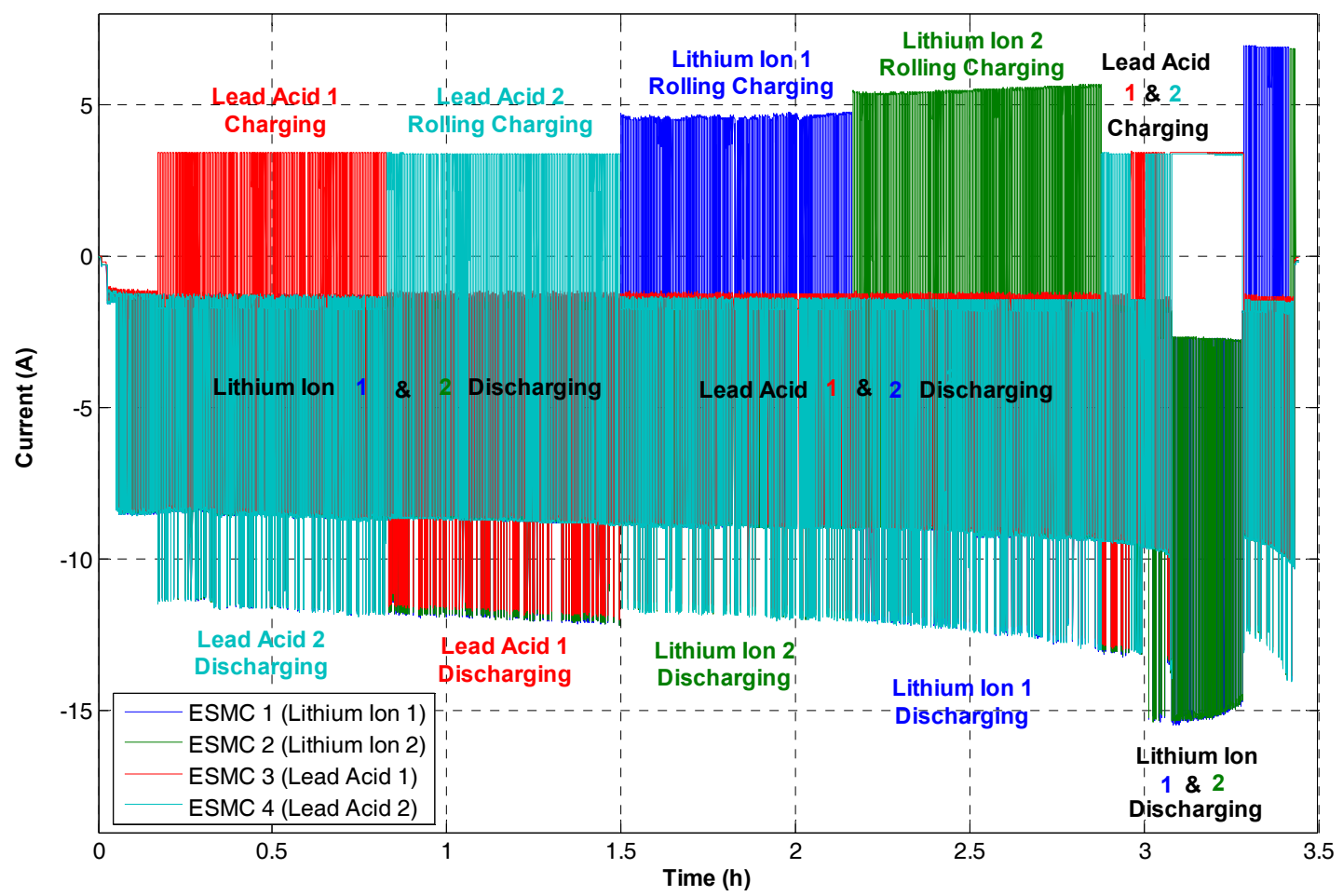

(b)

Figure 10.19. Test II with Rolling Charging: Individual Energy Storage Modules (a) Voltages and (b) Currents. 
Figure 10.19 depicts the voltage and current progressions of the HESS with an ESMC connected to each battery. The sequence begins by rolling charging one battery at a time. The lead acid batteries are rolling charged first, followed by each of the lithium ion batteries. Unfortunately, a heavier contribution by the lead acid batteries (a weaker source) while the lithium ion are rolling charged resulted in a low stack voltage. Since the system must shut down when any one ES device reaches its full discharge cutoff voltage, rolling charging is later replaced by constant charging for both lead acid batteries. During this period, the current contribution of the lithium ion batteries doubles, resulting in a quick degradation of their voltages. The energy output from the lithium ion and injection into the lead acid batteries is more clearly demonstrated in Figure 10.20. The heavy loading current resulted in only a $16 \mathrm{Ah}$ discharge output from the lithium ion modules and approximately $14 \mathrm{Ah}$ from the lead acid modules. This was expected as a result of heavier activation losses through the lead acid chemistry as was analyzed in previous chapters. After approximately $15 \mathrm{~min}$, the lead acid batteries are brought back into service, while both lithium ion batteries are placed in rolling charging. It is during this phase where the full discharge cutoff is reached after $207 \mathrm{~min}(3.45 \mathrm{~h})$.

The comparison depicted in Test II reveals the deployment of the ESMC can achieve a $26 \%$ increase in HESS service time through replenishing some of the lost energy online (while the stack was operating). This was not possible in the legacy system and could be of a significant importance for SPS in critical operating scenarios where it is not possible to shed vital loads [197]. Furthermore, the second scenario highlights the deployment of the ESMC is not limited to simply one charging mode or one device, but can utilize a compilation of charging and rolling charging measures to maintain system stability. 


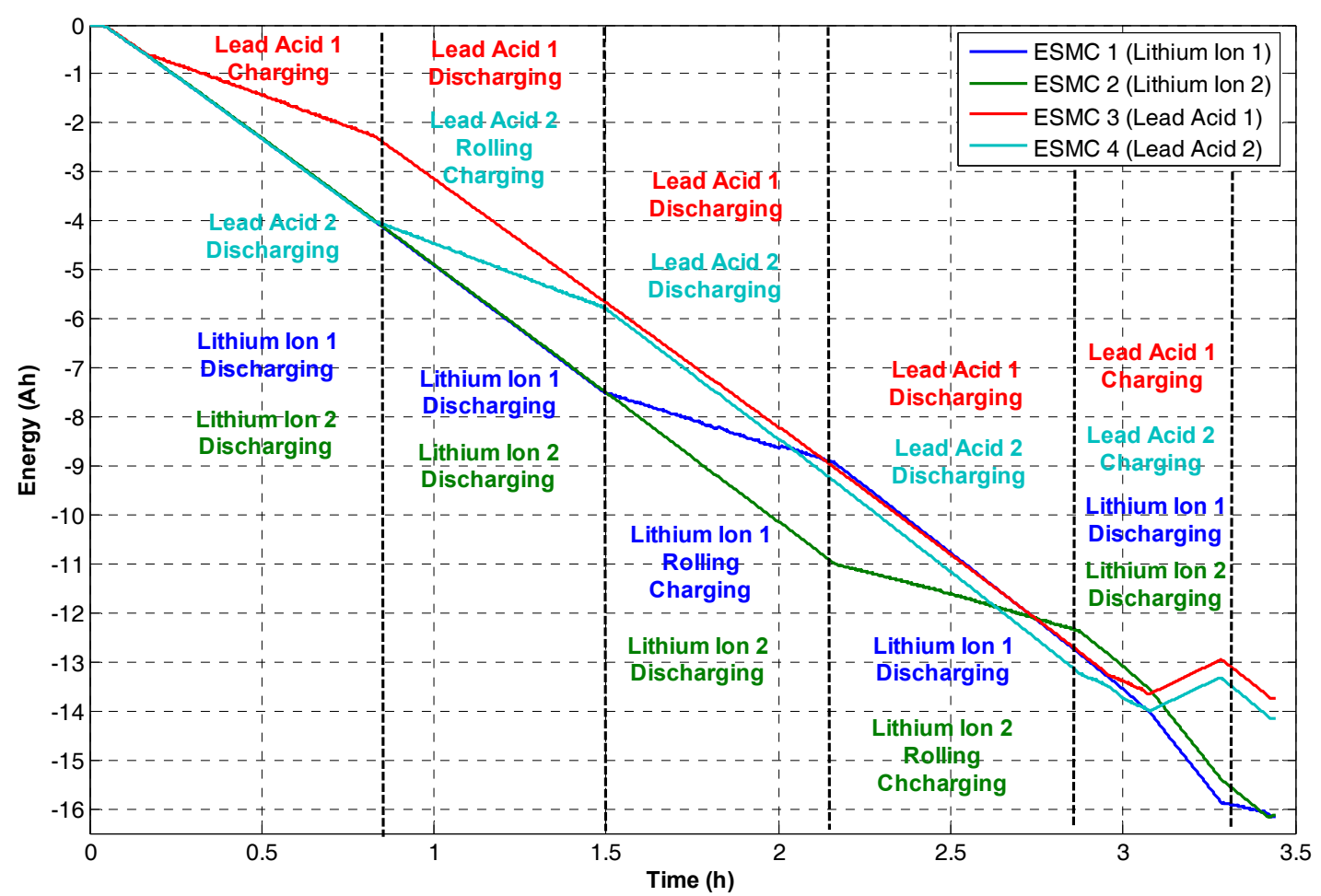

Figure 10.20. Test II with Rolling Charging: Energy Output from Individual Energy Storage Modules.

\subsubsection{Test III: 2 Lithium Ion Batteries, Lead Acid Battery, and a Supercapacitor without Rolling Charging}

In Test III, one of the remaining lead acid batteries was replaced with a SC presenting new challenges. To have an accurate comparison, this test is used as the base for the Case III HESS combination, since no rolling charging was applied. It can be seen from Figure 10.21 that the test was limited to less than 9 min. Figure 10.21(a) shows that the $\mathrm{SC}$ voltage decayed at a much higher rate than that of the batteries, reaching near $0 \mathrm{~V}$. This is explained by the vast difference in energy densities between the SC and the batteries, a comparison better illustrated by the SoC progressions in Figure 10.21(b). Since the SoC of the SC reached its full discharge cutoff after only $8 \min 40 \mathrm{~s}$, the HESS had to be disconnected. 


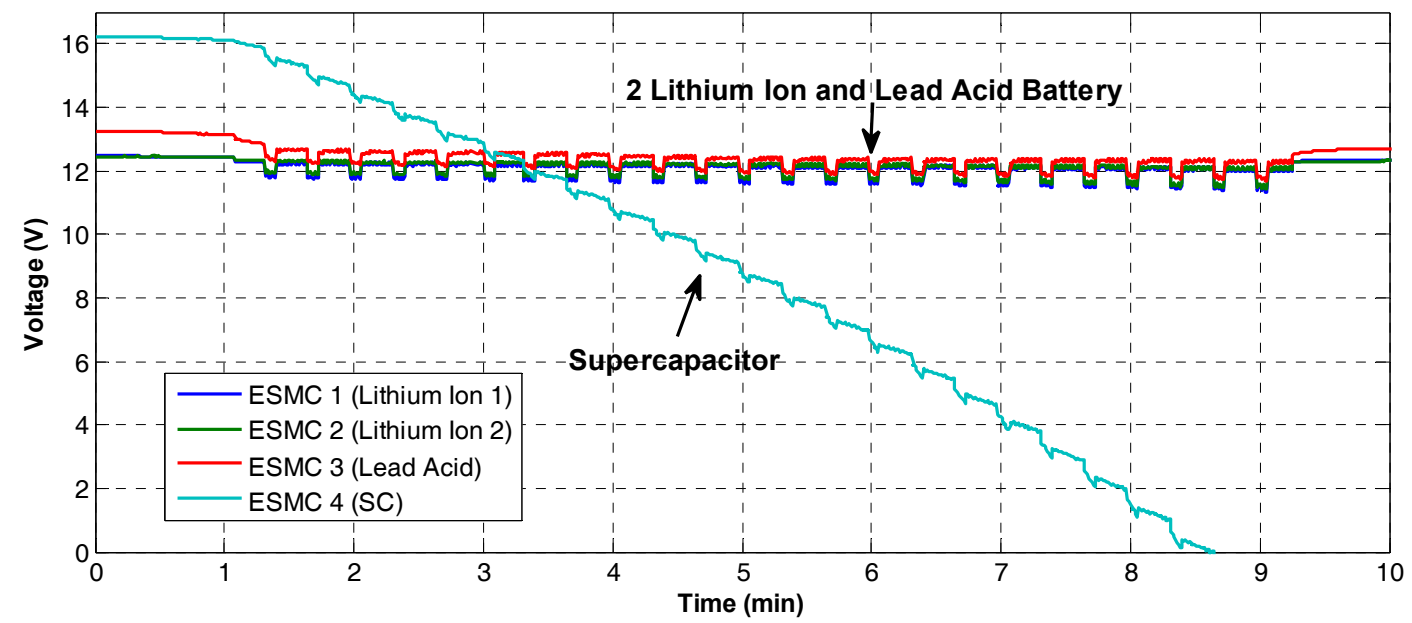

(a)

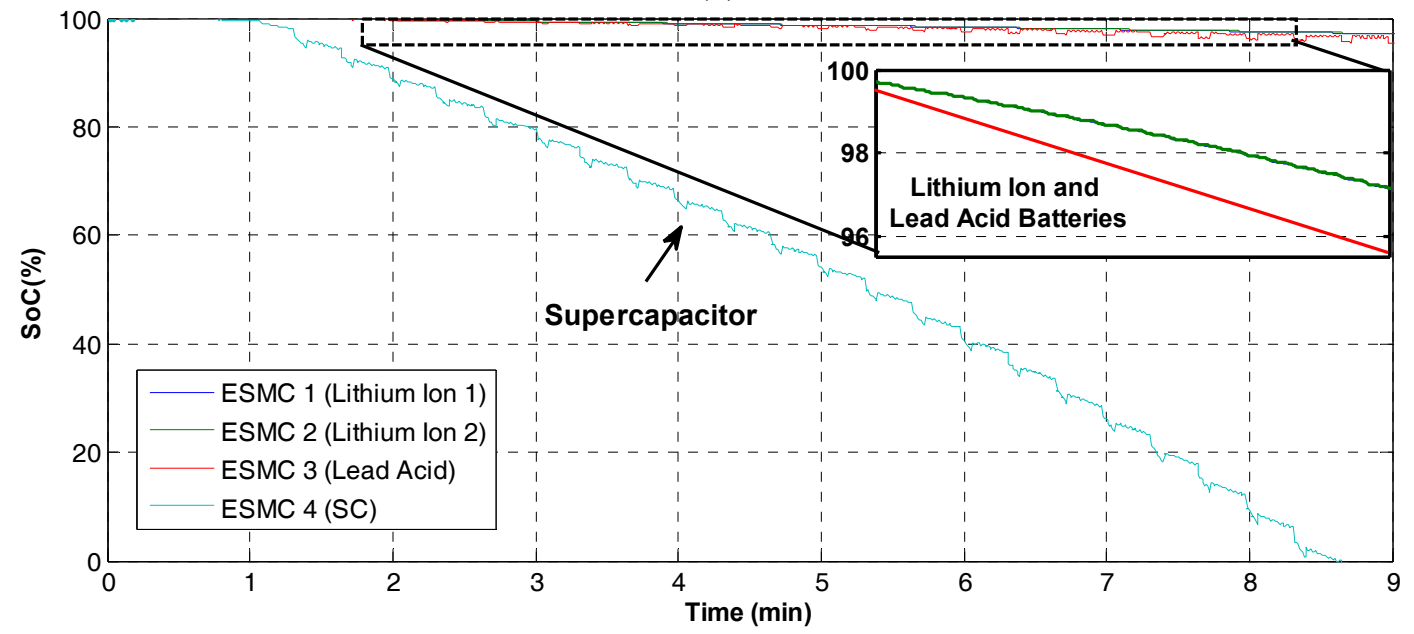

(b)

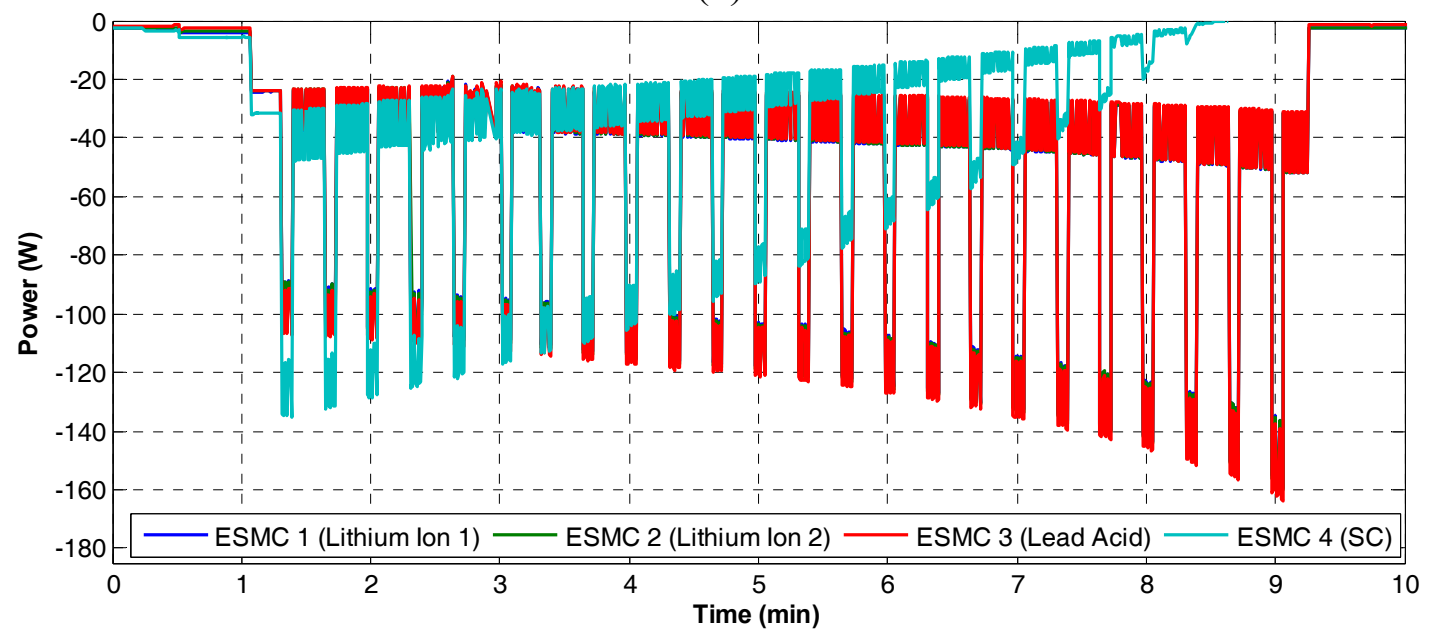

(c)

Figure 10.21. Test III: Individual Energy Storage Modules (a) Voltages, (b) State of Charges, and (c) Currents. 
Over the same period, the SoC of the lead acid dropped by just $4.5 \%$ and the lithium ion batteries by only $3 \%$. The lead acid SoC reduction rate is higher for the same reason as mentioned in the previous test, where heavy loading resulted in higher activation losses. Figure 10.21(c) shows that the power absorbed from the SC gradually decreased, which had to be compensated by an increase in the power injected by the batteries. If the HESS were to continue to operate past this point, all ES devices would require a $33 \%$ increase in their current contributions. This would result in a reduced runtime and would impact the long term SoH of the batteries, particularly the lead acid.

\subsubsection{Test IV: 2 Lithium Ion Batteries, Lead Acid Battery, and a Supercapacitor with Supercapacitor in Rolling Charging}

Due to SCs having a significantly lower energy density, they represent the weakest link in the Case III HESS. Thus, in Test IV they have been elected to be rolling charged, while a conservative approach is taken to maximize the $\mathrm{SoH}$ of the batteries by only dynamically charging the SC. Shown in Figure 10.22, the total test duration was $195 \mathrm{~min}(3.26 \mathrm{~h})$, where it can be seen that, as the voltage of the capacitor decayed to a pre-specified cutoff, it was decoupled from the stack, charged, and placed back in after achieving another full charge.

The SC voltage cutoff was preset to $8 \mathrm{~V}(50 \% \mathrm{SoC})$ in this scenario to avoid a major drop in the HESS array voltage. This process was repeated until one of the remaining batteries reached its full discharge voltage cutoff. The switching operation performed seamlessly without any noticeable impacts on the DC Bus. Figure 10.23 shows a comparison between the variable ESMC input array voltage and the resulting output DC Bus voltage. A zoom is provided revealing a maximum variation of the DC Bus of \pm 1.205 $\mathrm{V}$ and an average variation after the converter settles of only $\pm 0.358 \mathrm{~V}$. 


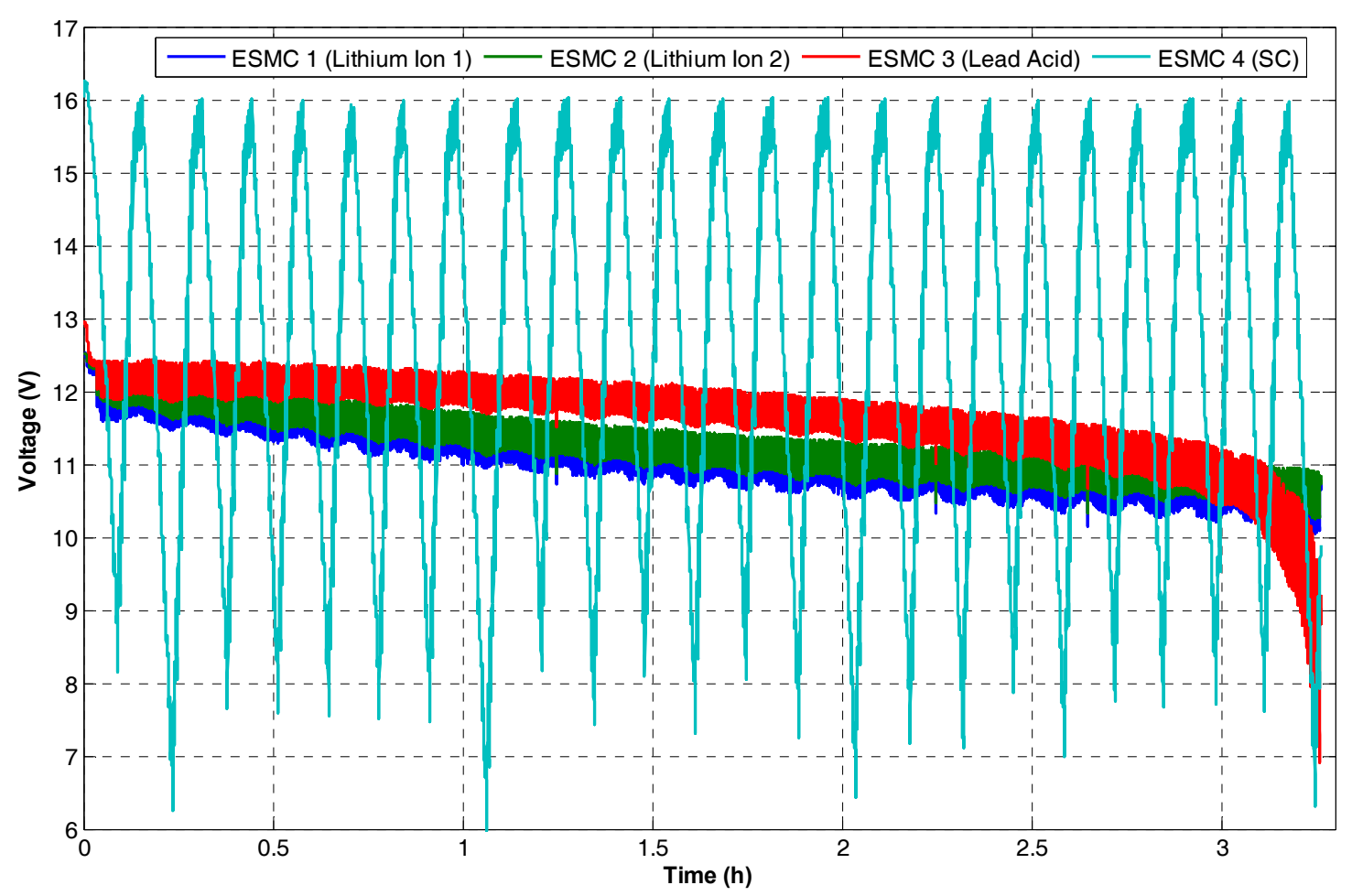

Figure 10.22. Test IV: Voltage from Individual Energy Storage Modules.

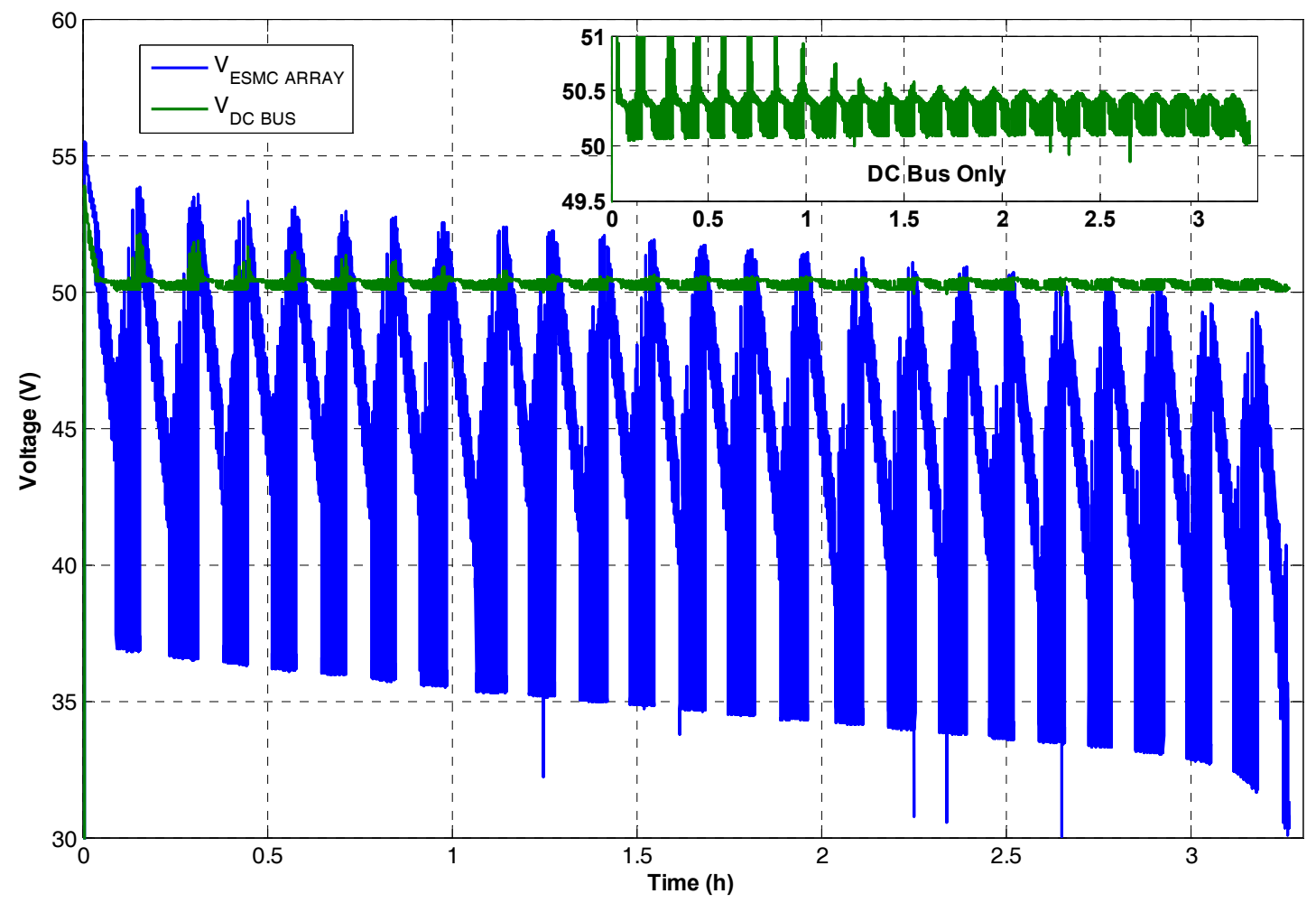

Figure 10.23. Test IV: Voltages from the ESMC Input Array and DC Bus. 


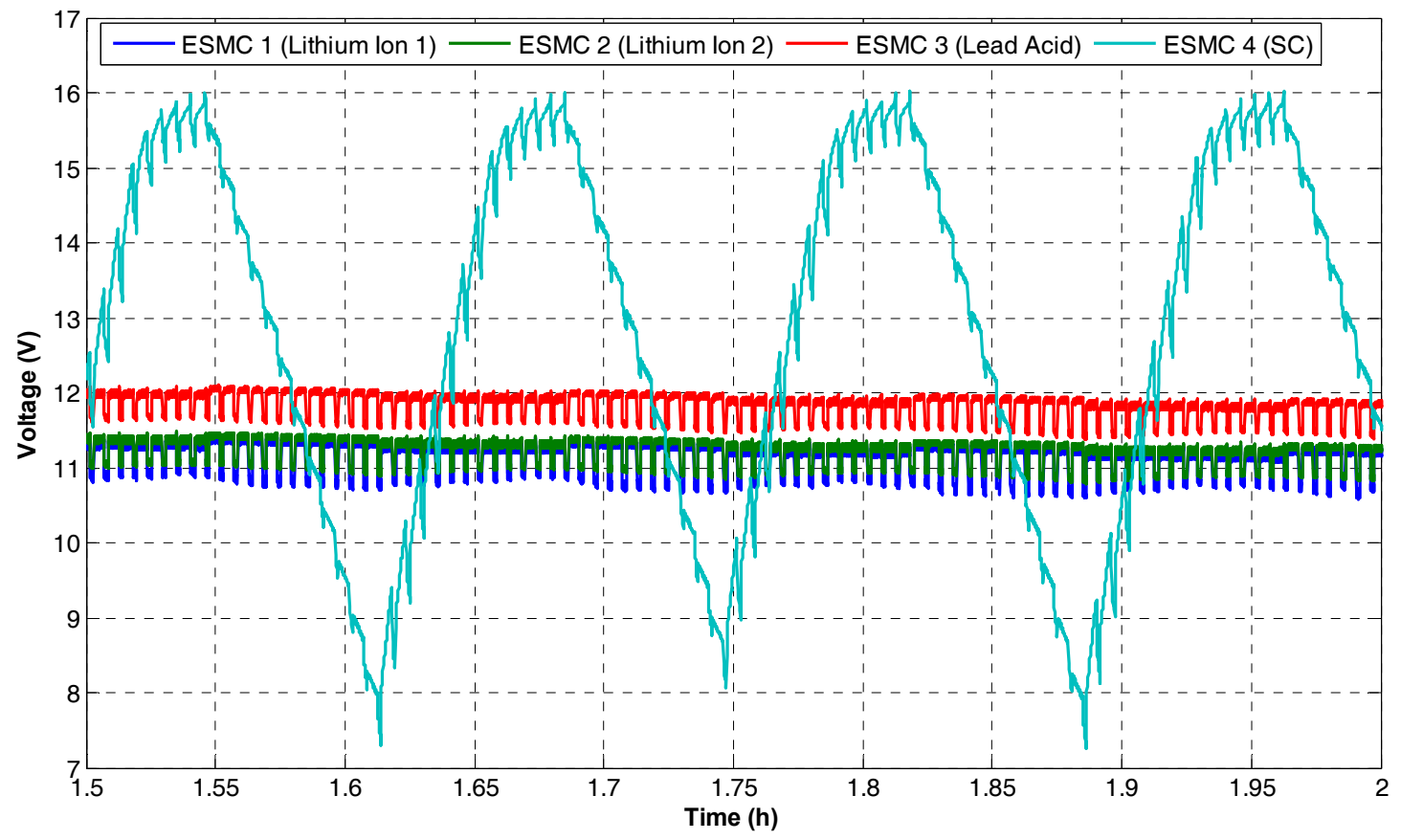

(a)

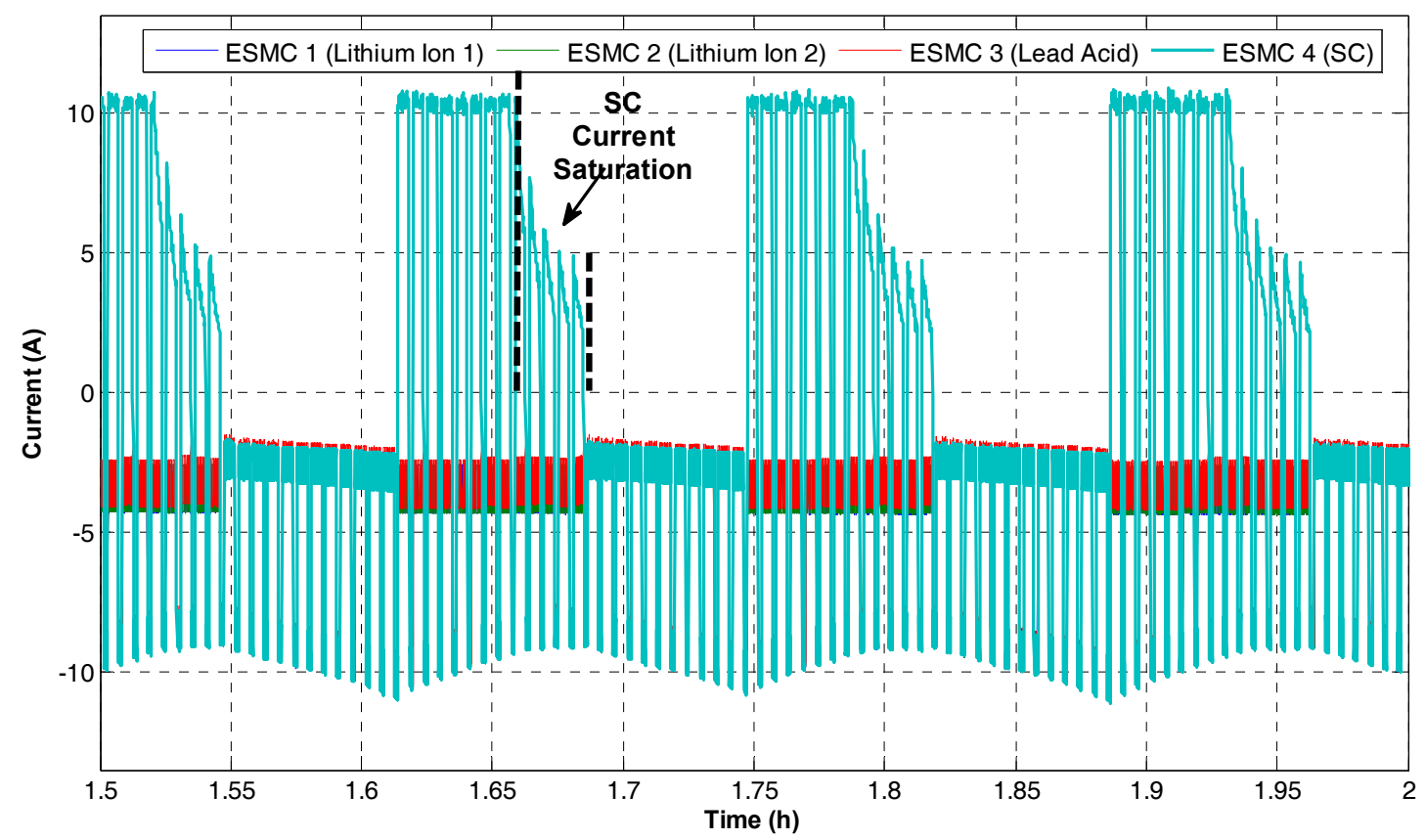

(b)

Figure 10.24. Test IV: 30 min Zoom from Individual Energy Storage Modules (a) Voltages and (b) Currents. 
Figure 10.24(a) shows a close-up of the SC voltage, depicting its wide variation as well as how it saturates when approaching a full charge. The impact of the saturation is more clearly demonstrated in Figure 10.24(b), where the absorbed SC current starts to decrease. An alternative solution to the energy mismatch problem is to increase the size of the SC; however, this would add weight and require more real estate. Since these are two factors which are tightly constrained in the modern SPS, the ESMC provides an effective solution.

\subsubsection{Test V: 2 Lithium Ion Batteries, a Lead Acid Battery, and a Supercapacitor all in Rolling Charging}

In the final test, the same configuration was utilized, except now rolling charging is applied to all four ES devices to achieve a maximum runtime and SoC balance amongst ES modules. The test duration was $25 \mathrm{~min}(0.42 \mathrm{~h})$, divided into seven intervals representing one single sequence that executes rolling charging once on all modules. This sequence could be repeated until the full discharge cutoff of the HESS has been reached. The seven intervals are as follows: 1) no charging of any ES module, 2) charging the SC, 3) charging the first lithium ion module, 4) charging the SC again, 5) charging the second lithium ion module, 6) charging the SC again, and finally, 7) charging the lead acid battery.

It can be seen from Figure 10.25(a) that the varying charging characteristics for all three ES types are met and the HESS operation is stable. Figure 10.25(b) illustrates the energy output from each ES module over the test. The energy output from the batteries following $25 \mathrm{~min}$ is approximately $2 \mathrm{Ah}$. Using the total energy output observed in the endurance test as a reference, this suggests this sequence could be repeated seven to eight times to cover a full discharge cycle. It is worthy to mention that arrows at the bottom of the figure indicate a current envelope, better realized by Figure 10.25(c) and Figure 10.26. 


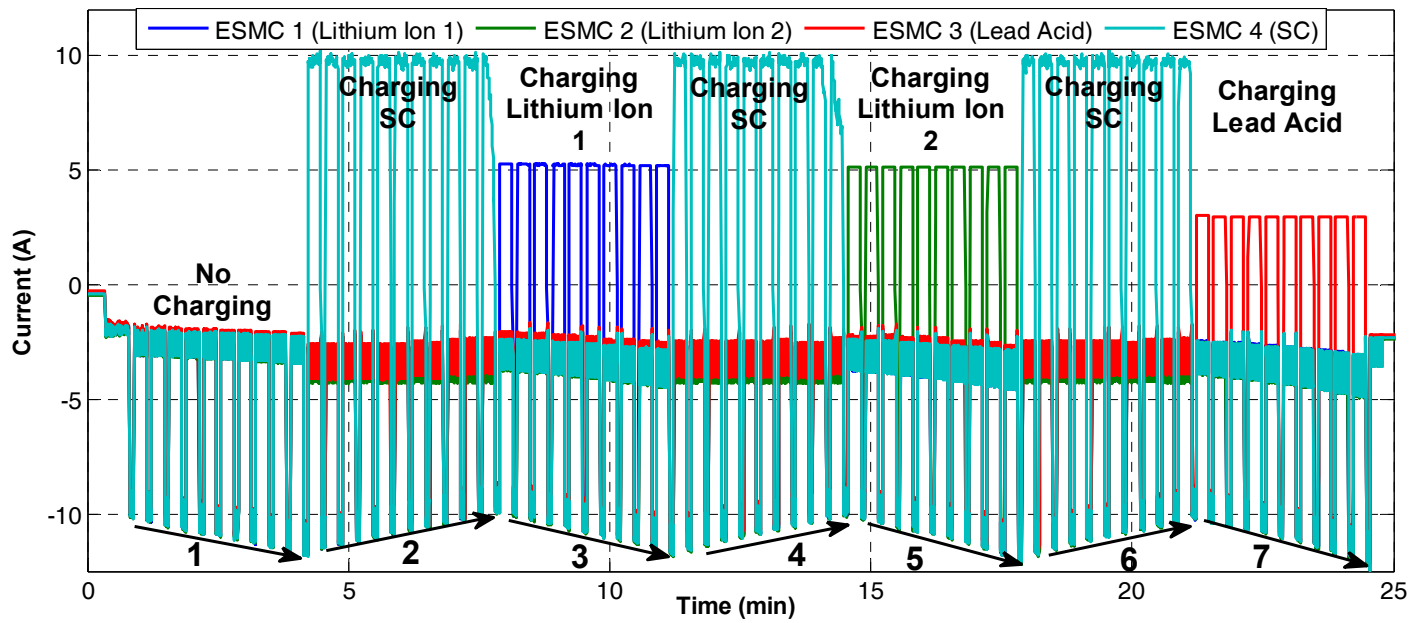

(a)

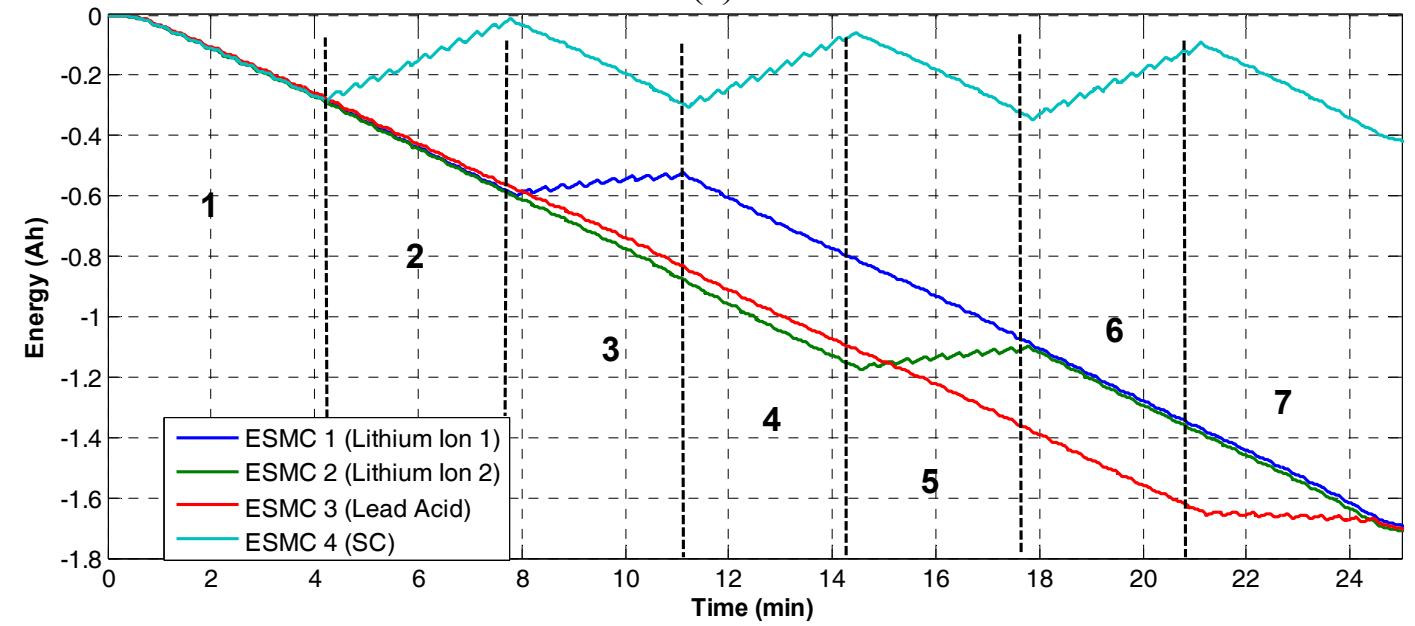

(b)

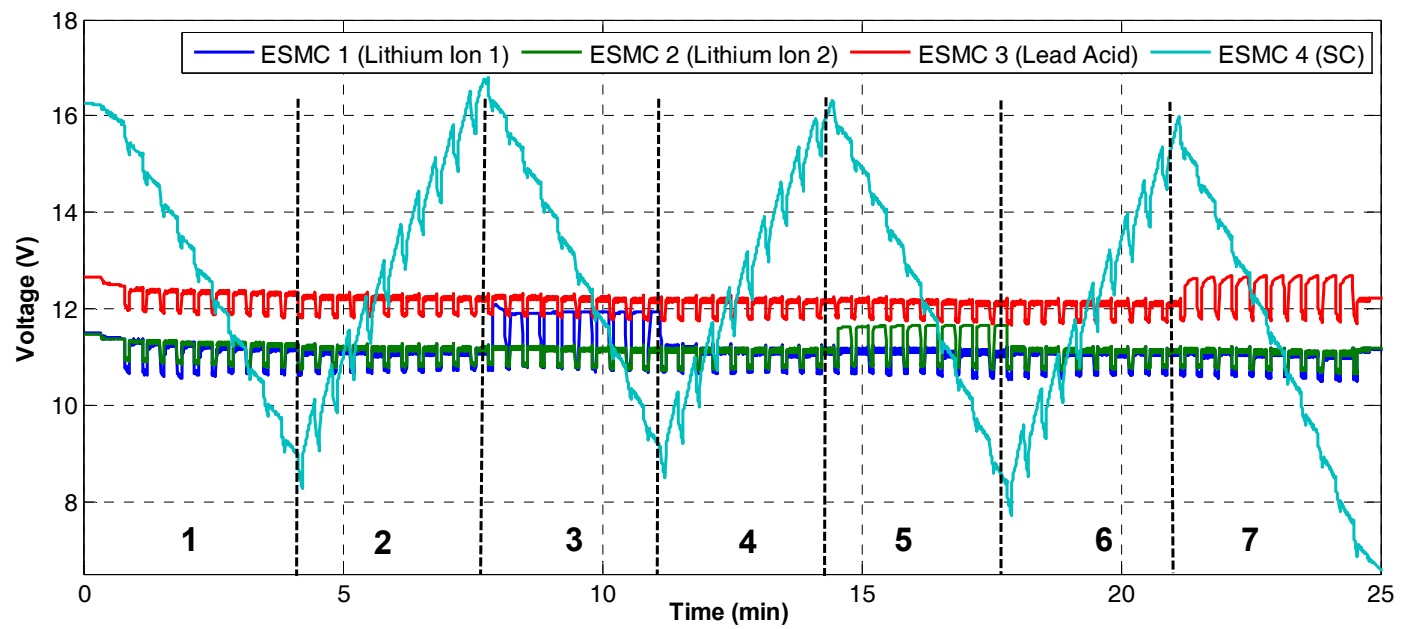

(c)

Figure 10.25. Test V: Individual Energy Storage Modules (a) Currents, (b) Voltages, and (c) Energy. 


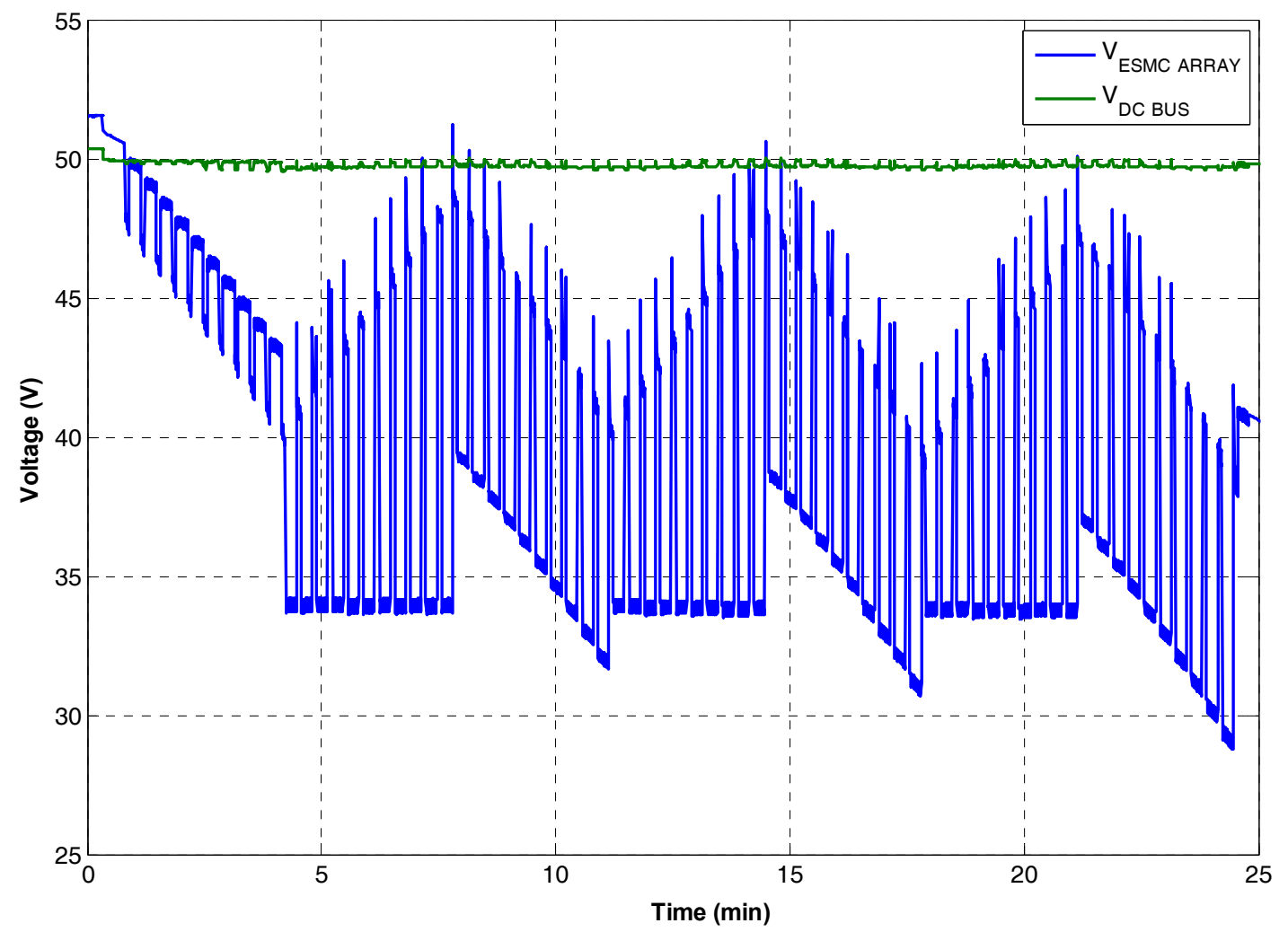

Figure 10.26. Test V: Voltages from the ESMC Input Array and DC Bus.

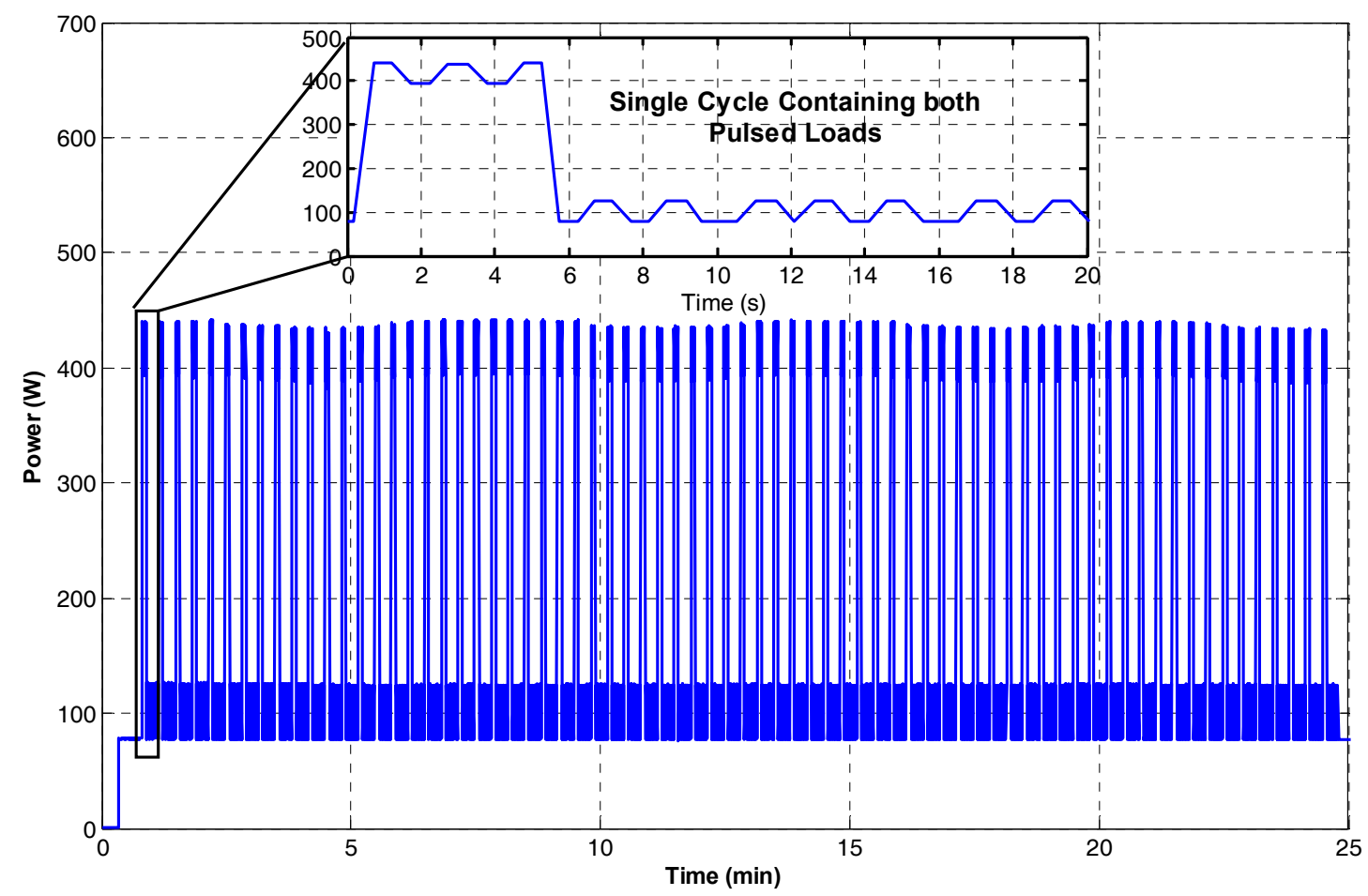

Figure 10.27. Test V: Power Output to the DC Bus. 
When charging the SC, its voltage quickly increases, which was hereby reflected back on the voltage of the entire stack. Since constant power loads were used, increasing the stack voltage resulted in decreased required current injection from each ES module. This is confirmed in Figure 10.27, where the constant and multiple pulsed load outputs to the DC Bus operated as expected.

The voltage of each individual ES module was shown in Figure 10.25(c). Over the course of the entire test, the SC voltage varied between $16.2 \mathrm{~V}$ and $8.0 \mathrm{~V}$. Test V further highlights the importance of individual monitoring and control of each ES module in a HESS. Figure 10.26 depicts a comparison of the array voltage from the primary side of the boost converter and the output DC Bus voltage. The array voltage experiences wide fluctuations due to coupling and decoupling of ES modules, however, these are not reflected back to the DC Bus due to the converter. Only minor voltage fluctuations are detected on the DC Bus and fall well within standard limits [65]. It should be noted that during all the performed tests, a basic boost converter similar to the architecture in the previous chapter was used.

\subsection{Summary}

In this chapter, the modeling and management of an advanced HESS was evaluated. The equivalent models for three different HESS containing combinations of lead acid and lithium ion batteries as well as SC ES were derived and verified experimentally. A coordinated control technique was introduced to handle the charging of different ES types, with a goal to extend the operating duration of the HESS array when exposed to one or more pulsed loads, common in modern SPS. Using an ESMC connected to each ES device, a single module is capable of being extracted from the array and connected to a charging 
bus to restore some of its lost energy during heavy loading periods. This system provided an effective solution to manage multiple ES types to serve multiple pulsed loads on a SPS platform.

Following the utilization, retrofitting, and upgrading of the initial ESMC control software, three novelties were explored in this chapter. First, the modeling and evaluation of multiple new series-configured HESS architectures were designed, tested, and evaluated. Second, multiple Naval SPS pulsed loads with varying frequencies and magnitudes were evaluated and normalized in a per-unit system to conduct scaled laboratory testing. Third, a specialized dispatch control scheme, coined as rolling charging, was designed to coordinate the charging and discharging of individual ES devices while the system was in operation to extend runtime and make tradeoffs in SoH impacts for the battery ES. The effectiveness and seamless operation of the system was verified extensively through hardware testing. In the next chapter, another HESS is introduced composed of a lithium ion battery and SC HESS and investigated for its usage in EV applications. A new control scheme is engineered with objectives to reduce the rate of $\mathrm{SoH}$ degradation of the lithium ion battery module, while improving the efficiency of charging by regenerative braking technology. Once again, ESMC devices are deployed to control the HESS and upgraded to support a parallel-configured system. 


\section{Chapter 11 Lithium Ion Battery - Supercapacitor Hybrid Energy Storage}

\section{Systems}

\subsection{Introduction}

The electric vehicle (EV) and transportation electrification has become a major topic of interest in this dissertation. Many of the metrics, hardware, and algorithms that have been developed and tested in previous chapters have placed a focus upon supporting their challenging loading profiles. From a systems and markets point-of-view, the goal has steered toward achieving an all-electric powertrain. This has also sparred interest in designing new electric motors and drivetrains to operate more efficiently, while accepting the challenge of reducing their size and weight, and still meeting safety regulations [198]. Directly in-line with the powertrain is a heightened interest into the batteries, or energy storage (ES) needed to deliver the adequate power and energy densities to meet or exceed that of the internal combustion engine [199]. In order to accomplish this feat, the market has broken these vehicles into three distinct categories: hybrid electric vehicles (HEVs), plug-in hybrid vehicles (PHEVs), and battery electric vehicles (BEVs) [200]. In each category, the importance of the battery bank increases until a full electric drivetrain is reached.

The HEV is primarily powered by a conventional internal combustion engine, but features two complimentary drive systems containing an electric motor connected to a battery bank as well. The battery bank can only receive energy through two methods: the combustion engine directly or regenerative braking. The PHEV operates primarily on the electric motor and battery bank, but is equipped with an internal combustion engine as well 
to take the place of the electric motor when the state of charge (SoC) of the battery bank is low. The battery bank on the PHEV is primarily charged through a grid connection, but like the HEV, can also be recharged using the combustion engine or regenerative braking. Finally, the BEV presents a fully electric drive train that relies solely on the battery bank. Without the presence of a combustion engine, there are only two methods to recharge the BEV: a grid charging connection or regenerative braking.

With both PHEVs and BEVs placing a heavy reliance on the battery bank, the installation of EV charging stations has been on a rise [201],[202]. Companies like NRG Energy have helped support the extension of charging stations by taking on the excessive investment costs for businesses and simply charging monthly rental fees in exchange for installation [203]. Other EV companies, such as Tesla, have deployed their own high-speed supercharging stations, with over 3,000 free to use by Tesla owners [204]. More than 1 million stations were already deployed in 2014 and it is forecasted to be well beyond 10 million by 2020 [205].

While high-speed charging of the battery bank will become crucial in the success of the BEV and PHEV, a focus has steered toward engineering of the ES system (ESS). Legacy systems, particularly HEVs, employed the usage of nickel metal hydride (NiMH) battery arrays [199]. This has since shifted to utilize primarily lithium ion compounds as a result of their high energy density and cycle life, though it is still limited to less than 5,000 cycles, even when the battery is managed conservatively [206]. A limited deep cycling life, combined with such a wide variance in the battery lifespan, or State of Health $(\mathrm{SoH})$, has opened the floor for research into alternative hybrid energy storage systems (HESS). In many cases, a single energy storage (ES) or battery solution is insufficient. As explored in 
previous chapters, HESS can help to reduce the burden upon the battery while possessing new characteristics that can further improve the performance over a single battery array. However, keen management and control are required to extend and preserve battery lifespans [190],[207]. In EV onboard applications, where size and weight are at a premium, a combination of a lithium ion battery bank with a supercapacitor (SC) bank presents one of the most practical choices.

In Reference [43], the challenges faced in integrating a HESS composed of a lithium ion battery and SC were discussed, where ineffective control resulted in extensive system losses. A rule-based algorithm was introduced to manage the power split between the battery and SC during a drive cycle, but the long-term impacts of battery cycling were not taken into consideration. Reference [208] looked at replacing the NiMH battery with a SC in a lead acid and NiMH battery HESS for an HEV. In this scenario, the vehicle would be capable of shutting off the combustion engine when it is stopped, and charge the SC using regenerative braking. However, the $\mathrm{SoH}$ consequences in cycling the battery were not investigated. In this chapter, a new management scheme for an EV lithium ion battery and SC HESS is developed and tested experimentally. Using the Department of Energy (DOE) PHEV Hybrid Pulsed Power Characterization (HPPC) test profile, standardized discharge drive and regenerative braking pulse tests are periodically applied to the HESS to evaluate its performance [209]. Using the HPPC profile, a legacy lithium ion battery and SC HESS is assessed for its energy harvesting efficiency and the cycling impacts on the battery.

\subsection{Mathematical Models}

In order to demonstrate differences in lithium ion battery and SC behavior and performance, an extensive analysis is first made into the lifetime performance of the 
common lithium ion cell. This analysis reveals how cycling contributes to the development of a solid electrolyte interface (SEI) layer, leading to capacity loss. Next, equivalent circuit models and cycling curves are depicted for both a common 4-cell lithium ion battery and 58 F SC module.

Table 11-1. Cycle Life until 30\% Capacity Loss $(70 \% \mathrm{SoH})$ as a Function of Depth of Discharge.

\begin{tabular}{c|c|c}
\hline \hline Energy Storage Type & Depth of Discharge & Discharge Cycles \\
\hline Supercapacitor & $100 \%$ & $500,000-1,000,000$ \\
\hline Lithium Ion Battery & $100 \%$ & $300-500$ \\
\hline Lithium Ion Battery & $50 \%$ & $1,200-1,500$ \\
\hline Lithium Ion Battery & $25 \%$ & $2,000-2,500$ \\
\hline Lithium Ion Battery & $10 \%$ & $3,750-4,700$ \\
\hline
\end{tabular}

\subsubsection{Lithium Ion Battery Cycle Life Analysis}

Lithium ion batteries have become commonplace for usage in PHEVs and BEVs as a result of severe SoH risks in the utilization of lead acid batteries under deep depth of discharge (DoD). As mentioned previously, the $\mathrm{SoH}$ of the lithium ion battery is dependent upon the operational current and the DoD. High operational currents generate excessive heat, which result in micofracturing of active materials inside the battery cell. Through active thermal control, these impacts can be reduced. However, its dependence on the DoD is fixed. Table 11-1 depicts the lithium ion battery cycle life as a function of the $\mathrm{DoD}$, as compared to the SC [26],[28],[210]. In general practice, estimations define the battery cycling life as the point where the usable battery capacity drops by $30 \%$ from its nameplate (70\% remaining capacity) [210]. For the purpose of this study, a cycle is defined as anytime a battery has three sequential changes in the direction of current (i.e. oxidation, reduction, 
and oxidation). In an EV application, $100 \%$ DoD and 10\% DoD are not practical, thus the battery lifetime is expected to be between 1,500 and 2,500 cycles.

Unfortunately, a precise figure of battery cycle life is not practical, as it would require a comprehensive history consisting of many variables. These variables not only include operational characteristics, but intricate manufacturing differences in the battery cell and quality of its materials. As previously mentioned, battery SoH has not yet been assigned a formal definition. In this dissertation, it has been viewed as a percentage of the remaining usable capacity versus the nameplate, thus a battery cycle life is designated to end when the SoH drops below 70\%.

A deeper investigation has been completed through an addition to the physics-based model (PBM) developed previously, which places a primary cause of capacity loss upon the development of electrochemical side reactions. As the battery is placed under numerous heavy charging and discharging cycles, an SEI layer develops, increasing the resistivity in lithium ion flow to and from the negative electrode [211],[212]. From a power engineering perspective, this can be viewed upon as an additional component within the electrochemical pseudo-2D (P2D) model acting solely as a parasitic layer. This layer, composed of degraded materials, grows slowly with every charging and discharging cycle. The current the SEI layer consumes results in an increased voltage drop during each cycle when the same load is applied.

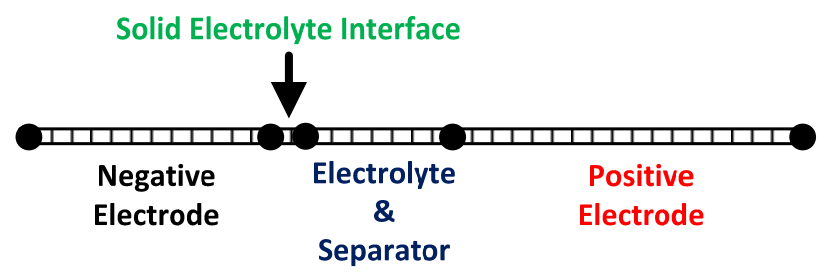

Figure 11.1. Modified Lithium Ion Battery Psuedo-2D Model Including Solid Electrolyte Interface. 
To demonstrate the effect of the SEI layer growth and resulting capacity loss experienced cycle-to-cycle, a modification has been made to the previous P2D battery cell cross-sectional model from Chapter 5, as shown in Figure 11.1. From a modeling perspective, growth of the SEI layer can be viewed as an additional electrochemical reaction, simultaneously occurring with the intercalation of lithium ions to inhibit operation. An additional parasitic lithium solvent reaction models the solvent $\mathrm{S}$ and product $\mathrm{P}$ formed during the reaction, demonstrated by the following chemical balance:

$$
\mathrm{S}+2 \mathrm{Li}^{+}+2 e^{-} \rightarrow \mathrm{P}
$$

Through accounting for the side reaction in Equation (11-1), the PBM can now include performance impacts caused by the expansion of graphite particles due to lithium intercalation. This process inherently exposes the graphite surface to the electrolyte, which hereby results in an increase in resistance across the SEI layer. The kinetics of the parasitic reaction are described by:

$$
i_{\text {loc,side }}=i_{0_{\text {side }}} \frac{c_{L i^{+}}}{c_{L i^{+}, \text {ref }}} e^{-\frac{F \eta}{2 R T}}
$$

where $c_{L i^{+}}$is the lithium ion concentration in the electrolyte, $c_{L i^{+}, \text {ref }}$ is the reference concentration, $R$ is the universal gas constant $(8.314 \mathrm{~J} / \mathrm{mol} \cdot \mathrm{K}), F$ is Faradays constant ( 96,485 Coulombs/mol), $T$ is the absolute temperature, and $\eta$ is the activation overpotential. Growth of the SEI layer thickness $\delta_{S E I}$ next to the negative graphite electrode is modeled by:

$$
\frac{\partial \delta_{S E I}}{\partial t}=-\frac{i_{\text {loc,side }} M_{P}}{2 F \rho_{P}}
$$

where $M_{P}$ is the molar weight $(0.1 \mathrm{~kg} / \mathrm{mol})$ and $\rho_{P}\left(2100 \mathrm{~kg} / \mathrm{m}^{3}\right)$ is the density of P formed by the reaction. The resistance of the SEI layer forming on the negative electrode is then 
calculated by the traditional form:

$$
R_{S E I}=\frac{\delta_{S E I}}{\kappa}
$$

where $\kappa$ is the conductivity of the SEI layer, obtained experimentally from Reference [211] to be $0.379 \mu \mathrm{S} / \mathrm{m}$.
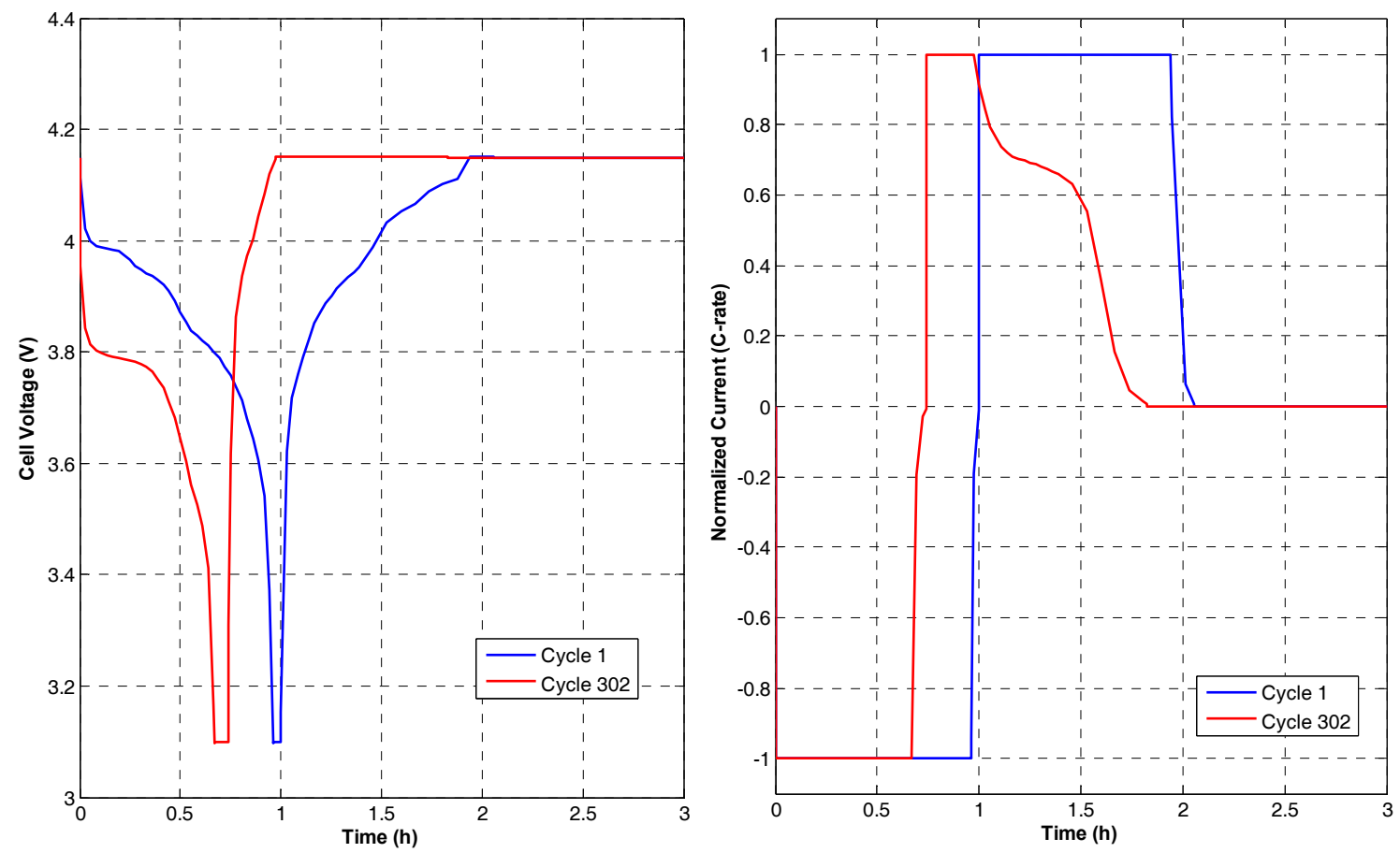

Figure 11.2. Voltage (top) and Current (bottom) Profiles for the Lithium Ion Battery Degradation Model in the First and Last Cycle.

The enhanced PBM is then exposed to 302 battery cycles down to $100 \%$ DoD per cycle before reaching 70\% SoH. Each cycle consists of a 1-hour Coulombic (1C) rate charge and discharge. The charging profile includes both constant current (CC) and constant voltage (CV) stages, starting at an initial current of $1 \mathrm{C}$ with a $\mathrm{CV}$ transition at $4.2 \mathrm{~V}$ until reaching a full charge current $\left(0.1 \mathrm{~A} / \mathrm{m}^{2}\right)$. Each charge and discharge stage is followed by a relaxation period at open circuit voltage $(\mathrm{OCV})$ until each total cycle equals $3 \mathrm{~h}$. 


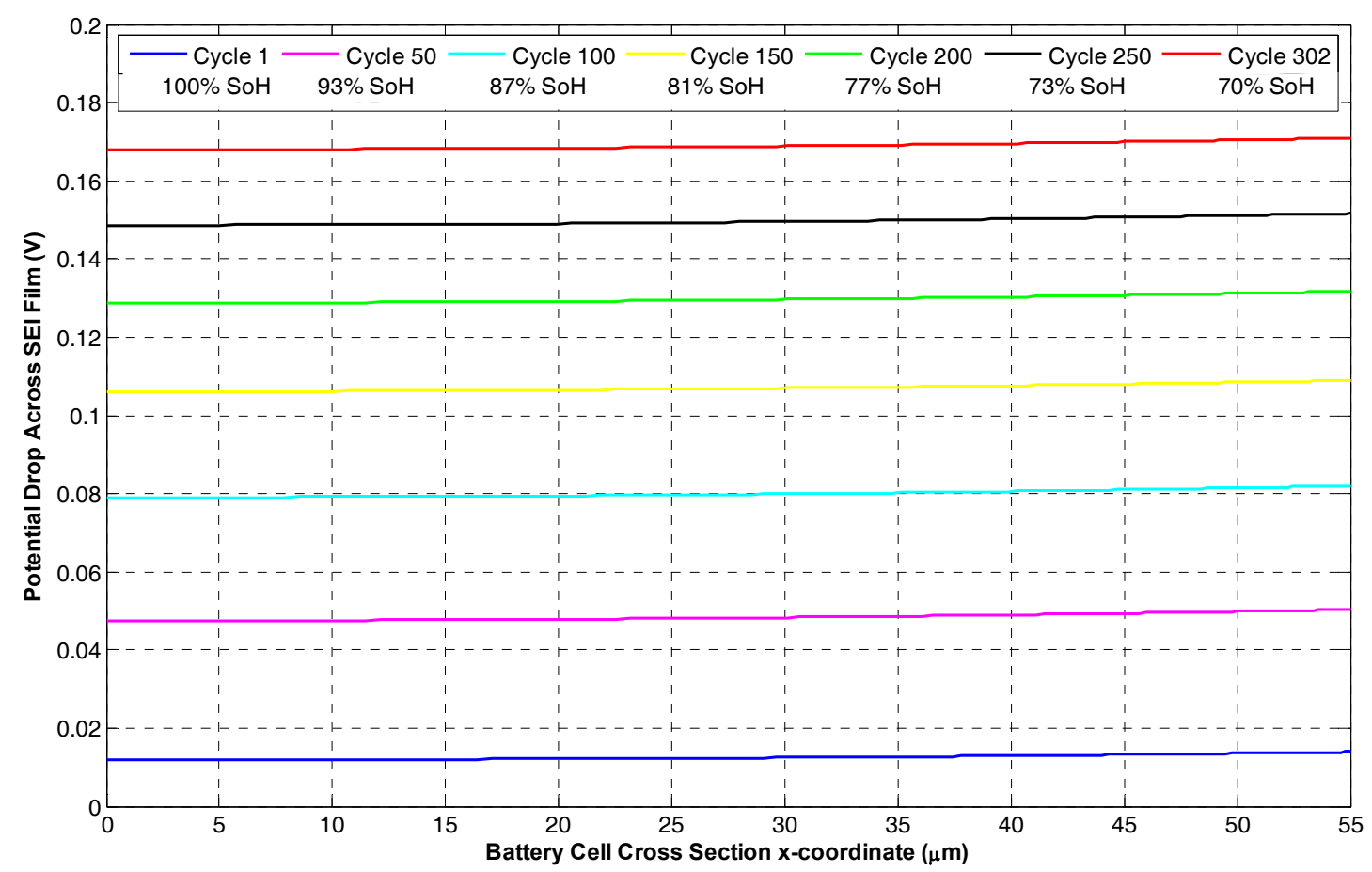

Figure 11.3. Cycle Comparison of Voltage Drop across the SEI Layer after $100 \mathrm{~s}$ into 1C Discharge Cycle.

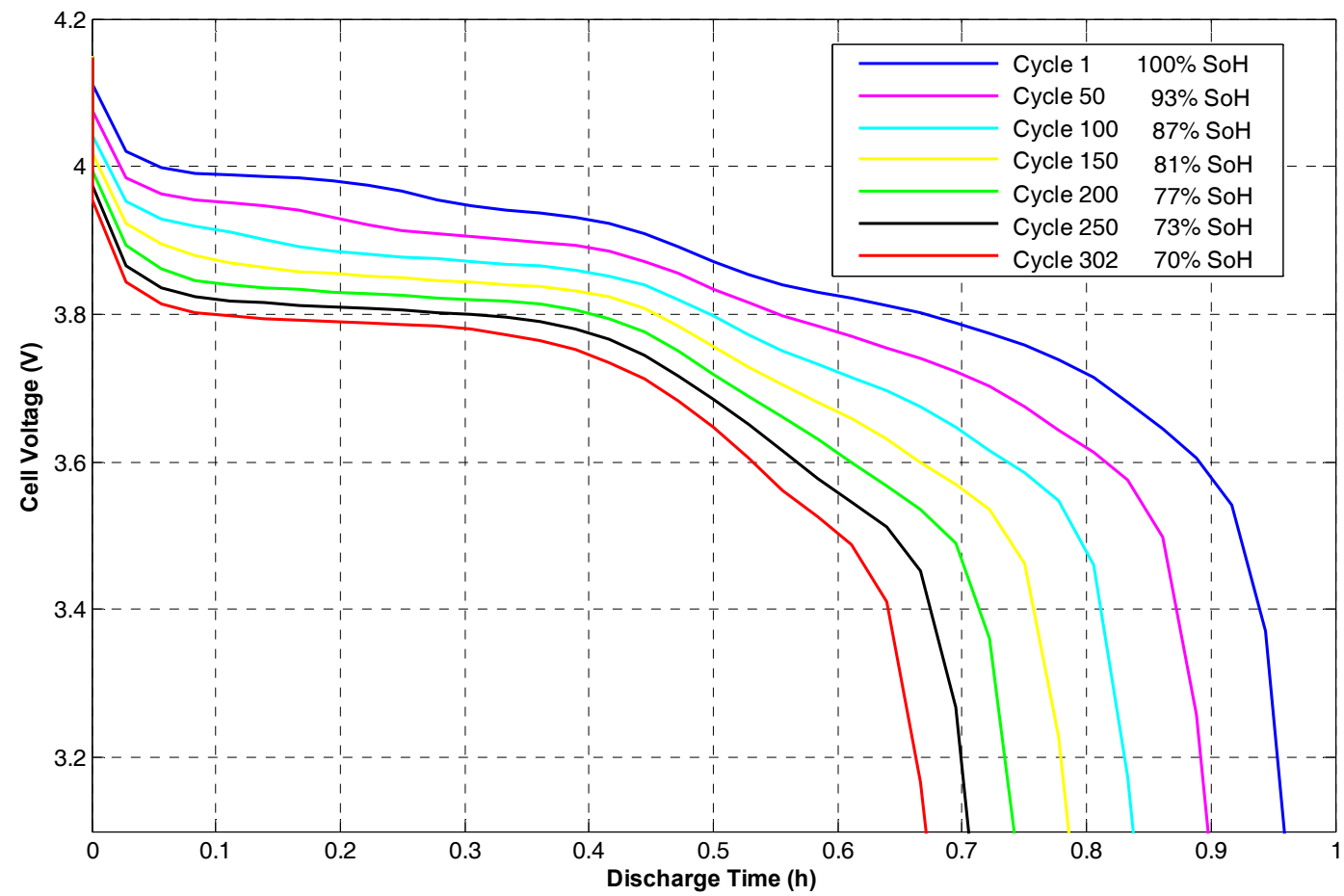

Figure 11.4. Discharge Voltage Profile Comparison and Capacity Retention. 
Figure 11.2 compares the battery cycle profile during the $1^{\text {st }}$ and $302^{\text {nd }}$ (last) cycle. One can notice a significant reduction in the discharge period in the $302^{\text {nd }}$ cycle, as the energy output has now been reduced by $30 \%$. This reduction is a result of an increased voltage drop across the SEI layer under load. This is better demonstrated in Figure 11.3, where the overpotential across the SEI layer is depicted at each position across the cell $100 \mathrm{~s}$ into the 1C discharge cycle. A closer look at the terminal voltage profile during dischages for every 50 cycles until reaching the $302^{\text {nd }}$ cycle is shown in Figure 11.4, revealing how the SEI growth results in a reduction of both the runtime and usable energy.

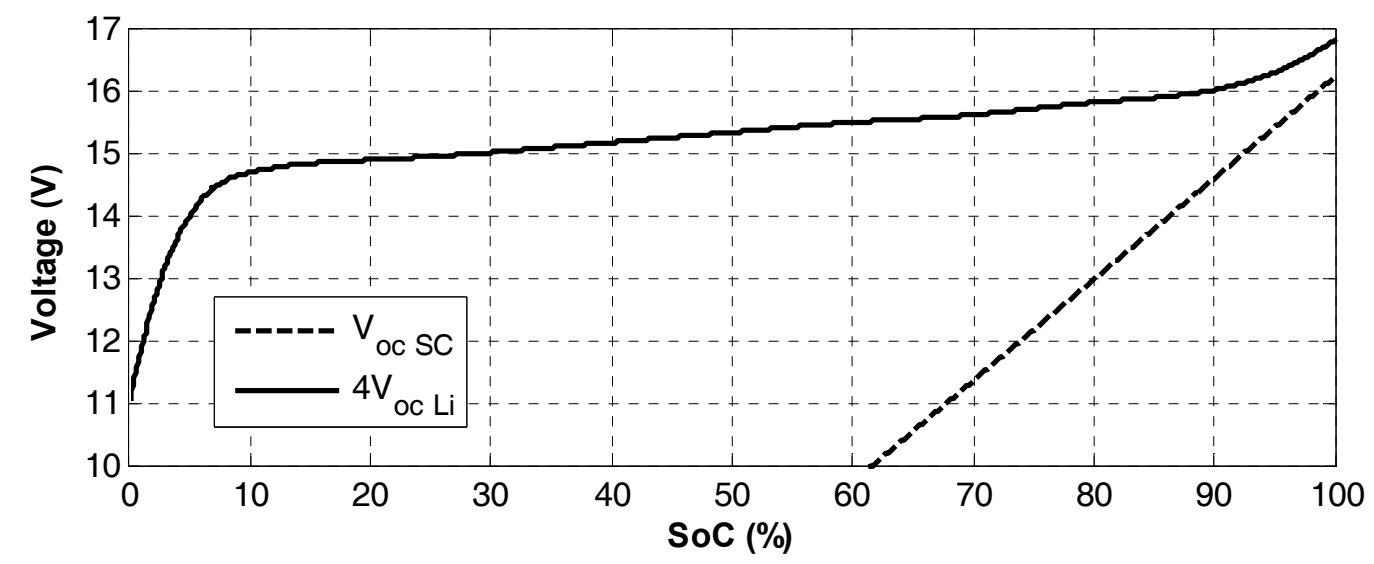

Figure 11.5. Open Circuit Voltage versus State of Charge for a 4-cell Lithium Ion Battery and $58 \mathrm{~F}(16.2 \mathrm{~V})$ Supercapacitor.

\subsubsection{Lithium Ion Battery Electric Vehicle Model}

The battery model for the EV is once again chosen, keeping the application and required level of computational analysis in consideration. Figure 11.5 provides a graphical representation of the relationship between the OCV for a 4-cell lithium ion battery $4 \mathrm{~V}_{\mathrm{Oc}} \mathrm{Li}$ and the SoC [199]. The SoC is driven by a number of nonlinear electrochemical processes, but remains relatively linear under the practical $10-90 \%$ SoC EV operating range when the SoH is high. Similar to the shipboard power system (SPS) profile, the HPPC test profile 
features changes in the seconds-range, thus a $1^{\text {st }}$-Order Randles equivalent circuit model is sufficient. However, unlike the SPS, in this case the equivalent circuit is broken into two parts to differentiate the dynamics of the battery in both charging and discharging modes.

Figure 11.6 depicts an enhanced equivalent circuit for the lithium ion battery, where resistance parameters change based on charging $R_{x \mathrm{c}}$ or discharging $R_{x \mathrm{~d}}$, respectively. Ideal diodes are present to enforce that charging and discharging cannot occur simultaneously. $R_{2 \mathrm{c}}$ and $R_{2 \mathrm{~d}}$ represent a varying ohmic resistance parameter during charging and discharging, $C_{1}$ represents the polarization capacitance, and $R_{1 \mathrm{c}}$ and $R_{1 \mathrm{~d}}$ represent a varying polarization resistance also observed during charging and discharging. The transfer function depicting the equivalent impedance is:

$$
\mathrm{Z}_{e q_{L i}}(s)=\left\{\begin{array}{lr}
\mathrm{R}_{2 \mathrm{c}}+\frac{\mathrm{R}_{1 \mathrm{c}}}{\mathrm{R}_{1 \mathrm{c}} \mathrm{C}_{1} \mathrm{~s}+1} & \text { charging } \\
\mathrm{R}_{2 \mathrm{~d}}+\frac{\mathrm{R}_{1 \mathrm{~d}}}{\mathrm{R}_{1 \mathrm{~d}} \mathrm{C}_{1} \mathrm{~s}+1} & \text { discharging }
\end{array}\right.
$$

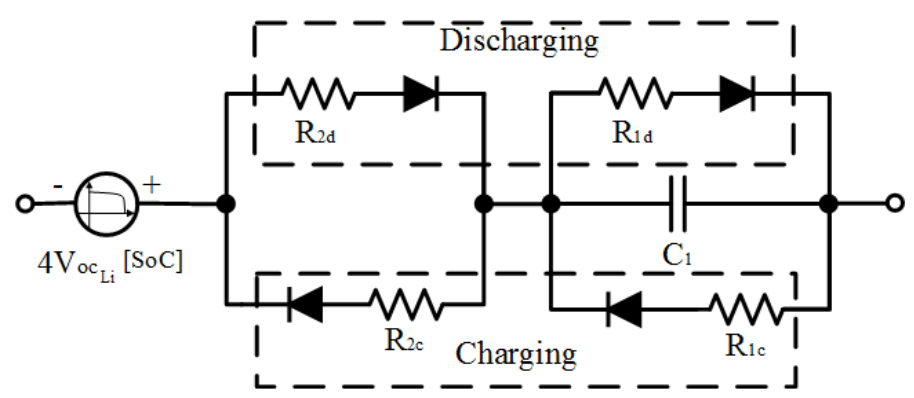

Figure 11.6. Lithium Ion Battery Equivalent Circuit Model Utilized for the Electric Vehicle.

\subsubsection{Supercapacitor Electric Vehicle Model}

In the previous chapter, the $\mathrm{SC}$ was introduced as a revolutionary improvement to the common capacitor as a result of its highly amplified surface area [28]. The SC has a significant advantage over a traditional electrochemical battery in terms of its power 
density, which is generally 100 times greater, but its energy density is reduced by nearly the same factor, making it infeasible for a direct replacement. Although the SC is an electrochemical ES device, no reaction takes place. This results in a lifespan magnitudes longer than a battery with little to no dependence upon its DoD [26],[28]. In the case of the SC, the same $2^{\text {nd }}$-Order model utilized in the SPS is sufficient. Depicted in Figure 11.7, a $2^{\text {nd }}$-Order model is suitable for the seconds-range load and charging timescale. As with the SPS, resistor-capacitor (RC) components are divided, modeling dynamics that stem from inside and outside of the core SC material [141]. The resistance and capacitance outside the electrode pore are denoted by $\mathrm{R}_{\mathrm{o}_{S C}}$ and $\mathrm{C}_{\mathrm{oSC}_{\mathrm{SC}}}$, while $\mathrm{R}_{\mathrm{i}_{\mathrm{SC}}}$ and $\mathrm{C}_{\mathrm{i}_{\mathrm{SC}}}$ represent the resistance and capacitance inside the pore, respectively. The transfer function depicting the $\mathrm{SC}$ equivalent impedance is thus:

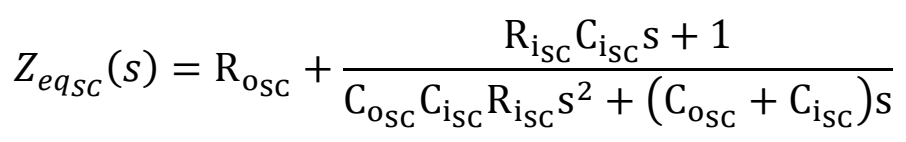

Figure 11.5 revealed a direct correlation between the SoC of the SC and the voltage. A comparison between the $\mathrm{OCV}$ of a $58 \mathrm{~F}(16.2 \mathrm{~V}) \mathrm{SC} \mathrm{V}_{\mathrm{oc}}$ exposes a significantly reduced operating voltage range compared to that of the 4-cell lithium ion battery $4 \mathrm{~V}_{\mathrm{oc}_{\mathrm{Li}}}$. In this case, the minimum operating $\mathrm{SoC}$ value of the $\mathrm{SC}$ is around $67 \%$, corresponding to $0 \%$ SoC on the lithium ion battery [195]. This further constrains the usable capacity to under only one-third of its theoretical value.

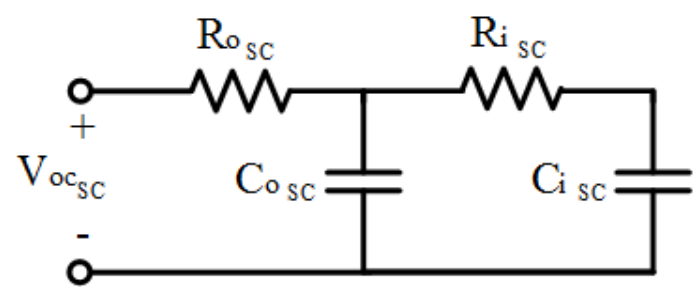

Figure 11.7. Supercapacitor Equivalent Circuit Model Utilized for the Electric Vehicle. 


\subsubsection{Hybrid Lithium Ion - Supercapacitor System}

The hybrid lithium ion and SC system utilized in this management scheme will operate under multiple states. Table 11-1 compared the cycling life of both lithium ion batteries and the SC, where the SC outweighed the lifespan of the lithium ion battery by more than 100 times. In terms of dynamics, the SC also has a much higher power density and can respond much quicker than that of a lithium ion battery. These traits reveal that a SC is a good fit to reduce the burden of placing additional cycles on a battery when the energy requirement is not as high. This would leave the lithium ion battery to source the base power. Under the HPPC test, three states are used: two dispatch either the lithium ion battery or the SC to the charge (regenerative braking) or the discharge (drive) pulses, and the final state features both the lithium ion battery and SC simultaneously connected.

In the third state, although the battery is still exposed to both regenerative braking and discharge pulses, placing a SC in parallel reduces and smoothens the current applied to the battery. Thus, cycling impacts on the battery are reduced. It is important to mention, however, that in terms of response time, a parallel-configured HESS is governed by the slowest source [161]. Since this state features the lithium ion battery and a SC simultaneously connected, a higher-order mathematical model is introduced, as shown in Equation (11-7).

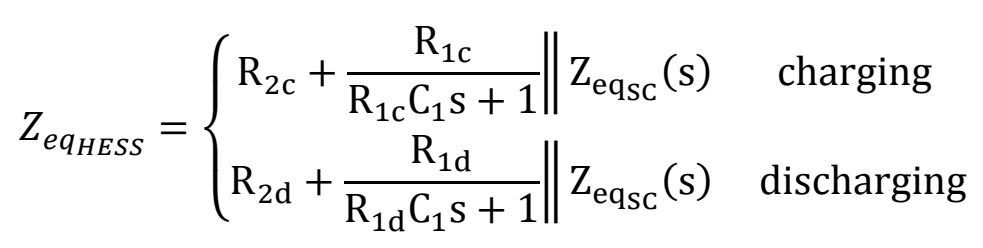




\subsection{Hybrid Pulse Power Characterization Test}

A standardized HPPC test procedure is used to demonstrate the advantages of replacing a traditional lithium ion array with a controlled lithium ion and SC HESS. Although HEVs have been available since the turn of the century, standardization of the battery array was relatively minimal. However, as the PHEV and BEV emerged, a need arose for establishing a standard to quantify and assess ES performance. In December 2010, the DOE Vehicle Technologies Program released an official "Battery Test Manual" based on technical targets to support the performance and life characterization of developed ES arrays [209].

The HPPC test, depicted in Figure 11.8, conducts 1-minute cycles using a normalized current, beginning with a 10 -second discharge (drive) at full test current, followed by a 40second rest, and a 10 -second charge (regenerative breaking) pulse set to $75 \%$ of the HPPC test current. The normalized HPPC current can be scaled based on a battery size factor (BSF) provided by the manufacturer. However, if a BSF is not provided, as in the case of this test, a $1 \mathrm{C}$ current can be used as the HPPC reference current. For this test, $6.4 \mathrm{Ah}$ lithium ion polymer batteries were used, thus the HPPC current is $6.4 \mathrm{~A}$ [164]. Hence, the discharge and charge pulses are set at $-6.4 \mathrm{~A}$ and $+4.8 \mathrm{~A}$, respectively.

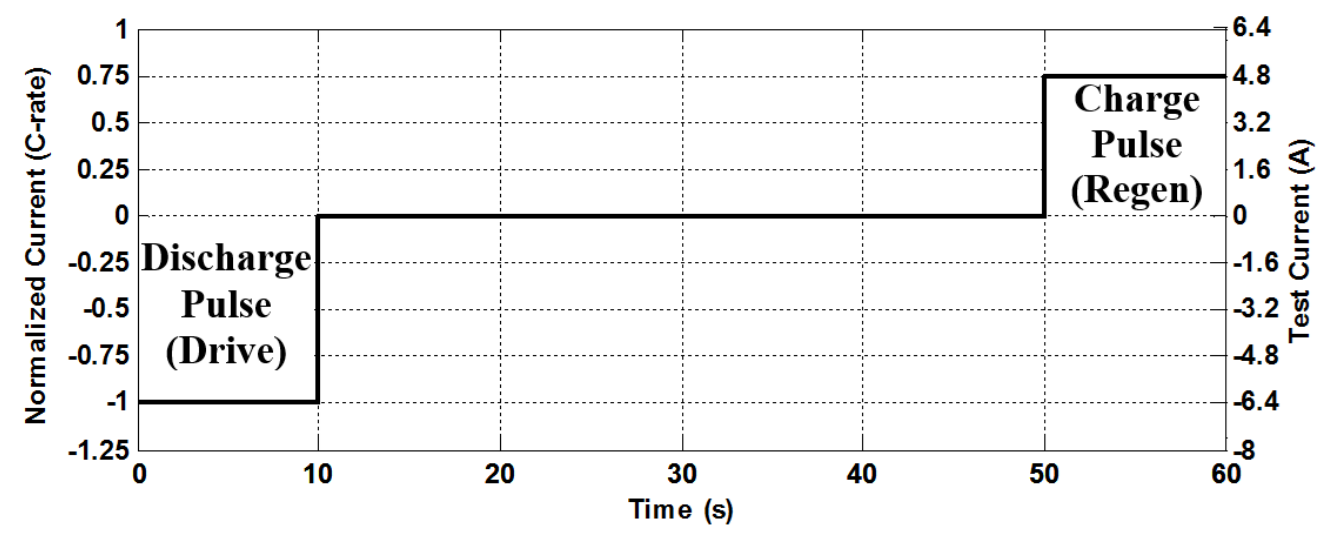

Figure 11.8. Hybrid Pulse Power Characterization Test Profile. 


\subsection{Proposed Hybrid Energy Storage Management Scheme}

The main objective of the proposed management scheme is to reduce the cycling and stresses on the lithium ion battery, thus increasing its lifetime. Another objective is to reduce the wasted energy that cannot be absorbed by the battery when it is at a high SoC due to electrochemical limitations. In order to manage the lithium ion battery and SC HESS, invoke the proper control sequence, and simulate the HPPC profile, Energy Storage Management Controllers (ESMC) were utilized. Shown in Figure 11.9, modifications were required in order to support a parallel-configured HESS array. The management scheme is broken into three different control states. However, since each ES type has a different discharge profile (Figure 11.5), switching between states is more complex. The states of the ESMC switches for each operation mode are shown in Table 11-2. The three states of proposed scheme are outlined as follows.

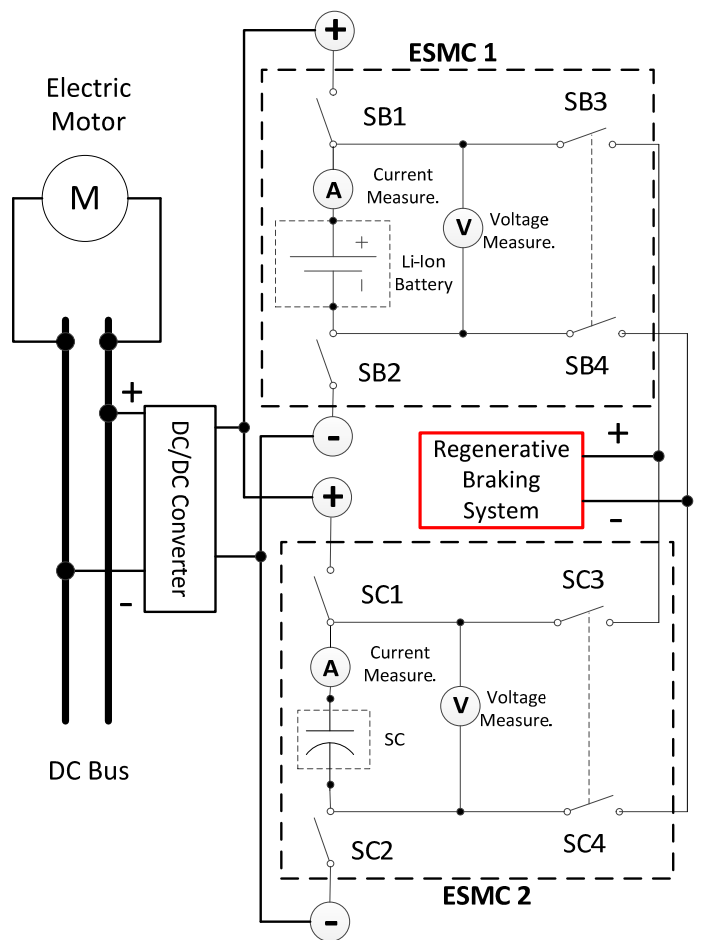

Figure 11.9. Schematic Diagram for Energy Storage Modular Controllers Connected in Parallel for Electric Vehicle Testing. 
Table 11-2. Switching States for Energy Storage Management Controllers in Each Operating Mode.

\begin{tabular}{cccc}
\hline \hline State & Mode & Open switches & Close switches \\
\hline \multirow{2}{*}{1} & Discharge & & \\
& Ideal & SB3, SB4, SC1, SC2 & SB1, SB2, SC3, SC4 \\
& Charge & & \\
2 & Discharge & SB3, SB4, SC3, SC4 & SB1, SB2, SC1, SC2 \\
& Ideal & SB3, SB4, SC3, SC4 & SB1, SB2, SC1, SC2 \\
& Charge & SB1, SB2, SC1, SC2 & SB3, SB4, SC3, SC4 \\
& & & \\
3 & Discharge & SB1, SB2, SC3, SC4 & SB3, SB4, SC1, SC2 \\
\hline \hline
\end{tabular}

\subsubsection{State 1}

In State 1, it is assumed that the lithium ion battery is fully charged and the SC is fully discharged. This scenario is similar to a typical case, where someone is driving to work in the morning and the battery is charged overnight. Since the SC has a high self-discharge rate, there is little justification to charging it in full or trickle charging it overnight. In this state, the discharge pulse is handled by the battery, while the charging pulse (regenerative braking) is handled by the SC. This only exposes the battery to a single cycle until the SC is fully charged, moving the system into State 2.

\subsubsection{State 2}

In State 2, the SC has reached a full charge. In order to continue harvesting energy from the regenerative breaking charge pulse, the SC and lithium ion battery are connected in parallel to absorb the discharging and charging pulses. In this stage, the SC helps to reduce battery current as it trickle charges near a full charge. However, in order to utilize the charging energy, the battery must be exposed to some cycling. State 2 continues until the lithium ion battery SoC drops below 50\%, where a progression into State 3 takes place. 


\subsubsection{State 3}

By the time the system progresses into State 3, the lithium ion battery has discharged considerably, but the SC SoC is still near $100 \%$ as a result of trickle charging in State 2. In this scheme, the charging pulse is now applied only to the battery and the discharge pulse is handled only by the SC. However, this state is limited in duration as a result of the reduced energy density and SoC-to-voltage relationship. Shown previously in Figure 11.5, the SC can only operate until its SoC has reached $\sim 60 \%$ SoC. Following this point, the output voltage through the boost converter would drop below a usable range. Once the SC reaches this point, the system returns to State 1 and progresses through each state again until the lithium ion battery bank has achieved the full discharge cut-off voltage.

\subsection{Experimental Results}

In order to investigate the feasibility of the proposed management scheme, a hardware setup has been established, as depicted in Figure 11.9. Two ESMCs are connected in parallel, where the first ESMC is connected to four 3.7 V, 6.4 Ah series-connected lithium ion cells to form the battery module. The second ESMC is connected to an SC array comprised from three BMOD0058-E016-B02, 16.2 V modules connected in parallel [195]. The same unidirectional DC-DC boost converter utilized previously in the SPS study is now used to interface the HESS to a DC bus, where the electric motor is modeled using a resistive load tuned to draw a $\mathrm{CC}$ during the drive pulses. The system is controlled in realtime using a slightly modified version of the LabVIEW platform introduced in the previous chapter, where the switching has been modified to match the states summarized in Table 11-2. However, since the scheme is simple, it could be easily embedded on the commercialized version of the ESMC. 


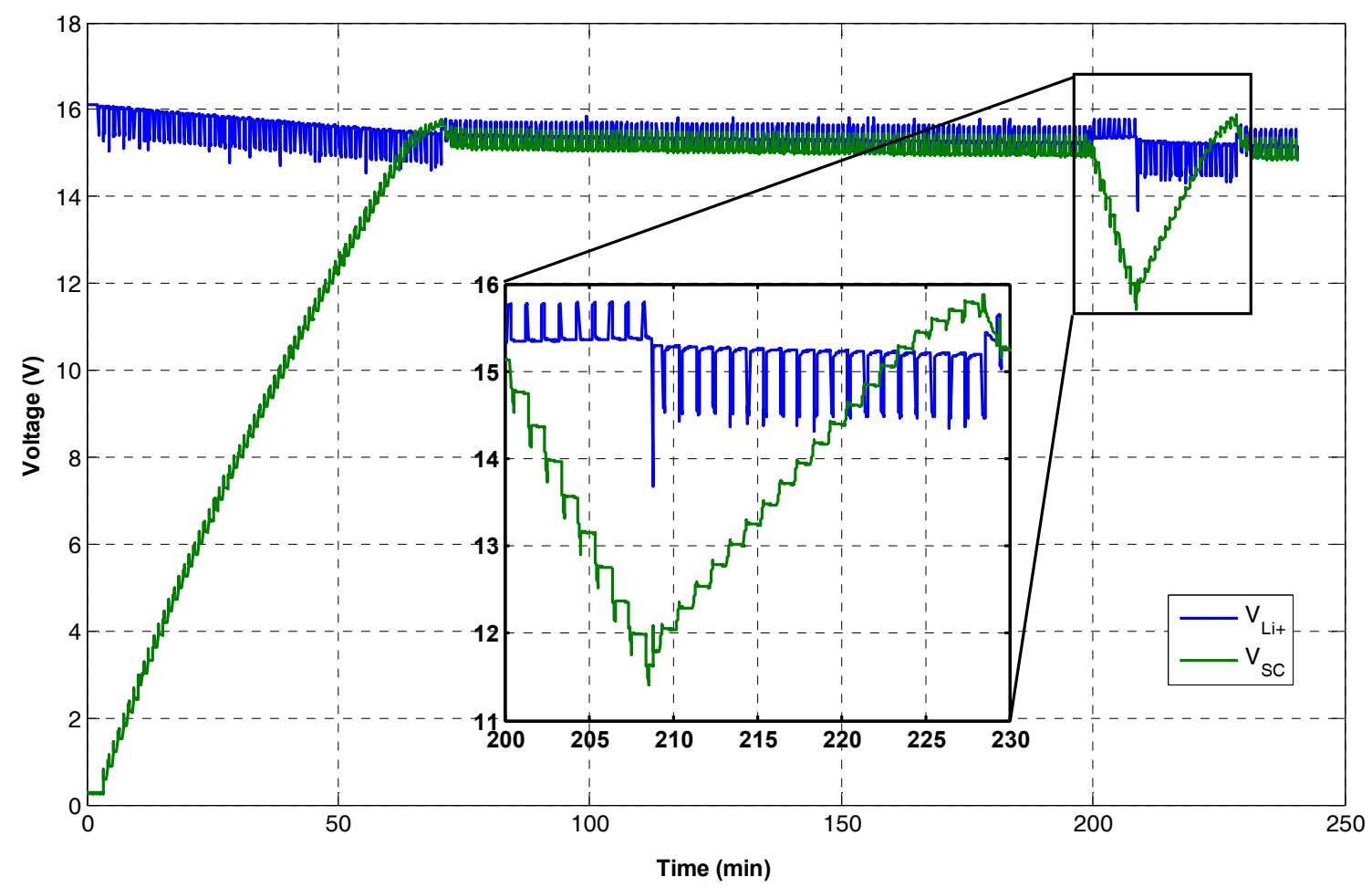

Figure 11.10. Individual Energy Storage Voltage Experimental Results.

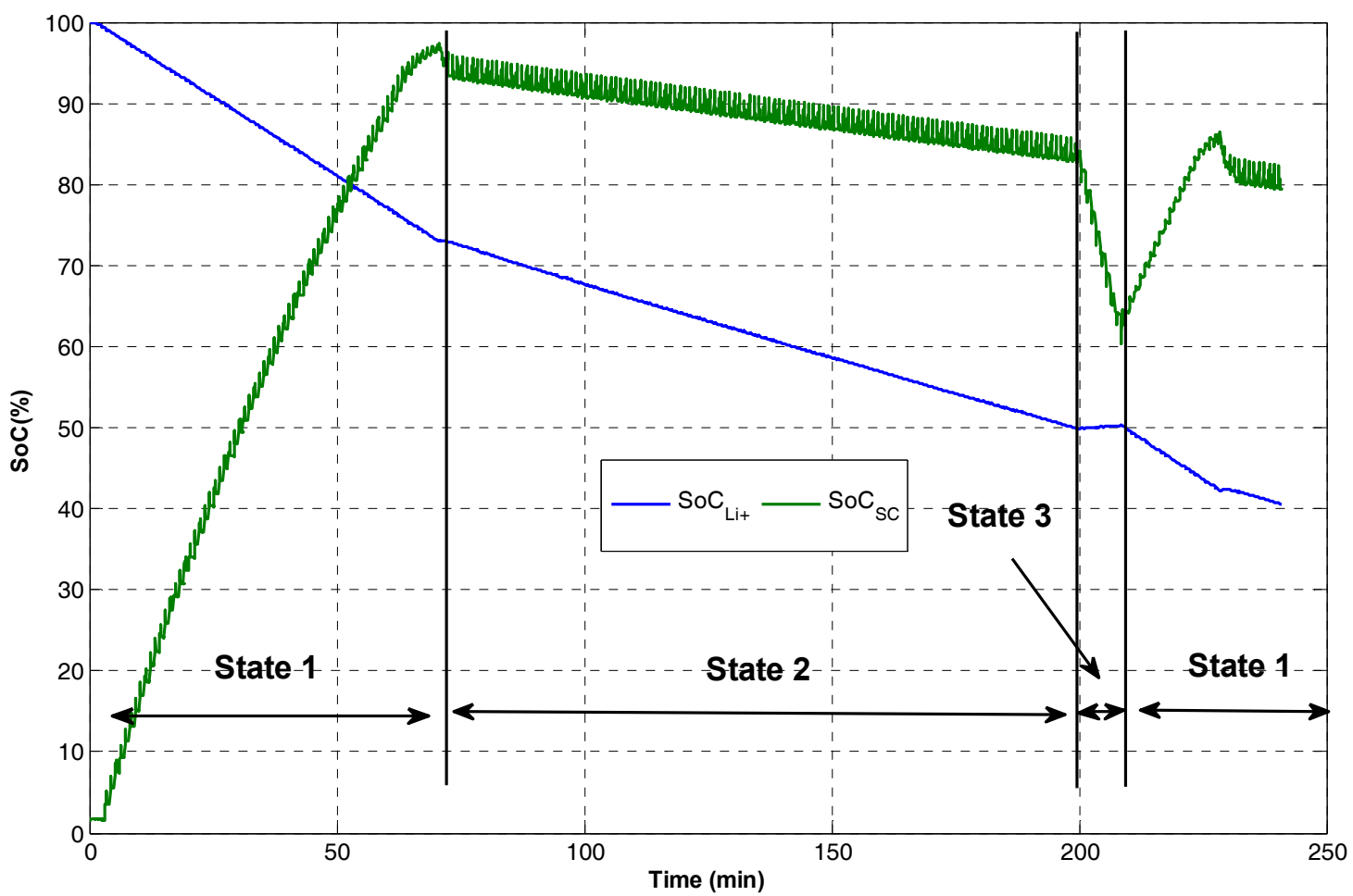

Figure 11.11. Individual Energy Storage State of Charges Experimental Results. 


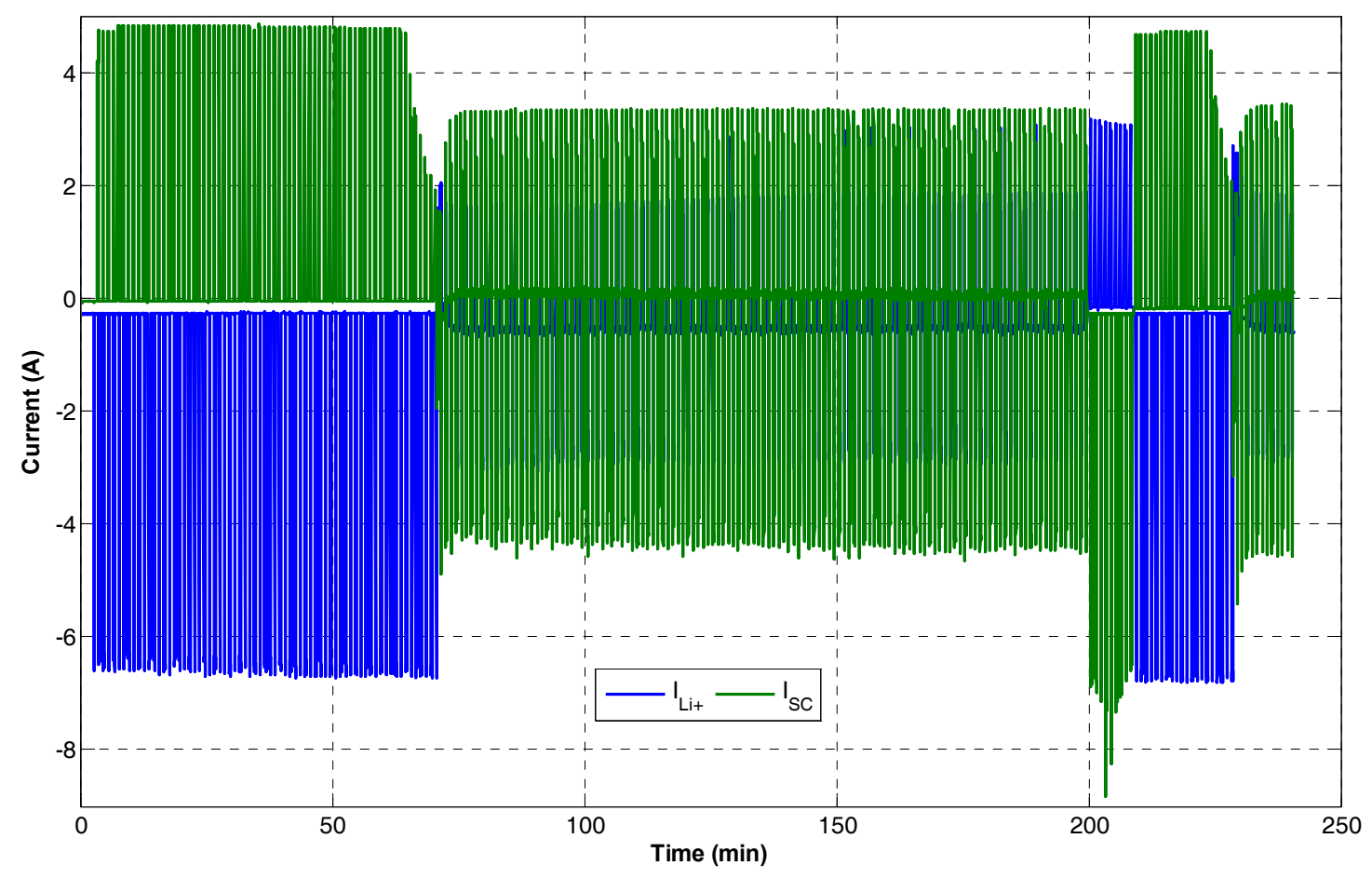

Figure 11.12. Individual Energy Storage Currents under Electric Vehicle Management Scheme.

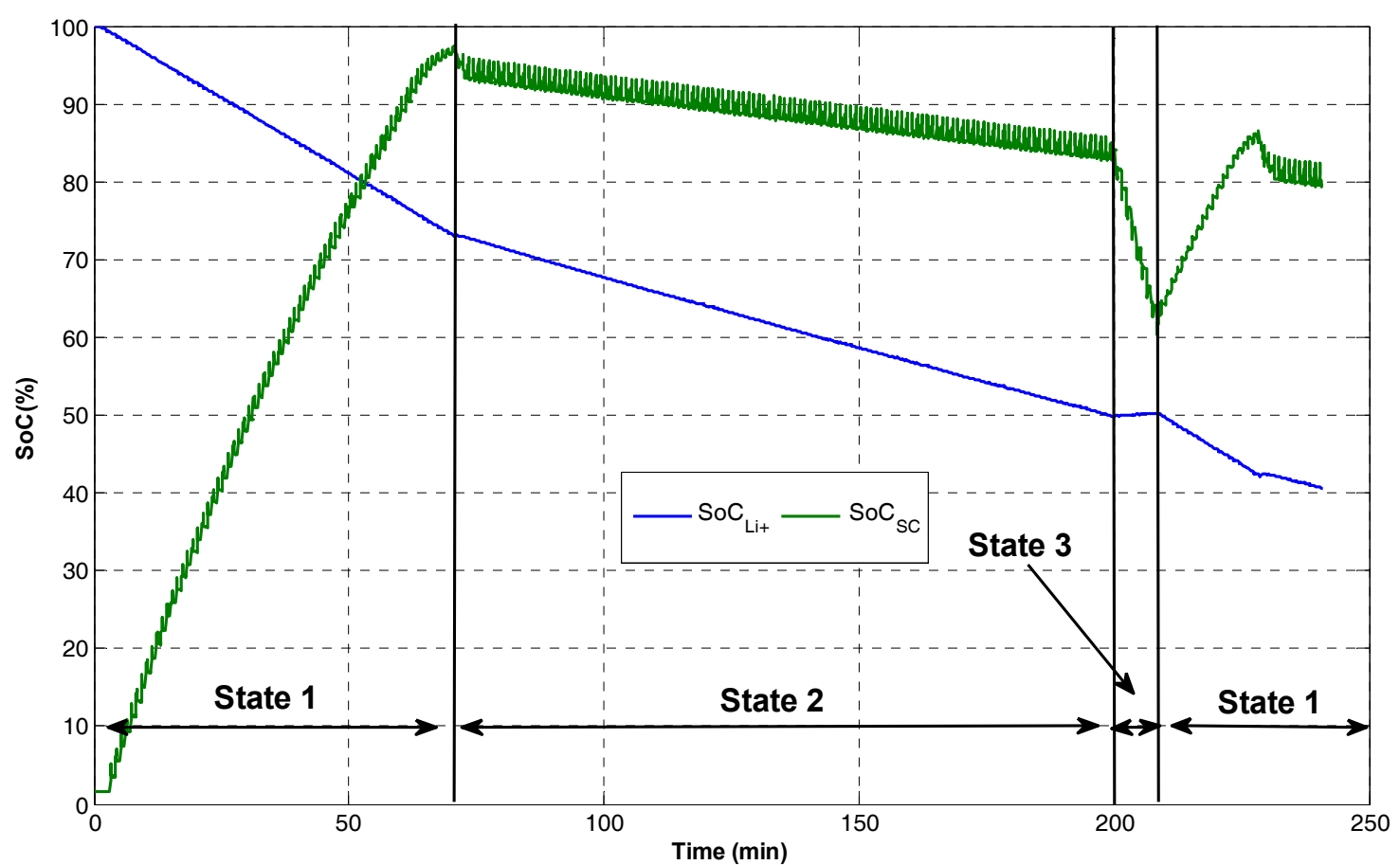

Figure 11.13. Individual Energy Storage Power under Electric Vehicle Management Scheme. 
The DOE standard HPPC profile operated for $225 \min (\sim 3.75 \mathrm{~h})$, passing through one complete management scheme cycle. In State 1, one can see the SC was charging from regenerative braking as its voltage and $\mathrm{SoC}$ increases. On the other hand, the battery voltage and SoC decreases at a high rate, since it is solely handling the load. Battery charging and SC discharging are indicated by positive and negative current injections, respectively. As opposed to the legacy design, the SC is now efficiently absorbing the regenerative braking energy, thus little energy is wasted. This consequently increases the overall efficiency of the EV drive system. Since during this state, the lithium ion battery bank was at a high SoC, it would be unable to enter a $\mathrm{CC}$ charging mode, which would prevent part of the regenerative breaking current from being absorbed. The overall current and power profiles from the lithium ion battery bank and SC are shown in Figure 11.12 and Figure 11.13, respectively. One can observe the SC absorbing approximately a $4.8 \mathrm{~A}$ charge, while the battery discharge is close to $6.4 \mathrm{~A}$. Since the SC starts fully discharged at $0 \mathrm{~V}$, the power injection increases linearly with every HPPC charge pulse until it reaches near a full charge. The SC is limited to a maximum of approximately $97 \% \mathrm{SoC}$ in order to more closely match the battery voltage prior to progressing into State 2 .

At the point where both voltages match, the system switches to State 2, where both ES devices are subjected to both charging and discharging pulses. By looking at State 2 in Figure 11.14 and analyzing the charging current injection for both elements, it can be seen that the SC injects more current than the battery at the beginning of the pulse. This is a result of the faster response time of the SC versus the battery. In this state, the $\mathrm{SC}$ is operating as an electric shock absorber, reducing the stresses on the battery. As expected, this reduced loading results in a slower voltage and lighter SoC reduction rate in the battery. 

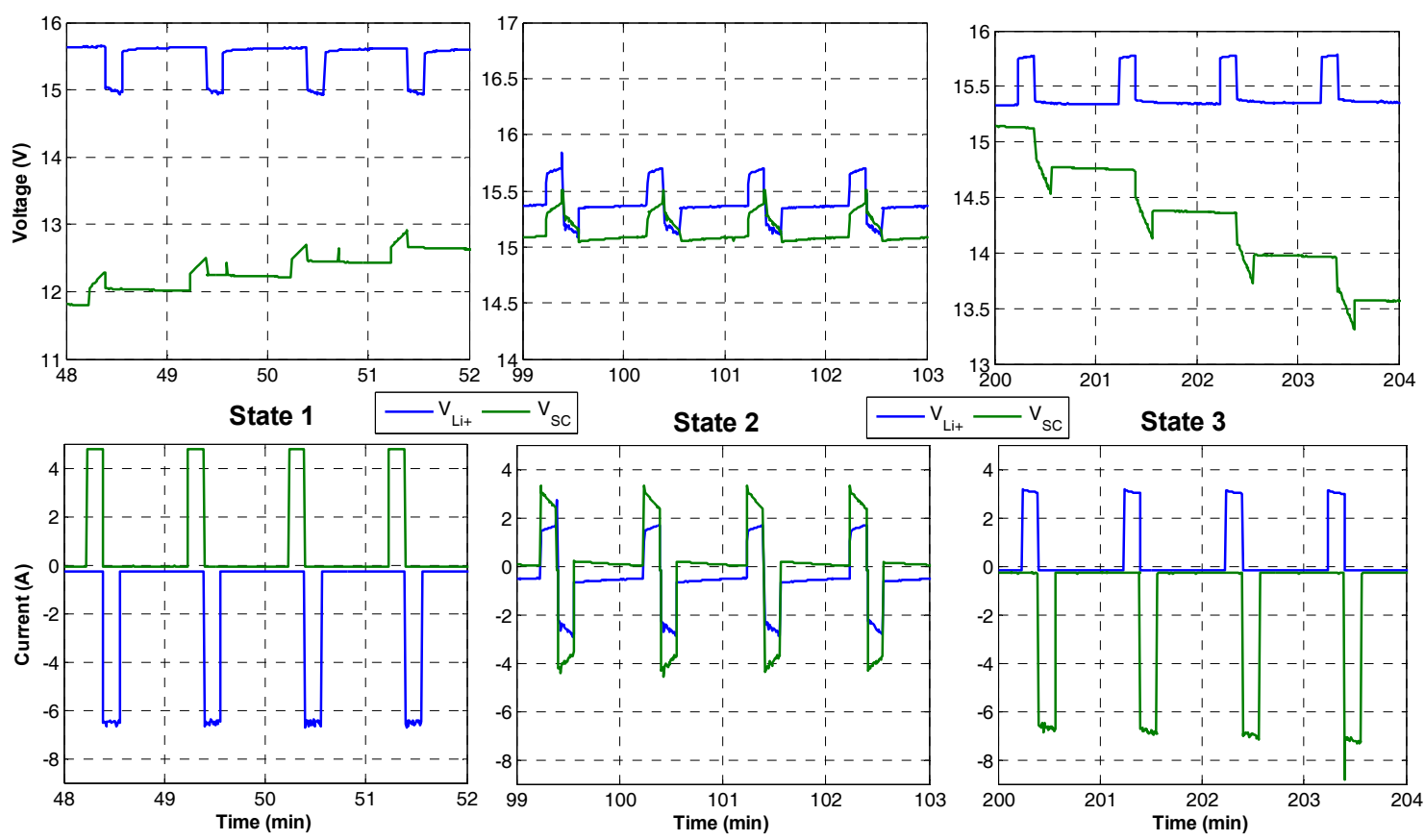

Figure 11.14. Four Minute Close-up of all States: Voltage (top) and Current (bottom), (b) State 2: Voltage (top) and Current (bottom).

Since the SoC of the SC only falls to around $80 \%$ prior to the end of State 2, its voltage is still relatively high, thus the $\mathrm{SC}$ handles the highest power variance, as shown in Figure 11.13. However, since the two waveforms overlap, the average power from the lithium ion battery and SC are close. State 3 is then initiated after 200 min, as the battery SoC reached its 50\% threshold, as depicted in Figure 11.11.

In State 3, the battery is now assigned to charge by regenerative breaking, while the SC supplies the motoring power. As can be seen, the current injections from State 1 are reversed as the SC current is negative, while the battery current is positive. Figure 11.10 demonstrated the drastic difference in the SC energy density with a zoom, as each motoring pulse results in a significant reduction in the SC voltage. This is more clearly shown in State 3 in Figure 11.14, where following each motoring pulse, the SC OCV drops by 
approximately $0.4 \mathrm{~V} /$ pulse. Longer periods could be achieved by increasing the capacity of the SC, given a weight versus energy trade-off is conducted for the EV.

Despite all the switching activities, it can be seen from Figure 11.15 that the DC bus voltage remained stable around the operating point (assumed to be $48 \mathrm{~V}$ in this test). As expected, the most challenging state was State 3, where a drop of the SC SoC to its minimum operating value of $60 \%$ resulted in a voltage sag on the HESS terminal voltage. However, this sag is compensated through the DC-DC converter. A zoom is provided in Figure 11.15 depicting the rapidly degrading ES array voltage input into the DC-DC converter, while the output remains relatively stable. After the SC SoC drops to $60 \%$, the system is switched back to State 1 for around $10 \mathrm{~min}$ to demonstrate how this management scheme can be set up in a repeated sequence, until both ES devices are discharged to their minimum SoC values.

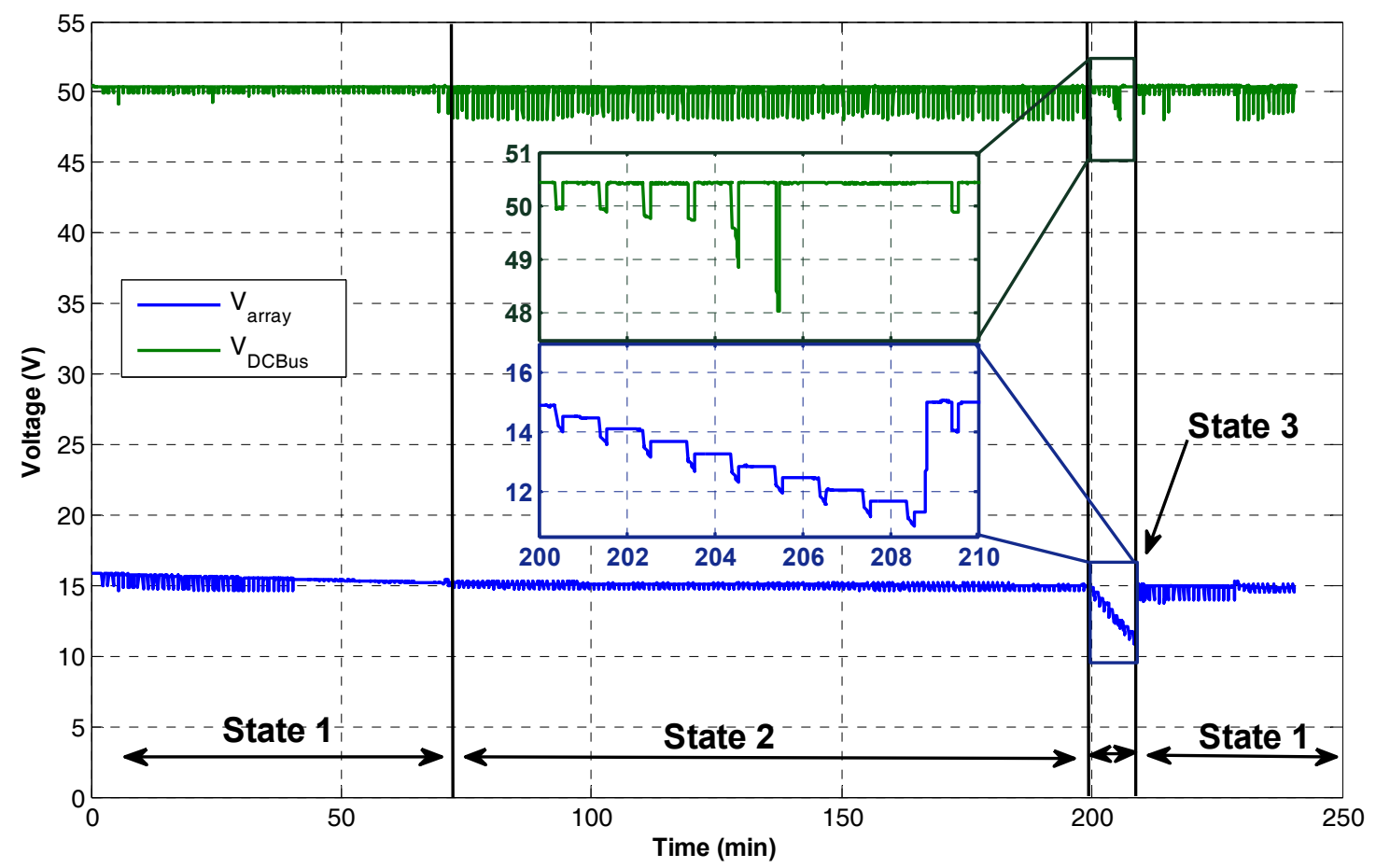

Figure 11.15. DC Bus and Energy Storage Array Voltages under Electric Vehicle Management Scheme. 


\subsection{Summary}

In this chapter, another example of HESS deployment is purposed for EV applications. This lithium ion battery and SC HESS has demonstrated how connecting different ES technologies in parallel with the proper control can be a feasible solution to improve the performance of an electrical propulsion system. Using an adequately designed HESS, the energy and power density, as well as the response of the system, can be improved, but must have adequate control. Furthermore, the combination of long duration discharge drive currents and short, high-powered charging currents from regenerative breaking places batteries under enormous stress, resulting in shorter lifetimes. An adequate management system utilizing ESMC devices can exploit the advantages of a HESS by understanding the mechanics of a lithium ion battery and SC HESS.

Following modifications to the ESMC control platform, a multi-state control and management scheme was designed to reduce cycling upon the lithium ion battery, while improving the efficiency of current injection from regenerative breaking pulses. The feasibility of the developed scheme was experimentally investigated using the DOE HPPC standard testing profile, where the charging and discharging of the EV HESS was handled dynamically by ESMC devices. It is anticipated that a scheme of this nature would increase the lifetime of the battery and the available "in road" service period for future EVs. In the following chapter, the HESS topic will continue to expand, focusing upon another unique HESS combination of a lead acid battery and flywheel ES. However, in this case, the HESS will not only be evaluated for its performance in power and energy delivery, but also how the lead acid battery array can serve to significantly improve the power quality of flywheel ES. 


\section{Chapter 12 Flywheel - Lead Acid Battery Hybrid Energy Storage Systems}

\subsection{Introduction}

Flywheel energy storage systems (FESS) have attracted new research attention in a number of different applications, one of which is in future naval platforms where the presence of heavy pulsed loads present a significant challenge [34]. Although the concept of the flywheel can be dated back to the early $20^{\text {th }}$ century, their assembly and particularly the applications to which they are used have changed dramatically. The flywheel has recently been deployed for a number of grid applications, space applications for NASA, and is the main component for regenerative breaking recovery in the Kinetic Energy Recovery System (KERS) deployed in the current Formula One racecar [213].

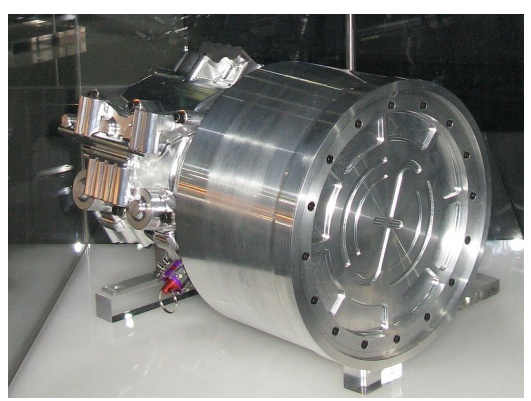

Figure 12.1. Formula One Kinetic Energy Recovery System Flywheel Energy Storage Device [213].

The FESS operates by mechanically storing kinetic energy in a rotating mass. Flywheels can be designed for low or high-speed operations, though low-speed applications have advantages of a lower cost, as they are able to be integrated using proven technologies [37]. Although low-speed flywheels have seen a wide range of usage, high speed operations have gained recent attention due to developments in relevant technology. Magnetic levitation, the introduction of composite materials, low-loss machinery, and 
power electronic switches have driven this progression. Through the replacement with new types of magnetic or superconducting materials, theoretical energy storage (ES) capacities can be increased as well [214],[215]. In Reference [40], a new model for a flywheel was proposed, where a superconducting magnetic bearing, together with a permanent magnet, was introduced. The system was able to increase the rotational speed of the flywheel and suppress vibrational aspects of the rotor, while also reducing the cost of cooling the motor.

While the materials to construct a flywheel are being improved, so have their power electronic and control integration algorithms in an aim to provide solutions to the intermittency associated with most renewable energy sources. In Reference [39], a FESS is connected to a wind farm through a solid state transformer to store excess wind power when generation levels are high, and provide a restoration measure during the time when the wind is at a deficit. Similar solutions have been proposed for usage in solar power applications [216].

Initial studies identified the flywheel to be a tool in improving land-grid power quality. This has since been extended to include its application on shipboard power systems (SPS) as well. Reference [38] focuses on voltage and power stability improvements by identifying a reduction in peak-to-peak transients, however the introduction of harmonics, or ripple frequencies as a result of operation, was not considered. Previously, in Reference [39], a Distribution Static Synchronous Compensator (DSSC) was coupled with a flywheel to provide active and reactive power assistance, as well as power factor correction and voltage control. Although the harmonics were addressed as a consequence of the control, the final system did not provide a full harmonic analysis. 
A majority of the networks identified are hybrid power systems, containing both an AC and a DC connection. In addressing harmonics, the same fundamental frequency from the AC connection can be applied to that of the analysis when referring to DC harmonic components. However, this technique becomes ineffective when the system does not contain an $\mathrm{AC}$ connection. The emerging electric vehicle (EV), in many cases, presents a purely DC bus [36],[217]. The interconnection of FESS and inducing of ripple voltages and currents present a much different problem in these scenarios. In Reference [36], an active power filter (APF) was designed to combat some of these issues. However, the frequency spectra was only viewed in terms of reduction in the overall Total Harmonic Distortion (THD) as opposed to identifying specific common frequency multiples that should be targeted when applying filtering methods. The onboard battery typically identifies a simple solution to this issue without the need for a separate APF. However, the required energy from the battery versus that of the flywheel must be quantified in order to adequately solve this problem, while preventing a significant increase in the system cost.

In this chapter, FESS will be reviewed for their strengths and weaknesses, as well as the advantages in the inclusion of a lead acid battery in parallel to design a hybrid ES system (HESS). A great deal of the problems present in the FESS are in terms of power quality, a problem that can be improved by the inclusion of a battery. Since the HESS operates primarily on a DC-only system, the detection and classification of power quality issues requires a new metric to specifically evaluate the ripple voltage frequencies contributing to a reduction in the power quality. As this metric is identified, the power quality issues associated with the HESS are evaluated. 

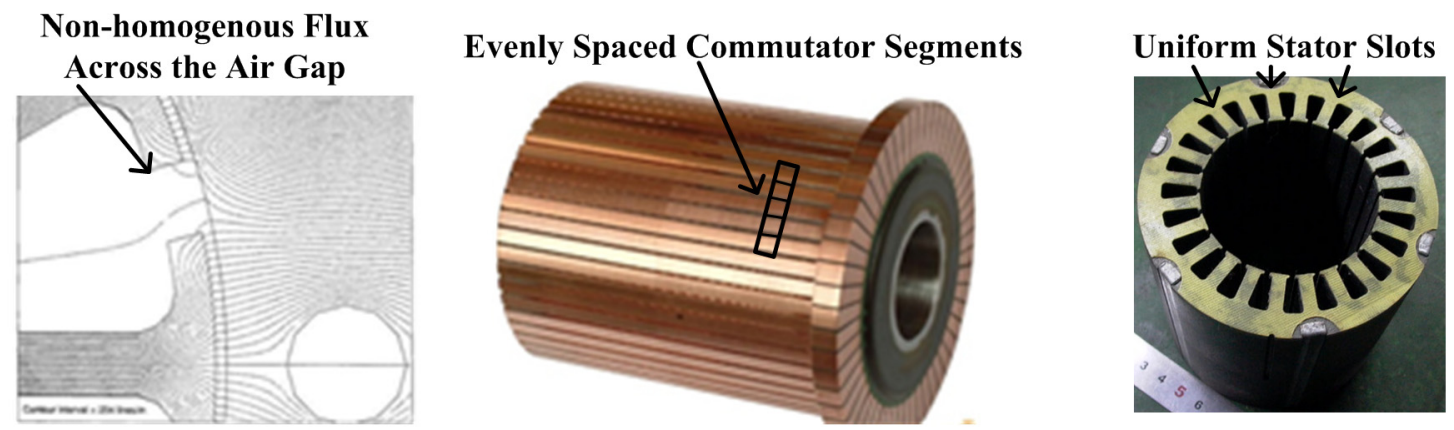

Figure 12.2. Significant Factors Inducing Ripple Frequencies in the DC Machine.

\subsection{Flywheel Energy Storage Systems}

The common FESS consists of a high inertia rotating mass and an electric machine, which can operate simultaneously as a motor during charge and generator during discharge. One of the disadvantages of the flywheel is its high self-discharging rate. Thus, the rotating parts are enclosed in a vacuum system to reduce friction losses. In the flywheel electromechanical system, the rotor is accelerated to high speed in order to store kinetic energy, a process which resembles charging. The amount of the stored energy $E$ can then be defined based on the common physics equation:

$$
E=\frac{1}{2} J \omega_{m}^{2}
$$

where the moment of inertia $J$ and square of the speed $\omega_{m}^{2}$ designates the energy density. As the speed of the flywheel is adjusted, the shaft power is adjusted proportionally. The shaft power $P$ is then:

$$
P=-J \omega_{m} \frac{d \omega_{m}}{d t}
$$

It can be concluded from Equations (12-1) and (12-2) that the ES capacity is a function of the operation of the flywheel over a wide range of speeds. One can also recognize that a steady-state operating point is not typical under normal operation. In this study, the ripple 
frequency levels in a hybrid flywheel and battery parallel combination are investigated. The ripple levels are determined at different levels of contribution from each ES device.

\subsection{Investigating Power Quality Issues}

Power quality metrics for the flywheel have begun to surface on the DC system, designating voltage ripple limitations to be no more than $2 \%$ of the DC value [218]. Three significant factors are involved in the production of induced ripple frequencies on the DC machine: non-homogenous flux distribution across the air gap, evenly-spaced commutator segments, and uniform slots on the stator [219]. Non-homogenous fields in the air gap are of typically low order. Conversely, slot ripple frequencies are typically of high order and can be obtained from the number of $S$ slots and $P$ poles.

Table 12-1. Hampden DYN-300X Motor Specifications.

\begin{tabular}{|l|r|r|}
\hline Nominal Voltage & $125 \mathrm{~V}$ \\
\hline Nominal Power & $3 \mathrm{hp}(2.24 \mathrm{~kW})$ \\
\hline Slots & 36 \\
\hline & Commutators \\
\hline Poles & 72 \\
\hline & Armature Current \\
\hline Rated Speed & $1400 \mathrm{RPM}(23.33 \mathrm{~Hz})$ \\
\hline
\end{tabular}

\subsubsection{Machine Speed Multiple}

In most HESS, a fundamental frequency is designated to conduct harmonic analysis, but in a DC-only system these components can appear ambiguous. Furthermore, the flywheel must operate at different speeds to share a load with the battery bank. To establish a metric to place meaning on specific ripple frequencies, a machine speed multiple (MSM) value has been defined, where the fundamental is equal to the mechanical rotation speed of the flywheel $f_{m}$. Using the MSM, a correlation is made between the spectral responses 
of the machine at each speed multiple. The MSM is calculated by these three factors based on the number of poles $P$, slots $S$, and commutator segments $C$ inside the machine. The $M S M$ general formula is:

$$
M S M=\left\{\begin{array}{l}
2 P(1+k) \\
C(1+k) \\
\left(\frac{2 k S}{P} \pm 1\right)
\end{array} \text { for } k=0,1,2 \ldots\right.
$$

where $k$ an integer. Target flywheel ripple frequencies $f_{f w}$ can then be determined by:

$$
f_{f w}=M S M f_{m}
$$

In this experiment, a Hampden DYN-300X DC machine is taking the place of the flywheel. Its specifications are outlined in Table 12-1.

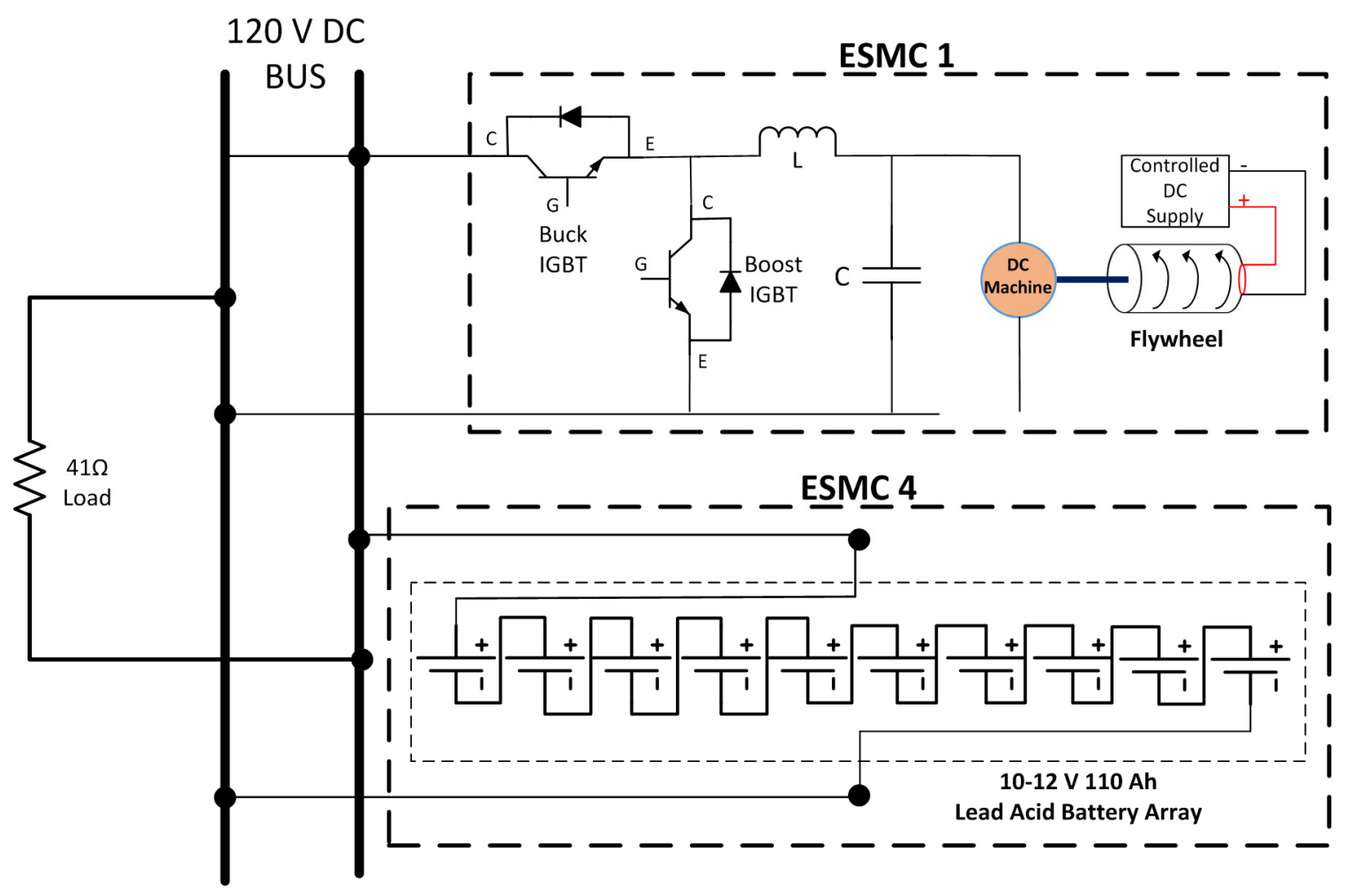

Figure 12.3. Flywheel - Lead Acid Battery Hybrid Energy Storage System. 


\subsubsection{Mathematical Modeling of the Hybrid Energy Storage System}

The flywheel HESS will be tested under four different levels of current contribution. Traditionally, the use of the flywheel under normal operating conditions would be expected to initially expend a great deal of energy, followed by a relatively short discharge period. This is investigated in the following experimental study, where a series-connected lead acid battery bank consisting of 10-12 V lead acid batteries with a 110 Amp-hour (Ah) capacity are connected in parallel to the flywheel [73]. The system under study is as shown in Figure 12.3, where the battery and flywheel are combined to form a HESS. A buck-boost DC-DC converter is placed between the flywheel and the DC bus to increase the output voltage from the flywheel during discharge, and reduce the DC bus voltage to the flywheel during charging.

\subsubsection{Power Quality Issues with Flywheel DC-DC Converter Interfacing}

The value of the DC-DC converter inductor is chosen based on the desired ripple current. It is usually recommended to operate the system at a ripple current equal to less than $20 \%$ of the average inductor current [163]. A higher input $V_{\text {in }}$ or output $V_{\text {out }}$ voltage also increases the ripple current, as depicted in Equation (12-5), where $V_{\text {in }}$ and $V_{\text {out }}$ are the input and output voltages of the flywheel driving converter, respectively, $f_{s}$ is the switching frequency $(\mathrm{Hz})$, and $L$ is the coil inductance.

$$
\Delta \mathrm{I}_{L}=\frac{1}{f_{S} L} V_{\text {out }}\left(1-\frac{V_{\text {out }}}{V_{\text {in }}}\right)
$$

Smaller inductance values will result in a higher output current slew rate and improve the load transient response of the converter, but would also result in a higher output voltage ripple. Conversely, larger inductance values reduce the ripple current, as well as core 
magnetic hysteresis losses, but will increase the size and weight of the converter. Moreover, it significantly limits the amount of transferred power.

\subsubsection{Battery Model and Characteristics for Power Quality Improvement}

As an alternative solution, capacitors are standard for storage and smoothening of the DC output in power converters. A comprehensive electrical lead acid battery model similar to that which was introduced previously in Chapter 9 is shown in Figure 12.4 [220]. Since the application in this case is not specified, the models have been optimized to serve both EV and SPS applications, thus the same model from Chapter 9 was utilized. This model partitions the battery into two parts: an energy and lifetime model, as well as an equivalent circuit model that models the voltage-current characteristics.

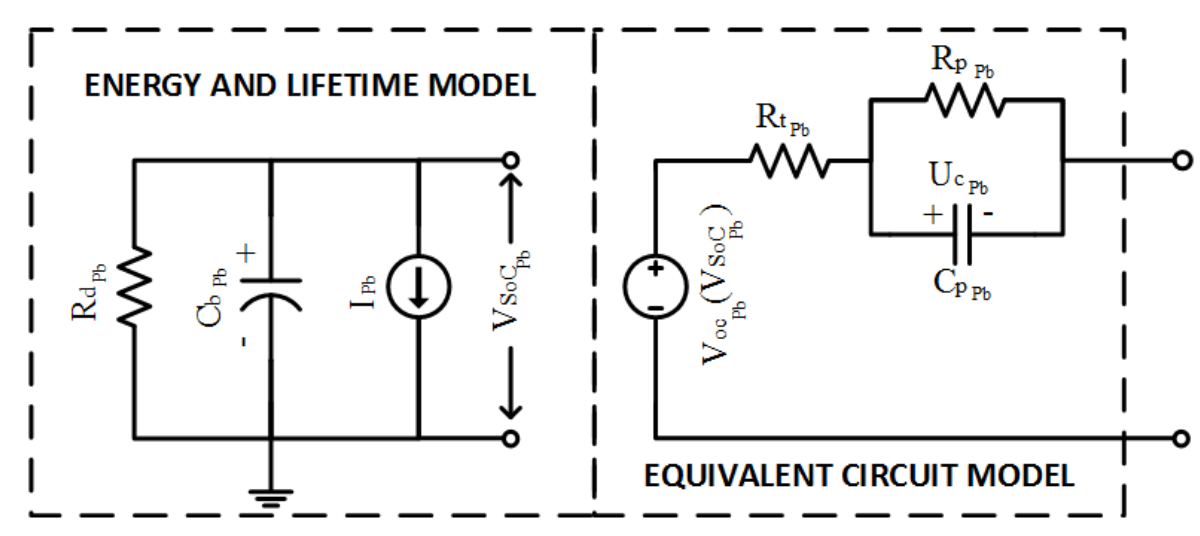

Figure 12.4. Lead Acid Battery Energy and Lifetime and Randles Circuit Models Utilized for Flywheel Integrated Hybrid Energy Storage Systems.

The energy and lifetime model from Reference [220] reveals that the energy stored inside the lead acid battery (denoted by the "Pb" subscript) can be represented as a large capacitance $\mathrm{C}_{\mathrm{b}_{\mathrm{Pb}}}$ in parallel with a large self-discharge resistor $\mathrm{R}_{\mathrm{d}_{\mathrm{Pb}}}$. Once again, in this application, the self-discharge resistor has been neglected since all tests are of short duration, as the battery will not be tested under an open circuit condition. A virtual 
capacitance $\mathrm{C}_{\mathrm{b}_{\mathrm{Pb}}}$ can therefore be calculated directly through using the battery capacity $\mathrm{E}_{\mathrm{b}_{\mathrm{Pb}}}$ (in $\left.\mathrm{Ah}\right)$ :

$$
\mathrm{C}_{\mathrm{b}_{\mathrm{Pb}}}=3600 \mathrm{~V}_{\mathrm{Pb}} \mathrm{E}_{\mathrm{b}_{\mathrm{Pb}}}
$$

where $V_{P b_{F C}}$ represents the full charge voltage of the battery module. The lead acid battery array utilized in this system is the same $10-12 \mathrm{~V} 110 \mathrm{Ah}$ stack that was utilized initially in Chapter 3. In this case, all 10 batteries are connected in series, thus the expected full charge open circuit voltage (OCV) is $128 \mathrm{~V}$, yielding an extremely large capacitance.

The equivalent circuit model portion is represented by the familiar $1^{\text {st }}$-Order Randles circuit model describing the behavior of the lead acid battery under load, where once again $R_{t_{P b}}$ represents the electrolytic resistance and $R_{p_{P b}}$ and $C_{p_{P b}}$ represent the polarization resistance and capacitance governing its impulse response. Although $C_{b_{P b}} \gg C_{p_{P b}}$, both play a role in smoothening the ripple current and voltage. This capacitance behaves as an additional filter in parallel with the flywheel converter.

\subsection{Experimental Test Setup}

To conduct the experimental testing, a National Instruments NI-9206 with 32 analog input channels in conjunction with the LabVIEW Development Platform was used [64]. An initial power quality test was conducted using a Fluke 435 Power Analyzer, as shown in Figure 12.5. Energy Storage Management System Controllers (ESMC) were utilized to interface the lead acid battery bank with the flywheel. However, in this case, no DC-DC converter was needed. Thus, both the battery (ESMC 1) and flywheel (ESMC 4) were connected directly to a $120 \mathrm{~V} \mathrm{DC}$ bus. An alternative control interface was designed, as dynamic switching was not required. This saved unnecessary computational overhead in 
order to construct a real-time Fast Fourier Transform (FFT) of the voltage and current signals, as shown in Figure 12.6.

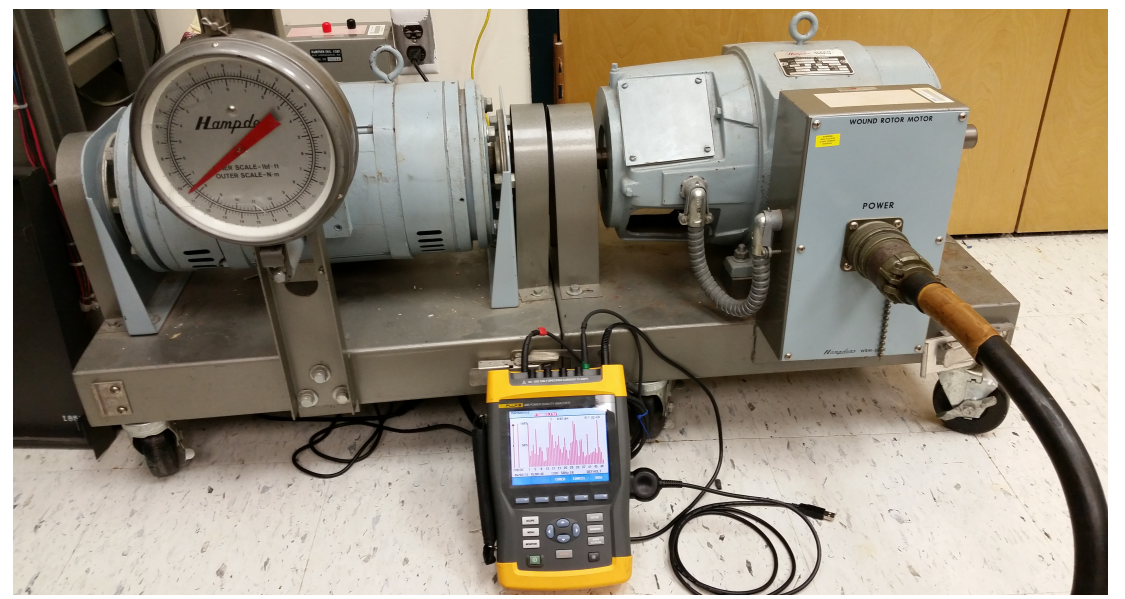

Figure 12.5. Initial Testing and Power Quality Analysis of the Flywheel.

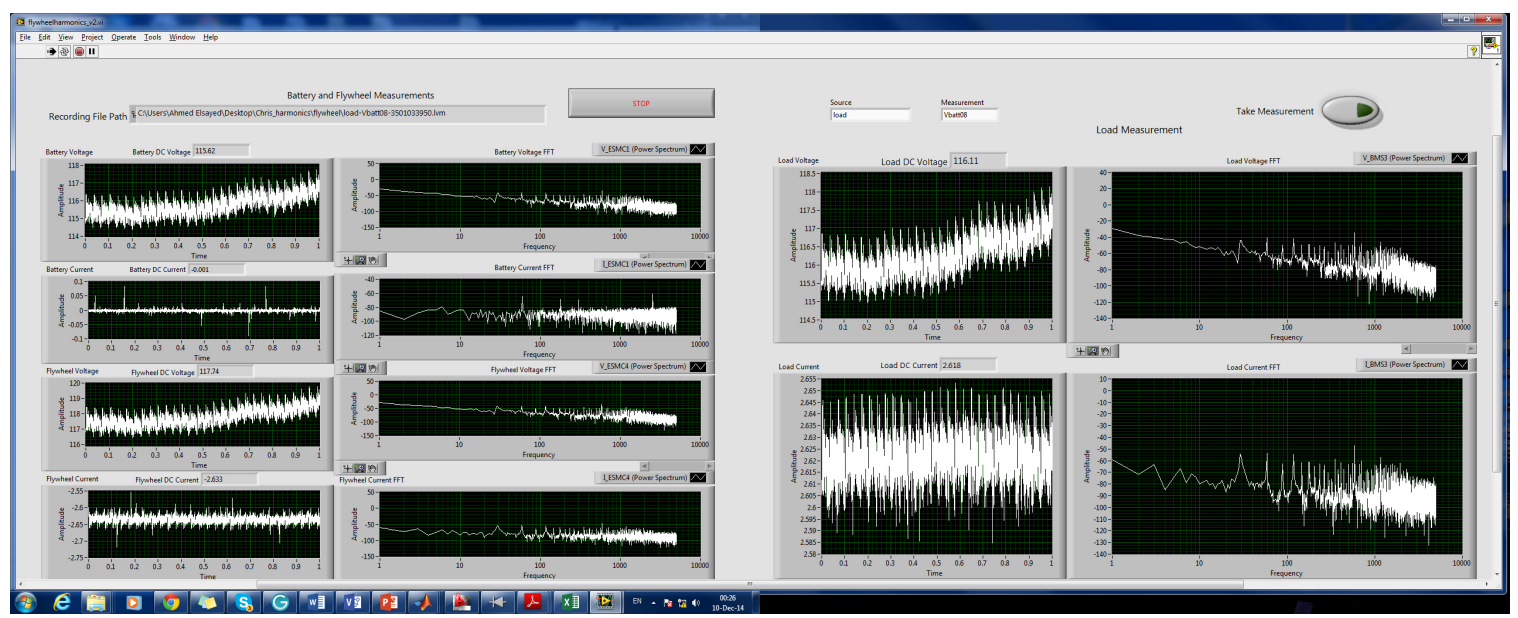

Figure 12.6. Custom LabVIEW Flywheel and Battery Data Acquisition Interface.

As shown previously in Figure 12.3, both the flywheel and lead acid battery are supplying a resistive load tuned to draw approximately 3 A at $120 \mathrm{~V}$. The ripple levels are then determined at 5\%,25\%, and 50\% current contribution from the battery. The remaining load current is supplied by the flywheel. During this test, the inertia coupled to the machine was large enough to supply the load over the entire test period (which is short), thus the flywheel does not require charging during the test. 
Voltage and current were measured through the use of two LEM Hall Effect sensors [62],[63]. In order to ensure high precision, a noise bias test was conducted first. To ensure the highest fidelity in frequency measurements, the NI-9206 frequency and sampling rate are set to their maximum at $10 \mathrm{kHz}$ and $10 \mathrm{kS} / \mathrm{s}$, respectively. Under the Nyquist criterion, the configuration provides an accurate Discrete Fourier Transform (DFT) of the signal to $5 \mathrm{kHz}$ and $5 \mathrm{kS} / \mathrm{s}$ [222]. Assuming $f(\mathrm{v})$ to be the continuous voltage signal under 5000 samples, the DFT or $F[n]$ is:

$$
F[n]=\sum_{k=0}^{N-1} \mathrm{~V}[k] e^{-j \frac{2 \pi n}{N T}} \text { where } 0 \leq n<N-1
$$

where $v[k]$ is the discrete voltage sequence, $N$ is the sample window of 5,000 samples, and $n$ is the sampling frequency.

\subsection{Experimental Testing}

The HESS is then tested under four different levels of contribution. Traditionally, the use of the flywheel in an EV or SPS application with a pulsed load would be expected to initially expend a great deal of energy, followed by a relatively short discharge period. This is comprehensively investigated in the following experimental study, where a seriesconnected lead acid battery bank of 10 batteries at $120 \mathrm{~V}$ are connected in parallel with a flywheel. The hardware investigation of this study is one of its most important contributions, since most of the machine models in commercially available software (such as MATLAB) neglect the effect of the internal construction of the machine. The DC output of a machine is represented as a clean, pure DC source, which is impractical. The following demonstrates battery support at $5 \%, 25 \%$, and $50 \%$ of the total energy delivered to the load. 

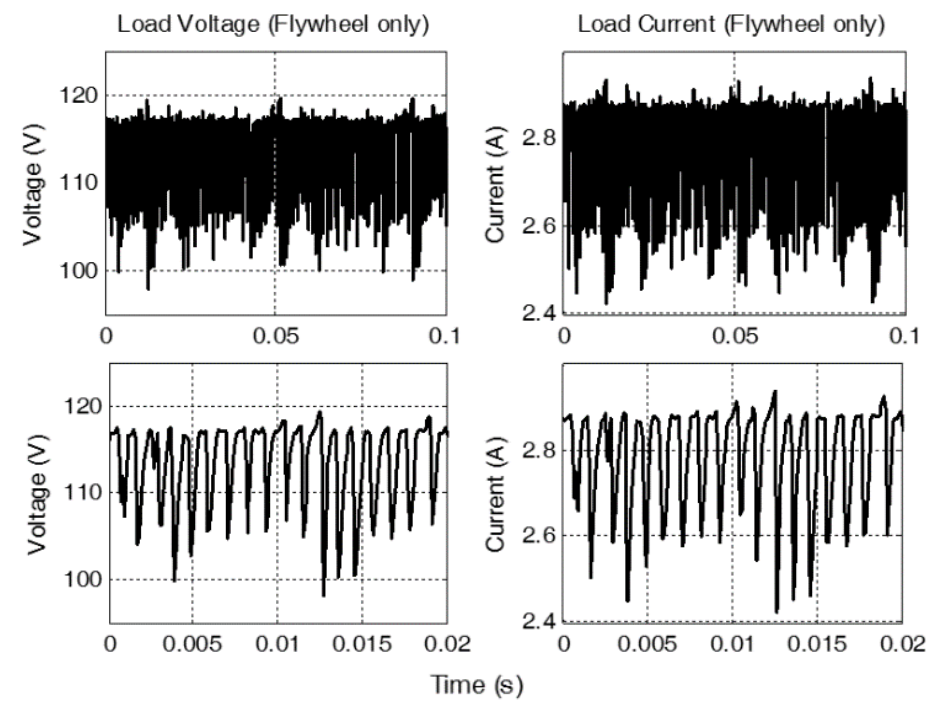

Figure 12.7. Voltage and Current of Flywheel Energy Storage Only.

\subsubsection{Flywheel System Only}

An initial test is conducted where the DC motor is connected directly to the load. The bus voltage is regulated near the terminal voltage of the battery bank, or approximately 116 V. The machine speed in this case was 773 RPM (12.88 Hz). Shown in Figure 12.7, both the voltage and current waveforms reveal a high level of noise, however, a closer inspection reveals detectable periodic contents. A close-up of the voltage and current in Figure 12.7 reveals a quasi-periodic square wave. This is a major feature, as it a direct relationship to the number of commutator segments in the machine. Figure 12.8 depicts the DFT of both the voltage and current waveforms shown under a dB-scale. A close correlation is identified between the two waveforms, which is to be expected under a linear load. The major difference is a shift in their biases. The current ripple frequencies are $30 \mathrm{~dB}$ lower than that of the voltage, which places them below the $2 \%$, or $-17 \mathrm{~dB}$ noise threshold. For this reason, as the HESS system is connected, only the voltage frequency spectra will be analyzed. A frequency spectra of the voltage in linear scale is shown in Figure 12.8, where 17 ripple frequencies exceed the $2 \%$ threshold. 
To profile ripple frequencies, the MSM is used and is shown in a linear scale in Figure 12.9. Table 12-2 provides a summary of all results for each case, where each frequency is identified with respect to a classification "ID". The ID column classifies the MSM into one of three categories discussed in Section 11.2, which are a result of DC machine power quality factors. IDs are correlated to their MSM and related to: a slot ripple frequency (S), non-homogenous flux across the air gap (A), or a result of the commutator (C).

The highest ripple frequency is present at the commutator multiple, or 72, producing $240 \%$ of the DC component. This is shown on the top plot in Figure 12.9, where the remaining ripple frequency magnitudes are significantly lower than that of the commutator. This scale is reduced below in Figure 12.9 to highlight the remaining components. In Table 12-2, each MSM investigated is shown in terms of each flywheel-battery combination. As the flywheel speed varies, the frequency shifts in a linear fashion across the chart, confirming the geometric correlation associated with each of the causal IDs.
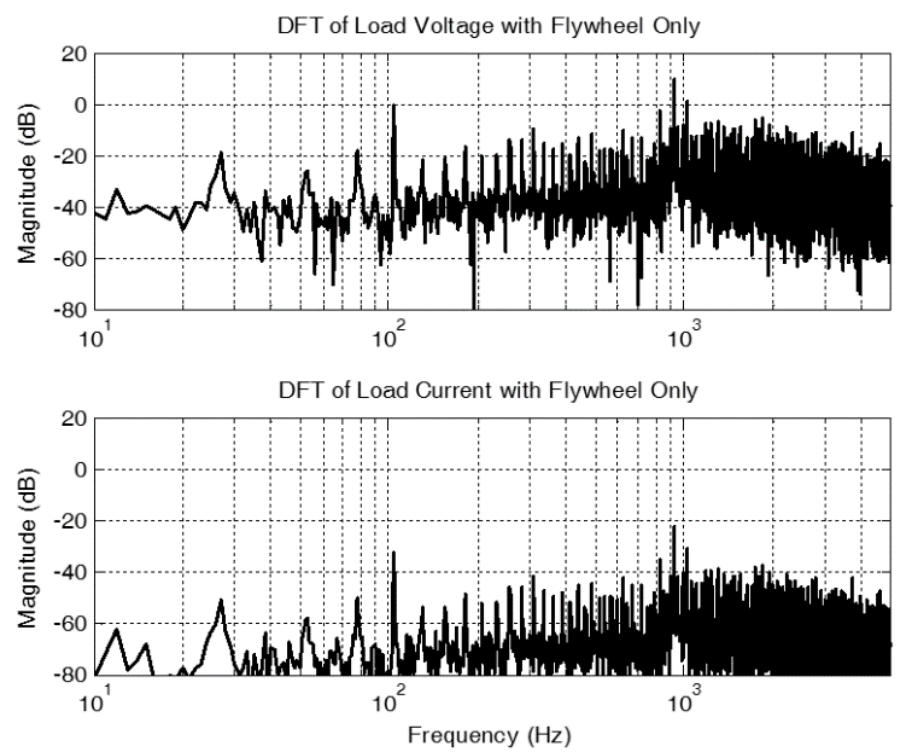

Figure 12.8. Discrete Fourier Transform of the Voltage and Current of the Flywheel Energy Storage. 

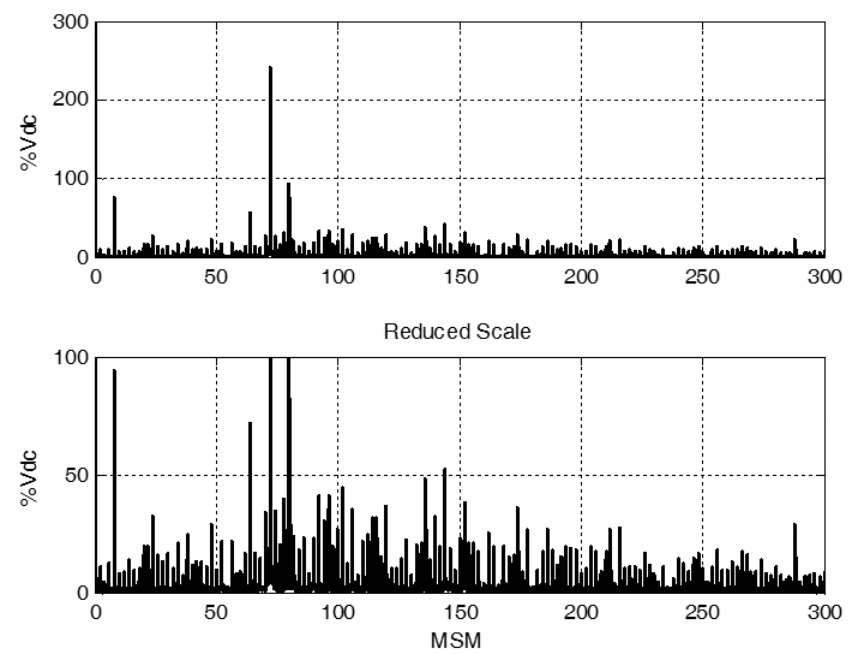

Figure 12.9. Voltage Ripple Distortion versus Percentage of the DC Component.

\subsubsection{5\% Battery / 95\% Flywheel Current Contribution}

The first test quantifies ripple frequency reductions under a minimum battery injection current. In this case, the flywheel is sourcing $95 \%$ of the load $(2.65 \mathrm{~A})$, while the battery bank contributes a mere 5\% (139 mA). The machine speed is held close to that of the flywheel-only case at 772 RPM $(12.86 \mathrm{~Hz})$. This already reveals a huge impact in reducing the magnitude of the ripple frequencies under a similar speed. Figure 12.10 depicts the original spectra to $5 \mathrm{kHz}$ in black, and the new HESS spectra in red. Figure 12.11 depicts the ripple voltage frequencies on the linear MSM scale, where the $2 \%$ threshold is identified. One can observe a $10 \mathrm{~dB}$ decrease in the overall ripple frequency noise bias. In Table 12-2, a column depicted the associated MSM frequencies, as well as the new voltage percentages at each multiple with respect to the fundamental value. A drastic reduction is observed in the commutator MSM, where its percentage is reduced from $87 \%$ to $7 \%$. Figure 12.12 highlights the ripple voltage percentage versus the DC component, as compared to the $2 \%$ threshold. A close-up depicts all frequencies that have fallen below this reference. 
Table 12-2. Power Quality Analysis of the Voltage at the Load.

\begin{tabular}{|c|c|c|c|c|c|c|c|c|c|c|c|c|}
\hline \multirow[b]{2}{*}{ MSM } & \multirow[b]{2}{*}{ ID } & \multicolumn{2}{|c|}{$\begin{array}{c}\text { Flywheel } \\
\text { Only } \\
773 \text { RPM }(\mathbf{1 2 . 8 8 H z})\end{array}$} & \multicolumn{3}{|c|}{$\begin{array}{c}\text { 95\% Flywheel } \\
5 \% \text { Battery } \\
772 \text { RPM (12.86 Hz) }\end{array}$} & \multicolumn{3}{|c|}{$\begin{array}{c}\text { 75\% Flywheel } \\
\text { 25\% Battery } \\
751 \text { RPM (12.52 Hz) }\end{array}$} & \multicolumn{3}{|c|}{$\begin{array}{c}\text { 50\% Flywheel } \\
50 \% \text { Battery } \\
729 \text { RPM (12.15 Hz) }\end{array}$} \\
\hline & & $f$ & $\% V_{D C}$ & $f$ & $\% V_{D C}$ & $\Delta \% V_{D C}$ & $f$ & $\% V_{D C}$ & $\Delta \% V_{D C}$ & $f$ & $\% V_{D C}$ & $\Delta \% V_{D C}$ \\
\hline 8 & $\mathrm{~A}$ & 103 & 87.9910 & 103 & 7.1228 & -80.868 & 100 & 1.0808 & -86.910 & 97 & 0.2579 & -87.733 \\
\hline 20 & $\mathrm{~S}$ & 257 & 4.3640 & 258 & 0.2716 & -4.092 & 251 & 0.0820 & -4.282 & 243 & 0.0075 & -4.356 \\
\hline 38 & $\mathrm{~S}$ & 489 & 6.1330 & 490 & 0.5282 & -5.604 & 477 & 0.0421 & -6.090 & 462 & 0.0122 & -6.120 \\
\hline 52 & $\mathrm{~S}$ & 669 & 5.6630 & 671 & 0.2309 & -5.432 & 652 & 0.0579 & -5.605 & 632 & 0.0054 & -5.657 \\
\hline 64 & $\mathrm{~A}$ & 824 & 53.4310 & 825 & 1.0518 & -52.379 & 803 & 0.4834 & -52.947 & 778 & 0.1049 & -53.326 \\
\hline 72 & $\mathrm{C}$ & 927 & 240.5100 & 929 & 3.4926 & -237.017 & 903 & 1.2199 & -239.290 & 875 & 0.5039 & -240.006 \\
\hline 80 & $\mathrm{~A}$ & 1030 & 142.2700 & 1032 & 2.1355 & -140.134 & 1004 & 0.5905 & -141.679 & 973 & 0.1183 & -142.151 \\
\hline 92 & $\mathrm{~S}$ & 1184 & 17.3290 & 1186 & 0.0816 & -17.247 & 1154 & 0.0375 & -17.291 & 1119 & 0.0093 & -17.319 \\
\hline 106 & $\mathrm{~S}$ & 1364 & 14.1000 & 1367 & 0.3774 & -13.722 & 1330 & 0.0353 & -14.064 & 1289 & 0.0080 & -14.092 \\
\hline 126 & $\mathrm{~S}$ & 1622 & 2.1500 & 1625 & 0.0435 & -2.106 & 1581 & 0.0033 & -2.146 & 1532 & 0.0052 & -2.144 \\
\hline 144 & $\mathrm{C}$ & 1854 & 29.9800 & 1857 & 0.1632 & -29.816 & 1807 & 0.0249 & -29.955 & 1751 & 0.0046 & -29.975 \\
\hline 162 & $\mathrm{~S}$ & 2085 & 6.4480 & 2089 & 0.0049 & -6.443 & 2032 & 0.0038 & -6.444 & 1970 & 0.0032 & -6.444 \\
\hline 178 & $\mathrm{~S}$ & 2291 & 7.3700 & 2296 & 0.0691 & -7.300 & 2233 & 0.0039 & -7.366 & 2164 & 0.0039 & -7.366 \\
\hline 196 & $\mathrm{~S}$ & 2523 & 3.6320 & 2528 & 0.0197 & -3.612 & 2459 & 0.0038 & -3.628 & 2383 & 0.0014 & -3.630 \\
\hline 216 & $\mathrm{C}$ & 2780 & 9.2500 & 2786 & 0.1811 & -9.068 & 2710 & 0.0579 & -9.192 & 2626 & 0.0113 & -9.238 \\
\hline 268 & $\mathrm{~S}$ & 3450 & 2.5890 & 3456 & 0.0067 & -2.582 & 3363 & 0.0021 & -2.586 & 3259 & 0.0006 & -2.588 \\
\hline 288 & $\mathrm{C}$ & 3707 & 8.4010 & 3714 & 0.0329 & -8.368 & 3613 & 0.0030 & -8.398 & 3502 & 0.0026 & -8.398 \\
\hline
\end{tabular}

*CODES FOR DESCRIBING RIPPLE FREQUENCY CAUSAL ID:

A (Non-homogeneous Flux across air gap), S (Slot), C (Commutator)* 


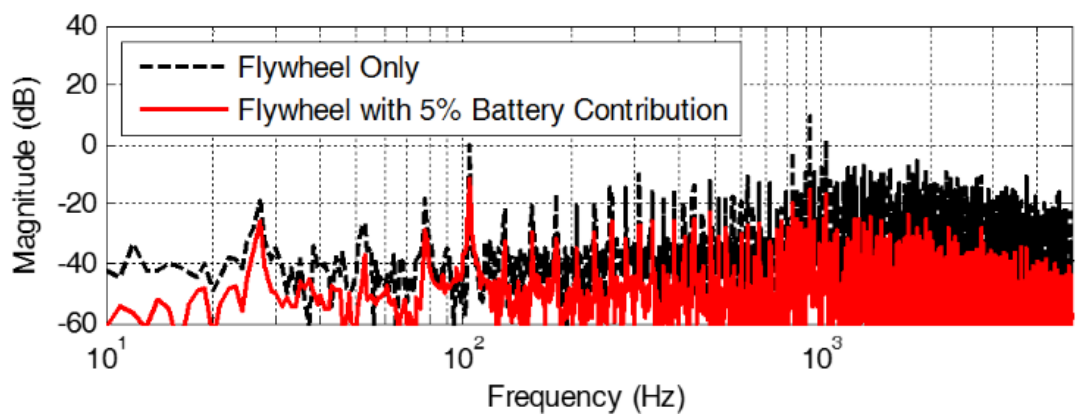

Figure 12.10. Discrete Fourier Transform of Load Voltage with 5\% Battery Current Contribution.

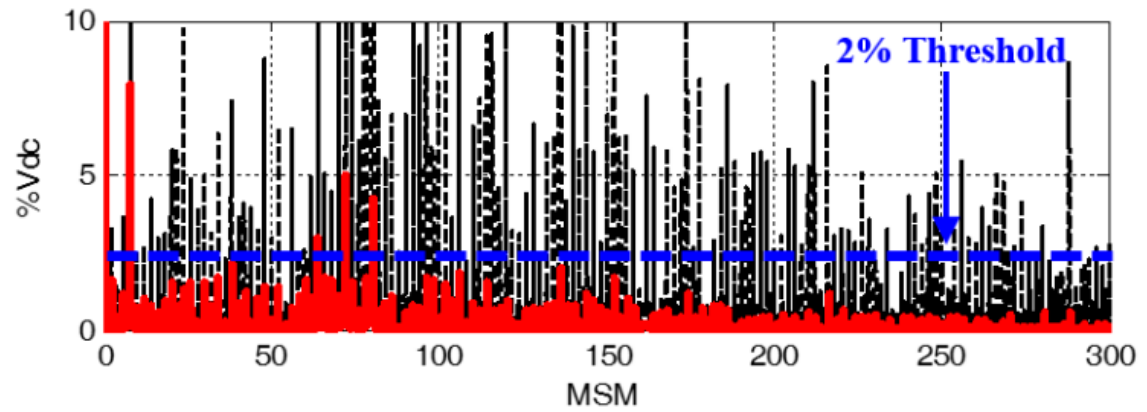

Figure 12.11. Voltage Percentage versus DC Component under 5\% Battery Current Contribution.

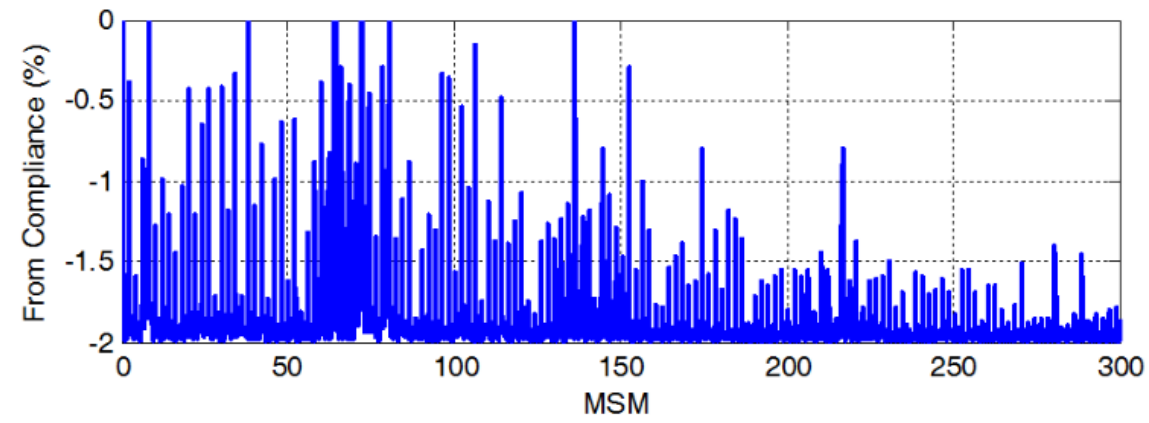

Figure 12.12. Departure of Ripple Voltage at each MSM from 2\% Compliance under 5\% Battery Current Contribution.

\subsubsection{5\% Battery / 75\% Flywheel Current Contribution}

In this case, the flywheel speed is reduced to allow for the battery bank current to begin injecting $25 \%$ of the load current. The flywheel current is reduced to $2.10 \mathrm{~A}(75 \%)$, while the battery bank increases its loading to $660 \mathrm{~mA}(25 \%)$. This results in a reduction of the machine speed to around 751 RPM (12.52 Hz). Figure 12.13 and Figure 12.14 once again 
depict the original spectra to $5 \mathrm{kHz}$ in black, and the new HESS spectra under $25 \%$ battery current contribution in red. Figure 12.13 features the magnitude reduction of each ripple voltage frequency in log scale, where Figure 12.14 displays the ripple voltage percentage versus the DC component over the new linear MSM scale. The $2 \%$ threshold marker is once again shown in blue.

Under a relatively small level of current, the overall spectral comparison reveals a 20 $\mathrm{dB}$ magnitude decrease in the overall ripple bias. Table 12-2 confirms that all ripple voltage percentages have been further reduced to meet the $2 \%$ threshold, as depicted in these figures. Figure 12.15 highlights the ripple voltage percentage versus the DC component, as compared to the $2 \%$ threshold. A close-up highlights that all frequencies have been reduced by $>0.5 \%$ from the threshold.

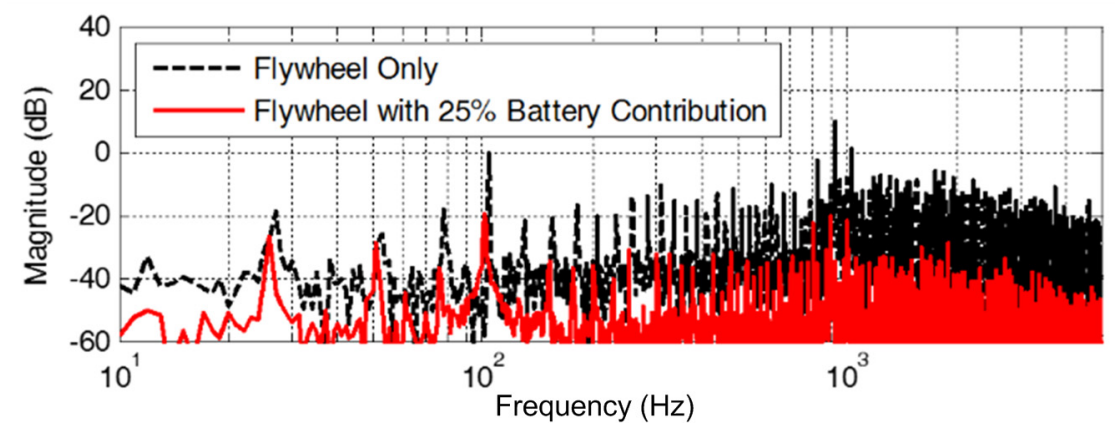

Figure 12.13. Discrete Fourier Transform of Load Voltage with 25\% Battery Current Contribution.

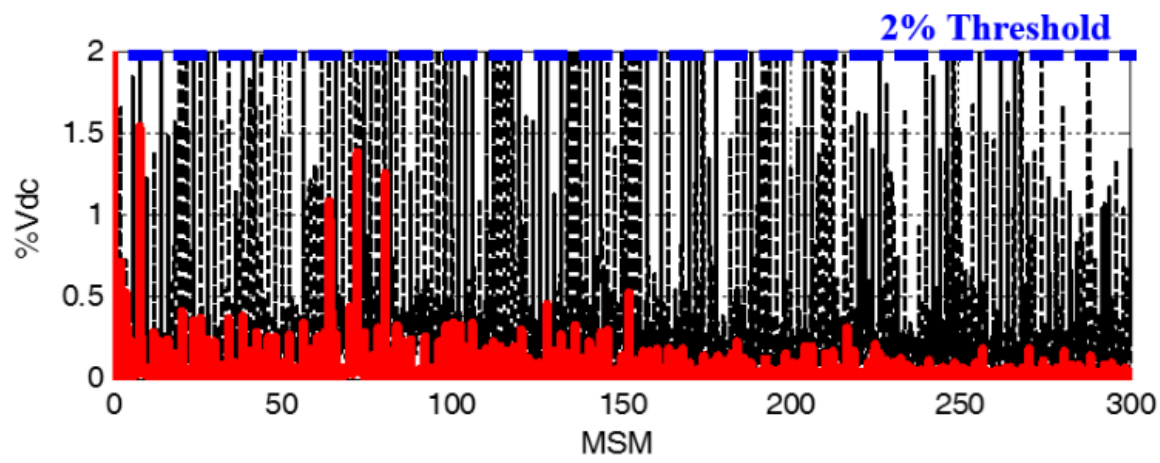

Figure 12.14. Voltage Percentage versus DC Component under 25\% Battery Current Contribution. 


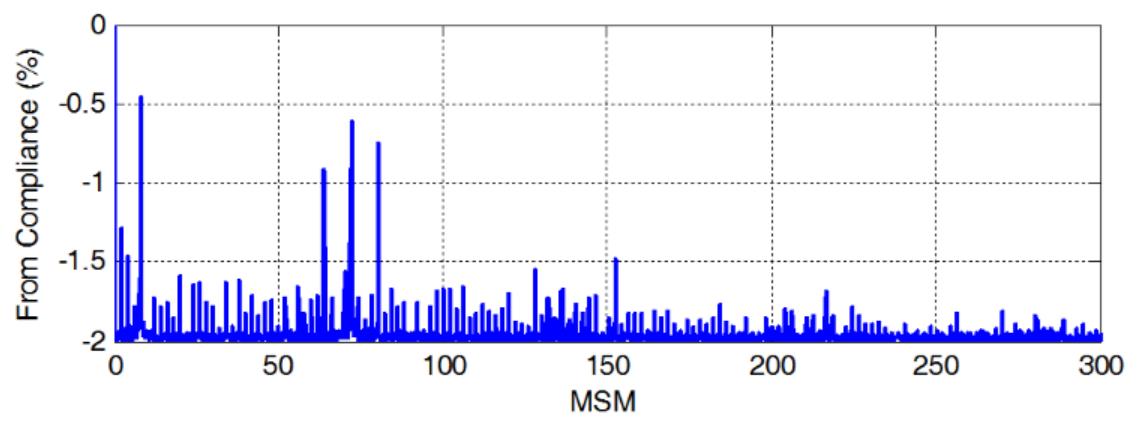

Figure 12.15. Departure from 2\% Compliance under 25\% Battery Contribution.

\subsubsection{0\% Battery / 50\% Flywheel Current Contribution}

The final case provides an equally-shared HESS energy supply case, where both the flywheel and battery are supporting $1.35 \mathrm{~A}$ (50\%), respectively. The machine speed is further reduced to 729 RPM $(12.15 \mathrm{~Hz})$ to maintain the energy output required. Figure 12.16 and Figure 12.17 depict the original spectra to $5 \mathrm{kHz}$ in black, and the new HESS spectra under $50 \%$ battery current contribution in red. The overall spectral bias is shown in Figure 12.16, revealing a $30 \mathrm{~dB}$ reduction from the case with the flywheel only. Figure 12.17 displays the ripple voltage percentage versus the DC component over a linear MSM scale. Since no frequencies approach $2 \%$, a threshold marker is not shown in this figure.

Although a $30 \mathrm{~dB}$ reduction is observed from the base flywheel case, when viewing this in the linear MSM scale in Figure 12.17, one can observe that this reduction does not provide a notable advantage except for in highly sensitive applications. This could be particularly of interest in some SPS, as some navy equipment and navigation electronics can be highly harmonic-sensitive [223]. From Table 12-2, one can see that all ripple frequencies have once again been reduced below $1 \%$, but only the $8^{\text {th }}$ and $72^{\text {nd }} M S M$ magnitudes have a noticeable decrease. 


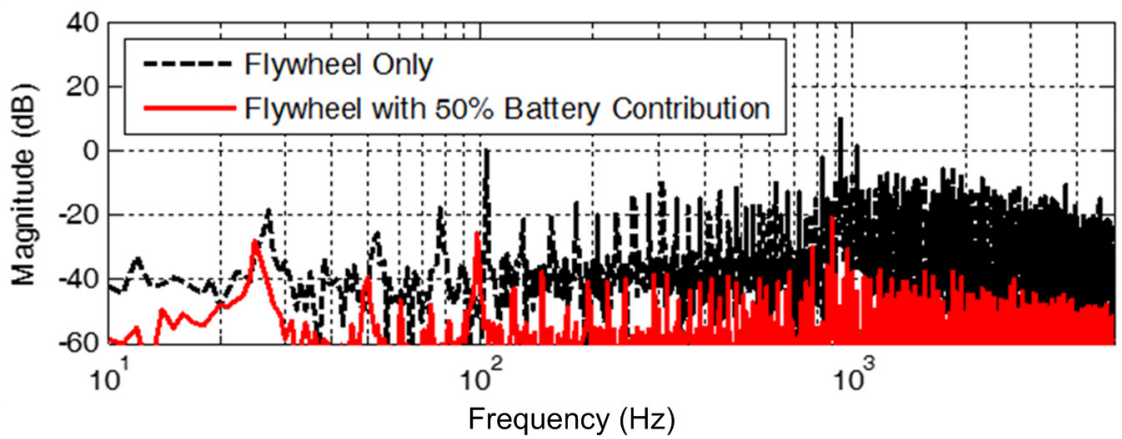

Figure 12.16. Discrete Fourier Transform of Load Voltage with 50\% Battery Current Contribution.

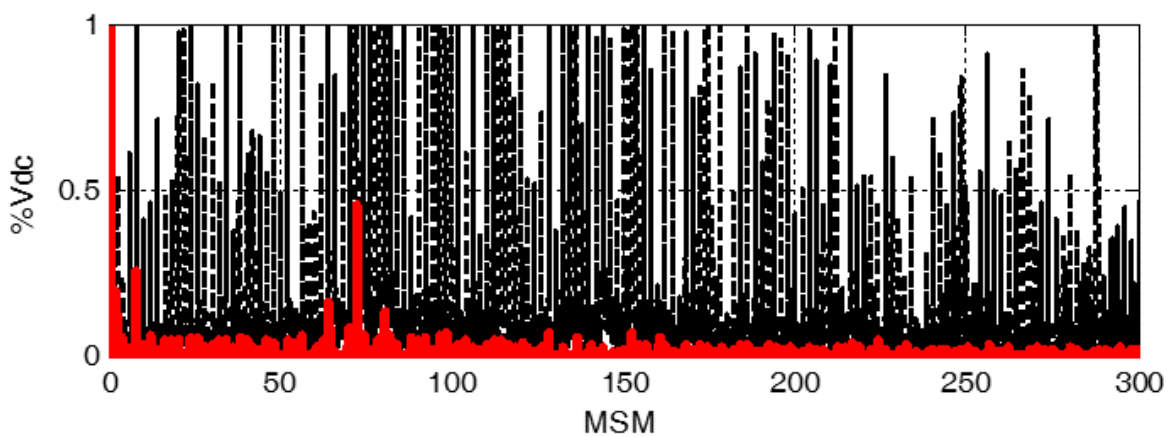

Figure 12.17. Voltage Percentage versus DC Component under 50\% Battery Current Contribution.

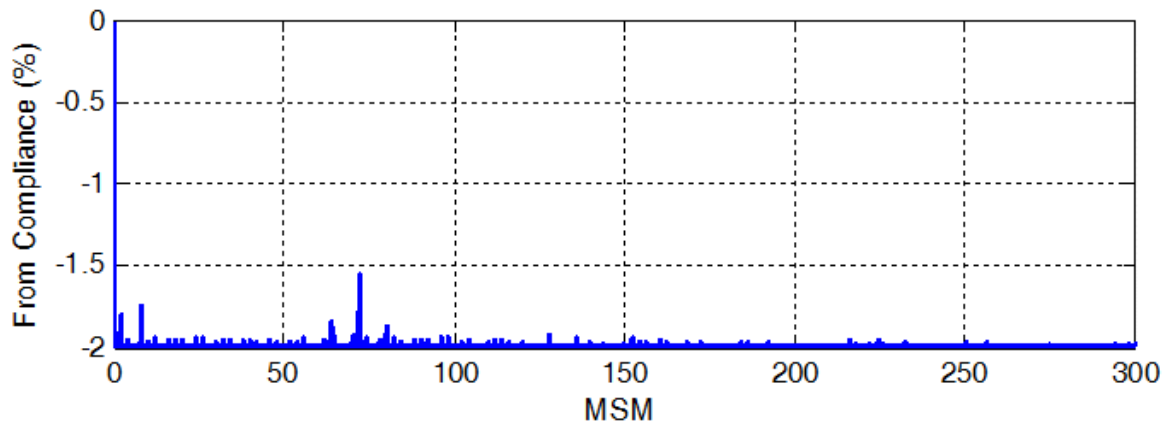

Figure 12.18. Departure from 2\% Compliance under 50\% Battery Current Contribution.

Shown in Figure 12.17, only four spectral frequencies (other than the DC component) are easily identified with the remaining components below $0.1 \%$. Figure 12.18 highlights the ripple voltage percentage versus the DC component, as compared to the $2 \%$ threshold, where the peak ripple voltage magnitude falls $1.5 \%$ below the threshold. This case proves that extracting more than $25 \%$ energy from the battery bank is unnecessary to significantly 
improve the power quality of the HESS unless sensitive applications are at stake. A correlation of these results to the ripple voltage requirement of the load can help to better balance the current contribution when operating a flywheel and battery HESS.

\subsection{Summary}

This chapter introduced the concept of FESS, as well as the advantages in the integration of a lead acid battery in parallel to form a HESS. In this chapter, the purpose of integrating a HESS took on a new approach, highlighting the advantage in integrating a battery to assist in improving the power quality. Voltage and current ripple frequencies induced while connected to a 3 A load were investigated. Common geometric and electromagnetic causes in generating these frequencies were discussed, while multiples of the flywheel rotation speed were interpreted by a new metric called the Machine Speed Multiple to explain the presence, location, and reduction of voltage ripple. Voltage ripple spectra from a flywheel-only system was compared to three different current contribution cases, including a battery bank connected in parallel. The ripple frequency reduction was identified at different MSMs, discovering that only a small contribution from a battery bank could result in a significant improvement in the power quality delivered to the load. Using the MSM as a frequency profiler, additional features could be added to an advanced controller such as the ESMC to correlate the target voltage ripple frequencies to the required design specification. In light of this study, a best combination and control scheme could be determined to reduce the overall voltage ripple frequencies for the HESS. 


\section{Chapter 13 Conclusions and Future Work}

\subsection{Conclusions}

The contributions of this dissertation are present in a wide range of areas related to modeling and control of energy storage (ES) and hybrid ES systems (HESS). With the advent of the Energy Storage Management Controller (ESMC), a number of avenues were opened in not only improving models of ES devices, but also the optimal selection and management of ES devices in HESS. The ESMC circuit topology along with many software solutions were developed and tested to manage individual ES devices, while providing total isolation by means of a bypass circuit. Meanwhile, the extracted ES can be connected to a dedicated charging circuit or fully extracted to conduct maintenance. A comprehensive analysis of the ESMC prototype, its components, and control were then assessed for their implementation into a comprehensive commercialized platform.

Development of the ESMC established a unique hardware and software platform that was vital in test and evaluation of not only sole ES devices, but particularly in complex HESS. In the first test scenario, testing was conducted upon lead acid batteries, where its features were demonstrated as well as what can be accomplished by providing individual ES charging terminals. Using unique capabilities of ESMC charging controller, a pulsed charging process was demonstrated to improve the SoH of a lead acid battery module. Without individual charging terminals and the capability to isolate and control individual modules, this process would not have been possible in an application that was also able to maintain system operation with remaining ES modules in the network. 
In the following chapters, a focus was placed upon how the software of the ESMC could be enhanced to provide better measures to estimate the State of Health $(\mathrm{SoH})$ and State of Charge (SoC) of ES devices. An introduction to the direct method of acquiring a real-time equivalent circuit through the use of electrochemical impedance spectroscopy (EIS) was explored, providing an overview of the challenges involved in its effective implementation on a real-time controller. A potential solution and circuit topology was proposed for its future implementation with the EMSC. Next, an alternative and low-cost equivalent circuit acquisition solution was proposed and tested using a pulsed load. There is an imperative need to provide a bridge between the depth of the electrochemical physics of the battery and the power engineering sector, a feat which was accomplished over the course of this work. Derived and verified through the utilization of pseudo 2D (P2D) physics-based models (PBM) of both lead acid and lithium ion batteries, a comprehensive hardware and software platform generated a tool to acquire a dynamic $1^{\text {st }}$ order equivalent circuit model that could also autonomously determine the battery chemistry. This battery management system was not limited to simply chemistry and equivalent circuit acquisition, but introduced a comprehensive operating platform that assessed SoH in two ways: through tracking of the equivalent circuit model cycle-to-cycle and tracking the latest usable energy. SoC metrics for both lead acid and lithium ion batteries were enhanced as well, with a particular focus upon improving the initial voltage-based SoC estimation.

Utilization and experimental fitting of the P2D PBM for each battery provided the basis to extend the lithium ion model into a comprehensive 3D PBM. In this work, the computational investment, accuracy, and unique capabilities provided by the 3D model were evaluated side-by-side with the P2D PBM. The 3D PBM provided a mechanism to 
study an aspect of battery ageing, or a reduction of the $\mathrm{SoH}$, by visualizing and quantifying the generation of undesired gradient currents across the lithium ion cell surface when operating at high levels of normalized cell current, or Columbic rates (C-rates). High Crates are common in shipboard power system (SPS) and electric vehicle (EV) applications, thus it is imperative to further understand the impacts from these operational scenarios. The development of gradient currents contribute to uneven thermodynamic and material stress, which can have long-term health impacts on a battery cell.

Next, many of the lessons learned from not only experimentation with EIS but also deployment of a pulsed load to extract equivalent circuit parameters, a dynamic $2^{\text {nd }}$ order equivalent circuit model was developed for a 51.8V $21 \mathrm{Amp}$-hour (Ah) lithium ion battery module. This $2^{\text {nd }}$ order dynamic model was able to capture a "fingerprint" of the battery so accurate simulations could be conducted for a wide range of applications, demonstrated particularly on an EV. The all-inclusive model does not carry with it a great deal of computational overhead and was implemented within the MATLAB/Simulink environment as a drop-in replacement for the SimPowerSystems battery block.

The dissertation then began to shift toward the development and implementation of HESS. This first studied basic interfacing power electronic converters between single ES devices, eventually honing in upon how the eventual replacement of legacy silicon-based switches with Gallium Nitride High Electron Mobility Transistors (GaN HEMT) could improve the system efficiency and performance. A PBM was developed and utilized to study how material and geometric adjustments to the switch structure could result in these devices handling higher voltage levels, which would lead to the application of GaN HEMT in many future applications. Next, three HESS applications were evaluated in detail 
utilizing features of the ESMC, which was tested and validated with the addition of lithium ion batteries, SC, and FESS. First, a major contribution integrated, modeled, tested, and evaluated three unique series-configured HESS to handle SPS loads using lead acid batteries, lithium ion batteries, and SC. The uniqueness in this work was in the development of specialized software that was able to apply a new control scheme called "rolling charging" to coordinate charging and discharging of individual ES units while in operation to extend the runtime while acknowledging SoH trade-offs.

SoH trade-offs remained a common theme in the following work, which studied the implementation of the lithium ion battery and SC HESS for EV applications. A particular focus was placed upon reducing the cycling of the lithium ion battery under traction applications involving both drive and regenerative braking (charge) currents. Once again, the ESMC was utilized, this time in a parallel-configured system, with specialized software to employ the Hybrid Pulsed Power Characterization (HPPC) test sequence representing an industry standard for EV ES and HESS performance evaluation. In addition to improving the SoH of the lithium ion battery, the control metric resulted in an increased energy harvesting efficiency from regenerative braking as well.

In the final work, a HESS consisting of a FESS and lead acid battery was tested and evaluated for its capability to improve the power quality over simply a FESS-only system. A metric was established to quantify and track ripple voltage frequencies on a primary DC network and use it to target and reduce electrical noise from the FESS. The ESMC software was once again extended to include a Fast Fourier Transform to conduct a live frequency analysis, where a linear load was utilized as reference to balance the current contribution between the battery and FESS. The lead acid battery contribution was tested at multiple 
levels of current concluding that even modest assistance to serve a load from the lead acid battery can result in a dramatic improvement of the power quality, particularly in harmonicsensitive applications.

\subsection{Future Work}

In this section, the doctoral work outlined in this dissertation is evaluated for both specific future research goals as well as large research areas that could continue work in this important field of study. With respect to large areas, a greater variety of HESS need to be tested and evaluated for their strengths and weaknesses beyond their basic characteristics as outlined in Chapter 1. The FESS, in particular, has not been exposed to a unique loading profile or comprehensively modeled in the simulation domain. In addition, there is always a desire to continuously increase the power and energy capacity of ES devices and HESS to exceed the typical laboratory-scale environment. As more realistic voltage and current levels can be achieved, a deeper and more accurate analysis of the system performance can be conducted. Furthermore, in much larger systems with thousands of battery cells or SC modules, not only would the behavior change, but this setup could expose new challenges that will need to be solved in future applications.

Lithium Ion Battery Performance and Degradation over its Full Lifespan: The evaluation of a full lithium ion battery lifespan can be accomplished in many ways, but in this case two approaches in particular can be taken. First, and the most simple, is applying accelerated ageing to the lithium ion battery through a high speed charging and discharging system. In this case, standard charging and discharging cycles could progress to more typical loading and charging scenarios. However, it is important to mention that even accelerated ageing does not capture the full story. Unfortunately, the most accurate 
procedure is to operate a battery from the beginning of its lifespan until its end with data recording. In this way, the most realistic loading and charging profiles, representative of real operating and environmental conditions, can give insight into how capacity fluctuation and impulse response of the battery voltage will change over time. This is already in progress, where a Samsung Galaxy S7 phone has been recording voltage and current data since it was first purchased in May 2017. A comprehensive performance evaluation and comparison of its charging and discharging cycles can be compared at the end of its life. Further Enhancements of the ESMC Device: This general category opens the avenue to many new research areas. First, the comprehensive autonomous battery management system platform from Chapter 5 could be implemented on the ESMC in conjunction with support for other ES devices. Second, a continued focus upon improving SoH estimation could include an extension socket or alternative version that includes on-board EIS measurements for either a full $1^{\text {st }}$ order equivalent circuit or at a minimum, the internal resistance of the ES device using the methods established in Chapter 4. Third, the ESMC has yet to be tested for other battery chemistries such as nickel-metal hydride, nickelcadmium, sodium sulfur, or emerging types of Lithium ion chemistries. Furthermore, integration with a unidirectional fuel cell could be evaluated as well. Finally, testing and development of the commercialized ESMC can be accomplished. Using the commercialized system with onboard microcontrollers, an extension can then be made into zonal platforms, where ES devices are located in multiple remote regions across a SPS platform or utility system.

Long-term SoH correlation to Lithium Ion Batteries: In Chapter 6, a 3D PBM visualized and quantified the generation of gradient currents on the lithium ion battery, which 
becomes apparent when the cell is operating at high C-rates. The long term impacts of gradient currents need to be evaluated as the battery ages, including how the magnitude and origin of these currents change, how operating voltage levels change, and how thermodynamic cycling will impact the active materials. A correlation between the thermodynamic cycling and material degradation models could enable a way to capture the thermodynamic expansion of each material inside the battery. This analysis, conducted over time, can help to estimate how active materials of the battery crack and eventually degrade. This would not only allow engineers to view how each material cracks and its thermodynamic stress independently, but also pinpoint how operating conditions (such as gradient currents) will contribute to nonlinear fracturing. In this way, one could forecast which materials would fail first.

Further Testing and Utilization of Experimentally Acquired Battery Models: In Chapter 7, a comprehensive $2^{\text {nd }}$ order dynamic battery equivalent circuit was acquired for a $51.8 \mathrm{~V} 21$ Ah lithium ion battery module. The long-term degradation of this module should be evaluated to provide a mechanism to capture the long-term impacts and include them in the final battery model. For EVs, the end of life is usually designated as a $30 \%$ loss of capacity [210]. A function could enhance this model to include a capacity loss trend equation, which provides a "fingerprint" for how this specific module will age. In this same analysis, the thermal impacts and its correlation to the $2^{\text {nd }}$ order dynamic equivalent circuit should be evaluated as well as how these would also impact the degradation model. Using the same hybrid procedure, hardware, and data acquisition platform to obtain the lithium ion cobalt module dynamic equivalent circuit, models for different types of lithium ion batteries (e.g. lithium ion phosphate) of similar voltage and capacity can be made in order 
to provide a performance evaluation between the two. This could also increase the number of lithium ion battery options for future utility grid, EV, and SPS applications. Moreover, there may also be a need to perform the same modeling for alternative chemistries as well. Extended Analysis of the Lithium Ion Battery and SC HESS: On the health front, an extension to the lithium ion battery and SC HESS work in Chapter 11 would apply the Hybrid Pulsed Power Characterization (HPPC) EV ES and HESS performance evaluation profile to the enhanced PBM. This research left off using a basic full charge and discharge cycling profile on the lithium ion battery as a basic metric to evaluate its cycling life. By including the additional physics element to model the degradation of the battery over time through the increase of thickness in the solid electrolyte interface (SEI) layer, the cycle life for the common lithium ion cobalt battery was depicted. This was initially used to demonstrate capacity loss over time. In a future work, the lithium ion battery can be coupled to the HPPC profile over time to evaluate how many cycles the battery can withstand under the charging and discharging HPPC profile over time. In the next step, the HESS can be tested under the same profile by either developing a PBM of the SC or through the extraction of the current profile placed on the battery as acquired by behavior acquired from previous experimentation.

Stronger Correlation between Gallium Nitride PBMs and Physical Switches: Future work needs to provide a better correlation between the GaN HEMT PBM and its power electronics Simulation Program with Integrated Circuit Emphasis (SPICE) models. This would begin with a particular study upon correlating the GaNSys GS66508T device to an accurate PBM. As mentioned in Chapter 9, this is challenging because specific dimensioning and material structures would need to be provided from the manufacturer. 
Work of this nature would require either working directly with the GaNSys fabrication team, or working with another company or agency that can provide these. In this way, a direct relationship could be made between the design and fabrication of the device and the PBM. Furthermore, an exchange could be accomplished between the PBM, as various other combinational structures could be simulated, similar to the process in Chapter 9, and returned to the same agency as a recommendation over how to fabricate the next device. Finally, with a strong correlation between the experimental and PBM, a version of the PBM could be used to replace legacy SPICE models in future power system simulations. Enhancing HESS Design and Analysis for SPS Applications: HESS SPS models can be extended to not only evaluate series-configured systems, but also parallel-configured. In addition, other ES devices can be tested as well including not only various types of other battery chemistries, but also FESS. Furthermore, with such a drastic difference in the energy density between the FESS and SC, an optimal sizing method could be established to better pair these devices with traditional batteries. The load side should be analyzed as well, looking at not only more types of pulsed loads that include more realistic loading profiles and sequences for naval equipment, but a more realistic representation of the hotel load as well and how minor variations in the profile can have impacts on the overall SPS. Since another aspect of this research provided an option that performed "rolling charging" only on the SC to protect the life of the battery ES devices, physics-based degradation models for both the lithium ion and lead acid could further quantify these impacts. This may also provide insight into the optimal rolling charging sequence. All of these different aspects could contribute to a variety of new test cases. 


\section{LIST OF REFERENCES}

[1] L. Xu, Z. Miao, and L. Fan, "Control of a battery system to improve operation of a microgrid," in 2012 IEEE Power and Energy Society General Meeting, 2012, pp. 18.

[2] M. C. Such and C. Hill, "Battery energy storage and wind energy integrated into the Smart Grid," in the 2012 Innovative Smart Grid Technologies (ISGT), Washington, DC, 16-20 Jan. 2012.

[3] Y. S. Wong, L. L. Lai, S. Gao, and K. T. Chau, "Stationary and mobile battery energy storage systems for smart grids," in 2011 4th International Conference on Electric Utility on 2011 4th International Conference on Electric Utility, Weihai, China, 06-09 Jul. 2011.

[4] Z. Miao, L. Xu, V. R. Disfani, and L. Fan, "An SOC-Based Battery Management System for Microgrids," IEEE Transactions on Smart Grid, vol. 5, no. 2, pp. 966973, Mar. 2014.

[5] P. Denholm, E. Ela, B. Kirby, and M. Milligan. "The Role of Energy Storage with Renewable Electricity Generation," NREL. Golden, CO. NREL/TP-6A2-47187. Jan. 2010.

[6] S. Yeleti and $\mathrm{Y}$. Fu, "Impacts of energy storage on the future power system," in NAPS 2010, Arlington, TX, 26-28 Sep 2010.

[7] G. S. Vassell, "Northeast Blackout of 1965," IEEE Power Eng. Review, vol. 11, no. 1, p. 4, Jan. 1991.

[8] J. E. Chadwick, "How a smarter grid could have prevented the 2003 U.S. cascading blackout," in 2013 IEEE Power and Energy Conference at Illinois (PECI), Champaign, IL, 22-23 Feb. 2013.

[9] J. L. Beven, L. A. Aliva, E. S. Blake, D. P. Brown, J. L. Franklin, R. D. Knabb, R. J. Pasch, J. R. Rhome, and S. R. Stewart, "Atlantic Hurricane Season of 2005," Mon. Wea. Rev., vol. 136, no. 3, pp. 1109-1173, Mar. 2008.

[10] Destroyers - DDG [Online]. United States Navy Fact File. Available: http://www.navy.mil/.

[11] A. Levin, "Boeing's Dreamliner Battery Fire Caused by Design, Probe Finds," Bloomberg, 01-Dec-2014. [Online].

Available: http://www.bloomberg.com/news/2014-12-02/. [Accessed: 31-Dec-2014].

[12] “Tesla Recalling 439 Roadsters for Fire Hazard," Wheels Blog. [Online]. Available: http://wheels.blogs.nytimes.com/2010/10/08/tesla-recalling-439-roadsters-for-firehazard/.

[13] D. Linden, T. B. Reddy, "Lead Acid Batteries" in Handbook of Batteries, $3^{\text {rd }}$ ed. New York, NY: McGraw-Hill, 2002, ch, 23. 
[14] A. Cooper and P. Moseley, "Progress in the Development of Lead-Acid Batteries for Hybrid Electric Vehicles," in IEEE Vehicle Power and Propulsion Conference, 2006. VPPC '06, 2006, pp. 1-6.

[15] Michael Koller, Theodor Borsche, Andreas Ulbig, Göran Andersson, "Review of grid applications with the Zurich $1 \mathrm{MW}$ battery energy storage system," Electric Power Systems Research, 120 (2015) 128-135.

[16] Maninder Singh, Luiz A.C. Lopes, Nayeem A. Ninad, "Grid forming Battery Energy Storage System (BESS) for a highly unbalanced hybrid mini-grid," Electric Power Systems Research, 127 (2015), 126-133.

[17] C. Pillot, "Micro hybrid, HEV, P-HEV and EV market 2012 \#x2013;2025 impact on the battery business," in Electric Vehicle Symposium and Exhibition (EVS27), 2013 World, 2013, pp. 1-6.

[18] Batteries: Lithium-ion vs AGM [Online]. Victron Energy. Available: https:/www.victronenergy.com/blog/2015/03/30/batteries-lithium-ion-vs-agm/.

[19] E. Wesoff, "Energy Storage at Grid Scale: A123 Gets Li-Ion to Market," 08-Jun2011. [Online]. Available: https://www.greentechmedia.com/articles/read/EnergyStorage-at-Grid-Scale-A123-Gets-Li-Ion-to-Market. [Accessed: 10-Feb-2017].

[20] G. Diaz, C. Gonzalez-Moran, J. Gomez-Aleixandre, A. Diez, Scheduling of droop coefficients for frequency and voltage regulation in isolated microgrids, IEEE Transactions on Power Systems, 25 (2010) 489-496.

[21] M. Doyle, J. Newman, A. S. Gozdz, C. N. Schmutz, and J.-M. Tarascon, "Comparison of Modeling Predictions with Experimental Data from Plastic Lithium Ion Cells," Journal of the Electrochemical Society, vol. 143, no. 6, pp. 1890-1903, Jun. 1996.

[22] "Types of Lithium-ion Batteries - Battery University." [Online]. Available: http://batteryuniversity.com/learn/article/types_of_lithium_ion. [Accessed: 23Nov-2016].

[23] J.-Y. Kim, J.-H. Jeon, S.-K. Kim, C. Cho, J. H. Park, H.-M. Kim, K.-Y. Nam, Cooperative Control Strategy of Energy Storage System and Microsources for Stabilizing the Microgrid during Islanded Operation, IEEE Transactions on Power Electronics, 25 (2010) 3037-3048.

[24] H. Karimi, H. Nikkhajoei, R. Iravani, Control of an Electronically-Coupled Distributed Resource Unit Subsequent to an Islanding Event, IEEE Transactions on Power Delivery, 23 (2008) 493-501.

[25] B. Sood, M. Osterman, and M. Pecht, "Health monitoring of lithium-ion batteries," in 2013 IEEE Symposium on Product Compliance Engineering (ISPCE), 2013, pp. $1-6$.

[26] "Supercapacitor Information - Battery University." [Online]. Available: http://batteryuniversity.com/learn/article/whats_the_role_of_the_supercapacitor. [Accessed: 20-Oct-2015]. 
[27] P. J. Grbović, "Ultra-Capacitor Energy Storage Devices", in Ultra-Capacitors in Power Conversion Systems, John Wiley \& Sons Ltd, 2014, pp. 22-77.

[28] F. Béguin, E. Frąckowiak, and A. Burke, "Testing of Electrochemical Capacitors", in Supercapacitors, Eds. Wiley-VCH Verlag GmbH \& Co. KGaA, 2013, pp. 437471.

[29] "Maxwell Ultracapacitors: Enabling Energy's Future," Maxwell Technologies. [Online]. Available: http://www.maxwell.com/products/ultracapacitors. [Accessed: 25-Aug-2017].

[30] J. P. Kelley, D. A. Wetz, J. A. Reed, I. J. Cohen, G. K. Turner, W. Lee, The impact of power quality when high power pulsed DC and continuous AC loads are simultaneously operated on a MicroGrid testbed, 2013 IEEE Electric Ship Technology Symposium, 2013, pp. 6-12.

[31] P. Thounthong, A. Luksanasakul, P. Koseeyaporn, and B. Davat, "Intelligent ModelBased Control of a Standalone Photovoltaic/Fuel Cell Power Plant With Supercapacitor Energy Storage," IEEE Transactions on Sustainable Energy, vol. 4, no. 1, pp. 240-249, Jan. 2013.

[32] B. Vural and C. S. Edrington, Ultra-capacitor based pulse power management in electrical ships, 2012 IEEE Transportation Electrification Conference and Expo (ITEC), 2012.

[33] A. Patil, "Flywheel Energy Storage (FES) Systems Market Analysis and In-depth Research on Size, Trends, Emerging Growth Factors and Forecasts 2022 | Green Mountain Outlook."

[34] J. McGroarty, J. Schmeller, R. Hockney and M. Polimeno, "Flywheel energy storage systems for electric start and an all-electric ship," IEEE Electric Ship Technologies Symposium, pp. 400-406, 2005.

[35] B. Bolund, H. Bernhoff, and M. Leijon, "Flywheel energy and power storage systems," Renewable and Sustainable Energy Reviews, vol. 11, no. 2, pp. 235-258, Feb. 2007.

[36] L. Zhang, L. Li, W. Cui, and S. Li, "Study on improvement of micro-grid's power quality based on APF and FESS," in the 2012 IEEE Innovative Smart Grid Technologies - Asia (ISGT Asia), 2012, pp. 1-6.

[37] S. Samineni, B. K. Johnson, H. L. Hess, and J. D. Law, "Modeling and analysis of a flywheel energy storage system for Voltage sag correction," IEEE Transactions on Industrial Applications, vol. 42, no. 1, pp. 42-52, Jan. 2006.

[38] C. Xie and C. Zhang, "Research on the Ship Electric Propulsion System Network Power Quality with Flywheel Energy Storage," in the 2010 Asia-Pacific Power and Energy Engineering Conference, 2010, pp. 1-3.

[39] G. O. Suvire and P. E. Mercado, "Improvement of power quality in wind energy applications using a DSTATCOM coupled with a Flywheel Energy Storage System," in the 2009 Brazilian Power Electronics Conference. COBEP '09. 
Brazilian, 2009, pp. 58-64

[40] M. Subkhan and M. Komori, "New Concept for Flywheel Energy Storage System Using SMB and PMB," IEEE Transactions on Applied Superconductivity, vol. 21, no. 3, pp. 1485-1488, Jun. 2011.

[41] I. Lahbib, A. Lahyani, A. Sari, P. Venet, Performance analysis of a lead-acid battery/supercapacitors hybrid and a battery stand-alone under pulsed loads, 2014 International Conference on Green Energy, 2014, pp. 273-278.

[42] M. Ibrahim, S. Jemei, G. Wimmer, and D. Hissel, "Nonlinear autoregressive neural network in an energy management strategy for battery/ultra-capacitor hybrid electrical vehicles," Electric Power Systems Research, vol. 136, pp. 262-269, Jul. 2016.

[43] P. J. Kollmeyer, L. W. Juang, and T. M. Jahns, "Loss optimization and ultracapacitor pack sizing for vehicles with battery/ultracapacitor hybrid energy storage," in 2014 IEEE Transportation Electrification Conference and Expo (ITEC), 2014, pp. 1-8.

[44] B. M. Huhman, J. M. Neri, D. A. Wetz, Design of a battery intermediate storage system for rep-rated pulsed power loads, 2013 IEEE Electric Ship Technologies Symposium (ESTS), 2013.

[45] G. L. Kusic, J. M. Heinzel, D. J. Hoffman, Monitoring pulsed power on ship electrical systems, 2013 IEEE Electric Ship Technologies Symposium (ESTS), 2013, pp. 13-20.

[46] H. Babazadeh, B. Asghari, and R. Sharma, "A new control scheme in a multi-battery management system for expanding microgrids," in Innovative Smart Grid Technologies 2014 (ISGT 2014), Washington, DC, 19-22 Feb. 2014.

[47] R.S. Treptow, "The Lead-Acid Battery: Its Voltage in Theory and in Practice," Journal of Chemical Education, vol. 79, no. 3, pp. 334-338, 2002.

[48] H. Chaoui and P. Sicard, "Hierarchical energy management scheme for multiple battery-based smart grids," in 2014 IEEE 23rd International Symposium on Industrial Electronics (ISIE), 2014, pp. 2020-2023.

[49] S. W. Moore and P. J. Schneider, "A review of cell equalization methods for lithium ion and lithium polymer battery systems," presented at the SAE 2001 World Congress, Detroit, MI, Mar. 2001.

[50] H. Qian, J. Zhang, J.-S. Lai, and W. Yu, "A high-efficiency grid-tie battery energy storage system," IEEE Transactions on Power Electronics, vol. 26, no. 3, pp. 886896, Mar. 2011.

[51] M. T. Lawder, B. Suthar, P. W. C. Northrop, S. De, C. M. Hoff, O. Leitermann, M. L. Crow, S. Santhanagopalan, and V. R. Subramanian, "Battery Energy Storage System (BESS) and Battery Management System (BMS) for Grid-Scale Applications," Proceedings of the IEEE, vol. 102, no. 6, pp. 1014-1030, Jun. 2014. 
[52] Jiapeng Wen, and Jiuchun Jiang, "Battery management system for the charge mode of quickly exchanging battery package," IEEE Vehicle Power and Propulsion Conference, 2008. VPPC '08, pp.1-4, 3-5 Sept. 2008.

[53] Wangbiao Qiu, and Zhiyuan Qiu, "Design for symmetrical management of storage battery expert system based on single battery," Proceedings of the 2006 IEEE International Conference on Mechatronics and Automation, pp.1141-1146, 25-28 June 2006.

[54] D. Tran and A. M. Khambadkone, "Energy Management for Lifetime Extension of Energy Storage System in Micro-Grid Applications," IEEE Transactions on Smart Grid, vol. 4, no. 3, pp. 1289-1296, Sep. 2013.

[55] S. Grillo, M. Marinelli, S. Massucco, and F. Silvestro, "Optimal Management Strategy of a Battery-Based Storage System to Improve Renewable Energy Integration in Distribution Networks," IEEE Transactions on Smart Grid, vol. 3, no. 2, pp. 950-958, Jun. 2012.

[56] G. Yifeng and Z. Chengning, "Study on the fast charging method of lead-acid battery with negative pulse discharge," in 2011 4th International Conference on Power Electronics Systems and Applications (PESA), 2011, pp. 1-4.

[57] W.-S. Jwo and W.-L. Chien, "Design and Implementation of a Charge Equalization Using Positive/Negative Pulse Charger," in the Conference Record of the 2007 IEEE Industry Applications Conference, 2007, pp. 1076-1081.

[58] M. Coleman, C. K. Lee, and W. G. Hurley, "State of Health Determination: Two Pulse Load Test for a VRLA Battery," in 37th IEEE Power Electronics Specialists Conference, 2006. PESC '06, 2006, pp. 1-6.

[59] Y. Shi, C. Ferone, C. Rao, and C. D. Rahn, "Nondestructive forensic pathology of Lead-Acid batteries," in American Control Conference (ACC), 2012, 2012, pp. $1350-1355$.

[60] T. K. Cheung, K. W. E. Cheng, H. L. Chan, Y. L. Ho, H. S. Chung, and K. P. Tai, "Maintenance techniques for rechargeable battery using pulse charging," in 2nd International Conference on Power Electronics Systems and Applications, 2006. ICPESA '06, 2006, pp. 205-208.

[61] K. Onda, M. Nakayama, K. Fukuda, K. Wakahara, and T. Araki, "Cell Impedance Measurement by Laplace Transformation of Charge or Discharge Current-Voltage," J. Electrochem. Soc., vol. 153, no. 6, pp. A1012-A1018, Jun. 2006.

[62] Current Transducer LA 25-NP, LEM USA, Inc., Milwaukee, WI, Nov. 2011.

[63] Voltage Transducer LV 25-P, LEM USA, Milwaukee, WI, Nov. 2011.

[64] NI 9206: 16-Channel Analog Input Module for Fuel Cells, National Instruments, Austin, TX, Feb. 2008.

[65] M. W. Earley, J. S. Sargent, "Storage Batteries" in National Electric Code 2011 Handbook, $12^{\text {th }}$ ed. Quincy, MA: National Fire Protection Association, 2010, ch. 4, sec. 480 . 
[66] Sangsun Kim, M. H. Todorovic, and P. N. Enjeti, "Three-phase active harmonic rectifier (AHR) to improve utility input current THD in telecommunication power distribution system," IEEE Transactions on Industry Applications, vol.39, no.5, pp.1414-1421, Sept.-Oct. 2003.

[67] Texas Instruments, “AMC1200/B Fully-Differential Isolation Amplifier," Dallas, TX. Available Online at: http://www.ti.com/lit/ds/symlink/amc1200.pdf

[68] Q. Wang, P. Ping, X. Zhao, G. Chu, J. Sun, and C. Chen, "Thermal runaway caused fire and explosion of lithium ion battery," Journal of Power Sources, vol. 208, pp. 210-224, Jun. 2012.

[69] Qt for developers by developers | The ultimate cross-platform development framework," Qt, 26-Jan-2016. [Online]. Available: https:/www.qt.io/developers/. [Accessed: 16-May-2017].

[70] STMicroelectronics, "Arm Cortex-M4 STM32F405xx STM32F407xx Datasheet", DocID022152 rev. 8, STMicroelectronics, Inc., Geneva, Switzerland, Sep. 2016. Available Online at: http://www.st.com.

[71] J. Kim, S. Lee, and B. Cho, "Discrimination of battery characteristics using discharging/charging voltage pattern recognition," in the 2009 IEEE Energy Conversion Congress and Exposition. ECCE 2009, 2009, pp. 1799-1805.

[72] D. Zhu, S. Yue, Y. Wang, Y. Kim, N. Chang, and M. Pedram, "Designing a residential hybrid electrical energy storage system based on the energy buffering strategy," in 2013 International Conference on Hardware/Software Codesign and System Synthesis (CODES+ISSS), 2013, pp. 1-9.B.

[73] UPG, "UB121100 12V 110 Ah SLA Battery Spec Sheet,” UPG No. 45824, Carrollton, TX. Available Online at: http://upgi.com/Themes/leanandgreen/images/UPG/ProductDownloads/45981.pdf.

[74] M. Farhadi and O. A. Mohammed, "Performance Enhancement of Actively Controlled Hybrid DC Microgrid Incorporating Pulsed Load," IEEE Transactions on Industry Applications, vol. 51, no. 5, pp. 3570-3578, Sep. 2015.

[75] MIL-STD-1399/390, military standard: interface standard for shipboard systems (section 390) electric power, direct current, (1987).

[76] M. T. Lawder, B. Suthar, P. W. C. Northrop, S. De, C. M. Hoff, O. Leitermann, M. L. Crow, S. Santhanagopalan, and V. R. Subramanian, "Battery Energy Storage System (BESS) and Battery Management System (BMS) for Grid-Scale Applications," Proceedings of the IEEE, vol. 102, no. 6, pp. 1014-1030, Jun. 2014.

[77] C. R. Rahn, C. Wang, "Estimation” in Battery Systems Engineering, 1st ed. West Sussex, UK: John Wiley \& Sons Ltd, 2013, ch, 7.

[78] R. Klein, N. A. Chaturvedi, J. Christensen, J. Ahmed, R. Findeisen, and A. Kojic, "Electrochemical Model Based Observer Design for a Li-Ion Battery," IEEE Transactions on Control Systems Technology, vol. 21, no. 2, pp. 289-301, 2013. 
[79] V. Spath, A. Jossen, H. Doring, and J. Garche, "The detection of the state of health of lead-acid batteries," in the 19th International Telecommunications Energy Conference, INTELEC '97, 1997, pp. 681-686.

[80] C. S. C. Bose and F. C. Laman, "Battery state of health estimation through coup de fouet," in Telecommunications Energy Conference, 2000. INTELEC. Twenty-second International, 2000, pp. 597-601.

[81] UPG, "Universal UB6120 TOY 6V 12 Ah SLA Battery Spec Sheet," UPG No. D5737, Carrollton, TX.

[82] M. Bhatt, W. G. Hurley, and W. Wolfle, "A new approach to intermittent charging of valve-regulated lead-acid batteries in standby applications," in Power Electronics Specialist Conference, 2003. PESC '03. 2003 IEEE 34th Annual, 2003, vol. 2, pp. 895-900 vol.2.

[83] B. Saha, K. Goebel, S. Poll, and J. Christophersen, "Prognostics methods for battery health monitoring using a bayesian framework," IEEE Transactions on Instrumentation and Measurement, vol. 58, no. 2, pp. 291-296, Feb. 2009.

[84] K. Goebel, B. Saha, A. Saxena, J. Celaya, and J. Christophersen, "Prognostics in battery health management," IEEE Instrumentation and Measurement Magazine, vol. 11, no. 4, pp. 33-40, Aug. 2008.

[85] M. V. Micea, L. Ungurean, G. N. Cârstoiu, and V. Groza, "Online state-of-health assessment for battery management systems," IEEE Instrumentation and Measurement Magazine, vol. 60, no. 6, pp. 1997-2006, Jun. 2011.

[86] M. Landi and G. Gross, "Measurement Techniques for Online Battery State of Health Estimation in Vehicle-to-Grid Applications," IEEE Transactions on Instrumentation and Measurement, vol. 63, no. 5, pp. 1224-1234, May 2014.

[87] A. Christensen and A. Adebusuyi, "Using on-board electrochemical impedance spectroscopy in battery management systems," in the Electric Vehicle Symposium and Exhibition (EVS27), Barcelona, Spain, 17-20 Nov. 2013.

[88] G. K. Lewis and W. Olbricht, "Cost-effective broad-band electrical impedance spectroscopy measurement circuit and signal analysis for piezo-materials and ultrasound transducers," Measurement Science and Technology, vol. 19, no. 10, p. 105102, Oct. 2008.

[89] H. J. B. V Pop, "State-of-the-art of battery state-of-charge determination. Measurement Science and Technology," vol. 16, no. 4, 2005.

[90] E. Barsoukov, J.R. MacDonald, "Measuring Techniques and Data Analysis" in Impedance Spectroscopy Theory, Experiment, and Applications, $2^{\text {nd }}$ Ed. Hoboken, NJ: John Wiley \& Sons, Inc., 2005, ch. 3.

[91] X. Gong, R. Xiong, and C. C. Mi, "Study of the Characteristics of Battery Packs in Electric Vehicles With Parallel-Connected Lithium-Ion Battery Cells," IEEE Transactions on Industry Applications, vol. 51, no. 2, pp. 1872-1879, Mar. 2015. 
[92] "Keysight / Agilent / HP 4294A Precision LCR Meter." [Online]. Available: http://www.bellnw.com/manufacturer/Agilent/4294A.htm. [Accessed: 09-May2017].

[93] "EIS Measurement Low Impedance Lithium Ion Battery." [Online]. Available: https://www.gamry.com/application-notes/EIS/eis-measurement-of-a-very-lowimpedance-lithium-ion-battery/. [Accessed: 09-May-2017].

[94] "VMP3 Multi-channel potentiostat," Bio-Logic Science Instruments.

[95] Analog Devices, "AD8065/AD8066 High Performance, 145 MHz FastFET Op Amps," Norwood, MA. Available Online at: http://www.farnell.com/datasheets/1759460.pdf.

[96] Linear Technology, "LT1116 12ns, Single Supply Ground-Sensing Comparator," Milpitas, CA. Available Online at: http://cds.linear.com/docs/en/datasheet/1116fb.pdf.

[97] "mikromedia for STM32 M4 - ARM Cortex-M4 Development Board." [Online]. Available: https://shop.mikroe.com/smart-displays/mikromedia/mikromedia3/stm32f4. [Accessed: 09-May-2017].

[98] "XR2206CP: EXAR Corporation: Monolithic Function Generator IC - XR-2206 : ICs \& Semiconductors." [Online]. Available: http://www.jameco.com/z/XR2206CP-EXAR-Corporation-Monolithic-FunctionGenerator-IC-XR-2206_34972.html. [Accessed: 09-May-2017].

[99] "74LV4052 - Dual 4-channel analog multiplexer/demultiplexer | Nexperia." [Online]. Available: http://www.nexperia.com/products/logic/switchesmultiplexers-de-multiplexers/digital-switches/series/74LV4052.html. [Accessed: 09-May-2017].

[100] W. M. H. R. Wickramasinghe, K. T. G. M. Duleeka, H. D. Kolamunna, and S. G. Abeyratne, "A power electronics assisted emergency vehicle starter," in Power Electronics and Motion Control Conference and Exposition (PEMC), 2014 16th International, 2014, pp. 283-288.

[101] S. Eaves and S. Mlyniec, "Lithium-ion batteries in telecom Hybrid Power Systems," in the IEEE $201133^{\text {rd }}$ International Telecommunications Energy Conference (INTELEC), 2011, pp. 1-3.

[102] "Tesla Powerwall." [Online]. Available: https://www.tesla.com/powerwall. [Accessed: 17-May-2017].

[103]B. B. McKeon, J. Furukawa, and S. Fenstermacher, "Advanced Lead Acid Batteries and the Development of Grid-Scale Energy Storage Systems," Proceedings of the IEEE, vol. 102, no. 6, pp. 951-963, Jun. 2014.

[104]H. J. Bergvekd, W.S. Kruijt, P.H.L. Notten., Battery Management System: Design by Modeling. Boston, MA: Kluwer Academic Publishers, 2002. ch. 2.

[105]R. Islam, S. Anwar, A. T. Kamal, S. N. Rahman, H. M. Faraby, H. Imtiaz, M. S. Jahan, and F. S. Tulip, "Battery Chemistry Detection Algorithm Implementable with 
Intelligent Systems: A Step towards the Development of a Novel Charger Applicable for Multi-Chemistry Environment," in the 2nd International Symposium on Intelligent Information Technology Application, 2008, vol. 3, pp. 805-810.

[106]M. A. U. S. Navaratne, R. V. Koswatta, and S. G. Abeyratne, "A simple battery chemistry identification and implementation technique for a self-adaptable charger," in the 2009 International Conference on Industrial and Information Systems (ICIIS), 2009, pp. 163-168.

[107]L. Tang, G. Rizzoni, and S. Onori, "Energy Management Strategy for HEVs Including Battery Life Optimization," IEEE Transactions on Transportation Electrification, vol. 1, no. 3, pp. 211-222, Oct. 2015.

[108]M. Gholizadeh and F. R. Salmasi, "Estimation of State of Charge, Unknown Nonlinearities, and State of Health of a Lithium-Ion Battery Based on a Comprehensive Unobservable Model," IEEE Transactions on Industrial Electronics, vol. 61, no. 3, pp. 1335-1344, Mar. 2014.

[109] C. R. Gould, C. M. Bingham, D. A. Stone, and P. Bentley, "New Battery Model and State-of-Health Determination Through Subspace Parameter Estimation and StateObserver Techniques, " IEEE Transactions on Vehicular Technology, vol. 58, no. 8, pp. 3905-3916, Oct. 2009.

[110]M. Coleman, W. G. Hurley, and C. K. Lee, "An Improved Battery Characterization Method Using a Two-Pulse Load Test," IEEE Transactions on Energy Conversion, vol. 23, no. 2, pp. 708-713, Jun. 2008.

[111]N. Doerry, J. Amy, and C. Krolick, "History and the Status of Electric Ship Propulsion, Integrated Power Systems, and Future Trends in the U.S. Navy," Proceedings of the IEEE, vol. 103, no. 12, pp. 2243-2251, Dec. 2015.

[112]D. G. Vutetakis and J. B. Timmons, "A comparison of lithium-ion and lead-acid aircraft batteries," in Power Systems Conference, November 11, 2008 - November 13, 2008.

[113]COMSOL. "Theory for the Lead Acid Battery Interface", in Batteries \& Fuel Cells User's Guide. Burlington, MA: COMSOL Inc., 2013, ch. 3, sec. 7, pp. 164-169.

[114]COMSOL. "Theory for the Lithium-Ion Battery Interface", in Batteries \& Fuel Cells User's Guide. Burlington, MA: COMSOL Inc., 2013, ch. 3, sec. 6, pp. 160-163.

[115]C. D. Rahn, C. Wang, "Governing Equations" in Battery Systems Engineering, $1^{\text {st }}$ ed. West Sussex, UK: John Wiley \& Sons, Ltd., 2013, ch. 3.

[116]M. Cugnet, S. Laruelle, S. Grugeon, B. Sahut, J. Sabatier, J.-M. Tarascon, and A. Oustaloup, "A Mathematical Model for the Simulation of New and Aged Automotive Lead-Acid Batteries," Journal of the Electrochemical Society, vol. 156, no. 12, p. A974, 2009.

[117]F. Baronti, W. Zamboni, N. Femia, H. Rahimi-Eichi, R. Roncella, S. Rosi, R. Saletti, and M. Y. Chow, "Parameter identification of Li-Po batteries in electric vehicles: A 
comparative study," in the 2013 IEEE International Symposium on Industrial Electronics (ISIE), 2013, pp. 1-7.

[118]G. L. Plett, "Extended Kalman filtering for battery management systems of LiPBbased HEV battery packs: Part 1. Background," Journal of Power Sources, vol. 134, no. 2, pp. 252-261, Aug. 2004.

[119]R. Xiong, H. He, F. Sun, and K. Zhao, "Evaluation on State of Charge Estimation of Batteries With Adaptive Extended Kalman Filter by Experiment Approach," IEEE Transactions on Vehicular Technology, vol. 62, no. 1, pp. 108-117, Jan. 2013.

[120]Y. Zhang, W. Song, S. Lin, and Z. Feng, "A novel model of the initial state of charge estimation for LiFePO4 batteries," Journal of Power Sources, vol. 248, pp. 10281033, Feb. 2014

[121]F. Feng, R. Lu, and C. Zhu, "A Combined State of Charge Estimation Method for Lithium-Ion Batteries Used in a Wide Ambient Temperature Range," Energies, vol. 7, no. 5, pp. 3004-3032, May 2014.

[122]X. Yi, "Strategy and temperature affect of the charging of a lithium-ion battery management system," in 2015 International Conference on Applied Science and Engineering Innovation (ASEI), 2015.

[123]K. S. Ng, C.-S. Moo, Y.-P. Chen, and Y.-C. Hsieh, "Enhanced coulomb counting method for estimating state-of-charge and state-of-health of lithium-ion batteries," Applied Energy, vol. 86, no. 9, pp. 1506-1511, Sep. 2009.

[124]Y.-H. Sun, H.-L. Jou, and J.-C. Wu, "Multilevel Peukert equations based residual capacity estimation method for lead-acid battery," in IEEE International Conference on Sustainable Energy Technologies, 2008. ICSET 2008, 2008, pp. 101-105.

[125]T. Kim, Y. Wang, Z. Sahinoglu, T. Wada, S. Hara, and W. Qiao, "A Rayleigh Quotient-Based Recursive Total-Least-Squares Online Maximum Capacity Estimation for Lithium-Ion Batteries," IEEE Transactions on Energy Conversion, vol. 30, no. 3, pp. 842-851, Sep. 2015.

[126]G. L. Plett, "Recursive approximate weighted total least squares estimation of battery cell total capacity," Journal of Power Sources, vol. 196, no. 4, pp. 2319 2331, Feb. 2011.

[127]T. Feng, L. Yang, X. Zhao, H. Zhang, and J. Qiang, "Online identification of lithium-ion battery parameters based on an improved equivalent-circuit model and its implementation on battery state-of-power prediction," Journal of Power Sources, vol. 281, pp. 192-203, May 2015.

[128]"Model 1761, Triple Output DC Power Supplies - B\&K Precision." [Online]. Available: http://www.bkprecision.com/products/power-supplies/1761-4-digittriple-output-dc-power-supply-20-35v-0-3a-12-65v-5a.html. [Accessed: 03-Apr2017].

[129]D. Linden, T. B. Reddy, "Lithium Ion Batteries" in Handbook of Batteries, 3rd ed. New York, NY: McGraw-Hill, 2002, ch, 35. 
[130] "Frequently Asked Questions (FAQs) on Power-Sonic Product Range." [Online]. Available: http://www.power-sonic.com/faq.php?ID=2. [Accessed: 18-May-2017].

[131]"Charging Lithium-Ion Batteries." [Online]. Available: http://batteryuniversity.com/learn/article/charging_lithium_ion_batteries. [Accessed: 02-Jun-2017].

[132] R. Gu, P. Malysz, H. Yang, and A. Emadi, "On the Suitability of ElectrochemicalBased Modeling for Lithium-ion Batteries," IEEE Transactions on Transportation Electrification, vol. PP, no. 99, pp. 1-1, 2016.

[133] Juchuan Li, A. K. Dozier, Yunchao Li, Fuqian Yang, and Yang-Tse Cheng, "Crack Pattern Formation in Thin Film Lithium-Ion Battery Electrodes," Journal of the Electrochemical Society, vol. 158, no. 6, pp. 689-94, Jun. 2011.

[134]"Galaxy S7 by Samsung and Adaptive Fast Charging Charger by Samsung Compatibility." [Online]. Available: https://gtrusted.com/how-the-samsunggalaxy-s7-edge-charges-quickly-with-the-15-watts-samsung-adaptive-fastcharging-usb-power-adapter. [Accessed: 02-Jun-2017].

[135]J. Vetter et al., "Ageing mechanisms in lithium-ion batteries," Journal of Power Sources, vol. 147, no. 1-2, pp. 269-281, Sep. 2005.

[136]R. Ahmed, M. E. Sayed, I. Arasaratnam, J. Tjong, and S. Habibi, "Reduced-Order Electrochemical Model Parameters Identification and SOC Estimation for Healthy and Aged Li-Ion Batteries Part I: Parameterization Model Development for Healthy Batteries," IEEE Journal of Emerging Selected Topics in Power Electronics., vol. 2, no. 3, pp. 659-677, Sep. 2014.

[137] G. Perez, I. Gandiaga, M. Garmendia, J. F. Reynaud, and U. Viscarret, "Modelling of Li-ion batteries dynamics using impedance spectroscopy and pulse fitting: EVs application," in Electric Vehicle Symposium and Exhibition (EVS27), 2013 World, 2013, pp. 1-9.

[138]Battery Space, Richmond, CA, USA. Polymer Lithium-ion Battery Product Specification Model: 8048168C, accessed on Feb. 15, 2017. [Online]. Available: http://www.batteryspace.com/prod-specs/4029.pdf.

[139]B. Bilgin et al., "Making the Case for Electrified Transportation," IEEE Transactions on Transportation Electrification, vol. 1, no. 1, pp. 4-17, Jun. 2015.

[140] "Electric Vehicles Market Size, Share, Growth And Forecast To 2022." [Online]. Available: http://www.credenceresearch.com/report/electric-vehicles-market. [Accessed: 29-Jul-2016].

[141]R. A. Dougal, L. Gao, S. Liu, "Ultracapacitor model with automatic order selection and capacity scaling for dynamic system simulation", Journal of Power Sources, 126 (2004) 250-257.

[142]D. Linden, T. B. Reddy, "Electrochemical Principles and Reactions" in Handbook of Batteries, $3^{\text {rd }}$ ed. New York, NY: McGraw-Hill, 2002, ch. 2. 
[143] S. Abu-Sharkh and D. Doerffel, "Rapid test and non-linear model characterisation of solid-state lithium-ion batteries," Journal of Power Sources, vol. 130, no. 1-2, pp. 266-274, May 2004.

[144] Y. Cao, R. C. Kroeze, and P. T. Krein, "Multi-Timescale Parametric Electrical Battery Model for Use in Dynamic Electric Vehicle Simulations," IEEE Transactions on Transportation Electrification, vol. PP, no. 99, pp. 1-1, 2016.

[145]Battery Space, "Lithium Ion Polymer Rechargeable Battery,” Model PL8048168, Richmond, CA. Available Online: http://www.batteryspace.com/.

[146] "Smart Charger for 48V SLA, 51.8V Li-Ion/LiMnNi, 51.2VLifePO4 Rechargeable Battery Pack," BatterySpace.com/AA Portable Power Corp. [Online]. Available: http://www.batteryspace.com/.

[147] “Electric Vehicles Market Size, Share, Growth And Forecast To 2022." [Online]. Available: http://www.credenceresearch.com/report/electric-vehicles-market. [Accessed: 29-Jul-2016].

[148]F. Musavi and W. Eberle, "Overview of wireless power transfer technologies for electric vehicle battery charging," IET Power Electronics, vol. 7, no. 1, pp. 60-66, Jan. 2014.

[149]“J2954A (WIP) Wireless Power Transfer for Light-Duty Plug-In/ Electric Vehicles and Alignment Methodology - SAE International." [Online]. Available: http://standards.sae.org/wip/j2954/. [Accessed: 10-Jul-2016].

[150]G. T. Bahouth, D. Murakhovskiy, K. H. Digges, H. Rist, and R. Wiik, "Opportunities for Reducing Far-Side Casualties," presented at the 24th International Technical Conference on the Enhanced Safety of Vehicles (ESV), 2015.

[151]O. US EPA, "Dynamometer Drive Schedules." [Online]. Available: https://www.epa.gov/vehicle-and-fuel-emissions-testing/dynamometer-driveschedules. [Accessed: 30-Jul-2016].

[152]M.-E. Choi, J.-S. Lee, and S.-W. Seo, "Real-Time Optimization for Power Management Systems of a Battery/Supercapacitor Hybrid Energy Storage System in Electric Vehicles," IEEE Transactions on Vehicular Technology, vol. 63, no. 8, pp. 3600-3611, Oct. 2014.

[153]A. A. S. Mohamed, F. G. N. de Almeida, and O. Mohammed, "Harmonics-based steady-state mathematical model of bidirectional inductive wireless power transfer system in V2G applications," in 2016 IEEE Transportation Electrification Conference and Expo (ITEC), 2016, pp. 1-6.

[154]A. A. S. Mohamed, A. Berzoy, and O. Mohammed, "Experimental Validation of Comprehensive Steady-state Analytical Model of Bidirectional WPT System in EVs Applications," IEEE Transactions on Vehicular Technology. 
[155]M. E. Choi, S. W. Kim, and S. W. Seo, "Energy Management Optimization in a Battery/Supercapacitor Hybrid Energy Storage System," IEEE Transactions on Smart Grid, vol. 3, no. 1, pp. 463-472, Mar. 2012.

[156]B. P. Roberts and C. Sandberg, "The Role of Energy Storage in Development of Smart Grids," Proceedings of the IEEE, vol. 99, no. 6, pp. 1139-1144, Jun. 2011.

[157] "High Voltage DC Power Supply - 2U XR Series: 6 kW, 8 kW, 10 kW | MagnaPower." [Online]. Available: http://www.magna-power.com/products/programmabledc-power-supplies/xr-series. [Accessed: 25-Jul-2017.

[158] “AeroWeb | DDG 1000 Zumwalt Class Destroyer,AeroWeb. [Online]. Available: http://www.bga-aeroweb.com/Defense/DDG-1000-Zumwalt-Destroyer.html. [Accessed: 04-Apr-2016].

[159] “The US Navys Dual Band Radars," Defense Industry Daily. [Online]. Available: http://www.defenseindustrydaily.com/the-us-navy-dual-band-radars-05393/. [Accessed: 25-Jul-2017].

[160] "US Navy tests its crazy electromagnetic railgun," CNET. [Online]. Available: https://www.cnet.com/news/us-navy-tests-its-crazy-electromagnetic-railgun/. [Accessed: 25-Jul-2017].

[161] L. Gao, R. A. Dougal, S. Liu, Power enhancement of an actively controlled battery/ultracapacitor hybrid, IEEE Transactions on Power Electronics, 20 (2005) 236-243.

[162] N. Mohan, "Design of Switching Power-Poles" in First Course on Power Electronics and Drives, 2003 ed. Minneapolis, MN: MNPERE, 2003, ch. 2, p. 2-11.

[163] D. W. Hart, "DC-DC Converters" in Power Electronics, New York, NY: McGrawHill, 2011, ch. 6, p. 207.

[164] Batteryspace.com, Lithium Ion Polymer Rechargeable Battery, Model HA055275, Richmond, CA. Available Online at: http://www.batteryspace.com/Prodspecs/A055275-21Ah.pdf.

[165] GaNSystems, "GS66508T Top-side cooled 650V E-mode GaN Transistor Preliminary Datasheet," Ottawa, ON. Available Online at: http://www.gansystems.com/.

[166] Infineon, "600V CoolMOS P6 Power Transistor IPx60R125P6," El Segundo, CA. Available Online at: https://www.infineon.com/.

[167] "Wiley: GaN Transistors for Efficient Power Conversion, 2nd Edition - Alex Lidow, Johan Strydom, Michael de Rooij, et al." [Online]. Available: http://www.wiley.com/ [Accessed: 19-Oct-2016].

[168] T. Ytterdal, Y. Cheng, and T.A. Tjeldly, "MOSFET Device Physics and Operation" in Device Modeling for Analog and RF CMOS Circuit Design, West Sussex, England: John Wiley \& Sons Ltd., 2003, ch. 1. 
[169] K. Shenai, "Switching Megawatts with Power Transistors," The Electrochemical. Society Interface, vol. 22, no. 1, pp. 47-53, Jan. 2013.

[170] D. Visalli, M. Van Hove, J. Derluyn, S. Degroote, M. Leys, K. Cheng, M. Germain, and G. Borghs, "AlGaN/GaN/AlGaN Double Heterostructures on Silicon Substrates for High Breakdown Voltage Field-Effect Transistors with low On-Resistance," Japanese Journal of Applied Physics, vol. 48, no. 4S, p. 04C101, Apr. 2009.

[171] F. Li, Q. H. Liu, and D. P. Klemer, "Numerical Simulation of high electron mobility transistors based on the spectral element Method," Journal of Applied Computational Electromagnetics, vol. 31, no. 10, pp. 1144-1150, 2016.

[172] G. Meneghesso, M. Meneghini, and E. Zanoni, "Breakdown mechanisms in AlGaN/GaN HEMTs: An overview," Japanese Journal of Applied Physics., vol. 53, no. 10, p. 100211, Sep. 2014.

[173] S. Vitanov et al., "Physics-Based Modeling of GaN HEMTs," IEEE Transactions on Electron Devices, vol. 59, no. 3, pp. 685-693, Mar. 2012.

[174] M. Kaddeche, A. Telia, and A. Soltani, "Study of field plate effects onAlGaN/GaN HEMTs," in 2009 International Conference on Microelectronics - ICM, 2009, pp. $362-365$.

[175] D. Visalli et al., "Experimental and simulation study of breakdown voltage enhancement of AlGaN/GaN heterostructures by Si substrate removal," Applied Physics Letters, vol. 97, no. 11, p. 113501, Sep. 2010.

[176] S. Karmalkar and U. K. Mishra, "Enhancement of breakdown voltage in AlGaN/GaN high electron mobility transistors using a field plate," IEEE Trans. Electron Devices, vol. 48, no. 8, pp. 1515-1521, Aug. 2001.

[177] B. Jogai, J. D. Albrecht, and E. Pan, "Effect of electromechanical coupling on the strain in AlGaN/GaN heterojunction field effect transistors," J. Appl. Phys., vol. 94, no. 6, pp. 3984-3989, Sep. 2003.

[178] S. K. Chung, "Analytic model for field-plate-edge breakdown of planar devices terminated with field plate and semiresistive layer," IEE Proc. - Sci. Meas. Technol., vol. 151, no. 1, pp. 21-24, Jan. 2004.

[179] K. K. Young, "Short-channel effect in fully depleted SOI MOSFETs," IEEE Trans. Electron Devices, vol. 36, no. 2, pp. 399-402, Feb. 1989.

[180] T. Jaster, A. Rowe, Z. Dong, Modeling and simulation of a hybrid electric propulsion system of a green ship, 2014 IEEE/ASME 10th International Conference on Mechatronic and Embedded Systems and Applications (MESA), 2014, pp. 1-6.

[181] M. Steurer, M. Andrus, J. Langston, L. Qi, S. Suryanarayanan, S. Woodruff, P. F. Ribeiro, Investigating the Impact of Pulsed Power Charging Demands on Shipboard Power Quality, IEEE Electric Ship Technologies Symposium, 2007. ESTS '07, pp. 315-321. 
[182] W. A. Walls, W. F. Weldon, S. B. Pratap, M. Palmer, D. Adams, Application of electromagnetic guns to future naval platforms, IEEE Transactions on Magnetics, vol. 35, no. 1, pp. 262-267, Jan. 1999.

[183] A. Neffati, M. Guemri, S. Caux, and M. Fadel, "Energy management strategies for multi source systems," Electric Power Systems Research, vol. 102, pp. 42-49, Sep. 2013.

[184] A. Esmaili, B. Novakovic, A. Nasiri, O. Abdel-baqi, A Hybrid System of Li-Ion Capacitors and Flow Battery for Dynamic Wind Energy Support, IEEE Transactions on Industry Applications, vol. 49, no. 4, pp. 1649-1657, Jul. 2013.

[185] A. Mohamed, V. Salehi, O. Mohammed, Real-Time Energy Management Algorithm for Mitigation of Pulse Loads in Hybrid Microgrids, IEEE Transactions on Smart Grid, vol. 3, no. 4, pp. 1911-1922, Dec. 2012.

[186] M. Guerrero, J. C. Vasquez, J. Matas, M. Castilla, L. G. de Vicuna, Control strategy for flexible microgrid based on parallel line-interactive UPS systems, IEEE Transactions on Industrial Electronics, 56 (2009) 726-736.

[187] M. Falahi, K. L. Butler-Purry, M. Ehsani, Reactive Power Coordination of Shipboard Power Systems in Presence of Pulsed Loads, IEEE Transactions on Power Systems, 28 (2013) 3675-3682.

[188] E. A. Lewis, M. Butcher, Managing multiple and varying energy demands in naval combatants with integrated electric propulsion, in the 2005 Pulsed Power Symposium, 2005, pp. 32/1-32/6.

[189] L. N. Domaschk, A. Ouroua, R. E. Hebner, O. E. Bowlin, W. B. Colson, Coordination of Large Pulsed Loads on Future Electric Ships, IEEE Transactions on Magnetics, 43 (2007) 450-455.

[190] T. Dragicevic, X. Lu, J.C. Vasquez, J.M. Guerrero, DC Microgrids-Part II: A Review of Power Architectures, Applications and Standardization Issues, IEEE Transactions on Power Electronics, 31 (2016) 3528-3549.

[191] Y. Tang, A. Khaligh, Bidirectional hybrid Battery/Ultracapacitor Energy Storage Systems for next generation MVDC shipboard power systems, 2011 IEEE Vehicle Power and Propulsion Conference (VPPC), 2011.

[192] J. Shen, A. Khaligh, A Supervisory Energy Management Control Strategy in a Battery/Ultracapacitor Hybrid Energy Storage System, IEEE Transactions on Transportation Electrification, 1 (2015) 223-231.

[193] PowerSonic, Rechargeable Sealed Lead Acid Battery, Model: PDC-12200 (12V$21 \mathrm{AH})$, San Diego, CA. Available Online at: http://www.powersonic.com/images/powersonic/sla_batteries/PDC-12200.pdf

[194] Batteryspace.com, Lithium Ion Polymer Rechargeable Battery, Model HA055275, Richmond, CA. Available Online at: http://www.batteryspace.com/Prodspecs/A055275-21Ah.pdf 
[195] Maxwell, 16V small cell module, Model: BMOD0058 E016 B02 (16.2V-58 F), San Diego, CA. Available Online at: http://www.maxwell.com/images/documents/datasheet_16v_small_cell_module.pdf

[196] A. T. Elsayed, C. R. Lashway, O. A. Mohammed, "Advanced Battery Management and Diagnostic System for Smart Grid Infrastructure," IEEE Transactions on Smart Grid, 7 (2016) 897-905.

[197] Y. Levron and D. Shmilovitz, "Power systems' optimal peak-shaving applying secondary storage," Electric Power Systems Research, vol. 89, pp. 80-84, Aug. 2012.

[198]K. T. Chau, C. C. Chan, and C. Liu, "Overview of Permanent-Magnet Brushless Drives for Electric and Hybrid Electric Vehicles," IEEE Transactions on Industry Electronics, vol. 55, no. 6, pp. 2246-2257, Jun. 2008.

[199]K. Young, C. Wang, L. Y. Wang, and K. Strunz, "Electric Vehicle Battery Technologies," in Electric Vehicle Integration into Modern Power Networks, R. Garcia-Valle and J. A. P. Lopes, Eds. Springer New York, 2013, pp. 15-56.

[200] “Types of Electric Vehicles.” [Online]. Available: http://electricvehicles.caa.ca/types-of-electric-vehicles/. [Accessed: 19-Oct-2015].

[201]D. Benzai and W. Zhiqiang, "Research on Electric-Vehicle Charging Station Technologies Based on Smart Grid," in Power and Energy Engineering Conference (APPEEC), 2011 Asia-Pacific, 2011, pp. 1-4.

[202] T. Dragicevic, S. Sucic, J.C. Vasquez, and J.M. Guerrero, "Flywheel-Based Distributed Bus Signalling Strategy for the Public Fast Charging Station," IEEE Trans. on Smart Grid, vol.5, no.6, pp.2825-2835, Nov. 2014.

[203] "The NRG eVgo Network | Electric Vehicle Network Providing Range Confidence," EVgo.

[204]"Supercharger | Tesla Motors." [Online]. Available: http://www.teslamotors.com/supercharger. [Accessed: 19-Oct-2015].

[205] "Global EV Charging Stations to Skyrocket by 2020, IHS Report Says | IHS Online Newsroom." [Online]. Available: http://press.ihs.com/pressrelease/automotive/global-ev-charging-stations-skyrocket-2020-ihs-report-says. [Accessed: 19-Oct-2015].

[206]M. Yilmaz and P. T. Krein, "Review of the Impact of Vehicle-to-Grid Technologies on Distribution Systems and Utility Interfaces," IEEE Transactions on Power Electronics, vol. 28, no. 12, pp. 5673-5689, Dec. 2013.

[207]T. P. Kohler, D. Buecherl, and H. Herzog, "Investigation of control strategies for hybrid energy storage systems in hybrid electric vehicles," in the 2009 IEEE Vehicle Power and Propulsion Conference. VPPC '09, 2009, pp. 1687-1693.

[208]A. Burke, M. Miller, H. Zhao, M. Radenbaugh, and Z. Liu, "Ultracapacitors in micro-and mild hybrids with lead-acid batteries: Simulations and laboratory and invehicle testing," in Electric Vehicle Symposium and Exhibition (EVS27), 2013 World, 2013, pp. 1-14. 
[209] U.S. Department of Energy Vehicle Technologies Program, "Battery Test Manual For Plug-In Hybrid Electric Vehicles, REVISION 0".

[210]"How to Prolong Lithium-based Batteries." [Online]. Available: http://batteryuniversity.com/learn/article/how_to_prolong_lithium_based_batteries . [Accessed: 02-Nov-2015].

[211]P. Ramadass, B. Haran, P. M. Gomadam, R. White, and B. N. Popov, "Development of First Principles Capacity Fade Model for Li-Ion Cells," J. Electrochem. Soc., vol. 151, no. 2, pp. A196-A203, Feb. 2004.

[212]G. Ning, R. E. White, and B. N. Popov, "A generalized cycle life model of rechargeable Li-ion batteries," Electrochimica Acta, vol. 51, no. 10, pp. 2012-2022, Feb. 2006.

[213] "Re-writing the F1 rulebook - Part 4: 'cleaner' cars, KERS and the return of slicks," Formula $1 \AA$ - The Official F1® Website. [Online]. Available: /en/latest/technical/2017/2/re-writing-the-f1-rulebook---part-4--cleaner-cars--kersand-the-.html. [Accessed: 28-Aug-2017].

[214] A. V. Filatov and E. H. Maslen, "Passive magnetic bearing for flywheel energy storage systems," IEEE Transactions on Magnetics, vol. 37, no. 6, pp. 3913-3924, Nov. 2001.

[215] F. N. Werfel, U. Floegel-Delor, T. Riedel, R. Rothfeld, D. Wippich, B. Goebel, G. Reiner, and N. Wehlau, "A Compact HTS 5 kWh/250 kW Flywheel Energy Storage System," IEEE Transactions on Applied Superconductivity, vol. 17, no. 2, pp. 21382141, Jun. 2007.

[216] S. Ye and B. Sun, "Application of Flywheel Battery in Solar Power System," in the 2009 International Conference on Energy and Environment Technology. ICEET '09, 2009, vol. 1, pp. 533-536.

[217] M. Jiang, J. Salmon, and A. M. Knight, "Design of a permanent magnet synchronous machine for a flywheel energy storage system within a hybrid electric vehicle," in the 2009 International Electric Machines and Drives Conference, IEMDC '09. 2009, pp. 1736-1742.

[218] Emerson, "Leibert FS Flywheel Energy Storage System Guide Specifications," SL29310_REV05_03-10, Columbus, OH.

[219] R. Haiduck, Y. Baghzouz, and R. F. Boehm, "Slot-harmonic effects on DC voltage of generator-rectifier set," in the 9th International Conference on Harmonics and Quality of Power, 2000., vol. 2, pp. 565-569 vol.2.

[220] M. Chen and G. A. Rincon-Mora, "Accurate electrical battery model capable of predicting runtime and I-V performance," IEEE Transactions on Energy Conversion, vol. 21, no. 2, pp. 504-511, Jun. 2006.

[221] Power Analyzer Fluke 435 Series II with Advanced Functions." [Online]. Available: http://en-us.fluke.com/products/power-quality-analyzers/fluke-435-iipower-quality.html. [Accessed: 26-Aug-2017]. 
[222] S. Sumathi, "Inputs and Outputs," in LabVIEW based Advanced Instrumentation Systems, London, England: Springer Berlin Heidelberg, 2007, ch. 4, sec. 4.3.3, pp. 180.

[223] M. R. Patel, "Clean Power Bus for Harmonic-Sensitive Loads" in Shipboard Electrical Power Systems, Boca Raton, FL: CRC Press, 2012, ch. 2. 


\section{VITA}

\section{CHRISTOPHER R. LASHWAY}

Born, St. Petersburg, FL

2003-2008

2008-2010

2013-2017

2010-2017

2017
B.Sc. University of Central Florida, Orlando, FL

M.Eng. Penn State Harrisburg, Middletown, PA

Graduate Assistant, Florida International University,

Miami, FL

Electrical Engineer, Naval Surface Warfare Center

Dahlgren Division, Dahlgren, VA

Dissertation Year Fellowship (DYF), Florida

International University, Miami, FL

\section{SELECTED PUBLICATIONS AND PRESENTATIONS \\ Format: Journal $[\mathrm{J}-n]$, Conference [C- $n]$, Patent [P- $n]$}

[J-1] C. R. Lashway and O. A. Mohammed, "Study of Battery Voltage Behavior Under Loading and Charging Conditions Using 3DFEM," IEEE Transactions on Magnetics, vol. 53, no. 6, pp. 1-5, Jun. 2017. DOI: 10.1109/TMAG.2017.2650908.

[J-2] C. R. Lashway, A. T. Elsayed, and O. A. Mohammed, "Hybrid energy storage management in ship power systems with multiple pulsed loads," Elsevier Electric Power Systems Research, vol. 141, pp. 50-62, 2016. DOI: 10.1016/j.epsr.2016.06.031.

[J-3] C. R. Lashway and O. A. Mohammed, "Adaptive Battery Management and Parameter Estimation Through Physics-Based Modeling and Experimental Verification," IEEE Transactions on Transportation Electrification, vol. 2, no. 4, pp. 454-464, Dec. 2016. DOI: 10.1109/TTE.2016.2558843

[J-4] A. A. S. Mohamed, C. R. Lashway, and O. Mohammed, "Modeling and Feasibility Analysis of Quasi-dynamic WPT System for EV Applications," IEEE Transactions on Transportation Electrification, vol. 3, no. 2, pp. 343-353, 2017. DOI: 10.1109/TTE.2017.2682111.

[J-5] A.T. Elsayed, C.R. Lashway, and O.A. Mohammed, "Advanced Battery Management and Diagnostic System for Smart Grid Infrastructure," IEEE Transactions on Smart Grid. 15 Mar 2015. DOI: 10.1109/TSG.2015.2418677. 
[C-1] A. Berzoy, C.R. Lashway, H. Moradisizkoohi, and O.A. Mohammed, "Breakdown Voltage Improvement and Analysis of GaN HEMTs through Field Plate Inclusion and Substrate Removal," accepted to the 5th Workshop on Wide Bandgap Power Devices and Applications, Albuquerque, NM, 30 Oct - 1 Nov 2017.

[C-2] C.R. Lashway and O.A. Mohammed, "Development and Implementation of a Comprehensive Battery Model for Electric Vehicle Simulations," accepted to the 2017 IEEE Vehicle Power and Propulsion Conference, Belfort, France, 11-14 Dec 2017.

[C-3] H.F. Habib, C.R. Lashway, and O.A. Mohammed, "On the Adaptive Protection of Microgrids: A Review on How to Mitigate Cyber Attacks and Communication Failures," presented at the 2017 IEEE Industry Applications Society Annual Meeting, Cincinnati, OH, 3 Oct 2017.

[C-4] C.R. Lashway, A. Berzoy, N. Elsayad, and O.A. Mohammed, "Breakdown Voltage Assessment of GaN HEMT Devices through Physics-Based Modeling," presented at the 2017 International Applied Computational Electromagnetics Society Symposium, Firenze, Italy, 26-30 Mar 2017.

[C-5] C.R. Lashway and O.A. Mohammed, "A coupled 3DFE/electrochemical model for the analysis of voltage behavior in batteries under loading and charging conditions," presented at the 17th Biennial Conference on Electromagnetic Field Computation, Miami, FL, 15 Nov 2016.

[C-6] C.R. Lashway, A. Elsayed, and O.A. Mohammed, "Management Scheme for Parallel Connected Hybrid Energy Storage in Electric Vehicles," presented at the 2016 IEEE Power \& Energy Society General Meeting, Boston, MA, 18 Jul 2016.

[C-7] C.R. Lashway, G. Constant, J. Theogene, and O.A. Mohammed, "A Real-time Circuit Topology for Battery Impedance Monitoring," presented at SoutheastCon 2016, Norfolk, VA, 01 Apr 2016.

[C-8] C.R. Lashway, A. Elsayed, and O.A. Mohammed, "DC Voltage Ripple Quantification for a Flywheel-Battery based Hybrid Energy Storage System," presented at the 2016 IEEE Applied Power Electronics Conference and Exposition, Long Beach, CA, 23 Mar 2016.

[C-9] C.R. Lashway and O.A. Mohammed, "Improved Estimation Methods for Lead Acid Battery Utility Arrays for Microgrids," presented at the IEEE PES Conference on Innovative Smart Grid Technologies, Washington, DC, 19 Feb. 2015.

[C-10] T. Ma, C.R. Lashway, Y. Song, and O.A. Mohammed, "Optimal Renewable Energy Farm and Energy Storage Sizing Method for Future Hybrid Power System," presented at the 17th International Conference on Electrical Machines and Systems, Hangzhou, China, 23 Oct 2014.

[P-1] A.T. Elsayad, C.R. Lashway, T. Youssef, and O.A. Mohammed, "Systems and Techniques for Energy Storage Regulation," Filed by FIU with the United States Patent and Trademark Office (USPTO), US Patent Number: 14/848,711, Awarded 15 Aug. 2017. 